\title{
Cochrane
}

Library

Cochrane Database of Systematic Reviews

\section{Reduction in saturated fat intake for cardiovascular disease} (Review)

Hooper L, Martin N, Jimoh OF, Kirk C, Foster E, Abdelhamid AS

Hooper L, Martin N, Jimoh OF, Kirk C, Foster E, Abdelhamid AS.

Reduction in saturated fat intake for cardiovascular disease.

Cochrane Database of Systematic Reviews 2020, Issue 5. Art. No.: CD011737.

DOI: 10.1002/14651858.CD011737.pub2.

www.cochranelibrary.com

Reduction in saturated fat intake for cardiovascular disease (Review) 
TABLE OF CONTENTS

HEADER

ABSTRACT

PLAIN LANGUAGE SUMMARY

SUMMARY OF FINDINGS

BACKGROUND

OBJECTIVES

METHODS

RESULTS

Figure 1.

Figure 2.

Figure 3.

Figure 4.

Figure 5.

Figure 6.

DISCUSSION

AUTHORS' CONCLUSIONS

ACKNOWLEDGEMENTS

REFERENCES

CHARACTERISTICS OF STUDIES

DATA AND ANALYSES

Analysis 1.1. Comparison 1: SFA reduction vs usual diet - primary outcomes, Outcome 1: ALL-CAUSE MORTALITY

Analysis 1.2. Comparison 1: SFA reduction vs usual diet - primary outcomes, Outcome 2: All-cause mortality, SA low summary risk of bias

Analysis 1.3. Comparison 1: SFA reduction vs usual diet - primary outcomes, Outcome 3: All-cause mortality, SA aim to reduce SFA

Analysis 1.4. Comparison 1: SFA reduction vs usual diet - primary outcomes, Outcome 4: All-cause mortality, SA statistically significant SFA reduction

Analysis 1.5. Comparison 1: SFA reduction vs usual diet - primary outcomes, Outcome 5: All-cause mortality, SA TC reduction ... Analysis 1.6. Comparison 1: SFA reduction vs usual diet - primary outcomes, Outcome 6: All-cause mortality, SA excluding WHI Analysis 1.7. Comparison 1: SFA reduction vs usual diet - primary outcomes, Outcome 7: All-cause mortality, SA Mantel-Haenszel fixed-effect

Analysis 1.8. Comparison 1: SFA reduction vs usual diet - primary outcomes, Outcome 8: All-cause mortality, SA Peto fixedeffect

Analysis 1.9. Comparison 1: SFA reduction vs usual diet - primary outcomes, Outcome 9: All-cause mortality, subgroup by any substitution

Analysis 1.10. Comparison 1: SFA reduction vs usual diet - primary outcomes, Outcome 10: All-cause mortality, subgroup by main substitution

Analysis 1.11. Comparison 1: SFA reduction vs usual diet - primary outcomes, Outcome 11: All-cause mortality, subgroup by duration

Analysis 1.12. Comparison 1: SFA reduction vs usual diet - primary outcomes, Outcome 12: All-cause mortality, subgroup by baseline SFA

Analysis 1.13. Comparison 1: SFA reduction vs usual diet - primary outcomes, Outcome 13: All-cause mortality, subgroup by SFA change

Analysis 1.14. Comparison 1: SFA reduction vs usual diet - primary outcomes, Outcome 14: All-cause mortality, subgroup by sex

Analysis 1.15. Comparison 1: SFA reduction vs usual diet - primary outcomes, Outcome 15: All-cause mortality, subgroup by CVD risk

Analysis 1.16. Comparison 1: SFA reduction vs usual diet - primary outcomes, Outcome 16: All-cause mortality, subgroup by TC reduction

Analysis 1.17. Comparison 1: SFA reduction vs usual diet - primary outcomes, Outcome 17: All-cause mortality, subgroup decade of publication

Analysis 1.18. Comparison 1: SFA reduction vs usual diet - primary outcomes, Outcome 18: CARDIOVASCULAR MORTALITY .... 
Analysis 1.19. Comparison 1: SFA reduction vs usual diet - primary outcomes, Outcome 19: CVD mortality, SA low summary risk of bias

Analysis 1.20. Comparison 1: SFA reduction vs usual diet - primary outcomes, Outcome 20: CVD mortality, SA aim to reduce SFA

Analysis 1.21. Comparison 1: SFA reduction vs usual diet - primary outcomes, Outcome 21: CVD mortality, SA statistically significant SFA reduction

Analysis 1.22. Comparison 1: SFA reduction vs usual diet - primary outcomes, Outcome 22: CVD mortality, SA TC reduction .... Analysis 1.23. Comparison 1: SFA reduction vs usual diet - primary outcomes, Outcome 23: CVD mortality, SA excluding WHI .... Analysis 1.24. Comparison 1: SFA reduction vs usual diet - primary outcomes, Outcome 24: CVD mortality, SA Mantel-Haenszel fixed-effect

Analysis 1.25. Comparison 1: SFA reduction vs usual diet - primary outcomes, Outcome 25: CVD mortality, SA Peto fixed-effect . Analysis 1.26. Comparison 1: SFA reduction vs usual diet - primary outcomes, Outcome 26: CVD mortality, subgroup by any substitution

Analysis 1.27. Comparison 1: SFA reduction vs usual diet - primary outcomes, Outcome 27: CVD mortality, subgroup by main substitution

Analysis 1.28. Comparison 1: SFA reduction vs usual diet - primary outcomes, Outcome 28: CVD mortality, subgroup by duration

Analysis 1.29. Comparison 1: SFA reduction vs usual diet - primary outcomes, Outcome 29: CVD mortality, subgroup by baseline SFA

Analysis 1.30. Comparison 1: SFA reduction vs usual diet - primary outcomes, Outcome 30: CVD mortality, subgroup by SFA change

Analysis 1.31. Comparison 1: SFA reduction vs usual diet - primary outcomes, Outcome 31: CVD mortality, subgroup by sex .... Analysis 1.32. Comparison 1: SFA reduction vs usual diet - primary outcomes, Outcome 32: CVD mortality, subgroup by CVD risk

Analysis 1.33. Comparison 1: SFA reduction vs usual diet - primary outcomes, Outcome 33: CVD mortality, subgroup by TC reduction

Analysis 1.34. Comparison 1: SFA reduction vs usual diet - primary outcomes, Outcome 34: CVD mortality, subgroup decade of publication

Analysis 1.35. Comparison 1: SFA reduction vs usual diet - primary outcomes, Outcome 35: COMBINED CARDIOVASCULAR EVENTS

Analysis 1.36. Comparison 1: SFA reduction vs usual diet - primary outcomes, Outcome 36: CVD events, SA low summary risk of bias

Analysis 1.37. Comparison 1: SFA reduction vs usual diet - primary outcomes, Outcome 37: CVD events, SA aim to reduce SFA ..

Analysis 1.38. Comparison 1: SFA reduction vs usual diet - primary outcomes, Outcome 38: CVD events, SA statistically significant SFA reduction

Analysis 1.39. Comparison 1: SFA reduction vs usual diet - primary outcomes, Outcome 39: CVD events, SA TC reduction ....... Analysis 1.40. Comparison 1: SFA reduction vs usual diet - primary outcomes, Outcome 40: CVD events, SA excluding WHI ..... Analysis 1.41. Comparison 1: SFA reduction vs usual diet - primary outcomes, Outcome 41: CVD events, SA Mantel-Haenszel fixed-effect

Analysis 1.42. Comparison 1: SFA reduction vs usual diet - primary outcomes, Outcome 42: CVD events, SA Peto fixed-effect ... Analysis 1.43. Comparison 1: SFA reduction vs usual diet - primary outcomes, Outcome 43: CVD events, SA excluding trials with additional interventions

Analysis 1.44. Comparison 1: SFA reduction vs usual diet - primary outcomes, Outcome 44: CVD events, subgroup by any substitution

Analysis 1.45. Comparison 1: SFA reduction vs usual diet - primary outcomes, Outcome 45: CVD events, subgroup by main substitution

Analysis 1.46. Comparison 1: SFA reduction vs usual diet - primary outcomes, Outcome 46: CVD events, subgroup by duration . Analysis 1.47. Comparison 1: SFA reduction vs usual diet - primary outcomes, Outcome 47: CVD events, subgroup by baseline SFA

Analysis 1.48. Comparison 1: SFA reduction vs usual diet - primary outcomes, Outcome 48: CVD events, subgroup by SFA change

Analysis 1.49. Comparison 1: SFA reduction vs usual diet - primary outcomes, Outcome 49: CVD events, subgroup by sex ........ Analysis 1.50. Comparison 1: SFA reduction vs usual diet - primary outcomes, Outcome 50: CVD events, subgroup by CVD risk .. Analysis 1.51. Comparison 1: SFA reduction vs usual diet - primary outcomes, Outcome 51: CVD events, subgroup by TC reduction 
Analysis 1.52. Comparison 1: SFA reduction vs usual diet - primary outcomes, Outcome 52: CVD events, subgroup decade of publication

Analysis 2.1. Comparison 2: SFA reduction vs usual diet - secondary health events, Outcome 1: MYOCARDIAL INFARCTION ...... Analysis 2.2. Comparison 2: SFA reduction vs usual diet - secondary health events, Outcome 2: MI, SA by low summary risk of bias

Analysis 2.3. Comparison 2: SFA reduction vs usual diet - secondary health events, Outcome 3: MI, SA aim to reduce SFA ........ Analysis 2.4. Comparison 2: SFA reduction vs usual diet - secondary health events, Outcome 4: MI, SA statistically significant SFA reduction

Analysis 2.5. Comparison 2: SFA reduction vs usual diet - secondary health events, Outcome 5: MI, SA by TC reduction ............ Analysis 2.6. Comparison 2: SFA reduction vs usual diet - secondary health events, Outcome 6: MI, SA excluding WHI .............. Analysis 2.7. Comparison 2: SFA reduction vs usual diet - secondary health events, Outcome 7: MI, SA Mantel-Haenszel fixedeffect

Analysis 2.8. Comparison 2: SFA reduction vs usual diet - secondary health events, Outcome 8: MI, SA Peto fixed-effect ........... Analysis 2.9. Comparison 2: SFA reduction vs usual diet - secondary health events, Outcome 9: MI, subgroup by any substitution

Analysis 2.10. Comparison 2: SFA reduction vs usual diet - secondary health events, Outcome 10: MI, subgroup by main substitution

Analysis 2.11. Comparison 2: SFA reduction vs usual diet - secondary health events, Outcome 11: MI, subgroup by duration ..... Analysis 2.12. Comparison 2: SFA reduction vs usual diet - secondary health events, Outcome 12: MI, subgroup by baseline SFA Analysis 2.13. Comparison 2: SFA reduction vs usual diet - secondary health events, Outcome 13: MI, subgroup by SFA change Analysis 2.14. Comparison 2: SFA reduction vs usual diet - secondary health events, Outcome 14: MI, subgroup by sex ............ Analysis 2.15. Comparison 2: SFA reduction vs usual diet - secondary health events, Outcome 15: MI, subgroup by CVD risk .... Analysis 2.16. Comparison 2: SFA reduction vs usual diet - secondary health events, Outcome 16: MI, subgroup by TC reduction Analysis 2.17. Comparison 2: SFA reduction vs usual diet - secondary health events, Outcome 17: MI, subgroup decade of publication

Analysis 2.18. Comparison 2: SFA reduction vs usual diet - secondary health events, Outcome 18: NON-FATAL MYOCARDIAL INFARCTION

Analysis 2.19. Comparison 2: SFA reduction vs usual diet - secondary health events, Outcome 19: Non-fatal MI, SA by low summary risk of bias

Analysis 2.20. Comparison 2: SFA reduction vs usual diet - secondary health events, Outcome 20: Non-fatal MI, SA aim to reduce SFA

Analysis 2.21. Comparison 2: SFA reduction vs usual diet - secondary health events, Outcome 21: Non-fatal MI, SA statistically significant SFA reduction

Analysis 2.22. Comparison 2: SFA reduction vs usual diet - secondary health events, Outcome 22: Non-fatal MI, SA by TC reduction

Analysis 2.23. Comparison 2: SFA reduction vs usual diet - secondary health events, Outcome 23: Non-fatal MI, SA excluding WHI

Analysis 2.24. Comparison 2: SFA reduction vs usual diet - secondary health events, Outcome 24: Non-fatal MI, SA MantelHaenszel fixed-effect

Analysis 2.25. Comparison 2: SFA reduction vs usual diet - secondary health events, Outcome 25: Non-fatal MI, SA Peto fixedeffect

Analysis 2.26. Comparison 2: SFA reduction vs usual diet - secondary health events, Outcome 26: Non-fatal MI, subgroup by any substitution

Analysis 2.27. Comparison 2: SFA reduction vs usual diet - secondary health events, Outcome 27: Non-fatal MI, subgroup by main substitution

Analysis 2.28. Comparison 2: SFA reduction vs usual diet - secondary health events, Outcome 28: Non-fatal MI, subgroup by duration

Analysis 2.29. Comparison 2: SFA reduction vs usual diet - secondary health events, Outcome 29: Non-fatal MI, subgroup by baseline SFA

Analysis 2.30. Comparison 2: SFA reduction vs usual diet - secondary health events, Outcome 30: Non-fatal MI, subgroup by SFA change

Analysis 2.31. Comparison 2: SFA reduction vs usual diet - secondary health events, Outcome 31: Non-fatal MI, subgroup by sex Analysis 2.32. Comparison 2: SFA reduction vs usual diet - secondary health events, Outcome 32: Non-fatal MI, subgroup by CVD risk 
Analysis 2.33. Comparison 2: SFA reduction vs usual diet - secondary health events, Outcome 33: Non-fatal MI, subgroup by TC reduction

Analysis 2.34. Comparison 2: SFA reduction vs usual diet - secondary health events, Outcome 34: Non-fatal MI, subgroup decade of publication

Analysis 2.35. Comparison 2: SFA reduction vs usual diet - secondary health events, Outcome 35: STROKE

Analysis 2.36. Comparison 2: SFA reduction vs usual diet - secondary health events, Outcome 36: Stroke, SA by low summary risk of bias

Analysis 2.37. Comparison 2: SFA reduction vs usual diet - secondary health events, Outcome 37: Stroke, SA aim to reduce SFA . Analysis 2.38. Comparison 2: SFA reduction vs usual diet - secondary health events, Outcome 38: Stroke, SA statistically significant SFA reduction

Analysis 2.39. Comparison 2: SFA reduction vs usual diet - secondary health events, Outcome 39: Stroke, SA by TC reduction .. Analysis 2.40. Comparison 2: SFA reduction vs usual diet - secondary health events, Outcome 40: Stroke, SA excluding WHI .... Analysis 2.41. Comparison 2: SFA reduction vs usual diet - secondary health events, Outcome 41: Stroke, SA Mantel-Haenszel fixed-effect

Analysis 2.42. Comparison 2: SFA reduction vs usual diet - secondary health events, Outcome 42: Stroke, SA Peto fixed-effect .. Analysis 2.43. Comparison 2: SFA reduction vs usual diet - secondary health events, Outcome 43: Stroke, subgroup by any substitution

Analysis 2.44. Comparison 2: SFA reduction vs usual diet - secondary health events, Outcome 44: Stroke, subgroup by main substitution

Analysis 2.45. Comparison 2: SFA reduction vs usual diet - secondary health events, Outcome 45: Stroke, subgroup by duration Analysis 2.46. Comparison 2: SFA reduction vs usual diet - secondary health events, Outcome 46: Stroke, subgroup by baseline SFA

Analysis 2.47. Comparison 2: SFA reduction vs usual diet - secondary health events, Outcome 47: Stroke, subgroup by SFA change

Analysis 2.48. Comparison 2: SFA reduction vs usual diet - secondary health events, Outcome 48: Stroke, subgroup by sex ..... Analysis 2.49. Comparison 2: SFA reduction vs usual diet - secondary health events, Outcome 49: Stroke, subgroup by CVD risk Analysis 2.50. Comparison 2: SFA reduction vs usual diet - secondary health events, Outcome 50: Stroke, subgroup by TC reduction

Analysis 2.51. Comparison 2: SFA reduction vs usual diet - secondary health events, Outcome 51: Stroke, subgroup decade of publication

Analysis 2.52. Comparison 2: SFA reduction vs usual diet - secondary health events, Outcome 52: CORONARY HEART DISEASE MORTALITY

Analysis 2.53. Comparison 2: SFA reduction vs usual diet - secondary health events, Outcome 53: CHD mortality, SA by low summary risk of bias

Analysis 2.54. Comparison 2: SFA reduction vs usual diet - secondary health events, Outcome 54: CHD mortality, SA aim to reduce SFA

Analysis 2.55. Comparison 2: SFA reduction vs usual diet - secondary health events, Outcome 55: CHD mortality, SA statistically significant SFA reduction

Analysis 2.56. Comparison 2: SFA reduction vs usual diet - secondary health events, Outcome 56: CHD mortality, SA by TC reduction

Analysis 2.57. Comparison 2: SFA reduction vs usual diet - secondary health events, Outcome 57: CHD mortality, SA excluding WHI

Analysis 2.58. Comparison 2: SFA reduction vs usual diet - secondary health events, Outcome 58: CHD mortality, SA MantelHaenszel fixed-effect

Analysis 2.59. Comparison 2: SFA reduction vs usual diet - secondary health events, Outcome 59: CHD mortality, SA Peto fixedeffect

Analysis 2.60. Comparison 2: SFA reduction vs usual diet - secondary health events, Outcome 60: CHD mortality, subgroup by any substitution

Analysis 2.61. Comparison 2: SFA reduction vs usual diet - secondary health events, Outcome 61: CHD mortality, subgroup by main substitution

Analysis 2.62. Comparison 2: SFA reduction vs usual diet - secondary health events, Outcome 62: CHD mortality, subgroup by duration

Analysis 2.63. Comparison 2: SFA reduction vs usual diet - secondary health events, Outcome 63: CHD mortality, subgroup by baseline SFA

Analysis 2.64. Comparison 2: SFA reduction vs usual diet - secondary health events, Outcome 64: CHD mortality, subgroup by SFA change 
Analysis 2.65. Comparison 2: SFA reduction vs usual diet - secondary health events, Outcome 65: CHD mortality, subgroup by sex

Analysis 2.66. Comparison 2: SFA reduction vs usual diet - secondary health events, Outcome 66: CHD mortality, subgroup by CVD risk

Analysis 2.67. Comparison 2: SFA reduction vs usual diet - secondary health events, Outcome 67: CHD mortality, subgroup by TC reduction

Analysis 2.68. Comparison 2: SFA reduction vs usual diet - secondary health events, Outcome 68: CHD mortality, subgroup decade of publication

Analysis 2.69. Comparison 2: SFA reduction vs usual diet - secondary health events, Outcome 69: CORONARY HEART DISEASE EVENTS

Analysis 2.70. Comparison 2: SFA reduction vs usual diet - secondary health events, Outcome 70: CHD events, SA by low summary risk of bias

Analysis 2.71. Comparison 2: SFA reduction vs usual diet - secondary health events, Outcome 71: CHD events, SA excluding WHI

Analysis 2.72. Comparison 2: SFA reduction vs usual diet - secondary health events, Outcome 72: CHD events, SA statistically significant SFA reduction

Analysis 2.73. Comparison 2: SFA reduction vs usual diet - secondary health events, Outcome 73: CHD events, SA by TC reduction

Analysis 2.74. Comparison 2: SFA reduction vs usual diet - secondary health events, Outcome 74: CHD events, SA aim to reduce SFA

Analysis 2.75. Comparison 2: SFA reduction vs usual diet - secondary health events, Outcome 75: CHD events, SA MantelHaenszel fixed-effect

Analysis 2.76. Comparison 2: SFA reduction vs usual diet - secondary health events, Outcome 76: CHD events, SA Peto fixedeffect

Analysis 2.77. Comparison 2: SFA reduction vs usual diet - secondary health events, Outcome 77: CHD events, subgroup by any substitution

Analysis 2.78. Comparison 2: SFA reduction vs usual diet - secondary health events, Outcome 78: CHD events, subgroup by main substitution

Analysis 2.79. Comparison 2: SFA reduction vs usual diet - secondary health events, Outcome 79: CHD events, subgroup by duration

Analysis 2.80. Comparison 2: SFA reduction vs usual diet - secondary health events, Outcome 80: CHD events, subgroup by baseline SFA

Analysis 2.81. Comparison 2: SFA reduction vs usual diet - secondary health events, Outcome 81: CHD events, subgroup by SFA change

Analysis 2.82. Comparison 2: SFA reduction vs usual diet - secondary health events, Outcome 82: CHD events, subgroup by sex Analysis 2.83. Comparison 2: SFA reduction vs usual diet - secondary health events, Outcome 83: CHD events, subgroup by CVD risk

Analysis 2.84. Comparison 2: SFA reduction vs usual diet - secondary health events, Outcome 84: CHD events, subgroup by TC reduction

Analysis 2.85. Comparison 2: SFA reduction vs usual diet - secondary health events, Outcome 85: CHD events, subgroup decade of publication

Analysis 2.86. Comparison 2: SFA reduction vs usual diet - secondary health events, Outcome 86: DIABETES DIAGNOSES ........ Analysis 3.1. Comparison 3: SFA reduction vs usual diet - secondary blood outcomes, Outcome 1: Total cholesterol, $\mathrm{mmol} / \mathrm{L} \quad . .$. Analysis 3.2. Comparison 3: SFA reduction vs usual diet - secondary blood outcomes, Outcome 2: TC, mmol/L, subgroup by any replacement

Analysis 3.3. Comparison 3: SFA reduction vs usual diet - secondary blood outcomes, Outcome 3: TC, mmol/L, subgroup by main replacement

Analysis 3.4. Comparison 3: SFA reduction vs usual diet - secondary blood outcomes, Outcome 4: LDL cholesterol, $\mathrm{mmol} / \mathrm{L} \quad$..... Analysis 3.5. Comparison 3: SFA reduction vs usual diet - secondary blood outcomes, Outcome 5: LDL, mmol/L, subgroup by any replacement

Analysis 3.6. Comparison 3: SFA reduction vs usual diet - secondary blood outcomes, Outcome 6: LDL, mmol/L, subgroup by main replacement

Analysis 3.7. Comparison 3: SFA reduction vs usual diet - secondary blood outcomes, Outcome 7: HDL cholesterol, $\mathrm{mmol} / \mathrm{L} \quad . .$. Analysis 3.8. Comparison 3: SFA reduction vs usual diet - secondary blood outcomes, Outcome 8: HDL, mmol/L, subgroup by any replacement 
Analysis 3.9. Comparison 3: SFA reduction vs usual diet - secondary blood outcomes, Outcome 9: HDL, mmol/L, subgroup by main replacement

Analysis 3.10. Comparison 3: SFA reduction vs usual diet - secondary blood outcomes, Outcome 10: Triglycerides, $\mathrm{mmol} / \mathrm{L} \quad \ldots .$. Analysis 3.11. Comparison 3: SFA reduction vs usual diet - secondary blood outcomes, Outcome 11: TG, mmol/L, subgroup by any replacement

Analysis 3.12. Comparison 3: SFA reduction vs usual diet - secondary blood outcomes, Outcome 12: TG, mmol/L, subgroup by main replacement

Analysis 3.13. Comparison 3: SFA reduction vs usual diet - secondary blood outcomes, Outcome 13: total cholesterol /HDL ratio

Analysis 3.14. Comparison 3: SFA reduction vs usual diet - secondary blood outcomes, Outcome 14: TC /HDL ratio, subgroup by any replacement

Analysis 3.15. Comparison 3: SFA reduction vs usual diet - secondary blood outcomes, Outcome 15: TC /HDL ratio, subgroup by main replacement

Analysis 3.16. Comparison 3: SFA reduction vs usual diet - secondary blood outcomes, Outcome 16: LDL /HDL ratio ............... Analysis 3.17. Comparison 3: SFA reduction vs usual diet - secondary blood outcomes, Outcome 17: Lp(a), mmol/L ................ Analysis 3.18. Comparison 3: SFA reduction vs usual diet - secondary blood outcomes, Outcome 18: Lp(a), mmol/L, subgroup by any replacement

Analysis 3.19. Comparison 3: SFA reduction vs usual diet - secondary blood outcomes, Outcome 19: Lp(a), mmol/L, subgroup by main replacement

Analysis 3.20. Comparison 3: SFA reduction vs usual diet - secondary blood outcomes, Outcome 20: Insulin sensitivity ........... Analysis 4.1. Comparison 4: SFA reduction vs usual diet - secondary outcomes including potential adverse effects, Outcome 1: Cancer diagnoses

Analysis 4.2. Comparison 4: SFA reduction vs usual diet - secondary outcomes including potential adverse effects, Outcome 2: Cancer deaths

Analysis 4.3. Comparison 4: SFA reduction vs usual diet - secondary outcomes including potential adverse effects, Outcome 3: Weight, $\mathrm{kg}$

Analysis 4.4. Comparison 4: SFA reduction vs usual diet - secondary outcomes including potential adverse effects, Outcome 4: BMI, kg/m2

Analysis 4.5. Comparison 4: SFA reduction vs usual diet - secondary outcomes including potential adverse effects, Outcome 5: Systolic Blood Pressure, $\mathrm{mmHg}$

Analysis 4.6. Comparison 4: SFA reduction vs usual diet - secondary outcomes including potential adverse effects, Outcome 6: Diastolic Blood Pressure, $\mathrm{mmHg}$

Analysis 4.7. Comparison 4: SFA reduction vs usual diet - secondary outcomes including potential adverse effects, Outcome 7: Quality of Life

ADDITIONAL TABLES

APPENDICES

WHAT'S NEW

HISTORY

CONTRIBUTIONS OF AUTHORS 
[Intervention Review]

\section{Reduction in saturated fat intake for cardiovascular disease}

Lee Hooper ${ }^{1}$, Nicole Martin ${ }^{2}$, Oluseyi F Jimoh ${ }^{1}$, Christian Kirk ${ }^{1}$, Eve Foster ${ }^{1}$, Asmaa S Abdelhamid ${ }^{1}$

${ }^{1}$ Norwich Medical School, University of East Anglia, Norwich, UK. 2Institute of Health Informatics Research, University College London, London, UK

Contact address: Lee Hooper, l.hooper@uea.ac.uk.

Editorial group: Cochrane Heart Group.

Publication status and date: New search for studies and content updated (no change to conclusions), published in Issue 5, 2020.

Citation: Hooper L, Martin N, Jimoh OF, Kirk C, Foster E, Abdelhamid AS. Reduction in saturated fat intake for cardiovascular disease. Cochrane Database of Systematic Reviews 2020, Issue 5. Art. No.: CD011737. DOI: 10.1002/14651858.CD011737.pub2.

Copyright @ 2020 The Cochrane Collaboration. Published by John Wiley \& Sons, Ltd.

\section{A B S T R A C T}

\section{Background}

Reducing saturated fat reduces serum cholesterol, but effects on other intermediate outcomes may be less clear. Additionally, it is unclear whether the energy from saturated fats eliminated from the diet are more helpfully replaced by polyunsaturated fats, monounsaturated fats, carbohydrate or protein.

\section{Objectives}

To assess the effect of reducing saturated fat intake and replacing it with carbohydrate (CHO), polyunsaturated (PUFA), monounsaturated fat (MUFA) and/or protein on mortality and cardiovascular morbidity, using all available randomised clinical trials.

\section{Search methods}

We updated our searches of the Cochrane Central Register of Controlled Trials (CENTRAL), MEDLINE (Ovid) and Embase (Ovid) on 15 October 2019, and searched Clinicaltrials.gov and WHO International Clinical Trials Registry Platform (ICTRP) on 17 October 2019.

\section{Selection criteria}

Included trials fulfilled the following criteria: 1) randomised; 2) intention to reduce saturated fat intake OR intention to alter dietary fats and achieving a reduction in saturated fat; 3) compared with higher saturated fat intake or usual diet; 4) not multifactorial; 5) in adult humans with or without cardiovascular disease (but not acutely ill, pregnant or breastfeeding); 6) intervention duration at least 24 months; 7) mortality or cardiovascular morbidity data available.

\section{Data collection and analysis}

Two review authors independently assessed inclusion, extracted study data and assessed risk of bias. We performed random-effects metaanalyses, meta-regression, subgrouping, sensitivity analyses, funnel plots and GRADE assessment.

\section{Main results}

We included 15 randomised controlled trials (RCTs) (16 comparisons, 59,000 participants), that used a variety of interventions from providing all food to advice on reducing saturated fat. The included long-term trials suggested that reducing dietary saturated fat reduced the risk of combined cardiovascular events by $21 \%$ (risk ratio (RR) $0.79 ; 95 \%$ confidence interval (CI) 0.66 to $0.93,11$ trials, 53,300 participants of whom $8 \%$ had a cardiovascular event, $I^{2}=65 \%$, GRADE moderate-quality evidence). Meta-regression suggested that greater reductions in saturated fat (reflected in greater reductions in serum cholesterol) resulted in greater reductions in risk of CVD events, explaining most heterogeneity between trials. The number needed to treat for an additional beneficial outcome (NNTB) was 56 in primary prevention trials, so 56 people need to reduce their saturated fat intake for four years for one person to avoid experiencing a CVD event. In secondary prevention trials, the NNTB was 32. Subgrouping did not suggest significant differences between replacement of saturated fat calories with polyunsaturated fat or carbohydrate, and data on replacement with monounsaturated fat and protein was very limited. 
We found little or no effect of reducing saturated fat on all-cause mortality (RR $0.96 ; 95 \% \mathrm{Cl} 0.90$ to $1.03 ; 11$ trials, 55,858 participants) or cardiovascular mortality (RR $0.95 ; 95 \% \mathrm{Cl} 0.80$ to $1.12,10$ trials, 53,421 participants), both with GRADE moderate-quality evidence.

There was little or no effect of reducing saturated fats on non-fatal myocardial infarction (RR $0.97,95 \% \mathrm{Cl} 0.87$ to 1.07 ) or CHD mortality (RR $0.97,95 \% \mathrm{Cl} 0.82$ to 1.16 , both low-quality evidence), but effects on total (fatal or non-fatal) myocardial infarction, stroke and CHD events (fatal or non-fatal) were all unclear as the evidence was of very low quality. There was little or no effect on cancer mortality, cancer diagnoses, diabetes diagnosis, HDL cholesterol, serum triglycerides or blood pressure, and small reductions in weight, serum total cholesterol, LDL cholesterol and BMI. There was no evidence of harmful effects of reducing saturated fat intakes.

\section{Authors' conclusions}

The findings of this updated review suggest that reducing saturated fat intake for at least two years causes a potentially important reduction in combined cardiovascular events. Replacing the energy from saturated fat with polyunsaturated fat or carbohydrate appear to be useful strategies, while effects of replacement with monounsaturated fat are unclear. The reduction in combined cardiovascular events resulting from reducing saturated fat did not alter by study duration, sex or baseline level of cardiovascular risk, but greater reduction in saturated fat caused greater reductions in cardiovascular events.

\section{PLAIN LANGUAGE SUMMARY}

\section{Effect of cutting down on the saturated fat we eat on our risk of heart disease}

\section{Review question}

We wanted to find out the effects on health of cutting down on saturated fat in our food (replacing animal fats and hard vegetable fats with plant oils, unsaturated spreads or starchy foods).

\section{Background}

Health guidance suggests that reducing the amount of saturated fat we eat, by cutting down on animal fats, is good for our health. We wanted to combine all available evidence to see whether following this advice leads to a reduced risk of dying or getting cardiovascular disease (heart disease or stroke).

\section{Study characteristics}

We assessed the effect of cutting down the amount of saturated fat we eat for at least two years on health outcomes including dying, heart disease and stroke. We only looked at studies of adults (18 years or older). They included men and women with and without cardiovascular disease. We did not include studies of acutely ill people or pregnant or breastfeeding women.

\section{Key results}

We found 15 studies with over 59,000 participants. The evidence is current to October 2019. The review found that cutting down on saturated fat led to a $21 \%$ reduction in the risk of cardiovascular disease (including heart disease and strokes), but had little effect on the risk of dying. The review found that health benefits arose from replacing saturated fats with polyunsaturated fat or starchy foods. The greater the decrease in saturated fat, and the more serum total cholesterol is reduced, the greater the protection from cardiovascular events. People who are currently healthy appear to benefit as much as those at increased risk of heart disease or stroke (people with high blood pressure, high serum cholesterol or diabetes, for example), and people who have already had heart disease or stroke. There was no difference in effect between men and women.

This means that, if 56 people without cardiovascular disease, or 32 people who already have cardiovascular disease, reduce their saturated fat for around 4 years, then one person will avoid a cardiovascular event (heart attack or stroke) they would otherwise have experienced.

\section{Quality of the evidence}

There is a large body of evidence assessing effects of reducing saturated fat for at least two years. These studies provide moderate-quality evidence that reducing saturated fat reduces our risk of cardiovascular disease. 
S U M MARY OF FINDINGS

Summary of findings 1. Effect of reducing saturated fat compared to usual saturated fat on CVD risk in adults (note: for the full set of GRADE tables see additional tables 24 to 28 )

Low saturated fat compared with usual saturated fat for CVD risk

Patient or population: people at any baseline risk of CVD

Intervention: lower saturated fat intake

Comparison: higher saturated fat intake

Settings: Any, including community-dwelling and institutions. Included RCTs were conducted in North America, Europe and Australia/New Zealand, no studies were carried out in industrialising or developing countries.

\begin{tabular}{|c|c|c|c|c|c|c|}
\hline \multirow[t]{2}{*}{ Outcomes } & \multirow{2}{*}{$\begin{array}{l}\text { Relative ef- } \\
\text { fect } \\
(95 \% \mathrm{CI})\end{array}$} & \multicolumn{2}{|c|}{$\begin{array}{l}\text { Anticipated absolute effects } \\
(95 \% \mathrm{CI})\end{array}$} & \multirow{2}{*}{$\begin{array}{l}\text { No of Partici- } \\
\text { pants } \\
\text { (studies) }\end{array}$} & \multirow{2}{*}{$\begin{array}{l}\text { Quality of the } \\
\text { evidence } \\
\text { (GRADE) }\end{array}$} & \multirow[t]{2}{*}{ Comments } \\
\hline & & $\begin{array}{l}\text { Risk with } \\
\text { higher SFA } \\
\text { intake }\end{array}$ & $\begin{array}{l}\text { Risk with } \\
\text { lower SFA in- } \\
\text { take }\end{array}$ & & & \\
\hline $\begin{array}{l}\text { All-cause mortality } \\
\text { follow-up mean duration } 56 \\
\text { months }^{1}\end{array}$ & $\begin{array}{l}\mathbf{R R} 0.96(0.90 \\
\text { to } 1.03)\end{array}$ & 62 per 1000 & $\begin{array}{l}60 \text { per } 1000 \\
\text { (56 to } 64)\end{array}$ & $\begin{array}{l}55,858 \\
(12)\end{array}$ & $\begin{array}{l}\oplus \oplus \oplus \ominus \\
\text { Moder- } \\
\text { ate }^{2,3,4,5,6}\end{array}$ & $\begin{array}{l}\text { Critical importance. Reducing saturated fat intake } \\
\text { probably makes little or no difference to all-cause } \\
\text { mortality. }\end{array}$ \\
\hline $\begin{array}{l}\text { Cardiovascular mortality } \\
\text { follow-up mean duration } 53 \\
\text { months }^{1}\end{array}$ & $\begin{array}{l}\mathbf{R R} 0.94(0.78 \\
\text { to } 1.13)\end{array}$ & 19 per 1000 & $\begin{array}{l}18 \text { per } 1000 \\
(15 \text { to } 22)\end{array}$ & $\begin{array}{l}53,421 \\
(11)\end{array}$ & $\begin{array}{l}\oplus \oplus \oplus \ominus \\
\text { Moder- } \\
\text { ate }^{2,3,4,6,7}\end{array}$ & $\begin{array}{l}\text { Critical importance. Reducing saturated fat intake } \\
\text { probably makes little or no difference to cardiovas- } \\
\text { cular mortality. }\end{array}$ \\
\hline $\begin{array}{l}\text { Combined cardiovascular } \\
\text { events } \\
\text { follow-up mean duration } 52 \\
\text { months }^{1}\end{array}$ & $\begin{array}{l}\mathbf{R R} \mathbf{0 . 7 9}(0.66 \\
\text { to } 0.93)\end{array}$ & 84 per 1000 & $\begin{array}{l}67 \text { per } 1000 \\
(56 \text { to } 79)\end{array}$ & $\begin{array}{l}53,300 \\
(12)\end{array}$ & $\begin{array}{l}\oplus \oplus \oplus \ominus \\
\text { Moder- } \\
\text { ate } \mathbf{4 , 8 , 9 , 1 0 , 1 1}\end{array}$ & $\begin{array}{l}\text { Critical importance. Reducing saturated fat intake } \\
\text { probably reduces cardiovascular mortality (to a } \\
\text { greater extent with greater cholesterol reduction). }\end{array}$ \\
\hline $\begin{array}{l}\text { Myocardial infarctions } \\
\text { follow-up mean duration } 55 \\
\text { months }\end{array}$ & $\begin{array}{l}\mathbf{R R} \mathbf{0 . 9 0}(0.80 \\
\text { to } 1.01)\end{array}$ & 32 per 1000 & $\begin{array}{l}29 \text { per } 1000 \\
(25 \text { to } 32 \text { ) }\end{array}$ & $\begin{array}{l}53,167 \\
(11)\end{array}$ & $\begin{array}{l}\oplus \ominus \odot \odot \\
\text { Very Low } \\
\mathbf{3 , 4 , 5 , 1 1 , 1 2}\end{array}$ & $\begin{array}{l}\text { Critical importance. The effect of reducing saturated } \\
\text { fat intake on risk of myocardial infarction is unclear } \\
\text { as the evidence is of very low quality. }\end{array}$ \\
\hline
\end{tabular}




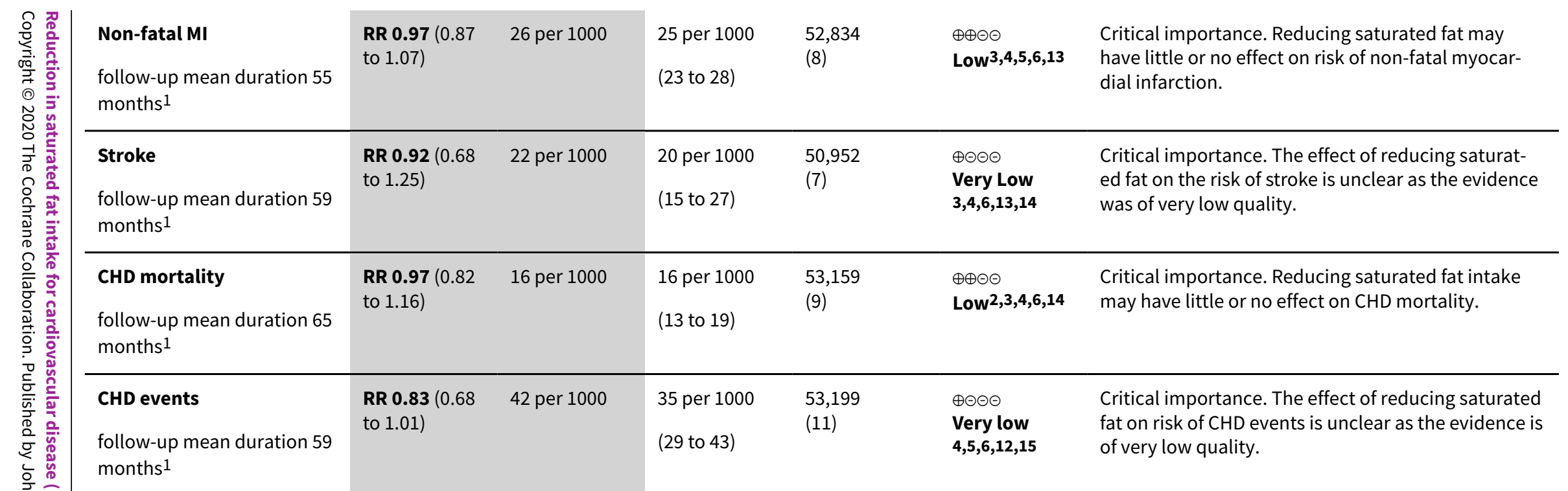

${ }^{*}$ The risk in the intervention group (and its 95\% confidence interval) is based on the assumed risk in the comparison group and the relative effect of the intervention (and its $95 \% \mathrm{Cl})$.

CI: Confidence interval; RR: Risk Ratio; CHD: coronary heart disease.

GRADE Working Group grades of evidence

High quality: Further research is very unlikely to change our confidence in the estimate of effect.

Moderate quality: Further research is likely to have an important impact on our confidence in the estimate of effect and may change the estimate.

Low quality: Further research is very likely to have an important impact on our confidence in the estimate of effect and is likely to change the estimate.

Very low quality: We are very uncertain about the estimate.

1 Minimum study duration was 24 months.

2 Risk of bias. Limiting trials to those at low summary risk of bias also suggested little or no effect. Not downgraded.

3 Inconsistency. We found no important heterogeneity; $\left.\right|^{2} \leq 30 \%$. Not downgraded.

4 Indirectness. These RCTs directly assessed the effect of lower vs higher saturated fat intake on health outcomes of interest. Participants included men and women with and without CVD at baseline (also some participants with CVD risk factors like diabetes, or at risk of cancers). However, no trials included participants from developing countries. Not downgraded.

5 Imprecision. The $95 \% \mathrm{Cl}$ includes both no effect and a benefit. Downgraded once.

6 Publication bias. The funnel plot, and comparison of fixed-and random-effects meta-analyses did not suggest major small-study (publication) bias. Not downgraded.

7 Imprecision. The $95 \% \mathrm{Cl}$ includes both harm and benefit. Downgraded once.

8 Risk of bias. Limiting trials to those at low summary risk of bias also suggested that reducing SFA reduced risk of CVD events. Not downgraded.

9 Inconsistency. Although heterogeneity was high, $\mathrm{I}^{2}=65 \%$, this was mostly explained by the degree of cholesterol-lowering (a dose effect). Not downgraded.

${ }^{10}$ Imprecision. The $95 \% \mathrm{Cl}$ includes only benefit. Not downgraded.

11 Publication bias. The funnel plot, and comparison of fixed-and random-effects meta-analyses suggested some small-study (publication) bias. Downgraded once.

12 Risk of bias. Limiting trials to those at low summary risk of bias moved the RR slightly towards 1.0, suggesting little or no effect on total MI. Downgraded once. 
13 Risk of bias. Limiting trials to those at low summary risk of bias moved the RR slightly away from 1.0, suggesting that reducing SFA reduces the risk of non-fatal MI. This was also seen in several other sensitivity analyses. Downgraded once.

14 Imprecision. The $95 \% \mathrm{Cl}$ includes both important benefits and important harms. Downgraded twice.

15 Inconsistency. Heterogeneity was high, $\mathrm{I}^{2}=65 \%$. Downgraded once. 


\section{B A C K G R O U N D}

In 1949, Ryle and Russell in Oxford documented a dramatic increase in coronary heart disease (CHD), and the Registrar General's Statistical Tables of 1920 to 1955 showed that there had been a 70fold increase in coronary deaths during this 35-year period (Oliver 2000; Ryle 1949). This sudden surge in coronary heart disease sparked research into its causes. A case-control study published in 1953 of 200 post-myocardial infarction patients and age-matched controls established that those with disease had higher low-density lipoprotein (LDL) cholesterol levels (Oliver 1953).

Meanwhile in 1949 in the USA, Gofman had separated lipids into lipoprotein classes through ultra centrifugation, describing the LDL as 'atherosclerogenic' (Gofman 1949). The following year Keys 1950 proposed that the concentration of plasma cholesterol was proportional to dietary saturated fatty acids (SFA) intake. This relationship was confirmed in work by Hegsted (Hegsted 1965; Hegsted 2000), who published an equation explaining the relationship in 1965 and subsequently in 2000. The equation suggests that dietary saturated fat increases serum cholesterol and so increases cardiovascular (CV) risk, while polyunsaturated fats (PUFA) reduce both. This has since been further refined:

$\Delta$ serum cholesterol (in $\mathrm{mg} / \mathrm{dL}$ ) $=2.16{ }^{*} \Delta$ dietary saturated fat intake (as percentage of energy) $-1.65^{*} \Delta$ dietary PUFA intake (as percentage of energy, $\% \mathrm{E}$ ) $+6.77^{*} \Delta$ dietary cholesterol intake (in units of $100 \mathrm{mg} /$ day) -0.53

The Seven Countries Study compared CHD mortality in 12,000 men aged 40 to 59 in seven countries, and found positive correlations between CHD mortality and total fat intake in 1970, then in 1986 between CHD mortality and saturated fat intake (Keys 1986; Thorogood 1996). A migrant study of Japanese men living in different cultures confirmed in 1974 that men in California had the diet richest in saturated fat and cholesterol, and the highest CHD rates, those in Hawaii had intermediate saturated fat and CHD rates, and those in Japan had a diet lowest in saturated fat and cholesterol, and the least CHD (Kagan 1974; Robertson 1977). However, systematic reviews of the observational data have not confirmed these early studies. Skeaff 2009 included 28 USA and European cohorts (including 6600 CHD deaths among 280,000 participants) investigating the effects of total, saturated, monounsaturated, trans and omega- 3 fats on CHD deaths and events. They found no clear relationship between total, saturated or monounsaturated fat (MUFA) intake and coronary heart disease events or deaths. There was evidence that trans fats increased both coronary heart disease events and deaths, and that total PUFAs and omega-3 fats decreased them. Intervention studies are needed to clarify cause and effect, to ensure that confounding is not hiding true relationships, or suggesting relationships where they do not exist. Trials also directly address the issue of whether altering dietary saturated fat in adults is helpful in reducing the risk of CVD in the general population and in those at high risk. Intervention trials are crucial in forming the basis of evidence-based practice in this area.

Most intervention studies have assessed effects of dietary interventions on risk factors for heart disease, and separate work ties the effect of altering these risk factors to changes in disease incidence and mortality. Systematic reviews in this area follow the same pattern. There are systematic reviews of the effect of dietary fat advice on serum lipid levels (Brunner 1997; Clarke 1997; Denke
1995; Kodama 2009; Malhotra 2014; Mensink 1992; Mensink 2003; Rees 2013; Weggemans 2001; Yu-Poth 1999), suggesting that dietary changes cause changes in serum lipids. There are also systematic reviews on the effect of lipid level alterations on CV morbidity and mortality (Briel 2009; De Caterina 2010; Law 1994; Robinson 2009; Rubins 1995; Walsh 1995), suggesting that changes in lipids do affect CVD risk. Other risk factors dealt with in a similar way are blood pressure (Bucher 1996; Law 1991; Shah 2007), body weight or fatness (Astrup 2000; Hession 2009; SIGN 1996), angiographic measurements (Marchioli 1994), antioxidant intake (Ness 1997), metabolic profile (Kodama 2009) and alcohol intake (Rimm 1996). A problem with this two-level approach is that any single dietary alteration may have effects over a wide range of risk factors for CVD. An example of this is the choice of substitution of saturated fats by carbohydrate, PUFAs, MUFAs or protein in the diet. This choice may alter lipid profile, and may also affect blood pressure, body weight, oxidative state, rate of cholesterol efflux from fibroblasts, insulin resistance, post-prandial triacylglycerol response, blood clotting factors, and platelet aggregation. There may also be further risk factors of which we are not yet aware. Evidence of beneficial effect on one risk factor does not rule out an opposite effect on another unstudied risk factor, and therefore an overall null (or harmful) effect of intervention. While understanding the effects of dietary advice on intermediate risk factors helps to ensure diets are truly altered by advice, and illuminates mechanisms, the best way of combining the effects on all of these risk factors is to not study risk factors, but to study the effects of dietary change on important outcomes, on CV morbidity and mortality, and on total mortality.

Substantial randomised controlled trial data on the effects of dietary fat on mortality and morbidity do exist and have been previously reviewed (Abdelhamid 2020; Abdelhamid 2018; Abdelhamid 2019; Brainard 2020; Brown 2019; Deane 2019; Hanson 2020; Hooper 2018; Hooper 2019; Hooper 2012). A recent very large trial, the Women's Health Initiative, that included over 2000 women with, and over 48,000 women without, CVD at baseline for over eight years (WHI 2006)) has raised many questions about both the effects of fat on health and on how we best conduct research to understand the relationship (Astrup 2011; Michels 2009; Prentice 2007; Stein 2006; Yngve 2006). We incorporated these findings into an update of a Cochrane review on dietary fat and CVD risk with a search in 2010 (Hooper 2012), finding reductions in cardiovascular events in studies that modified dietary fat, and in studies of at least two years' duration, but not in studies of fat reduction or studies with less than two years' follow-up.

\section{Why it is important to do this review}

Public health dietary advice on prevention of cardiovascular disease (CVD) has changed over time, with a focus on fat modification during the 1960 s and fat reduction during the 1990s following the introduction of USA and UK dietary guidance on fat reduction, limiting saturated fat intake to $10 \%$ of energy (Harcombe 2015). In 2006, recommendations by the American Heart Association suggested that, among other dietary measures, Americans should "limit intake of saturated fat to 7\% of energy, trans fat to $1 \%$ of energy, and cholesterol to $300 \mathrm{mg} /$ day by choosing lean meats and vegetable alternatives, fat-free (skim) or low-fat ( $1 \%$ fat) dairy products and minimise intake of partially hydrogenated fats" (Lichtenstein 2006). Current American Heart Association guidelines suggest that Americans should "Aim for a dietary pattern that achieves $5 \%$ to $6 \%$ of calories from 
saturated fat" and "Reduce percent of calories from saturated fat" (both graded as strong evidence on the basis of effects on serum lipids - trials with cardiovascular outcomes are not referenced or discussed, Eckel 2013). European guidance on the treatment of dyslipidaemia is similarly based on dietary effects on lipids, recommending reduction in saturated fats (ESC/EAS 2011) and referencing Mensink 2003, while the Joint British Societies' guidance on preventing CVD recommends a healthy diet including low saturated fat intake (Mach 2019), referencing a variety of evidence including several recent systematic reviews. This is reflected in UK Scientific Advisory Committee on Nutrition recommendations that "dietary reference value for saturated fats remains unchanged: the [population] average contribution of saturated fatty acids to [total] dietary energy be reduced to no more than about $10 \%$ ", and that "saturated fats are substituted with unsaturated fats. More evidence is available supporting substitution with PUFA than substitution with MUFA" (SACN 2019).

Recent UK National Institute for Health and Care Excellence(NICE) guidance suggests that for people at high risk of or with CVD that they "eat a diet in which total fat intake is $30 \%$ or less of total energy intake, saturated fats are $7 \%$ or less of total energy intake, intake of dietary cholesterol is less than $300 \mathrm{mg} /$ day and where possible saturated fats are replaced by monounsaturated and polyunsaturated fats". This statement was based on long-term randomised controlled trials reporting hard outcomes, and NICE separately assessed effects of high polyunsaturated diets, including four of the trials included in this review (NICE 2014).

We were interested in assessing the direct evidence from trials of the effects of reducing saturated fats, and considering what macronutrients the saturated fats were replaced by, updating Hooper 2015a. This update also supports a request from the World Health Organization Nutrition Guidance Expert Advisory Group (WHO NUGAG) to more accurately assess effects of reducing saturated fats on all-cause mortality, CV morbidity and other health outcomes, and to consider the differential effects on health outcomes of replacement of the energy from saturated fat by other fats, carbohydrates or protein.

\section{O B JE C T IVES}

To assess the effect of reducing saturated fat intake and replacing it with carbohydrate ( $\mathrm{CHO}$ ), polyunsaturated (PUFA) or monounsaturated fat (MUFA) and/or protein on mortality and cardiovascular morbidity, using all available randomised clinical trials.

Additional World Health Organization Nutrition Guidance Expert Advisory Group (WHO NUGAG) specific questions included:

1. In adults, what is the effect in the population of reduced percentage of energy $(\% \mathrm{E})$ intake from saturated fatty acids (SFA) relative to higher intake for reduction in risk of noncommunicable diseases (NCDs)?

2. What is the effect on coronary heart disease mortality and coronary heart disease events?

3. What is the effect in the population of replacing SFA with polyunsaturated fats (PUFAs), monounsaturated fats (MUFAs), carbohydrates $(\mathrm{CHO})$ (refined versus unrefined), protein or trans fatty acids (TFAs) relative to no replacement for reduction in risk of NCDs?
4. What is the effect in the population of consuming $<10 \% \mathrm{E}$ as SFA relative to $>10 \% \mathrm{E}$ as SFA for reduction in risk of NCDs?

5. What is the effect in the population of a reduction in $\% E$ from SFA from $10 \%$ in gradual increments relative to higher intake for reduction in risk of NCDs?

\section{METHODS}

\section{Criteria for considering studies for this review \\ Types of studies}

Randomised controlled trials only. We accepted randomisation of individuals, or of larger groups (clusters) where there were at least six of these groups randomised. We excluded studies where allocation was not truly randomised (e.g. divisions based on days of the week or first letter of the family name), or where allocation was not stated as randomised, and no further information was available from the authors.

\section{Types of participants}

We included studies of adults (18 years or older, no upper age limit) at any risk of cardiovascular disease, with or without existing cardiovascular disease, using or not using lipid-lowering medication. Participants could be of either gender, but we excluded those who were acutely ill, pregnant or lactating.

\section{Types of interventions}

We included randomised controlled trials stating an intention to reduce saturated fat (SFA) intake (by suggesting appropriate nutrient-based or food-based aims) OR which provided a general dietary aim, such as improving heart health or reducing total fat, that also achieved a statistically significant saturated fat reduction $(P<0.05)$ during the trial in the intervention arm compared with the control arm. The intervention had to be dietary advice, supplementation of fats, oils or modified or low-fat foods, or a provided diet, compared to higher saturated fat intake which could be usual diet, higher saturated fat, placebo or a control diet. Intended duration of the dietary intervention was at least two years (24 months or 104 weeks).

\section{Types of outcome measures}

\section{Primary outcomes}

- All-cause mortality (deaths from any cause)

- Cardiovascular (CVD) mortality (deaths from myocardial infarction, stroke, and/or sudden death)

- Combined CVD events. These included data available on number of people experiencing any of the following: cardiovascular death, cardiovascular morbidity (non-fatal myocardial infarction, angina, stroke, heart failure, peripheral vascular events, atrial fibrillation) and unplanned cardiovascular interventions (coronary artery bypass surgery or angioplasty).

To meet our inclusion criteria, trials had to report either deaths or CVD events. These could be reported as serious adverse events (SAEs) or via communication with authors.

\section{Secondary outcomes}

- Additional health events; the outcomes CHD mortality and CHD events were added at the request of the WHO NUGAG group, 
and were not present in the original overarching systematic review. For each of these, we assessed number of participants experiencing any of these:

* Myocardial infarction, total (fatal and non-fatal)

* Myocaridal infarction, non-fatal

* Stroke

* CHD mortality, which includes death from myocardial infarction or sudden CVD death

* CHD events, which include any of the following: fatal or nonfatal myocardial infarction, angina or sudden CVD death

* type II diabetes incidence

- Blood measures including serum blood lipids

* total cholesterol (TC, $\mathrm{mmol} / \mathrm{L}$ )

* low-density lipoprotein (LDL) cholesterol, mmol/L

* high-density lipoprotein (HDL) cholesterol, $\mathrm{mmol} / \mathrm{L}$

* triglyceride (TG), $\mathrm{mmol} / \mathrm{L}$

* TG/HDL ratio

* LDL/HDL ratio

* total/HDL ratio

* lipoprotein (a) (Lp(a)), mmol/L

* insulin sensitivity including glucose tolerance (homeostatic model assessment (HOMA), intravenous glucose tolerance test (IV-GTT), clamp, glycosylated haemoglobin (HbA1C))

- Other outcomes including adverse effects reported by study authors

* cancer diagnoses

* cancer deaths

* body weight, $\mathrm{kg}$

* body mass index (BMI, $\left.\mathrm{kg} / \mathrm{m}^{2}\right)$

* systolic blood pressure (sBP, $\mathrm{mmHg}$ )

* diastolic blood pressure (dBP, $\mathrm{mmHg}$ )

* quality of life (any measure)

As all trials collect data on deaths and cardiovascular events (as serious adverse events if not as planned outcome measures), we only included trials where we knew that at least one primary outcome occurred, by communication with authors if necessary. Where we knew that at least one primary outcome occurred, we included the study even where we were unable to use that data in meta-analysis. We excluded studies where we knew that no primary outcome events occurred (for a study to be excluded in this way the paper needed to be very explicit about the lack of all outcomes or we received confirmation from the authors) and this was noted as the reason for exclusion. Lack of a single primary outcome only occurs in very small studies or in young cohorts, so omitting these studies will make no difference to effect sizes and very little difference to absolute effect sizes (NNTs etc). All other trials were considered unclear and where we could not gain clarification on events from authors, they were classified as "awaiting assessment".

For composite outcomes (like CVD events), we worked to collect data on the number of participants in each arm who experienced any type of CVD event, and did not double-count people (so that a person experiencing a stroke and two heart attacks during a trial was counted as one person experiencing CVD events, not as three CVD events).
We extracted event and continuous outcome data for the latest time point available within the trial, and always at least 24 months from inception. We collected change data (with a measure of variance) for continuous outcomes where these were available, and end data where change data were not provided in usable format.

\section{Search methods for identification of studies}

\section{Electronic searches}

The updated searches were run on 15 October 2019 on the following databases:

- CENTRAL (Issue 10 of 12, 2019, Cochrane Library)

- MEDLINE (Epub Ahead of Print, In-Process \& Other Non-Indexed Citations, MEDLINE Daily and MEDLINE, Ovid, 1946 to October 14, 2019)

- Embase (Ovid, 1980 to 2019 week 41).

For this update, we introduced searches of two trials registers on 17 October 2019; Clinicaltrials.gov (www.clinicaltrials.gov) and WHO International Clinical Trials Registry Platform (ICTRP) (apps.who.int/trialsearch/). The searches are described in Appendix 1. The RCT filter for MEDLINE was the Cochrane sensitivity and precision-maximising RCT filter (Lefebvre 2011), and for Embase, terms as recommended in the Cochrane Handbook were applied (Lefebvre 2011).

As we were updating another Cochrane review relating to dietary fat (Hooper 2015b) at the same time, results of the searches for both reviews were combined and de-duplicated before assessment of titles and abstracts.

The search to 2014 is described in Hooper 2015a, and previous searches in Hooper 2012.

\section{Searching other resources}

We searched for recent publications of the included studies, to ensure the best possible data set for each study.

\section{Data collection and analysis}

\section{Selection of studies}

Search results were loaded into Covidence software. All authors independently assessed titles and abstracts from the search, differences were resolved by discussion and, when the findings were not clear cut, the full text was collected for assessment. We only rejected articles on initial screen if the author could determine from the title and abstract that the article was not a report of a randomised controlled trial; the trial did not address a low or modified fat diet; the trial was exclusively in children less than 18 years old, pregnant women or the critically ill; the trial was of less than 24 months duration; or the intervention was multifactorial. When we could not reject a title/abstract with certainty, we obtained the full text of the article for further evaluation.

\section{Data extraction and management}

We used a data extraction form designed for earlier versions of this review. We extracted data concerning participants, interventions and outcomes, trial quality characteristics (Chalmers 1990), data on potential effect modifiers including participants' baseline risk of cardiovascular disease, trial duration, intensity of intervention (dietary advice, diet provided, dietary advice 
plus supplementation, supplementation alone), medications used (particularly lipid-lowering medication) and smoking status, numbers of events and total participant years in trial. Where provided, we collected data on risk factors for cardiovascular disease including blood pressure, lipids and weight.

We defined baseline risk of cardiovascular disease as follows: high risk are participants with existing vascular disease including a history of myocardial infarction, stroke, peripheral vascular disease, angina, heart failure or previous coronary artery bypass grafting or angioplasty; moderate risk are participants with a familial risk, dyslipidaemia, diabetes mellitus, hypertension, chronic renal failure; low risk are other participants or mixedpopulation groups. Those at low or moderate risk combined are primary prevention trials.

Data were extracted independently in duplicate by AA, FOJ and/or $\mathrm{LH}$, alongside assessment of risk of bias.

\section{Assessment of risk of bias in included studies}

We carried out 'Risk of bias' assessment independently in duplicate as part of data extraction. We assessed trial risk of bias using the Cochrane tool for assessment of risk of bias (Higgins 2011). For included RCTs, we also assessed whether each study:

1. was free of systematic differences in care,

2. aimed to reduce SFA intake,

3. achieved SFA reduction, or

4. achieved total serum cholesterol reduction.

We used the category 'other bias' to note any further issues of methodological concern. Funding was not formally a part of our assessment of bias in RCTs as it is not a core part of the Cochrane 'Risk of bias' tool, but was reported in the Characteristics of included studies.

Two authors (LH, NM) independently extracted validity data from studies identified by the previous search, and resolved differences by discussion.

Poorly concealed allocation is associated with a $40 \%$ greater effect size (Schulz 1995), so randomisation and allocation concealment are core issues for all trials. Lack of blinding is associated with bias, though smaller levels of bias than lack of allocation concealment (Savovic 2012), especially in studies with objectively measured outcomes (Wood 2008).

For this review, we introduced the concept of summary risk of bias for whole trials. We considered dietary advice or all-food-provided type trials to be at low summary risk of bias where we judged randomisation, allocation concealment, and blinding of outcome assessors to be adequate. Summary risk of bias was considered moderate to high in all other included trials.

\section{Measures of treatment effect}

The effect measures of choice were risk ratios (RR) for dichotomous data and mean difference (MD) for continuous data.

\section{Unit of analysis issues}

We did not include any cluster-randomised trials in this review, as no relevant studies included at least six clusters.
Where there was more than one relevant intervention arm but only one control arm, we either pooled the relevant intervention arms to create a single pairwise comparison (where the intervention arms were equivalently appropriate for this review) as described in the Cochrane Handbook (Higgins 2011), or we excluded intervention arms that were not appropriate for this review, or less appropriate than another arm. When two arms were appropriate for different subgroups (Rose corn oil 1965; Rose olive 1965), then we used the control group once with each intervention arm, and divided the number of events in the control group, and the number of participants in the control group, evenly between the two study comparisons.

In the previous version of this review, data for WHI 2006 were presented separately for those without baseline CVD, and with baseline CVD, for most outcomes. This has been altered in this version of the review, so that both sets of data are presented as a single trial except when subgrouping by CVD risk. This has the effect of representing this study in the same way others are represented (which is appropriate), and slightly reducing the weight of the WHI 2006 study in random-effects meta-analysis, altering the numbers in the analysis.

When assessing event data, we aimed to assess number of participants experiencing an event (rather than numbers of events), to avoid counting more than one outcome event for any one individual within any one comparison. Where we were unclear (for example, where a paper reported numbers of myocardial infarcts but not by arm), we asked authors for further information.

\section{Dealing with missing data}

Where trials satisfied the inclusion criteria of our review but did not report mortality and morbidity, or not by study arm, we tried to contact study authors. This allowed inclusion of studies that would otherwise have had to be excluded. We excluded studies which were otherwise relevant but where we could not establish the presence or absence of primary outcomes, despite multiple attempts at author contact.

It was often unclear whether data on primary or secondary outcome events may still have been missing, and so we did not impute data for this review.

Where included studies used methods to infer missing data (such as carrying the latest measurement forward), then we used these data in analyses. Where this was not done, we used the data as presented.

\section{Assessment of heterogeneity}

We examined heterogeneity using the $\mathrm{I}^{2}$ test, and considered it important where greater than 50\% (Higgins 2003; Higgins 2011). Where we identified important clinical or unexplained statistical heterogeneity, we did not pool but instead summarised the studies in a narrative format. We used the assessment of heterogeneity in our GRADE assessments, so that the quality of evidence was downgraded where heterogeneity was important, and not explained by subgrouping or meta-regression.

\section{Assessment of reporting biases}

We used funnel plots to examine the possibility of small study bias, including publication bias (Egger 1997), for the primary outcomes of total mortality and combined cardiovascular events. For this 
update, we also compared findings of fixed- and random-effects meta-analysis since the two methods weight small trials differently, and different effect sizes suggest potential small study bias (Page 2019).

\section{Data synthesis}

We carried out data synthesis in the absence of clinical heterogeneity. We used numbers of events in each study arm, and total number of participants randomised, where extracted, and Mantel-Haenszel random-effects meta-analysis carried out in Review Manager 5 software, to assess risk ratios. We extracted event and continuous outcome data for the latest time point available within the trial, and always at least 24 months from inception.

We excluded trials where we knew that there were no events in either group. Where trials ran one control group and more than one included intervention group, we used data from the intervention group providing the comparison that best assessed the effect of altering dietary fat. Where the intervention groups appeared equal in this respect, we merged the intervention groups (simply added for dichotomous data, and using the techniques described in Higgins 2011 for continuous data). We had planned that if we identified trials randomised by cluster we would reduce the participant numbers to an "effective sample size" (as described by Hauck 1991); however, we found none that were both included and had cardiovascular events or deaths.

To assess the WHO NUGAG question on the effect of consuming $<10 \% \mathrm{E}$ as SFA relative to $>10 \% \mathrm{E}$ as SFA on the risk of noncommunicable diseases (NCDs) in the population, we combined studies with a control group saturated fat intake of $>$ $10 \% \mathrm{E}$ and an intervention group saturated fat intake of $<10 \% \mathrm{E}$. To assess the effect of a reduction in \%E from SFA from $10 \%$ in gradual increments relative to higher intake, we repeated this with saturated fat cut-offs between $7 \% \mathrm{E}$ and $13 \% \mathrm{E}$.

\section{Subgroup analysis and investigation of heterogeneity}

Prespecified analyses included:

Effects of SFA reduction compared with usual or standard diet on all (primary and secondary) outcomes and potential adverse effects. This main analysis addressed the main objective of the review and the first WHO specific question.

\section{Prespecified subgroups for all outcomes included:}

- energy substitution - we intended to subgroup studies according to the main energy replacement for SFA - PUFA, MUFA, CHO (refined or unrefined), protein, trans fats, a mixture of these, or unclear. However, when we presented these data to the WHO NUGAG group, they suggested that this subgrouping be altered. They suggested that we use all studies where SFA was reduced and any of PUFA, MUFA, CHO or protein were statistically significantly increased $(P<0.05)$ in the intervention compared to the control group to assess the effects of replacement by each, regardless of whether or not it constituted the main replacement for SFA. This meant that some studies appeared in more than one subgroup. As there were almost no data in the studies on trans fats, or on refined and unrefined carbohydrates, we did not include a trans group or distinguish by carbohydrate type. This subgrouping addresses the main objective of the review, and the third WHO specific question.
Further subgroups, run for primary and CVD health-related secondary outcomes only, included:

\section{Prespecified:}

- Baseline SFA intake, represented by control group SFA intake (up to $12 \% \mathrm{E}$ from SFA, > 12 to $15 \% \mathrm{E},>15$ to $18 \% \mathrm{E},>18 \% \mathrm{E}$ from SFA, or unclear)

- Sex (men, women and mixed populations)

- Baseline CVD risk (low-risk or general populations, moderaterisk populations which were defined by risk factors for CVD such as hypertension or diabetes, high-risk populations with existing CVD at baseline)

- Duration in study (mean duration in trial up to 24 months, > 24 to 48 months, $>48$ months, and unclear). Duration was a prespecified subgroup that we used in earlier versions of this review to separate studies with duration of less than two years from those of at least two years. As we have excluded shorter studies from this review, and have access to longer studies, we have explored duration over longer time spans. As some long studies had a high proportion of participants whose time in trial was censored, and we wanted to express mean experience of the trial, we used mean duration of participants in the study, rather than the formal study duration for this subgrouping, so that some two-year intervention trials, because they had some deaths or dropouts, had a mean duration in trial of 21 or 22 months.

\section{WHO NUGAG added subgroups:}

- Degree of SFA reduction, represented by the difference between SFA intake in the intervention and control groups during the study (up to $4 \% \mathrm{E}$ from SFA reduction achieved, $>4$ to $8 \%$ reduction achieved, $>8 \%$ reduction achieved, unclear). We prespecified that we intended to explore the degree of SFA reduction in meta-regression, but its addition as a subgroup was post hoc, and requested by the WHO NUGAG group.

- Serum total cholesterol reduction achieved (reduced by a mean of at least $0.2 \mathrm{mmol} / \mathrm{L}$, reduced by less than $0.2 \mathrm{mmol} / \mathrm{L}$ or unclear). We prespecified that we intended to explore the degree of serum total cholesterol reduction in meta-regression.

- Ethnic group. Insufficient information was presented to make this feasible. Hence, we report ethnicity information in the Characteristics of included studies.

We explored the effects of different levels of SFA, PUFAs, MUFAs and total dietary fats, and $\mathrm{CHO}$ achieved in trials (all as difference between the intervention and control groups, as $\% \mathrm{E}$, and for SFA as a percentage of SFA in the intervention compared with control), baseline SFA intake (as \%E), change in total cholesterol (difference between intervention and control groups, in $\mathrm{mmol} / \mathrm{L}$ ), sex, study duration in months, and baseline CVD risk using metaregression on total cardiovascular events. We performed randomeffects meta-regression (Berkley 1995) using the STATA command metareg (Sharp 1998; Sterne 2001; Sterne 2009).

To explore the WHO NUGAG specific question about the effect of the population consuming $<10 \% \mathrm{E}$ as SFA relative to $>10 \% \mathrm{E}$ SFA, we assessed effects of all studies where the mean assessed intervention SFA intake was $<10 \% \mathrm{E}$ and the mean control SFA intake was $>10 \% \mathrm{E}$. We explored the effect of reduction of $\% \mathrm{E}$ from SFA in gradual increments by using cut-offs of $7 \% \mathrm{E}$ (where 
all studies with a mean intervention SFA intake $<7 \% \mathrm{E}$ and mean control SFA intake $>7 \%$ E were pooled), $8 \%, 9 \%, 10 \%, 11 \%, 12 \%$ and $13 \%$. We omitted studies where SFA intakes were not reported from these analyses. For each primary outcome, we plotted the pooled risk ratio of that outcome against the cut-off, \%E from SFA.

\section{Referee-added subgroups:}

In response to the suggestion of a referee of this systematic review, and to better understand the effect of use of statins since the 1990s, we subgrouped studies by decade of publication.

\section{Sensitivity analysis}

We carried out sensitivity analyses for primary outcomes assessing the effect of:

1. Excluding studies which did not state an aim to reduce SFA

2. Excluding studies which did not report SFA intake during the trial, or did not find a statistically significant reduction in SFA in the intervention compared to the control

3. Excluding studies where total cholesterol (TC) was not reduced (statistically significant reduction of TC, or of LDL where TC was not reported (considered reduced where $P<0.05$ ), or where reduction was not at least $0.2 \mathrm{mmol} / \mathrm{L}$ in intervention compared to control where variance was not reported)

4. Excluding the largest study (WHI 2006)

5. Analysis run with Mantel-Haenszel fixed-effect model

6. Analysis run with Peto fixed-effect model

For this update we also introduced sensitivity analysis excluding trials not at low summary risk of bias. We used results of these analysis to inform GRADE assessment of risk of bias.

\section{GRADE}

All primary outcomes, and secondary additional health events, were represented in the 'Summary of findings' table, and underwent GRADE assessment. The GRADE Working Group has developed a common, sensible and transparent approach to grading quality of evidence and strength of recommendations (www.gradeworkinggroup.org/; GRADE 2004). The evidence within this systematic review was first assessed using the GRADE system by the review authors and then discussed and modified by the WHO NUGAG group.

Outcome data were interpreted as follows:
1. Is there an effect? (options were 'increased risk', 'decreased risk', or 'little or no effect'). Our main outcome measure was RR so we decided on existence of an effect using RR. RR $>8 \%(R R<0.92$ or $>1.08$ ) for the highest quality evidence suggested increased or decreased risk (otherwise little or no effect). The presence or not of an effect was decided on the RR for the main analysis and sensitivity analyses, the highest quality evidence (the main analysis, the sensitivity analyses of trials at low summary risk of bias and at low risk of compliance problems).

2. For continuous outcomes, reducing SFA was considered to have little or no effect unless effect sizes represented at least $5 \%$ change from baseline (or $2 \%$ in the case of cumulative outcomes such as adiposity).

3. Quality of evidence was assessed using GRADE assessment (GRADE 2004) for key outcomes. We used the five GRADE considerations (risk of bias, consistency of effect, imprecision, indirectness and publication bias) to assess the quality of the body of evidence as it related to the studies that contributed data to the meta-analyses for the prespecified outcomes. We used methods and recommendations described in Section 8.5 and Chapter 12 of the Cochrane Handbook for Systematic Reviews of Interventions (Higgins 2011), plus GRADEpro GDT software (GRADEpro 2015). We justified all decisions to downgrade the quality of studies using footnotes and made comments to aid reader's understanding of the review.

4. Where there was a suggested effect, the size of effect was assessed using the number needed to treat for an additional beneficial outcome (NNTB), number needed to treat for an additional harmful outcome (NNTH) or absolute risk reduction (ARR).

\section{RES U LTS}

\section{Description of studies}

\section{Results of the search}

Figure 1 displays the flow diagram for inclusion of studies. We assessed the 7991 titles and abstracts from the updated electronic search, as well as assessing the 8930 titles and abstracts from the search for our sister review (Hooper 2015b), which de-duplicated to 15,314 titles and abstracts. Of these, 530 were considered potentially relevant to one or both reviews, so were collected as full text. Ten publications were considered relevant for this systematic review, and these were grouped into: 
Figure 1. Study flow diagram for this systematic review (update searches run October 2019).

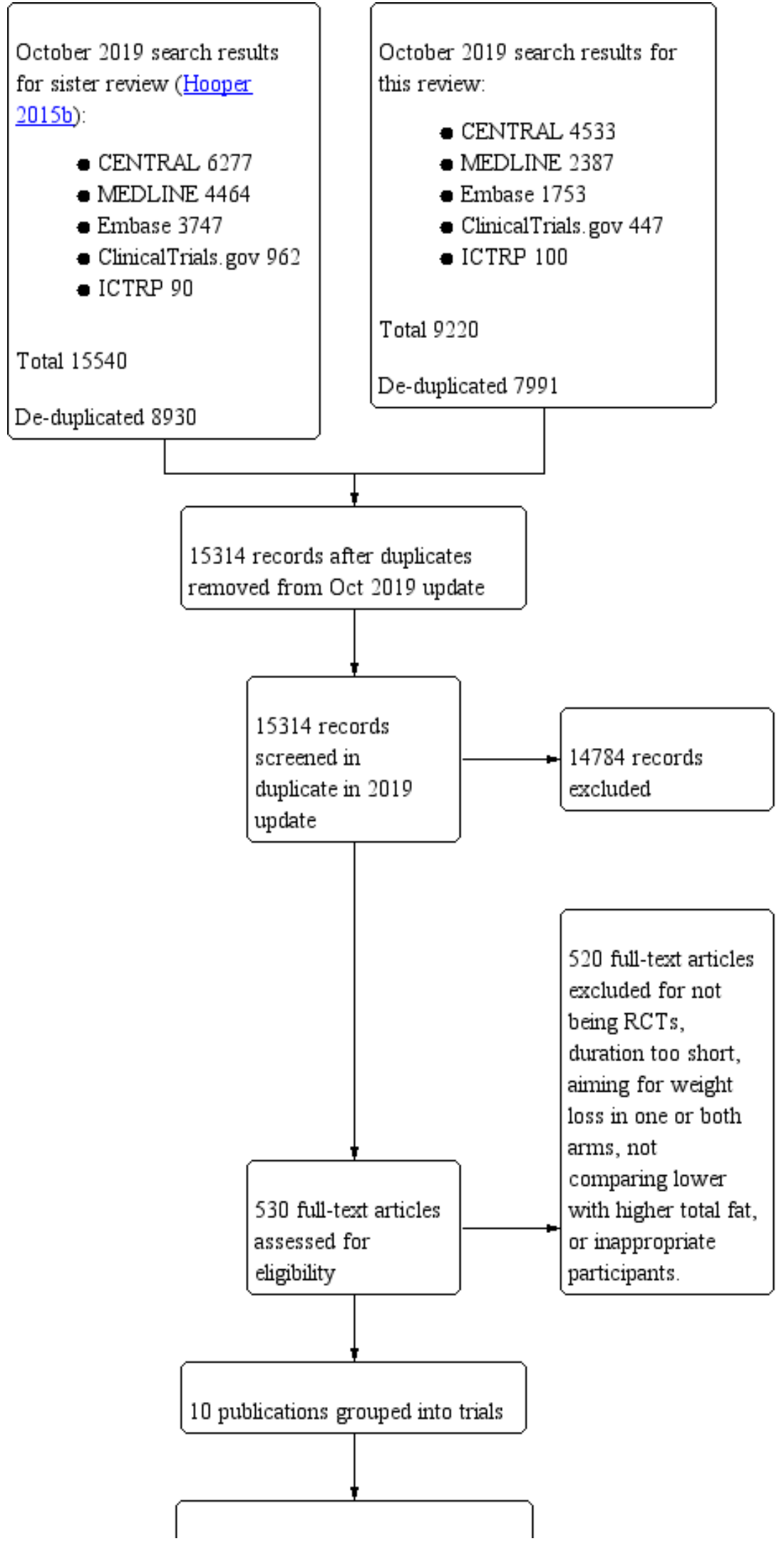

$48 \mathrm{RCT}$ s in overarching review (Hooper 2012) plus 1459 titles and abstracts from the March 2011 searches assessed for previous version of this review ( 2015a)

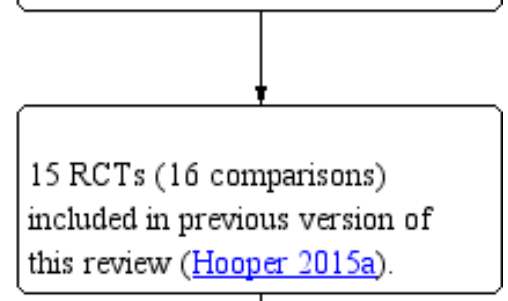


Figure 1. (Continued)

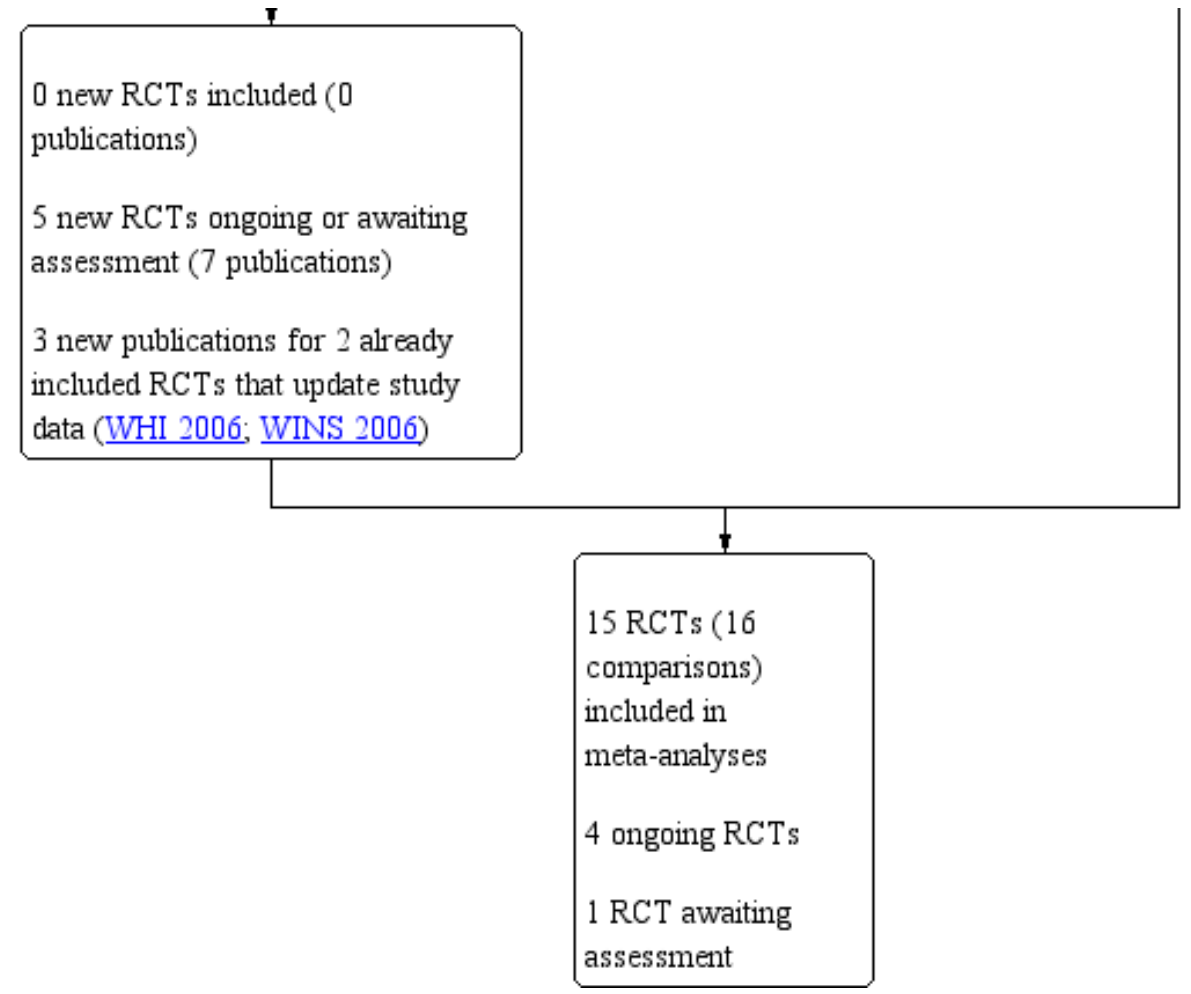

- three new publications for two already included trials (WHI 2006; WINS 2006),

- two publications for one study awaiting assessment (not enough details to confirm inclusion, ICFAMED), and

- five publications for four ongoing trials (ENAbLE due unclear; NCT02481466 due 2020; NCT02938832 due 2023; NEW Soul Study due 2022).

There were no new included trials, but there were new data for WHI 2006 and WINS 2006, as well as the ongoing studies and the study awaiting assessment. This resulted in an updated review including 15 RCTs (16 comparisons as the Rose trial has two comparisons, Rose corn oil 1965 and Rose olive 1965).

\section{Included studies}

We included 15 randomised controlled trials (RCTs) in the review (Included studies), and describe them in Characteristics of included studies. The interventions are compared in Table 1.

The main study papers ranged in publication date from 1965 to 2006, but with supplementary publications included up to 2019. The RCTs were conducted in North America (six), Europe (seven), and Australia/New Zealand (two); no studies were carried out in industrialising or developing countries. Six RCTs included only people at high risk of cardiovascular disease, four at moderate risk, and four at low risk (three with raised cancer risk or cancer diagnosis, one with no specific health risks), while one trial included participants at low and high CVD risk (WHI 2006, Table 1; this trial made assessments in each of these groups). Seven studies included only men, three only women, and five both men and women. However, as the largest trial (WHI 2006) was in women only, women are the largest group represented. Trial duration ranged from two to more than eight years, with a mean duration of 4.7 years.

The form of interventions varied (Table 1 ). Interventions were of advice to alter intake in 15 of the 16 intervention arms, and additional supplements such as oil or other foods were provided in three trials (four arms: MRC 1968; Oslo Diet-Heart 1966; Rose corn oil 1965; Rose olive 1965), while all food was provided in a residential facility in one RCT (Veterans Admin 1969). Of the $15 \mathrm{arms}$ with an advice element, most interventions were delivered face-toface, but this was unclear in three arms (Houtsmuller 1979; Rose corn oil 1965; Rose olive 1965). Advice was provided individually in nine intervention arms (followed by later group sessions in two arms), in groups only in two trials (Ley 2004; WHI 2006), and was unclear in three RCTs (Black 1994; Houtsmuller 1979; Rose corn oil 1965; Rose olive 1965). Advice was provided by a dietitian in nine arms, a nutritionist in one, a trained nurse in one and was unclear in four. Frequency of study visits for advice and follow-up varied between three times in the first year and twice annually thereafter up to 18 sessions in the first year and quarterly maintenance visits thereafter.

Of the 15 included studies (16 intervention arms), 11 RCTs (12 comparisons) provided data on all-cause mortality (including 55,858 participants and 3518 deaths), 10 RCTs (11 comparisons) on CV mortality (53,421 participants and 1096 cardiovascular deaths), and 11 RCTs (12 comparisons) on combined cardiovascular CVD events (53,300 participants, of whom 4476 participants experienced at least one CVD event) (Table 2). In two included studies, it was clear that events had occurred, but it was not clear in which arm(s) the events had occurred (Oxford Retinopathy 1978; Simon 1997), so that we could not include the data in the metaanalyses. Secondary health events and other secondary outcomes 
were reported in varying number of studies (between 1 and 15 studies reported on any single outcome, see Table 2 and Table 3).

\section{Excluded studies}

We excluded 520 full-text publications at this update, having assessed the full texts in duplicate. We describe the reasons for some of these exclusions in Characteristics of excluded studies tables. We excluded 29 studies where data on events were not reported in publications and contact with authors confirmed that there had been no deaths or cardiovascular events, where contact with authors confirmed that data were not available, or where we could not establish contact with authors.

\section{Risk of bias in included studies}

We display 'Risk of bias' assessments in the individual included study arms in Figure 2.

Figure 2. Methodological quality summary: review authors' judgements about each methodological quality item for each included study. Please note that while Rose 1965 (Rose corn oil 1965; Rose olive 1965) appears twice in this 
summary, it is a single trial. Rose 1965 was a 3-arm trial and we have used the two intervention arms separately in the review.

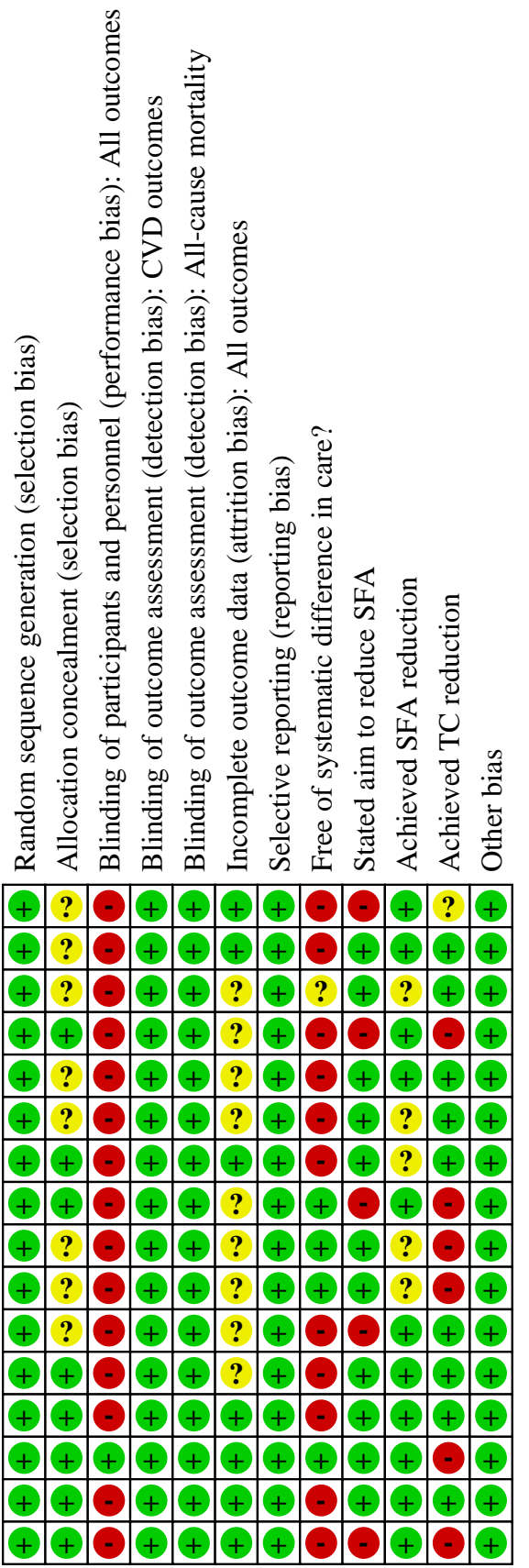

\section{Allocation}

All the included trials were randomised controlled trials, and some detail of the randomisation process was provided for all studies, so all were considered at low risk of bias. We excluded those with detected pseudo-random allocation (for example where participants are randomised according to birth date or alphabetically from their name). We judged allocation concealment

to be well done in eight RCTs (eight comparisons, Ley 2004; Oslo Diet-Heart 1966; Oxford Retinopathy 1978; STARS 1992; Sydney Diet-Heart 1978; Veterans Admin 1969; WHI 2006; WINS 2006), and unclear in the remainder. 


\section{Blinding}

Blinding of participants is not easy in dietary studies, as the participants usually have to follow instructions to attain the specific dietary goals. However, it is feasible in some circumstances, including when food is provided via an institutional setting, or meals provided at a central setting and remaining meals packed to take away. It can also be achieved through use of a trial shop, where very specific food-based dietary advice is provided for all participants, or where the same dietary advice is provided to both groups but a different supplement (e.g. dietary advice to reduce fats, then provision of different oils or fats) is provided. Where participants are not blinded, it is difficult to ensure that study staff, healthcare providers and outcome assessors are blinded. The single RCT that appears to have had adequate participant and study personnel blinding was Veterans Admin 1969, and we judged blinding of participants to be inadequate in the remaining studies.

Blinding of outcome assessment was assessed separately for mortality and CVD outcomes. Blinding is not relevant in assessing all-cause mortality, so all trials were considered at low risk of bias for detection bias for this outcome. For CVD outcomes, nine trials were at low risk of detection bias, one was at high risk and the remainder were unclear.

\section{Incomplete outcome data}

Assessing whether incomplete outcome data had been addressed was difficult, as the primary outcomes for this review (mortality and cardiovascular events) were often reported as dropouts and exclusions from the original studies, rather than as the primary outcomes of these trials. When mortality or cardiovascular events or both were noted in any one study, it is still feasible that some participants left that study feeling unwell or because the diet was inconvenient, so were simply lost to follow-up from the perspective of the study, and later died or experienced a cardiovascular event. However, six of the studies checked medical records or death registers to ensure that such events were all collected (Black 1994, DART 1989; Oslo Diet-Heart 1966; Sydney Diet-Heart 1978; Veterans Admin 1969; WINS 2006). Within one study, there was extensive tracking of medical records, with assessment of health status by blinded trained adjudicators (WHI 2006), so few major events were likely to have been missed. In the other eight studies, it is not possible to know whether additional deaths or cardiovascular events occurred, that were not counted or ascertained within this review.

\section{Selective reporting}

Assessment of selective reporting is difficult when the outcome of interest was simply considered a cause of dropouts in most included studies. We tried to contact all of the trialists to ask about deaths and outcome events, but it is possible that some trialists did not reply as they felt that their data did not reflect the expected or hoped-for pattern of events. All of the included studies have either reported that the participants did not experience any of our primary outcomes, have published their outcome data, or have provided the data they did possess. For this reason, we have graded all the included studies as at low risk of selective reporting.

\section{Other potential sources of bias}

Systematic differences in care. We assessed the studies for risk of bias in relation to systematic differences in care. The three
RCTs (four comparisons) that appeared at low risk of systematic differences in care between the study arms included Rose corn oil 1965; Rose olive 1965; Oxford Retinopathy 1978; Veterans Admin 1969, while 11 RCTs clearly did have differences in care, such as differential time provided for those on the intervention to learn a new diet, and/or differential medical follow-up, and one was unclear (Houtsmuller 1979).

Aim to reduce saturated fat. As several studies did not provide clear aims for their interventions (other than to alter specific dietary components, for example), we assessed whether the study stated an aim to reduce saturated fat. Ten RCTs (11 comparisons) clearly aimed to reduce saturated fat in their intervention arms, either directly or indirectly, for example, by stating food goals (DART 1989; Houtsmuller 1979; Moy 2001; MRC 1968; Oslo Diet-Heart 1966; Rose corn oil 1965; Rose olive 1965; STARS 1992; Sydney Diet-Heart 1978; Veterans Admin 1969; WHI 2006), while the remaining five did not (although they did achieve SFA reduction).

Successful saturated fat reduction. Eleven RCTs (11 comparisons) assessed SFA intake during the study period and showed that SFA intake in the intervention arm was statistically significantly lower than that in the control arm (Black 1994; DART 1989; Ley 2004; Moy 2001; Oxford Retinopathy 1978; Simon 1997; STARS 1992; Sydney Diet-Heart 1978; Veterans Admin 1969; WHI 2006; WINS 2006). The remaining studies did not report SFA intake, so we rated them as unclear.

Successful cholesterol reduction. We would expect saturated fat reduction to be reflected in total or LDL cholesterol reductions, which may be more accurate assessments than self-reported saturated fat intake. Nine RCTs (10 comparisons) provided information on serum total or LDL cholesterol levels in the intervention and control arms during the study, and found a reduction in the intervention arm compared to the control $(P<$ 0.05 , or where variances were not provided showed a reduction of at least $0.2 \mathrm{mmol} / \mathrm{L}$ in the mean intervention measure compared with control). The studies that successfully reduced serum total cholesterol in lower saturated fat arms compared with higher saturated fat arms were DART 1989; Houtsmuller 1979; Simon 1997; STARS 1992; Sydney Diet-Heart 1978; WHI 2006, while Moy 2001 did not report total cholesterol (TC) but showed statistically significant reductions in LDL, and two studies (MRC 1968; Oslo Diet-Heart 1966) did not report variances but did reduce mean TC in the intervention arm compared with control by at least $0.2 \mathrm{mmol} / \mathrm{L}$. One study (Black 1994) did not report lipid levels during the study, while five others did report lipid levels but did not suggest clear differences between lower and higher saturated fat arms (Ley 2004; Oxford Retinopathy 1978; Rose corn oil 1965; Rose olive 1965; Veterans Admin 1969; WINS 2006).

Dietary changes other than saturated fat. Some trials were partially confounded by aiming to make dietary changes other than those directly related to dietary fat intakes; for example, some studies encouraged intervention participants to make changes to their fat intake as well as changes to fruit and vegetable or fibre or salt intakes. In these studies, any effect on outcomes could be a result of other dietary changes, not of changes in saturated fat intake. The 11 studies (12 comparisons) that appeared free of such differences included Black 1994; DART 1989; Houtsmuller 1979; Ley 2004; MRC 1968; Oxford Retinopathy 1978; Rose corn oil 1965; Rose olive 1965; Simon 1997; Sydney Diet-Heart 1978; Veterans Admin 1969; WINS 2006. This factor was not considered alongside others 
in the formal risk of bias assessment (Figure 2) so is described here. We did not identify any further methodological issues.

Summary risk of bias. We considered dietary advice or all-foodprovided type trials to be at low summary risk of bias where we judged randomisation, allocation concealment, and blinding of outcome assessors to be adequate. For CVD outcomes, five trials were assessed as at low summary risk of bias: Ley 2004; Sydney Diet-Heart 1978; Veterans Admin 1969; WHI 2006; WINS 2006. For all-cause mortality (and lipid outcomes) where blinding of outcome assessors is not important, a further three trials were also at low summary risk of bias, eight in total: Ley 2004; Oslo Diet-Heart 1966; Oxford Retinopathy 1978; STARS 1992; Sydney Diet-Heart 1978; Veterans Admin 1969; WHI 2006; WINS 2006.

\section{Effects of interventions}

See: Summary of findings 1 Effect of reducing saturated fat compared to usual saturated fat on CVD risk in adults (note: for the full set of GRADE tables see additional tables 24 to 28)

\section{Primary outcomes}

\section{All-cause mortality}

GRADE assessment suggests that reducing saturated fat intake probably makes little or no difference to all-cause mortality (moderate-quality evidence, downgraded once for imprecision).

There was little or no effect of lower saturated fat compared to higher saturated fat intake on mortality (risk ratio (RR) $0.96,95 \%$ confidence interval $(\mathrm{Cl}) 0.90$ to $1.03, \mathrm{I}^{2}=2 \%, 55,858$ participants, 3518 deaths, 11 RCTs, $P_{\text {effect }}=0.42$, Analysis 1.1 ). This lack of effect was confirmed in sensitivity analyses including only trials at low summary risk of bias (Analysis 1.2), that aimed to reduce saturated fat (Analysis 1.3), that significantly reduced saturated fat intake (Analysis 1.4), that achieved a reduction in total or LDL cholesterol (Analysis 1.5), excluding the largest trial (WHI 2006, Analysis 1.6), or analysing using Mantel-Haenszel or Peto fixed-effect analysis (Analysis 1.7; Analysis 1.8).

Small study bias was assessed using a funnel plot and comparing the results of fixed- and random-effects meta-analysis. The funnel plot did not suggest any small study bias (Figure 3), and the results of fixed- and random-effects meta-analyses were very similar, suggesting that small study bias was not an issue.

\section{Figure 3. Funnel plot of comparison: fat modification or reduction vs usual diet - total mortality.}

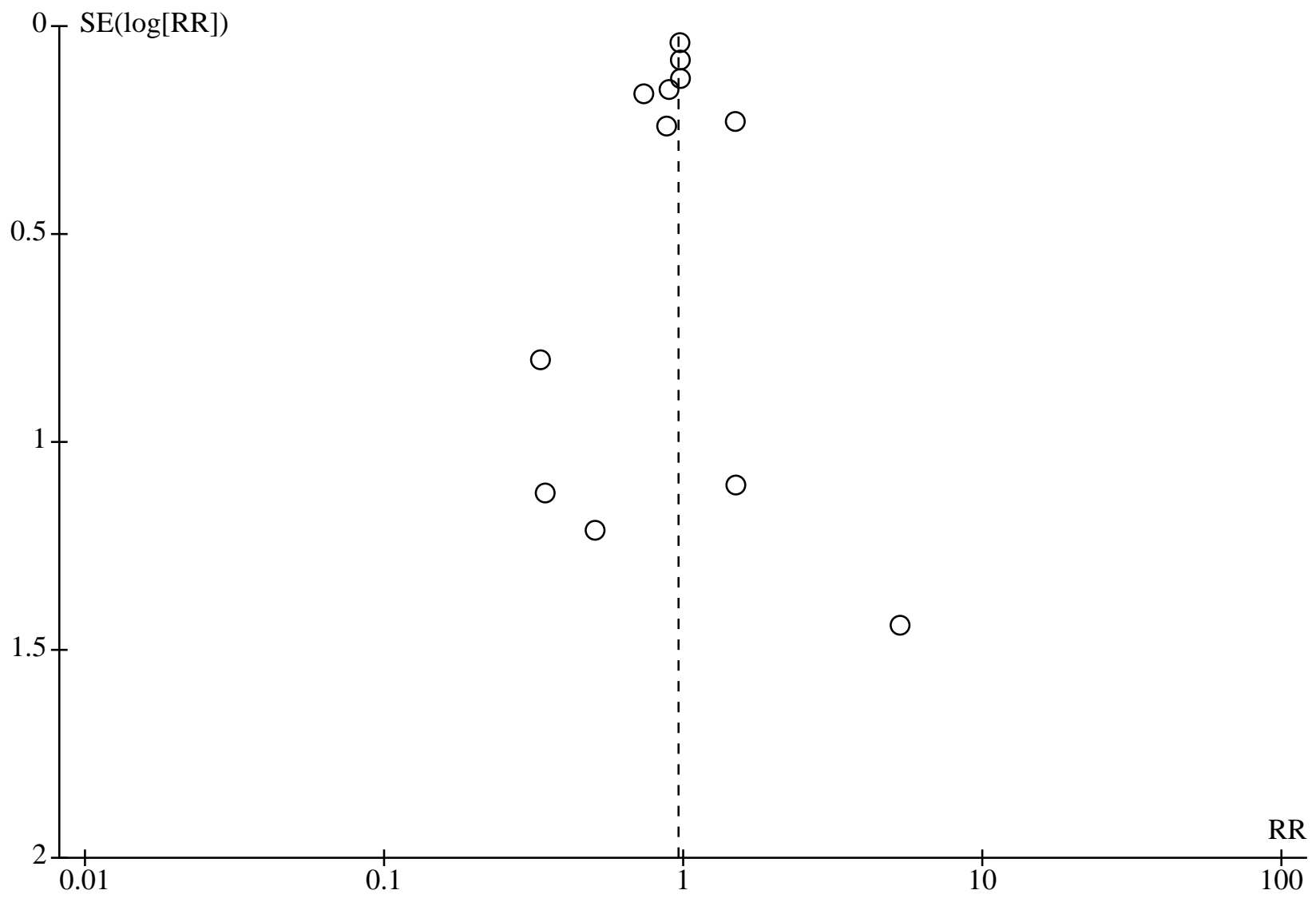

There was little or no effect, regardless of what nutrients were used to replace the saturated fat removed, including replacement with PUFA, MUFA, CHO and/or protein (Analysis 1.9). Effects did not differ by main substitution (Analysis 1.10), study duration (Analysis 1.11), baseline saturated fat intake (Analysis 1.12), degree of difference in saturated fat between arms (Analysis 1.13), participant sex

Reduction in saturated fat intake for cardiovascular disease (Review) 
(Analysis 1.14), by baseline CVD risk (Analysis 1.15), by degree of cholesterol reduction (Analysis 1.16) or by decade of publication (Analysis 1.17, $\mathrm{Chi}^{2}$ test for differences between subgroups all $\mathrm{P}>$ 0.05).

\section{Cardiovascular mortality}

GRADE assessment suggests that reducing saturated fat intake probably makes little or no difference to cardiovascular mortality (moderate-quality evidence, downgraded once for imprecision).

There was little or no effect of SFA reduction on cardiovascular mortality (RR $0.95,95 \% \mathrm{Cl} 0.80$ to $1.12, \mathrm{I}^{2}=30 \%, 10 \mathrm{RCTs}, 53,421$ participants, 1096 cardiovascular deaths, Analysis 1.18). This lack of effect was confirmed in sensitivity analyses limiting to trials at low summary risk of bias (Analysis 1.19), explicitly aiming to reduce saturated fat (Analysis 1.20), achieving statistically significant saturated fat reduction (Analysis 1.21), achieving cholesterol reduction (Analysis 1.22), or running fixed-effect analysis (Analysis 1.24; Analysis 1.25). However, excluding the largest single trial (WHI 2006) suggested that reducing saturated fat intake reduced the risk of CVD mortality (Analysis 1.23).

The funnel plot did not suggest small study bias (Figure 4), and the similarity in effect sizes between fixed-and random-effects analysis suggests that small study bias is not important here.

\section{Figure 4. Funnel plot of comparison: fat modification or reduction vs usual diet - cardiovascular mortality}

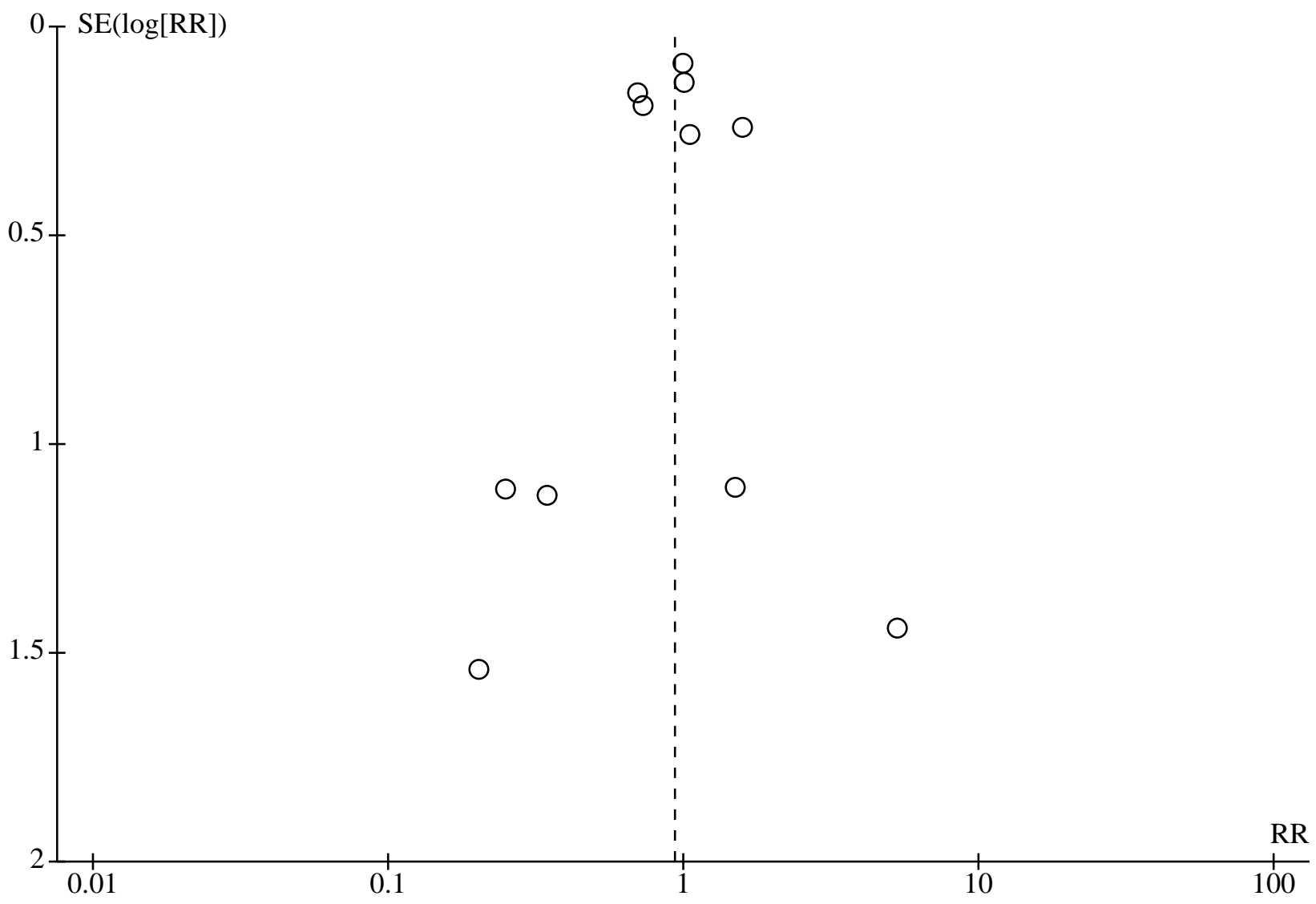

Subgrouping did not suggest important effects of reduced SFA on cardiovascular mortality, regardless of what was substituted for SFA (Analysis 1.26). When subgrouping by main substitution (Analysis 1.27), duration (Analysis 1.28), baseline SFA intake (Analysis 1.29), by difference in SFA (Analysis 1.30), participant sex (Analysis 1.31), baseline CVD risk (Analysis 1.32), or degree of cholesterol reduction (Analysis 1.33), there were no statistically significant differences between subgroups. There was a marginally significant difference between subgroups when ordered by decade of publication, but no clear pattern of effect, so we assumed the effect was probably spurious (Analysis 1.34). Additionally, effects did not appear to relate to statin use, as there was a reduction in risk of CVD mortality in studies published in the 1960s and a marginal increase in risk in the one trial published during the 1970s (although the $95 \%$ confidence interval did include 1.0), both well before statins were in common use (the $4 \mathrm{~S}$ trial which first showed that use of statins reduced mortality was published in 1994, 4S 1994).

\section{Cardiovascular events}

GRADE assessment suggests that reducing SFA intake probably reduces cardiovascular events, to a greater extent with greater cholesterol reduction (moderate-quality evidence, downgraded once for publication bias).

There was a $21 \%$ reduction in cardiovascular events in people who had reduced SFA compared with those on higher SFA (RR 0.79, 95\% 
Cl 0.66 to $0.93, \mathrm{I}^{2}=65 \%, 11 \mathrm{RCTs}, 53,300$ participants, 4476 people with cardiovascular events, $P_{\text {effect }}=0.006$, Analysis 1.35 ). This protective effect was confirmed in sensitivity analyses including only trials at low summary risk of bias (Analysis 1.36), that aimed to reduce saturated fat (Analysis 1.37), that significantly reduced saturated fat intake (Analysis 1.38), that achieved a reduction in total or LDL cholesterol (Analysis 1.39), or excluding the largest trial (WHI 2006, Analysis 1.40). Analysing using Mantel-Haenszel or Peto fixed-effect analysis suggested more marginal protection (Analysis 1.41; Analysis 1.42).
A funnel plot did not suggest severe small-study bias, but it is possible that a few small studies with more cardiovascular events in the intervention groups may be missing from the review (Figure 5). Fixed-effect analyses suggested smaller effects (Analysis 1.41; Analysis 1.42), again suggesting that smaller studies with more cardiovascular events in the intervention groups may be missing. Adding any such studies back would tend to moderate the protective effect of reducing SFA.

Figure 5. Funnel plot of comparison: fat modification or reduction vs usual diet - combined cardiovascular events.

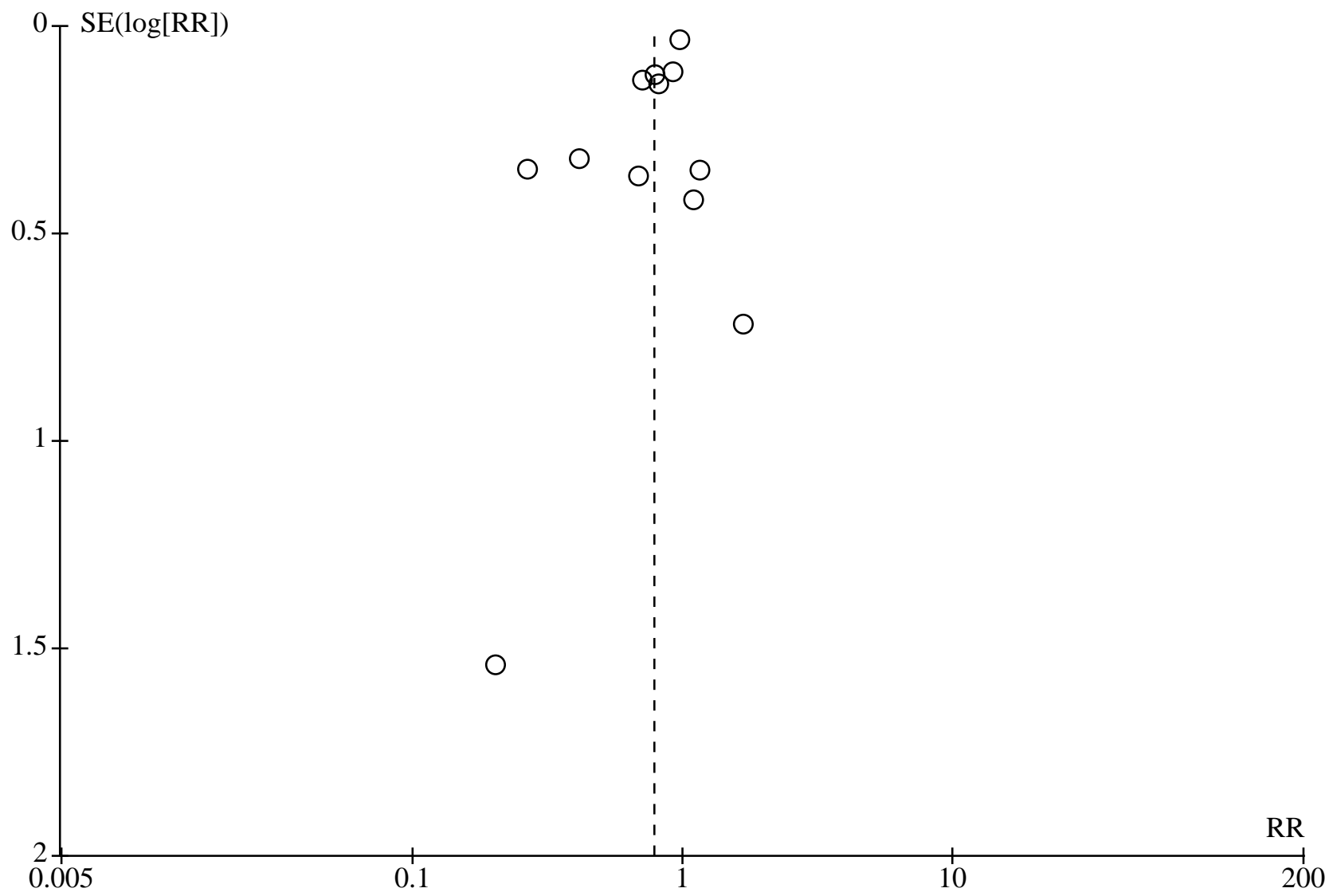

When we subgrouped according to replacement for SFA, the PUFA replacement group suggested a $27 \%$ reduction in cardiovascular events, while there were no clear effects of other replacement groups (Analysis 1.44). However, there were no statistically significant differences between subgroups by main replacement (Analysis 1.45), by study duration (Analysis 1.46), or baseline CVD risk (Analysis 1.50). When subgrouping, there was a suggestion of greater effects when baseline SFA was higher (Analysis 1.47), with greater reduction of SFA (Analysis 1.48), in men rather than women (Analysis 1.49), and with greater cholesterol reduction (Analysis 1.51). There were different effects by decade of publication, but no suggestion of a trend or a change following wider introduction of statins in the mid-1990s (Analysis 1.52).

We further explored the effects of dietary fats on cardiovascular events, by using meta-regression of the difference between the control and intervention of total fat intake, SFA intake, MUFA intake, PUFA intake, $\mathrm{CHO}$ intake (all by percentage of energy (\%E)), serum total cholesterol (in $\mathrm{mmol} / \mathrm{L}$ ) achieved in trials, as well as baseline SFA intake, sex, study duration in months, and CVD risk of participants at baseline (Table 4). As we included only 13 studies for this outcome, we ran meta-regressions exploring single explanatory factors at once, and as data were limited, with many studies not reporting dietary intake data, these analyses were limited in power to assess outcomes. The data suggested that greater reductions in total serum cholesterol levels reduced CVD events more. Greater baseline SFA intake and greater reduction in SFA were also associated with greater improvement in CVD events with SFA reduction, and increases in PUFA and MUFA intakes were slightly protective of CVD events, but none of these relationships were statistically significant. Overall, the relationship with serum total cholesterol was clearest $(P=0.04$, accounting for $99 \%$ of between-study variation). Sex, study duration and 
baseline cardiovascular risk did not appear to influence effect size. Apparent heterogeneity was accounted for by a dose-effect; where SFA reduction resulted in greater serum cholesterol reduction, the reduction in CVD events was greater.

This $21 \%$ reduction in risk of CVD events translated into a number needed to treat for an additional beneficial outcome (NNTB) of 56 in primary prevention trials, so that 56 people need to reduce their saturated fat intake over around four years for one person to avoid experiencing a CVD event. In secondary prevention trials, the NNTB was 32.

\section{Secondary outcomes - health events}

\section{Myocardial Infarction (fatal and non-fatal)}

GRADE assessment suggested that the effect of reducing saturated fat intake on risk of myocardial infarction is unclear as the evidence was of very low-quality (downgraded once each for risk of bias, imprecision and publication bias).

There was a small protective effect of SFA reduction on myocardial infarction (fatal and non-fatal, RR $0.90,95 \% \mathrm{Cl} 0.80$ to 1.01 , $\left.\right|^{2}=$ $10 \%, 10 \mathrm{RCTs}$ (11 comparisons) including 53,167 participants, 1714 people experiencing MI, Analysis 2.1). This protective effect was slightly modified in sensitivity analyses, and confirmed in analyses limited to trials that aimed to reduce saturated fat (Analysis 2.3), that achieved a reduction in total or LDL cholesterol (Analysis 2.5), and excluding the largest trial (WHI 2006, Analysis 2.6). Sensitivity analyses including only trials at low summary risk of bias (RR $0.93,95 \% \mathrm{Cl} 0.81$ to 1.08 , Analysis 2.2 ), that significantly reduced saturated fat intake (Analysis 2.4), analysed using Mantel-Haenszel or Peto fixed-effect analysis (Analysis 2.7; Analysis 2.8) suggested little or no effect, though risk ratios were still all $<1.0$.

The funnel plot was difficult to interpret, but did not raise major concerns about small-study bias (not shown). While effects of random- and fixed-effect meta-analysis were only slightly different, they fell each side of the line suggesting an effect (Analysis 2.7; Analysis 2.8). There may be a small amount of small study bias.

The protective effect of replacing SFA with PUFA appeared to explain the reduction in MI (Analysis 2.9), but there were no statistically significant differences between subgroups by replacement (Analysis 2.10), duration (Analysis 2.11), baseline SFA intake (Analysis 2.12), change in SFA intake (Analysis 2.13), participant sex (Analysis 2.14), baseline CVD risk (Analysis 2.15), cholesterol reduction (Analysis 2.16) or decade of publication (Analysis 2.17).

\section{Myocardial Infarction (non-fatal only)}

GRADE assessment suggests that reducing saturated fat may have little or no effect on risk of non-fatal myocardial infarction (lowquality evidence, downgraded once each for risk of bias and imprecision).

There was no clear effect of SFA reduction compared to usual diet on non-fatal myocardial infarction (RR $0.97,95 \% \mathrm{Cl} 0.87$ to $1.07, \mathrm{I}^{2}$ $=0 \%, 7$ RCTs, 52,834 participants, 1385 people with at least one non-fatal MI, Analysis 2.18). This lack of effect was not altered in sensitivity analyses retaining only those that aimed to reduce SFA (Analysis 2.20), those showing a reduction in serum cholesterol (Analysis 2.22), or fixed-effect analysis (Analysis 2.24; Analysis 2.25). However, sensitivity analyses retaining only trials at low summary risk of bias (RR $0.89,95 \% \mathrm{Cl} 0.58$ to 1.35 , Analysis 2.19), those showing a significant reduction in SFA (Analysis 2.21), and omitting the largest trial (WHI 2006, Analysis 2.23) all suggested a reduction in non-fatal MI with reduced SFA.

The funnel plot did not raise major concerns about small-study bias (not shown), and effects of fixed-and random-effects analyses were very similar, reinforcing the lack of small study bias.

Subgrouping by any replacement for SFA suggested reductions in non-fatal MI when replaced by PUFA, but not other replacements (Analysis 2.26). Subgrouping by main substitution (Analysis 2.27), duration (Analysis 2.28), baseline SFA intake (Analysis 2.29), degree of SFA reduction (Analysis 2.30), sex (Analysis 2.31), baseline CVD risk (Analysis 2.32), degree of cholesterol reduction (Analysis 2.33) and decade of publication (Analysis 2.34) did not suggest significant differences between subgroups.

\section{Stroke (any type, fatal or non-fatal)}

GRADE assessment suggests that the effect of reducing SFA intake on stroke is unclear as the evidence is of very low-quality (downgraded twice for imprecision and once for risk of bias).

As data on stroke were sparse, it was not possible to tease out differential effects on ischaemic or haemorrhagic strokes, or whether a stroke was fatal. For this analysis, we combined all stroke data from any study. There was little or no effect of SFA reduction compared to usual diet on stroke of any type with any outcome (RR $0.92,95 \% \mathrm{Cl} 0.68$ to $1.25, \mathrm{I}^{2}=9 \%, 7$ RCTs, 50,952 participants, 1118 people with stroke, Analysis 2.35). This lack of effect was not altered in sensitivity analyses retaining only those that aimed to reduce SFA (Analysis 2.37), those showing a reduction in serum cholesterol (Analysis 2.39), or fixed-effect analysis (Analysis 2.41; Analysis 2.42). However, for sensitivity analyses retaining only trials at low summary risk of bias (RR $0.76,95 \% \mathrm{Cl} 0.42$ to 1.38 , Analysis 2.36), those showing a significant reduction in SFA (Analysis 2.38), and omitting the largest trial (WHI 2006, Analysis 2.40) the best estimate of effect always suggested a reduction in stroke with reduced SFA, though they were not statistically significant.

We did not create a funnel plot as the analysis only included data from seven RCTs, however RRs generated using fixed-effect analyses were much closer to 1.0 than the random-effects metaanalysis (suggesting a small amount of publication bias), though both suggested little or no effect.

Subgrouping by any substitution for SFA suggested reduction in risk of stroke whether SFA was replaced by PUFA, CHO or protein (Analysis 2.43). Subgrouping did not suggest significant differences between subgroups by main substitution (Analysis 2.44), duration (Analysis 2.62), baseline SFA (Analysis 2.46), SFA change (Analysis 2.47), sex (Analysis 2.48), CVD risk (Analysis 2.49), cholesterol reduction (Analysis 2.50) or decade of publication (Analysis 2.51).

\section{Coronary heart disease (CHD) mortality}

GRADE assessment suggests that reducing saturated fat intake may have little or no effect on CHD mortality (low-quality evidence, downgraded twice for imprecision).

Eight RCTs (9 comparisons) suggest little or no effect of reducing saturated fat on risk of CHD mortality (RR $0.97,95 \% \mathrm{Cl} 0.82$ to 1.16 , $1^{2}=28 \%, 53,159$ participants, 927 people died of coronary heart 
disease, Analysis 2.52), and this was not altered in any sensitivity analyses (Analysis 2.53; Analysis 2.54; Analysis 2.55; Analysis 2.56; Analysis 2.57; Analysis 2.58; Analysis 2.59).

We did not create a funnel plot as the analysis only included data from seven RCTs, but the results of fixed- and random-effects analyses were nearly identical, suggesting that small study bias is not an issue here.

There was no suggestion of an effect of reducing SFA on CHD mortality regardless of what replaced the SFA (Analysis 2.60). There were no statistically significant differences between subgrouping in any analysis (Analysis 2.61; Analysis 2.62; Analysis 2.63; Analysis 2.64; Analysis 2.65; Analysis 2.66; Analysis 2.67; Analysis 2.68).

\section{Coronary heart disease events}

GRADE assessment suggested that the effect of reducing saturated fat on CHD events is unclear as the evidence is of very lowquality (downgraded once each for imprecision, risk of bias and inconsistency).

There was the suggestion of a $17 \%$ reduction in CHD events as a result of saturated fat reduction in the main analysis (RR $0.83,95 \%$ $\mathrm{Cl} 0.68$ to $1.01, \mathrm{I}^{2}=62 \%, 53,199$ participants, 2261 people had at least one coronary heart disease event in 10 RCTs, Analysis 2.69). This did not differ in sensitivity analyses (Analysis 2.74; Analysis 2.72; Analysis 2.73; Analysis 2.71; Analysis 2.75; Analysis 2.76) except when limiting to trials at low summary risk of bias (RR $0.92,95 \% \mathrm{Cl}$ 0.77 to 1.10 , Analysis 2.70 ).

The funnel plot did not appear unbalanced, and the results of fixed- and random-effects analyses were different, though both suggested that reducing SFA resulted in lower risk of CHD.

Subgrouping by any replacement for SFA suggested that replacement by PUFA may lead to reduced risk of $\mathrm{CHD}$ events (Analysis 2.77). There were no statistically significant differences between any other subgroups (Analysis 2.78; Analysis 2.79; Analysis 2.80; Analysis 2.81; Analysis 2.82; Analysis 2.83; Analysis 2.84) except by decade of publication, though this did not suggest any sequence or step change (Analysis 2.85).

\section{Type 2 diabetes, new diagnoses}

Only one RCT reported on diagnosis of diabetes (WHI 2006). There was little or no effect of reducing SFA intakes on diagnosis of diabetes in this study (RR $0.96,95 \% \mathrm{Cl} 0.90$ to $1.02,48,835$ participants, 3342 developed diabetes, Analysis 2.86). WHI 2006 was assessed at low summary risk of bias, aimed to reduce SFA, and demonstrated significant SFA and cholesterol reduction. With only one trial, we were not able to assess publication bias or carry out subgrouping.

\section{Secondary outcomes - blood levels}

\section{Serum blood lipids}

Total cholesterol (TC): There was a reduction in TC in participants with reduced SFA compared to higher SFA (mean difference (MD) $-0.24 \mathrm{mmol} / \mathrm{L}, 95 \% \mathrm{Cl}-0.36$ to $-0.13, \mathrm{I}^{2}=60 \%, 13 \mathrm{RCTs}, 7115$ participants, Analysis 3.1). We did not conduct sensitivity analyses or most subgroupings on secondary outcomes, but there was no clear differential effect on TC depending on the replacement for SFA (PUFA, MUFA, CHO or a mixture, Analysis 3.2; Analysis 3.3).
The funnel plot did not raise concerns about small-study bias (not shown).

Low-density lipoprotein (LDL): There was a reduction in LDL in participants with reduced SFA compared to higher SFA (MD $-0.19 \mathrm{mmol} / \mathrm{L}, 95 \% \mathrm{Cl}-0.33$ to $-0.05, \mathrm{I}^{2}=37 \%, 5 \mathrm{RCTs}, 3291$ participants, Analysis 3.4). There was no clear differential effect on LDL depending on the replacement for SFA (PUFA, MUFA, CHO or a mixture, Analysis 3.5; Analysis 3.6). We could not interpret the funnel plot due to sparsity of studies (not shown).

High-density lipoprotein (HDL): There was little or no effect of reducing SFA intakes on $\mathrm{HDL}$ (MD $-0.01 \mathrm{mmol} / \mathrm{L}, 95 \% \mathrm{Cl}-0.02$ to 0.01 , $\mathrm{I}^{2}=0 \%, 7 \mathrm{RCTs}, 5147$ participants, Analysis 3.7). There was no clear differential effect on HDL depending on the replacement for SFA (PUFA, MUFA, CHO or a mixture, Analysis 3.8; Analysis 3.9). We could not interpret the funnel plot due to sparsity of studies (not shown).

Triglycerides (TG): There was little or no effect of reducing SFA intakes on TG (MD $-0.08 \mathrm{mmol} / \mathrm{L}, 95 \% \mathrm{Cl}-0.21$ to $0.04, \mathrm{I}^{2}=51 \%$, 7 RCTs, 3845 participants, Analysis 3.10). There was no clear differential effect on TG depending on the replacement for SFA (PUFA, MUFA, CHO or a mixture, Analysis 3.11; Analysis 3.12). We could not interpret the funnel plot due to sparsity of studies (not shown).

TG/HDL ratio: We did not find any studies that reported TG/HDL ratio.

TC/HDL ratio: Only three RCTs reported on TC/HDL ratio. There was little or no effect of reducing SFA intakes on TC/HDL (MD -0.10, $95 \% \mathrm{Cl}-0.33$ to $0.13, \mathrm{I}^{2}=24 \%, 2985$ participants, Analysis 3.13). There were no clear differential effects of replacement on TC/HDL (Analysis 3.14; Analysis 3.15). We could not interpret the funnel plot due to sparsity of studies (not shown).

LDL/HDL ratio: Only one RCT reported on LDL/HDL ratio. There was no clear effect of reducing SFA intakes on LDL/HDL in this study (MD $-0.36,95 \% \mathrm{Cl}-0.92$ to $0.20,50$ participants, Analysis 3.16$)$. This study replaced SFA with $\mathrm{CHO}$ (mainly) and PUFA.

Lipoprotein (a) (Lp(a)): Only two RCTs reported on lipoprotein (a), but these included 28,820 participants. There was little or no effect of reducing SFA intakes on $\mathrm{Lp}(\mathrm{a})$ (MD $0.00,95 \% \mathrm{Cl}-0.00$ to $0.00, \mathrm{I}^{2}$ $=0 \%$, Analysis 3.17). There was no suggestion of differential effects of replacement on Lp(a) (Analysis 3.18; Analysis 3.19). We could not interpret the funnel plot due to sparsity of studies (not shown).

Homeostatic model assessment (HOMA): Only one RCT reported on the effects of reducing SFA on insulin resistance using HOMA. There was little or no effect of reducing SFA intakes compared to usual diet on HOMA in this study (MD $-0.00,95 \% \mathrm{Cl}-0.04$ to 0.04 , 2832 participants, Analysis 3.20).

Glucose at two hours post-glucose tolerance test (GTT): Only three RCTs reported on glucose two hours post-GTT. There was a reduction in glucose after reducing SFA intakes compared to usual diet (MD $-1.69 \mathrm{mmol} / \mathrm{L}, 95 \% \mathrm{Cl}-2.55$ to $-0.82, \mathrm{I}^{2}=45 \%, 249$ participants, Analysis 3.20). We could not interpret the funnel plot due to sparsity of studies (not shown).

HbA1c (glycosylated haemoglobin): HbA1c was not measured in any included RCT. 


\section{Secondary outcomes - other outcomes and potential harms}

There was little or no effect of reducing SFA intakes on cancer diagnoses of any type (RR $0.94,95 \% \mathrm{Cl} 0.83$ to $1.07, \mathrm{I}^{2}=33 \%$, 4 RCTs, 52,294 participants, 5476 cancer diagnoses, Analysis 4.1); cancer deaths $\left(\mathrm{RR} 1.00,95 \% \mathrm{Cl} 0.61\right.$ to $1.64, \mathrm{I}^{2}=49 \%, 5 \mathrm{RCTs}$, 52,283 participants, 2472 cancer deaths, Analysis 4.2); systolic blood pressure (MD $-0.19 \mathrm{mmHg}, 95 \% \mathrm{Cl}-1.36$ to $0.97, \mathrm{I}^{2}=0 \%, 5$ RCTs, 3812 participants, Analysis 4.5); diastolic blood pressure (MD $-0.36 \mathrm{mmHg}, 95 \% \mathrm{Cl}-1.03$ to $0.32, \mathrm{I}^{2}=0 \%, 5 \mathrm{RCTs}, 3812$ participants, Analysis 4.6).

There was evidence that reducing SFA intake resulted in small reductions in body weight (MD $-1.97 \mathrm{~kg}, 95 \% \mathrm{Cl}-3.67$ to $-0.27, \mathrm{I}^{2}=$ 72\%, 6 RCTs, 4541 participants, Analysis 4.3), and body mass index (MD $-0.50,95 \% \mathrm{Cl}-0.82$ to $-0.19, \mathrm{I}^{2}=55 \%, 6$ RCTs, 5553 participants, Analysis 4.4).

Only one RCT reported assessing quality of life. The Women's Health Initiative (WHI 2006) assessed quality of life at baseline using the SF-36 tool). They found that being in the lower SFA arm resulted in a small improvement in Global Quality of Life at trial close-out (on a scale of 0 worst to 10 best, MD $0.04,95 \% \mathrm{Cl} 0.01$ to 0.07 , Analysis 4.7 ). This very small effect (less than $1 \%$ change) was statistically significant but unlikely to be relevant to individuals. However, it suggests no reduction in quality of life in those reducing their saturated fat.

\section{Other results}

To assess the effect in the population of consuming $<10 \% \mathrm{E}$ as SFA relative to $>10 \% \mathrm{E}$ as SFA for reduction in risk of noncommunicable diseases (NCDs), we combined studies with a control group saturated fat intake of $>10 \% \mathrm{E}$ and an intervention group saturated fat intake of $<10 \% \mathrm{E}$ for all-cause mortality, cardiovascular and coronary heart disease mortality and events, myocardial infarctions, non-fatal myocardial infarctions, and stroke. To assess the effect in the population of a reduction in \%E from SFA from $10 \%$ in gradual increments relative to higher intake we repeated this with saturated fat cut-offs between $7 \% \mathrm{E}$ and $13 \% \mathrm{E}$. The data for these cut-offs are shown in Table 5, and were plotted for a visual overview (Figure 6). The figure suggests reductions in cardiovascular outcomes in studies where saturated fat intake was greater than $10 \% \mathrm{E}$ in control groups, and less than $10 \% \mathrm{E}$ in intervention groups.

Figure 6. Exploration of saturated fat cut-offs

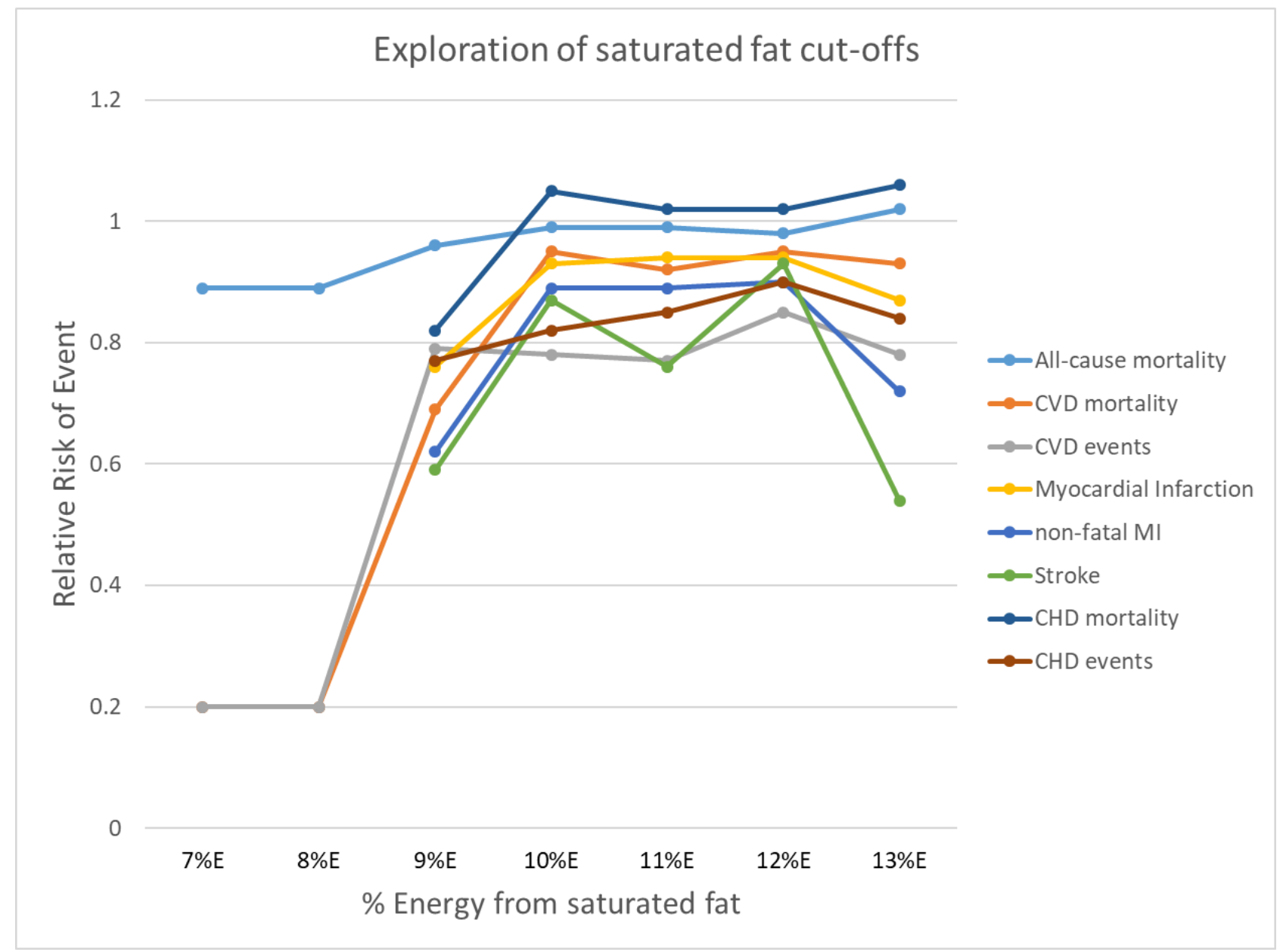


Additional WHO NUGAG specific questions:

\section{In adults what is the effect in the population of reduced percentage of energy (\%E) intake from saturated fatty acids (SFA) relative to higher intake for reduction in risk of noncommunicable diseases (NCDS)?}

We found that reducing saturated fat for at least two years suggested no clear effects on all-cause or cardiovascular mortality, but a $21 \%$ reduction in combined cardiovascular events. Heterogeneity in this result was partially explained by greater reductions in cardiovascular events in studies with greater serum total cholesterol reductions (implying greater reductions in SFA intake). Effects of reducing SFA on other cardiovascular and cancer outcomes were either very small or unclear (as the evidence was of very low quality), but it should be noted that risk ratios were all 1.0 or lower - no harm was indicated. Effects on NCD risk factors were small but positive (serum total cholesterol, LDL cholesterol, systolic and diastolic blood pressure, weight and $\mathrm{BMI}$ ) or neutral (HDL cholesterol and TGs).

\section{What is the effect of reducing SFA on coronary heart disease mortality and coronary heart disease events?}

We found little or no effect of reducing SFA on non-fatal MI and CHD events, but the evidence on MI, stroke and CHD events was of very low quality. However, all risk ratios were less than 1.0.

What is the effect in the population of replacing SFA with PUFAs, MUFAs, $\mathrm{CHO}$ (refined versus unrefined), protein or trans fatty acids (TFAs) relative to no replacement for reduction in risk of NCDs?

We found greater reductions in cardiovascular events in studies that replaced saturated fats by PUFAs or $\mathrm{CHO}$ than in studies with replacement with MUFAs or protein, where there was little evidence of any effect.

\section{What is the effect of replacing some saturated fat with PUFA on risk of CVD in adults?}

There is moderate-quality evidence that replacing saturated fat with PUFA probably reduces the risk of CVD events. Replacing SFA with PUFA also appears to reduce the risk of total MI, non-fatal $\mathrm{MI}$, stroke and CHD events, but has little or no effect on all-cause mortality, CVD mortality and CHD mortality.

\section{What is the effect of replacing some saturated fat with MUFA on risk of CVD in adults?}

The evidence for effects of replacing SFA with MUFA was very limited, so assessment of health effects was not possible.

\section{What is the effect of replacing some saturated fat with $\mathrm{CHO}$ on risk of CVD in adults?}

While studies that replaced SFA with $\mathrm{CHO}$ reduced CVD events and stroke, effects on all-cause mortality and other CVD outcomes suggested little or no effect.

\section{What is the effect of replacing some saturated fat with protein on risk of CVD in adults?}

There was no evidence suggesting that replacing SFA with protein reduced all-cause mortality or any CVD outcomes, but the evidence was limited.
What is the effect in the population of consuming $<10 \% E$ as SFA relative to $>10 \% E$ as SFA for reduction in risk of NCDs?

Cut-off data were difficult to interpret, and confidence intervals were wide, but they suggested greater reductions in cardiovascular events in studies where saturated fat intake was greater than $10 \% \mathrm{E}$ in control groups, and less than $10 \% \mathrm{E}$ in intervention groups (see Figure 6).

What is the effect in the population of a reduction in \%E from SFA from $10 \%$ in gradual increments relative to higher intake for reduction in risk of NCDs?

The data from RCTs are too limited to be able to address this question.

\section{DISCUSSION}

\section{Summary of main results}

This systematic review of long-term randomised controlled trials of SFA reduction suggests that reducing saturated fat for at least two years probably has little or no effect on all-cause or cardiovascular mortality, but probably caused a $21 \%\left(95 \% \mathrm{Cl} 7\right.$ to $34 \%, 1^{2}=$ $65 \%$, moderate-quality evidence) reduction in people experiencing cardiovascular events. The heterogeneity in this relationship was explained by greater reduction in CVD events in trials with greater serum cholesterol lowering. This effect on cardiovascular events was retained in sensitivity analyses. Subgrouping suggested that there was a $27 \%(95 \% \mathrm{Cl} 8$ to $42 \%)$ reduction in cardiovascular events in studies that replaced saturated fats by PUFAs, and a $16 \%(95 \% \mathrm{Cl}-6$ to $33 \%)$ reduction in studies replacing with $\mathrm{CHO}$, with little information on the effect of replacing with MUFAs or protein. The difference between subgroups was not statistically significant. We could not explore data on trans fats. Meta-regression and subgrouping suggested that greater reductions in SFA intake, greater reductions in total serum cholesterol levels, higher baseline SFA intake and greater increases in PUFA and MUFA intakes reduced CVD events more, but the strongest factor was the degree of cholesterol lowering. This clearly indicates that the cardiovascular effects of reducing saturated fat rely on changes in atherosclerosis via serum cholesterol. The degree of cholesterol lowering reflects greater reduction in SFA and greater increase in PUFA (Hegsted 2000).

There may be little or no effect of SFA reduction on non-fatal MI or CHD mortality (both low-quality evidence), and effects on fatal and non-fatal MI, stroke and CHD events were unclear (as the evidence was of very low-quality). However, risk ratios were less than 1.00 for all of these. While we found small reductions in body weight and body mass index with advice to reduce saturated fats, there was little or no effect on diabetes diagnoses, cancer diagnoses or cancer deaths, or on systolic or diastolic blood pressure.

Reducing saturated fat caused reductions in serum total and LDL cholesterol, which did not differ according to type of replacement. There was little or no effect of saturated fat reduction on serum HDL cholesterol or triglyceride. Data on lipid ratios, Lp(a) and HOMA were very limited and effects unclear, but SFA reduction appears to reduce glucose two hours after a glucose load. 


\section{Overall completeness and applicability of evidence}

The review included adult participants at varying levels of risk of cardiovascular disease, men and women, with mean ages from 46 to 66 years at baseline, in free-living and institutional settings, and across the past 50 years. All the studies were conducted in industrialised countries, and no data were available from developing or transitional countries. The effectiveness of SFA reduction has been well assessed, with trials of at least 24 months including more than 50,000 participants for all primary and secondary CVD outcomes. Three thousand five hundred and eighteen participants in the included trials died, 1096 died of a cardiovascular cause, and 4476 experienced at least one cardiovascular event.

The external validity of the review in industrialised countries, men and women, people with low, moderate and high risk of cardiovascular disease was high, but it is not clear how this evidence relates to diets in developing and transitional countries.

\section{Quality of the evidence}

All 15 trials (16 comparisons) included were randomised controlled trials, allocation concealment was judged well done in eight RCTs and blinding of outcome assessors adequate in nine trials assessing CVD outcomes (and all trials assessing all-cause mortality). Blinding of participants is difficult and expensive in dietary fat trials, but was adequate in one trial. We judged incomplete outcome data not to be a problem in seven RCTs, and selective reporting was not a problem in any trial. Three trials were free of differences in care between the intervention and control arms, 10 RCTs stated an aim to reduce saturated fat, 11 showed evidence they had reduced SFA intake (all studies did one or the other), and nine studies showed clear reductions in total cholesterol. Five trials were at low summary risk of bias.

The lack of blinding of participants in most dietary trials is unlikely to alter outcome assessment when outcomes include death and cardiovascular events, but lack of blinding in the participants may have led those in the control groups to alter their lifestyle and dietary practices (for example, feeling that they have not been helped to reduce their cardiovascular risk, they may act to reduce their own risk by altering other lifestyle behaviours such as smoking or exercise, leading to a potential lessening of the apparent effect of the dietary intervention). Systematic differences in care between arms may have led to intervention groups receiving additional support in areas like self efficacy and gaining support from new social circles, potentially beneficial to health regardless of dietary fat intake, or gaining additional healthcare professional time, possibly leading to earlier diagnosis and treatment of other risk factors such as raised blood pressure. Additional dietary messages such as those around fruit and vegetable intake, fibre, alcohol and sugars, present in many studies, may have been protective, or may have diluted the effect or attainability or both of the saturated fat goals.

The quality of evidence balances the uncertainty over allocation concealment, lack of blinding and presence of systematic differences in care and additional dietary differences between arms (Figure 2) with the scale and consistency of the evidence across studies and across decades, despite very different designs and design flaws. For this reason, there is moderate-quality evidence that the interventions that reduce dietary saturated fat intake reduce the risk of cardiovascular events.

\section{Complex interventions}

With complex interventions, such as dietary interventions, there are additional questions that need to be asked about included studies. Important issues to consider include defining the intervention, searching for and identifying all relevant studies, selecting studies for inclusion and data synthesis (Lenz 2007; Sheppherd 2009), as well as questions around whether the intended intervention was realised in study participants during the study.

For this review, we have worked to define the interventions clearly (see Characteristics of included studies), providing information on the type of intervention, stating the study aims and methods for each arm and the assessed total and saturated fat intakes attained within the study. However, while we have characterised the interventions, no two studies that reduced SFA had exactly the same dietary goals for the intervention groups. Methods of attaining the dietary goals varied from providing a whole diet over several years (in studies based in institutions) to providing advice on diet alongside supplementary foods such as margarines or oils, to providing dietary advice with or without supplementary support in the way of group sessions, cooking classes, shopping tours, feedback, self-efficacy sessions and/or individual counselling. We aimed to use this variety to support generalisability for the effects of the interventions.

We aimed to identify all the relevant studies through use of a broad search strategy, which was time-consuming. However, we believe that we have included most relevant trials. We also carefully defined acceptable interventions for each arm, to simplify decisions on inclusion, and the two independent assessors often agreed. We augmented data synthesis by subgrouping and meta-regression, to help us understand the effects of individual elements of dietary fat changes.

A study that sets out to assess the effect of a $30 \%$ reduction in saturated fat intake may attain this level of reduction in some participants, exceed it in some and not achieve it at all in others. The actual mean change attained in the intervention group may be less dramatic than that aimed for, and the participants in the control group may also reduce their saturated fat intake by a small amount, narrowing the difference in saturated fat between the groups further and so reducing the scale of any outcome. This can be dealt with in the systematic review if we meta-regress the difference in saturated fat intake between the intervention and control group with the scale of the outcome (assuming a linear dose response), still allowing us to understand the effect of altering saturated fat intake. However, it is difficult to measure actual saturated fat intake achieved. Some trials did not report it, either because they did not assess it, or did assess it but didn't report this relatively uninteresting outcome. Other trials did report the results of asking people what they were eating, using a food frequency questionnaire or several 24-hour food recalls. However, there is good evidence to believe that asking people how they are eating may produce somewhat biased information (Kristal 2005; Schatzkin 2003), and this may be a greater problem where the participant has been recently urged to eat in a particular way, as in a dietary trial. Assessment of change in total cholesterol is a way to get over self-reporting of dietary intake as reducing saturated 
fat reduces total and LDL cholesterol. This review suggests that the relationship between saturated fat reduction and CVD events is moderated by the degree of cholesterol lowering, which is exactly what would be expected of a true effect.

The interventions used in the studies included in this review were varied, with some participants given all their food over a long period of time in an institutional setting, while most participants were given advice on how to achieve dietary changes, with or without the support of supplements such as oils and foods (Table 1). Advice was provided by a variety of health professionals, and with different levels of intensity. The effect of this was that different degrees of saturated fat reduction were achieved in different studies. The level of compliance with interventions involving long-term behaviour change, such as those used in these studies, can vary widely. This is likely to attenuate the pooled effect and bias it towards the null. Insofar as we were able to understand this issue, subgrouping and meta-regression suggested that greater reductions in saturated fats were associated with greater reductions in the risk of cardiovascular disease events. This suggestion of a dose response strengthens our belief that there is a true effect of reducing saturated fat on CVD events.

\section{Potential biases in the review process}

In compiling the included studies, we worked hard to locate randomised studies that altered dietary SFA intake for at least 24 months, even when cardiovascular events were not reported in study publications, or where such events were reported incidentally as reasons for participant dropouts. We attempted to contact all authors of potentially includable studies to verify the presence or absence of our outcomes. In many studies, no outcomes relevant to this review occurred or were recorded, and the numbers of events occurring within single studies varied from none to over 2500 deaths, over 500 cardiovascular deaths, and over 3000 participants experiencing at least one cardiovascular event (all within WHI 2006, the largest single study with almost 50,000 female participants for many years).

The number of cardiovascular deaths across the review was relatively small (1096), so while we can be quite confident in reporting a reduction in participants experiencing cardiovascular events (4476 events) with SFA reduction, and a lack of effect on total mortality (3518 deaths) within the studies' time scales, the effect on cardiovascular mortality is less clear. The risk ratio of 0.94 (95\% $\mathrm{Cl} 0.78$ to 1.13 , Analysis 1.18) may translate into a small protective effect, but this is unclear.

The lack of effect on individual cardiovascular events is harder to explain; there were 1714 people experiencing MIs, 1118 strokes and 1385 non-fatal MIs, 2472 cancer deaths, 3342 diabetes diagnoses and 5476 cancer diagnoses. Lack of clear effects on any of these outcomes is surprising, given the effects on total cardiovascular events, but may be due to the relatively short timescale of the included studies, compared to a usual lifespan during which risks of chronic illnesses develop over decades, and to relatively small reductions in saturated fat (and serum cholesterol) in some trials. There are difficulties in finding data on the number of people experiencing composite end points such as cardiovascular events. This end point represents the number of people experiencing any of the following: cardiovascular death, cardiovascular morbidity (non-fatal myocardial infarction, angina, stroke, heart failure, peripheral vascular events, atrial fibrillation) and unplanned cardiovascular interventions (coronary artery bypass surgery or angioplasty). Adding up the number of events is easy, but a single participant may have experienced a stroke, an $\mathrm{MI}$ and atrial fibrillation during a trial - and we need to take care not to count this individual three times. So finding such composite end point data involves using the best published composite end point data and supplementing this with author contact where possible. We have underestimated such composite end points rather than overestimated them where exact data are not available. Added to this complex picture, it needs to be remembered that definitions and diagnoses of some end points have altered over time.

Where the funnel plots and comparison of fixed- and randomeffects meta-analyses suggest small-study bias, we have downgraded the quality of the evidence in GRADE, but effects of any such small study bias appear small.

Some trials were partially confounded by aiming to make dietary changes other than those directly related to dietary fat intakes; for example, some studies encouraged intervention participants to make changes to their fat intake as well as changes to fruit and vegetable, fibre or salt intakes. In these studies, any effect on outcomes could be a result of other dietary changes, not of changes in saturated fat intake. The 11 studies (12 comparisons) that appeared free of such differences included Black 1994; DART 1989; Houtsmuller 1979; Ley 2004; MRC 1968; Oxford Retinopathy 1978; Rose corn oil 1965; Rose olive 1965; Simon 1997; Sydney Diet-Heart 1978; Veterans Admin 1969; WINS 2006. On the basis of reviewer comments, we assessed effects of reducing saturated fat intake on combined CVD events including only these trials free of additional interventions. Omitting trials with additional interventions (Oslo Diet-Heart 1966; STARS 1992; WHI 2006) leaves eight studies (nine arms) randomising 3998 participants of whom 750 experienced a CVD event, suggesting a similar reduction in CVD events (RR $0.80,95 \% \mathrm{Cl} 0.64$ to $0.99, \mathrm{I}^{2}=48 \%$, Analysis 1.43 ) to the main analysis (RR $0.79,95 \% \mathrm{Cl} 0.66$ to $0.93, \mathrm{I}^{2}=65 \%,>53,000$ participants randomised, Analysis 1.35). This suggests that effects on combined CVD events are not driven by interventions other than reductions in saturated fats and any energy replacements.

One surprising element of this review is the lack of new trials identified in the update, and small numbers of potential ongoing trials. This is likely to be because well-powered trials on cardiovascular end points will need to be large and carried out over several years, so expensive. As the effects of saturated fats are felt to be established and understood, trialists and funders may feel that the money would be better invested in answering other questions. For most of the ongoing trials, information is limited and these trials may or may not be included when fully published. Perhaps the current evidence set is as definitive as we will achieve during the 'statin era'.

\section{Agreements and disagreements with other studies or reviews}

In this review, saturated fat reduction had little or no effect on all-cause or cardiovascular mortality but did appear to reduce the risk of cardiovascular events by $21 \%$, although effects on $\mathrm{MI}$ and stroke individually were less clear. This result was rather different from those of Siri-Tarino 2010, who systematically reviewed cohort studies that assessed relationships between saturated fat and cardiovascular events. They included 21 studies and did not find associations between saturated fat intake and cardiovascular 
disease (RR 1.0, 95\% $\mathrm{Cl} 0.89$ to 1.11). However, this meta-analysis has been criticised (Katan 2010; Scarborough 2010; Stamler 2010), as results of half of the studies included in their meta-analysis were adjusted for serum cholesterol concentrations, while there is an established relation between saturated fat intake and cholesterol level. The studies included in the meta-analysis also varied widely in the method used to assess intake, as half of the studies collected one-day intake data. However, as with our review, they found no relationship between saturated fat intake and coronary heart disease (RR 1.07, 95\% Cl 0.96 to 1.19) or stroke (RR 0.81, 95\% Cl 0.62 to 1.05 , Siri-Tarino 2010).

In this review, we found that replacing saturated fat with PUFAs (a modified-fat diet) appeared more protective of cardiovascular events than replacement with carbohydrates (a low-fat diet, Analysis 1.44; Analysis 1.45). This was similar to results within our closely allied systematic review assessing health effects of total fat reduction, where modified-fat diets were protective and low-fat diets were not (Hooper 2012). Meta-regression did not suggest any relationship between either PUFAs or MUFAs and cardiovascular events in this review, although the analysis was underpowered. Alonso 2006 suggested a protective role for MUFA from olive oil, but not from meat sources (the main source of MUFA in the USA and Northern Europe). Our systematic review was not able to explore this issue as we included only one small study (underpowered to assess health outcomes on its own) that replaced SFA with MUFA, using an olive oil supplement (Rose olive 1965). A review by Mozaffarian 2010, which again included very similar studies to the last version of this review, with the Finnish Mental Hospital study and Women's Health Initiative data added, stated that their findings provided evidence that consuming PUFAs in place of saturated fat would reduce coronary heart disease. However, their evidence for this was limited, as they found that modifying fat reduced the risk of myocardial infarction or coronary heart disease death (combined) by $19 \%$ (similar to our result). As the mean increase in PUFAs in these studies was 9.9\% of energy, they infer an effect of increasing PUFAs by $5 \%$ of energy of $10 \%$ reduction in risk of myocardial infarction or coronary heart disease death. They provided no suggestion or evidence of a relationship between degree of PUFAs increase and level of risk reduction. Another review carried out during updating of the Nordic Nutritional Recommendations (Schwab 2014) included observational as well as intervention studies, and concluded that there was convincing evidence that partial replacement of SFA with PUFA decreases risk of CVD while replacement with $\mathrm{CHO}$ is associated with increased CVD risk. The review included studies performed solely in white participants or with a clear white majority.

Within the meta-regression, we hoped to combine studies that effectively altered saturated fat by different degrees, so that studies that reduced saturated fat very little and studies that reduced it a great deal would all offer data points for the meta-regression against mortality and morbidity end points, and similarly for total fat, polyunsaturated, monounsaturated and trans fats. Unfortunately many of the included studies did not report data on assessed dietary intake during the trial, reducing the quantity of data available to understand the relationships. Another limitation in understanding effects of individual classes of fatty acids on mortality and morbidity (both in trials and in observational studies) was our ability to correctly assess participants' intake. We could overcome this by using biomarkers such as serum LDL cholesterol (differences between the LDL concentration in the intervention and control arms could be seen as a reasonable and independent approximation of saturated fat intake); however, as many studies were carried out in the 1960 s to 1990 s, few measured and reported LDL cholesterol. We used meta-regression with serum total cholesterol (although this is a composite marker and so less related to saturated fat intake), but although this was available for more studies than LDL it was not available for all studies. Despite the limited data, there was a clear suggestion from meta-regression that there was greater reduction of risk of cardiovascular events in studies with greater total serum cholesterol reduction, supporting the central role of serum lipids in the link between dietary saturated fats and cardiovascular events.

\section{Participants' level of risk}

As the rate of events is higher in high-risk groups (by definition), it should require smaller sample sizes and shorter follow-up to observe an effect of an intervention in a high-risk group of participants (Davey Smith 1993). There have been suggestions that randomised controlled trials are unsuitable for assessing the effectiveness of interventions with very modest levels of effect in low-risk populations, because of the huge numbers of personyears of observation needed to gain sufficient statistical power to avoid Type II errors (Ebrahim 1997). However, with the publication of the Women's Health Initiative trial (WHI 2006) we now have data on more people experiencing cardiovascular events who were originally at low risk of cardiovascular disease than in people with moderate or high risk. The same is true for cardiovascular deaths and total mortality.

When end points such as total mortality are used, the situation becomes more difficult, as in low-risk groups the proportion of deaths which are unrelated to cardiovascular disease (and perhaps unlikely to be influenced by dietary fat changes) rises, again diluting any differences in the numbers of deaths between intervention and control groups. It is more likely that changes in cardiovascular deaths will be seen than in total mortality. The trend is certainly in this direction, with the pooled risk ratio for total mortality 0.96 ( $95 \% \mathrm{Cl} 0.90$ to 1.03 , Analysis 1.1), and for cardiovascular mortality RR 0.94 (95\% Cl 0.78 to 1.13 , Analysis 1.18). Our best estimate is that SFA reduction results in a reduction of $6 \%$ in deaths due to cardiovascular disease, and a reduction of $4 \%$ in total deaths, but these are small effects with wide confidence intervals.

The high-risk participants all showed evidence of cardiovascular disease at baseline. Under current guidelines, most high-risk participants with raised lipid levels should be on lipid-lowering medication (Grundy 2019; NICE 2014; O'Gara 2014). This raises the question of whether there is any additional advantage of adherence to a reduced SFA diet in addition to statin therapy. Little evidence exists at present to answer this question. However, in all parts of the world where drug budgets are restricted and use of lipidlowering medication remains rationed even for those at high risk, the use of reduced SFA diets would appear to be a cost-effective option leading to considerable reductions in cardiovascular events for populations (and so in health budgets) in only a few years.

Low-risk participants are unlikely to be on lipid-lowering medication under current guidelines. The suggestion of protection of low-risk individuals from cardiovascular events with a reduction of roughly $21 \%$ of events in just a few years of intervention, as there is no evidence that effects in the low-CVD-risk group are different from effects in the higher-risk groups, would appear to merit 
continued public health action. Recent guidelines recommend saturated fat reduction in general populations (SACN 2019).

A factor that may affect participant risk of cardiovascular disease, and also the effectiveness of reducing saturated fat intake, that has altered over time is the level of use of statins to control serum lipids in people at moderate and high risk of CVD. The 4S 1994 trial, which was the first trial to show that use of statins could reduce mortality in people with coronary heart disease, was published in 1994 and led to an explosion of the use of statins. For most health outcomes, we saw no clear effect of a decade of publication on risk, but for combined CVD events and CHD events, there were differences between subgroups. For combined CVD events, there were reductions in risk with reduced saturated fat intakes in the 1960s, 1970s and 1990s (both trials published early in the decade), but no clear effect of reducing saturated fat in the 1980s (one trial with 283 events) or 2000s (three large trials). It is possible (but not clear) that participants in the trials published in the 2000s were protected by higher levels of statin use (statins were allowed in participants in the largest trial, WHI 2006).

\section{AUTHORS' CONCLUSIONS}

\section{Implications for practice}

Evidence supports the reduction of saturated fat to reduce risk of combined cardiovascular events in people with and without existing cardiovascular disease, in men and women, over at least two years and in industrialised countries. Little or no effect of saturated fat reduction was seen on all-cause and cardiovascular mortality, at least on this timescale.

Practical ways to achieve reductions in dietary saturated fat include switching to lower fat dairy foods and cutting off meat fats, as well as reducing intake of foods high in saturated fats such as cakes, biscuits, pies and pastries, butter, ghee, lard, palm oil, sausages and cured meats, hard cheese, cream, ice cream, milkshakes and chocolate (for further details see NHS 2020).

\section{Implications for research}

To complement this review of long-term RCTs, we need reviews of metabolic studies to clarify the effects of specific replacements for saturated fat in the diet, and systematic reviews of cohort studies to clarify longer-term effects of saturated fat reductions.

The financial implications (costs and savings) of appropriate advice and legislation to modify fat intake in those at various levels of cardiovascular risk should be assessed and reflected in health policy. Whilst interventions to alter dietary fat intake in individuals at high cardiovascular risk have been fairly successful, such health promotion initiatives in the general population have been less successful. Further work is needed to help high- and low-risk individuals to make effective changes to reduce saturated fat and to maintain these changes over their lifetimes. Research into the effects of legislation to alter fat contents of foods, improved labelling, pricing initiatives and improved availability of healthier foods, linking food production and processing into the health agenda, may yield huge advances in this area.

It is not clear whether there is an additional benefit of reducing saturated fat in those at high risk of cardiovascular disease who are on lipid-lowering medication. Further research to examine the need for maintenance of reduced saturated fat whilst on lipid-lowering medication would be useful, but not as useful as understanding specific dietary fat replacements for saturated fat. However, we did not identify any relevant ongoing trials in our searches.

All future trials should be of at least 2 years duration (preferably longer), employ excellent methodology in terms of randomisation and allocation concealment, blinding of outcome assessors, highquality assessment of macronutrients and micronutrients during the trial in both arms, and equivalent attention and health professional time to participants in both arms.

\section{ACKNOWLEDGEMENTS}

We gratefully acknowledge the help of the following investigators in providing information about their own and others' trials (whether eventually included or excluded): H Arnesen (Ullevål University Hospital), AV Astrup (University of Copenhagen), K Aziz (NICVD, Karachi), F Azizi (Research Institute for Endocrine Sciences, Tehran), SAA Beresford (University of Washington), HS Black (Baylor College of Medicine), BPM Bloemberg (National Institute of Public Health and Environmental Protection, Netherlands), DJ Bowen (Fred Hutchinson Cancer Research Center, Seattle), NF Boyd (University of Toronto), GA Bray (Pennington Biomedical Research Center, Baton Rouge), ML Burr (University of Wales), G Carruba (University of Palermo), L Castagnetta (University of Palermo), JL Curzio (University of Glasgow), RF DeBusk (Stanford University), C Defoort (Méditerranée University), Z Djuric (Wayne State University), A Due (University of Copenhagen), S Druker (University of Massachusetts), RPF Dullaart (University Hospital, Groningen), GE Eyssen (University of Toronto), TM Hayes (University Hospital of Wales, Cardiff), JA Heady (retired, formerly of MRC Social Medicine Research Unit), J Hebert (University of South Carolina), RJ Heine (Free University Hospital, Amsterdam), M-L Hellenius (Karolinska Institute), RF Heller (University of Newcastle), TDR Hockaday (retired, formerly Radcliffe Infirmary), L-E Holm (Swedish Radiation Protection Institute), DJ Hyman (Baylor College of Medicine, Houston), AFM Kardinaal (University of Wageningen), $F$ Khan (Ninewells Hospital and Medical School, Dundee), RH Knopp (University of Washington), D Lairon (Méditerranée University), MEJ Lean (University of Glasgow), B Leelarthaepin (University of Sydney), P Leren (University of Oslo), A Lindman (University of Oslo), S Mackey (Stanford University), R MacLennan (retired, formerly of Queensland Institute of Medical Research), F Macrae (Royal Melbourne Hospital), JI Mann (University of Otago), J Marniemi (Social Insurance Institute), K McManus (Harvard Medical School), RP Mensink (Maastricht University), PA Metcalf (University of Auckland), A Michalsen (University Duisburg-Essen), TF Moy (Johns Hopkins University), AR Ness (University of Bristol), I Okene (University of Massachusetts), GS Oostenbrug (Maastricht University), J Pierce (University of California, San Diego), SD Poppitt (University of Auckland), RJ Reber (University of Illinois), JE Reseland (University of Oslo), BM Retzlaff (University of Washington), AA Rivellese (Federico II University, Naples), P Roderick (University of Southampton), DP Rose (American Health Foundation), FM Sacks (Harvard School of Public Health), WHM Saris (University of Maastricht), ES Sarkkinen (University of Kuopio), A Schatzkin (National Cancer Institute), B Seppelt (German Institute of Human Nutrition), MS Simon (Wayne State University), B Smith (University of Kentucky), E Søndergaard (Svendborg Hospital, Svendborg), AS St. Leger (University of Manchester), VJ Stevens (Kaiser Permanente Centre for Health Research), A Stoddard (University of Massachusetts), LP Svetkey (Duke 
University Medical Center), LC Tapsell (University of Wollongong), BC Tilley (Medical University of South Carolina), $\mathrm{H}$ van den Berg (TNO Nutrition and Food Research Institute), W van Herpen (Unilever), $\mathrm{K}$ van het Hof (Unilever, Rotterdam), MW Verheijden (Wageningen University), GF Watts (University Hospital of Perth), AS Wierzbichi (St. Thomas's Hospital, London), PT Williams (Stanford University), RR Wing (University of Pittsburgh), PD Wood (Stanford University), I Zazpe (University of Navarra), PL Zock (Wageningen Centre for Food Studies).

Thank you to the peer reviewers who have contributed to this review update: Dr Dimitrios Koutoukidis of Nuffield Department of Primary Care Health Sciences, Zhou-Qing Kang of Liaoning Provincial Hospital of Geriatrics, Kristina Petersen of Pennsylvania State University, and the additional referee (who preferred to remain anonymous) as well as to the Cochrane editors Sarah Hodgkinson and Karen Rees, and Charlene Bridges who expertly ran our searches.

We also gratefully acknowledge the expertise and help of the following who contributed to previous iterations of this systematic review: S Adams (Royal Free Hospital, London), B Anagnostelis (Royal Free Hospital, London), M Brand (Cochrane Hypertension Group), R Clarke (Universtiy of Oxford), D Darrah-Morgan (Russian translation), A Donner (University of Western Ontario), D Fagard (University of East Anglia for duplication of inclusion and data extraction), Shweta Gidwani (University of Manchester), G Gubitz (Cochrane Stroke Group), M Haugh (Cochrane Renal Group), IU Haq (Northern General Hospital, Sheffield), J Hooper (Danish, Swedish and Norwegian translation), BK Hurley (Italian translation), L Jones (Systematic Reviews Training Unit, London), SPH Keen (Cochrane Diabetes Group), S Logan (Systematic Reviews Training Unit, London), LI Mennen (INSERM), T Moore (Cochrane Heart Group), J Muscroft (German and French translation), HL Newmark (Rutgers), E Royle (Cochrane Peripheral Vascular Diseases Group), I Tumur (Pfizer Ltd), AS Truswell (University of Sydney), M Turner (Chinese translation), JM Walsh (University of California), A Wierzbicki (St. Thomas' Hospital, London), WC Willett (Harvard School of Public Health), AF Winder (University of London).

Many thanks to those people who contributed to, and were co-authors of, previous versions of this review (Hooper 2000; Hooper 2001; Hooper 2012; Hooper 2015a): Julian PT Higgins, Shah Ebrahim, George Davey Smith, Rudolph Riemersma, Nigel E Capps, Carolyn Summerbell, Rachel Thompson, Helen Moore, Diredre Sills, Felicia Roberts, Dorotheé Fagard and Gillian Clements.

Finally, many thanks for the members of the WHO NUGAG committee who took time to debate and discuss this review, and ensure that it was addressing the questions that WHO NUGAG were addressing. Members of this NUGAG subcommittee included Nahla C Houalla (American University of Beirut), Jim Mann (University of Otago), Barbara O Schneeman (University of California at Davis), Mary R L'Abbé (University of Toronto), Murray Skeaff (University of Otago), Due Li (Zhejiang University), Russell de Souza (McMaster University), Ibrahim Elmadfa (University of Vienna). Thank you too to all of the NUGAG group members, and members of WHO including Jason Montez, Chizuru Nishida and Emma Kennedy. 


\section{R E F E R E N C E S}

\section{References to studies included in this review}

Black 1994 \{published and unpublished data\}

* Black HS, Herd JA, Goldberg LH, , Thornby JI, Rosen T, et al. Effect of a low-fat diet on the incidence of actinic keratosis. New England Journal of Medicine 1994; 330(18):1272-5.

Black HS, Thornby JI, , Goldberg LH, Herd JA, Rosen T, et al. Evidence that a low-fat diet reduces the occurrence of nonmelanoma skin cancer. International Journal of Cancer 1995; 62(2):165-9.

Jaax S, Scott LW, , Thornby JI, Black HS. General guidelines for a low-fat diet effective in the management and prevention of nonmelanoma skin cancer. Nutrition and Cancer 1997; 27(2):150-6.

\section{DART 1989 \{published and unpublished data\}}

* Burr ML, Fehily AM, Gilbert JF, Rogers S, Holliday RM, Sweetnam PM, et al. Effects of changes in fat, fish, and fibre intakes on death and myocardial reinfarction: diet and reinfarction trial (DART). Lancet 1989; 2(8666):757-61.

Burr ML, Fehily AM, Rogers S, Welsby E, King S, Sandham S. Diet and reinfarction trial (DART): design, recruitment, and compliance. European Heart Journal 1989; 10(6):558-67.

Burr ML, Fehily AM. Fish and the heart. Lancet 1989; ii:1450-2.

Burr ML, Holliday RM, Fehily AM, Whitehead PJ. Haematological prognostic indices after myocardial infarction: evidence from the diet and reinfarction trial (DART). European Heart Journal 1992; 13(2):166-70.

Burr ML, Sweetham PM, Fehily AM. Diet and reinfarction [letter]. European Heart Journal 1994; 15(8):1152-3.

Fehily AM, Vaughan-Williams E, Shiels K, Williams AH, Horner M, Bingham $G$, et al. The effect of dietary advice on nutrient intakes: evidence from the diet and reinfarction trial (DART). Journal of Human Nutrition \& Dietetics 1989; 2(4):225-5.

\section{Houtsmuller 1979 \{published data only\}}

Houtsmuller AJ, Van Hal-Ferwerda J, Zahn KJ, Henkes HE. Favourable influences of linoleic acid on the progression of diabetic micro- and macroangiopathy. Nutrition and Metabolism 1980; 24(Suppl 1):105-18.

Houtsmuller AJ, Van Hal-Ferwerda J, Zahn KJ, Henkes HE. Influence of different diets on the progression of diabetic retinopathy. Progress in Food Nutritition and Science 1980; 4(5):41-6.

* Houtsmuller AJ, Zahn KJ, Henkes HE. Unsaturated fats and progression of diabetic retinopathy. Documenta Ophthalmologia 1979; 48(2):363-71.

\section{Ley 2004 \{published and unpublished data\}}

* Ley SJ, Metcalf PA, Scragg RK, Swinburn BA. Long-term effects of a reduced fat diet intervention on cardiovascular disease risk factors in individuals with glucose intolerance. Diabetes Research and Clinical Practice 2004; 63(2):103-12.

Swinburn BA, Metcalf PA, Ley SJ. Long-term (5-year) effects of a reduced-fat diet intervention in individuals with glucose intolerance. Diabetes Care 2001; 24(4):619-24.

Swinburn BA, Woollard GA, Chang EC, Wilson MR. Effects of reduced-fat diets consumed ad libitum on intake of nutrients particularly antioxidant vitamins. Journal of the American Dietetic Association 1999; 99(11):1400-5.

Moy 2001 \{published and unpublished data\} Moy TF, Yanek LR, Raqueno JV, Bezirdjian PJ, Blumenthal RS, Wilder LB, et al. Dietary counseling for high blood cholesterol in families at risk of coronary disease. Preventive Cardiology 2001; 4(4):158-64.

\section{MRC 1968 \{published and unpublished data\}}

Ederer F, Leren P, Turpeinen O, . Cancer among men on cholesterol lowering diets: experience of five clinical trials. Lancet 1971; 2(7717):203-6.

Heady JA. Are PUFA harmful? British Medical Journal 1974; 1(898):115-6.

* . Controlled trial of soya-bean oil in myocardial infarction. Lancet 1968; 2(570):693-9.

Oslo Diet-Heart 1966 \{published and unpublished data\}

Leren P. Prevention of coronary heart disease, some results from the Oslo secondary and primary intervention studies. Journal of the American College of Nutrition 1989; 8(5):407-10.

Leren P. The Oslo diet-heart study. Eleven year report. Circulation 1970; 42(5):935-42.

Leren P. The effect of a cholesterol lowering diet in male survivors of myocardial infarction. A controlled clinical trial) [Virkningen av cholesterolsenkende diett hos menn som har gjennomgatt hjerteinfarkt. Et kontrollert klinisk fors/ok]. Nordisk Medicin 1967; 77(21):658-61.

* Leren P. The effect of plasma cholesterol lowering diet in male survivors of myocardial infarction. A controlled clinical trial. Acta Medica Scandinavia 1966; 466 Suppl:1-92.

Leren P. The effect of plasma-cholesterol-lowering diet in male survivors of myocardial infarction. A controlled clinical trial. Bulletin of the New York Academy of Medicine 1968; 44(8):1012-20.

\section{Oxford Retinopathy 1978 \{published and unpublished data\}}

Coppack SW, Doll HA, Pim B, Hockaday TD. Intravenous glucose tolerance and mortality in non-insulin-dependant diabetes mellitus. Quarterly Journal of Medicine 1990; 75(277):451-60.

Hillson RM, Hockaday TD, Mann JI, Newton DJ. Hyperinsulinaemia is associated with development of ECG abnormalities in diabetics. Diabetes Research 1984; 1(3):143-9. 
* Hockaday TD, Hockaday JM, Mann JI, Turner RC. Prospective comparison of modified fat-high-carbohydrate with standard low-carbohydrate dietary advice in the treatment of diabetes: one year follow-up study. British Journal of Nutrition 1978; 39(2):357-62.

Howard-Williams J, Patel P, Jelfs R, Carter RD, Awdry P, Bron A, et al. Polyunsaturated fatty acids and diabetic retinopathy. British Journal of Ophthalmology 1985; 69(1):15-8.

Lopez-Espinoza I, Howard WJ, Mann JI, Carter RD, Hockaday TD. Fatty acid composition of platelet phospholipids in non-insulindependent diabetics randomized for dietary advice. British Journal of Nutrition 1984; 52(1):41-7.

\section{Rose corn oil 1965 \{published data only\}}

Rose GA, Thomson WB, Williams RT. Corn oil in treatment of ischaemic heart disease. British Medical Journal 1965; 1(5449):1531-3.

\section{Rose olive 1965 \{published data only\}}

Rose GA, Thomson WB, Williams RT. Corn oil in treatment of ischaemic heart disease. British Medical Journal 1965; 1(5449):1531-3.

\section{Simon 1997 \{published and unpublished data\}}

Djuric Z, Heilbrun LK, Reading BA, Boomer A, Valeriote FA, Martino $S$. Effects of a low fat diet on levels of oxidative damage to DNA in human peripheral nucleated blood cells. Journal of the National Cancer Institute 1991; 83(11):766-9.

Djuric Z, Martino S, Heilbrun LK, Hart RW. Dietary modulation of oxidative DNA damage. Advances In Experimental Medicine and Biology 1994; 354:71-83.

Kasim SE, Martino S, , Khilnani S, Boomer A, Depper J, et al. Dietary and anthropometric determinants of plasma lipoproteins during a long-term low-fat diet in healthy women. American Journal of Clinical Nutrition 1993; 57(2):146-53.

* Simon MS, Heilbrun LK, Boomer A, Kresge C, Depper J, Kim PN, et al. A randomised trial of a low-fat dietary intervention in women at high risk for breast cancer. Nutrition and Cancer 1997; 27(2):136-42.

\section{STARS 1992 \{published and unpublished data\}}

Blann AD, Jackson P, Bath PM, Watts GF. Von Willebrand factor, a possible indicator of endothelial cell damage, decreases during long-term compliance with a lipid-lowering diet. Journal of Internal Medicine 1995; 237(6):557-61.

Watts GF, Brunt JN, Coltart DJ, Lewis B. The St. Thomas Atherosclerosis Regression Study (STARS). Atherosclerosis 1992; 97:231.

Watts GF, Jackson P, Burke V, Lewis B. Dietary fatty acids and progression of coronary artery disease in men. American Journal of Clinical Nutrition 1996; 64(2):202-9.

Watts GF, Jackson P, Mandalia S, Brunt JN, Lewis ES, Coltart DJ, et al. Nutrient intake and progression of coronary artery disease. American Journal of Cardiology 1994; 73(5):328-32.
* Watts GF, Lewis B, Brunt JN, Lewis ES, Coltart DJ, Smith LD, et al. Effects on coronary artery disease of lipid-lowering diet, or diet plus cholestyramine, in the St Thomas' Atherosclerosis Regression Study (STARS). Lancet 1992; 339(8793):563-9.

Watts GF, Lewis B, Brunt JNH, Swan AV. Coronary atheroma regression trials. Lancet 1992; 339(i):1241-3.

Watts GF, Lewis B, Jackson P, Burke V, Lewis ES, Brunt JN, et al. Relationships between nutrient intake and progression/ regression of coronary atherosclerosis as assessed by serial quantitative angiography. Canadian Journal of Cardiology 1995; 11(Suppl G):110G-4G.

Watts GF, Mandalia S, Brunt JN, Slavin BM, Coltart DJ, Lewis B. Independent associations between plasma lipoprotein subfraction levels and the course of coronary artery disease in the St. Thomas' Atherosclerosis Regression Study (STARS). Metabolism: Clinical and Experimental 1993; 42(11):1461-7.

Watts GF, Mandalia S, Slavin BM, Brunt JN, Coltart DJ, Lewis B. Metabolic determinants of the course of coronary artery disease in men. Clinical Chemistry 1994; 40(12):2240-6.

Watts GF. Nutritional, metabolic, and genetic determinants of the progression of coronary heart disease. STARS Group. Journal of Cardiovascular Pharmacology 1995; 25(Suppl 4):S11-9.

Sydney Diet-Heart 1978 \{published and unpublished data\} Blacket RB, Leelarthaepin B, McGilchrist C, Palmer AJ, Woodhill JM. The synergistic effect of weight loss and changes in dietary lipids on the serum cholesterol of obese men with hypercholesterolaemia: implications for prevention of coronary heart disease. Australian and New Zealand Journal of Medicine 1979; 9(5):521-9.

Ramsden CE, Zamora D, Leelarthaepin B, Majchrzak-Hong SF, Faurot KR, Suchindran CM, et al. Use of dietary linoleic acid for secondary prevention of coronary heart disease and death: evaluation of recovered data from the Sydney Diet Heart Study and updated meta-analysis. BMJ 2013; 346:e8707. [DOI: 10.1136/bmj.e8707]

* Woodhill JM, Palmer AJ, Leelarthaepin B, McGilchrist C, Blacket RB. Low fat, low cholesterol diet in secondary prevention of coronary heart disease. Advances in Experimental Medicine and Biology 1978; 109:317-30.

\section{Veterans Admin 1969 \{published data only\}}

Dayton S, Hashimoto S, Dixon W, Pearce ML. Composition of lipids in human serum and adipose tissue during prolonged feeding of a diet high in unsaturated fat. Journal of Lipid Research 1966; 7(1):103-11.

Dayton S, Hashimoto S, Pearce ML. Adipose tissue linoleic acid as a criterion of adherence to a modified diet. Journal of Lipid Research 1967; 8(5):508-10.

Dayton S, Hashimoto S, Pearce ML. Influence of a diet high in unsaturated fat upon composition of arterial tissue and atheromata in man. Circulation 1965; 32(6):911-24. 
Dayton S, Hashimoto S, Rosenblum D, Pearce M. Vitamin E status of humans during prolonged feeding of unsaturated fats. Journal of Laboratory and Clinical Medicine 1965; 65(5):739-47.

Dayton S, Pearce ML, Goldman H, Harnish A, Plotkin D, Shickman M, et al. Controlled trial of a diet high in unsaturated fat for prevention of atherosclerotic complications. Lancet 1968; 2(577):1060-2.

* Dayton S, Pearce ML, Hashimoto S, Dixon WJ, Tomayasu U. A controlled clinical trial of a diet high in unsaturated fat in preventing complications of atherosclerosis. Circulation 1969; 15(1, Suppl 2):II-1-63.

Dayton S, Pearce ML, Hashimoto S, Fakler LJ, Hiscock E, Dixon WJ. A controlled clinical trial of a diet high in unsaturated fat. New England Journal of Medicine 1962; 266:1017-23.

Dayton S, Pearce ML. Diet and atherosclerosis. Lancet 1970; 1(644):473-4.

Dayton S, Pearce ML. Diet and cardiovascular diseases. Lancet 1969; 1(584):51-2.

Dayton S, Pearce ML. Diet high in unsaturated fat: a controlled clinical trial. Minnesota Medicine 1969; 52(8):1237-42.

Dayton S, Pearce ML. Prevention of coronary heart disease and other complications of atherosclerosis by modified diet. American Journal of Medicine 1969; 46(5):751-62.

Dayton S, Pearce ML. Trial of unsaturated-fat diet. Lancet 1968 2(581):1296-7.

Hiscock E, Dayton S, Pearce ML, Hashimoto S. A palatable diet high in unsaturated fat. Journal of the American Dietetic Association 1962; 40:427-31.

Pearce ML, Dayton S. Incidence of cancer in men on a diet high in polyunsaturated fat. Lancet 1971; 1(697):464-7.

Sturdevant RA, Pearce ML, Dayton S. Increased prevalence of cholelithiasis in men ingesting a serum-cholesterol-lowering diet. New England Journal of Medicine 1973; 288(1):24-7.

Tompkins MJ, Dayton S, Pearce ML. Effect of long-term feeding of various fats on whole blood clotting times in men. Journal of Laboratory and Clinical Medicine 1964; 64(5):763-72.

WHI 2006 \{published data only\}

ZZZ <accessionId ref="info:x-wiley/clinicalTrialsGov/

NCT00000611"> ZZZNCT00000611

Anderson G, Cummings S, Freedman LS, Furberg C, Henderson M, Johnson SR, et al. Design of the Women's Health Initiative clinical trial and observational study. Controlled Clinical Trials 1998; 19(1):61-109.

Anderson GL, Manson J, Wallace R, Lund B, Hall D, Davis S, et al. Implementation of the Women's Health Initiative study design. Annals of Epidemiology 2003; 13(9 Suppl):S5-17.

Beresford SA, Johnson KC, Ritenbaugh C, Lasser NL, Snetselaar LG, Black HR, et al. Low-fat dietary pattern and risk of colorectal cancer: the Women's Health Initiative Randomized Controlled Dietary Modification Trial. JAMA 2006; 295(6):643-54.
Bowen D, Ehret C, Pedersen M, Snetselaar L, Johnson M, Tinker $L$, et al. Results of an adjunct dietary intervention program in the Women's Health Initiative. Journal of the American Dietetic Association 2002; 102(11):1631-7.

Carty CL, Kooperberg C, Neuhouser ML, Tinker L, Howard B, Wactawski-Wende J, et al. Low-fat dietary pattern and change in body-composition traits in the Women's Health Initiative Dietary Modification Trial. American Journal of Clinical Nutrition 2011; 93(3):516-24.

Curb JD, McTiernan A, Heckbert SR, Kooperberg C, Stanford J, Nevitt M, et al. Outcomes ascertainment and adjudication methods in the Women's Health Initiative. Annals of Epidemiology 2003; 13(9 Suppl):S122-8.

Hays J, Hunt JR, Hubbell FA, Anderson GL, Limacher M, Allen C et al. The Women's Health Initiative recruitment methods and results. Annals of Epidemiology 2003; 13(9 Suppl):S18-77.

Hebert JR, Patterson RE, Gorfine M, Ebbeling CB, St Jeor ST, Chlebowski RT, et al. Differences between estimated caloric requirements and self-reported caloric intake in the Women's Health Initiative. Annals of Epidemiology 2003; 13(9):629-37.

Howard BV, Curb JD, Eaton CB, Kooperberg C, Ockene J, Kostis JB, et al. Low-fat dietary pattern and lipoprotein risk factors: the Women's Health Initiative dietary modification trial. American Journal of Clinical Nutrition 2010; 91(4):860-74.

Howard BV, Manson JE, Stefanick ML, Beresford SA, Frank G, Jones B, et al. Low-fat dietary pattern and weight change over 7 years: the Women's Health Initiative Dietary Modification Trial. JAMA 2006; 295(1):39-49.

Howard BV, Van Horn L, Hsia J, Manson JE, Stefanick ML, Wassertheil-Smoller S, et al. Low-fat dietary pattern and risk of cardiovascular disease: the Women's Health Initiative Randomized Controlled Dietary Modification Trial. JAMA 2006; 295(6):655-66.

Howard BV. Dietary fat and cardiovascular disease: putting the Women's Health Initiative in perspective. Nutrition Metabolism \& Cardiovascular Diseases 2007; 17(3):171-4.

Neuhouser ML, Tinker L, Shaw PA, Schoeller D, Bingham SA, Horn LV, et al. Use of recovery biomarkers to calibrate nutrient consumption self-reports in the Women's Health Initiative. American Journal of Epidemiology 2008; 167(10):1247-59.

Patterson RE, Kristal A, Rodabough R, Caan B, Lillington L, Mossavar-Rahmani $Y$, et al. Changes in food sources of dietary fat in response to an intensive low-fat dietary intervention: early results from the Women's Health Initiative. Journal of the American Dietetic Association 2003; 103(4):454-60.

Patterson RE, Kristal AR, Tinker LF, Carter RA, Bolton MP, GursCollins T, et al. Measurement characteristics of the Women's Health Initiative food frequency questionnaire. Annals of Epidemiology 1999; 9(3):178-87.

Prentice RL, Aragaki AK, Howard BV, Chlebowski RT, Thomson CA, Van Horn L, et al. Low-fat dietary pattern among postmenopausal women influences long-term cancer, 
cardiovascular disease, and diabetes outcomes. Journal of Nutrition 2019; 149(9):1565-74.

* Prentice RL, Aragaki AK, Van Horn L, Thomson CA, Beresford SAA, Robinson J, et al. Low-fat dietary pattern and cardiovascular disease: results from the Women's Health Initiative randomized controlled trial. American Journal of Clinical Nutrition 2017; 106(1):35-43.

Prentice RL, Caan B, Chlebowski RT, Patterson R, Kuller LH, Ockene JK, et al. Low-fat dietary pattern and risk of invasive breast cancer: the Women's Health Initiative Randomized Controlled Dietary Modification Trial. JAMA 2006; 295(6):629-42.

Prentice RL, Thomson CA, Caan B, Hubbell FA, Anderson GL, Beresford SA, et al. Low-fat dietary pattern and cancer incidence in the Women's Health Initiative Dietary Modification Randomized Controlled Trial. Journal of the National Cancer Institute 2007; 99(20):1534-43.

Ritenbaugh C, Patterson RE, Chlebowski RT, Caan B, FelsTinker L, Howard B, et al. The Women's Health Initiative Dietary Modification trial: overview and baseline characteristics of participants. Annals of Epidemiology 2003; 13(9 Suppl):S87-97.

Robinson JG, Wallace R, Safford MM, Pettinger M, Cochrane B, Ko MG, et al. Another treatment gap: restarting secondary prevention medications: the Women's Health Initiative. Journal of Clinical Lipidology 2010; 4(1):36-45.

Rossouw JE, Finnegan LP, Harlan WR, Pinn VW, Clifford C, McGowan JA. The evolution of the Women's Health Initiative: perspectives from the NIH. Journal of the American Medical Women's Association 1995; 50(2):50-5.

The Women's Health Initiative Study Group. Design of the Women's Health Initiative clinical trial and observational study. Controlled Clinical Trials 1998; 19(1):61-109.

Tinker LF, Bonds DE, Margolis KL, Manson JE, Howard BV, Larson J, et al. Low-fat dietary pattern and risk of treated diabetes mellitus in postmenopausal women: the Women's Health Initiative randomized controlled dietary modification trial. Archives of Internal Medicine 2008; 168(14):1500-11.

Tinker LF, Perri MG, Patterson RE, Bowen DJ, McIntosh M, Parker LM, et al. The effects of physical and emotional status on adherence to a low-fat dietary pattern in the Women's Health Initiative. Journal of the American Dietetic Association 2002; 102(6):789-800.

Tinker LF, Rosal MC, Young AF, Perri MG, Patterson RE, Van Horn L, et al. Predictors of dietary change and maintenance in the Women's Health Initiative Dietary Modification Trial. Journal of the American Dietetic Association 2007; 107(7):1155-66.

Women's Health Initiative Study Group. Dietary adherence in the Women's Health Initiative Dietary Modification Trial. Journal of the American Dietetic Association 2004; 104(4):654-8.

\section{WINS 2006 \{published and unpublished data\}}

Chlebowski RT, Blackburn GL, Buzzard IM, Rose DP, Martino S, Khandekar JD, et al. Adherence to a dietary fat intake reduction program in postmenopausal women receiving therapy for early breast cancer. The Women's Intervention Nutrition Study. Journal of Clinical Oncology 1993; 11(11):2072-80.

* Chlebowski RT, Blackburn GL, Thomson CA, Nixon DW, Shapiro A, Hoy MK, et al. Dietary fat reduction and breast cancer outcome: interim efficacy results from the Women's Intervention Nutrition study. Journal of the National Cancer Institute 2006; 98(24):1767-76.

Chlebowski RT, Blackburn GL. Final survival analysis from the randomized Womens Intervention Nutrition Study (WINS) evaluating dietary intervention as adjuvant breast cancer therapy. Cancer Research 2015; 75(9 Supplement):S5-08.

Chlebowski RT, Rose D, Blackburn G, Buzzard M, Khandekar J, York $\mathrm{R}$, et al. Feasibility of using dietary fat intake reduction in adjuvant breast cancer management. Proceedings of American Society of Clinical Oncology 1991; 86:86.

Chlebowski RT, Rose DP, Buzzard IM, Blackburn GL, York M, , et al. Dietary fat reduction in adjuvant breast cancer therapy: current rationale and feasibility issues. Adjuvant: the Cancer Journal 1990; 6:357-63.

Hoy MK, Winters BL, Chlebowski RT, Papoutsakis C, Shapiro A, Lubin MP, et al. Implementing a low-fat eating plan in the Women's Intervention Nutrition Study. Journal of the American Dietetic Association 2009; 109(4):688-96.

Rose DP, Chlebowski RT, Connolly JM, Jones LA, Wynder EL. Effects of tamoxifen adjuvant therapy and a low-fat diet on serum binding proteins and estradiol bioavailability in postmenopausal breast cancer patients. Cancer Research 1992; 52(19):5386-90.

Rose DP, Connolly JM, Chlebowski RT, Buzzard IM, Wynder EL. The effects of a low-fat dietary intervention and tamoxifen adjuvant therapy on the serum estrogen and sex hormonebinding globulin concentrations of postmenopausal breast cancer patients. Breast Cancer Research \& Treatment 1993; 27(3):253-62.

Wynder EL, Cohen LA, Winters BL. The challenges of assessing fat intake in cancer research investigations. Journal of the American Dietetic Association 1997; 97 (7 Suppl):S5-8.

\section{References to studies excluded from this review}

Agewall 2001 \{published data only\}

Agewall S. Multiple risk intervention trial in high risk hypertensive men: comparison of ultrasound intima-media thickness and clinical outcome during 6 years of follow-up. Journal of Internal Medicine 2001; 249(4):305-14.

\section{Ammerman 2003 \{published data only\}}

Ammerman AS, Keyserling TC, Atwood JR, Hosking JD, Zayed H, Krasny C. A randomized controlled trial of a public health nurse directed treatment program for rural patients with high blood cholesterol. Preventive Medicine 2003; 36(3):340-51. 
Anderson 1990 \{published and unpublished data\}

* Anderson JW, Garrity TF, Smith BM, Whitis SE. Follow-up on a clinical trial comparing the effects of two lipid lowering diets. Arteriosclerosis 1990; 10(5):882a.

Anderson JW, Garrity TF, Wood CL, Whitis SE, Smith BM, Oeltgen PR. Prospective, randomized, controlled comparison of the effects of low-fat and low-fat plus high-fiber diets on serum lipid concentrations. American Journal of Clinical Nutrition 1992; 56(5):887-94.

\section{Aquilani 2000 \{published data only\}}

Aquilani R, Tramarin R, Pedretti RF, Bertolotti G, Sommaruga M, Mariani $P$, et al. Can a very-low-fat diet achieve cholesterol goals in CAD? Cardiology Review 2000; 17(10):36-40.

\section{Arntzenius 1985 \{published data only\}}

Arntzenius AC, Kromhout D, Barth JD, Reiber JH, Bruschke AV, Buis B, et al. Diet, lipoprotiens and progression of coronary atherosclerosis: the Leiden Intervention Trial. New England Journal of Medicine 1985; 312(13):805-8.

\section{Aro 1990 \{published data only\}}

Aro A, Ahola I, Jauhiainen M, Mutanen M, Valsta LM. Effects of plasma phospholipid fatty acids of rapeseed oil and sunflower oil diets [Abstract]. Arteriosclerosis 1990; 10:877a.

\section{ASSIST 2001 \{published data only\}}

Moher M, Yudkin P, Wright L, Turner R, Fuller A, Schofield T, et al. Cluster randomised controlled trial to compare three methods of promoting secondary prevention of coronary heart disease in primary care. BMJ 2001; 322(7298):1338.

\section{Australian Polyp Prev 95 \{published and unpublished data\}}

* MacLennan R, Macrae F, Bain C, Battistutta D, Chapuis P, Gratten $\mathrm{H}$, et al. Randomized trial of intake of fat, fiber, and beta carotene to prevent colorectal adenomas. The Australian Polyp Prevention Project. Journal of the National Cancer Institute 1995; 87(23):1760-6.

MacLennan R. Effect of fat, fibre and beta-carotene on colorectal adenomas after 24 months. Gastroenterology 1991; 100:A382.

Macrae FA, Hughes NR, Bhathal PS, Tay D, Selbie L, MacLennan R. Dietary suppression of rectal epthelial cell proliferation. Gastroenterology 1991; 100:A383.

\section{Azadbakht 2007 \{published and unpublished data}

Azadbakht L, Mirmiran P, Esmaillzadeh A, Azizi F. Better dietary adherence and weight maintenance achieved by a longterm moderate fat diet. British Journal of Nutrition 2007; 97(2):399-404.

\section{Bakx 1997 \{published data only\}}

Bakx JC, Stafleu A, Van Staveren WA, Van den Hoogen HJ, Van Weel C. Long-term effect of nutritional counseling: a study in family medicine. American Journal of Clinical Nutrition 1997; 65(6 Suppl):1946S-50S.

\section{Ball 1965 \{published data only\}}

Ball KP, Hanington E, McAllen PM, Pilkington TR, Richards JM, Sharland DE, et al. Low-fat diet in myocardial infarction: a controlled trial. Lancet 1965; 2(411):501-4.

\section{Barnard 2009 \{published data only\}}

Barnard ND, Cohen J, Jenkins DJ, Turner-McGrievy G, Gloede L, Green A, et al. A low-fat vegan diet and a conventional diabetes diet in the treatment of type 2 diabetes: a randomized, controlled, 74-wk clinical trial. American Journal of Clinical Nutrition 2009; 89(5):1588S-96S.

Barndt 1977 \{published data only\}

Barndt R, Blankenhorn CH, Crawford DW, Brooks SH. Regression and progression of early femoral atherosclerosis in treated hyperlipidaemic patients. Annals of Internal Medicine 1977; 86(2):139-46.

\section{Baron 1990 \{published data only\}}

Baron JA, Gleason R, Crowe B, Mann JI. Preliminary trial of the effect of general practice based nutritional advice. British Journal of General Practice 1990; 40(333):137-41.

\section{Barr 1990 \{published data only\}}

Barr SL, Ramakrishnan R, Holleran S. A 30\% fat diet high in polyunsaturates and a $30 \%$ fat diet high in monounsaturates both lower total and low density lipoprotein cholesterol levels in normal males [Abstract]. Arteriosclerosis 1990; 10:872a.

\section{Barsotti 1991 \{published data only\}}

Barsotti A. Modern trends in the therapy of arteriosclerosis in the light of new physiopathological findings [Moderne tendenze della terapia dell'arteriosclerosi alla luce delle nuove acquisizioni fisiopatologiche]. Cardiologia 1991; 36(12 Suppl 1):33-48.

\section{Baumann 1982 \{published data only\}}

Baumann J, Martschick R. Therapy of hyperlipidemia with xanthinol nicotinate as opposed to low fat diet [Therapie der hyperlipidämie mit xantinolnicotinat gegenüber fettarmer diät]. Die Medizinische Welt 1982; 33(4):139-41.

\section{BDIT Pilot Studies 1996 \{published and unpublished data\}}

Boyd NF, Cousins M, Beaton M, Fishell E, Wright B, Fish E, et al. Clinical trial of low-fat, high-carbohydrate diet in subjects with mammographic dysplasia: report of early outcomes. Journal of the National Cancer Institute 1988; 80(15):1244-8.

Boyd NF, Cousins M, Beaton M, Han L, McGuire V.

Methodological issues in clinical trials of dietary fat reduction in patients with breast dysplasia. Progress in Clinical and Biological Research 1986; 222:117-24.

Boyd NF, Cousins M, Beaton M, Kriukov V, Lockwood G, Tritchler D. Quantitative changes in dietary fat intake and serum cholesterol in women: results from a randomized, controlled trial. American Journal of Clinical Nutrition 1990; 52(3):470-6.

Boyd NF, Cousins M, Kriukov V. A randomised controlled trial of dietary fat reduction: the retention of subjects and characteristics of drop outs. Journal of Clinical Epidemiology 1992; 45(1):31-8. 
Boyd NF, Cousins M, Lockwood G, Tritchler D. Dietary fat and breast cancer risk: the feasibility of a clinical trial of breast cancer prevention. Lipids 1992; 27(10):821-6.

Boyd NF, Cousins M, Lockwood G, Tritchler D. The feasibility of testing experimentally the dietary fat-breast cancer hypothesis. Progress in Clinical and Biological Research 1990; 346:231-41.

* Boyd NF, Martin LJ, Beaton M, Cousins M, Kriukov V. Longterm effects of participation in a randomized trial of a low-fat, high-carbohydrate diet. Cancer Epidemiology, Biomarkers \& Prevention 1996; 5(3):217-22.

Lee-Han H, Cousins M, Beaton M, McGuire V, Kriukov V, Chipman $\mathrm{M}$, et al. Compliance in a randomized clinical trial of dietary fat reduction in patients with breast dysplasia. American Journal of Clinical Nutrition 1988; 48(3):575-86.

Beckmann 1995 \{published data only\}

Beckmann SL, Os I, Kjeldsen SE, Eide IK, Westheim AS, Hjermann I. Effect of dietary counselling on blood pressure and arterial plasma catecholamines in primary hypertension. American Journal of Hypertension 1995; 8(7):704-11.

\section{beFIT 1997 \{published and unpublished data\}}

Retzlaff BM, Walden CE, McNeney WB, Buck BL, McCann BS, Knopp RH. Nutritional intake of women and men on the NCEP Step I and Step II diets. Journal of the American College of Nutrition 1997; 16(1):52-61.

* Walden CE, Retzlaff BM, Buck BL, McCann BS, Knopp RH. Lipoprotein lipid response to the National Cholesterol Education Program Step II diet by hypercholesterolemic and combined hyperlipidemic women and men. Arteriosclerosis, Thrombosis and Vascular Biology 1997; 17(2):375-82.

Walden CE, Retzlaff BM, Buck BL, Wallick S, McCann BS, Knopp RH. Differential effect of National Cholesterol Education Program (NCEP) Step II Diet on HDL cholesterol, its subfractions, and apoprotein A-1 levels in hypercholesterolemic women and men after 1 year: the beFIT study. Arteriosclerosis, Thrombosis and Vascular Biology 2000; 20(6):1580-7.

\section{Beresford 1992 \{published data only\}}

Beresford SA, Farmer EM, Feingold L, Graves KL, Sumner SK, Baker RM. Evaluation of a self-help dietary intervention in a primary care setting. American Journal of Public Health 1992; 82(1):79-84.

\section{Bergstrom 1967 \{published data only\}}

Bergstrom G, Svanborg A. Dietary treatment of acute myocardial infarction. Acta Medica Scandinavica 1967; 181(6):717-21.

\section{Bierenbaum 1963 \{published data only\}}

Bierenbaum ML, Fleischman Al, Raichelson RI, Hayton T, Watson P. Ten year experience of modified fat diets on younger men with coronary heart disease. Lancet 1973; 1(7817):1404-7.

Bierenbaum ML, Green DP, Florin A, Fleischman AI, Caldwell AB. Modified-fat dietary management of the young male with coronary disease. A five-year report. JAMA 1967; 202(13):1119-23.
* Bierenbaum ML, Green DP, Gherman C, Florin A, Caldwell AB. The effects of two low fat dietary patterns on the blood cholesterol levels of young male coronary patients. Journal of Chronic Diseases 1963; 16:1073-83.

\section{Bloemberg 1991 \{published and unpublished data\}}

Bloemberg BP, Kromhout D, Goddijn HE, Jansen A, Obermannde Boer GL. The impact for the guidelines for a healthy diet of the Netherlands Nutrition Council on total and high density lipoprotein cholesterol in hypercholesterolemic free living men. American Journal of Epidemiology 1991; 134(1):39-48.

\section{Bloomgarden 1987 \{published data only\}}

Bloomgarden ZT, Karmally W, Metzger MJ, Brothers M, Nechemias C, Bookman J, et al. Randomized, controlled trial of diabetic patient education: improved knowledge without improved metabolic status. Diabetes Care 1987; 10(3):263-72.

\section{Bonk 1975 \{published data only\}}

Bonk S, Hubotter E, Nickel C, Stocksmeier U, Vahey P, Volk I, et al. Myocardial infarct patients with and without intensive nutrition consultation over several years-- comparison of physiological and social variables [Herzinfarktpatienten mit und ohne mehrjährige intensive ernährungsberatung - vergleich von physiologischen und sozialen variablen]. Infusionstherapie und Klinische Ernährung 1975; 2(4):290-6.

\section{Bonnema 1995 \{published data only\}}

Bonnema SJ, Jespersen LT, Marving J, Gregersen G. Supplementation with olive oil rather than fish oil increases small arterial compliance in diabetic patients. Diabetes, Nutrition and Metabolism Clinical and Experimental 1995; 8:81-7.

\section{Bosaeus 1992 \{published data only\}}

Bosaeus I, Belfrage L, Lindgren C, Andersson H. Olive oil instead of butter increases net cholesterol excretion from the small bowel. European Journal of Clinical Nutrition 1992; 46(2):111-5.

Boyd 1988 \{published and unpublished data\}

Boyd NF, McGuire V, Shannon P, Cousins M, Kriukov V, Mahoney $L$, et al. Effect of a low-fat high-carbohydrate diet on symptoms of cyclical mastopathy. Lancet 1988; 2(8603):128-32.

\section{BREACPNT \{published data only\}}

. The breast cancer personalized nutrition study (BREACPNT). clinicaltrials.gov/ct2/show/NCT04079270 (first posted 5 September 2019).

Brehm 2009 \{published data only (unpublished sought but not used)\}

Brehm BJ, Lattin BL, Summer SS, Boback JA, Gilchrist GM, Jandacek RJ, et al. One-year comparison of a highmonounsaturated fat diet with a high-carbohydrate diet in type 2 diabetes. Diabetes Care 2009; 32(2):215-20.

\section{Brensike 1982 \{published data only\}}

Brensike JF, Kelsey SF, Passamani ER, Fisher MR, Richardson JM, Loh IK, et al. National Heart, Lung, and Blood Institute type II Coronary Intervention Study: design, methods, and baseline characteristics. Controlled Clinical Trials 1982; 3(2):91-111. 
BRIDGES 2001 \{published and unpublished data\}

Hebert JR, Ebbeling CB, Olendzki BC, Hurley TG, Ma Y, Saal N, et al. Change in women's diet and body mass following intensive intervention for early-stage breast cancer. Journal of the American Dietetic Association 2001; 101(4):421-31.

Broekmans 2003 \{published and unpublished data\} Broekmans WM, Klöpping-Ketelaars IA, Weststrate JA, Tijburg LB, Van Poppel G, Vink AA, et al. Decreased carotenoid concentrations due to dietary sucrose polyesters do not affect possible markers of disease risk in humans. Journal of Nutrition 2003; 133(3):720-6.

\section{Brown 1984 \{published data only\}}

Brown GD, Whyte L, Gee MI, Crockford PM, Grace M, Oberle K, et al. Effects of two "lipid-lowering" diets on plasma lipid levels of patients with peripheral vascular disease. Journal of the American Dietetic Association 1984; 84(5):546-50.

\section{Bruce 1994 \{published data only\}}

Bruce SL, Grove SK. The effect of a coronary artery risk evaluation program on serum lipid values and cardiovascular risk levels. Applied Nursing Research 1994; 7(2):67-74.

\section{Bruno 1983 \{published data only\}}

Bruno R, Arnold C, Jacobson L, Winick M, Wynder E. Randomized controlled trial of a nonpharmacologic cholesterol reduction program at the worksite. Preventive Medicine 1983; 12(4):523-32.

\section{Butcher 1990 \{published data only\}}

Butcher LA, O'Dea K, Sinclair AJ, Parkin JD, Smith IL, Blombery P. The effects of very low fat diets enriched with fish or kangaroo meat on cold-induced vasoconstriction and platelet function. Prostaglandins, Leukotrienes, and Essential Fatty Acids 1990; 39(3):221-6.

\section{Byers 1995 \{published data only\}}

Byers T, Mullis R, Anderson J, Dusenbury L, Gorsky R, Kimber C, et al. The costs and effects of a nutritional education program following work-site cholesterol screening. American Journal of Public Health 1995; 85(5):650-5.

\section{Caggiula 1996 \{published data only\}}

Caggiula AW, Watson JE, Kuller LH, Olson MB, Milas NC, Berry M, et al. Cholesterol-lowering intervention program. Effect of the step I diet in community office practices. Archives of Internal Medicine 1996; 156(11):1205-13.

\section{Canadian DBCP 1997 \{published data only (unpublished sought} but not used)\}

Boyd NF, Greenberg C, Lockwood G, Little L, Martin L, Byng J, et al. Effects at two years of a low-fat, high-carbohydrate diet on radiologic features of the breast: results from a randomized trial. Journal of the Nationl Cancer Institute 1997; 89(7):488-96.

\section{CARMEN 2000 \{published and unpublished data\}}

Poppitt SD, Keogh GF, Prentice AM, Williams DE, Sonnemans HM, Valk EE, et al. Long-term effects of ad libitum low-fat, high-carbohydrate diets on body weight and serum lipids in overweight subjects with metabolic syndrome. American Journal of Clinical Nutrition 2002; 75(1):11-20.

Raben A, Astrup A, Vasilaras TH, Prentice AM, , Formiguera X, et al. The CARMEN study [CARMEN-studiet]. Ugeskrift für Laeger 2002; 164(5):627-31.

* Saris WH, Astrup A, Prentice AM, Zunft HJ, Formiguera X, Verboeket-van de Venne WP, et al. Randomized controlled trial of changes in dietary carbohydrate/fat ratio and simple vs complex carbohydrates on body weight and blood lipids: the CARMEN study. International Journal of Obesity 2000; 24(10):1310-8.

Saris WH, Astrup A, Prentice AM, Zunft HJ, Formiguera X. CARMEN Project: European multicentre study on the impact of dietary fat/CHO ratio and simple/complex $\mathrm{CHO}$ changes on long term weight control in overweight subjects. International Journal of Obesity 1997; 21(Suppl 2):S71.

Vasilaras TH, Astrup A, Raben A. Micronutrient intake in overweight subjects is not deficient on an ad libitum fatreduced, high-simple carbohydrate diet. European Journal of Clinical Nutrition 2004; 58(2):326-36.

\section{CARMEN substudy 2002 \{published and unpublished data\}}

Poppitt SD, Keogh GF, Prentice AM, Williams DEM, Sonnemans HMW, Valk EEJ, et al. Long-term effects of ad libitum low-fat, high-carbohydrate diets on body weight and serum lipids in overweight subjects with metabolic syndrome. American Journal of Clinical Nutrition 2002; 75(1):11-20.

\section{Casas-Agustench 2013 \{published data only\}}

Casas-Agustench P, Molina S, Espinosa-Salinas I, Olivares M, Reglero G, Ordovás JM, et al. SELP variant modulates plasma HDL-C responses in subjects with moderate cardiovascular risk after skimmed milk consumption. FASEB Journal 2013; 27(1 suppl):640.21.

Loria-Kohen V, Espinosa-Salinas I, Ramirez de Molina A, CasasAgustench P, Herranz J, Molina S, et al. A genetic variant of PPARA modulates cardiovascular risk biomarkers after milk consumption. Nutrition 2014; 30(10):1144-50.

\section{Cerin 1993 \{published data only\}}

Cerin A, Collins A, Landgren BM, Eneroth P. Hormonal and biochemical profiles of premenstrual syndrome. Treatment with essential fatty acids. Acta Obstetricia et Gynecologica Scandinavica 1993; 72(5):337-43

\section{Chan 1993 \{published data only\}}

Chan JK, McDonald BE, Gerrard JM, Bruce VM, Weaver BJ, Holub BJ. Effect of dietary alpha-linolenic acid and its ratio to linolenic acid on platelet and plasma fatty acids and thrombogenesis. Lipids 1993; 28(9):811-7.

\section{Chapman 1950 \{published data only\}}

Chapman CB, Gibbons T, Henschel A. The effect of the rice-fruit diet on the composition of the body. New England Journal of Medicine 1950; 243(23):899-905. 
Charbonnier 1975 \{published data only\}

Charbonnier A, Nepveux P, Fluteau G, Fluteau D. Immediate effects of ingestion of olive oil on the principal lipid constituents of the plasma. Comparison with other edible fats. Médecine \& Chirurgie Digestives 1975; 4 Suppl 2:73-9.

\section{Cheng 2004 \{published data only\}}

Cheng C, Graziani C, Diamond JJ. Cholesterol-lowering effect of the Food for Heart Nutrition Education Program. Journal of the American Dietetic Association 2004; 104(12):1868-72.

\section{Chiostri 1988 \{published data only\}}

Chiostri JE, Kwiterovich PO. Effect of American Heart Association Phase 2 diet versus eater's choice based diet on hypercholesterolaemia. Circulation 1988; 78(4):II-385.

\section{Choudhury 1984 \{published data only\}}

Choudhury S, Jackson P, Katan MB, Marenah CB, Cortese C, Miller NE, et al. A multifactorial diet in the management of hyperlipidaemia. Atherosclerosis 1984; 50(1):93-103.

\section{Clark 1997 \{published data only\}}

Clark M, Ghandour G, Miller NH, Taylor CB, Bandura A, DeBusk RF. Development and evaluation of a computer-based system for dietary management of hyperlipidemia. Journal of the American Dietetic Association 1997; 97(2):146-50.

\section{Clifton 1992 \{published data only\}}

Clifton PM, Wight MB, Nestel PJ. Is fat restriction needed with HMGCoA reductase inhibitor treatment? Atherosclerosis 1992; 93(1-2):59-70.

\section{Cobb 1991 \{published data only\}}

Cobb MM, Teitelbaum HS, Breslow JL. Lovastatin efficacy in reducing low-density lipoprotein cholesterol levels on high- vs low-fat diets. JAMA 1991; 265(8):997-1001.

\section{Cohen 1991 \{published data only\}}

Cohen MD, D'Amico FJ, Merenstein JH. Weight reduction in obese hypertensive patients. Family Medicine 1991; 23(1):25-8

\section{Cole 1988 \{published data only\}}

Cole TG, Schmeisser D, Prewitt TE. AHA phase 3 diet reduces cholesterol in moderately hypercholesterolemic premenopausal women [Abstract]. Circulation 1988; 78(4):II-73.

\section{Colquhoun 1990 \{published data only\}}

Colquhoun DM, Moores D, Somerset SM. Comparison of the effects of an avocado enriched and American Heart Association diets on lipid levels [Abstract]. Arteriosclerosis 1990; 10:875a.

\section{Consolazio 1946 \{published data only\}}

Consolazio FC, Forbes WH. The effects of high fat diet in a temperate environment. Journal of Nutrition 1946; 32:195-204.

\section{Cox 1996 \{published data only\}}

Cox RH, Gonzales-Vigilar MC, Novascone MA, Silva-Barbeau I. Impact of a cancer intervention on diet-related cardiovascular disease risks of white and African-American EFNEP clients. Journal of Nutrition Education 1996; 28:209-18.

\section{Croft 1986 \{published data only\}}

Croft PR, Brigg D, Smith S, Harrison CB, Branthwaite A, Collins MF. How useful is weight reduction in the management of hypertension? Journal of the Royal College of General Practitioners 1986; 36(291):445-8.

Curzio 1989 \{published and unpublished data\}

Curzio JL, Kennedy SS, Elliott HL, Farish E, Barnes JF, Howie CA, et al. Hypercholesterolaemia in treated hypertensives: a controlled trial of intensive dietary advice. Journal of Hypertension 1989; 7 (6 Suppl):S254-5.

Dalgard 2001 \{published data only\} Dalgard C, Thuroe A, Haastrup B, Haghfelt T, Stender S. Saturated fat intake is reduced in patients with ischemic heart disease 1 year after comprehensive counseling but not after brief counseling. Journal of the American Dietetic Association 2001; 101(12):1420-9.

\section{Da Qing IGT 1997 \{published data only\}}

Pan XR, Li GW, Hu YH, Wang JX, Yang WY, An ZX, et al. Effects of diet and exercise in preventing NIDDM in people with impaired glucose tolerance. The Da Qing IGT and Diabetes Study. Diabetes Care 1997; 20(4):537-44.

\section{DAS 2000 \{published data only\}}

Bovbjerg VE, McCann BS, Brief DJ, Follette WC, Retzlaff BM, Dowdy AA, et al. Spouse support and long-term adherence to lipid-lowering diets. American Journal of Epidemiology 1995; 141(5):451-60.

* Knopp RH, Retzlaff B, Walden C, Fish B, Buck B, McCann B. One-year effects of increasingly fat-restricted, carbohydrateenriched diets on lipoprotein levels in free-living subjects. Proceedings of the Society for Experimental Biology \& Medicine 2000; 225(3):191-9.

Knopp RH, Walden CE, McCann BS, Retzlaff B, Dowdy A, Gey G, et al. Serial changes in lipoprotein cholesterol in hypercholesterolemic men treated with alternative diets [abstract]. Arteriosclerosis 1989; 9:745A.

Knopp RH, Walden CE, Retzlaff BM, McCann BS, Dowdy AA, Albers JJ, et al. Long-term cholesterol-lowering effects of 4 fat-restricted diets in hypercholesterolaemic and combined hyperlipidaemic men: the Dietary Alternatives Study. JAMA 1997; 278(18):1509-15.

Walden CE, McCann BS, Retzlaff B, Dowdy A, Hanson M, Fish B, et al. Alternative fat-restricted diets for hypercholesterolemia and combined hyperlipidemia: feasibility, design, subject recruitment, and baseline characteristics of the Dietary Alternatives study. Journal of the American College of Nutrition 1991; 10(5):429-42.

\section{DASH 1997 \{published data only\}}

* Appel LJ, Moore TJ, Obarzanek E, Vollmer WM, Svetkey LP, Sacks FM, et al, DASH Collaborative Research Group. A clinical trial of the effects of dietary patterns on blood pressure. New England Journal of Medicine 1997; 336(16):1117-24. 
Blackburn GL. Functional foods in the prevention and treatment of disease: significance of the Dietary Approaches to Stop Hypertension Study. American Journal of Clinical Nutrition 1997; 66(5):1067-71.

\section{Davey Smith 2005 \{published data only\}}

Davey Smith G, Bracha Y, Svendsen KH, Neaton JD, Haffner SM, Kuller $\mathrm{LH}$, et al. Incidence of type 2 diabetes in the randomized multiple risk factor intervention trial. Annals of Internal Medicine 2005; 142(5):313-22.

\section{De Boer 1983 \{published data only\}}

De Boer AC, Turek JV, Pannebakker MA, Den Ottolander GJ. The effect of diets high in polyunsaturated and high in saturated fatty acids on blood lipids and platelet tests in patients with coronary artery disease (CAD) [abstract]. Thrombosis and Haemostasis 1983; 50:96.

\section{De Bont 1981 \{published and unpublished data\}}

De Bont AJ, Baker IA, St Leger AS, Sweetnam PM, Wragg KG, Stephens SM, et al. A randomised controlled trial of the effect of low fat diet advice on dietary response in insulin independent diabetic women. Diabetologia 1981; 21(6):529-33.

\section{DeBusk 1994 \{published data only\}}

DeBusk RF, Miller NH, Superko HR, Dennis CA, Thomas RJ, Lew HT, et al. A case-management system for coronary risk factor modification after acute myocardial infarction [see comments]. Annals of Internal Medicine 1994; 120(9):721-9.

\section{DEER 1998 \{published data only (unpublished sought but not} used)\}

Stefanick ML, Mackey S, Sheehan RD, Ellsworth N, Haskell WL, Wood PD. Effects of diet and exercise in men and postmenopausal women with low levels of HDL cholesterol and high levels of LDL cholesterol. New England Journal of Medicine 1998; 339(1):12-20.

\section{Delahanty 2001 \{published data only\}}

Delahanty LM, Hayden D, Ammerman A, Nathan DM. Medical nutrition therapy for hypercholesterolemia positively affects patient satisfaction and quality of life outcomes. Annals of Behavioral Medicine 2002; 24(4):269-78.

* Delahanty LM, Sonnenberg LM, Hayden D, Nathan DM. Clinical and cost outcomes of medical nutrition therapy for hypercholesterolemia: a controlled trial. Journal of the American Dietetic Association 2001; 101(9):1012-23.

\section{Delius 1969 \{published data only\}}

Delius L. Treatment of hypotensive circulatory disorder [Die behandlung der hypotonen kreislaufregulationsstörung]. Deutsche Medizinische Wochenschrift 1969; 94(42):2172-3.

\section{Demark 1990 \{published data only\}}

Demark WW, Bowering J, Cohen PS. Reduced serum cholesterol with dietary change using fat-modified and oat bran supplemented diets. Journal of the American Dietetic Association 1990; 90(2):223-9.

\section{Dengel 1995 \{published data only\}}

Dengel JL, Katzel LI, Goldberg AP. Effect of an American Heart Association diet, with or without weight loss, on lipids in obese middle-aged and older men. American Journal of Clinical Nutrition 1995; 62(4):715-21.

\section{Denke 1994 \{published data only\}}

Denke MA, Grundy SM. Individual responses to a cholesterol lowering diet in 50 men with moderate hypercholesterolaemia. Archives of Internal Medicine 1994; 154(3):17-25.

Diabetes CCT 1995 \{published data only\}

The Diabetes Control and Complications Trial (DCCT) Research Group. Effect of intensive diabetes management on macrovascular events and risk factors in the Diabetes Control and Complications Trial. American Journal of Cardiology 1995; 75(14):894-903.

Diet \& Hormone Study 2003 \{published data only (unpublished sought but not used)\}

Gann PH, Chatterton RT, Gapstur SM, Liu K, Garside D, Giovanazzi S, et al. The effects of a low-fat/high-fiber diet on sex hormone levels and menstrual cycling in premenopausal women: a 12-month randomized trial (the Diet and Hormone Study). Cancer 2003; 98(9):1870-9.

\section{DIET 1998 \{published data only\}}

Dornelas EA, Wylie-Rosett J, Swencionis C. The DIET study: long term outcomes of a cognitive-behavioural weight control intervention in independent-living elders. Journal of the American Dietetic Association 1998; 98(11):1276-81.

\section{Ding 1992 \{published data only\}}

Ding Q. Clinical study of qianxining in the treatment of 60 cases of yang hyperactivity due to yin deficiency type of hypertension. Chung Kuo Chung Hsi I Chieh Ho Tsa Chih 1992; 12(7):409-11, 388-9.

\section{DIPI 2018 \{published data only\} \\ ZZZ <accessionId ref="info:x-wiley/clinicalTrialsGov/ NCT02062424"> ZZZNCT02062424}

Arentoft JL, Hoppe C, Andersen EW, Overvad K, Tetens I. Associations between adherence to the Danish Food-Based Dietary Guidelines and cardiometabolic risk factors in a Danish adult population: the DIPI study. British Journal of Nutrition 2018; 119(6):664-73.

DIRECT 2009 \{published data only (unpublished sought but not used)\}

* Ben-Avraham S, Harman-Boehm I, Schwarzfuchs D, Shai I. Dietary strategies for patients with type 2 diabetes in the era of multi-approaches; review and results from the Dietary Intervention Randomized Controlled Trial (DIRECT). Diabetes Research and Clinical Practice 2009; 86(Suppl 1):S41-8.

Resch KL. Dietary Intervention Randomized Controlled Trial (DIRECT) group: weight loss with a low-

carbohydrate, Mediterranean, or low-fat diet. Forschende Komplementarmedizin (2006) 2008; 15(6):351-2. 
Shai I, Gepner Y, Shelef I, Schwarzfuchs D, Zelicha H, Tene L, et al. Precision nutrition. Obesity Facts 2019; 12:1.

Shai I, Schwarzfuchs D, Henkin Y, Shahar DR, Witkow S, Greenberg I, et al. Weight loss with a low-carbohydrate, Mediterranean, or low-fat diet. New England Journal of Medicine 2008; 359(3):229-41.

Shai I, Spence JD, Schwarzfuchs D, Henkin Y, Parraga G, Rudich A, et al. Dietary intervention to reverse carotid atherosclerosis. Circulation 2010; 121(10):1200-8.

Shai I. The effect of low-carb, Mediterranean and low-fat diets on renal function; a 2-year dietary intervention randomized controlled trial (DIRECT). Obesity Facts 2012; 5:19.

\section{Dobs 1991 \{published data only\}}

Dobs AS, Sarma PS, Wilder L. Lipid-lowering diets in patients taking pravastatin, a new HMG-CoA reductase inhibitor: compliance and adequacy. American Journal of Clinical Nutrition 1991; 54(4):696-700.

\section{DO IT 2006 \{published and unpublished data\}}

Berstad P, Seljeflot I, Veierod MB, Hjerkinn EM, Arnesen H, Pedersen JI, et al. Supplementation with fish oil affects the association between very long-chain $n-3$ polyunsaturated fatty acids in serum non-esterified fatty acids and soluble vascular cell adhesion molecule-1. Clinical Science 2003; 105(1):13-20.

Ellingsen I, Hjerkinn EM, Seljeflot I, Arnesen H, Tonstad S, Ellingsen I, et al. Consumption of fruit and berries is inversely associated with carotid atherosclerosis in elderly men. British Journal of Nutrition 2008; 99(3):674-81 Erratum in: British Journal of Nutrition 2008; 99(3): 697.

Ellingsen I, Seljeflot I, Arnesen H, Tonstad S, , , et al. Vitamin $C$ consumption is associated with less progression in carotid intima media thickness in elderly men: a 3-year intervention study. Nutrition Metabolism \& Cardiovascular Diseases 2009; 19(1):8-14.

Furenes EB, Seljeflot I, Solheim S, Hjerkinn EM, Arnesen H. Long-term influence of diet and/or omega-3 fatty acids on matrix metalloproteinase- 9 and pregnancy-associated plasma protein-A in men at high risk of coronary heart disease. Scandinavian Journal of Clinical \& Laboratory Investigation 2008; 68(3):177-84.

* Hjerkinn EM, Abdelnoor M, Breivik L, Bergengen L, Ellingsen I, Seljeflot I, et al. Effect of diet or very long chain omega-3 fatty acids on progression of atherosclerosis, evaluated by carotid plaques, intima-media thickness and by pulse wave propagation in elderly men with hypercholesterolaemia. European Journal of Cardiovascular Prevention \& Rehabilitation 2006; 13(3):325-33.

Hjerkinn EM, Seljeflot I, Ellingsen I, Berstad P, Hjermann I, Sandvik L, et al. Influence of long-term intervention with dietary counselling, long-chain n-3 fatty acid supplements, or both on circulating markers of endothelial activation in men with longstanding hyperlipidemia. American Journal of Clinical Nutrition 2005; 81(3):583-9.
Lindman AS, Pedersen JI, Hjerkinn EM, Arnesen $\mathrm{H}$, Veierod MB, Ellingsen I, et al. The effects of long-term diet and omega-3 fatty acid supplementation on coagulation factor VII and serum phospholipids with special emphasis on the R353Q polymorphism of the FVII gene. Thrombosis \& Haemostasis 2004; 91(6):1097-104.

Troseid M, Arnesen H, Hjerkinn EM, Seljeflot I. Serum levels of interleukin-18 are reduced by diet and $n-3$ fatty acid intervention in elderly high-risk men. Metabolism: Clinical \& Experimental 2009; 58(11):1543-9.

Troseid M, Seljeflot I, Hjerkinn EM, Arnesen H. Interleukin-18 is a strong predictor of cardiovascular events in elderly men with the metabolic syndrome: synergistic effect of inflammation and hyperglycemia. Diabetes Care 2009; 32(3):486-92.

\section{Due 2008 \{published and unpublished data\}}

Bladbjerg EM, Larsen TM, Due A, Jespersen J, Stender S, Astrup A. Postprandial coagulation activation in overweight individuals after weight loss: acute and long-term effects of a high-monounsaturated fat diet and a low-fat diet. Thrombosis Research 2014; 133(3):327-33.

Due A, Larsen TM, Hermansen K, Stender S, Holst JJ, Toubro S, et al. Comparison of the effects on insulin resistance and glucose tolerance of 6-mo high-monounsaturated-fat, low-fat, and control diets. American Journal of Clinical Nutrition 2008; 87(4):855-62.

* Due A, Larsen TM, Mu H, Hermansen K, Stender S, Astrup A. Comparison of 3 ad libitum diets for weight-loss maintenance, risk of cardiovascular disease, and diabetes: a 6-mo randomized, controlled trial. American Journal of Clinical Nutrition 2008; 88(5):1232-41.

Rasmussen LG, Larsen TM, Mortensen PK, Due A, Astrup A. Effect on 24-h energy expenditure of a moderate-fat diet high in monounsaturated fatty acids compared with that of a low-fat, carbohydrate-rich diet: a 6-mo controlled dietary intervention trial. American Journal of Clinical Nutrition 2007; 85(4):1014-22.

Sloth B, Due A, Larsen TM, Holst JJ, Heding A, Astrup A, et al. The effect of a high-MUFA, low-glycaemic index diet and a lowfat diet on appetite and glucose metabolism during a 6-month weight maintenance period. British Journal of Nutrition 2009; 101(12):1846-58.

\section{Duffield 1982 \{published data only\}}

* Duffield RG, Lewis B, Miller NE, Jamieson CW, Brunt JN, Colchester AC. Treatment of hyperlipidaemia retards progression of symptomatic femoral atherosclerosis. A randomised controlled trial. Lancet 1983; 2(8351):639-42.

Duffield RG, Miller NE, Jamieson CW, Lewis B. A controlled trial of plasma lipid reduction in peripheral atherosclerosis - an interim report. British Journal of Surgery 1982; 69(Suppl):S3-S5.

Dullaart 1992 \{published and unpublished data\}

Dullaart RP, Beusekamp BJ, Meijer S, Hoogenberg K, Van Doormaal JJ, Sluiter WJ. Long-term effects of linoleic-acidenriched diet on albuminuria and lipid levels in type 1 (insulin- 
dependent) diabetic patients with elevated urinary albumin excretion. Diabetologia 1992; 35(2):165-72.

Eating Patterns 1997 \{published and unpublished data\} Beresford SA, Curry SJ, Kristal AR, Lazovich D, Feng Z, Wagner EH. A dietary intervention in primary care practice: the Eating Patterns Study. American Journal of Public Health 1997; 87(4):610-6.

\section{Ehnholm 1982 \{published data only\}}

Ehnholm C, Huttunen JK, Pietinen P, Leino U, Mutanen M, Kostiainen $\mathrm{E}$, et al. Effect of diet on serum lipoproteins in a population with a high risk of coronary heart disease. New England Journal of Medicine 1982; 307(14):850-5.

\section{Ehnholm 1984 \{published data only\}}

Ehnholm C, Huttunen JK, Pietinen P, Leino U, Mutanen M, Kostiainen $\mathrm{E}$, et al. Effect of a diet low in saturated fatty acids on plasma lipids, lipoproteins, and HDL subfractions. Arteriosclerosis 1984; 4(3):265-9.

\section{Eisenberg 1990 \{published data only\}}

Eisenberg S. The effect of dietary substitution of monounsaturated fatty acids with carbohydrates on lipoprotein levels, structure, and function in a free-living population [abstract]. Arteriosclerosis 1990; 10:872A.

\section{Elder 2000 \{published data only\}}

Elder JP, Candelaria JI, Woodruff SI, Criqui MH, Talavera GA, Rupp JW. Results of language for health: cardiovascular disease nutrition education for Latino English-as-a-second-language students. Health Education \& Behavior 2000; 27(1):50-63.

\section{Ellegard 1991 \{published data only\}}

Ellegard L, Bosaeus I. Sterol and nutrient excretion in ileostomists on prudent diets. European Journal of Clinical Nutrition 1991; 45(9):451-7.

\section{Esposito 2003 \{published data only\}}

Esposito K, Pontillo A, Di Palo C, Giugliano G, Masella M, Marfella R, et al. Effect of weight loss and lifestyle changes on vascular inflammatory markers in obese women: a randomized trial. JAMA 2003; 289(14):1799-804.

\section{Esposito 2004 \{published data only\}}

Esposito K, Marfella R, Ciotola M, Di Palo C, Giugliano F, Giugliano G, et al. Effect of a Mediterranean-style diet on endothelial dysfunction and markers of vascular inflammation in the metabolic syndrome: a randomized trial. JAMA 2004; 292(12):1440-6.

\section{EUROACTION 2008 \{published data only\}}

Wood DA, Kotseva K, Connolly S, Jennings C, Mead A, Jones J, et al. Nurse-coordinated multidisciplinary, familybased cardiovascular disease prevention programme (EUROACTION) for patients with coronary heart disease and asymptomatic individuals at high risk of cardiovascular disease: a paired, cluster-randomised controlled trial. Lancet 2008; 371(9629):1999-2012.

\section{FARIS 1997 \{published data only\}}

Goble A, Jackson B, Phillips P, Race E, Oliver RG, Worcester MC. The Family Atherosclerosis Risk Intervention Study (FARIS): risk factor profiles of patients and their relatives following an acute cardiac event. Australian and New Zealand Journal of Medicine 1997; 27(5):568-77.

\section{Fasting HGS 1997 \{published data only\}}

Dyson PA, Hammersley MS, Morris RJ, Holman RR, Turner RC. The Fasting Hyperglycaemia Study: II. Randomized controlled trial of reinforced healthy-living advice in subjects with increased but not diabetic fasting plasma glucose. Metabolism 1997; 46(12 Suppl 1):50-5.

\section{Ferrara 2000 \{published data only\}}

Ferrara LA, Raimondi AS, D'Episcopo L, Guida L, Dello Russo A, Marotta T. Olive oil and reduced need for antihypertensive medications. Archives of Internal Medicine 2000; 160(6):837-42.

Fielding 1995 \{published data only\}

Fielding CJ, Havel RJ, Todd KM, Yeo KE, Schloetter MC, Weinberg V, et al. Effects of dietary cholesterol and fat saturation on plasma lipoproteins in an ethnically diverse population of healthy young men. Journal of Clinical Investigation 1995; 95(2):611-8.

\section{Finnish Diabet Prev 2000 \{published data only\}}

Uusitupa M, Louheranta A, Lindstrom J, Valle T, Sundvall J, Eriksson J, et al. The Finnish Diabetes Prevention Study. British Journal of Nutrition 2000; 83(Suppl 1):S137-42.

Finnish Mental Hosp 1972 \{published data only\}

* Miettinen M, Turpeinen O, Karvonen MJ, Elosuo R, Paavilainen E. Effect of cholesterol-lowering diet on mortality from coronary heart-disease and other causes: a twelve-year clinical trial in men and women. Lancet 1972; 2(782):835-8.

Miettinen M, Turpeinen O, Karvonen MJ, Pekkarinen M, Paavilainen E, Elosuo R. Dietary prevention of coronary heart disease in women: the Finnish Mental Hospital study. International Journal of Epidemiology 1983; 12(1):17-25.

Turpeinen O, Miettinen M, Karvonen M, Roine P, Pekkarinen M, Lehtosuo EJ, et al. Dietary prevention of coronary heart disease: long-term experiment. I. Observations on male subjects. American Journal of Clinical Nutrition 1968; 21(4):255-76.

\section{Fisher 1981 \{published data only\}}

Fisher EA, Breslow JL, Zannis VI, Shen G, Blum CB. Dietary saturated fat, not cholesterol, affects plasma lipids and Apo E. Arteriosclerosis 1981; 1(5):364a.

FIT Heart 2011 \{published data only\}

Mochari-Greenberger H, Terry MB, Mosca L. Does stage of change modify the effectiveness of an educational intervention to improve diet among family members of hospitalized cardiovascular disease patients? Journal of the American Dietetic Association 2010; 110(7):1027-35.

* Mochari-Greenberger H, Terry MB, Mosca L. Gender, age and race/ethnicity do not modify the effectiveness of a diet intervention among family members of hospitalized 
cardiovascular disease patients. Journal of Nutrition Education and Behavior 2011; 43(5):366-73.

Fleming 2002 \{published data only\}

Fleming RM. The effect of high-, moderate-, and low-fat diets on weight loss and cardiovascular disease risk factors. Preventive Cardiology 2002; 5(3):110-5.

\section{Fortmann 1988 \{published data only\}}

Fortmann SP, Haskell WL, Wood PD. Effects of weight loss on clinic and ambulatory blood pressure in normotensive men. American Journal of Cardiology 1988; 62(1):89-93.

\section{Foster 2003 \{published data only\}}

Foster GD, Wyatt HR, Hill JO, McGuckin BG, Brill C, Mohammed BS, et al. A randomized trial of a low-carbohydrate diet for obesity. New England Journal of Medicine 2003; 348(21):2082-90.

\section{Frenkiel 1986 \{published data only\}}

Frenkiel PG, Lee DW, Cohen H, Gilmore CJ, Resser K, Bonorris GG, et al. The effect of diet on bile acid kinetics and biliary lipid secretion in gallstone patients treated with ursodeoxycholic acid. American Journal of Clinical Nutrition 1986; 43(2):239-50.

\section{FRESH START 2007 \{published data only\}}

Denmark-Wahnefried W, Clipp EC, Lipkus IM, Lobach D, Snyder DC, Sloane R, et al. Main outcomes of the FRESH START trial: a sequentially tailored, diet and exercise mailed print intervention among breast and prostate cancer survivors. Journal of Clinical Oncology 2007; 25(19):2709-18.

\section{Gambera 1995 \{published data only\}}

Gambera PJ, Schneeman BO, Davis PA. Use of the Food Guide Pyramid and US Dietary Guidelines to improve dietary intake and reduce cardiovascular risk in active-duty Air Force members. Journal of the American Dietetic Association 1995; 95(11):1268-73.

\section{Gaullier 2007 \{published data only\}}

, Halse J, Hoivik HO, Hoye K, Syvertsen C, Nurminiemi M, et al. Six months supplementation with conjugated linoleic acid induces regional-specific fat mass decreases in overweight and obese. British Journal of Nutrition 2007; 97:550-60.

\section{Ginsberg 1988 \{published data only\}}

Ginsberg H. Both a high monounsaturated fat diet and the step 1 AHA diet significantly reduce plasma cholesterol levels in healthy males [abstract]. Circulation 1988; 78:II73.

\section{Gjone 1972 \{published data only\}}

Gjone E, Nordoy A, Blomhoff JP, Wiencke I. The effects of unsaturated and saturated dietary fats on plasma cholesterol, phospholipids and lecithin: cholesterol acyltransferase activity. Acta Medica Scandinavica 1972; 191(6):481-4.

\section{Glatzel 1966 \{published data only\}}

Glatzel $H$. The relationship between postprandial triglyceridemia and the fat content of the basic diet [Die
Abhängigkeit der postcenalen triglyceridamie vom fettgehalt der grundkost]. Klinische Wochenschrift 1966; 44(5):283-4.

Goodpaster 1999 \{published data only\}

Goodpaster BH, Kelley DE, Wing RR, Meier A, Thaete FL. Effects of weight loss on regional fat distribution and insulin sensitivity in obesity. Diabetes 1999; 48(4):839-47.

Grundy 1986 \{published data only\}

Grundy SM, Nix D, Whelan MF, Franklin L. Comparison of three cholesterol-lowering diets in normolipidaemic men. JAMA 1986; 256(17):2351-5

Hardcastle 2008 \{published data only\}

Hardcastle S, Taylor A, Bailey M, Castle R. A randomised controlled trial on the effectiveness of a primary health care based counselling intervention on physical activity, diet and CHD risk factors. Patient Education \& Counseling 2008; 70(1):31-9.

\section{Harris 1990 \{published data only\}}

Harris WS, Feldman EB. Intensive dietary intervention in hypercholesterolemic patients. Observed versus predicted changes in cholesterol levels [abstract]. Arteriosclerosis 1990; 10:853A.

\section{Hartman 1993 \{published data only\}}

Hartman T, McCarthy P, Himes J. Use of eating pattern messages to evaluate changes in eating behaviors in a worksite cholesterol education program. Journal of the American Dietetic Association 1993; 93(10):1119-23.

\section{Hartwell 1986 \{published data only\}}

Hartwell SL, Kaplan RM, Wallace JP. Comparison of behavioral interventions for control of type II diabetes mellitus. Behavior Therapy 1986; 17:447-61.

Hashim 1960 \{published data only\} Hashim SA, Arteaga A, Van Itallie TB. Effect of saturated medium-chain triglyceride on serum-lipids in man. Lancet 1960; 1(7134):1105-7.

Haufe 2011 \{published and unpublished data\}

* Haufe S, Engeli S, Kast P, Böhnke J, Utz W, Haas V, et al. Randomized comparison of reduced fat and reduced carbohydrate hypocaloric diets on intrahepatic fat in overweight and obese human subjects. Hepatology 2011; 53(5):1504-14.

Haufe S, Utz W, Engeli S, Kast P, Böhnke J, Pofahl M, et al. Left ventricular mass and function with reduced-fat or reducedcarbohydrate hypocaloric diets in overweight and obese subjects. Hypertension 2012; 59(1):70-5.

Haynes 1984 \{published data only\}

Haynes RB, Harper AC, Costley SR, Johnston M, Logan AG, Flanagan PT, et al. Failure of weight reduction to reduce mildly elevated blood pressure: a randomized trial. Journal of Hypertension 1984; 2(5):535-9. 
Heber 1991 \{published data only\}

Heber D, Ashley JM, Leaf DA, Barnard JA. Reduction of serum estradiol in postmenopausal women given free access to low-fat high carbohydrate diet. Nutrition 1991; 7(2):137-41.

\section{Heine 1989 \{published and unpublished data\}}

Heine RJ, Mulder C, Popp-Snijders C, Van der Meer J, Van der Veen EA. Linoleic-acid-enriched diet: long-term effects on serum lipoprotein and apolipoprotein concentration and insulin sensitivity in noninsulin-dependent diabetic patients. American Journal of Clinical Nutrition 1989; 49(3):448-56.

Hellenius 1995 \{published and unpublished data\} Hellenius ML, Brismar KE, Berglund BH, De Faire U. Effects on glucose tolerance, insulin secretion, insulin-like growth factor 1 and its binding protein, IGFBP-1, in a randomized controlled diet and exercise study in healthy, middle-aged men. Journal of Internal Medicine 1995; 238(2):121-30.

Hellenius ML, Dahlof C, Aberg H, Krakau I, De Faire U. Quality of life is not negatively affected by diet and exercise intervention in healthy men with cardiovascular risk factors. Quality of Life Research 1995; 4(1):13-20.

Hellenius ML, De Faire U, Berglund B, Hamsten A, Krakau I. Diet and exercise are equally effective in reducing risk for cardiovascular disease. Results of a randomized controlled study in men with slightly to moderately raised cardiovascular risk factors. Atherosclerosis 1993; 103(1):81-91.

Hellenius ML, Krakau I, De Faire U. Favourable long-term effects from advice on diet and exercise given to healthy men with raised cardiovascular risks. Nutrition, Metabolism and Cardiovascular Diseases 1997; 7:293-300.

* ,. Prevention of Cardiovascular Disease: Studies on the Role of Diet and Exercise in the Prevention of Cardiovascular Disease among Middle-Aged Men [PhD thesis]. Huddinge, Sweden: Karolinska Intitute, 1995.

Naslund GK, Fredrikson M, Hellenius ML, De Faire U. Effect of diet and physical exercise intervention programmes on coronary heart disease risk in smoking and non-smoking men in Sweden. Journal of Epidemiology and Community Health 1996; 50(2):131-6.

\section{Heller 1993 \{published and unpublished data\}}

* Heller RF, Knapp JC, Valenti LA, Dobson AJ. Secondary prevention after acute myocardial infarction. American Journal of Cardiology 1993; 72(11):759-62.

Heller RF, Walker RJ, Boyle CA, O'Connell DL, Rusakaniko S, Dobson AJ. A randomised controlled trial of a dietary advice program for relatives of heart attack victims. Medical Journal of Australia 1994; 161(9):529-31.

\section{Hildreth 1951 \{published data only\}}

Hildreth EA, Mellinkoff SM, Blair GW, Hildreth DM. The effect of vegetable fat ingestion on human serum cholesterol concentration. Circulation 1951; 3(5):641-6.
Holm 1990 \{published data only (unpublished sought but not used)\}

* Holm LE, Nordevang E, Ikkala E, Hallstrom L, Callmer E. Dietary intervention as adjuvant therapy in breast cancer patients - a feasibility study. Breast Cancer Research and Treatment 1990; 16(2):103-9.

Nordevang E, Callmer E, Marmur A, Holm LE. Dietary intervention in breast cancer patients: effects on food choice. European Journal of Clinical Nutrition 1992; 46(6):387-96.

Nordevang E, Ikkala E, Callmer E, Hallstrom L, Holm LE. Dietary intervention in breast cancer patients: effects on dietary habits and nutrient intake. European Journal of Clinical Nutrition 1990; 44(9):681-7.

Horlick 1957 \{published data only\}

* Horlick L, Craig BM. Effect of long-chain polyunsaturated and saturated fatty acids on the serum-lipids of man. Lancet 1957; 2:566-9.

\section{Horlick 1960 \{published data only\}}

Horlick L, O'Neil JB. Effect of modified egg-yolk fats on bloodcholesterol levels [letter]. Lancet 1960; 1:438.

Howard 1977 \{published data only\}

Howard AN, Marks J. Hypocholesterolaemic effect of milk [letter]. Lancet 1977; 2(8031):255-6.

\section{Hunninghake 1990 \{published data only\}}

Hunninghake DB, Laskarzewski PM. Gender difference in the response to lovastatin administration with and without a cholesterol lowering diet [abstract]. Arteriosclerosis 1990; 10:786A.

\section{Hutchison 1983 \{published data only\}}

Hutchison K, Oberle K, Crockford P, Grace M, Whyte L, Gee M, et al. Effects of dietary manipulation on vascular status of patients with peripheral vascular disease. JAMA 1983; 249(24):3326-30.

Hyman 1998 \{published and unpublished data\}

Hyman DJ, Ho KS, Dunn K, Simons-Morton D. Dietary intervention for cholesterol reduction in public clinic patients. American Journal of Preventive Medicine 1998; 15(2):139-45.

\section{lacono 1981 \{published data only\}}

lacono JM, Judd JT, Marshall MW, Canary JJ, Dougherty RM, Mackin JF, et al. The role of dietary essential fatty acids and prostaglandins in reducing blood pressure. Progress in Lipid Research 1981; 20:349-64.

IMPACT 1995 \{published data only\}

Fielding JE, Mason T, Kinght K, Klesges R, Pelletier KR. A randomized trial of the IMPACT worksite cholesterol reduction program. American Journal of Preventive Medicine 1995; 11(2):120-3

Iso 1991 \{published data only\}

Iso H, Konishi M, Terao A, Kiyama M, Tanigaki M, Baba M, et al. A community-based education program for serum cholesterol reduction in urban hypercholesterolemic persons: 
comparison of intensive and usual education groups. Nippon.Koshu.Eisei.Zasshi [Japanese Journal of Public Health] 1991; 38(9):751-61.

\section{Ives 1993 \{published data only\}}

Ives DG, Kuller LH, Traven ND. Use and outcomes of a cholesterol-lowering intervention for rural elderly subjects. American Journal of Preventive Medicine 1993; 9(5):274-81.

\section{Jalkanen 1991 \{published data only\}}

Jalkanen L. The effect of a weight reduction program on cardiovascular risk factors among overweight hypertensives in primary health care. Scandinavian Journal of Social Medicine 1991; 19(1):66-71.

\section{Jerusalem Nut 1992 \{published data only\}}

Berry EM, Eisenberg S, Friedlander Y, Harats D, Kaufmann NA, Norman Y, et al. Effects of diets rich in monounsaturated fatty acids on plasma lipoproteins: the Jerusalem Nutrition Study. II. Monounsaturated fatty acids vs carbohydrates. American Journal of Clinical Nutrition 1992; 56(2):394-403.

\section{Jula 1990 \{published data only\}}

Jula A, Ronnemaa T, Rastas M, Karvetti RL, Maki J. Longterm nonpharmacological treatment for mild to moderate hypertension. Journal of Internal Medicine 1990; 227(6):413-21.

\section{Junker 2001 \{published data only\}}

Junker R, Pieke B, Schulte H, Nofer R, Neufeld M, Assmann G, et al. Changes in hemostasis during treatment of hypertriglyceridemia with a diet rich in monounsaturated and $\mathrm{n}-3$ polyunsaturated fatty acids in comparison with a low-fat diet. Thrombosis Research 2001; 101(5):355-66.

\section{Karmally 1990 \{published data only\}}

Karmally W, Carpentiri C, Viscardi T, Cheverez V, Holleran S, Ramakrishnan $\mathrm{R}$, et al. Replacing monounsaturated by polyunsaturated fatty acids within an AHA step I diet does not affect the plasma levels or metabolism of low density and high density lipoproteins in normal men [abstract]. Arteriosclerosis 1990; 10:877A.

\section{Karvetti 1992 \{published data only\}}

Karvetti RL, Hakala P. A seven-year follow-up of a weight reduction programme in Finnish primary health care. European Journal of Clinical Nutrition 1992; 46(10):743-52.

\section{Kastarinen 2002 \{published data only\}}

Kastarinen MJ, Puska PM, Korhonen MH, Mustonen JN, Salomaa VV, Sundvall JE, et al. Non-pharmacological treatment of hypertension in primary health care: a 2-year open randomized controlled trial of lifestyle intervention against hypertension in eastern Finland. Journal of Hypertension 2002; 20(12):01.

\section{Kather 1985 \{published data only\}}

Kather $\mathrm{H}$, Wildenberg $\mathrm{U}$, Wieland E. Influence of different dietary conditions in ideal-weight subjects on serum levels of free fatty acids and of glycerol in vivo and on lipid mobilization in vitro [abstract]. European Journal of Clinical Investigation 1985; 15(Suppl 1):

\section{Katzel 1995 \{published data only\}}

Katzel LI, Bleecker ER, Colman EG, Rogus EM, Sorkin JD, Goldberg AP. Effects of weight loss vs aerobic exercise training on risk factors for coronary disease in healthy, obese, middleaged and older men. A randomized controlled trial [see comments]. JAMA 1995; 274(24):1915-21.

\section{Kawamura 1993 \{published data only\}}

Kawamura M, Akasaka T, Kasatsuki T, Nakajima J, Onodera S, Fujiwara T, et al. Blood pressure is reduced by short-time calorie restriction in overweight hypertensive women with a constant intake of sodium and potassium. Journal of Hypertension 1993; 11(Suppl 5):S320-1.

Keidar 1988 \{published data only\} Keidar S, Krul ES, Goldberg AC, Bateman J, Schonfield G. Fatfree diet modulates epitope expression of LDL-apo [abstract]. Arteriosclerosis 1988; 8:565A.

\section{Kempner 1948 \{published data only\}}

Kempner W. Treatment of hypertensive vascular disease with rice diet. American Journal of Medicine 1948; 4(4):545-77.

Keys 1957a \{published data only\}

Keys A, Anderson JT, Grande F. Serum-cholesterol response to dietary fat [letter]. Lancet 1957; 1:787.

\section{Keys 1957b \{published data only\}}

Keys A, Anderson JT, Grande F. Essential fatty acids, degree of unsaturation, and effect of corn (maize) oil on the serumcholesterol level in man. Lancet 1957; 1(6959):66-8.

Keys 1957c \{published data only\}

Keys A. Prediction of serum-cholesterol responses of man to changes in fats in the diet. Lancet 1957; 2:959-66.

Khan 2003 \{published and unpublished data\}

Khan F, Elherik K, Bolton-Smith C, Barr R, Hill A, Murrie I, et al. The effects of dietary fatty acid supplementation on endothelial function and vascular tone in healthy subjects. Cardiovascular Research 2003; 59(4):955-62.

King 2000 \{published data only\}

King S, David S, Newton H, Hevey D, Rafferty F, Horgan JH. The effect of dietary modification on the training outcome and body composition in patients undergoing undergoing a cardiac rehabilitation programme. Coronary Health Care 2000; 4(2):76-81.

\section{Kingsbury 1961 \{published data only\}}

Kingsbury KJ, Morgan DM, Aylott C, Emmerson R. Effects of ethyl arachidonate, cod-liver oil, and corn oil on the plasmacholesterol level: a comparison in normal volunteers. Lancet 1961; 1(7180):739-41.

KNOTA \{published data only\}

ZZZ <accessionId ref="info:x-wiley/clinicalTrialsGov/ NCT00692536"> ZZZNCT00692536

Andersson J, Mellberg C, Otten J, Ryberg M, Rinnstrom D, Larsson C, et al. Left ventricular remodelling changes without 
concomitant loss of myocardial fat after long-term dietary intervention. International Journal of Cardiology 2016; 216:92-6.

Blomquist C, Chorell E, Ryberg M, Mellberg C, Larsson C, Lindahl $B$, et al. Beneficial effects on fatty acid composition and indices of fatty acid desaturase activity with a paleolithic-type diet during a two-year intervention in obese postmenopausal women. Endocrine Reviews 2016; 37(2 Suppl 1):SUN 575.

Blomquist C, Chorell E, Ryberg M, Mellberg C, Worrsjo E, Makoveichuk E, et al. Decreased lipogenesis-promoting factors in adipose tissue in postmenopausal women with overweight on a Paleolithic-type diet. European Journal of Nutrition 2018; 57(8):2877-86.

Franklin KA, Eriksson M, Larsson C, Lindahl B, Mellberg C, Sahlin C, et al. Palaeolithic diet and obstructive sleep apnoea in overweight females: a randomised controlled trial. European Respiratory Journal 2016; 48:PA2376.

* Mellberg C, Sandberg S, Ryberg M, Eriksson M, Brage S, Larsson C, et al. Long-term effects of a Palaeolithic-type diet in obese postmenopausal women: a 2-year randomized trial. European Journal of Clinical Nutrition 2014; 68(3):350-7.

Otten J, Mellberg C, Ryberg M, Sandberg S, Kullberg J, Lindahl B, et al. Strong and persistent effect on liver fat with a Paleolithic diet during a two-year intervention. International Journal of Obesity 2016; 40(5):747-53.

Otten J, Mellberg C, Sandberg S, Hauksson J, Larsson C, Lindahl B, et al. Decrease of visceral adipose tissue and liver fat during diet intervention: a predictor of insulin sensitivity improvement? Diabetes 2014; 63:A16-.

Otten J, Ryberg M, Mellberg C, Andersson T, Chorell E, Lindahl B, et al. Postprandial levels of GLP-1, GIP and glucagon after 2 years of weight loss with a Paleolithic diet: a randomised controlled trial in healthy obese women. European Journal of Endocrinology 2019; 180(6):417-27.

Otten J, Ryberg M, Mellberg C, Lindahl B, Larsson C, Juul Holst J, et al. Weight loss by two different diets increases the postprandial response of GLP-1 but only the Paleolithic diet increases the postprandial response of GIP. Diabetologia 2017; 60(1):S233-.

Otten J, Stomby A, Ryberg M, Svensson M, Hauksson J, Olsson T. Effects of a paleolithic diet with and without supervised exercise on liver fat and insulin sensitivity: a randomised controlled trial in individuals with type 2 diabetes. Diabetologia 2016; 59(1):S10-.

Otten J, Stomby A, Waling M, Isaksson A, Soderstrom I, Ryberg $\mathrm{M}$, et al. $\mathrm{A}$ heterogeneous response of liver and skeletal muscle fat to the combination of a Paleolithic diet and exercise in obese individuals with type 2 diabetes: a randomised controlled trial. Diabetologia 2018; 61(7):1548-59.

Otten J, Stomby A, Waling M, Isaksson A, Tellstrom A, LundinOlsson L, et al. Benefits of a Paleolithic diet with and without supervised exercise on fat mass, insulin sensitivity, and glycemic control: a randomized controlled trial in individuals with type 2 diabetes. Diabetes/Metabolism Research and
Reviews 2017; 33(1):e2828. [DOI: https://doi.org/10.1002/ dmrr.2828]

Stomby A, Otten J, Ryberg M, Nyberg L, Olsson T, Boraxbekk CJ. A paleolithic diet with and without combined aerobic and resistance exercise increases functional brain responses and hippocampal volume in subjects with type 2 diabetes. Frontiers in Aging Neuroscience 2017; 9(DEC):391.

Stomby A, Simonyte K, Mellberg C, Ryberg M, Stimson RH, Larsson C, et al. Diet-induced weight loss has chronic tissuespecific effects on glucocorticoid metabolism in overweight postmenopausal women. International Journal of Obesity 2015; 39(5):814-9.

\section{Koopman 1990 \{published data only\}}

Koopman H, Spreeuwenberg C, Westerman RF, Donker AJ. Dietary treatment of patients with mild to moderate hypertension in a general practice: a pilot intervention study (2). Beyond three months. Journal of Human Hypertension 1990; 4(4):372-4.

\section{Koranyi 1963 \{published data only\}}

Koranyi A. Prophylaxis and treatment of the coronary syndrome. Therapia Hungarica 1963; 11:17-20.

\section{Korhonen 2003 \{published data only\}}

Korhonen M, Kastarinen M, Uusitupa M, Puska P, Nissinen A. The effect of intensified diet counselling on the diet of hypertensive subjects in primary health care: a 2-year open randomized controlled trial of lifestyle intervention against hypertension in eastern Finland. Preventive Medicine 2003; 36(1):8-16.

\section{Kriketos 2001 \{published data only\}}

Kriketos AD, Robertson RM, Sharp TA, Drougas H, Reed GW, Storlien $\mathrm{LH}$, et al. Role of weight loss and polyunsaturated fatty acids in improving metabolic fitness in moderately obese, moderately hypertensive subjects. Journal of Hypertension 2001; 19(10):1745-54.

\section{Kris 1994 \{published data only\}}

Kris EP, Mustad VA. Chocolate feeding studies: a novel approach for evaluating the plasma lipid effects of stearic acid. American Journal of Clinical Nutrition 1994; 60(6 Suppl):1029S-36S.

\section{Kristal 1997 \{published data only\}}

Kristal AR, Shattuck AL, Bowen DJ, Sponzo RW, Nixon DW. Feasibility of using volunteer research staff to deliver and evaluate a low-fat dietary intervention: the American Cancer Society Breast Cancer Dietary Intervention Project. Cancer Epidemiology, Biomarkers and Prevention 1997; 6(6):459-67.

\section{Kromhout 1987 \{published data only\}}

Kromhout D, Arntzenius AC, Kempen-Voogd N, Kempen HJ, Barth JD, Van der Voort HA, et al. Long-term effects of linoleic-acid enriched diet, changes in body weight and alcohol consumption on serum total and HDL-cholesterol. Atherosclerosis 1987; 66(1-2):99-105. 
Kummel 2008 \{published data only\}

Kummel MV. Effects of an intervention on health behaviors of older coronary artery bypass (CAB) patients. Archives of Gerontology and Geriatrics 2008; 2(2):227-44.

\section{Laitinen 1993 \{published data only\}}

Laitinen JH, Ahola IE, Sarkkinen ES, Winberg RL, Harmaakorpi IP, Uusitupa MI. Impact of intensified dietary therapy on energy and nutrient intakes and fatty acid composition of serum lipids in patients with recently diagnosed non-insulin-dependent diabetes mellitus. Journal of the American Dietetic Association 1993; 93(3):276-83.

\section{Laitinen 1994 \{published data only\}}

Laitinen J, Uusitupa M, Ahola I, Siitonen O. Metabolic and dietary determinants of serum lipids in obese patients with recently diagnosed non-insulin-dependent diabetes. Annals of Medicine 1994; 26(2):119-24.

\section{Lean 1997 \{published and unpublished data\}}

Han TS, Richmond P, Avenell A, Lean ME. Waist circumference reduction and cardiovascular benefits during weight loss in women. International Journal of Obesity and Related Metabolic Disorders 1997; 21(2):127-34.

* Lean ME, Han TS, Prvan T, Richmond PR, Avenell A. Weight loss with high and low carbohydrate $1200 \mathrm{kcal}$ diets in free living women. European Journal of Clinical Nutrition 1997; 51(4):243-8.

\section{Leduc 1994 \{published data only\}}

Leduc CP, Cherniak D, Faucher J. Effectiveness of a group dietary intervention on hypercholesterolaemia: a randomised controlled clinical trial [poster abstract]. Atherosclerosis 1994; 19:149.

\section{Lewis 1958 \{published data only\}}

Lewis B. Effect of certain dietary oils on bile-acid secretion and serum-cholesterol. Lancet 1958; 1(7030):1090-2.

\section{Lewis 1981 \{published data only\}}

Lewis B, Hammett F, Katan M, Kay RM, Merkx I, Nobels A, et al. Towards an improved lipid-lowering diet: additive effects of changes in nutrient intake. Lancet 1981; 2(8259):1310-3

\section{Lewis 1985 \{published data only\}}

Lewis B. Randomised controlled trial of the treatment of hyperlipidaemia on progression of atherosclerosis. Acta Medica Scandinavica 1985; 701(Suppl):53-7.

\section{Lichtenstein 2002 \{published data only\}}

Lichtenstein AH, Ausman LM, Jalbert SM, Vilella-Bach M, Jauhiainen M, McGladdery S, et al. Efficacy of a therapeutic lifestyle change/step 2 diet in moderately hypercholesterolemic middle-aged and elderly female and male subjects. Journal of Lipid Research 2002; 43(2):264-73.

\section{Lim 2010 \{published data only\}}

Lim SS, Noakes M, Keogh JB, Clifton PM. Long-term effects of a low carbohydrate, low fat or high unsaturated fat diet compared to a no-intervention control. Nutrition, Metabolism, and Cardiovascular Diseases 2010; 20(8):599-607.

Linko 1957 \{published data only\}

Linko E. Vegetable oils and serum cholesterol: short-term experiments with rapeseed and sunflower oils. Acta Medica Scandinavica 1957; 159(6):475-88.

Lipid Res Clinic 1984 \{published data only\}

Gordon DJ, Salz KM, Roggenkamp KJ. Dietary determinants of plasma cholesterol change in the recruitment phase of the Lipid Research Clinics Coronary Primary Prevention Trial. Arteriosclerosis 1982; 2(6):537-48.

* Lipid Research Clinics. The Lipid Research Clinics Coronary Primary Prevention Trial results. I. Reduction in incidence of coronary heart disease. JAMA 1984; 251(3):351-64.

Lipid Research Clinics. The Lipid Research Clinics Coronary Primary Prevention Trial results. II. The relationship of reduction in incidence of coronary heart disease to cholesterol lowering. JAMA 1984; 251(3):365-74.

\section{Little 1990 \{published data only\}}

Little P, Girling G, Hasler A, Craven A, Trafford A. The effect of a combination low sodium, low fat, high fibre diet on serum lipids in treated hypertensive patients. European Journal of Clinical Nutrition 1990; 44(4):293-300.

\section{Little 2004 \{published data only\}}

Little P, Kelly J, Barnett J, Dorward M, Margetts B, Warm D, et al. Randomised controlled factorial trial of dietary advice for patients with a single high blood pressure reading in primary care. BMJ 2004; 328(7447):1054.

\section{Lottenberg 1996 \{published data only\}}

Lottenberg AM, Nunes VS, Lottenberg SA, Shimabukuro AF, Carrilho AJ, Malagutti S, et al. Plasma cholesteryl ester synthesis, cholesteryl ester transfer protein concentration and activity in hypercholesterolemic women: effects of the degree of saturation of dietary fatty acids in the fasting and postprandial states. Atherosclerosis 1996; 126(2):265-75.

\section{Luszczynska 2007 \{published data only\}}

Luszczynska A, Scholz U, Sutton S. Planning to change diet: a controlled trial of an implementation intentions training intervention to reduce saturated fat intake among patients after myocardial infarction. Journal of Psychosomatic Research 2007; 63(5):491-7.

\section{Lyon Diet Heart 1994 \{published data only\}}

* De Lorgeril M, Renaud S, Mamelle N, Salen P, Martin JL, Monjaud I, et al. Mediterranean alpha-linolenic acid-rich diet in secondary prevention of coronary heart disease. Lancet 1994; 343(8911):1454-9.

De Lorgeril M, Salen P, Caillat-Vallet E, , Barthelemy JC, Mamelle N. Control of bias in dietary trial to prevent coronary recurrences: the Lyon Diet Heart study. European Journal of Clinical Nutrition 1997; 51(2):116-22. 
De Lorgeril M, Salen P, , Monjaud I, Delaye J, Mamelle N. Mediterranean diet, traditional risk factors, and the rate of cardiovascular complications after myocardial infarction: final report of the Lyon Diet Heart study. Circulation 1999; 99(6):779-85.

De Lorgeril M, Salen P, Martin JL, Mamelle N, Monjaud I, Touboul P, et al. Effect of a Mediterranean type of diet on the rate of cardiovascular complications in patients with coronary artery disease. insights into the cardioprotective effect of certain nutriments. Journal of the American College of Cardiology 1996; 28(5):1103-8.

De Lorgeril M, Salen P, Martin JL, Monjaud I, Boucher P, Mamelle N. Mediterranean dietary pattern in a randomised trial. Archives of Internal Medicine 1998; 158(11):1181-7.

De Lorgeril M, Salen P. Mediterranean diet in secondary prevention of coronary heart disease. Australian Journal of Nutrition and Dietetics 1998; 55(Suppl):s16-s20.

Renaud S, De Lorgeril M, Delaye J, Guidollet J, Jacquard F, Mamelle $\mathrm{N}$, et al. Cretan Mediterranean diet for prevention of coronary heart disease. American Journal of Clinical Nutrition 1995; 61 (6 Suppl):1360S-7S.

\section{Lysikova 2003 \{published data only\}}

Lysikova SL, Pogozheva AV, Akol'zina SE, Vasil'ev AV, Vorob'eva LS. The study of the clinical potency of antiatherogenic diet containing flavonoids in cardiovascular patients. Voprosy Pitaniia 2003; 72(3):8-11.

Macdonald 1972 \{published data only\}

Macdonald I. Relationship between dietary carbohydrates and fats in their influence on serum lipid concentrations. Clinical Science 1972; 43(2):265-74.

\section{Mansel 1990 \{published data only\}}

Mansel RE, Harrison BJ, Melhuish J, Sheridan W, Pye JK, Pritchard G, et al. A randomized trial of dietary intervention with essential fatty acids in patients with categorized cysts. Annals of the New York Academy of Sciences 1990; 586:288-94.

\section{MARGARIN 2002 \{published data only\}}

Bemelmans WJ, Broer J, Feskens EJ, Smit AJ, Muskiet FA, Lefrandt JD, et al. Effect of an increased intake of alphalinolenic acid and group nutritional education on cardiovascular risk factors: the Mediterranean Alpha-linolenic Enriched Groningen Dietary Intervention (MARGARIN) study. American Journal of Clinical Nutrition 2002; 75(2):221-7.

\section{Marniemi 1990 \{published and unpublished data\}}

Hakala P, Karvetti RL. Weight reduction on lactovegetarian and mixed diets. European Journal of Clinical Nutrition 1989; 43(6):421-30

\footnotetext{
* Marniemi J, Seppanen A, Hakala P. Long-term effects on lipid metabolism of weight reduction on lactovegetarian and mixed diet. International Journal of Obesity 1990; 14(2):113-25.
}

\section{Mattson 1985 \{published data only\}}

Mattson FH, Grundy SM. Comparison of effects of dietary saturated, monounsaturated and polyunsaturated fatty acids on plasma lipids and lipoproteins in man. Journal of Lipid Research 1985; 26(2):194-202.

\section{McAuley 2005 \{published and unpublished data\}}

* McAuley KA, Hopkins CM, Smith KJ, McLay RT, Williams SM, Taylor RW, et al. Comparison of a high-fat and high-protein diets with a high-carbohydrate diet in insulin-resistant obese women. Diabetologia 2005; 48:8-16.

McAuley KA, Smith KJ, Taylor RW, McLay RT, Williams SM, Mann JI. Long-term effects of popular dietary approaches on weight loss and features of insulin resistance. International Journal of Obesity 2006; 30(2):342-9.

\section{McCarron 1997 \{published data only\}}

McCarron DA, Oparil S, Chait A, Haynes RB, Kris EP, Stern JS, et al. Nutritional management of cardiovascular risk factors. A randomized clinical trial. Archives of Internal Medicine 1997; 157(2):169-77.

\section{McCarron 2001 \{published data only\}}

McCarron DA, Reusser ME. Reducing cardiovascular disease risk with diet. Obesity Research 2001; 9(Suppl 4):335S-40S.

McKeown-Eyssen 1994 \{published and unpublished data\} McKeown-Eyssen GE, Bright SE, Bruce WR, Jazmaji V. A randomized trial of a low fat high fibre diet in the recurrence of colorectal polyps. Journal of Clinical Epidemiology 1994; 47(5):525-36.

\section{McManus 2001 \{published and unpublished data\}}

McManus K, Antinoro L, Sacks F. Randomized controlled trial of a moderate-fat low-energy diet compared with a low fat, lowenergy diet for weight loss in overweight adults. International Journal of Obesity and Related Metabolic Disorders 2001; 25(10):1503-11.

\section{McNamara 1981 \{published data only\}}

McNamara DJ, Kolb R, Parker T, Batwin H, Brown C, Samuel P, et al. Diet and cholesterol homeostasis in men [abstract]. Arteriosclerosis 1981; 1:369A.

\section{MeDiet 2002 \{published and unpublished data\}}

Carruba G, Granata OM, Pala V, Campisi I, Agostara B, Cusimano R, et al. A traditional Mediterranean diet decreases endogenous estrogens in healthy postmenopausal women. Nutrition and Cancer 2006; 56(2):253-9.

* Castagnetta L, Granata OM, Cusimano R, Ravazzolo B, Liquori M, Polito L, et al. The Mediet Project. Annals of the New York Academy of Science 2002; 963:282-9.

Granata OM, Traina A, Ramirez S, Campisi I, Zarcone M, Amodio R, et al. Dietary enterolactone affects androgen and estrogen levels in healthy postmenopausal women. Annals of the New York Academy of Science 2009; 1155:232-6. 
MEDINA \{published data only\}

Papamiltiadous ES, Roberts SK, Nicoll AJ, Ryan MC, Itsiopoulos C, Salim A, et al. A randomised controlled trial of a Mediterranean Dietary Intervention for Adults with Non Alcoholic Fatty Liver Disease (MEDINA): study protocol. BMC Gastroenterology 2016; 16:14.

\section{Medi-RIVAGE 2004 \{published and unpublished data\}}

Borel P, Moussa M, Reboul E, Lyan B, Defoort C, VincentBaudry S, et al. Human fasting plasma concentrations of vitamin $\mathrm{E}$ and carotenoids, and their association with genetic variants in apo C-III, cholesteryl ester transfer protein, hepatic lipase, intestinal fatty acid binding protein and microsomal triacylglycerol transfer protein. British Journal of Nutrition 2009; 101(5):680-7.

Borel P, Moussa M, Reboul E, Lyan B, Defoort C, VincentBaudry $S$, et al. Human plasma levels of vitamin $E$ and carotenoids are associated with genetic polymorphisms in genes involved in lipid metabolism. Journal of Nutrition 2007; 137(12):2653-9.

Gastaldi M, Diziere S, Defoort C, Portugal H, Lairon D, Darmon M, et al. Sex-specific association of fatty acid binding protein 2 and microsomal triacylglycerol transfer protein variants with response to dietary lipid changes in the 3-mo MediRIVAGE primary intervention study. American Journal of Clinical Nutrition 2007; 86(6):1633-41.

* Vincent S, Gerber M, Bernard MC, Defoort C, Loundou A, Portugal $\mathrm{H}$, et al. The Medi-RIVAGE study (Mediterranean Diet, Cardiovascular Risks and Gene Polymorphisms): rationale, recruitment, design, dietary intervention and baseline characteristics of participants. Public Health Nutrition 2004; 7(4):531-42.

Vincent-Baudry S, Defoort C, Gerber M, Bernard MC, Verger P, Helal O, et al. The Medi-RIVAGE study: reduction of cardiovascular disease risk factors after a 3-mo intervention with a Mediterranean-type diet or a low-fat diet. American Journal of Clinical Nutrition 2005; 82(5):964-71.

Mensink 1987 \{published data only\}

Mensink RP, Katan MB. Effect of monounsaturated fatty acids versus complex carbohydrates on high-density lipoproteins in healthy men and women. Lancet 1987; 1(8525):122-5.

\section{Mensink 1989 \{published data only\}}

Mensink RP, Katan MB. Effect of a diet enriched with monounsaturated or polyunsaturated fatty acids on levels of low density and high density lipoprotein cholesterol in healthy women and men. New England Journal of Medicine 1989; 321(7):436-41.

\section{Mensink 1990a \{published data only\}}

Mensink RP, Katan MB. Effect of dietary trans fatty acids on high density and low density lipoprotein cholesterol levels in healthy subjects. New England Journal of Medicine 1990; 323(7):439-45.

\section{Mensink 1990b \{published and unpublished data\}}

Mensink RP. Effect of Monounsaturated Fatty Acids on HighDensity and Low-Density Lipoprotein Cholesterol Levels and
Blood Pressure in Healthy Men and Women. Wageningen, Netherlands: Wageningen University and Research Centre, 1990.

Metroville Health 2003 \{published data only (unpublished sought but not used)\}

Aziz KU, Dennis B, Davis CE, Sun K, Burke G, Manolio T, et al. Efficacy of CVD risk factor modification in a lower-middle class community in Pakistan: the Metroville Health Study. Asia Pacific Journal of Public Health 2003; 15(1):30-6.

Michalsen 2006 \{published and unpublished data\}

Michalsen A, Lehmann N, Pithan C, Knoblauch NT, Moebus S, Kannenberg $\mathrm{F}$, et al. Mediterranean diet has no effect on markers of inflammation and metabolic risk factors in patients with coronary artery disease. European Journal of Clinical Nutrition 2006; 60(4):478-85.

\section{Miettinen 1994 \{published data only\}}

Miettinen TA, Vanhanen H. Dietary sitostanol related to absorption, synthesis and serum level of cholesterol in different apolipoprotein E phenotypes. Atherosclerosis 1994; 105(2):217-26.

\section{Millar 1973 \{published data only\}}

Millar JH, Zilkha KJ, Langman MJ, Payling-Wright H, Smith AD, Belin J, et al. Double-blind trial of linoleate supplementation of the diet in multiple sclerosis. BMJ 1973; 1(5856):765-8.

Miller 1998 \{published data only\}

Miller ER, Appel LJ, Risby TH. Effect of dietary patterns on measures of lipid peroxidation: results from a randomised clinical trial. Circulation 1998; 98(22):2390-5.

\section{Miller 2001 \{published and unpublished data\}}

Miller SL, Reber RJ, Chapman-Novakofski K. Prevalence of CVD risk factors and impact of a two-year education program for premenopausal women. Women's Health Issues 2001; 11(6):486-93.

\section{Milne 1994 \{published data only\}}

Milne RM, Mann JI, Chisholm AW, Williams SM. Long-term comparison of three dietary prescriptions in the treatment of NIDDM. Diabetes Care 1994; 17(1):74-80.

\section{Minnesota Coronary 1989 \{published data only\}}

Brewer ER, Ashman PL, Kuba K. The Minnesota Coronary Survey: composition of diets, adherence and serum lipid response [Abstract]. Circulation 1975; 51 and 52(Suppl II):269.

Dawson EA, Gatewood LC. The Minnesota Coronary Survey: methodology and characteristics of the population [Abstract]. Circulation 1975; 51 and 52(Suppl II):271.

* , Dawson EA, Ashman PL, Gatewood LC, Bartsch GE, Kuba K, et al. Test of effect of lipid lowering by diet on cardiovascular risk. The Minnesota Coronary Survey. Arteriosclerosis 1989; 9(1):129-35.

, Dawson EA, Kuba K, Brewer ER, Gatewood LC, Bartsch GE. The Minnesota Coronary Survey: effect of diet on cardiovascular events and deaths [abstract]. Circulation 1975; 51 and 52(Suppl II):4. 
Minnesota HHP 1990 \{published data only\}

Murray DM, Kurth C, Mullis R, Jeffery RW. Cholesterol reduction through low-intensity interventions: results from the Minnesota Heart Health Program. Preventive Medicine 1990; 19(2):181-9.

\section{Mojonnier 1980 \{published data only\}}

Mojonnier ML, Hall Y, Berkson DM, Robinson E, Wethers B, Pannbacker $B$, et al. Experience in changing food habits of hyperlipidaemic men and women. Journal of the American Dietetic Association 1980; 77(2):140-8.

\section{Mokuno 1988 \{published data only\}}

Mokuno H, Yamada N, Sugimoto T. Cholesterol free diet in heterozygous familial hypercholesterolaemia: significant lowering effect on plasma cholesterol (abstract). Arteriosclerosis 1988; 8(5):590a.

\section{Mortensen 1983 \{published data only\}}

Mortensen JZ, Schmidt EB, Nielsen AH, Dyerberg J. The effect of $\mathrm{N}-6$ and N-3 polyunsaturated fatty acids on hemostasis, blood lipids and blood pressure. Thrombosis and Haemostasis 1983; 50(2):543-6.

\section{Mottalib 2018 \{published data only\}}

Mottalib A, Mitri J, Salsberg V, Ashrafzadeh S, Elseaidy T, Tomah S, et al. Effect of dairy consumption and Its fat content on glycemic control and cardiovascular risk factors in patients with type 2 diabetes - a randomized controlled study. Diabetes 2018; 67(Suppl 1):760-P.

\section{MRFIT substudy 1986 \{published data only\}}

Daniel GJ, Dolecek TA, Caggiula AW, Van Horn LV, Epley L, Randall BL. Increasing the use of meatless meals: a nutrition intervention substudy in the Multiple Risk Factor Intervention Trial (MRFIT). Journal of the American Dietetic Association 1986; 86(6):778-81.

\section{MSDELTA 1995 \{published data only\}}

Ginsberg HN. New directions in dietary studies and heart disease: the National Heart, Lung and Blood Institute sponsored multicenter study of diet effects on lipoproteins and thrombogenic activity. Advances In Experimental Medicine and Biology 1995; 369:241-7.

\section{MSFAT 1997 \{published and unpublished data\}}

* Van het Hoff K, Weststrate JA, Van den Berg H, Velthuis-te Wierik EJ, De Graaf C, Zimmermanns NJ, et al. A long-term study on the effect of spontaneous consumption of reduced fat products as part of a normal diet on indicators of health. International Journal of Food Sciences and Nutrition 1997; 48(1):19-29.

Velthuis-te Wierik EJ, Van Leeuwen RE, Hendriks HF, Verhagen H, Loft S, Poulsen HE, et al. Short-term moderate energy restriction does not affect indicators of oxidative stress and genotoxicity in humans. Journal of Nutrition 1995; 125(10):2631-9.

Velthuis-te Wierik EJ, Van den Berg H, Weststrate JA, Van het Hoff KH, De Graaf C. Consumption of reduced-fat products: effects on parameters of anti-oxidative capacity. European Journal of Clinical Nutrition 1996; 50(4):214-9.

Weststrate JA, Van het Hof KH, Van den Berg H, Velthuis-te Wierik EJ, De Graaf C, Zimmermanns NJ, et al. A comparison of the effect of free access to reduced fat products or their full fat equivalents on food intake, body weight, blood lipids and fat-soluble antioxidants levels and haemostasis variables. European Journal of Clinical Nutrition 1998; 52(6):389-95.

\section{Mujeres Felices 2003 \{published data only\}}

* Fitzgibbon ML, Gapstur SM, Knight SJ. Mujeres felices por ser saludables: a breast cancer risk reduction program for Latino women. Preventive Medicine 2003; 36(5):536-46.

Fitzgibbon ML, Gapstur SM, Knight SJ. Results of Mujeres Felices por ser Saludables: a dietary/breast health randomized clinical trial for Latino women. Annals of Behavioral Medicine 2004; 28(2):95-104.

\section{Mutanen 1997 \{published data only\}}

Mutanen M. Comparison between dietary monounsaturated and polyunsaturated fatty acids as regards diet-related diseases. Biomedicine and Pharmacotherapy 1997; 51(8):314-7.

\section{Muzio 2007 \{published data only\}}

Muzio F, Mondazzi L, Harris WS, Sommariva D, Branchi A. Effects of moderate variations in the macronutrient content of the diet on cardiovascular disease risk factors in obese patients with the metabolic syndrome. American Journal of Clinical Nutrition 2007; 86(4):946-51.

Naglak 2000 \{published data only (unpublished sought but not used)\}

Naglak MC, Mitchell DC, Shannon BM, Pearson TA, Harkness WL, Kris-Etherton PM. Nutrient adequacy of diets of adults with hypercholesterolemia after a cholesterol-lowering intervention: long term assessment. Journal of the American Dietetic Association 2000; 100(11):1385-91.

\section{NAS 1987 \{published data only\}}

Chlebowski RT, Nixon DW, Blackburn GL, Jochimsen P, Scanlon EF, Insull W, et al. A breast cancer Nutrition Adjuvant Study (NAS): protocol design and initial patient adherence. Breast Cancer Research and Treatment 1987; 10(1):21-9.

\section{National Diet Heart 1968 \{published data only\}}

Baker BM, , Keys A, Kinsell LW, Page IH, Stamler J, et al. The National Diet-Heart Study: an initial report. JAMA 1963; 185:105-6.

Brown HB. The National Diet Heart Study - implications for dietitians and nutritionists. Journal of the American Dietetic Association 1968; 52(4):279-87.

Page IH, Brown HB. Some observations on the National DietHeart Study. Circulation 1968; 37(3):313-5.

* The National Diet-Heart Study Research Group. The National Diet-Heart Study final report. Circulation 1968; 37(II):1-428.

The National Diet-Heart Study Research Group. The National Diet-Heart Study. Nutrition Reviews 1968; 26(5):133-6. 
NCEP weight 1991 \{published and unpublished data\}

Wood PD, Stefanick ML, Williams PT, Haskell WL. The effects on plasma lipoproteins of a prudent weight-reducing diet, with or without exercise, in overweight men and women. New England Journal of Medicine 1991; 325(7):461-6.

NCT01954472 \{published data only\}

ZZZ <accessionId ref="info:x-wiley/clinicalTrialsGov/ NCT01954472"> ZZZNCT01954472

. Enhanced multicenter dietary portfolio study (EDP8). clinicaltrials.gov/ct2/show/NCT01954472 (first posted 1 October 2013).

\section{NCT03068078 \{published data only\}}

- A reduced-carbohydrate diet high in monounsaturated fats in type 2 diabetes (ReDuCtion). clinicaltrials.gov/ct2/show/ NCT03068078 (first posted 1 March 2017).

Neil 1995 \{published data only\}

Neil HA, Roe L, Godlee RJ, Moore JW, Clark GM, Brown J, et al. Randomised trial of lipid lowering dietary advice in general practice: the effects on serum lipids, lipoproteins, and antioxidants [see comments]. BMJ 1995; 310(6979):569-73.

\section{Neverov 1997 \{published data only\}}

Neverov NI, Kaysen GA, Tareyeva IE. Effect of lipid-lowering therapy on the progression of renal disease in nondiabetic nephrotic patients. Contributions to Nephrology 1997; 120:68-78.

\section{Next Step 1995 \{published and unpublished data\}}

Tilley BC, Vernon SW, Glanz K, Myers R, Sanders K, Lu M, et al. Worksite cancer screening and nutrition intervention for highrisk auto workers: design and baseline findings of the Next Step Trial. Preventive Medicine 1997; 26(2):227-35.

* Tilley BC, Vernon SW, Myers R, Glanz K, Lu M, Sanders K, et al. Planning the next step. A screening promotion and nutrition intervention trial in the work site. Annals of the New York Academy of Sciences 1995; 768:296-9.

\section{Nordoy 1971 \{published data only\}}

Nordoy A, Rodset JM. The influence of dietary fats on platelets in man. Acta Medica Scandinavica 1971; 190(1-2):27-34.

\section{Norway Veg Oil 1968 \{published data only\}}

Natvig H, Borchgrevink CF, Dedichen J, Owren PA, Schiotz EH, Westlund K. A controlled trial of the effect of linolenic acid on incidence of coronary heart disease: the Norwegian Vegetable Oil Experiment of 1965-66. Scandinavian Journal of Clinical and Laboratory Investigation 1968; 105(Suppl):1-20.

Nutri-AGEs 2015 \{published data only\}

. Effect of low-glycemic index Mediterranean diet on ages (Nutri_AGEs). clinicaltrials.gov/ct2/show/NCT02353416 (first posted 2 February 2015)

Nutrition Breast Health \{published and unpublished data\} Djuric Z, Poore KM, Depper JB, Uhley VE, Lababidi S, Covington $\mathrm{C}$, et al. Methods to increase fruit and vegetable intake with and without a decrease in fat intake: compliance and effects on body weight in the Nutrition and Breast Health Study. Nutrition and Cancer 2002; 43(2):141-51.

O'Brien 1976 \{published data only\}

O'Brien JR, Etherington MD, Jamieson S. Effect of a diet of polyunsaturated fats on some platelet-function tests. Lancet 1976; 2(7993):995-6.

\section{ODES 2006 \{published data only\}}

Anderssen S, Holme I, Urdal P, Hjermann I. Diet and exercise intervention have favourable effects on blood pressure in mild hypertensives: the Oslo Diet and Exercise Study (ODES). Blood Pressure 1995; 4(6):343-9.

Anderssen SA, Hjermann I, Urdal P, Torjesen PA, Holme I. Improved carbohydrate metabolism after physical training and dietary intervention in individuals with the "atherothrombogenic syndrome". Oslo Diet and Exercise Study (ODES). A randomized trial. Journal of Internal Medicine 1996; 240(4):203-9.

* Holme I, Haaheim LL, Tonstad S, Hjermann I, Holme I, Haaheim LL, et al. Effect of dietary and antismoking advice on the incidence of myocardial infarction: a 16-year follow-up of the Oslo Diet and Antismoking Study after its close. Nutrition Metabolism \& Cardiovascular Diseases 2006; 16(5):330-8.

ODES Investigators. The Oslo Diet and Exercise Study (ODES): design and objectives. Controlled Clinical Trials 1993; 14(3):229-43.

Rokling-Andersen $\mathrm{MH}$, Reseland JE, Veierod MB, Anderssen SA, Urdal $P$, et al. Effects of long-term exercise and diet intervention on plasma adipokine concentrations. American Journal of Clinical Nutrition 2007; 86(5):1293-301.

Torjesen PA, Birkeland KI, Anderssen SA, Hjermann I, Holme I, Urdal P. Lifestyle changes may reverse development of the insulin resistance syndrome. The Oslo Diet and Exercise Study: a randomized trial. Diabetes Care 1997; 20(1):26-31.

\section{Oldroyd 2001 \{published data only\}}

Oldroyd JC, Unwin NC, White M, Mathers JC, Alberti KG. Randomised controlled trial evaluating lifestyle interventions in people with impaired glucose tolerance. Diabetes Research \& Clinical Practice 2006; 72(2):117-27.

* Oldroyd JC. Randomised controlled trial evaluating the effectiveness of behavioural interventions to modify cardiovascular risk factors in men and women with impaired glucose tolerance: outcomes at 6 months. Diabetes Research and Clinical Practice 2001; 52(1):29-43.

\section{Ole Study 2002 \{published and unpublished data\}}

* Bray GA, Lovejoy JC, Most-Windhauser M, Smith SR, Volaufova J, Denkins Y, et al. A 9-mo randomized clinical trial comparing fat-substituted and fat-reduced diets in healthy obese men: the Ole Study. American Journal of Clinical Nutrition 2002; 76(5):928-34.

Lovejoy JC, Bray GA, Lefevre M, Smith SR, Most MM, Denkins YM, et al. Consumption of a controlled low-fat diet containing olestra for 9 months improves health risk factors in conjunction 
with weight loss in obese men: the Ole Study. International Journal of Obesity and Related Metabolic Disorders 2003; 27(10):1242-9.

\section{OLIVE 1997 \{published data only (unpublished sought but not} used)\}

Colquhoun DM, Somerset S, Glasziou PP, Richards D, Weyers J. Comparison of an olive oil enriched diet to a low fat diet intervention study using vascular endpoints: assessed by repeat quantitative angiography (OLIVE study). Australian Journal of Nutrition and Dietetics 1998; 55(Suppl 4):S24-9.

* Colquhoun DM. Rationale and design of the "OLIVE" study (Comparison of an Olive oil enriched to a low fat diet intervention study using vascular endpoints) [Abstract]. In: 11th International Symposium on Atherosclerosis; 1997 October; Paris. 1997:326.

\section{ORIGIN 2008 \{published data only\}}

Gerstein H, Yusuf S, Riddle MC, Ryden L, Bosch J. Rationale, design, and baseline characteristics for a large international trial of cardiovascular disease prevention in people with dysglycemia: the ORIGIN Trial (Outcome Reduction with an Initial Glargine Intervention). American Heart Journal 2008; 155(1):26-32.

\section{Oslo Study 2004 \{published data only\}}

* Hjerkinn EM, Sandvik L, Hjermann I, Arnesen H. Effect of diet intervention on long-term mortality in healthy middleaged men with combined hyperlipidaemia. Journal of Internal Medicine 2004; 255(1):68-73.

Hjermann I, Leren P, Norman N, Helgeland A, Holme I. Serum insulin response to oral glucose load during a dietary intervention trial in healthy coronary high risk men: the Oslo study. Scandinavian Journal of Clinical and Laboratory Investigation 1980; 40(1):89-94.

Hjermann I, Velve BK, Holme I, Leren P. Effect of diet and smoking intervention on the incidence of coronary heart disease. Report from the Oslo Study Group of a randomised trial in healthy men. Lancet 1981; 2(8259):1303-10.

Hjermann I. Intervention of smoking and eating habits in healthy men carrying high risk for coronary heart disease. The Oslo Study. Acta Medica Scandinavica 1981; 651(Suppl):281-4.

Hjermann I. Smoking and diet intervention in healthy coronary high risk men. Methods and 5-year follow-up of risk factors in a randomized trial. The Oslo study. Journal of the Oslo City Hospitals 1980; 30(1):3-17.

\section{Pascale 1995 \{published data only\}}

Pascale RW, Wing RR, Butler BA, Mullen M, Bononi P. Effects of a behavioral weight loss program stressing calorie restriction versus calorie plus fat restriction in obese individuals with NIDDM or a family history of diabetes. Diabetes Care 1995; 18(9):1241-8.

\section{PEP 2001 \{published data only\}}

Ohrig E, Geib HC, , Schwandt P. The prevention education program (PEP) Nuremberg: design and baseline data of a family oriented intervention study. International Journal of Obesity 2001; 25(Suppl 1):S89-92.

PHYLLIS 1993 \{published data only\}

Bond GM, Crepaldi G, Zanchetti A, Avogaro P, Marubini E, Maseri A, et al. Plaque hypertension lipid-lowering Italian study (PHYLLIS): a protocol for non-invasive evaluation of carotid atherosclerosis in hypercholesterolaemic hypertensive subjects. Journal of Hypertension 1993; 11(Suppl 5):S314-5.

Pilkington 1960 \{published and unpublished data\}

Pilkington TR, Stafford JL, Hankin VS, Simmonds FM, Koerselman HB. Practical diets for lowering serum lipids. British Medical Journal 1960; 1(5165):23-5.

Polyp Prevention 1996 \{published and unpublished data\} Lanza E, Schatzkin A, Ballard BR, Clifford DC, Paskett E, Hayes D, et al. The polyp prevention trial II: dietary intervention program and participant baseline dietary characteristics. Cancer Epidemiology, Biomarkers and Prevention 1996; 5(5):385-92.

* Schatzkin A, Lanza E, Freedman LS, Tangrea J, Cooper MR, Marshall JR, et al. The polyp prevention trial I: rationale, design, recruitment, and baseline participant characteristics. Cancer Epidemiology, Biomarkers and Prevention 1996; 5(5):375-83.

POUNDS LOST 2009 \{published and unpublished data\}

Anton SD, Gallagher J, Carey VJ, Laranjo N, Cheng J, Champagne CM, et al. Diet type and changes in food cravings following weight loss: findings from the POUNDS LOST Trial. Eating \& Weight Disorders 2012; 17(2):e101-8.

Anton SD, LeBlanc E, Allen HR, Karabetian C, Sacks F, Bray G, et al. Use of a computerized tracking system to monitor and provide feedback on dietary goals for calorie-restricted diets: the POUNDS LOST study. Journal of Diabetes Science \& Technology 2012; 6(5):1216-25.

Bray GA, Smith SR, DeJonge L, De Souza R, Rood J, Champagne CM, et al. Effect of diet composition on energy expenditure during weight loss: the POUNDS LOST Study. International Journal of Obesity 2012; 36(3):448-55.

De Jonge L, Bray GA, Smith SR, Ryan DH, De Souza RJ, Loria CM, et al. Effect of diet composition and weight loss on resting energy expenditure in the POUNDS LOST study. Obesity 2012; 20(12):2384-9.

De Souza RJ, Bray GA, Carey VJ, Hall KD, LeBoff MS, Loria CM, et al. Effects of 4 weight-loss diets differing in fat, protein, and carbohydrate on fat mass, lean mass, visceral adipose tissue, and hepatic fat: results from the POUNDS LOST trial. American Journal of Clinical Nutrition 2012; 95(3):614-25.

Mattei J, Qi Q, Hu FB, Sacks FM, Qi L, Mattei J, et al. TCF7L2 genetic variants modulate the effect of dietary fat intake on changes in body composition during a weight-loss intervention. American Journal of Clinical Nutrition 2012; 96(5):1129-36.

Mirzaei K, Xu M, Qi Q, De Jonge L, Bray GA, Sacks F, et al. Variants in glucose- and circadian rhythm-related genes affect the response of energy expenditure to weight-loss diets: the 
POUNDS LOST Trial. American Journal of Clinical Nutrition 2014; 99(2):392-9.

Nicklas JM, Sacks FM, Smith SR, LeBoff MS, Rood JC, Bray GA, et al. Effect of dietary composition of weight loss diets on highsensitivity c-reactive protein: the randomized POUNDS LOST trial. Obesity 2013; 21(4):681-9.

Qi Q, Bray GA, Hu FB, Sacks FM, Qi L, Qi Q, et al. Weight-loss diets modify glucose-dependent insulinotropic polypeptide receptor rs2287019 genotype effects on changes in body weight, fasting glucose, and insulin resistance: the Preventing Overweight Using Novel Dietary Strategies trial. American Journal of Clinical Nutrition 2012; 95(2):506-13.

Qi Q, Bray GA, Smith SR, Hu FB, Sacks FM, Qi L, et al. Insulin receptor substrate 1 gene variation modifies insulin resistance response to weight-loss diets in a 2-year randomized trial: the Preventing Overweight Using Novel Dietary Strategies (POUNDS LOST) trial. Circulation 2011; 124(5):563-71.

Qi Q, Xu M, Wu H, Liang L, Champagne CM, Bray GA, et al. IRS1 genotype modulates metabolic syndrome reversion in response to 2-year weight-loss diet intervention: the POUNDS LOST trial. Diabetes Care 2013; 36(11):3442-7.

* Sacks FM, Bray GA, Carey VJ, Smith SR, Ryan DH, Anton SD, et al. Comparison of weight-loss diets with different compositions of fat, protein, and carbohydrates. New England Journal of Medicine 2009; 360(9):859-73.

Williamson DA, Anton SD, Han $\mathrm{H}$, Champagne CM, Allen R, LeBlanc $E$, et al. Adherence is a multi-dimensional construct in the POUNDS LOST trial. Journal of Behavioral Medicine 2010; 33(1):35-46.

Williamson DA, Anton SD, Han H, Champagne CM, Allen R, LeBlanc E, et al. Early behavioral adherence predicts short and long-term weight loss in the POUNDS LOST study. Journal of Behavioral Medicine 2010; 33(4):305-14.

Xu M, Qi Q, Liang J, Bray GA, Hu FB, Sacks FM, et al. Genetic determinant for amino acid metabolites and changes in body weight and insulin resistance in response to weight-loss diets: the Preventing Overweight Using Novel Dietary Strategies (POUNDS LOST) trial. Circulation 2013; 127(12):1283-9.

Zhang X, Qi Q, Bray GA, Hu FB, Sacks FM, Qi L, et al. APOA5 genotype modulates 2-y changes in lipid profile in response to weight-loss diet intervention: the Pounds Lost Trial. American Journal of Clinical Nutrition 2012; 96(4):917-22.

Zhang X, Qi Q, Zhang C, Smith SR, Hu FB, Sacks FM, et al. FTO genotype and 2-year change in body composition and fat distribution in response to weight-loss diets: the POUNDS LOST trial. Diabetes 2012; 61(11):3005-11 Erratum in: Diabetes. 2013 Feb;62(2):662; Note: Smith, SR [added]; Bray, GA [added].

\section{PREDIMED 2008 \{published data only (unpublished sought but not} used)\}

Buil-Cosiales P, Irimia P, Ros E, Riverol M, Gilabert R, MartinezVila $E$, et al. Dietary fibre intake is inversely associated with carotid intima-media thickness: a cross-sectional assessment in the PREDIMED study. European Journal of Clinical Nutrition 2009; 63(10):1213-9.

Castañer O, Corella D, Covas MI, Sorlí JV, Subirana I, Flores-Mateo $\mathrm{G}$, et al. In vivo transcriptomic profile after a Mediterranean diet in high-cardiovascular risk patients: a randomized controlled trial. American Journal of Clinical Nutrition 2013; 98(3):845-53.

Díaz-López A, Bulló M, , Guasch-Ferré M, Ros E, Basora J, et al. Effects of mediterranean diets on kidney function: a report from the PREDIMED trial. American Journal of Kidney Diseases 2012; 60(3):380-9.

Damasceno NRT, Sala-Vila A, Cofán M, Pérez-Heras AM, Fitó M, Ruiz-Gutiérrez V, et al. Mediterranean diet supplemented with nuts reduces waist circumference and shifts lipoprotein subfractions to a less atherogenic pattern in subjects at high cardiovascular risk. Atherosclerosis 2013; 230(2):347-53.

Estruch R, Martínez-González MA, Corella D, Salas-Salvadó J, Ruiz-Gutiérrez V, Covas MI, et al. Effects of a Mediterranean-style diet on cardiovascular risk factors: a randomized trial. Annals of Internal Medicine 2006; 145(1):1-11.

* Estruch R, Ros E, Salas-Salvadó J, , Corella D, Arós F, et al, PREDIMED Study Investigators. Primary prevention of cardiovascular disease with a Mediterranean diet. New England Journal of Medicine 2013; 368(14):1279-90. [DOI: 10.1056/ NEJMoa1200303]

Fernández-Real JM, Bulló M, Moreno-Navarrete JM, Ricart W, Ros E, Estruch R, et al. A Mediterranean diet enriched with olive oil is associated with higher serum total osteocalcin levels in elderly men at high cardiovascular risk. Journal of Clinical Endocrinology \& Metabolism 2012; 97(10):3792-8.

Guasch-Ferré M, Bulló M, Babio N, Martínez-González MA, Estruch R, Covas MI, et al. Mediterranean diet and risk of hyperuricemia in elderly participants at high cardiovascular risk. Journals of Gerontology Series A - Biological Sciences \& Medical Sciences 2013; 68(10):1263-70.

Hu EA, Toledo E, Diez-Espino J, Estruch R, Corella D, SalasSalvado J, et al. Lifestyles and risk factors associated with adherence to the Mediterranean diet: a baseline assessment of the PREDIMED trial. PIOS One 2013; 8(4):e60166.

Martínez-Lapiscina EH, Clavero P, Toledo E, Estruch R, SalasSalvadó J, San Julián B, et al. Mediterranean diet improves cognition: the PREDIMED-NAVARRA randomised trial. Journal of Neurology, Neurosurgery, and Psychiatry 2013; 84(12):1318-25.

Martínez-Lapiscina EH, Clavero P, Toledo E, San Julián B, Sanchez-Tainta A, Corella D, et al. Virgin olive oil supplementation and long-term cognition: the PredimedNavarra randomized, trial. Journal of Nutrition, Health and Aging 2013; 17(6):544-52.

Mitjavila MT, Fandos M, Salas-Salvadó J, Covas MI, Borrego S, Estruch R, et al. The Mediterranean diet improves the systemic lipid and DNA oxidative damage in metabolic syndrome individuals. A randomized, controlled, trial. Clinical Nutrition 2013; 32(2):172-8. 
Nordmann AJ, Suter-Zimmermann K, Bucher HC, Shai I, Tuttle KR, Estruch R, et al. Meta-analysis comparing Mediterranean to low-fat diets for modification of cardiovascular risk factors. American Journal of Medicine 2011; 124(9):841-51.

Prieto RM, Fiol M, Perello J, Estruch R, Ros E, Sanchis P, et al. Effects of Mediterranean diets with low and high proportions of phytate-rich foods on the urinary phytate excretion. European Journal of Nutrition 2010; 49(6):321-6.

Razquin C, Martinez JA, Martinez-Gonzalez MA, Bes-Rastrollo M, Fernández-Crehuet J, Marti A. A 3-year intervention with a Mediterranean diet modified the association between the rs9939609 gene variant in FTO and body weight changes. International Journal of Obesity 2010; 34(2):266-72.

Razquin C, Martinez JA, Martinez-Gonzalez MA, FernándezCrehuet J, Santos JM, Marti A. A Mediterranean diet rich in virgin olive oil may reverse the effects of the -174 G/C IL6 gene variant on 3-year body weight change. Molecular Nutrition \& Food Research 2010; 54 Suppl 1:S75-82.

Razquin C, Martinez JA, Martinez-Gonzalez MA, Mitjavila MT, Estruch R, Marti A, et al. A 3 years follow-up of a Mediterranean diet rich in virgin olive oil is associated with high plasma antioxidant capacity and reduced body weight gain. European Journal of Clinical Nutrition 2009; 63(12):1387-93.

Razquin C, Martinez JA, Martinez-Gonzalez MA, SalasSalvado J, Estruch R, Marti A. A 3-year Mediterranean-style dietary intervention may modulate the association between adiponectin gene variants and body weight change. European Journal of Nutrition 2010; 49:311-9.

Ruiz-Canela M, Estruch R, Corella D, Salas-Salvadó J, MartínezGonzález MA. Association of Mediterranean diet with peripheral artery disease: the PREDIMED randomized trial. JAMA 2014; 311(4):415-7.

Sánchez-Taínta A, Estruch R, Bulló M, Corella D, Gómez-Gracia E, Fiol M, et al. Adherence to a Mediterranean-type diet and reduced prevalence of clustered cardiovascular risk factors in a cohort of 3,204 high-risk patients. European Journal of Cardiovascular Prevention \& Rehabilitation 2008; 15(5):589-93.

Sánchez-Villegas A, Galbete C, Martínez-González MA, Martinez JA, Razquin C, Salas-Salvadó J, et al. The effect of the Mediterranean diet on plasma brain-derived neurotrophic factor (BDNF) levels: the PREDIMED-NAVARRA randomized trial. Nutritional Neuroscience 2011; 14(5):195-201.

Sánchez-Villegas A, Martínez-González MA, Estruch R, SalasSalvadó J, Corella D, Covas MI, et al. Mediterranean dietary pattern and depression: the PREDIMED randomized trial. BMC Medicine 2013; 11:208.

Sala-Vila A, Romero-Mamani ES, Gilabert R, Núñez I, De la Torre R, Corella D, et al. Changes in ultrasound-assessed carotid intima-media thickness and plaque with a mediterranean diet: a substudy of the PREDIMED trial. Arteriosclerosis, Thrombosis, and Vascular Biology 2014; 34(2):439-45.
Salas-Salvadó J, Bulló M, Babio N, , Ibarrola-Jurado N, Basora J, et al. Reduction in the incidence of type 2 diabetes with the Mediterranean diet: results of the PREDIMED-Reus nutrition intervention randomized trial. Diabetes Care 2011; 34:14-9.

Salas-Salvadó J, Bulló M, Estruch R, Ros E, Covas MI, IbarrolaJurado N, et al. Prevention of diabetes with Mediterranean diets: a subgroup analysis of a randomized trial. Annals of Internal Medicine 2014; 160(1):1-10.

Salas-Salvadó J, Fernandez-Ballart J, Ros E, MartínezGonzález MA, Fito M, Estruch R, et al. Effect of a Mediterranean diet supplemented with nuts on metabolic syndrome status: one-year results of the PREDIMED randomized trial. Archives of Internal Medicine 2008; 168(22):2449-58.

Salas-Salvado J, Garcia-Arellano A, Estruch R, MarquezSandoval F, Corella D, Fiol M, et al. Components of the Mediterranean-type food pattern and serum inflammatory markers among patients at high risk for cardiovascular disease. European Journal of Clinical Nutrition 2008; 62(5):651-9.

Schröder H, De la Torre R, Estruch R, Corella D, MartínezGonzález MA, Salas-Salvadó J, et al. Alcohol consumption is associated with high concentrations of urinary hydroxytyrosol. American Journal of Clinical Nutrition 2009; 90(5):1329-35.

Schröder H, Fitó M, Estruch R, Martínez-González MA, Corella D, Salas-Salvadó J, et al. A short screener is valid for assessing Mediterranean diet adherence among older Spanish men and women. Journal of Nutrition 2011; 141(6):1140-5.

Solá R, Fitó M, Estruch R, Salas-Salvadó J, Corella D, De La Torre R, et al. Effect of a traditional Mediterranean diet on apolipoproteins B, A-I, and their ratio: a randomized, controlled trial. Atherosclerosis 2011; 218(1):174-80.

Toledo E, Delgado-Rodríguez M, Estruch R, Salas-Salvadó J, Corella D, Gomez-Gracia E, et al. Low-fat dairy products and blood pressure: follow-up of 2290 older persons at high cardiovascular risk participating in the PREDIMED study. British Journal of Nutrition 2009; 101(1):59-67.

Toledo E, Hu FB, Estruch R, Buil-Cosiales P, Corella D, SalasSalvadó J, et al. Effect of the Mediterranean diet on blood pressure in the PREDIMED trial: results from a randomized controlled trial. BMC Medicine 2013; 11:207.

Urpi-Sarda M, Casas R, Chiva-Blanch G, Romero-Mamani ES, Valderas-Martínez P, Arranz S, et al. Virgin olive oil and nuts as key foods of the Mediterranean diet effects on inflammatory biomakers related to atherosclerosis. Pharmacological Research 2012; 65(6):577-83.

Waterhouse AL. Resveratrol metabolites in urine as biomarker of wine intake in free-living subjects: the PREDIMED Study. Free Radical Biology \& Medicine 2009; 46(12):1561.

Zamora-Ros R, Urpi-Sarda M, Lamuela-Raventos RM, Estruch R, Martínez-González MA, Bulló M, et al. Resveratrol metabolites in urine as a biomarker of wine intake in free-living subjects: the PREDIMED Study. Free Radical Biology \& Medicine 2009; 46(12):1562-6. 
Zazpe I, Estruch R, Toledo E, Sánchez-Taínta A, Corella D, Bulló M, et al. Predictors of adherence to a Mediterranean-type diet in the PREDIMED trial. European Journal of Nutrition 2010; 49(2):91-9.

Zazpe I, Sánchez-Taínta A, Estruch R, Lamuela-Raventos RM, Schröder H, Salas-Salvadó J, et al. A large randomized individual and group intervention conducted by registered dietitians increased adherence to Mediterranean-type diets: the PREDIMED study. Journal of the American Dietetic Association 2008; 108(7):1134-44.

\section{PREMIER 2003 \{published and unpublished data\}}

* Appel LJ, Champagne CM, Harsha DW, Cooper LS, Obarzanek E, Elmer PJ, et al. Effects of comprehensive lifestyle modification on blood pressure control: main results of the PREMIER clinical trial. JAMA 2003; 289(16):2083-93.

Elmer PJ, Obarzanek E, Vollmer WM, Simons-Morton D, Stevens VJ, Young DR, et al. Effects of comprehensive lifestyle modification on diet, weight, physical fitness, and blood pressure control: 18-month results of a randomized trial. Annals of Internal Medicine 2006; 144(7):485-95.

Ledikwe JH, Rolls BJ, Smiciklas-Wright H, Mitchell DC, Ard JD, Champagne $\mathrm{C}$, et al. Reductions in dietary energy density are associated with weight loss in overweight and obese participants in the PREMIER trial. American Journal of Clinical Nutrition 2007; 85(5):1212-21.

Lien LF, Brown AJ, Ard JD, Loria C, Erlinger TP, Feldstein AC, et al. Effects of PREMIER lifestyle modifications on participants with and without the metabolic syndrome. Hypertension 2007; 50(4):609-16

Lin PH, Appel LJ, Funk K, Craddick S, Chen C, Elmer P, et al. The PREMIER intervention helps participants follow the Dietary Approaches to Stop Hypertension dietary pattern and the current Dietary Reference Intakes recommendations. Journal of the American Dietetic Association 2007; 107(9):1541-51.

Lin PH, Miwa S, Li YJ, Wang Y, Levy E, Lastor K, et al. Factors influencing dietary protein sources in the PREMIER trial population. Journal of the American Dietetic Association 2010; 110(2):291-5.

Maruthur NM, Wang NY, Appel LJ. Lifestyle interventions reduce coronary heart disease risk: results from the PREMIER Trial. Circulation 2009; 119(15):2026-31.

McGuire HL, Svetkey LP, Harsha DW, Elmer PJ, Appel LJ, Ard JD. Comprehensive lifestyle modification and blood pressure control: a review of the PREMIER trial. Journal of Clinical Hypertension 2004; 6(7):383-90.

Obarzanek E, Vollmer WM, Lin PH, Cooper LS, Young DR, Ard JD, et al. Effects of individual components of multiple behavior changes: the PREMIER trial. American Journal of Health Behavior 2007; 31(5):545-60.

Svetkey LP, Erlinger TP, Vollmer WM, Feldstein A, Cooper LS, Appel LJ, et al. Effect of lifestyle modifications on blood pressure by race, sex, hypertension status, and age. Journal of Human Hypertension 2005; 19(1):21-31.
Svetkey LP, Harsha DW, Vollmer WM, Stevens VJ, Obarzanek E, Elmer PJ, et al. Premier: a clinical trial of comprehensive lifestyle modification for blood pressure control: rationale, design and baseline characteristics. Annals of Epidemiology 2003; 13(6):462-71.

Young DR, Coughlin J, Jerome GJ, Myers V, Chae SE, Brantley PJ. Effects of the PREMIER interventions on health-related quality of life. Annals of Behavioral Medicine 2010; 40(3):302-12.

Pritchard 2002 \{published data only\}

Pritchard JE, Nowson CA, Billington T, Wark JD. Benefits of a year-long workplace weight loss program on cardiovascular risk factors. Nutrition and Dietetics 2002; 59(2):87-96.

Puget Sound EP 2000 \{published and unpublished data\} Kristal AR, Curry SJ, Shattuck AL, Feng Z, Li S. A randomized trial of a tailored, self-help dietary intervention: the Puget Sound Eating Patterns Study. Preventive Medicine 2000; 31(4):380-9.

Rabast 1979 \{published data only\}

Rabast U, Schonborn J, Kasper H. Dietetic treatment of obesity with low and high-carbohydrate diets: comparative studies and clinical results. International Journal of Obesity 1979; 3(3):201-11.

Rabkin 1981 \{published data only\}

Rabkin SW, Boyko E, Streja DA. Relationship of weight loss and cigarette smoking to changes in high-density lipoprotein cholesterol. American Journal of Clinical Nutrition 1981; 34(9):1764-8

\section{Radack 1990 \{published data only\}}

Radack K, Deck C, Huster G. The comparative effects of n-3 and n-6 polyunsaturated fatty acids on plasma fibrinogen levels: a controlled clinical trial in hypertriglyceridemic subjects. Journal of the American College of Nutrition 1990; 9(4):352-7.

\section{Rasmussen 1995 \{published data only\}}

Rasmussen OW, Thomsen $\mathrm{CH}$, Hansen KW, Vesterlund M, Winther E, Hermansen K. Favourable effect of olive oil in patients with non-insulin-dependent diabetes. The effect on blood pressure, blood glucose and lipid levels of a high-fat diet rich in monounsaturated fat compared with a carbohydrate-rich diet [Gunstig virkning af olivenolie hos ikkeinsulinkraevende diabetikere. Virkningen pa blodtryk, blodglukose og lipidniveauer af en dioet med et hojt indhold af monoumoettet fedt sammenlignet med en kulhydratrig dioet]. Ugeskrift for Laeger 1995; 157(8):1028-32.

\section{Reaven 2001 \{published data only\}}

Reaven GM, Abbasi F, Bernhart S, Coulston A, Darnell B, Dashti N, et al. Insulin resistance, dietary cholesterol, and cholesterol concentration in postmenopausal women. Metabolism: Clinical \& Experimental 2001; 50(5):594-7.

\section{Reid 2002 \{published data only\}}

Reid R, Fodor G, Lydon-Hassen K, D'Angelo MS, McCrea J, Bowlby $\mathrm{M}$, et al. Dietary counselling for dyslipidemia in primary care: results of a randomized trial. Canadian Journal of Dietetic Practice \& Research 2002; 63(4):169-75. 
Renaud 1986 \{published data only\}

Renaud S, Godsey F, Dumont E, Thevenon C, Ortchanian E, Martin JL. Influence of long-term diet modification on platelet function and composition in Moselle farmers. American Journal of Clinical Nutrition 1986; 43(1):136-50.

Rivellese 1994 \{published and unpublished data\}

Rivellese AA, Auletta P, Marotta G, Saldalamacchia G, Giacco A, Mastrilli V, et al. Long term metabolic effects of two dietary methods of treating hyperlipidaemia. BMJ 1994; 308(6923):227-31.

\section{Rivellese 2003 \{published data only\}}

Rivellese AA, Maffettone A, Vessby B, Uusitupa M, Hermansen K, Berglund $L$, et al. Effects of dietary saturated, monounsaturated and $\mathrm{n}-3$ fatty acids on fasting lipoproteins, LDL size and postprandial lipid metabolism in healthy subjects. Atherosclerosis 2003; 167(1):149-58

\section{Roderick 1997 \{published and unpublished data\}}

Roderick P, Ruddock V, Hunt P, Miller G. A randomized trial to evaluate the effectiveness of dietary advice by practice nurses in lowering diet-related coronary heart disease risk. British Journal of General Practice 1997; 47(414):7-12.

\section{Roman CHD prev 1986 \{published data only\}}

* Research Group of the Rome Project of Coronary Heart Disease Prevention. Eight-year follow-up results from the Rome Project of Coronary Heart Disease Prevention. Preventive Medicine 1986; 15(2):176-91.

Research Group of the Rome Project of Coronary Heart Disease Prevention. The Roman Coronary Disease Prevention Project: effectiveness of intervention and reduction of mortality over a 10-year period [II Progetto Romano di Prevenzione della Cardiopatia Coronarica: efficacia dell'intervento e riduzione della mortalita in 10 anni]. Giornale Italiano di Cardiologia 1986; 16(3):196-202.

\section{Rose 1987 \{published data only\}}

Rose DP, Boyar AP, Cohen C, Strong LE. Effect of a low fat diet on hormone levels in women with cystic breast disease. I. Serum steroids and gonadotropins. Journal of the National Cancer Institute 1987; 78(4):623-6.

\section{Sarkkinen 1995 \{published and unpublished data\}}

Makinen E, Uusitupa MI, Pietinen P, Aro A, Penttila I. Long term effects of three fat modified diets on serum lipids in free living hypercholesterolaemic subjects (abstract). European Heart Journal 1991; 12:162.

* Sarkkinen E. Long-Term Feasibility and Effects of Three Different Fat-Modified Diets in Free-Living Hypercholesterolemic Subjects [PhD thesis]. Kuopio, Finland: University of Kuopio, 1995.

Sarkkinen ES, Agren JJ, Ahola I, Ovaskainen ML, Uusitupa MI. Fatty acid composition of serum cholesterol esters, and erythrocyte and platelet membranes as indicators of long-term adherence to fat-modified diets. American Journal of Clinical Nutrition 1994; 59(2):364-70.
Sarkkinen ES, Uusitupa MI, Nyyssonen K, Parviainen M, Penttila I, Salonen JT. Effects of two low-fat diets, high and low in polyunsaturated fatty acids, on plasma lipid peroxides and serum vitamin E levels in free-living hypercholesterolaemic men. European Journal of Clinical Nutrition 1993; 47(9):623-30.

Sarkkinen ES, Uusitupa MI, Pietinen P, Aro A, Ahola I, Penttila I, et al. Long-term effects of three fat-modified diets in hypercholesterolemic subjects. Atherosclerosis 1994; 105(1):9-23.

Uusitupa MI, Sarkkinen ES, Torpstrom J, Pietinen P, Aro A. Long-term effects of four fat-modified diets on blood pressure. Journal of Human Hypertension 1994; 8(3):209-18.

\section{Schaefer 1995a \{published data only\}}

Schaefer EJ, Lichtenstein AH, Lamon-Fava S, McNamara JR, Schaefer MM, Rasmussen $\mathrm{H}$, et al. Body weight and low density lipoprotein cholesterol changes after consumption of a low fat ad libitum diet. JAMA 1995; 274(18):1450-5.

\section{Schaefer 1995b \{published data only\}}

Schaefer EJ, Lichtenstein AH, Lamon-Fava S, Contois JH, Li Z, Rasmussen H, et al. Efficacy of a National Cholesterol Education Program Step 2 diet in normolipidaemic and hypercholesterolaemic middle-aged and elderly men and women. Arteriosclerosis, Thrombosis, and Vascular Biology 1995; 15(8):1079-85.

\section{Schectman 1996 \{published data only\}}

Schectman G, Wolff N, Byrd JC, Hiatt JG, Hartz A. Physician extenders for cost-effective management of hypercholesterolemia. Journal of General Internal Medicine 1996; 11(5):277-86

\section{Schlierf 1995 \{published data only\}}

Schlierf G, Schuler G, Hambrecht R, Niebauer J, Hauer K, Vogel G, et al. Treatment of coronary heart disease by diet and exercise. Journal of Cardiovascular Pharmacology 1995; 25(Suppl 4):S32-4.

\section{Seppanen-Laakso 1992 \{published data only\}}

Seppanen-Laakso T, Vanhanen H, Laakso I, Kohtamaki H, Viikari J. Replacement of butter on bread by rapeseed oil and rapeseed oil-containing margarine: effects on plasma fatty acid composition and serum cholesterol. British Journal of Nutrition 1992; 68:639-54.

\section{Seppelt 1996 \{published and unpublished data\}}

Seppelt B, Weststrate JA, Reinert A, Johnson D, Luder W, Zunft HJ. Long-term effects of nutrition with fat-reduced foods on energy consumption and body weight [Langzeiteffekte einer ernährung mit fettreduzierten lebensmitteln auf die energieaufnahme und das körpergewicht]. Zeitschrift für Ernährungswissenschaft 1996; 35(4):369-77.

\section{Singh 1991 \{published data only\}}

Singh RB, Rastogi SS, Sircar AR. Dietary strategies for risk-factor modification to prevent cardiovascular diseases. Nutrition 1991; 7(3):210-4. 
Singh 1992 \{published data only\}

Singh RB, Niaz MA, Agarwal P, Begom R, Rastogi SS. Effect of antioxidant-rich foods on plasma ascorbic acid, cardiac enzyme, and lipid peroxide levels in patients hospitalized with acute myocardial infarction. Journal of the American Dietetic Association 1995; 95(7):775-80.

Singh RB, Niaz MA, Ghosh S. Effect on central obesity and associated disturbances of low-energy, fruit- and vegetableenriched prudent diet in north Indians. Postgraduate Medical Journal 1994; 70(830):895-900.

* Singh RB, Rastogi SS, Verma R, Bolaki L, Singh R. An Indian experiment with nutritional modulation in acute myocardial infarction. American Journal of Cardiology 1992; 69(9):879-85.

Singh RB, Rastogi SS, Verma R, Laxmi B, Singh R, Ghosh S, et al. Randomised controlled trial of cardioprotective diet in patients with recent acute myocardial infarction: results of one year follow up. BMJ 1992; 304(6833):1015-9.

\section{Sirtori 1992 \{published data only\}}

Sirtori CR, Gatti E, Tremoli E, Galli C, Gianfranceschi G, Franceschini $\mathrm{G}$, et al. Olive oil, corn oil, and n-3 fatty acids differently affect lipids, lipoproteins, platelets, and superoxide formation in type II hypercholesterolemia. American Journal of Clinical Nutrition 1992; 56(1):113-22.

\section{SLIM 2008 \{published data only\}}

Roumen C, Corpeleijn E, Feskens EJ, Mensink M, Saris WH, Blaak EE, et al. Impact of 3-year lifestyle intervention on postprandial glucose metabolism: the SLIM study. Diabetic Medicine 2008; 25(5):597-605.

\section{Sopotsinskaia 1992 \{published data only\}}

Sopotsinskaia EB, Balitskii KP, Tarutinov VI, Zhukova VM, Semenchuk DD, Kozlovskaia SG, et al. Experience with the use of a low-calorie diet in breast cancer patients to prevent metastasis [Opyt primeneniia nizkokaloriinoi diety u bol'nykh rakom molochnoi zhelezy s tsel'iu profilaktiki metastazi]. Voprosy Onkologii 1992; 38(5):592-9.

\section{Soul Food Light \{published data only\}}

Anderson-Loftin W, Barnett S, Bunn P, Sullivan P, Hussey J, Tavakoli A. Soul Food Light: culturally competent diabetes education. Diabetes Educator 2005; 31(4):555-63.

\section{Stanford NAP 1997 \{published data only\}}

Howard PB, Winkleby MA, Albright CL, Bruce B, Fortmann SP. The Stanford Nutrition Action Program: a dietary fat intervention for low-literacy adults. American Journal of Public Health 1997; 87(12):1971-6.

\section{Stanford Weight 1994 \{published and unpublished data\}}

Williams PT, Krauss RM, Stefanick ML, Vranizan KM, Wood PD. Effects of low-fat diet, calorie restriction, and running on lipoprotein subfraction concentrations in moderately overweight men. Metabolism 1994; 43(5):655-63.

\section{Starmans 1995 \{published data only\}}

Starmans KM, Lustermans FT, Kragten HA, Struijker BH, Rilla H. Lowering cholesterol in patients with mild hypercholesterolaemia does not improve functional properties of large arteries [abstract]. Netherlands Journal Of Medicine 1995; 46:A70.

\section{Steinbach 1996 \{published data only\}}

Steinbach M. A Romanian contribution to the epidemiology and prevention of cardiovascular diseases. Romanian Journal of Internal Medicine 1996; 34(1-2):137-48.

\section{Steptoe 2001 \{published data only\}}

Steptoe A, Kerry S, Rink E, Hilton S. The impact of behavioral counselling on stage of change in fat intake, physical activity, and cigarette smoking in adults at increased risk of coronary heart disease. American Journal of Public Health 2001; 91(2):265-9.

\section{Stevens 2002 \{published and unpublished data\}}

Stevens VJ, Glasgow RE, Toobert DJ, Karanja N, Smith KS. Oneyear results from a brief, computer-assisted intervention to decrease consumption of fat and increase consumption of fruits and vegetables. Preventive Medicine 2003; 36(5):594-600.

* Stevens VJ, Glasgow RE, Toobert DJ, Karanja N, Smith KS. Randomized trial of a brief dietary intervention to decrease consumption of fat and increase consumption of fruits and vegetables. American Journal of Health Promotion 2002; 16(3):129-34.

\section{Stevenson 1988 \{published data only\}}

Stevenson DW, Darga LL, Spafford TR, Ahmad N, Lucas CP. Variable effects of weight loss on serum lipids and lipoproteins in obese patients. International Journal of Obesity 1988; 12(6):495-502.

\section{Strychar 2009 \{published and unpublished data\}}

Strychar I, Cohn JS, Renier G, Rivard M, Aris-Jilwan N, Beauregard $\mathrm{H}$, et al. Effects of a diet higher in carbohydrate/ lower in fat versus lower in carbohydrate/higher in monounsaturated fat on postmeal triglyceride concentrations and other cardiovascular risk factors in type 1 diabetes. Diabetes Care 2009; 32(9):1597-9.

\section{Sweeney 2004 \{published data only\}}

Sweeney M. Effects of very low-fat diets on anginal symptoms. Medical Hypotheses 2004; 63(3):553.

Søndergaard 2003 \{published and unpublished data\} Søndergaard E, Møller JE, Egstrup K. Effect of dietary intervention and lipid-lowering treatment on brachial vasoreactivity in patients with ischemic heart disease and hypercholesterolemia. American Heart Journal 2003; 145(5):E19.

TAIM 1992 \{published data only\}

Davis BR, Blaufox MD, Hawkins CM, Langford HG, Oberman A, Swencionis $C$, et al. Trial of antihypertensive interventions and management. Design, methods, and selected baseline results. Controlled Clinical Trials 1989; 10(1):11-30.

Davis BR, Blaufox MD, Oberman A, Wassertheil SS, Zimbaldi N, Cutler JA, et al. Reduction in long-term antihypertensive medication requirements. Effects of weight reduction by dietary 
intervention in overweight persons with mild hypertension. Archives of Internal Medicine 1993; 153(15):1773-82.

Davis BR, Oberman A, Blaufox MD, Wassertheil SS, Hawkins CM, Cutler JA, et al. Effect of antihypertensive therapy on weight loss. The Trial of Antihypertensive Interventions and Management Research Group. Hypertension 1992; 19(4):393-9.

Langford HG, Davis BR, Blaufox D, Oberman A, WassertheilSmoller S, Hawkins M, et al. Effect of drug and diet treatment of mild hypertension on diastolic blood pressure. The TAIM Research. Hypertension 1991; 17(2):210-7.

Oberman A, Wassertheil-Smoller S, Langford HG, Blaufox MD, Davis BR, Blaszkowski T, et al. Pharmacologic and nutritional treatment of mild hypertension: changes in cardiovascular risk status. Annals of Internal Medicine 1990; 112(2):89-95.

Wassertheil-Smoller S, Davis BR, Breuer B, Chee JC, Oberman A, Blaufox MD. Differences in precision of dietary estimates among different population subgroups. Annals of Epidemiology 1993; 3(6):619-28.

* Wassertheil-Smoller S, Oberman A, Blaufox MD, Davis B, Langford $\mathrm{H}$. The Trial of Antihypertensive Interventions and Management (TAIM) study. Final results with regard to blood pressure, cardiovascular risk, and quality of life. American Journal of Hypertension 1992; 5(1):37-44.

Wylie-Rosett J, Wassertheil-Smoller S, Blaufox MD, Davis BR, Langford HG, Oberman A, et al. Trial of antihypertensive intervention and management: greater efficacy with weight reduction than with a sodium-potassium intervention. Journal of the American Dietetic Association 1993; 93(4):408-15.

Tapsell 2004 \{published data only (unpublished sought but not used)\}

Tapsell LC, Hokman A, Sebastiao A, Denmeade S, Martin G, Calvert GD, et al. The impact of usual dietary patterns, selection of significant foods and cuisine choices on changing dietary fat under 'free living' conditions. Asia Pacific Journal of Clinical Nutrition 2004; 13(1):86-91.

\section{THIS DIET 2008 \{published data only\}}

Tuttle KR, Shuler LA, Packard DP, Milton JE, Daratha KB, Bibus DM, et al. Comparison of low-fat versus Mediterraneanstyle dietary intervention after first myocardial infarction (from the Heart Institute of Spokane Diet Intervention and Evaluation Trial). American Journal of Cardiology 2008; 101(11):1523-30.

\section{TOHP I 1992 \{published data only\}}

Kumanyika SK, Hebert PR, Cutler JA, Lasser VI, Sugars CP, Steffen Batey L, et al. Feasibility and efficacy of sodium reduction in the Trials of Hypertension Prevention, phase I. Hypertension 1993; 22(4):502-12.

Satterfield S, Cutler JA, Langford HG, Applegate WB, Borhani NO, Brittain E, et al. Trials of hypertension prevention. Annals of Epidemiology 1991; 1(5):455-71.

Stevens VJ, Corrigan SA, Obarzanek E, Bernauer E, Cook NR, Hebert P, et al, the TOHP Collaborative Research Group.
Weight loss intervention in phase I of the trials of hypertension prevention. Archives of Internal Medicine 1993; 153(7):849-58.

* The Trials of Hypertension Prevention Collaborative Research Group. The effects of nonpharmacologic interventions on blood pressure of persons with high normal levels. Results of the Trials of Hypertension Prevention, Phase I. JAMA 1992; 267(9):1213-20.

Whelton PK, Hebert PR, Cutler J, Applegate WB, Eberlein KA, Klag MJ, et al. Baseline characteristics of participants in phase I of the Trials of Hypertension Prevention. Annals of Epidemiology 1992; 2(3):295-310.

Whelton PK, Kumanyika SK, Cook NR, Cutler JA, Borhani NO, Hennekens $\mathrm{CH}$, et al. Efficacy of nonpharmacologic interventions in adults with high-normal blood pressure: results from phase 1 of the Trials of Hypertension Prevention. American Journal of Clinical Nutrition 1997; 65(2 Suppl):652S-60S.

\section{TONE 1997 \{published data only\}}

Whelton PK, Appel LJ, Espeland MA, Applegate WB, EttingerWH J, Kostis JB, et al. Sodium reduction and weight loss in the treatment of hypertension in older persons: a randomized controlled trial of nonpharmacologic interventions in the elderly (TONE). JAMA 1998; 279(11):839-46.

* Whelton PK, Babnson J, Appel LJ, Charleston J, Cosgrove N, Espeland MA, et al. Recruitment in the Trial of NOnpharmacologic intervention in the Elderly (TONE). Journal of the American Geriatrics Society 1997; 45(2):185-93.

Toobert 2003 \{published data only\}

Toobert DJ, Glasgow RE, Strycker LA, , Radcliffe JL, Wander RC, et al. Biologic and quality-of-life outcomes from the Mediterranean Lifestyle Program: a randomized clinical trial. Diabetes Care 2003; 26(8):2288-93.

\section{Towle 1994 \{published data only\}}

Towle LA, Bergman EA, Joseph E. Low-fat bison-hybrid ground meat has no effects on serum lipid levels in a study of 12 men. Journal of the American Dietetic Association 1994; 94(5):546-8.

TRANSFACT 2006 \{published data only\}

* Chardigny JM, Malpuech-Brugere C, Dionisi F, Bauman DE, German B, Mensink RP, et al. Rationale and design of the TRANSFACT project phase I: a study to assess the effect of the two different dietary sources of trans fatty acids on cardiovascular risk factors in humans. Contemporary Clinical Trials 2006; $27(4): 364-73$.

Chardigny JM. Do trans fatty acids from industrially produced sources and from natural sources have the same effect on cardiovascular disease risk factors in healthy subjects? Results of the Trans Fatty Acids Collaboration (TRANSFACT) study. American Journal of Clinical Nutrition 2008; 108(3):558-66.

\section{Treatwell 1992 \{published and unpublished data\}}

Sorensen G, Morris DM, Hunt MK, Hebert JR, Harris DR, Stoddard A, et al. Work-site nutrition intervention and employees' dietary habits: the Treatwell program. American Journal of Public Health 1992; 82(6):877-80. 
Tromsø Heart 1989 \{published data only\}

Knutsen SF, Knutsen R. The Tromsø Heart Study: family approach to intervention on CHD. Feasibility of risk factor reduction in high-risk persons--project description. Scandinavian Journal of Social Medicine 1989; 17(1):109-19.

Troyer 2010 \{published and unpublished data\}

Racine EF, Lyerly J, Troyer JL, Warren-Findlow J, McAuley WJ. The influence of home-delivered dietary approaches to stop hypertension meals on body mass index, energy intake, and percent of energy needs consumed among older adults with hypertension and/or hyperlipidemia. Journal of the Academy of Nutrition \& Dietetics 2012; 112(11):1755-62.

* Troyer JL, Racine EF, Ngugi GW, McAuley WJ. The effect of home-delivered Dietary Approach to Stop Hypertension (DASH) meals on the diets of older adults with cardiovascular disease. American Journal of Clinical Nutrition 2010; 91(5):1204-12.

UK PDS 1996 \{published data only\}

* Turner R, Cull C, Holman R. United Kingdom Prospective Diabetes Study 17: a 9-year update of a randomized, controlled trial on the effect of improved metabolic control on complications in non-insulin-dependent diabetes mellitus. Annals of Internal Medicine 1996; 124(1 Pt 2):136-45.

Turner RC, Holman RR. Lessons from UK prospective diabetes study. Diabetes Research and Clinical Practice 1995; 28(Suppl):S151-7.

\section{Urbach 1952 \{published data only\}}

Urbach R, Hildreth EA, Wackerman MT. The therapeutic uses of low fat, low cholesterol diets: I. Treatment of essential familial xanthomatosis. Journal of Clinical Nutrition 1952; 1(1):52-6.

\section{Uusitupa 1993 \{published data only\}}

Uusitupa M, Laitinen J, Siitonen O, Vanninen E, Pyorala K. The maintenance of improved metabolic control after intensified diet therapy in recent type 2 diabetes. Diabetes Research and Clinical Practice 1993; 19(3):227-38.

VASTKOST 2012 \{published data only\}

ZZZ <accessionId ref="info:x-wiley/clinicalTrialsGov/ NCT01005498"> ZZZNCT01005498

* Guldbrand H, Dizdar B, Bunjaku B, Lindstrom T, BachrachLindstrom M, Fredrikson M, et al. In type 2 diabetes, randomisation to advice to follow a low-carbohydrate diet transiently improves glycaemic control compared with advice to follow a low-fat diet producing a similar weight loss. Diabetologia 2012; 55(8):2118-27.

Guldbrand H, Lindstrom T, Dizdar B, Bunjaku B, Ostgren CJ, Nystrom $\mathrm{FH}$, et al. Randomization to a low-carbohydrate diet advice improves health related quality of life compared with a low-fat diet at similar weight-loss in type 2 diabetes mellitus. Diabetes Research \& Clinical Practice 2014; 106(2):221-7.

Jonasson L, Guldbrand H, Lundberg AK, Nystrom FH. Advice to follow a low-carbohydrate diet has a favourable impact on lowgrade inflammation in type 2 diabetes compared with advice to follow a low-fat diet. Annals of Medicine 2014; 46(3):182-7.

\section{Vavrikova 1958 \{published data only\}}

Vavrikova J. Essential fatty acids, lipid metabolism, and atherosclerosis [letter]. Lancet 1958; 1:1337.

Verheiden 2003 \{published data only (unpublished sought but not used)\}

Verheiden MW, Van der Veen JE, Van Zadelhoff WM, Bakx C, Koelen MA, Van den Hoogen $\mathrm{HJ}$, et al. Nutrition guidance in Dutch family practice: behavioural determinants of reduction in fat consumption. American Journal of Clinical Nutrition 2003; 77(Suppl):1058S-64S.

WAHA 2016 \{published and unpublished data\} ZZZ <accessionId ref="info:x-wiley/clinicalTrialsGov/ NCT01634841"> ZZZNCT01634841

Bitok E, Jaceldo-Siegl K, Rajaram S, Serra-Mir M, Roth I, FeitasSimoes T, et al. Favourable nutrient intake and displacement with long-term walnut supplementation among elderly: results of a randomised trial. British Journal of Nutrition 2017; 118(3):201-9. [DOI: 10.1017/S0007114517001957]

Bitok E, Rajaram S, Ros E. Does a daily walnut supplement given for a year result in body weight gain? FASEB Journal 2016; 30:1157.5.

Huey L, Bitok E, Kazzi N. Dietary compliance of walnut or no walnut intake in a 1-year randomized intervention trial among free-living elderly in the Walnuts and Healthy Aging Study (WAHA). FASEB Journal 2016; 30:1157.10.

Rajaram S, Valls-Pedret C, Cofan M, Sabate J, Serra-Mir M, PerezHeras AM, et al. The Walnuts and Healthy Aging Study (WAHA): protocol for a nutritional intervention trial with walnuts on brain aging. Frontiers in Aging Neuroscience 2016; 8:333. [PMID: 28119602]

* Ros E, Rajaram S, Sala-Vila A, Serra-Mir M, Valls-Pedret C, Cofán M, et al. Effect of a 1-year walnut supplementation on blood lipids among older individuals: findings from the Walnuts and Healthy Aging (WAHA) study. FASEB Journal 2016; 30(1 Suppl):293.4.

\section{Wass 1981 \{published data only\}}

Wass VJ, Jarrett RJ, Meilton V, Start MK, Mattock M, Ogg CS, et al. Effect of a long-term fat-modified diet on serum lipoprotein levels of cholesterol and triglyceride in patients. Clinical Science 1981; 60(1):81-6.

\section{Wassertheil 1985 \{published data only\}}

Wassertheil SS, Blaufox MD, Langford HG, Oberman A, Cutter G, Pressel S. Prediction of response to sodium intervention for blood pressure control. Journal of Hypertension 1986; 4(5 Suppl):S343-6.

* Wassertheil SS, Langford HG, Blaufox MD, Oberman A, Hawkins M, Levine B, et al. Effective dietary intervention in hypertensives: sodium restriction and weight reduction. Journal of the American Dietetic Association 1985; 85(4):423-30.

WATCH 1999 \{published and unpublished data\}

Ockene IS, Hebert JR, Ockene JK, Saperia GM, Stanek E, Nicolosi R, et al. Effect of a physician-delivered nutrition 
counselling training and an office-support program on saturated fat intake, weight, and serum lipid measurements in a hyperlipidemic population: Worcester Area Trial for Counseling in Hyperlipidemia. Archives of Internal Medicine 1999; 159(7):725-31.

\section{Watts 1988 \{published data only\}}

Watts GF, Ahmed W, Quiney J, Houlston R, Jackson P, Iles C, et al. Effective lipid lowering diets including lean meat. BMJ (Clinical Research Ed) 1988; 296(6617):235-7.

\section{Weintraub 1992 \{published data only\}}

Weintraub M, Sundaresan PR, Schuster B. Long-term weight control study. VII (weeks 0 to 210). Serum lipid changes. Clinical Pharmacology and Therapeutics 1992; 51(5):634-41.

\section{Westman 2006 \{published data only\}}

Westman EC, , Olsen MK, Dudley T, Guyton JR. Effect of a lowcarbohydrate, ketogenic diet program compared to a low-fat diet on fasting lipoprotein subclasses. International Journal of Cardiology 2006; 110(2):212-6.

\section{Weststrate 1998 \{published data only\}}

Weststrate JA, Meijer GW. Plant sterol enriched margarines and reduction of plasma total-and LDL-cholesterol concentrations in normocholesterolaemic and mildly hypercholesterolaemic subjects. European Journal of Clinical Nutrition 1998; 52(5):334-43.

\section{WHEL 2007 \{published data only\}}

Bardwell WA, Profant J, Casden DR, Dimsdale JE, Ancoli-Israel S, Natarajan L, et al. The relative importance of specific risk factors for insomnia in women treated for early-stage breast cancer. Psycho-Oncology 2008; 17(1):9-18.

Caan BJ, Flatt SW, Rock CL, Ritenbaugh C, Newman V, Pierce JP, et al, Women's Healthy Eating and Living Group. Low-energy reporting in women at risk for breast cancer recurrence. Cancer Epidemiology, Biomarkers \& Prevention 2000; 9(10):1091-7.

Gold EB, Flatt SW, Pierce JP, Bardwell WA, Hajek RA, Newman VA, et al. Dietary factors and vasomotor symptoms in breast cancer survivors: the WHEL Study. Menopause 2006; 13(3):423-33.

Gold EB, Pierce JP, Natarajan L, Stefanick ML, Laughlin GA, Caan BJ, et al. Dietary pattern influences breast cancer prognosis in women without hot flashes: the Women's Healthy Eating and Living trial. Journal of Clinical Oncology 2009; 27(3):352-9.

Hernandez-Valero MA, Thomson CA, Hernandez M, Tran T, Detry MA, Theriault RL, et al. Comparison of baseline dietary intake of Hispanic and matched non-Hispanic white breast cancer survivors enrolled in the Women's Healthy Eating and Living study. Journal of the American Dietetic Association 2008; 108(8):1323-9.

Hong S, Bardwell WA, Natarajan L, Flatt SW, Rock CL, Newman VA, et al. Correlates of physical activity level in breast cancer survivors participating in the Women's Healthy Eating and Living (WHEL) study. Breast Cancer Research \& Treatment 2007; 101(2):225-32.

Hyder JA, Thomson CA, Natarajan L, Madlensky L, Pu M, Emond J, et al. Adopting a plant-based diet minimally increased food costs in WHEL Study. American Journal of Health Behavior 2009; 33(5):530-9.

Madlensky L, Natarajan L, Flatt SW, Faerber S, Newman VA, Pierce JP, et al. Timing of dietary change in response to a telephone counseling intervention: evidence from the WHEL study. Health Psychology 2008; 27(5):539-47.

Mortimer JE, Flatt SW, Parker BA, Gold EB, Wasserman L, Natarajan L, et al. Tamoxifen, hot flashes and recurrence in breast cancer. Breast Cancer Research \& Treatment 2008; 108(3):421-6.

Newman VA, Thomson CA, Rock CL, Flatt SW, Kealey S, Bardwell WA, et al. Achieving substantial changes in eating behavior among women previously treated for breast cancer: an overview of the intervention. Journal of the American Dietetic Association 2005; 105(3):382-91.

Paxton RJ, Jones LA, Chang S, Hernandez M, Hajek RA, Flatt SW, et al. Was race a factor in the outcomes of the Women's Health Eating and Living Study? Cancer 2011; 117(16):3805-13.

Pierce JP, Faerber S, Wright FA, Rock CL, Newman V, Flatt SW, et al. A randomized trial of the effect of a plant-based dietary pattern on additional breast cancer events and survival: the Women's Healthy Eating and Living (WHEL) Study. Controlled Clinical Trials 2002; 23(6):728-56.

Pierce JP, Natarajan L, Caan BJ, Flatt SW, Kealey S, Gold EB, et al. Dietary change and reduced breast cancer events among women without hot flashes after treatment of early-stage breast cancer: subgroup analysis of the Women's Healthy Eating and Living Study. American Journal of Clinical Nutrition 2009; 89(5):1565S-71S.

* Pierce JP, Natarajan L, Caan BJ, Parker BA, Greenberg ER, Flatt SW, et al. Influence of a diet very high in vegetables, fruit, and fiber and low in fat on prognosis following treatment for breast cancer: the Women's Healthy Eating and Living (WHEL) randomized trial. JAMA 2007; 298(3):289-98.

Pierce JP, Natarajan L, Sun S, Al-Delaimy W, Flatt SW, Kealey S, et al. Increases in plasma carotenoid concentrations in response to a major dietary change in the Women's Healthy Eating and Living study. Cancer Epidemiology, Biomarkers \& Prevention 2006; 15(10):1886-92.

Pierce JP, Newman VA, Flatt SW, Faerber S, Rock CL, Natarajan L, et al. Telephone counseling intervention increases intakes of micronutrient- and phytochemical-rich vegetables, fruit and fiber in breast cancer survivors. Journal of Nutrition 2004; 134(2):452-8.

Pierce JP. A randomized trial of the effect of a plant-based dietary pattern on additional breast cancer events and survival: the Women's Healthy Eating and Living (WHEL) Study. Controlled Clinical Trials 2002; 23(6):728-56. 
Pierce JP. Diet and breast cancer prognosis: making sense of the Women's Healthy Eating and Living and Women's Intervention Nutrition Study trials. Current Opinion in Obstetrics \& Gynecology 2009; 21(1):86-91.

Rock CL, Flatt SW, Laughlin GA, Gold EB, Thomson CA, Natarajan L, et al. Reproductive steroid hormones and recurrence-free survival in women with a history of breast cancer. Cancer Epidemiology, Biomarkers \& Prevention 2008; 17(3):614-20.

Rock CL, Flatt SW, Newman V, Caan BJ, Haan MN, Stefanick ML, et al. Factors associated with weight gain in women after diagnosis of breast cancer. Women's Healthy Eating and Living Study Group. Journal of the American Dietetic Association 1999; 99(10):1212-21.

Rock CL, Flatt SW, Thomson CA, Stefanick ML, Newman VA, Jones $\mathrm{L}$, et al. Plasma triacylglycerol and HDL cholesterol concentrations confirm self-reported changes in carbohydrate and fat intakes in women in a diet intervention trial. Journal of Nutrition 2004; 134(2):342-7.

Rock CL, Natarajan L, Pu M, Thomson CA, Flatt SW, Caan BJ, et al. Longitudinal biological exposure to carotenoids is associated with breast cancer-free survival in the Women's Healthy Eating and Living Study. Cancer Epidemiology, Biomarkers \& Prevention 2009; 18(2):486-94.

Saquib N, Flatt SW, Natarajan L, Thomson CA, Bardwell WA, Caan $B$, et al. Weight gain and recovery of pre-cancer weight after breast cancer treatments: evidence from the Women's Healthy Eating and Living (WHEL) study. Breast Cancer Research \& Treatment 2007; 105(2):177-86.

Saxe GA, Madlensky L, Kealey S, Wu DP, Freeman KL, Pierce JP, et al. Disclosure to physicians of CAM use by breast cancer patients: findings from the Women's Healthy Eating and Living Study. Integrative Cancer Therapies 2008; 7(3):122-9.

\section{WHO primary prev 1979 \{published data only\}}

WHO Primary prevention trial. Primary prevention of ischaemic heart disease: WHO coordinated cooperative trial. A summary report. Bulletin Of The World Health Organization 1979; 57(5):801-5.

\section{WHT 1990 \{published and unpublished data\}}

Bowen D, Clifford CK, Coates R, Evans M, Feng Z, Fouad M, et al. The Women's Health Trial feasibility study in minority populations: design and baseline descriptions. Annals of Epidemiology 1996; 6(6):507-19.

Bowen D. The role of participation in the Women's Health trial: feasibility study in minority populations. Preventive Medicine 2000; 31(5):474-80.

Bowen DJ, Kestin M, McTiernan A, Carrell D, Green P. Effects of dietary fat intervention on mental health in women. Cancer Epidemiology, Biomarkers and Prevention 1995; 4(5):555-9.

Gorbach SL, Morrill LA, Woods MN, Dwyer JT, Selles WD, Henderson $\mathrm{M}$, et al. Changes in food patterns during a low-fat dietary intervention in women. Journal of the American Dietetic Association 1990; 90(6):802-9.

Henderson MM, Kushi LH, Thompson DJ, Gorbach SL, Clifford CK, Insull W, et al. Feasibility of a randomized trial of a low-fat diet for the prevention of breast cancer: dietary compliance in the Women's Health Trial Vanguard Study. Preventive Medicine 1990; 19(2):115-33.

* Insull W, Henderson MM, Prentice RL, Thompson DJ, Clifford C, Goldman S, et al. Results of a randomized feasibility study of a low-fat diet. Archives of Internal Medicine 1990; 150(2):421-7.

Kristal AR, White E, Shattuck AL, Curry S, Anderson GL, Fowler A, et al. Long-term maintenance of a low-fat diet: durability of fatrelated dietary habits in the Women's Health Trial. Journal of the American Dietetic Association 1992; 92(5):553-9.

Prentice RL, Kakar F, Hursting S, Sheppard L, Klein R, Kushi LH. Aspects of the rationale for the Women's Health Trial. Journal of the National Cancer Institute 1988; 80(11):802-14.

Self S, Prentice R, Iverson D, Henderson M, Thompson D, Byar D, et al. Statistical design of the Women's Health Trial. Controlled Clininical Trials 1988; 9(2):119-36.

Sheppard L, Kristal AR, Kushi LH. Weight loss in women participating in a randomised trial of low-fat diets. American Journal of Clinical Nutrition 1991; 54(5):821-8.

Urban N, Baker M. The Women's Health Trial as an investment. Medical Decision Making 1989; 9(1):59-64.

White E, Shattuck AL, Kristal AR, Urban N, Prentice RL, Henderson MM, et al. Maintenance of a low-fat diet: follow-up of the Women's Health Trial. Cancer Epidemiology, Biomarkers and Prevention 1992; 1(4):315-23.

\section{WHT Feasibility 2003 \{published and unpublished data\}}

Bowen D, Clifford CK, Coates R, Evans M, Feng Z, Fouad M, et al. The Women's Health Trial Feasibility Study in Minority Populations: design and baseline descriptions. Annals of Epidemiology 1996; 6(6):507-19.

* Hall WD, Feng Z, George VA, Lewis CE, Oberman A, Huber M, et al. Low-fat diet: effect on anthropometrics, blood pressure, glucose, and insulin in older women. Ethnicity and Disease 2003; 13(3):337-43.

\section{Wilke 1974 \{published data only\}}

Wilke $\mathrm{H}$, Frahm H. Influence of low-caloric-diet and dtriiodothyronine on serum lipids and body weight (author's trans) [Verhalten der serumlipide und des körpergewichts unter reduktionsdiät und medikamentöser behandlung mit dtrijodthyronin]. Medizinische Klinik 1974; 69(48):1986-9.

\section{Williams 1990 \{published data only\}}

Williams PT, Krauss RM, Vranizan KM, Wood PS. Changes in lipoprotein subfractions during diet-induced and exerciseinduced weight loss in moderately overweight men. Circulation 1990; 81(4):1293-304. 
Williams 1992 \{published data only\}

Williams PT, Krauss RM, Vranizan KM, Albers JJ, Wood PD. Effects of weight-loss by exercise and by diet on apolipoproteins AI and A-II and the particle-size distribution of high-density lipoproteins in men. Metabolism: Clinical and Experimental 1992; 41(4):441-9.

\section{Williams 1994 \{published data only\}}

Williams PT, Stefanick ML, Vranizan KM, Wood PD. The effects of weight loss by exercise or by dieting on plasma high-density lipoprotein (HDL) levels in men with low, intermediate, and normal-to-high HDL at baseline. Metabolism 1994; 43(7):917-24.

\section{Wilmot 1952 \{published data only\}}

Wilmot VA, Swank RL. The influence of low fat diet on blood lipid levels in health and in multiple sclerosis. American Journal of the Medical Sciences 1952; 223(1):25-34.

Wing 1998 \{published data only\}

Wing RR, Venditti E, Jakicic JM, Polley BA, Lang W. Lifestyle intervention in overweight individuals with a family history of diabetes. Diabetes Care 1998; 21(3):350-9.

WINS UK 2011 \{published and unpublished data\}

Parry BM, Milne JM, Yadegarfar G, Rainsbury RM. Dramatic dietary fat reduction is feasible for breast cancer patients: results of the randomised study, WINS (UK) - stage 1. European Journal of Surgical Oncology 2011; 37(10):848-55.

\section{WOMAN 2007 \{published data only\}}

Kuller LH, Kriska AM, Kinzel LS, Simkin-Silverman LR, SuttonTyrrell K, Johnson BD, et al. The clinical trial of Women On the Move through Activity and Nutrition (WOMAN) study. Contemporary Clinical Trials 2007; 28(4):370-81.

\section{Wood 1988 \{published data only\}}

Wood PD, Stefanick ML, Dreon DM, Frey HB, Garay SC, Williams PT, et al. Changes in plasma lipids and lipoproteins in overweight men during weight loss through dieting as compared with exercise. New England Journal of Medicine 1988; 319(18):1173-9.

\section{Woollard 2003 \{published data only\}}

Woollard J, Burke V, Beilin LJ, Verheijden M, Bulsara MK. Effects of a general practice-based intervention on diet, body mass index and blood lipids in patients at cardiovascular risk. Journal of Cardiovascular Risk 2003; 10(1):31-40.

\section{Working Well 1996 \{published data only\}}

Sorensen G, Thompson B, Glanz K, Feng Z, Kinne S, DiClemente $C$, et al. Work site-based cancer prevention: primary results from the Working Well Trial. American Journal of Public Health 1996; 86(7):939-47.

\section{Zock 1995 \{published and unpublished data\}}

Zock PL, Mensink RP, Harryvan J, De Vries JH, Katan MB. Fatty acids in serum cholesteryl esters as quantitative biomarkers of dietary intake in humans. American Journal of Epidemiology 1997; 145(12):1114-22.
* Zock PL. Dietary Fatty Acids and Risk Factors for Coronary Heart Disease: Controlled Studies in Healthy Volunteers [PhD thesis]. Wageningen, Netherlands: Landbouwuniversiteit te Wageningen, 1995.

\section{References to studies awaiting assessment}

\section{ICFAMED \{published data only\}ISRCTN27497769}

Lapetra J, Lozano-Rodríguez JM, Caballero-Valderrama MR, Santos-Lozano JM, Ortega-Calvo M, García de la Corte FJ, et al. Effect of a Mediterranean diet on the primary prevention of atrial fibrillation and major cardiovascular events in hypertensive patients with high cardiovascular risk: results of ICFAMED study. In: Obesity and Nutrition in the 21st Century. VIII Symposium Ciber Fisiopatología de la Obesidad y Nutrición; 2017; Madrid. Madrid: Ciber Fisiopatología de la Obesidad y Nutrición, 2017:71.

* Lapetra J, Lozano-Rodriguez JM, Miro-Moriano L, OrtegaCalvo M, Santos-Lozano JM, Garcia-Corte FJ, et al. Effect of a Mediterranean diet on the primary prevention of atrial fibrillation and major cardiovascular events in hypertensive patients with high cardiovascular risk: results of ICFAMED randomized trial. European Journal of Clinical Investigation 2018; 48:182-3

\section{References to ongoing studies}

ENAbLE due unclear \{published data only\}

English C, Patterson A, MacDonald-Wicks L, Attia J, Callister R, Hillier S, et al. ENAbLE: Secondary prevention of stroke. A physical activity and diet trial protocol. Abstracts Presented at the SMART STROKES 2019 Conference, 8-9 August 2019, Hunter Valley, NSW. International Journal of Stroke 2019; 14(1 Suppl):12. [DOI: 10.1177/1747493019858233]

\section{NCT02481466 due 2020 \{published data only\}}

. The combined portfolio diet and exercise study (PortfolioEx). clinicaltrials.gov/ct2/show/NCT02481466 (first posted 25 June 2015).

\section{NCT02938832 due 2023 \{published data only\}}

. Does the advice to eat a Mediterranean diet with low carbohydrate intake, compared with a low-fat diet, reduce diabetes and cardiovascular disease? clinicaltrials.gov/ct2/ show/NCT02938832 (first posted 19 October 2016).

NEW Soul Study due 2022 \{published data only\} ZZZ <accessionId ref="info:x-wiley/clinicalTrialsGov/ NCT03354377"> ZZZNCT03354377

. Nutritious Eating With Soul (The NEW Soul Study). https:// clinicaltrials.gov/ct2/show/NCT03354377 (first posted 27 November 2017).

* Turner-McGrievy G, Wilcox S, Frongillo EA, Murphy A, Hutto B, Williams K, et al. The Nutritious Eating with Soul (NEW Soul) Study: Study design and methods of a two-year randomized trial comparing culturally adapted soul food vegan vs. omnivorous diets among African American adults at risk for heart disease. Contemporary Clinical Trials 2020; 88:105897. 


\section{Additional references}

\section{S 1994}

Scandinavian Simvastatin Survival Study Group. Randomised trial of cholesterol lowering in 4444 patients with coronary heart disease: the Scandinavian Simvastatin Survival Study (4S). Lancet 1994; 344(8934):1383-9.

\section{Abdelhamid 2018}

Abdelhamid AS, Martin N, Bridges C, Brainard JS, Wang X, Brown TJ, et al. Polyunsaturated fatty acids for the primary and secondary prevention of cardiovascular disease.

Cochrane Database of Systematic Reviews 2018, Issue 11. [DOI: 10.1002/14651858.CD012345.pub2] [CD012345]

\section{Abdelhamid 2019}

Abdelhamid AS, Hooper L, Sivakaran R, Hayhoe R, Welch AA, PUFAH Group. The relationship between omega-3, omega- 6 and total polyunsaturated fat and musculoskeletal health and functional status in adults: a systematic review and meta-analysis of RCTs. Calcified Tissue International 2019; 105(4):353-72. [DOI: 10.1007/s00223-019-00584-3]

\section{Abdelhamid 2020}

Abdelhamid AS, Brown TJ, Brainard JS, Biswas P, Thorpe GC, Moore HJ, Deane KHO, Summerbell CD, Worthington HV, Song F, Hooper L. Omega-3 fatty acids for the primary and secondary prevention of cardiovascular disease. Cochrane Database of Systematic Reviews 2020, Issue 3. [DOI: 10.1002/14651858.CD003177.pub5] [CD003177]

\section{Alonso 2006}

, Ruiz-Gutierrez V, . Monounsaturated fatty acids, olive oil and blood pressure: epidemiological, clinical and experimental evidence. Public Health Nutrition 2006; 9(2):251-7.

\section{Astrup 2000}

Astrup A, Ryan L, Grunwald GK, Storgaard M, Saris W, Melanson $\mathrm{E}$, et al. The role of dietary fat in body fatness: evidence from a preliminary meta-analysis of ad libitum lowfat dietary intervention studies. British Journal of Nutrition 2000; 83(Suppl 1):S25-32.

\section{Astrup 2011}

Astrup A, Dyerberg J, Elwood P, Hermansen K, Hu FB, Jakobsen $\mathrm{MU}$, et al. The role of reducing intakes of saturated fat in the prevention of cardiovascular disease: where does the evidence stand in 2010? American Journal of Clinical Nutrition 2011; 93(4):684-8.

\section{Berkley 1995}

Berkley CS, Hoaglin DC, Mosteller F, Colditz GA. A randomeffects regression model for meta-analysis. Statistics in Medicine 1995; 14(4):395-411.

\section{Blann 1995}

Blann AD, Jackson P, Bath PM, Watts GF. Von Willebrand factor, a possible indicator of endothelial cell damage, decreases during long-term compliance with a lipid-lowering diet. Journal of Internal Medicine 1995; 237(6):557-61.

\section{Brainard 2020}

Brainard J, Jimoh OF, Deane K, Biswas P, Donaldson D, Maas K, et al. Omega-3, omega-6, and polyunsaturated fat for cognition: systematic review and meta-analysis of randomized trials. Journal of the American Medical Directors Association 2020; 15 April 2020 [Epub ahead of print]. [DOI: 10.1016/ j.jamda.2020.02.022]

\section{Briel 2009}

Briel M, Ferreira-Gonzalez I, You JJ, Karanicolas PJ, Akl EA, Wu P, et al. Association between change in high density lipoprotein cholesterol and cardiovascular disease morbidity and mortality: systematic review and meta-regression analysis. BMJ 2009; 338:b92. [DOI: 10.1136/bmj.b92]

\section{Brown 2019}

Brown TJ, Brainard J, Song F, Wang X, Abdelhamid A, Hooper L. Omega-3, omega-6, and total dietary polyunsaturated fat for prevention and treatment of type 2 diabetes mellitus: systematic review and meta-analysis of randomised controlled trials. BMJ 2019; 366:14697.

\section{Brunner 1997}

Brunner E, White I, Thorogood M, Bristow A, Curle D, Marmot M. Can dietary interventions change diet and cardiovascular risk factors? A meta-analysis of randomized controlled trials. American Journal of Public Health 1997; 87(9):1415-22.

\section{Bucher 1996}

Bucher HC, Cook RJ, Guyatt GH, Lang JD, Cook DJ, Hatala R, et al. Effects of dietary calcium supplementation on blood pressure: a meta-analysis of randomised controlled trials. JAMA 1996; 275(13):1016-22.

\section{Chalmers 1990}

Chalmers I, Adams M, Dickersin K, Hetherington J, TarnowMordi W, Meinert C, et al. A cohort study of summary reports of controlled trials. JAMA 1990; 263(10):1401-5.

\section{Clarke 1997}

Clarke R, Frost C, Collins R, Appleby P, Peto R. Dietary lipids and blood cholesterol: quantitative meta-analysis of metabolic ward studies. BMJ 1997; 314(7074):112-7.

\section{Davey Smith 1993}

Davey Smith G, Song F, Sheldon TA. Cholesterol lowering and mortality: the importance of considering initial level of risk. BMJ 1993; 306(6889):1367-73.

\section{Dayton 1965}

Dayton S, Hashimoto S, Rosenblum D, Pearce M. Vitamin E status of humans during prolonged feeding of unsaturated fats. Journal of Laboratory and Clinical Medicine 1965; 65(5):739-47.

\section{De Caterina 2010}

De Caterina R, Scarano M, Marfisi RM, Lucisano G, Palma F, Tatasciore A, et al. Cholesterol-lowering interventions and stroke. Journal of the American College of Cardiology 2010; 55(3):198-211. 


\section{Deane 2019}

Deane KHO, Jimoh OF, Biswas P, O'Brien A, Hanson S, Abdelhamid AS, et al. Omega-3 and polyunsaturated fat for prevention of depression and anxiety symptoms: systematic review and meta-analysis of randomised trials. British Journal of Psychiatry 2019 Oct 24 [E-pub ahead of print]. [DOI: 10.1192/ bjp.2019.234]

\section{Denke 1995}

Denke MA. Cholesterol-lowering diets. A review of the evidence. Archives of Internal Medicine 1995; 155(1):17-26.

\section{Ebrahim 1997}

Ebrahim S, Davey Smith G. Systematic review of randomised controlled trials of multiple risk factor interventions for preventing coronary heart disease. BMJ 1997; 314(7095):1666.

\section{Eckel 2013}

Eckel RH, Jakicic JM, Ard JD, De Jesus JM, Houston Miller N, Hubbard VS, et al. 2013 AHA/ACC guideline on lifestyle management to reduce cardiovascular risk: a report of the American College of Cardiology/American Heart Association Task Force on Practice Guidelines. Circulation 2013; 129(25 suppl 2):S76-99. [DOI: 10.1161/01.cir.0000437740.48606.d1]

\section{Egger 1997}

Egger M, Davey Smith G, Schneider M, Minder C. Bias in meta-analysis detected by a simple graphical test. BMJ 1997; 315(7109):629-34.

\section{ESC/EAS 2011}

Task Force for the management of dyslipidaemias of the European Society of Cardiology (ESC) and the European Atherosclerosis Society (EAS). ESC/EAS Guidelines for the management of dyslipidaemias. European Heart Journal 2011; 32(14):1769-1818. [DOI: 10.1093/eurheartj/ehr158]

\section{Gofman 1949}

Gofman JW, Lindgren FT, Elliott HA. Ultracentrifugal studies of lipoproteins of human serum. Journal of Biological Chemistry 1949; 179(2):973-9.

\section{GRADE 2004}

Atkins D, Best D, Briss PA, Eccles M, Falck-Ytter Y, Flottorp S, et al. Grading quality of evidence and strength of recommendations. BMJ 2004; 328(7454):1490.

\section{GRADEpro 2015 [Computer program]}

McMaster University and Evidence Prime Inc GRADEpro GDT. https://gradepro.org/: McMaster University and Evidence Prime Inc, 2015

\section{Grundy 2019}

Grundy SM, Stone NJ, Bailey AL, Beam C, Birtcher KK, Blumenthal RS, et al. 2018 AHA/ACC/AACVPR/AAPA/ABC/ ACPM/ADA/AGS/APhA/ASPC/NLA/PCNA Guideline on the Management of Blood Cholesterol. Journal of the American College of Cardiology 2019; 73(24):e285-350.

\section{Hanson 2020}

Hanson S, Thorpe G, Winstanley L, Abdelhamid AS, Hooper L, PUFAH Group. Omega-3, omega- 6 and total dietary polyunsaturated fat on cancer incidence: systematic review and meta-analysis of randomised trials. British Journal of Cancer 2020; 122:1260-70. [DOI: 10.1038/s41416-020-0761-6]

\section{Harcombe 2015}

Harcombe Z, Baker JS, Cooper SM, Davies B, Sculthorpe N, DiNicolantonio JJ, et al. Evidence from randomised controlled trials did not support the introduction of dietary fat guidelines in 1977and 1983: a systematic review and meta-analysis. Open Heart 2015; 2(1):e000196. [DOI: 10.1136/openhrt-2014-000196]

\section{Hauck 1991}

Hauck WW, Gilliss CL, Donner A, Gortner S. Randomisation by cluster. Nursing Research 1991; 40(6):356-8.

\section{Hegsted 1965}

Hegsted DM, McGandy RB, Myers ML, Stare FJ. Quantitative effects of dietary fat on serum cholesterol in man. American Journal of Clinical Nutrition 1965; 17(5):281-95.

\section{Hegsted 2000}

Hegsted DM. From chick nutrition to nutrition policy. Annual Review of Nutrition 2000; 20:1-19.

\section{Hession 2009}

Hession M, Rolland C, Kulkarni U, Wise A, Broom J. Systematic review of randomized controlled trials of low-carbohydrate vs. low-fat/low-calorie diets in the management of obesity and its comorbidities. Obesity Reviews 2009; 10(1):36-50.

\section{Higgins 2003}

Higgins JPT, Thompson SG, Deeks JJ, Altman DG. Measuring inconsistency in meta-analyses. BMJ 2003; 327(7414):557-60.

\section{Higgins 2011}

Higgins JPT, . Cochrane Handbook for Systematic Reviews of Interventions Version 5.1.0 [updated March 2011]. The Cochrane Collaboration, 2011. Available from handbook.cochrane.org.

\section{Hooper 2015b}

Hooper L, Abdelhamid A, Bunn D, Brown T, Summerbell CD, Skeaff CM. Effects of total fat intake on body weight. Cochrane Database of Systematic Reviews 2015, Issue 8. [DOI: 10.1002/14651858.CD011834]

\section{Hooper 2018}

Hooper L, Al-Khudairy L, Abdelhamid AS, Rees K, Brainard JS, Brown TJ, et al. Omega- 6 fats for the primary and secondary prevention of cardiovascular disease. Cochrane Database of Systematic Reviews 2018, Issue 11. [DOI: 10.1002/14651858.CD011094.pub3] [CD011094]

\section{Hooper 2019}

Hooper L, Abdelhamid A, Brainard J, Deane KHO, Song F. Creation of a database to assess effects of omega-3, omega- 6 and total polyunsaturated fats on health: database and methodology for a set of reviews. BMJ Open 2019; 9(5):e029554. [DOI: 10.1136/bmjopen-2019-029554] 


\section{Howard 2010}

Howard BV, Curb JD, Eaton CB, Kooperberg C, Ockene J, Kostis JB, et al. Low-fat dietary pattern and lipoprotein risk factors: the Women's Health Initiative dietary modification trial. American Journal of Clinical Nutrition 2010; 91(4):860-74.

\section{Kagan 1974}

Kagan A, Harris BR, , Johnson KG, Kato H, Syme SL, et al. Epidemiologic studies of coronary heart disease and stroke in Japanese men living in Japan, Hawaii and California: demographic, physical, dietary and biochemical characteristics. Journal of Chronic Disease 1974; 27(7-8):345-64.

\section{Kasim 1993}

Kasim SE, Martino S, , Khilnani S, Boomer A, Depper J, et al. Dietary and anthropometric determinants of plasma lipoproteins during a long-term low-fat diet in healthy women. American Journal of Clinical Nutrition 1993; 57(2):146-53.

\section{Katan 2010}

Katan MB, Brouwer IA, Clarke R, Geleijnse JM, Mensink RP. Saturated fat and heart disease. American Journal of Clinical Nutrition 2010; 92(2):459-60; author reply 460-1.

\section{Keys 1950}

Keys A, Mickelsen O, Miller EV, Carleton B. The relation in man between cholesterol levels in the diet and in the blood. Science 1950; 112(2899):79-81.

\section{Keys 1986}

Keys A, Menotti A, Karvonen MJ, Aravanis C, Blackburn H, Buzina RM, et al. The diet and 15-year death rate in the seven countries study. American Journal of Epidemiology 1986; 124(6):903-15.

\section{Kodama 2009}

Kodama S, Saito K, Tanaka S, Maki M, Yachi Y, Sato M, et al. Influence of fat and carbohydrate proportions on the metabolic profile in patients with type 2 diabetes: a meta-analysis. Diabetes Care 2009; 32(5):959-65.

\section{Kristal 2005}

Kristal AR, Peters U, Potter JD. Is it time to abandon the food frequency questionnaire? Cancer Epidemiology, Biomarkers and Prevention 2005; 14(12):2826-8.

\section{Law 1991}

Law MR, Frost CD, Wald NJ. By how much does dietary salt restriction lower blood pressure? BMJ 1991; 302(6780):811-24.

\section{Law 1994}

Law MR, Wald NJ, Thompson SG. By how much and how quickly does reduction in serum cholesterol concentration lower risk of ischaemic heart disease? BMJ 1994; 308(6925):367-73.

\section{Lefebvre 2011}

Lefebvre C, Manheimer E, Glanville J. Chapter 6: Searching for studies. In: Higgins JPT, Green S (editors). Cochrane Handbook for Systematic Reviews of Interventions Version 5.1.0 [updated March 2011]. The Cochrane Collaboration, 2011. Available from handbook.cochrane.org.

\section{Lenz 2007}

Lenz M, Steckelberg A, Richter B, Mühlhauser I. Meta-analysis does not allow appraisal of complex interventions in diabetes and hypertension self-management: a methodological review. Diabetologia 2007; 50(7):1375-83.

\section{Lichtenstein 2006}

Lichtenstein AH, Appel LJ, Brands M, Carnethon M, Daniels S, Franch $\mathrm{HA}$, et al. Diet and lifestyle recommendations revision 2006: a scientific statement from the American Heart Association Nutrition Committee. Circulation 2006; 114(1):82-96.

\section{Lopez-Espinoza 1984}

Lopez-Espinoza I, Howard WJ, Mann JI, Carter RD, Hockaday TD. Fatty acid composition of platelet phospholipids in non-insulindependent diabetics randomized for dietary advice. British Journal of Nutrition 1984; 52(1):41-7.

\section{Mach 2019}

Mach F, Baigent C, Catapano AL, Koskinas KC, Casula M, Badimon L, et al. 2019 ESC/EAS guidelines for the management of dyslipidaemias: lipid modification to reduce cardiovascular risk. Atherosclerosis 2019; 290:140-205.

\section{Malhotra 2014}

Malhotra A, Shafiq N, Arora A, Singh M, Kumar R, Malhotra S. Dietary interventions (plant sterols, stanols, omega-3 fatty acids, soy protein and dietary fibers) for familial hypercholesterolaemia. Cochrane Database of Systematic Reviews 2014, Issue 6. [DOI: 10.1002/14651858.CD001918.pub3]

\section{Marchioli 1994}

Marchioli R, Prieto JC, Tognoni G. Surrogate end-points: the case of trials on coronary atherosclerotic plaque regression. Clinical Trials and Meta-Analyses 1994; 29(2-3):139-76.

\section{Mensink 1992}

Mensink RP, Katan MB. Effect of dietary fatty acids on serum lipids and lipoproteins: a meta-analysis of 27 trials. Arteriosclerosis and Thrombosis 1992; 12(8):911-9.

\section{Mensink 2003}

Mensink RP, Zock PL, Kester ADM, Katan MB. Effects of dietary fatty acids and carbohydrates on the ratio of serum total to HDL cholesterol and on serum lipids and apolipoproteins: a metaanalysis of 60 controlled trials. American Journal of Clinical Nutrition 2003; 77(5):1146-55.

\section{Michels 2009}

Michels KB, Willett WC. The Women's Health Initiative randomized controlled dietary modification trial: a postmortem. Breast Cancer Research and Treatment 2009; 114(1):1-6.

\section{Mozaffarian 2010}

Mozaffarian D, Micha R, Wallace S. Effects of coronary heart disease of increasing polyunsaturated fat in place of saturated fat: a systematic review and meta-analysis of randomized controlled trials. PLOS Medicine 2010; 7(3):e1000252. 


\section{Ness 1997}

Ness AR, Powles JW. Fruit and vegetables, and cardiovascular disease: a review. International Journal of Epidemiology 1997; 26(1):1-13.

\section{NHS 2020}

NHS. How to eat less saturated fat. www.nhs.uk/live-well/eatwell/eat-less-saturated-fat/ (accessed 11 Feb 2020).

\section{NICE 2014}

National Institute of Health and Care Excellence. Lipid modification: cardiovascular risk assessment and the modification of blood lipids for the primary and secondary prevention of cardiovascular disease. www.nice.org.uk/ guidance/cg181/resources/guidance-lipid-modificationcardiovascular-risk-assessment-and-the-modification-ofblood-lipids-for-the-primary-and-secondary-prevention-ofcardiovascular-disease-pdf (accessed 29th Dec 2019).

\section{O'Gara 2014}

O'Gara PT, Kushner FG, Ascheim DD, , Chung MK, De Lemos JA, et al. 2013 ACCF/AHA guideline for the management of STelevation myocardial infarction: a report of the American College of Cardiology Foundation/American Heart Association Task Force on Practice Guidelines. Circulation 2013; 127(4):e362-e425. [DOI: 10.1161/CIR.0b013e3182742cf6 ]

\section{Oliver 1953}

Oliver MF, Boyd GS. The plasma lipids in coronary artery disease. British Heart Journal 1953; 15(4):387-90.

\section{Oliver 2000}

Oliver MF. Pioneer research in Britain into atherosclerosis and coronary heart disease - an historical review. Atherosclerosis 2000; 150(1):1-12.

\section{Page 2019}

Page MJ, Higgins JPT, Sterne JAC. Chapter 13: Assessing risk of bias due to missing results in a synthesis. In: Higgins JPT, Thomas J, Chandler J, Cumpston M, Li T, Page MJ, Welch VA (editors). Cochrane Handbook for Systematic Reviews of Interventions Version 6.0 (updated July 2019). Cochrane 2019. Available from www.training.cochrane.org/handbook.

\section{Patterson 2003}

Patterson RE, Kristal A, Rodabough R, Caan B, Lillington L, Mossavar-Rahmani Y, et al. Changes in food sources of dietary fat in response to an intensive low-fat dietary intervention: early results from the Women's Health Initiative. Journal of the American Dietetic Association 2003; 103(4):454-60.

\section{Prentice 2007}

Prentice RL. Observational studies, clinical trials, and the Women's Health Initiative. Lifetime Data Analysis 2007; 13(4):449-62.

\section{Rees 2013}

Rees K, Dyakova M, Wilson N, Ward K, Thorogood M, Brunner E. Dietary advice for reducing cardiovascular risk. Cochrane Database of Systematic Reviews 2013, Issue 12. [DOI: 10.1002/14651858.CD002128.pub5]

\section{Rimm 1996}

Rimm EB, Klatsky A, Grobbee D, Stampfer MJ. Review of moderate alcohol consumption and reduced risk of coronary heart disease: is the effect due to beer, wine or spirits? BMJ 1996; 312(7033):731-6.

\section{Robertson 1977}

Robertson TL, Kato H, Rhoads GG, Kagan A, Marmot M, Syme SL, et al. Epidemiologic studies of coronary heart disease and stroke in Japanese men living in Japan, Hawaii and California. Incidence of myocardial infarction and death from coronary heart disease. American Journal of Cardiology 1977; 39(2):239-43.

\section{Robinson 2009}

Robinson JG, Wang S, Smith BJ, Jacobson TA. Meta-analysis of the relationship between non-high-density lipoprotein cholesterol reduction and coronary heart disease risk. Journal of the American College of Cardiology 2009; 53(4):316-22.

\section{Rubins 1995}

Rubins HB. Cholesterol in patients with coronary heart disease: how low should we go? Journal of General Internal Medicine 1995; 10(8):464-71.

\section{Ryle 1949}

Ryle JA, Russell WT. The natural history of coronary disease. A clinical and epidemiological study. British Heart Journal 1949; 11(4):370.

\section{SACN 2019}

Scientific Advisory Committee on Nutrition. Saturated fat and health. www.gov.uk/government/publications/saturated-fatsand-health-sacn-report (accessed prior to 30 April 2020).

\section{Savovic 2012}

Savovic J, Jones H, Altman D, Harris R, Jüni P, Pildal J, et al. Influence of reported study design characteristics on intervention effect estimates from randomised controlled trials: combined analysis of meta-epidemiological studies. Health Technology Assessment 2012; 16:1-82.

\section{Scarborough 2010}

Scarborough P, Rayner M, Van Dis I, Norum K. Meta-analysis of effect of saturated fat intake on cardiovascular disease: overadjustment obscures true associations. American Journal of Clinical Nutrition 2010; 92(2):458-9; author reply 459.

\section{Schatzkin 2003}

Schatzkin A, Kipnis V, Carroll RJ, Midthune D, Subar AF, Bingham S, et al. A comparison of a food frequency questionnaire with a 24-hour recall for use in an epidemiological cohort study: results from the biomarkerbased Observing Protein and Energy Nutrition (OPEN) Study. International Journal of Epidemiology 2003; 32(6):1054-62.

\section{Schulz 1995}

Schulz KF, Chalmers I, Hayes RJ, Altman DG. Empirical evidence of bias. Dimensions of methodological quality associated with estimates of treatment effects in controlled trials. JAMA 1995; 273(5):408-12. 


\section{Schwab 2014}

Schwab U, Lauritzen L, Tholstrup T, Haldorssoni T, Riserus U, Uusitupa M, et al. Effect of the amount and type of dietary fat on cardiometabolic risk factors and risk of developing type-2 diabetes, cardiovascular disease, and cancer: a systematic review. Food \& Nutrition Research 2014; 58:25145. [DOI: 10.3402/fnr.v58.25145]

\section{Shah 2007}

Shah M, Adams-Huet B, Garg A. Effect of high-carbohydrate or high-cis-monounsaturated fat diets on blood pressure: a meta-analysis of intervention trials. American Journal of Clinical Nutrition 2007; 85(5):1251-6.

\section{Sharp 1998}

Sharp S. Meta-analysis regression. Stata Technical Bulletin 1998; 42:16-22.

\section{Sheppherd 2009}

Shepperd S, Lewin S, Straus S, Clarke M, Eccles MP, Fitzpatrick $R$, et al. Can we systematically review studies that evaluate complex interventions? PLOS Medicine 2009; 6(8):e1000086.

\section{SIGN 1996}

Scottish Intercollegiate Guidelines Network. Management of obesity (national guideline). https://www.sign.ac.uk/sign-115management-of-obesity (accessed prior to 30 April 2020);115.

\section{Siri-Tarino 2010}

Siri-Tarino PW, Sun Q, Hu FB, Krauss RM. Meta-analysis of prospective cohort studies evaluating the association of saturated fat with cardiovascular disease. American Journal of Clinical Nutrition 2010; 91(3):535-46.

\section{Skeaff 2009}

Skeaff CM, Miller J. Dietary fat and coronary heart disease: summary of evidence from prospective cohort and randomised controlled trials. Annals of Nutrition and Metabolism 2009; 55(1-3):173-201.

\section{Stamler 2010}

Stamler J. Diet-heart: a problematic revisit. American Journal of Clinical Nutrition 2010; 91(3):497-9.

\section{Stein 2006}

Stein K. After the media feeding frenzy: whither the Women's Health Initiative Dietary Modification Trial? Journal of the American Dietetic Association 2006; 106(6):794-800.

\section{Sterne 2001}

Sterne JA, Bradburn MJ, Egger M. Meta-analysis in STATA. In: Egger M, Davey Smith G, Altman DG, editors(s). Systematic Reviews in Health Care: Meta-analysis in Context. London: BMJ Books, 2001.

\section{Sterne 2009}

Sterne JA. Meta-Analysis in Stata: an Updated Collection from the Stata Journal. Texas, USA: STATA Press, 2009.

\section{Thorogood 1996}

Thorogood M. Nutrition. In: Lawrence M, Neil A, Mant D, Fowler G, editors(s). Prevention of Cardiovascular Disease: an Evidence-Based Approach. Kings Lynn: Oxford University Press, 1996:54-66.

\section{Walsh 1995}

Walsh JME, Grady D. Treatment of hyperlipidaemia in women. JAMA 1995; 274(14):1152-8.

\section{Weggemans 2001}

Weggemans RM, Zock PL, Katan MB. Dietary cholesterol from eggs increases the ratio of total cholesterol to high-density lipoprotein cholesterol in humans: a meta-analysis. American Journal of Clinical Nutrition 2001; 73(5):885-91.

\section{Wood 2008}

Wood L, Egger M, Gluud LL, Schulz K, Jüni P, Altman DG, et al. Empirical evidence of bias in treatment effect estimates in controlled trials with different interventions and outcomes: meta-epidemiological study. British Medical Journal 2008; 336:601-5.

\section{Yngve 2006}

Yngve A, Hambraeus L, Lissner L, Serra Majem L, Vaz de Almeida MD, Berg C, et al. Invited Commentary. The Women's Health Initiative. What is on trial: nutrition and chronic disease? Or misinterpreted science, media havoc and the sound of silence from peers. Public Health Nutrition 2006; 9(2):269-72.

\section{Yu-Poth 1999}

Yu-Poth S, Zhao G, Etherton T, Naglak M, Jonnalagadda S, KrisEtherton PM. Effects of the National Cholesterol Education Program's Step I and Step II dietary intervention programs on cardiovascular disease risk factors: a meta-analysis. American Journal of Clinical Nutrition 1999; 69(4):632-46.

\section{References to other published versions of this review Hooper 2000}

Hooper L, Summerbell CD, Higgins JPT, Thompson RL, Clements G, Capps N, et al. Reduced or modified dietary fat for preventing of cardiovascular disease. Cochrane Database of Systematic Reviews 2000, Issue 2. [DOI: 10.1002/14651858.CD002137]

\section{Hooper 2001}

Hooper L, Summerbell CD, Higgins JPT, Thompson RL, Capps N, Davey Smith G, et al. Dietary fat intake and prevention of cardiovascular disease: systematic review. BMJ 2001; 322(7289):757-63.

\section{Hooper 2011}

Hooper L, Summerbell CD, Thompson R, Sills D, Roberts FG, Moore $\mathrm{HJ}$, et al. Reduced or modified dietary fat for preventing cardiovascular disease. Cochrane Database of Systematic Reviews 2011, Issue 7. [DOI: 10.1002/14651858.CD002137.pub2] 


\section{Hooper 2012}

Hooper L, Summerbell CD, Thompson R, Sills D, Roberts FG, Moore $\mathrm{HJ}$, et al. Reduced or modified dietary fat for preventing cardiovascular disease. Cochrane Database of Systematic Reviews 2012, Issue 5. [DOI: 10.1002/14651858.CD002137.pub3]

\section{Hooper 2015a}

Hooper L, Martin N, Jimoh OF, Abdelhamid AS. Reduction in saturated fat intake for cardiovascular disease. Cochrane Database of Systematic Reviews 2015, Issue 6. [DOI: 10.1002/14651858.CD011737]

* Indicates the major publication for the study

CHARACTERISTICS OF STUDIES

Characteristics of included studies [ordered by study ID]

Black 1994

\section{Study characteristics}

Methods RCT

Summary risk of bias: moderate to high

Participants People with non-melanoma skin cancer (USA)

CVD risk: low

Control: randomised 67 , analysed 58

Intervention: randomised 66, analysed 57

Mean years in trial: 1.9

$\%$ male: control $67 \%$, intervention $54 \%$

Age: mean control 52.3 (SD 13.2), intervention 50.6 (SD 9.7)

Ethnicity: white 100\% (excluded from study if of Asian, Black, Hispanic or American Indian ancestry)

Statins use allowed: Unclear

$\%$ taking statins: Not reported

Reduced fat vs usual diet

Control aims: no dietary advice

Intervention aims: total fat 20\%E, protein $15 \% \mathrm{E}, \mathrm{CHO} 65 \% \mathrm{E}$

Control methods: no dietary change, 4-month intervals clinic examination by dermatologist

Intervention methods: $8 \mathrm{x}$ weekly classes plus monthly follow-up sessions, with behavioural techniques being taught following individual approach (not clear if in a group or individual). 4-month intervals clinic examination by dermatologist

Intervention delivered face-to-face by a dietitian

Total fat intake, \%E ("during study" months 4 - 24): cont 37.8 (SD 4.1), int 20.7 (SD 5.5) (mean difference $-17.10,95 \% \mathrm{Cl}-18.88$ to -15.32 ) significant reduction

Saturated fat intake, \%E ("during study", months 4 - 24): cont 12.8 (SD 2.0), int 6.6 (SD 1.8), (mean difference $-6.20,95 \% \mathrm{Cl}-6.90$ to -5.50 ) significant reduction

PUFA intake, \%E ("during study", months 4 - 24): cont 7.8 (SD 1.4), int 4.5 (SD 1.3), (mean difference $-3.30,95 \% \mathrm{Cl}-3.79$ to -2.81 ) significant reduction

PUFA n-3 intake: not reported

PUFA n-6 intake: Linoleic acid, cont 16.9 (SD 5.6) g, int 8.5 (SD 3.3) g

MUFA intake, \%E ("during study", months 4 - 24): cont 14.4 (SD 1.7), int 7.6 (SD 2.2), (mean difference $-6.80,95 \% \mathrm{Cl}-7.52$ to -6.08$)$ significant reduction 
Black 1994 (Continued)

CHO intake, \%E ("during study", months 4 - 24): cont 44.6 (SD 6.9), int 60.3 (SD 6.3), (mean difference

$15.70,95 \% \mathrm{Cl} 13.29$ to 18.11 ) significant increase

Protein intake, \%E ("during study", months 4 - 24): cont 15.7 (SD 2.4), int 17.7 (SD 2.2), (mean difference

$2.00,95 \% \mathrm{Cl} 1.16$ to 2.84 ) significant increase

Trans fat intake: not reported

Replacement for saturated fat: $\mathrm{CHO}$ and protein (by dietary aims and achievements), main is $\mathrm{CHO}$

Style: diet advice

Setting: community

Stated trial outcomes: incidence of actinic keratosis and non-melanoma skin cancer
Data available on total mortality? yes
Cardiovascular mortality? yes
Events available for combined cardiovascular events: cardiovascular deaths
Secondary outcomes: cancer deaths (none)
Tertiary outcomes: none (weight data provided, but no variance info)

Notes

Study duration 24 months.

Study aim was to achieve low-fat diet, but the study achieved a statistically significant reduction in saturated fat intake in the low-fat group compared to control.

\section{SFA reduction achieved.}

\section{Total serum cholesterol: not reported}

At 2 years control $-1.5 \mathrm{~kg} \mathrm{n}=50$ ?, intervention $-1 \mathrm{~kg} \mathrm{n}=51$ ?

Trial dates: Study dates not reported (but still recruiting at first publication in 1994)

Funding: National Cancer Institute

Declarations of Interest of primary researchers: none stated, all authors worked for academic or health institutions

\section{Risk of bias}

\begin{tabular}{lll}
\hline Bias & Authors' judgement & Support for judgement \\
\hline $\begin{array}{l}\text { Random sequence genera- } \\
\text { tion (selection bias) }\end{array}$ & Low risk & "list of randomly generated numbers" \\
\hline $\begin{array}{l}\text { Allocation concealment } \\
\text { (selection bias) }\end{array}$ & Unclear risk & Allocation method not clearly described \\
\hline $\begin{array}{l}\text { Blinding of participants } \\
\text { and personnel (perfor- } \\
\text { mance bias) }\end{array}$ & High risk & Dietary advice provided, so participants not blinded \\
All outcomes & \\
\hline
\end{tabular}

\begin{tabular}{|c|c|c|}
\hline $\begin{array}{l}\text { Blinding of outcome as- } \\
\text { sessment (detection bias) }\end{array}$ & Low risk & $\begin{array}{l}\text { "examined .... by dermatologists unaware of their treatment assignments". } \\
\text { Deaths (all-cause and CVD) not considered relevant to the intervention }\end{array}$ \\
\hline
\end{tabular}

CVD outcomes

Blinding of outcome as-
sessment (detection bias) $\quad$ Low risk Blinding is not relevant in assessment of mortality


Black 1994 (Continued)

All-cause mortality

Incomplete outcome data Low risk Low risk for all-cause and CVD mortality. Unclear for other outcomes
(attrition bias)
(attrition bias)

All outcomes

\begin{tabular}{lll}
\hline $\begin{array}{l}\text { Selective reporting (re- } \\
\text { porting bias) }\end{array}$ & Low risk & Not relevant for primary and secondary outcomes as all trialists asked for data \\
\hline $\begin{array}{l}\text { Free of systematic differ- } \\
\text { ence in care? }\end{array}$ & High risk & $\begin{array}{l}\text { Minor, all have 4-monthly clinic visits, the intervention group had 8 behaviour- } \\
\text { al technique classes that the control group did not have }\end{array}$ \\
\hline Stated aim to reduce SFA & High risk & Aim to reduce SFA not stated \\
\hline Achieved SFA reduction & Low risk & Statistically significant SFA reduction achieved \\
\hline Achieved TC reduction & Unclear risk & Not reported \\
\hline Other bias & Low risk & None noted \\
\hline
\end{tabular}

DART 1989

\section{Study characteristics}

\begin{tabular}{ll}
\hline Methods & Factorial RCT \\
& Diet And Reinfarction Trial (DART) \\
& Summary risk of bias: moderate to high \\
\hline Participants & Men recovering from an MI (UK) \\
CVD risk: high \\
Control: randomised 1015, analysed unclear \\
Intervention: randomised 1018, analysed unclear \\
Mean years in trial: control 1.9, randomised 1.9 \\
\% male: $100 \%$ \\
Age: mean control 56.8, intervention 56.4 (< 70$)$ \\
Ethnicity: not stated \\
Statins use allowed? Unclear, but there do not appear to have been any medication-based exclusion \\
criteria and included participants were taking anti-hypertensives, anti-anginals, anti-coagulants, an- \\
ti-platelet, digoxin and "other cardiac drugs". \\
\% taking statins: Not reported, but only 5.4\% were taking "other cardiac drugs" which may have includ- \\
ed statins.
\end{tabular}

\section{Interventions $\quad$ Reduced and modified fat vs usual diet}

Control aims: no dietary advice on fat, weight reducing advice if $\mathrm{BMI}>30$

Intervention aims: reduce fat intake to $30 \% \mathrm{E}$, increase $\mathrm{P} / \mathrm{S}$ to 1.0 , weight-reducing advice if $\mathrm{BMI}>30$

Note: This was a factorial trial, and so some in each group were randomised to increased fatty fish and/ or increased cereal fibre.

Control methods: dietitians provided 'sensible eating' advice without specific information on fats. 
Intervention methods: dietitians provided the participants and their wives with initial individual advice and a diet information sheet; participants were revisited for further advice, recipes, encouragement at $1,3,6,9,12,15,18$ and 21 months.

Intervention delivered individually face-to-face by a dietitian

Total fat intake, \%E (through study): cont 35 (SD 6), int 31 (SD 7) (mean difference $-4.00,95 \% \mathrm{Cl}-4.57$ to -3.43) significant reduction

Saturated fat intake, \%E (through study): cont 15 (SD3), int 11 (SD3), (mean difference $-4.00,95 \%$ $\mathrm{Cl}-4.26$ to -3.74 ) significant reduction

PUFA intake (through study) 肴: cont 7 (SD unclear), int 9 (SD unclear), (mean difference $2.00,95 \% \mathrm{Cl}$ 1.57 to 2.43 assuming SDs of 5) significant increase

PUFA n-3 intake: EPA, cont 0.6 (SD 0.7) g/wk, Int 2.4 (SD 1.4) g/wk

PUFA n-6 intake: not reported

MUFA intake (through study) $\underset{\star}{*}$ : cont 13 (SD unclear), int 11 (SD unclear) (mean difference $-2.00,95 \%$ $\mathrm{Cl}-2.43$ to -1.57 assuming SDs of 5 ) significant reduction

CHO intake (through study): cont 44 (SD 6), int 46 (SD 7) (mean difference 2.00, 95\% $\mathrm{Cl} 1.43$ to 2.57) significant increase

Protein intake (through study): cont 17 (SD 4), int 18 (SD 4) (mean difference $1.00,95 \% \mathrm{Cl} 0.65$ to 1.35 ) significant increase

Trans fat intake: not reported

Replacement for saturated fat: PUFA and CHO (by dietary aims), PUFA, CHO and protein (by dietary achievements), main PUFA

Style: diet advice

Setting: community

Est imated by subtraction (assuming total fat = SFA + PUFA + MUFA) or using the ratio (assuming P/ $\mathrm{S}=\mathrm{PUFA} / \mathrm{SFA}$ )

Outcomes

Stated trial outcomes: mortality, reinfarction

Data available on total mortality? yes

Cardiovascular mortality? yes

Events available for combined cardiovascular events: cardiovascular deaths (including stroke deaths) plus non-fatal MI

Secondary outcomes: cancer deaths, total MI, non-fatal MI, CHD mortality, CHD events (total MI)

Tertiary outcomes: total and HDL cholesterol

Study duration: 24 months

Study aim was to achieve low fat diet with raised $\mathrm{P} / \mathrm{S}$ ratio and saturated fat intake in the intervention group was significantly lower than in the control group.

\section{SFA reduction aimed and achieved.}

Total serum cholesterol, difference between intervention and control, $\mathrm{mmol} / \mathrm{L}:-0.26(95 \% \mathrm{Cl}$ -0.36 to -0.16$)$, statistically significant reduction

Trial dates: Study dates not reported (published in 1989)

Funding: Welsh Scheme for the Development of Health and Social Research, Welsh Heart Research Foundation, Flora Project, Health Promotion Research Trust. (Seven Seas Health Care and Duncan 
Flockhart provided the MaxEPA capsules and Norgene provided 'Fybranta' tablets - but these were not used in the comparison discussed in this systematic review)

Declarations of Interest of primary researchers: none stated, all authors worked for academic or health institutions

\section{Risk of bias}

\begin{tabular}{|c|c|c|}
\hline Bias & Authors' judgement & Support for judgement \\
\hline $\begin{array}{l}\text { Random sequence genera- } \\
\text { tion (selection bias) }\end{array}$ & Low risk & Randomised using sealed envelopes \\
\hline $\begin{array}{l}\text { Allocation concealment } \\
\text { (selection bias) }\end{array}$ & Unclear risk & Unclear if envelopes were opaque \\
\hline $\begin{array}{l}\text { Blinding of participants } \\
\text { and personnel (perfor- } \\
\text { mance bias) } \\
\text { All outcomes }\end{array}$ & High risk & $\begin{array}{l}\text { Very difficult to blind trials where participants need to make their own dietary } \\
\text { changes }\end{array}$ \\
\hline $\begin{array}{l}\text { Blinding of outcome as- } \\
\text { sessment (detection bias) } \\
\text { CVD outcomes }\end{array}$ & Low risk & $\begin{array}{l}\text { Quote: "outcome assessors were not aware of study allocation" (Prof Burr, per- } \\
\text { sonal communication). Method of blinding not stated }\end{array}$ \\
\hline $\begin{array}{l}\text { Blinding of outcome as- } \\
\text { sessment (detection bias) } \\
\text { All-cause mortality }\end{array}$ & Low risk & Blinding is not relevant in assessment of mortality \\
\hline $\begin{array}{l}\text { Incomplete outcome data } \\
\text { (attrition bias) } \\
\text { All outcomes }\end{array}$ & Low risk & $\begin{array}{l}\text { GPs contacted for information on mortality and morbidity when participants } \\
\text { did not attend }\end{array}$ \\
\hline $\begin{array}{l}\text { Selective reporting (re- } \\
\text { porting bias) }\end{array}$ & Low risk & $\begin{array}{l}\text { Not relevant for primary and secondary outcomes as we asked all trialists for } \\
\text { data }\end{array}$ \\
\hline $\begin{array}{l}\text { Free of systematic differ- } \\
\text { ence in care? }\end{array}$ & High risk & $\begin{array}{l}\text { Different levels of advice appear to have been provided. See control and inter- } \\
\text { vention methods in the Interventions section of the table of Characteristics of } \\
\text { included studies }\end{array}$ \\
\hline Stated aim to reduce SFA & Low risk & Aim to reduce SFA stated \\
\hline Achieved SFA reduction & Low risk & SFA reduction achieved \\
\hline Achieved TC reduction & Low risk & Statistically significant TC fall \\
\hline Other bias & Low risk & None noted \\
\hline
\end{tabular}

Houtsmuller 1979

\section{Study characteristics}

\begin{tabular}{ll}
\hline Methods & RCT \\
& Summary risk of bias: moderate to high \\
\hline Participants & Adults with newly-diagnosed diabetes (the Netherlands) \\
\hline
\end{tabular}

Reduction in saturated fat intake for cardiovascular disease (Review) 
Houtsmuller 1979 (Continued)

CVD risk: moderate

Control: 51 randomised, unclear how many analysed (all analysed re deaths)

Intervention: 51 randomised, unclear how many analysed (all re deaths)

Mean years in trial: unclear (max duration 6 years)

$\%$ male: $56 \%$ overall

Age: mean unclear

Baseline total fat intake: unclear

Baseline saturated fat intake: unclear

Ethnicity: not stated

Statins use allowed? Unclear

$\%$ taking statins: Not reported (probably none as too early, pre-1980)

Interventions

Modified fat vs usual diet

Control aims: SFA 35\%E, CHO 50\%E, protein $15 \% \mathrm{E}$

Intervention aims: total fat $40 \% \mathrm{E}, 1 / 3$ linoleic acid, $\mathrm{CHO} 45 \% \mathrm{E}$, protein $15 \% \mathrm{E}$

Control methods: unclear, surveyed by dietitian

Intervention methods: unclear, surveyed by dietitian

Intervention appears to be delivered by dietitian but no clear details on format or frequency

Total fat intake: not reported

\section{Saturated fat intake: not reported (mean difference unclear)}

PUFA intake: not reported

PUFA n-3 intake: not reported

PUFA n-6 intake: not reported

MUFA intake: not reported

$\mathrm{CHO}$ intake: not reported

Protein intake: not reported

Trans fat intake: not reported

Replacement for saturated fat: mainly PUFA (based on dietary aims)

Style: diet advice?

Setting: community

Outcomes

Stated trial outcomes: progression of diabetic retinopathy

Data available on total mortality? no

Cardiovascular mortality? no

Events available for combined cardiovascular events: total MI and angina

Secondary outcomes: total cholesterol, TGs (data read off graph), CHD mortality (fatal MI), CHD events (MI, angina)

Notes

Study duration 6 years. Study aim was for control group to take $35 \% \mathrm{E}$ as saturated fat, and the intervention group $40 \% \mathrm{E}$ from fat, of which $33 \%$ was from linoleic acid (so saturated fat $<27 \% \mathrm{E}$ ), but saturated fat intake during trial not reported 
Houtsmuller 1979 (Continued)

\section{SFA reduction aimed (unclear whether achieved).}

Total serum cholesterol, difference between intervention and control, $\mathrm{mmol} / \mathrm{L}:-0.47(95 \% \mathrm{Cl}$ -0.76 to -0.18$)$, statistically significant reduction

Trial dates: Study recruitment 1973 to (unclear)

Funding: Dutch Heart Foundation

Declarations of Interest of primary researchers: none stated, all authors worked for academic or health institutions

\section{Risk of bias}

\begin{tabular}{|c|c|c|}
\hline Bias & Authors' judgement & Support for judgement \\
\hline $\begin{array}{l}\text { Random sequence genera- } \\
\text { tion (selection bias) }\end{array}$ & Low risk & Participants matched in pairs then randomised \\
\hline $\begin{array}{l}\text { Allocation concealment } \\
\text { (selection bias) }\end{array}$ & Unclear risk & Allocation method not clearly described \\
\hline $\begin{array}{l}\text { Blinding of participants } \\
\text { and personnel (perfor- } \\
\text { mance bias) } \\
\text { All outcomes }\end{array}$ & High risk & Unclear, though unlikely as dietary advice provided \\
\hline $\begin{array}{l}\text { Blinding of outcome as- } \\
\text { sessment (detection bias) } \\
\text { CVD outcomes }\end{array}$ & Unclear risk & Blinding of outcome assessors not mentioned \\
\hline $\begin{array}{l}\text { Blinding of outcome as- } \\
\text { sessment (detection bias) } \\
\text { All-cause mortality }\end{array}$ & Low risk & Blinding is not relevant in assessment of mortality \\
\hline $\begin{array}{l}\text { Incomplete outcome data } \\
\text { (attrition bias) } \\
\text { All outcomes }\end{array}$ & Unclear risk & $\begin{array}{l}\text { Unclear, deaths, cancer and CV events are dropouts, trialists asked for data - } \\
\text { unclear if any data missing }\end{array}$ \\
\hline $\begin{array}{l}\text { Selective reporting (re- } \\
\text { porting bias) }\end{array}$ & Low risk & $\begin{array}{l}\text { Not relevant for primary and secondary outcomes as we asked all trialists for } \\
\text { data }\end{array}$ \\
\hline $\begin{array}{l}\text { Free of systematic differ- } \\
\text { ence in care? }\end{array}$ & Unclear risk & $\begin{array}{l}\text { Level and type of intervention unclear. See control and intervention methods } \\
\text { in the Interventions section of the table of Characteristics of included studies }\end{array}$ \\
\hline Stated aim to reduce SFA & Low risk & Aim to reduce SFA stated \\
\hline Achieved SFA reduction & Unclear risk & SFA intake not reported \\
\hline Achieved TC reduction & Low risk & Statistically significant TC fall \\
\hline Other bias & Low risk & None noted \\
\hline
\end{tabular}

\section{Study characteristics}


Ley 2004 (Continued)
Methods
RCT
Summary risk of bias: low

\section{Participants}

People with impaired glucose intolerance or high normal blood glucose (New Zealand) CVD risk: moderate

Control: unclear how many randomised (176 between both groups), unclear how many analysed (112 between both groups at 5 years)

Intervention: as above

Mean years in trial: 4.1 over whole trial

$\%$ male: control $80 \%$, intervention $68 \%$

Age: mean control 52.0 (SE 0.8), intervention 52.5 (SE 0.8)

Ethnicity: European $67 \%$ int, $77 \%$ control, Maori $11 \%$ int, $7 \%$ control, Pacific islander $20 \%$ int, $13 \%$ control, Other $3 \%$ int, $4 \%$ control (outcomes not provided by ethnicity)

Statins use allowed? Unclear

$\%$ taking statins: Not reported

Reduced fat vs usual diet

Control aims: usual diet

Intervention aims: reduced fat diet (no specific goal stated)

Control methods: usual intake plus general advice on healthy eating consistent with the New Zealand guidelines and standard dietary information for people with nutrition-related problems upon entering the trial

Intervention methods: monthly small group meetings to follow a 1-year structured programme aimed at reducing fat in the diet, includes education, personal goal-setting, self-monitoring

Total fat intake, \%E (at 1 year): int 26.1 (SD 7.7), cont 33.6 (SD 7.8) (mean difference $-7.50,95 \% \mathrm{Cl}-10.37$ to -4.63 ) significant reduction

Intervention delivered in small face-to-face groups but unclear by whom

Saturated fat intake, \%E (at 1 year): cont 13.4 (SD 4.7), int 10.0 (SD 4.2) (mean difference -3.40, $95 \% \mathrm{Cl}-5.05$ to -1.75 ) significant reduction

PUFA intake, \%E (at 1 year): cont 4.8 (SD 1.6), int 4.0 (SD 1.4) (mean difference $-0.80,95 \% \mathrm{Cl}-1.36$ to

-0.24) significant reduction

PUFA n-3 intake: not reported

PUFA n-6 intake: not reported

MUFA intake, \%E (at 1 year): cont 11.8 (SD 3.1), int 8.9 (SD 2.8) (mean difference $-2.90,95 \% \mathrm{Cl}-3.99$ to -1.81) significant reduction

CHO intake, \%E (at 1 year): cont 45.8 (SD 10.9), int 54.2 (SD 10.5) (mean difference $8.40,95 \% \mathrm{Cl} 4.44$ to 12.36) significant increase

Protein intake, \%E (at 1 year): cont 16.6 (SD 3.9), int 18.4 (SD 3.5), (mean difference $1.80,95 \% \mathrm{Cl} 0.43$ to 3.17) significant increase

Trans fat intake: not reported

Replacement for saturated fat: carbohydrate and protein (based on dietary achievements)

Style: diet advice

Setting: community 
Ley 2004 (Continued)
Outcomes
Stated trial outcomes: lipids, glucose, blood pressure
Data available on total mortality? yes
Cardiovascular mortality? yes
Events available for combined cardiovascular events: MI, angina, stroke, heart failure
Secondary outcomes: total MI, stroke, cancer diagnoses, cancer deaths, CHD events (MI or angina)
Tertiary outcomes: weight, total, LDL and HDL cholesterol, TGs, BP

\section{Study duration over 4 years}

Study aim was to reduce total fat (not saturated fat), but saturated fat intake in the intervention group was significantly lower than in the control group.

\section{SFA reduction achieved}

Total serum cholesterol, difference between intervention and control, $\mathrm{mmol} / \mathrm{L}:-0.05$ ( $95 \% \mathrm{Cl}$ -0.46 to 0.36 ), NO statistically significant reduction and smaller than 0.20

Trial dates: Recruitment 1988 to 1990

Funding: National Heart Foundation of New Zealand, Auckland Medical Research Foundation, Lotteries Medical Board and the Health Research Council of New Zealand

Declarations of Interest of primary researchers: none stated, all authors worked for academic or health institutions

\section{Risk of bias}

\begin{tabular}{lll}
\hline Bias & Authors' judgement & Support for judgement \\
\hline $\begin{array}{l}\text { Random sequence genera- } \\
\text { tion (selection bias) }\end{array}$ & Low risk & $\begin{array}{l}\text { Unmarked opaque envelopes were opened by the person recruiting, unable to } \\
\text { alter allocation later (trial author stated in their reply to us that randomisation } \\
\text { and preparation of the envelopes was by people not involved in recruitment). }\end{array}$ \\
\hline $\begin{array}{l}\text { Allocation concealment } \\
\text { (selection bias) }\end{array}$ & Low risk & $\begin{array}{l}\text { Unmarked opaque envelopes were opened by the person recruiting, unable to } \\
\text { alter allocation later. }\end{array}$ \\
\hline $\begin{array}{l}\text { Blinding of participants } \\
\begin{array}{l}\text { and personnel (perfor- } \\
\text { mance bias) } \\
\text { All outcomes }\end{array}\end{array}$ & High risk & Dietary advice, not blinded \\
\hline
\end{tabular}

Blinding of outcome as-
sessment (detection bias) $\quad$ Low risk Trial authors stated that outcome assessors were blinded.

$$
\text { CVD outcomes }
$$

\begin{tabular}{lll}
\hline $\begin{array}{l}\text { Blinding of outcome as- } \\
\text { sessment (detection bias) } \\
\text { All-cause mortality }\end{array}$ & Low risk & Blinding is not relevant in assessment of mortality. \\
\hline $\begin{array}{l}\text { Incomplete outcome data } \\
\text { (attrition bias) } \\
\text { All outcomes }\end{array}$ & Unclear risk & $\begin{array}{l}\text { Unclear, deaths, cancer and CV events are dropouts, trialists were asked for } \\
\text { data - unclear if any data missing }\end{array}$ \\
\hline $\begin{array}{l}\text { Selective reporting (re- } \\
\text { porting bias) }\end{array}$ & Low risk & $\begin{array}{l}\text { Not relevant for primary and secondary outcomes as we asked all trialists for } \\
\text { data }\end{array}$ \\
\hline $\begin{array}{l}\text { Free of systematic differ- } \\
\text { ence in care? }\end{array}$ & High risk & $\begin{array}{l}\text { See control and intervention methods in the Interventions section of the table } \\
\text { of Characteristics of included studies }\end{array}$ \\
\hline
\end{tabular}


Ley 2004 (Continued)

\begin{tabular}{lll} 
Stated aim to reduce SFA & High risk & Aim to reduce SFA not stated \\
\hline Achieved SFA reduction & Low risk & SFA reduction achieved \\
\hline Achieved TC reduction & High risk & TC fall small (0.05 mmol/L only) and not statistically significant \\
\hline Other bias & Low risk & None noted \\
\hline
\end{tabular}

Moy 2001

\section{Study characteristics}

Methods RCT

Summary risk of bias: moderate to high

Participants

Middle-aged siblings of people with early CHD, with at least 1 CVD risk factor (USA)

CVD risk: moderate

Control: randomised 132, analysed 118

Intervention: randomised 135, analysed 117

Mean years in trial: 1.9

$\%$ male: control $49 \%$, intervention $55 \%$

Age: control mean 45.7 (SD 7), intervention 46.2 (SD 7)

Ethnicity: African-American 18\% int, 25\% control (remainder of group ethnicity not described, and outcomes not presented by ethnicity)

Statins use allowed? Unclear (raised LDL cholesterol was a condition of entry, so use of statins probably minimal)

$\%$ taking statins: Not reported

Reduced fat intake vs usual diet

Control aim: usual care

Intervention aim: total fat $40 \mathrm{~g} / \mathrm{d}$ or less

Control methods: usual physician care with risk factor management at 0, 1 and 2 years

Intervention methods: Individualised counselling by trained nurse, appointments 6 - 8 weekly for 2 years

Intervention delivered individually, face-to-face by a trained nurse.

Total fat intake, \%E (at 2 years): int 34.1 (SD unclear), cont 38.0 (SD unclear) (mean difference -3.90, $95 \% \mathrm{Cl}-6.46$ to -1.34 assuming SDs of 10 ) significant reduction

Saturated fat intake, \%E (at 2 years): int 11.5 (SD unclear), cont 14.4 (SD unclear) (mean difference $-2.90,95 \% \mathrm{Cl}-4.18$ to -1.62 assuming SDs of 5 ) significant reduction

PUFA intake: not reported

PUFA n-3 intake: not reported

PUFA n-6 intake: not reported

MUFA intake: not reported

CHO intake: not reported 
Moy 2001 (Continued)

Protein intake: not reported

Trans fat intake: not reported

Replacement for saturated fat: unclear

Style: diet advice

Setting: community

Stated trial outcomes: dietary intake
Data available on total mortality? yes, no deaths
Cardiovascular mortality? yes, no deaths
Events available for combined cardiovascular events: total MI, stroke, unstable angina, PVD and PTCA
Secondary outcomes: cancer diagnoses (no events), cancer deaths (none), stroke, total and non-fatal
MI, CHD mortality (none), CHD events (MI or angina)
Tertiary outcomes: BMI, HDL and LDL cholesterol, TG

\begin{tabular}{|c|c|}
\hline \multirow[t]{7}{*}{ Notes } & Study duration 2 years \\
\hline & $\begin{array}{l}\text { Study aim was to reduce total fat based on ATPII dietary guidelines, and preliminary work established } \\
\text { that this intervention reduced saturated fat and dietary cholesterol, and saturated fat intake was signif- } \\
\text { icantly lower than in the control group }\end{array}$ \\
\hline & SFA reduction aimed and achieved \\
\hline & $\begin{array}{l}\text { Total serum cholesterol not reported, but LDL was, difference between intervention and control, } \\
\mathrm{mmol} / \mathrm{L}:-0.29(95 \% \mathrm{Cl}-0.54 \text { to }-0.04) \text {, statistically significant reduction }\end{array}$ \\
\hline & Trial dates: Study recruitment 1991 to 1994 \\
\hline & $\begin{array}{l}\text { Funding: National Institute of Nursing Research, General Clinical Research Center of the National Insti- } \\
\text { tutes of Health }\end{array}$ \\
\hline & $\begin{array}{l}\text { Declarations of Interest of primary researchers: none stated, all authors worked for academic or health } \\
\text { institutions }\end{array}$ \\
\hline
\end{tabular}

Risk of bias

\begin{tabular}{|c|c|c|}
\hline Bias & Authors' judgement & Support for judgement \\
\hline $\begin{array}{l}\text { Random sequence genera- } \\
\text { tion (selection bias) }\end{array}$ & Low risk & $\begin{array}{l}\text { Randomly assigned via computerised schema after all eligible siblings from a } \\
\text { family had been screened }\end{array}$ \\
\hline $\begin{array}{l}\text { Allocation concealment } \\
\text { (selection bias) }\end{array}$ & Unclear risk & Allocation method not clearly described \\
\hline $\begin{array}{l}\text { Blinding of participants } \\
\text { and personnel (perfor- } \\
\text { mance bias) } \\
\text { All outcomes }\end{array}$ & High risk & Participants clear about their allocation \\
\hline $\begin{array}{l}\text { Blinding of outcome as- } \\
\text { sessment (detection bias) } \\
\text { CVD outcomes }\end{array}$ & High risk & Trialists clear about allocation \\
\hline $\begin{array}{l}\text { Blinding of outcome as- } \\
\text { sessment (detection bias) } \\
\text { All-cause mortality }\end{array}$ & Low risk & Blinding is not relevant in assessment of mortality. \\
\hline
\end{tabular}


Moy 2001 (Continued)

$\begin{array}{ll}\text { Incomplete outcome data } & \text { Unclear risk } \\ \text { (attrition bias) } & \begin{array}{l}\text { Unclear, deaths, cancer and CV events are dropouts, trialists were asked for } \\ \text { data - unclear if any data missing }\end{array} \\ \text { All outcomes } & \end{array}$

All outcomes

Selective reporting (re- Low risk $\quad$ Not relevant for primary and secondary outcomes as all trialists asked for data
porting bias)

\begin{tabular}{lll}
\hline $\begin{array}{l}\text { Free of systematic differ- } \\
\text { ence in care? }\end{array}$ & High risk & $\begin{array}{l}\text { Differences in frequency of follow-up, but unclear what differences in care oc- } \\
\text { curred between the physician and nurse-led care. See control and interven- } \\
\text { tion methods in Interventions section of the table of Characteristics of includ- } \\
\text { ed studies }\end{array}$ \\
\hline Stated aim to reduce SFA & Low risk & Aim to reduce SFA stated \\
\hline Achieved SFA reduction & Low risk & SFA reduction achieved \\
\hline Achieved TC reduction & Low risk & Statistically significant LDL fall (though TC not reported) \\
\hline Other bias & Low risk & None noted \\
\hline
\end{tabular}

MRC 1968

\section{Study characteristics}

\begin{tabular}{ll}
\hline Methods & RCT \\
Medical Research Council (MRC) \\
Summary risk of bias: moderate to high \\
\hline
\end{tabular}

Pree-living men who have survived a first MI (UK)
CVD risk: high
Control: randomised 194, analysed 181 at 2 years
Intervention: randomised 199, analysed 172 at 2 years
Mean years in trial: control 3.7, intervention 3.8
\% male: 100
Age: unclear (all < 60)
Ethnicity: not stated
Statins use allowed? Unclear (anti-coagulants allowed, but few other medications appear to have been
used)
\% taking statins: Not reported (probably none as too early, pre-1980)

Interventions

Modified fat vs usual diet

Control aims: usual diet

Intervention aims: reduce dietary fat to $35 \mathrm{~g}$ fat per day, add $84 \mathrm{~g}$ soya oil per day

Control methods: usual diet plus reducing diet (reduced $\mathrm{CHO}$ ) for weight management for overweight men

Intervention methods: instructed to follow a dietary regimen removing saturated fat from the diet plus daily dose of $85 \mathrm{~g}$ soya oil; half of it had to be taken unheated. Reduced CHO diet for weight management in overweight men

Intervention appears to be delivered and supervised by trial dietitian but unclear how often. 
MRC 1968 (Continued)

Total fat intake, \%E (at 3.5 years): int 46 (SD unclear), cont 43 (SD unclear) (mean difference 3.00, 95\%

$\mathrm{Cl} 0.91$ to 5.09 assuming SDs of 10) significant increase

\section{Saturated fat intake: not reported (mean difference unclear)}

PUFA intake: not reported

PUFA n-3 intake: not reported

PUFA n-6 intake: not reported

MUFA intake: not reported

$\mathrm{CHO}$ intake: not reported

Protein intake: not reported

Trans fat intake: not reported

\section{Replacement for saturated fat: mainly PUFA (based on dietary goals)}

Style: diet advice \& supplement (soy oil)

Setting: community

Stated trial outcomes: MI or sudden death
Data available on total mortality? yes
Cardiovascular mortality? yes
Events available for combined cardiovascular events: cardiovascular deaths and fatal or non-fatal MI
Secondary outcomes: total and non-fatal MI, stroke, cancer deaths, CHD mortality, CHD events (CHD
mortality or non-fatal MI)
Tertiary outcomes: none (data for weight, total cholesterol and BP, but no variance info)

Notes Study duration over 6 years

Study aim: for intervention "saturated fats were replaced by polyunsaturated fats", but saturated fat intakes during trial were not reported.

\section{SFA reduction aimed}

Total serum cholesterol, difference between intervention and control, mmol/L: -0.64 (95\% Cl unclear), reduction $>0.20$

For all, data at 4 years, control $n=89$, intervention $n=88$

Weight change: control $-3 \mathrm{~kg}$, intervention $0 \mathrm{~kg}$

Total cholesterol change: control $-0.47 \mathrm{mmol} / \mathrm{L}$, intervention $-1.11 \mathrm{mmol} / \mathrm{L}$

Systolic BP change: control $0 \mathrm{mmHg}$, intervention $+2 \mathrm{mmHg}$

Diastolic BP change: control $+3 \mathrm{mmHg}$, intervention $-1 \mathrm{mmHg}$

Trial dates: Study recruitment 1960 to 1965, analysed 1967

Funding: Medical Research Council

Declarations of Interest of primary researchers: none stated, all authors worked for academic or health institutions.

\section{Risk of bias}

Bias Authors' judgement Support for judgement


MRC 1968 (Continued)

Random sequence genera- Low risk Quote: "using random numbers, by blocks within hospitals" tion (selection bias)

Allocation concealment $\quad$ Unclear risk $\quad$ Not described
(selection bias)

\begin{tabular}{|c|c|c|}
\hline $\begin{array}{l}\text { Blinding of participants } \\
\text { and personnel (perfor- } \\
\text { mance bias) } \\
\text { All outcomes }\end{array}$ & High risk & $\begin{array}{l}\text { Big changes to fat intake in intervention group while control group ate their } \\
\text { usual diet }\end{array}$ \\
\hline
\end{tabular}

\begin{tabular}{lll}
\hline Blinding of outcome as- & Low risk & Quote: "Suspected relapses were assessed at regular intervals by a review \\
sessment (detection bias) & committee unaware of the patients diet group".
\end{tabular}

CVD outcomes

$\begin{array}{ll}\text { Blinding of outcome as- } & \text { Low risk } \quad \text { Blinding is not relevant in assessment of mortality. }\end{array}$

All-cause mortality

\begin{tabular}{|c|c|c|}
\hline $\begin{array}{l}\text { Incomplete outcome data } \\
\text { (attrition bias) } \\
\text { All outcomes }\end{array}$ & Unclear risk & $\begin{array}{l}\text { Data collection was thorough, but some participants dropped out and contact } \\
\text { was lost, so some events may have been missed. }\end{array}$ \\
\hline $\begin{array}{l}\text { Selective reporting (re- } \\
\text { porting bias) }\end{array}$ & Low risk & $\begin{array}{l}\text { Not relevant for primary and secondary outcomes as all trialists were asked for } \\
\text { data }\end{array}$ \\
\hline $\begin{array}{l}\text { Free of systematic differ- } \\
\text { ence in care? }\end{array}$ & High risk & $\begin{array}{l}\text { Unlikely as control group continued diet as usual, intervention group were } \\
\text { likely to have had additional contact. See control and intervention methods in } \\
\text { the Interventions section of the table of Characteristics of included studies }\end{array}$ \\
\hline Stated aim to reduce SFA & Low risk & Aim to reduce SFA stated \\
\hline Achieved SFA reduction & Unclear risk & SFA intake not reported \\
\hline Achieved TC reduction & Low risk & $\begin{array}{l}\text { Although statistical significance was not reported or calculable, TC in the in- } \\
\text { tervention group was } 0.64 \mathrm{mmol} / \mathrm{L} \text { lower than in the control group, a large fall } \\
\text { (and almost certainly statistically significant). }\end{array}$ \\
\hline Other bias & Low risk & None noted \\
\hline
\end{tabular}

\section{Oslo Diet-Heart 1966}

\section{Study characteristics}

\begin{tabular}{ll}
\hline Methods & RCT \\
Oslo Diet-Heart Trial \\
Summary risk of bias: moderate to high for CVD outcomes, low for all-cause mortality \\
\hline
\end{tabular}

$\begin{array}{ll}\text { Participants } & \text { Men with previous MI (Norway) } \\ \text { CVD risk: high } \\ \text { Control: randomised 206, analysed } 148 \text { (at } 5 \text { years) } \\ \text { Intervention: randomised 206, analysed } 152 \text { (at } 5 \text { years) } \\ \text { Mean years in trial: control 4.3, intervention } 4.3 \\ \text { \% male: } 100\end{array}$


Oslo Diet-Heart 1966 (Continued)

Age: mean control 56.3, intervention 56.2 (all 30 - 67)

Ethnicity: ethnicity not mentioned

Statins use allowed? Unclear (medications not mentioned as exclusion criteria, most appeared to be on anti-coagulant medications, statins not mentioned)

\% taking statins: Not reported (probably none as too early, pre-1980)

Interventions

Modified fat diet vs control

Control aims: no dietary advice but direct questions answered, supplement = 1 vitamin tablet daily Intervention aims: reduce meat and dairy fats, increase fish, vegetables, supplement - 1 vitamin tablet daily, $0.5 \mathrm{~L}$ soy bean oil per week (free to $25 \%$ of participants), sardines in cod liver oil (free at certain times to encourage compliance)

Control methods: usual diet

Intervention methods: continuous instruction and supervision by dietitian, including home visits, letters and phone calls

Total fat intake: unclear (note - intake of total fat, carbohydrate, protein and sugar was assessed in 17 "especially conscientious and positive" as well as intelligent dieters, but this was not reported here as unlikely to be representative, and lacking control group data)

\section{Saturated fat intake: unclear (mean difference unclear)}

PUFA intake: unclear

PUFA n-3 intake: not reported

PUFA n-6 intake: not reported

MUFA intake: unclear

CHO intake: unclear

Protein intake: unclear

Trans fat intake: unclear

\section{Replacement for saturated fat: PUFA (based on dietary goals)}

Style: diet advice and supplement (food)

Setting: community

Stated trial outcomes: coronary heart disease morbidity and mortality
Data available on total mortality? yes
Cardiovascular mortality? yes
Events available for combined cardiovascular events: total MI, sudden death, stroke, angina
Secondary outcomes: non-fatal and total MI, stroke, CHD mortality (fatal MI and sudden death), CHD
events (MI, angina and sudden death)
Tertiary outcomes: weight, total cholesterol, systolic and diastolic BP (but no variance information was
provided)

Notes

Study duration over 4 years

Study aim was to reduce serum cholesterol by a diet "low in saturated fats and in cholesterol, and rich in highly unsaturated fats", saturated fat intakes during study were not reported

SFA reduction aimed (reduction unclear as not measured except in a highly compliant subgroup) 
Oslo Diet-Heart 1966 (Continued)

Total serum cholesterol, difference between intervention and control, $\mathrm{mmol} / \mathrm{L}:-1.07(95 \% \mathrm{Cl}$ unclear), reduction $>0.20$

Weight change from baseline was $-0.5 \mathrm{~kg}$ in the control group ( $\mathrm{n}$ 155), $-2.5 \mathrm{~kg}$ in the intervention group $(\mathrm{n} \sim 160)$ at 51 months

Total cholesterol change from baseline was $-0.46 \mathrm{mmol} / \mathrm{L}$ in the control group and $-1.53 \mathrm{mmol} / \mathrm{L}$ in the intervention group at 51 months

Systolic BP at baseline was $153.8 \mathrm{mmHg}$ in control and 159.0 in intervention, and mean SBP through trial was $154.3 \mathrm{mmHg}$ in control and $158.2 \mathrm{mmHg}$ in the intervention group.

Diastolic BP at baseline was $93.5 \mathrm{mmHg}$ in control and $97.1 \mathrm{mmHg}$ in intervention, through trial mean $\mathrm{dBP}$ was $95.5 \mathrm{mmHg}$ in control and $98.6 \mathrm{mmHg}$ in intervention participants

Trial dates: Recruitment 1956 to 1958

Funding: Det Norske Råd for Hjerte- og karsyk-dommer, A/S Freia Chokoladefabriks Arbeidsfond for Ernærings-forskning, JL Tiedemanns Tobaksfabrik Joh H Andresens medisinske fond, plus A/S Farmacöytisk Industri provided a multivitamin free of charge, DE-NO-FA and Lillleborg Fabriker provided soy bean oil at reduced prices, the Research Laboratory of the Norwegian Canning Industry, Stavanger Preserving $\mathrm{Co}$ and Kommendal Packing Comp provided Norwegian sardines in cod liver oil free to those in the intervention group.

Declarations of Interest of primary researchers: none stated, all authors worked for academic or health institutions.

\section{Risk of bias}

\begin{tabular}{|c|c|c|}
\hline Bias & Authors' judgement & Support for judgement \\
\hline $\begin{array}{l}\text { Random sequence genera- } \\
\text { tion (selection bias) }\end{array}$ & Low risk & "table of random numbers used", by Prof Knut Westlund \\
\hline $\begin{array}{l}\text { Allocation concealment } \\
\text { (selection bias) }\end{array}$ & Low risk & $\begin{array}{l}\text { Randomisation appears to have occurred before medical examination with- } \\
\text { in the study, so was not affected by participant characteristics and was con- } \\
\text { cealed. }\end{array}$ \\
\hline $\begin{array}{l}\text { Blinding of participants } \\
\text { and personnel (perfor- } \\
\text { mance bias) } \\
\text { All outcomes }\end{array}$ & High risk & Participants were aware of their allocation as was the main trialist. \\
\hline $\begin{array}{l}\text { Blinding of outcome as- } \\
\text { sessment (detection bias) } \\
\text { CVD outcomes }\end{array}$ & Unclear risk & $\begin{array}{l}\text { Outcomes were categorised by a diagnostic board, but their blinded status } \\
\text { was unclear. }\end{array}$ \\
\hline $\begin{array}{l}\text { Blinding of outcome as- } \\
\text { sessment (detection bias) } \\
\text { All-cause mortality }\end{array}$ & Low risk & Blinding is not relevant in assessment of mortality. \\
\hline $\begin{array}{l}\text { Incomplete outcome data } \\
\text { (attrition bias) } \\
\text { All outcomes }\end{array}$ & Low risk & $\begin{array}{l}\text { The participants who could not be directly followed up for the } 5 \text { years were fol- } \\
\text { lowed until death or study end through personal interviews, or contact with } \\
\text { their physicians or relatives. }\end{array}$ \\
\hline $\begin{array}{l}\text { Selective reporting (re- } \\
\text { porting bias) }\end{array}$ & Low risk & $\begin{array}{l}\text { Not relevant for primary and secondary outcomes as all trialists were asked for } \\
\text { data }\end{array}$ \\
\hline
\end{tabular}


Oslo Diet-Heart 1966 (Continued)

Free of systematic differ- High risk ence in care?

Dietetic input level very different, although medical care appeared similar. See control and intervention methods in the Interventions section of the table of

Characteristics of included studies

\begin{tabular}{lll}
\hline Stated aim to reduce SFA & Low risk & Aim to reduce SFA stated \\
\hline Achieved SFA reduction & Unclear risk & SFA intake not reported \\
\hline Achieved TC reduction & Low risk & $\begin{array}{l}\text { Although statistical significance was not reported or calculable, TC in the in- } \\
\text { tervention group was } 1.07 \text { mmol/L lower than in the control group, a large fall } \\
\text { (and almost certainly statistically significant). }\end{array}$ \\
\hline Other bias & Low risk & None noted
\end{tabular}

\section{Oxford Retinopathy 1978}

\section{Study characteristics}

Methods RCT

Summary risk of bias: moderate to high for CVD outcomes, low for all-cause mortality

Participants Newly-diagnosed non-insulin-dependent diabetics (UK)

CVD risk: moderate

Control: number randomised unclear (249 split between the 2 groups, 125?), number analysed for mortality unclear (all but 2 overall at 16 years)

Intervention: number randomised unclear (249 split between the 2 groups, 125?), number analysed as above

Mean years in trial: overall 9.3?

$\%$ male: overall $49 \%$

Age: mean overall $47.1($ all $<65)$

Ethnicity: not stated

Statins use allowed? Unclear

$\%$ taking statins: Not reported (probably none as too early, pre-1980)

Interventions $\quad$ Reduced and modified dietary fat vs average diet

Control aims: total fat 40\%E, PUFA $12 \% \mathrm{E}$, protein $20 \% \mathrm{E}, \mathrm{CHO} 40 \% \mathrm{E}$ (reducing simple sugars), $1500 \mathrm{kcal} /$ day

Intervention aims: total fat 26\%E, PUFA 16\%E, protein 20\%E, CHO 54\%E (reducing simple sugars), 1500 $\mathrm{kcal} / \mathrm{day}$

Control methods: dietary advice from diabetes dietitian

Intervention methods: dietary advice from diabetes dietitian

Total fat intake, \%E (at 7 - 9 years)§: int 32 (SD unclear), cont 41 (SD unclear) (mean difference -9.00, $95 \% \mathrm{Cl}-11.48$ to -6.52 assuming SDs of 10 ) significant reduction

Saturated fat intake, \%E (at 7 - 9 years)§: int 10.7 (SD unclear), cont 20.4 (SD unclear) (mean difference $-9.70,95 \% \mathrm{Cl}-10.94$ to -8.46 assuming SD of 5) significant reduction

PUFA intake, \%E (at 7 - 9 years)§: int 11.8 (SD unclear), cont 2.1 (SD unclear) (mean difference 9.70, 95\%

$\mathrm{Cl} 8.46$ to 10.94 assuming SDs of 5) significant increase

PUFA n-3 intake: not reported 
Oxford Retinopathy 1978 (Continued)

PUFA n-6 intake: not reported

MUFA intake, \%E (at 7 - 9 years)§: int 9.5 (SD unclear), cont 18.6 (SD unclear) (mean difference -9.10, $95 \% \mathrm{Cl}-10.34$ to 7.86 assuming SDs of 5) significant reduction

Carbohydrate intake: not reported

Protein intake: not reported

Trans fat intake: not reported

Replacement for saturated fat: PUFA and CHO (based on dietary goals and achievements)

Style: diet advice

Setting: community (outpatients clinic)

§validity of these data is questionable as it represents only 3 intervention and 3 control participants. Source: Lopez-Espinoza 1984

Stated trial outcomes: retinopathy
Data available on total mortality? yes, but unable to ascertain from which intervention groups (34
deaths at 10 years)
Cardiovascular mortality? no
Events available for combined cardiovascular events: none
Secondary outcomes: none
Tertiary outcomes: BMI, total cholesterol

Study aim was to reduce total fat and increase PUFAs (so reducing saturates), and saturated fat intake in the intervention group was significantly lower than in the control group

\section{SFA reduction achieved}

Total serum cholesterol, difference between intervention and control, mmol/L: 0.07 (95\% $\mathrm{Cl}-0.34$ to 0.48 ), NO statistically significant reduction and smaller than 0.20

Trial dates: Recruitment 1973 to 1976

Funding: Oxford Diabetes Trust, British Diabetic Association, International Sugar Research Foundation Inc

Declarations of Interest of primary researchers: none stated, all authors worked for academic or health institutions.

\section{Risk of bias}

\begin{tabular}{lll}
\hline Bias & Authors' judgement & Support for judgement \\
\hline $\begin{array}{l}\text { Random sequence genera- } \\
\text { tion (selection bias) }\end{array}$ & Low risk & $\begin{array}{l}\text { "random number sequence, provided and allotted by a separate agency" (Prof } \\
\text { Richard Peto) }\end{array}$ \\
\hline $\begin{array}{l}\text { Allocation concealment } \\
\text { (selection bias) }\end{array}$ & Low risk & $\begin{array}{l}\text { "random number sequence, provided and allotted by a separate agency" (Prof } \\
\text { Richard Peto) }\end{array}$ \\
\hline $\begin{array}{l}\text { Blinding of participants } \\
\begin{array}{l}\text { and personnel (perfor- } \\
\text { mance bias) }\end{array}\end{array}$ & High risk \\
\begin{tabular}{l} 
All outcomes \\
\hline
\end{tabular}
\end{tabular}


Oxford Retinopathy 1978 (Continued)
Blinding of outcome as-
Unclear risk
Unclear whether physicians blinded to allocation sessment (detection bias) CVD outcomes

\begin{tabular}{lll}
\hline $\begin{array}{l}\text { Blinding of outcome as- } \\
\text { sessment (detection bias) } \\
\text { All-cause mortality }\end{array}$ & Low risk & Blinding is not relevant in assessment of mortality. \\
\hline $\begin{array}{l}\text { Incomplete outcome data } \\
\text { (attrition bias) } \\
\begin{array}{l}\text { All outcomes } \\
\text { Selective reporting (re- }\end{array}\end{array}$ & Unclear risk & $\begin{array}{l}\text { Unclear, deaths, cancer and CV events are dropouts - unclear if any data miss- } \\
\text { ing }\end{array}$ \\
\hline $\begin{array}{l}\text { pree of systematic differ- } \\
\text { ence in care? }\end{array}$ & Low risk & $\begin{array}{l}\text { Not relevant for primary and secondary outcomes as all trialists were asked for } \\
\text { data }\end{array}$ \\
\hline $\begin{array}{l}\text { Stated aim to reduce SFA } \\
\text { Achieved SFA reduction }\end{array}$ & High risk & $\begin{array}{l}\text { Dietetic advice for both groups. See control and intervention methods in the } \\
\text { Interventions section of the table of Characteristics of included studies }\end{array}$ \\
\hline Achieved TC reduction & High risk & Aim to reduce SFA not stated \\
\hline \begin{tabular}{l} 
Other bias \\
\hline
\end{tabular} & Low risk & SFA reduction achieved \\
\hline
\end{tabular}

Rose corn oil 1965

\section{Study characteristics}

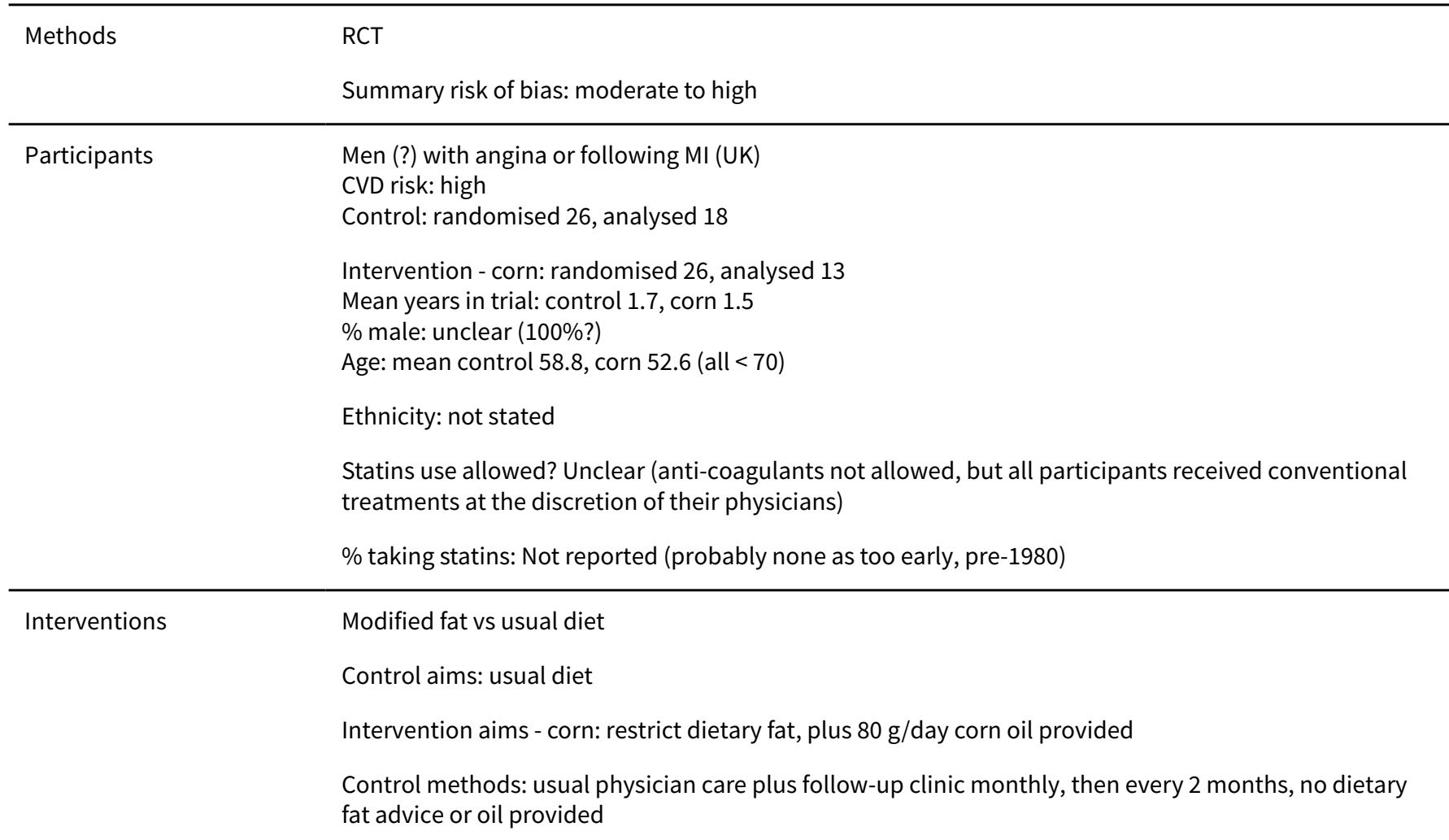


Intervention methods: usual physician care plus follow-up clinic monthly, then every 2 months, dietary fat advice plus oil provided

Unclear how the advice was delivered or by whom

Total fat intake, \%E (at 18 months): corn 50.5 (SD unclear), cont 32.6 (SD unclear) (mean difference $17.90,95 \% \mathrm{Cl} 10.77$ to 25.03 assuming SDs of 10 ) significant increase

Saturated fat intake: unclear (mean difference unclear)

PUFA intake: unclear

PUFA n-3 intake: not reported

PUFA n-6 intake: not reported

MUFA intake: unclear

$\mathrm{CHO}$ intake, \%E (at 18 months): corn 36.5 (SD unclear), cont 51.5 (|SD unclear) (mean difference -15.00, $95 \% \mathrm{Cl}-29.27$ to -0.73 assuming SDs of 20 ) significant reduction

Protein intake, \%E (at 18 months): corn 11.0 (SD unclear), cont 13.2 (SD unclear) (mean difference -2.20, $95 \% \mathrm{Cl}-5.77$ to 1.37 assuming SDs of 5) no significant difference

Trans fat intake: unclear

Replacement for saturated fat: mainly PUFA (based on aims and achievements)

Style: diet advice and supplement (oil)

Setting: community

Outcomes Stated trial outcomes: cardiac events

Data available on total mortality? yes

Cardiovascular mortality? yes

Events available for combined cardiovascular events: cardiovascular deaths, non-fatal MI, angina, stroke

Secondary outcomes: stroke (none), non-fatal and total MI, CHD mortality (fatal MI and sudden death), CHD events (all MI and sudden death)

Tertiary outcomes: total cholesterol

Study aim was to reduce total fat (by restricting fatty meat, sausages, pastry, ice cream, cheese, cake, milk, eggs and butter) and prescribe vegetable oil (so reducing saturates), but saturated fat intakes during intervention were not reported.

\section{SFA reduction aimed (but unclear whether achieved as SFA intake not reported)}

Total serum cholesterol, difference between intervention and control, $\mathrm{mmol} / \mathrm{L}:-\mathbf{0 . 5 8}(\mathbf{9 5 \%} \mathrm{Cl}$ -1.42 to 0.26 ), NO statistically significant reduction but $>0.20$

Trial dates: unclear, published in 1965

Funding: probably unfunded (they thank the Paddington General Hospital for clinic facilities, and St Mary's and Paddington General Hospital physicians for referral of patients, but no funding acknowledged)

Declarations of Interest of primary researchers: none stated, all authors worked for academic or health institutions. 
Rose corn oil 1965 (Continued)

\begin{tabular}{lll} 
Bias & Authors' judgement & Support for judgement \\
\hline $\begin{array}{l}\text { Random sequence genera- } \\
\text { tion (selection bias) }\end{array}$ & Low risk & $\begin{array}{l}\text { Trial was stated as "randomised" but without further detail, apart from use of } \\
\text { a sealed envelope as below. }\end{array}$ \\
\hline $\begin{array}{l}\text { Allocation concealment } \\
\text { (selection bias) }\end{array}$ & Unclear risk & $\begin{array}{l}\text { When a new participant was accepted for the trial a sealed envelope was } \\
\text { opened containing the allocation instructions. In the case of participants allo- } \\
\text { cated to an oil group, the instructions referred only to a code number. }\end{array}$
\end{tabular}

\begin{tabular}{|c|c|c|}
\hline $\begin{array}{l}\text { Blinding of participants } \\
\text { and personnel (perfor- } \\
\text { mance bias) } \\
\text { All outcomes }\end{array}$ & High risk & $\begin{array}{l}\text { The physicians in charge knew which participants were receiving oil, but they } \\
\text { did not know until the end of the trial the kind of oil that they were receiving. }\end{array}$ \\
\hline
\end{tabular}

\begin{tabular}{|c|c|c|}
\hline $\begin{array}{l}\text { Blinding of outcome as- } \\
\text { sessment (detection bias) } \\
\text { CVD outcomes }\end{array}$ & Low risk & $\begin{array}{l}\text { The electrocardiograms were assessed without the knowledge of the partici- } \\
\text { pant's treatment group. }\end{array}$ \\
\hline
\end{tabular}

Blinding of outcome as- $\quad$ Low risk $\quad$ Blinding is not relevant in assessment of mortality.
sessment (detection bias)
All-cause mortality

\begin{tabular}{|c|c|c|}
\hline $\begin{array}{l}\text { Incomplete outcome data } \\
\text { (attrition bias) } \\
\text { All outcomes }\end{array}$ & Unclear risk & Some lost to follow-up by 2 years, so some events may have been missed \\
\hline $\begin{array}{l}\text { Selective reporting (re- } \\
\text { porting bias) }\end{array}$ & Low risk & $\begin{array}{l}\text { Not relevant for primary and secondary outcomes as all trialists were asked for } \\
\text { data. }\end{array}$ \\
\hline $\begin{array}{l}\text { Free of systematic differ- } \\
\text { ence in care? }\end{array}$ & Low risk & $\begin{array}{l}\text { All received conventional treatments at the discretion of the physicians, all at- } \\
\text { tended a special follow-up clinic. See control and intervention methods in the } \\
\text { Interventions section of the table of Characteristics of included studies }\end{array}$ \\
\hline Stated aim to reduce SFA & Low risk & Aim to reduce SFA stated \\
\hline Achieved SFA reduction & Unclear risk & SFA intake not reported \\
\hline Achieved TC reduction & High risk & $\begin{array}{l}\text { Although the TC in the intervention group was } 0.58 \mathrm{mmol} / \mathrm{L} \text { lower than in the } \\
\text { control group, this was not statistically significant in this small study. }\end{array}$ \\
\hline Other bias & Low risk & None noted \\
\hline
\end{tabular}

Rose olive 1965

\section{Study characteristics}

\begin{tabular}{ll}
\hline Methods & RCT \\
& Summary risk of bias: moderate to high \\
\hline Participants & Men (?) with angina or following MI (UK) \\
CVD risk: high & Control: randomised 26 , analysed 18 \\
& Intervention - olive: randomised 28 , analysed 12 \\
& Mean years in trial: control 1.7 , olive 1.5
\end{tabular}


Rose olive 1965 (Continued)

$\%$ male: unclear $(100 \% ?)$

Age: mean control 58.8, olive 55.0 (all $<70$ )

Ethnicity: Not stated

Statins use allowed? Unclear (anti-coagulants not allowed, but all participants received conventional treatments at the discretion of their physicians)

\% taking statins: Not reported (probably none as too early, pre-1980)

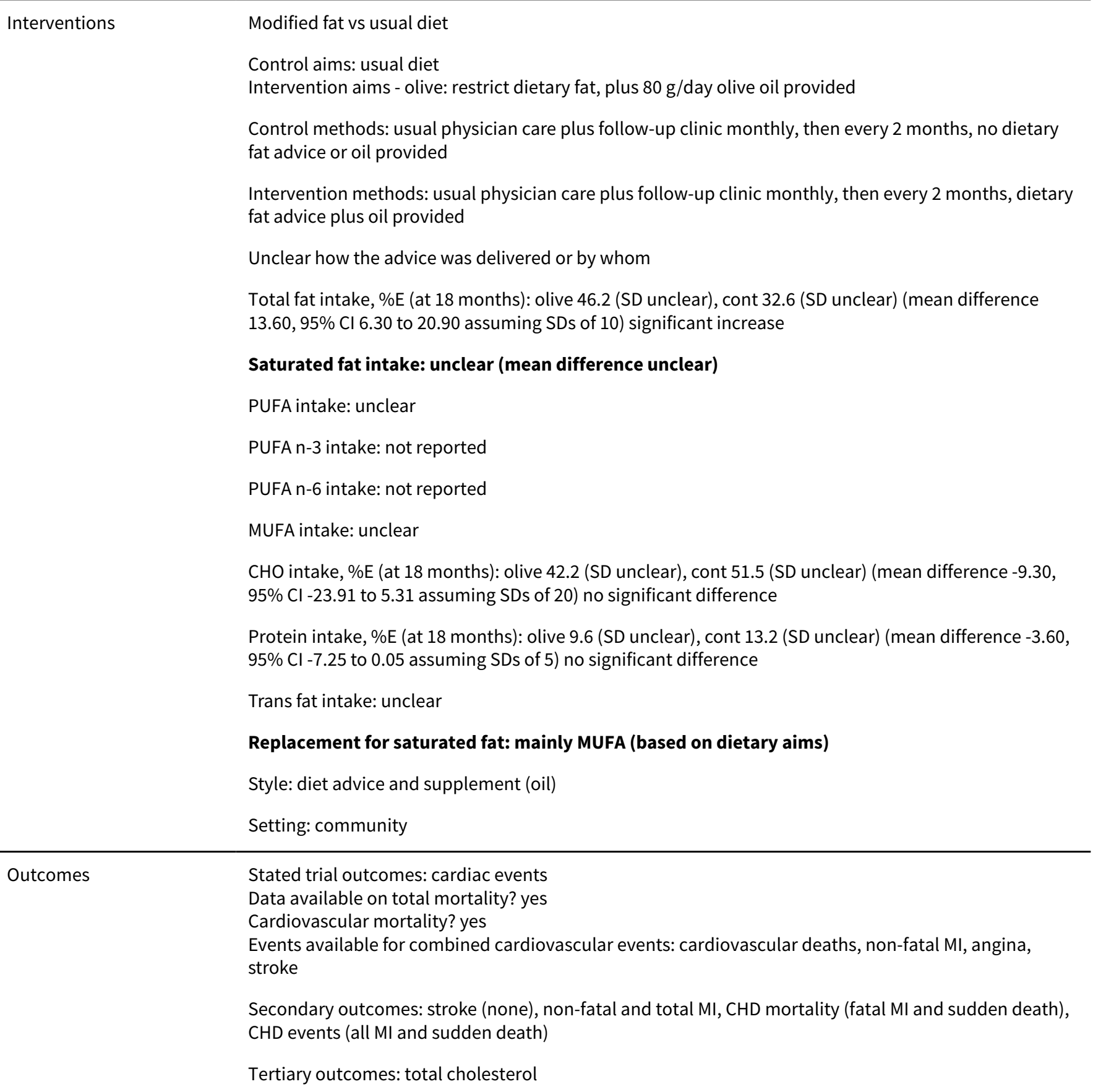

Notes Study duration 2 years 
Study aim was to reduce total fat (by restricting fatty meat, sausages, pastry, ice cream, cheese, cake, milk, eggs and butter) and prescribe vegetable oil (so reducing saturates), but saturated fat intakes during intervention were not reported

\section{SFA reduction aimed (but unclear whether achieved as SFA intake not reported)}

Total serum cholesterol, difference between intervention and control, mmol/L: 0.30 (95\% Cl -0.93 to 1.53), NO statistically significant reduction, mean total cholesterol rose

Trial dates: unclear, published in 1965

Funding: probably unfunded (they thank the Paddington General Hospital for clinic facilities, and St Mary's and Paddington General Hospital physicians for referral of patients, but no funding acknowledged)

Declarations of Interest of primary researchers: none stated, all authors worked for academic or health institutions.

\section{Risk of bias}

\begin{tabular}{lll}
\hline Bias & Authors' judgement & Support for judgement \\
\hline $\begin{array}{l}\text { Random sequence genera- } \\
\text { tion (selection bias) }\end{array}$ & Low risk & $\begin{array}{l}\text { Trial was stated as "randomised" but without further detail, apart from use of } \\
\text { a sealed envelope as below. }\end{array}$ \\
\hline
\end{tabular}

\begin{tabular}{|c|c|c|}
\hline $\begin{array}{l}\text { Allocation concealment } \\
\text { (selection bias) }\end{array}$ & Unclear risk & $\begin{array}{l}\text { When a new participant was accepted for the trial a sealed envelope was } \\
\text { opened containing the allocation instructions. In the case of participants allo- } \\
\text { cated to an oil group, the instructions referred only to a code number. }\end{array}$ \\
\hline
\end{tabular}

\begin{tabular}{ll}
\hline $\begin{array}{l}\text { Blinding of participants } \\
\text { and personnel (perfor- }\end{array}$ & High risk
\end{tabular}$\quad \begin{aligned} & \text { The physicians in charge knew which participants were receiving oil, but they } \\
& \text { did not know until the end of the trial the kind of oil that they were receiving. }\end{aligned}$
mance bias)

All outcomes

\begin{tabular}{|c|c|c|}
\hline $\begin{array}{l}\text { Blinding of outcome as- } \\
\text { sessment (detection bias) }\end{array}$ & Low risk & $\begin{array}{l}\text { The electrocardiograms were assessed without the knowledge of the partici- } \\
\text { pant's treatment group. }\end{array}$ \\
\hline
\end{tabular}

CVD outcomes

Blinding of outcome as-
sessment (detection bias)
All-cause mortality

Blinding is not relevant in assessment of mortality.

\begin{tabular}{lll}
\hline $\begin{array}{l}\text { Incomplete outcome data } \\
\text { (attrition bias) }\end{array}$ & Unclear risk & Some lost to follow-up by 2 years, so some events may have been missed \\
All outcomes & \\
\hline $\begin{array}{l}\text { Selective reporting (re- } \\
\text { porting bias) }\end{array}$ & Low risk & $\begin{array}{l}\text { Not relevant for primary and secondary outcomes as all trialists were asked for } \\
\text { data. }\end{array}$
\end{tabular}

\begin{tabular}{lll}
\hline $\begin{array}{l}\text { Free of systematic differ- } \\
\text { ence in care? }\end{array}$ & Low risk & $\begin{array}{l}\text { All received conventional treatments at the discretion of the physicians, all at- } \\
\text { tended a special follow-up clinic. See control and intervention methods in the } \\
\text { Interventions section of the table of Characteristics of included studies }\end{array}$ \\
\hline Stated aim to reduce SFA & Low risk & Aim to reduce SFA stated \\
\hline Achieved SFA reduction & Unclear risk & SFA intake not reported \\
\hline Achieved TC reduction & High risk & $\begin{array}{l}\text { Although the TC in the intervention group was } 0.58 \text { mmol/L lower than in the } \\
\text { control group, this was not statistically significant in this small study. }\end{array}$ \\
\hline
\end{tabular}


Rose olive 1965 (Continued)
Other bias
Low risk
None noted

Simon 1997

\section{Study characteristics}

\begin{tabular}{ll}
\hline Methods & RCT \\
& Summary risk of bias: moderate to high
\end{tabular}

Women with a high risk of breast cancer (USA)
CVD risk: low
Control: randomised 96, analysed 75
Intervention: randomised 98, analysed 72
Mean years in trial: control 1.8, intervention 1.7
\% male: 0
Age: mean control 46, intervention 46
Ethnicity: White $89 \%$, African-American 9\%, Hispanic 2\%
Statins use allowed? No (those on lipid-lowering medications were excluded)
\% taking statins: $0 \%$

Interventions

Reduced fat vs usual diet

Control aims: usual diet

Intervention aims: total fat $15 \% \mathrm{E}$

Control methods: continued usual diet

Intervention methods: Bi-weekly individual dietetic appointments over 3 months followed by monthly individual or group appointments, including education, goal-setting, evaluation, feedback and selfmonitoring

Intervention delivered face-to-face by a dietitian

Total fat intake, \%E (at 12 months)§: int 17.6 (SD 5.8), cont 33.8 (SD 7.4) (mean difference -16.20, 95\% Cl -18.34 to -14.06$)$ significant reduction

\section{Saturated fat intake, \%E (at 12 months)§: int 6.0 (SD 3.0), cont 12.1 (SD 5.2) (mean difference $-6.10,95 \% \mathrm{Cl}-7.47$ to -4.73 ) significant reduction}

PUFA intake, \%E (at 12 months)§: int 3.8 (SD 1.7), cont 7.3 (SD 4.1) (mean difference -3.50, 95\% Cl -4.51 to -2.49 ) significant reduction

PUFA n-3 intake: not reported

PUFA n-6 intake: not reported

MUFA intake, \%E (at 12 months)§: int 6.1 (SD 3.0), cont 12.8 (SD 6.3) (mean difference -6.70, 95\% Cl -8.29 to -5.11) significant reduction

$\mathrm{CHO}$ intake: not reported

Protein intake: not reported

Trans fat intake: not reported

Replacement for saturated fat: unclear, either carbohydrate or protein (based on aims and achievements) 
Simon 1997 (Continued)

\section{Style: diet advice}

Setting: community

§Kasim 1993

\begin{tabular}{|c|c|}
\hline Outcomes & $\begin{array}{l}\text { Stated trial outcomes: intervention feasibility } \\
\text { Data available on total mortality? yes ( } 2 \text { deaths, but not clear in which arms) } \\
\text { Cardiovascular mortality? no } \\
\text { Events available for combined cardiovascular events: none } \\
\text { Secondary outcomes: cancer diagnosis ( } 8 \text { diagnoses, but not clear in which arms) } \\
\text { Tertiary outcomes: weight, total, LDL and HDL cholesterol, TGs }\end{array}$ \\
\hline Notes & $\begin{array}{l}\text { Study duration } 2 \text { years } \\
\text { Study aim was to reduce total fat to } 15 \% E \text { (saturated fat not mentioned), but saturated fat intake in the } \\
\text { intervention group was significantly lower than in the control group } \\
\text { SFA reduction achieved } \\
\text { Total serum cholesterol, difference between intervention and control, } \mathbf{m m o l} / \mathbf{L}: \mathbf{- 0 . 3 4}(\mathbf{9 5 \%} \mathbf{~ C l} \\
\mathbf{- 0 . 6 4} \text { to -0.04), statistically significant reduction } \\
\text { Trial dates: Recruitment } 1987 \text { to } 1989 \\
\text { Funding: Marilyn J Smith Fund, Harper-Grace Hospitals, the Wesley Foundation, National Cancer Insti- } \\
\text { tute, Karmanos Cancer Institute Core Grant, the United Foundation of Detroit } \\
\text { Declarations of Interest of primary researchers: none stated, all authors worked for academic or health } \\
\text { institutions except PN Kim who was affiliated with Wesley Health Strategies (now Health Strategies, } \\
\text { which offers a "full-service health and fitness centre with an educated fitness staff and spacious work- } \\
\text { out areas", see healthstrategiesfitness.com/) }\end{array}$ \\
\hline
\end{tabular}

\section{Risk of bias}

\begin{tabular}{|c|c|c|}
\hline Bias & Authors' judgement & Support for judgement \\
\hline $\begin{array}{l}\text { Random sequence genera- } \\
\text { tion (selection bias) }\end{array}$ & Low risk & $\begin{array}{l}\text { Randomisation method not clearly described, but participants were stratified } \\
\text { by age and randomised (block size } 2 \text { ). }\end{array}$ \\
\hline $\begin{array}{l}\text { Allocation concealment } \\
\text { (selection bias) }\end{array}$ & Unclear risk & Allocation method not clearly described \\
\hline $\begin{array}{l}\text { Blinding of participants } \\
\text { and personnel (perfor- } \\
\text { mance bias) } \\
\text { All outcomes }\end{array}$ & High risk & Participants knew their allocation. \\
\hline $\begin{array}{l}\text { Blinding of outcome as- } \\
\text { sessment (detection bias) } \\
\text { CVD outcomes }\end{array}$ & Unclear risk & Unclear whether physicians knew allocations \\
\hline $\begin{array}{l}\text { Blinding of outcome as- } \\
\text { sessment (detection bias) } \\
\text { All-cause mortality }\end{array}$ & Low risk & Blinding is not relevant in assessment of mortality. \\
\hline $\begin{array}{l}\text { Incomplete outcome data } \\
\text { (attrition bias) } \\
\text { All outcomes }\end{array}$ & Unclear risk & $\begin{array}{l}\text { Unclear, deaths, cancer and CV events are dropouts - unclear if any data miss- } \\
\text { ing }\end{array}$ \\
\hline
\end{tabular}


Simon 1997 (Continued)

\begin{tabular}{lll}
$\begin{array}{l}\text { Selective reporting (re- } \\
\text { porting bias) }\end{array}$ & Low risk & $\begin{array}{l}\text { Not relevant for primary and secondary outcomes as all trialists were asked for } \\
\text { data }\end{array}$ \\
\hline $\begin{array}{l}\text { Free of systematic differ- } \\
\text { ence in care? }\end{array}$ & High risk & $\begin{array}{l}\text { Very different contact time with dietitian, but medical appointments same in } \\
\text { both groups. See control and intervention methods in the Interventions sec- } \\
\text { tion of the table of Characteristics of included studies }\end{array}$ \\
\hline Stated aim to reduce SFA & High risk & Aim to reduce SFA not stated \\
\hline Achieved SFA reduction & Low risk & SFA reduction achieved \\
\hline Achieved TC reduction & Low risk & Statistically significant TC fall \\
\hline Other bias & Low risk & None noted
\end{tabular}

\section{STARS 1992}

\section{Study characteristics}

$\begin{array}{ll}\text { Methods } & \text { RCT } \\ \text { St Thomas' Atherosclerosis Regression Study (STARS) } \\ \text { Summary risk of bias: moderate to high for CVD outcomes, low for all-cause mortality }\end{array}$

Men with angina referred for angiography (UK)
CVD risk: high
Control: unclear how many randomised (30?), analysed 24
Intervention: unclear how many randomised (30?), analysed 26
Mean years in trial: control 2.9, intervention 3.0
\% male: $100 \%$
Age: mean control 53.9, intervention 48.9 (all <66)
Ethnicity: not stated
Statins use allowed? No (1 arm of the trial, not described here, prescribed cholestyramine)
\% taking statins: $0 \%$

Interventions

Reduced and modified fat diet vs usual diet

Control aims: no diet intervention but advised to lose weight if $\mathrm{BMI}>25$

Intervention aims: total fat 27\%E, SFA 8 - 10\%E, omega-3 and omega-6 PUFA $8 \%$ E, increase in plant-derived soluble fibre, dietary cholesterol $100 \mathrm{mg} / 1000 \mathrm{kcal}$, advised to lose weight if BMI > 25

Control methods: usual care but no formal dietetic counselling. They were counselled against smoking if appropriate and advised about daily exercise level.

Intervention methods: Usual care plus dietetic individual assessment of diet and advice. Further dietetic counselling and food stuffs were given to participants who did not achieve or maintain certain levels of serum cholesterol reduction

Initial intervention was delivered individually face-to-face by a dietitian and follow-up by a clinician.

Total fat intake, \%E (through study): int 27 (SD 7), cont 37 (SD 5) (mean difference $-10.00,95 \% \mathrm{Cl}-13.35$ to -6.65 ) significant reduction

Saturated fat intake, \%E (through study): int 9 (SD 3), cont 16 (SD 4) (mean difference $-7.00,95 \%$ $\mathrm{Cl}-8.97$ to -5.03 ) significant reduction 
PUFA intake, \%E (through study)§: int 7 (SD 2), cont 5 (SD 2) (mean difference $2.00,95 \% \mathrm{Cl} 0.89$ to 3.11 ) significant increase

PUFA n-3 intake: not reported

PUFA n-6 intake: not reported

MUFA intake, \%E (through study)§: int 10 (SD 4), cont 17 (SD 5) (mean difference -7.00, 95\% Cl -9.52 to -4.48) significant reduction

$\mathrm{CHO}$ intake, \%E (through study)§: int 49 (SD 7), cont 41 (SD 7) (mean difference 8.00, $95 \% \mathrm{Cl} 4.12$ to

11.88) significant increase

Protein intake, \%E (through study)§: int 19 (SD 4), cont 18 (SD 2) (mean difference $1.00,95 \% \mathrm{Cl}-0.73$ to

2.73) no significant effect

Trans fat intake: not reported

Replacement for saturated fat: $\mathrm{CHO}$ and PUFA (based on aims and achievements)

Style: diet advice

Setting: community

§Blann 1995

Stated trial outcomes: angiography
Data available on total mortality? yes
Cardiovascular mortality? yes
Events available for combined cardiovascular events: cardiovascular deaths, non-fatal MI, angina,
stroke, CABG, angioplasty, stroke, total MI, CHD events, plus cancer deaths (none)
Secondary outcomes: total, HDL, LDL cholesterol, TGs, total/HDL and LDL/HDL ratios, 2-hour post-load
glucose (weight and BP "remained similar" but were not reported, Lp(a) reported but as geometric
means)

Notes Study duration: 3 years

Study aim was to reduce saturated fats (to $8-10 \% \mathrm{E}$ ), and saturated fat intake in the intervention group was significantly reduced

\section{SFA reduction aimed and achieved}

Total serum cholesterol, difference between intervention and control, $\mathrm{mmol} / \mathrm{L}:-0.76(95 \% \mathrm{Cl}$ -1.19 to -0.33$)$, statistically significant reduction

Trial dates: Study dates not reported (published in 1992)

Funding: Unilever plc, the Chemical Pathology Fund of St Thomas' Hospital, and Bristol-Meyers Ltd

Declarations of Interest of primary researchers: none stated, all authors worked for academic or health institutions.

\section{Risk of bias}

\begin{tabular}{lll}
\hline Bias & Authors' judgement & Support for judgement \\
\hline $\begin{array}{l}\text { Random sequence genera- } \\
\text { tion (selection bias) }\end{array}$ & Low risk & "blinded random cards issued centrally by statistician advisor" \\
\hline $\begin{array}{l}\text { Allocation concealment } \\
\text { (selection bias) }\end{array}$ & Low risk & "blinded random cards issued centrally by statistician advisor" \\
\hline
\end{tabular}


STARS 1992 (Continued)

Blinding of participants High risk Participant blinding: inadequate and personnel (performance bias)

All outcomes

\begin{tabular}{|c|c|c|}
\hline $\begin{array}{l}\text { Blinding of outcome as- } \\
\text { sessment (detection bias) } \\
\text { CVD outcomes }\end{array}$ & Unclear risk & Physician blinding: unclear \\
\hline $\begin{array}{l}\text { Blinding of outcome as- } \\
\text { sessment (detection bias) } \\
\text { All-cause mortality }\end{array}$ & Low risk & Blinding is not relevant in assessment of mortality. \\
\hline $\begin{array}{l}\text { Incomplete outcome data } \\
\text { (attrition bias) } \\
\text { All outcomes }\end{array}$ & Unclear risk & $\begin{array}{l}\text { Unclear, deaths, cancer and CV events are dropouts - unclear if any data miss- } \\
\text { ing }\end{array}$ \\
\hline $\begin{array}{l}\text { Selective reporting (re- } \\
\text { porting bias) }\end{array}$ & Low risk & $\begin{array}{l}\text { Not relevant for primary and secondary outcomes as all trialists were asked for } \\
\text { data }\end{array}$ \\
\hline $\begin{array}{l}\text { Free of systematic differ- } \\
\text { ence in care? }\end{array}$ & High risk & $\begin{array}{l}\text { Usual care in both groups, dietetic counselling only in the intervention group. } \\
\text { See control and intervention methods in the Interventions section of the table } \\
\text { of Characteristics of included studies }\end{array}$ \\
\hline Stated aim to reduce SFA & Low risk & Aim to reduce SFA stated \\
\hline Achieved SFA reduction & Low risk & SFA reduction achieved \\
\hline Achieved $\mathrm{TC}$ reduction & Low risk & Statistically significant TC fall \\
\hline Other bias & Low risk & None noted \\
\hline
\end{tabular}

Sydney Diet-Heart 1978

\section{Study characteristics}

\begin{tabular}{ll}
\hline Methods & RCT \\
& Sydney Diet-Heart Trial \\
& Summary risk of bias: moderate to high \\
\hline Participants & Men with previous MI (Australia) \\
CVD risk: high & Control: randomised 237, analysed 221 at 2 years \\
Intervention: randomised 221, analysed 205 at 2 years & Mean years in trial: control 4.3, intervention 4.3 \\
& $\%$ male: 100 \\
& Age: mean control 49.1 (SD 6.5), intervention 48.7 (SD 6.8) \\
& Ethnicity: not stated \\
& Statins use allowed? Unclear (use of medication did not appear to be an exclusion criteria) \\
& $\%$ taking statins: Not reported (probably none as too early, pre-1980) \\
\hline Interventions & Modified fat diet vs usual diet \\
\hline
\end{tabular}


Sydney Diet-Heart 1978 (Continued)

Control aims: reduction in energy if overweight, no other specific dietary advice, allowed to use PUFA margarine instead of butter

Intervention aims: SFA $10 \%$ E, PUFA $15 \%$ E, reduction in energy if overweight, dietary chol $<300 \mathrm{mg} /$ day

Control methods: no specific dietary instruction (except re weight)

Intervention methods: advised and tutored individually, diet assessed 3 times in 1st year and twice annually thereafter

Intervention was delivered face-to-face individually but unclear by whom

Total fat intake, \%E ("during follow-up"): int 38.3 (SD 5.9), cont 38.1 (SD 5.4) (mean difference 0.20, 95\% $\mathrm{Cl}-0.88$ to 1.28$)$ no significant difference

Saturated fat intake, \%E ("during follow-up"): int 9.8 (SD 2.6), cont 13.5 (SD 3.2) (mean difference $-3.70,95 \% \mathrm{Cl}-4.25$ to -3.15 ) significant reduction

PUFA intake, \%E ("during follow-up"): int 15.1 (SD 4.3), cont 8.9 (SD 3.5) (mean difference 6.20, 95\% Cl 5.45 to 6.95$)$ significant increase

PUFA n-3 intake: not reported

PUFA n-6 intake: not reported

MUFA intake, \%E ("during follow-up"): int 11.5 (SD 2.1), cont 13.8 (SD 2.5) (mean difference -2.30, 95\%

$\mathrm{Cl}-2.74$ to -1.86$)$ significant reduction

CHO intake, \%E ("during follow-up"): int 40.9 (SD 7.3), cont 40.3 (SD 7.3) (mean difference $0.60,95 \% \mathrm{Cl}$ -0.79 to 1.99$)$ no significant difference

Protein intake, \%E ("during follow-up"): int 15.2 (SD 2.8), cont 15.7 (SD 3.4) (mean difference -0.50, 95\% $\mathrm{Cl}-1.09$ to 0.09$)$ no significant difference

Trans fat intake: not reported

Primary replacement for saturated fat: mainly PUFA (based on dietary aims and achievements)

Style: diet advice

Setting: community

Outcomes Stated trial outcomes: cardiovascular mortality and morbidity

Data available on total mortality? yes

Cardiovascular mortality? yes (exact events included not stated)

Events available for combined cardiovascular events: none

Secondary outcomes: CHD deaths (exact events included not stated)

Tertiary outcomes: total cholesterol, TG, BMI, sBP, dBP

Notes Study duration 7 years

Study aim was saturated fat $10 \% \mathrm{E}$, and saturated fat intake in the intervention group was less than $80 \%$ of that in the control $(73 \%)$

\section{SFA reduction aimed and achieved}

Total serum cholesterol, difference between intervention and control, $\mathrm{mmol} / \mathrm{L:}-\mathbf{- 0 . 3 0}(95 \% \mathrm{Cl}$ -0.51 to -0.09$)$, statistically significant reduction

Trial dates: Recruitment 1966 to [unclear] and followed for 2 to 7 years

Funding: Life Insurance Medical Research Fund of Australia and New Zealand 
Sydney Diet-Heart 1978 (Continued)

Declarations of Interest of primary researchers: none stated, all authors worked for academic or health institutions.

\section{Risk of bias}

\begin{tabular}{|c|c|c|}
\hline Bias & Authors' judgement & Support for judgement \\
\hline $\begin{array}{l}\text { Random sequence genera- } \\
\text { tion (selection bias) }\end{array}$ & Low risk & $\begin{array}{l}\text { Quote: "table of random numbers ... generated by a research assistant and } \\
\text { was concealed until after medical evaluations and testing at baseline were } \\
\text { completed". }\end{array}$ \\
\hline $\begin{array}{l}\text { Allocation concealment } \\
\text { (selection bias) }\end{array}$ & Low risk & As above \\
\hline $\begin{array}{l}\text { Blinding of participants } \\
\text { and personnel (perfor- } \\
\text { mance bias) } \\
\text { All outcomes }\end{array}$ & High risk & $\begin{array}{l}\text { Very difficult to blind trials where participants need to make their own dietary } \\
\text { changes }\end{array}$ \\
\hline $\begin{array}{l}\text { Blinding of outcome as- } \\
\text { sessment (detection bias) } \\
\text { CVD outcomes }\end{array}$ & Low risk & Initially masked to group assignment (though success of blinding not checked) \\
\hline $\begin{array}{l}\text { Blinding of outcome as- } \\
\text { sessment (detection bias) } \\
\text { All-cause mortality }\end{array}$ & Low risk & Blinding is not relevant in assessment of mortality. \\
\hline $\begin{array}{l}\text { Incomplete outcome data } \\
\text { (attrition bias) } \\
\text { All outcomes }\end{array}$ & Low risk & Survival analysis used \\
\hline $\begin{array}{l}\text { Selective reporting (re- } \\
\text { porting bias) }\end{array}$ & Low risk & $\begin{array}{l}\text { Not relevant for primary and secondary outcomes as all trialists were asked for } \\
\text { data }\end{array}$ \\
\hline $\begin{array}{l}\text { Free of systematic differ- } \\
\text { ence in care? }\end{array}$ & High risk & $\begin{array}{l}\text { Advice and follow-up in intervention group, not in control. See control and in- } \\
\text { tervention methods in the Interventions section of the table of Characteristics } \\
\text { of included studies }\end{array}$ \\
\hline Stated aim to reduce SFA & Low risk & Aim to reduce SFA stated \\
\hline Achieved SFA reduction & Low risk & SFA reduction achieved \\
\hline Achieved $\mathrm{TC}$ reduction & Low risk & Statistically significant TC fall \\
\hline Other bias & Low risk & None noted \\
\hline
\end{tabular}

\section{Veterans Admin 1969}

\section{Study characteristics}

\begin{tabular}{ll}
\hline Methods & RCT \\
& Summary risk of bias: moderate to high \\
\hline Participants & $\begin{array}{l}\text { Men living at the Veterans Administration Center (USA) } \\
\text { CVD risk: low }\end{array}$ \\
\hline
\end{tabular}


Veterans Admin 1969 (Continued)

Control: randomised 422, analysed 422

Intervention: randomised 424, analysed 424

Mean years in trial: control 3.7, intervention 3.7

$\%$ male: 100

Age: mean control 65.6, intervention 65.4 (all 54 - 88)

Ethnicity: White 90\%, African-American 7\%, Asian 1\%, Mexican 1\%, other $1 \%$

Statins use allowed? Unclear (only 4 participants were taking nicotinic acid, 17 diuretics, 56 digitalis, none on heparin)

\% taking statins: Not reported (probably none as too early, pre-1980)

Interventions
Modified fat vs usual diet

Control aims: provided, total fat $40 \% \mathrm{E}$

Intervention aims: total fat $40 \% \mathrm{E}, 2 / 3$ of SFA replaced by unsaturated fats, dietary chol reduced

Control methods: whole diet provided

Intervention methods: whole diet provided

Total fat intake, \%E (during trial): int 38.9 (SD unclear), cont 40 (SD unclear) (mean difference -1.10, $95 \% \mathrm{Cl}-2.45$ to 0.25 assuming SDs of 10 ) no significant difference

Saturated fat intake, \%E (during trial): int 8.3 (SD unclear), cont 18.5 (SD unclear) (mean difference $-10.20,95 \% \mathrm{Cl}-10.87$ to -9.53 assuming SDs of 5 ) significant reduction

PUFA intake, \%E (during trial)§: int 16.0 (SD ?), cont 4.9 (SD 0.10) (mean difference $11.10,95 \% \mathrm{Cl} 10.62$ to 11.58 assuming missing SD was 5) significant increase

PUFA n-3 intake: not reported

PUFA n-6 intake: not reported

MUFA intake, \%E (during trial) ) $95 \% \mathrm{Cl}-3.87$ to -2.53$)$ significant reduction

$\mathrm{CHO}$ intake, \%E (during trial) $\stackrel{\star}{*}$ : not reported, approx int 45.9, cont 44.8 (mean difference 1.10, 95\%

$\mathrm{Cl}-1.60$ to 3.80 assuming SDs of 20 ) no significant difference

Protein intake, \%E (during trial)§: int 15.2 (SD ?), cont 15.2 (SD ?) (mean difference $0.00,95 \% \mathrm{Cl}-0.67$ to 0.67 assuming SDs of 5) no significant difference

Trans fat intake: not reported

\section{Replacement for saturated fat: mainly PUFA (based on dietary aims and achievements)}

Style: diet provided

Setting: residential institution

§Dayton 1965

${ }_{*}^{\star}$ Est imated by subtraction (assuming total fat = SFA + PUFA + MUFA or energy intake = energy from fat $+\mathrm{CHO}+$ protein)
Stated trial outcomes: mortality, heart disease

Data available on total mortality? yes

Cardiovascular mortality? yes

Events available for combined cardiovascular events: sudden death, definite MI, definite stroke, angina, PVD events

Secondary outcomes: cancer deaths, cancer diagnoses, stroke, non-fatal MI, total MI, CHD deaths (fatal $\mathrm{MI}$ and sudden death due to $\mathrm{CHD}$ ), $\mathrm{CHD}$ events (any MI or sudden death due to $\mathrm{CHD}$ ) 
Veterans Admin 1969 (Continued)

Tertiary outcomes: none (some data on total cholesterol, but no variance info)

Notes Study duration over 8 years

Study aim was to replace $66 \%$ of saturated fat by unsaturated fats, and saturated fat intake in the intervention group was significantly lower than in control

\section{SFA reduction aimed and achieved}

Total serum cholesterol, difference between intervention and control, $\mathrm{mmol} / \mathrm{L}:-0.37(95 \% \mathrm{Cl}$ -0.77 to 0.03 ), NO statistically significant reduction but reduction $>0.20$

Trial dates: Recruitment 1959 to 1967

Funding: Veterans Administration, Arthur Dodd Fuller Foundation, National Heart Institute, Los Angeles County Heart Association, plus gifts of foods from Mazola corn oil and Mazola margarine, the National Soybean Processors Association, Pitman-Moore Company (Emdee margarine) and Hi-Saff Imitation Icecream from Frozen Desserts Company. Edgmar Farms donated milk refrigeration equipment.

Declarations of Interest of primary researchers: none stated, all authors worked for academic or health institutions.

\section{Risk of bias}

\begin{tabular}{lll}
\hline Bias & Authors' judgement & Support for judgement \\
\hline $\begin{array}{l}\text { Random sequence genera- } \\
\text { tion (selection bias) }\end{array}$ & Low risk & Quote: "table of random numbers used" \\
\hline $\begin{array}{l}\text { Allocation concealment } \\
\text { (selection bias) }\end{array}$ & Low risk & Extensive baseline assessment before randomisation \\
\hline $\begin{array}{l}\text { Blinding of participants } \\
\begin{array}{l}\text { and personnel (perfor- } \\
\text { mance bias) }\end{array}\end{array}$ & Low risk \\
$\begin{array}{l}\text { All outcomes } \\
\end{array}$ & & \\
\hline
\end{tabular}

\begin{tabular}{|c|c|c|}
\hline $\begin{array}{l}\text { Blinding of outcome as- } \\
\text { sessment (detection bias) } \\
\text { CVD outcomes }\end{array}$ & Low risk & Physician knowledge of allocation was assessed and found similar to random. \\
\hline
\end{tabular}

Blinding of outcome as-
$\begin{aligned} & \text { sessment (detection bias) } \\ & \text { All-cause mortality }\end{aligned}$

\begin{tabular}{lll}
\hline $\begin{array}{l}\text { Incomplete outcome data } \\
\text { (attrition bias) } \\
\text { All outcomes }\end{array}$ & Low risk & All followed up via Veterans Admin system \\
\hline $\begin{array}{l}\text { Selective reporting (re- } \\
\text { porting bias) }\end{array}$ & Low risk & $\begin{array}{l}\text { Not relevant for primary and secondary outcomes as all trialists were asked for } \\
\text { data }\end{array}$ \\
\hline $\begin{array}{l}\text { Free of systematic differ- } \\
\text { ence in care? }\end{array}$ & Low risk & $\begin{array}{l}\text { All ate centre food as usual. See control and intervention methods in the Inter- } \\
\text { ventions section of the table of Characteristics of included studies }\end{array}$ \\
\hline $\begin{array}{l}\text { Stated aim to reduce SFA } \\
\text { Achieved SFA reduction }\end{array}$ & Low risk & Aim to reduce SFA stated \\
\hline Achieved TC reduction & High risk & SFA reduction achieved \\
\hline \hline
\end{tabular}


Veterans Admin 1969 (Continued)
Other bias
Low risk
None noted

WHI 2006

\section{Study characteristics}

\begin{tabular}{ll}
\hline Methods & RCT \\
& Women's Health Initiative (WHI) \\
Summary risk of bias: low
\end{tabular}

Participants

Postmenopausal women aged 50 - 79 with or without CVD at baseline (USA)

CVD risk: low in those without CVD at baseline, high in those with CVD

Control without CVD at baseline: randomised 29,294, analysed 29,294

Intervention without CVD at baseline: randomised 19,541, analysed 19,541

Control with CVD at baseline: randomised 1369, analysed 1369

Intervention with CVD at baseline: randomised 908, analysed 908

Mean years in trial: control 8.1, intervention 8.1

$\%$ male: 0

Age: mean (both with and without CVD at baseline) int 62.3 (SD 6.9), control 62.3 (SD 6.9)

Ethnicity (women both with and without CVD at baseline): white $82 \%$, black $11 \%$, Asian or Pacific Islander $2 \%$, unknown $1 \%$, American Indian or Alaskan native $<1 \%$. No statistically significant effects of the intervention on CHD events was seen for any ethnic subgroup.

Statins use allowed? Yes

$\%$ taking statins: $12 \%$ of women recruited were on lipid-lowering medication (these were a mixture of participants with and without CVD at baseline).

Control: diet-related education materials

Intervention: low-fat diet (20\%E from fat), reduce saturated fat to $7 \% \mathrm{E}$ with increased fruit and vegetables

Control methods: given copy of 'Dietary Guidelines for Americans'

Intervention methods: 18 group sessions with trained and certified nutritionists in the 1st year, quarterly maintenance sessions thereafter, focusing on diet and behaviour modification

Intervention delivered face-to-face in a group by nutritionists

Intake data all relate to the full WHI cohort (not divided by whether participants have CVD at baseline or not)

Total fat intake, \%E (at 6 years): int 28.8 (SD 8.4), cont 37.0 (SD 7.3) (mean difference $-8.20,95 \% \mathrm{Cl}-8.34$ to -8.06) significant reduction

\section{Saturated fat intake, \%E (at 6 years): int 9.5 (SD3.2), cont 12.4 (SD3.1) (mean difference -2.90, $95 \% \mathrm{Cl}-2.96$ to -2.84 for full WHI population) significant reduction}

PUFA intake, \%E (at 6 years)§: int 6.3 (SD?), cont 7.6 (SD?) (mean difference $-1.30,95 \% \mathrm{Cl}-1.72$ to -0.88 assuming missing SDs were 5) significant reduction

PUFA n-3 intake: not reported

PUFA n-6 intake: not reported 
WHI 2006 (Continued)

MUFA intake, \%E (at 6 years)§: int 11.1 (SD?), cont 14.3 (SD?) (mean difference $-3.20,95 \% \mathrm{Cl}-3.62$ to

-2.78 assuming unclear SDs were 5 ) significant reduction

CHO intake, \%E (at 6 years)§: int 53.9 (SD?), cont 46.3 (SD?) (mean difference 7.60, 95\% Cl 5.91 to 9.29 assuming SDs of 20) significant increase

Protein intake, \%E (at 6 years)§: int 17.7 (SD?), cont 17.0 (SD?) (mean difference $0.70,95 \% \mathrm{Cl} 0.28$ to

1.12 assuming SDs of 5) significant increase

Trans fat intake, \%E (at 6 years)§: int 1.8 (SD?), cont 2.4 (SD?) (mean difference unclear, no SDs assumed)

Replacement for saturated fat: mainly carbohydrate, some protein (based on dietary achievement)

Style: dietary advice

Setting: community

$\S$ Amongst the 881 intervention and 1373 control participants with blood samples at baseline, with or without CVD at baseline (Howard 2010)

Data available on total mortality? yes*

Cardiovascular mortality? yes

Events available for combined cardiovascular events: $\mathrm{CHD}$, stroke, heart failure, angina, peripheral vascular disease, revascularisation, pulmonary embolism, DVT

Secondary outcomes: cancer deaths ${ }^{\star}$, cancer diagnoses ${ }^{\star}$, stroke, non-fatal MI, diabetes diagnosis*

Tertiary outcomes: weight, BMI, total, LDL and HDL cholesterol, TGs, systolic and diastolic BP (Lp(a) and HOMA reported as geometric means)

* these are only available for the whole cohort, not split between low and high CVD risk groups

Notes Study duration over 8 years

Study aim was to reduce total fat to $20 \% \mathrm{E}$, reduce saturated fat to $7 \% \mathrm{E}$ and increase fruit and vegetable intake (Patterson 2003), and saturated fat intake in the intervention group was significantly lower than in control

\section{SFA reduction aimed and achieved}

Total serum cholesterol, difference between intervention and control, $\mathrm{mmol} / \mathrm{L}:-0.09(95 \% \mathrm{Cl}$ -0.15 to -0.02 ), statistically significant reduction

Trial dates: Recruitment was between 1993 and 1998

Funding: National Heart, Lung and Blood Institute of the National Institutes of Health

Declarations of Interest of primary researchers: Declarations varied from paper to paper, but this is a typical one from Beresford 2006 "Dr Black has received research grants from Pfizer and AstraZeneca, was on the speakers bureaus for Pfizer, Novartis, Sanofi-Aventis, Bristol-Meyers Squibb, Searle, Pharmacia, and Boehringer and served as a consultant of on an advisory board for Myogen, Merck Sharp and Dohme, Novartis, Mylan-Bertek, Pfizer, Bristol-Meyers Squibb, and Sanofi-Aventis. Dr Howard has served on the advisory boards of Merck, Schering Plough, and the Egg Nutrition Council, has received research support from Merck and Pfizer, and has consulted for General Millls. Dr Assaf is an employee of Pfizer. No other disclosures were reported."

\section{Risk of bias}

Bias Authors' judgement Support for judgement


WHI 2006 (Continued)

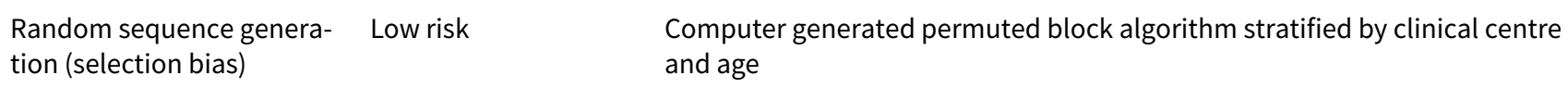

Allocation concealment Low risk Allocations developed by the WHI Clinical Coordinating Center

(selection bias)

Blinding of participants High risk Participants aware of allocation
and personnel (perfor-
mance bias)
All outcomes

\begin{tabular}{lll}
\hline $\begin{array}{l}\text { Blinding of outcome as- } \\
\text { sessment (detection bias) }\end{array}$ & Low risk & $\begin{array}{l}\text { Trained clinic staff, who were responsible for anthropometric assessments and } \\
\text { administration of FFQs, were blinded to treatment assignments to the extent } \\
\text { practical. The dietary intervention staff did not conduct clinical assessments, } \\
\text { and clinic staff were not permitted to } \\
\text { participate in any intervention activities; participants were instructed not to } \\
\text { discuss nutrition activities with clinic staff. }\end{array}$
\end{tabular}

Blinding of outcome as- Low risk Blinding not relevant for mortality assessment
sessment (detection bias)

All-cause mortality

Incomplete outcome data Low risk ITT analysis
(attrition bias)
All outcomes

\begin{tabular}{|c|c|c|}
\hline $\begin{array}{l}\text { Selective reporting (re- } \\
\text { porting bias) }\end{array}$ & Low risk & $\begin{array}{l}\text { Trials register 1999, study completion } 2005 \text {, but outcomes not stated in trials } \\
\text { register. However, outcomes were well published; trialists were asked for data. }\end{array}$ \\
\hline $\begin{array}{l}\text { Free of systematic differ- } \\
\text { ence in care? }\end{array}$ & High risk & $\begin{array}{l}\text { Intervention participants received } 18 \text { group sessions with behavioural mod- } \\
\text { ification plus quarterly maintenance sessions thereafter; control groups re- } \\
\text { ceived a leaflet. See control and intervention methods in the Interventions } \\
\text { section of the table of Characteristics of included studies }\end{array}$ \\
\hline Stated aim to reduce SFA & Low risk & Aim to reduce SFA stated \\
\hline Achieved SFA reduction & Low risk & SFA reduction achieved \\
\hline Achieved TC reduction & Low risk & Statistically significant TC fall \\
\hline Other bias & Low risk & None noted \\
\hline
\end{tabular}

WINS 2006

\section{Study characteristics}

\begin{tabular}{ll}
\hline Methods & RCT \\
& Women's Intervention Nutrition Study (WINS) \\
& Summary risk of bias: low \\
\hline Participants & Women with localised resected breast cancer (USA) \\
CVD risk: low \\
Control: 1462 randomised, 1462 analysed
\end{tabular}


WINS 2006 (Continued)

Intervention: 975 randomised, 975 analysed

Mean years in trial: overall 5.0

$\%$ men: 0

Age: control mean 58.5 ( $95 \% \mathrm{Cl} 43.6$ to 73.4 ), intervention mean 58.6 (95\% Cl 44.4 to 72.8 ) (all postmenopausal)

Ethnicity: $85 \%$ white, 5\% black, 4\% Hispanic, 5\% Asian or Pacific Islander, < 1\% American Indian or unknown (no outcome data based on ethnicity)

Statins use allowed? Not stated (statins not mentioned in inclusion or exclusion criteria within trial protocol)

$\%$ taking statins: Not reported

Control aims: minimal nutritional counselling focused on nutritional adequacy Intervention aims: total fat $15-20 \% \mathrm{E}$

Control methods: 1 baseline dietetic session plus 3-monthly sessions

Intervention methods: 8 bi-weekly individual dietetic sessions plus 3-monthly contact and optional monthly group sessions, incorporating individual fat gram goals, social cognitive theory, self-monitoring, goal-setting, modelling, social support and relapse prevention and management

Intervention was delivered face-to-face individually by trained dietitian

Total fat intake, \%E (at 1 year): int 20.3 (SD 8.1), cont 29.2 (SD 7.4) (mean difference $-8.90,95 \% \mathrm{Cl}-9.53$ to -8.27 )

Total fat intake, \%E (at 5 years): int 23.2 (SD 8.4) $n=380$, cont 31.2 (SD 8.9) $n=648$ (mean difference $-8.00,95 \% \mathrm{Cl}-9.09$ to -6.91$)$ significant reduction

Saturated fat intake ${ }^{\star}, \%$ (at 1 year): int 6.4 (SD 0.14 [4.4]), cont 9.8 (SD 0.15 [5.7]) (mean difference $-3.40,95 \% \mathrm{Cl}-3.80$ to -3.00 assuming reported $\mathrm{SDs}$ were actually $\mathrm{SEs}$ ) significant reduction

PUFA intake ${ }^{\star}, \%$ (at 1 year): int 4.5 (SD 0.09 (2.8)), cont 6.4 (SD 0.10 (3.8)) (mean difference -1.90, 95\% $\mathrm{Cl}-2.16$ to -1.64$)$ significant reduction

PUFA n-3 intake: not reported by study arm

PUFA $n-6$ intake: not reported by study arm

MUFA intake ${ }^{\star}, \% \mathrm{E}$ (at 1 year): int 7.6 (SD 0.14 (4.4)), cont 11.5 (SD 0.16 (6.1)) (mean difference -3.90, 95\%

$\mathrm{Cl}-4.32$ to -3.48$)$ significant reduction

CHO intake, \%E (at 6 months): int 60.8 (SD 19.6), cont 50.5 (SD 14.8) (mean difference 10.30, 95\% Cl 8.85 to 11.75 ) significant increase

Protein intake, \%E (at 6 months): int 19.1 (SD 5.2), cont 17.6 (SD 4.1) (mean difference 1.50, 95\% Cl 1.11 to 1.89 ) significant increase

Trans fat intake: not reported

Replacement for saturated fat: $\mathrm{CHO}$ and protein (based on dietary achievement)

Style: dietary advice

Setting: community

${ }^{\star}$ SDs appear incorrect, probably SEs? 
WINS 2006 (Continued)

Data available on total mortality? yes

Cardiovascular mortality? no

Events available for combined cardiovascular events: none

Secondary outcomes: cancer diagnoses

Tertiary outcomes: weight, BMI, total cholesterol

Study duration 5 years
Study aim was to reduce total fat to $15-20 \% \mathrm{E}$
SFA reduction achieved
Total serum cholesterol, difference between intervention and control, $\mathrm{mmol} / \mathrm{L}:-0.14(95 \% \mathrm{Cl}$
$-\mathbf{0 . 3 4}$ to 0.05$),$ NO statistically significant reduction and reduction $<0.20$

Trial dates: Recruitment 1994 to 2001

Funding: National Cancer Institute, Breast Cancer Research Foundation, American Institute for Cancer Research

Declarations of Interest of primary researchers: none stated, all authors worked for academic or health institutions except that Njeri Karanja worked for Kaiser Permanente Center for Health Research, Bette Caan for Kaiser Permanente Medical Group, and Barbara L Winters for Campbell's Soup Company.

\section{Risk of bias}

\begin{tabular}{|c|c|c|}
\hline Bias & Authors' judgement & Support for judgement \\
\hline $\begin{array}{l}\text { Random sequence genera- } \\
\text { tion (selection bias) }\end{array}$ & Low risk & $\begin{array}{l}\text { Random stratified permuted block design, carried out at the statistical coordi- } \\
\text { nating centre of WINS }\end{array}$ \\
\hline $\begin{array}{l}\text { Allocation concealment } \\
\text { (selection bias) }\end{array}$ & Low risk & $\begin{array}{l}\text { Random stratified permuted block design, carried out at the statistical coordi- } \\
\text { nating centre of WINS }\end{array}$ \\
\hline $\begin{array}{l}\text { Blinding of participants } \\
\text { and personnel (perfor- } \\
\text { mance bias) } \\
\text { All outcomes }\end{array}$ & High risk & Not for dietary advice and participants \\
\hline $\begin{array}{l}\text { Blinding of outcome as- } \\
\text { sessment (detection bias) } \\
\text { CVD outcomes }\end{array}$ & Low risk & All outcomes assessed by the blinded outcome committee \\
\hline $\begin{array}{l}\text { Blinding of outcome as- } \\
\text { sessment (detection bias) } \\
\text { All-cause mortality }\end{array}$ & Low risk & Outcome assessors blinded \\
\hline $\begin{array}{l}\text { Incomplete outcome data } \\
\text { (attrition bias) } \\
\text { All outcomes }\end{array}$ & Low risk & All assessed \\
\hline $\begin{array}{l}\text { Selective reporting (re- } \\
\text { porting bias) }\end{array}$ & Low risk & $\begin{array}{l}\text { Not relevant for primary and secondary outcomes as all trialists were asked for } \\
\text { data }\end{array}$ \\
\hline $\begin{array}{l}\text { Free of systematic differ- } \\
\text { ence in care? }\end{array}$ & High risk & $\begin{array}{l}\text { Differences in attention - more time for those in intervention group. See con- } \\
\text { trol and intervention methods in the Interventions section of the table of Char- } \\
\text { acteristics of included studies }\end{array}$ \\
\hline
\end{tabular}


WINS 2006 (Continued)

\begin{tabular}{lll} 
Stated aim to reduce SFA & High risk & Aim to reduce SFA not stated \\
\hline Achieved SFA reduction & Low risk & SFA reduction achieved \\
\hline Achieved TC reduction & High risk & No statistically significant TC fall \\
\hline Other bias & Low risk & None noted \\
\hline
\end{tabular}

$\% \mathrm{E}$ : percent of total energy intake

ATPII: Adult treatment panel II

BMI: body mass index (weight in $\mathrm{kg} /$ height in $\mathrm{m}$, squared)

BP: blood pressure

CABG: coronary artery bypass graft

CHD: coronary heart disease

$\mathrm{CHO}$ : carbohydrate

chol: cholesterol

$\mathrm{Cl}$ : confidence interval

cont: control group

CVD: cardiovascular disease

DART: Diet And Reinfarction Trial

dBP: diastolic blood pressure

DVT: deep vein thrombosis

EPA: eicosapentaenoic acid

GPs: general practitioners

HDL: high density lipoprotein

HOMA: homeostatic model assessment

int: intervention group

ITT: Intention to treat analysis

LDL: low density lipoprotein

Lp(a): lipoprotein (a)

MI: myocardial infarction

MRC: Medical Research Council

MUFA: monounsaturated fat

$\mathrm{P} / \mathrm{S}$ : polyunsaturated/saturated fat ratio

PCTA: percutaneous transluminal coronary angioplasty

PUFA: polyunsaturated fat

PVD: peripheral vascular disease

$\mathrm{RCT}$ : randomised controlled trial

SBP: systolic blood pressure

SD: standard deviation

SE: standard error

SFA: saturated fats

STARS: St Thomas' Atherosclerosis Regression Study

TC: total cholesterol

TG: triglyceride

vs: versus

WHI: Women's Health Initiative

WINS: Women's Intervention Nutrition Study

Characteristics of excluded studies [ordered by study ID] 


\begin{tabular}{|c|c|}
\hline Study & Reason for exclusion \\
\hline Agewall 2001 & Multifactorial intervention \\
\hline Ammerman 2003 & No appropriate control group (and not low fat vs modified fat) \\
\hline Anderson 1990 & Follow-up less than 24 months \\
\hline Aquilani 2000 & No appropriate control group (and not low fat vs modified fat) \\
\hline Arntzenius 1985 & No appropriate control group (and not low fat vs modified fat) \\
\hline Aro 1990 & Intervention and randomised follow-up less than 6 months \\
\hline ASSIST 2001 & Intervention was not dietary fat modification or low fat diet. \\
\hline Australian Polyp Prev 95 & $\begin{array}{l}\text { Neither mortality nor cardiovascular morbidity data available (only decided after contact with at } \\
\text { least } 1 \text { author) }\end{array}$ \\
\hline Azadbakht 2007 & Follow-up less than 24 months \\
\hline Bakx 1997 & Multifactorial intervention \\
\hline Ball 1965 & $\begin{array}{l}\text { Study aim was to assess effects of a low-fat diet and methods stated that the "nature of the fat con- } \\
\text { sumed was not altered". Saturated fat content of diet was not reported. }\end{array}$ \\
\hline Barnard 2009 & Weight reduction encouraged in the conventional diet, but not in the vegan diet arm \\
\hline Barndt 1977 & No appropriate control group (and not low fat vs modified fat) \\
\hline Baron 1990 & Multifactorial intervention \\
\hline Barr 1990 & Intervention and randomised follow-up less than 6 months \\
\hline Barsotti 1991 & $\begin{array}{l}\text { Complex paper in Italian; unclear whether cardiovascular events occurred; contact with authors } \\
\text { not established }\end{array}$ \\
\hline Baumann 1982 & Intervention and randomised follow-up less than 6 months \\
\hline BDIT Pilot Studies 1996 & $\begin{array}{l}\text { Study aim was to reduce total fat intake to } 15 \% \text { E with no specific intervention on saturated fat. Sat- } \\
\text { urated fat in intervention group was more than } 80 \% \text { of that in the control group. }\end{array}$ \\
\hline Beckmann 1995 & Intervention was not dietary fat modification or low-fat diet. \\
\hline beFIT 1997 & Follow-up less than 24 months \\
\hline Beresford 1992 & Intervention and randomised follow-up less than 6 months \\
\hline Bergstrom 1967 & Intervention and randomised follow-up less than 6 months \\
\hline Bierenbaum 1963 & No appropriate control group (and not low fat vs modified fat) \\
\hline Bloemberg 1991 & $\begin{array}{l}\text { Neither mortality nor cardiovascular morbidity data available (only decided after contact with at } \\
\text { least } 1 \text { author) }\end{array}$ \\
\hline Bloomgarden 1987 & Multifactorial intervention \\
\hline
\end{tabular}




\begin{tabular}{|c|c|}
\hline Study & Reason for exclusion \\
\hline Bonk 1975 & Trial, unclear if randomised; contact could not be established with trialists \\
\hline Bonnema 1995 & No appropriate control group (and not low fat vs modified fat) \\
\hline Bosaeus 1992 & Intervention and randomised follow-up less than 6 months \\
\hline Boyd 1988 & Follow-up less than 24 months \\
\hline BREACPNT & $\begin{array}{l}\text { Individual microbiome-based dietary advice vs Mediterranean diet (no suggestion of saturated fat } \\
\text { reduction in either arm) }\end{array}$ \\
\hline Brehm 2009 & Unclear whether any relevant events occurred, not able to contact trialists \\
\hline Brensike 1982 & No appropriate control group (and not low fat vs modified fat) \\
\hline BRIDGES 2001 & Follow-up less than 24 months \\
\hline Broekmans 2003 & Intervention was not dietary fat modification or low fat diet. \\
\hline Brown 1984 & No appropriate control group (and not low fat vs modified fat) \\
\hline Bruce 1994 & No appropriate control group (and not low fat vs modified fat) \\
\hline Bruno 1983 & Multifactorial intervention \\
\hline Butcher 1990 & Intervention and randomised follow-up less than 6 months \\
\hline Byers 1995 & No appropriate control group (and not low fat vs modified fat) \\
\hline Caggiula 1996 & No appropriate control group (and not low fat vs modified fat) \\
\hline Canadian DBCP 1997 & Unable to establish contact with authors to provide data on numbers of deaths and CV events \\
\hline CARMEN 2000 & Follow-up less than 24 months \\
\hline CARMEN substudy 2002 & Follow-up less than 24 months \\
\hline Casas-Agustench 2013 & Less than 24 months duration \\
\hline Cerin 1993 & Intervention and randomised follow-up less than 6 months \\
\hline Chan 1993 & Intervention and randomised follow-up less than 6 months \\
\hline Chapman 1950 & Intervention and randomised follow-up less than 6 months \\
\hline Charbonnier 1975 & Intervention and randomised follow-up less than 6 months \\
\hline Cheng 2004 & Intervention and randomised follow-up less than 6 months \\
\hline Chiostri 1988 & Intervention and randomised follow-up less than 6 months \\
\hline Choudhury 1984 & Intervention and randomised follow-up less than 6 months \\
\hline Clark 1997 & Multifactorial intervention \\
\hline
\end{tabular}




\begin{tabular}{|c|c|}
\hline Study & Reason for exclusion \\
\hline Clifton 1992 & Intervention and randomised follow-up less than 6 months \\
\hline Cobb 1991 & Intervention and randomised follow-up less than 6 months \\
\hline Cohen 1991 & Intervention was not dietary fat modification or low fat diet. \\
\hline Cole 1988 & Intervention and randomised follow-up less than 6 months \\
\hline Colquhoun 1990 & Intervention and randomised follow-up less than 6 months \\
\hline Consolazio 1946 & Intervention and randomised follow-up less than 6 months \\
\hline Cox 1996 & Multifactorial intervention \\
\hline Croft 1986 & Intervention was not dietary fat modification or low fat diet. \\
\hline Curzio 1989 & Follow-up less than 24 months \\
\hline Da Qing IGT 1997 & Intervention was not dietary fat modification or low-fat diet. \\
\hline Dalgard 2001 & No appropriate control group (and not low fat vs modified fat) \\
\hline DAS 2000 & No appropriate control group (and not low fat vs modified fat) \\
\hline DASH 1997 & Intervention and randomised follow-up less than 6 months \\
\hline Davey Smith 2005 & Multifactorial intervention \\
\hline De Boer 1983 & Intervention and randomised follow-up less than 6 months \\
\hline De Bont 1981 & Neither mortality nor cardiovascular morbidity data available as study data have been lost \\
\hline DeBusk 1994 & Multifactorial intervention \\
\hline DEER 1998 & Duration 1 year only \\
\hline Delahanty 2001 & No appropriate control group (and not low fat vs modified fat) \\
\hline Delius 1969 & Intervention was not dietary fat modification or low fat diet. \\
\hline Demark 1990 & Intervention and randomised follow-up less than 6 months \\
\hline Dengel 1995 & No appropriate control group (and not low fat vs modified fat) \\
\hline Denke 1994 & Intervention and randomised follow-up less than 6 months \\
\hline Diabetes CCT 1995 & Intervention was not dietary fat modification or low fat diet. \\
\hline Diet \& Hormone Study 2003 & Duration 1 year only \\
\hline DIET 1998 & Multifactorial intervention \\
\hline Ding 1992 & Intervention and randomised follow-up less than 6 months \\
\hline DIPI 2018 & Less than 24 months duration \\
\hline
\end{tabular}




\begin{tabular}{|c|c|}
\hline Study & Reason for exclusion \\
\hline DIRECT 2009 & $\begin{array}{l}\text { Unable to establish contact with authors to establish whether relevant events occurred; multiple } \\
\text { publications checked and no relevant outcomes found }\end{array}$ \\
\hline DO IT 2006 & $\begin{array}{l}\text { Intervention aim was for a "mediterranean diet" with total fat } 27-30 \% \mathrm{E} \text {, protein } 15-18 \% \mathrm{E}, \mathrm{CHO} 50 \\
\text { - 55\%E, no specific aim to reduce saturated fat (though polyunsaturated margarine given to inter- } \\
\text { vention group), and intervention group saturated fat was more than } 80 \% \text { of that in the control. }\end{array}$ \\
\hline Dobs 1991 & No appropriate control group (and not low fat vs modified fat) \\
\hline Due 2008 & Follow-up less than 24 months \\
\hline Duffield 1982 & Multifactorial intervention \\
\hline Dullaart 1992 & Study authors confirmed that no deaths or cardiovascular events occurred during the study. \\
\hline Eating Patterns 1997 & $\begin{array}{l}\text { Neither mortality nor cardiovascular morbidity data available (only decided after contact with at } \\
\text { least } 1 \text { author) }\end{array}$ \\
\hline Ehnholm 1982 & Intervention and randomised follow-up less than 6 months \\
\hline Ehnholm 1984 & Intervention and randomised follow-up less than 6 months \\
\hline Eisenberg 1990 & Intervention and randomised follow-up less than 6 months \\
\hline Elder 2000 & No appropriate control group (and not low fat vs modified fat) \\
\hline Ellegard 1991 & Intervention and randomised follow-up less than 6 months \\
\hline Esposito 2003 & No appropriate control group (and not low fat vs modified fat) \\
\hline Esposito 2004 & Unable to establish contact with authors to assess whether any relevant events occurred \\
\hline EUROACTION 2008 & Multifactorial intervention \\
\hline FARIS 1997 & Multifactorial intervention \\
\hline Fasting HGS 1997 & No appropriate control group (and not low fat vs modified fat) \\
\hline Ferrara 2000 & No appropriate control group (and not low fat vs modified fat) \\
\hline Fielding 1995 & Intervention and randomised follow-up less than 6 months \\
\hline Finnish Diabet Prev 2000 & Multifactorial intervention \\
\hline Finnish Mental Hosp 1972 & Not randomised (cluster-randomised, but < 6 clusters) \\
\hline Fisher 1981 & Intervention and randomised follow-up less than 6 months \\
\hline FIT Heart 2011 & $\begin{array}{l}\text { Authors confirmed that differences between intervention and control groups included smoking } \\
\text { and physical activity, as well as dietary changes. }\end{array}$ \\
\hline Fleming 2002 & No appropriate control group (and not low fat vs modified fat) \\
\hline Fortmann 1988 & Intervention was not dietary fat modification or low fat diet. \\
\hline
\end{tabular}




\begin{tabular}{|c|c|}
\hline Study & Reason for exclusion \\
\hline Foster 2003 & Weight reduction in 1 arm but not the other \\
\hline Frenkiel 1986 & Follow-up less than 24 months \\
\hline FRESH START 2007 & Participants were newly diagnosed with cancer. \\
\hline Gambera 1995 & Intervention and randomised follow-up less than 6 months \\
\hline Gaullier 2007 & No appropriate control group (and not low fat vs modified fat) \\
\hline Ginsberg 1988 & Intervention and randomised follow-up less than 6 months \\
\hline Gjone 1972 & Intervention and randomised follow-up less than 6 months \\
\hline Glatzel 1966 & No appropriate control group (and not low fat vs modified fat) \\
\hline Goodpaster 1999 & No appropriate control group (and not low fat vs modified fat) \\
\hline Grundy 1986 & Intervention and randomised follow-up less than 6 months \\
\hline Hardcastle 2008 & Multifactorial intervention \\
\hline Harris 1990 & Intervention and randomised follow-up less than 6 months \\
\hline Hartman 1993 & No appropriate control group (and not low fat vs modified fat) \\
\hline Hartwell 1986 & No appropriate control group (and not low fat vs modified fat) \\
\hline Hashim 1960 & Intervention and randomised follow-up less than 6 months \\
\hline Haufe 2011 & $\begin{array}{l}\text { Aim was to reduce total fat or reduce carbohydrate, but no saturated fat aims were stated, and ef- } \\
\text { fects of the diets on saturated fat intakes were unclear. }\end{array}$ \\
\hline Haynes 1984 & Intervention was not dietary fat modification or low fat diet. \\
\hline Heber 1991 & Intervention and randomised follow-up less than 6 months \\
\hline Heine 1989 & $\begin{array}{l}\text { Neither mortality nor cardiovascular morbidity data available (only decided after contact with at } \\
\text { least } 1 \text { author) }\end{array}$ \\
\hline Hellenius 1995 & The study aimed for weight loss in 1 arm and not in the comparison arm. \\
\hline Heller 1993 & $\begin{array}{l}\text { Neither mortality nor cardiovascular morbidity data available (only decided after contact with at } \\
\text { least } 1 \text { author) }\end{array}$ \\
\hline Hildreth 1951 & No appropriate control group (and not low fat vs modified fat) \\
\hline Holm 1990 & $\begin{array}{l}\text { Neither mortality nor cardiovascular morbidity data available (only decided after contact with at } \\
\text { least } 1 \text { author) }\end{array}$ \\
\hline Horlick 1957 & Intervention and randomised follow-up less than 6 months \\
\hline Horlick 1960 & Intervention and randomised follow-up less than 6 months \\
\hline Howard 1977 & Intervention and randomised follow-up less than 6 months \\
\hline
\end{tabular}




\begin{tabular}{|c|c|}
\hline Study & Reason for exclusion \\
\hline Hunninghake 1990 & Intervention and randomised follow-up less than 6 months \\
\hline Hutchison 1983 & No appropriate control group (and not low fat vs modified fat) \\
\hline Hyman 1998 & $\begin{array}{l}\text { Neither mortality nor cardiovascular morbidity data available (only decided after contact with at } \\
\text { least } 1 \text { author) }\end{array}$ \\
\hline Iacono 1981 & Not randomised; Intervention and randomised follow-up less than 6 months \\
\hline IMPACT 1995 & Multifactorial intervention \\
\hline Iso 1991 & No appropriate control group (and not low fat vs modified fat) \\
\hline Ives 1993 & Multifactorial intervention \\
\hline Jalkanen 1991 & Multifactorial intervention \\
\hline Jerusalem Nut 1992 & Intervention and randomised follow-up less than 6 months \\
\hline Jula 1990 & Multifactorial intervention \\
\hline Junker 2001 & Intervention and randomised follow-up less than 6 months \\
\hline Karmally 1990 & Intervention and randomised follow-up less than 6 months \\
\hline Karvetti 1992 & Multifactorial intervention \\
\hline Kastarinen 2002 & Multifactorial intervention \\
\hline Kather 1985 & Intervention and randomised follow-up less than 6 months \\
\hline Katzel 1995 & Intervention was not dietary fat modification or low fat diet. \\
\hline Kawamura 1993 & Intervention and randomised follow-up less than 6 months \\
\hline Keidar 1988 & Intervention and randomised follow-up less than 6 months \\
\hline Kempner 1948 & No appropriate control group (and not low fat vs modified fat) \\
\hline Keys $1957 a$ & Intervention and randomised follow-up less than 6 months \\
\hline Keys 1957b & Intervention and randomised follow-up less than 6 months \\
\hline Keys 1957c & Intervention and randomised follow-up less than 6 months \\
\hline Khan 2003 & $\begin{array}{l}\text { Neither mortality nor cardiovascular morbidity data available (only decided after contact with at } \\
\text { least } 1 \text { author) }\end{array}$ \\
\hline King 2000 & Intervention and randomised follow-up less than 6 months \\
\hline Kingsbury 1961 & Intervention and randomised follow-up less than 6 months \\
\hline KNOTA & Numerous publications checked, but no relevant outcome data found. Trialists not contacted. \\
\hline Koopman 1990 & Intervention and randomised follow-up less than 6 months \\
\hline
\end{tabular}




\begin{tabular}{|c|c|}
\hline Study & Reason for exclusion \\
\hline Koranyi 1963 & Unclear whether randomised, unable to contact authors to discuss \\
\hline Korhonen 2003 & Multifactorial intervention \\
\hline Kriketos 2001 & Intervention and randomised follow-up less than 6 months \\
\hline Kris 1994 & Intervention and randomised follow-up less than 6 months \\
\hline Kristal 1997 & Multifactorial intervention \\
\hline Kromhout 1987 & No appropriate control group (and not low fat vs modified fat) \\
\hline Kummel 2008 & Intervention was not dietary fat modification or low-fat diet. \\
\hline Laitinen 1993 & Multifactorial intervention \\
\hline Laitinen 1994 & Multifactorial intervention \\
\hline Lean 1997 & Follow-up less than 24 months \\
\hline Leduc 1994 & Multifactorial intervention \\
\hline Lewis 1958 & Intervention and randomised follow-up less than 6 months \\
\hline Lewis 1981 & Intervention and randomised follow-up less than 6 months \\
\hline Lewis 1985 & Multifactorial intervention \\
\hline Lichtenstein 2002 & Intervention and randomised follow-up less than 6 months \\
\hline $\operatorname{Lim} 2010$ & $\begin{array}{l}\text { Unable to establish contact with authors to gain access to data on health outcomes (none reported } \\
\text { in paper) }\end{array}$ \\
\hline Linko 1957 & Intervention and randomised follow-up less than 6 months \\
\hline Lipid Res Clinic 1984 & No appropriate control group (and not low fat vs modified fat) \\
\hline Little 1990 & Intervention and randomised follow-up less than 6 months \\
\hline Little 2004 & Intervention was not dietary fat modification or low-fat diet. \\
\hline Lottenberg 1996 & Intervention and randomised follow-up less than 6 months \\
\hline Luszczynska 2007 & No appropriate control group (and not low fat vs modified fat) \\
\hline Lyon Diet Heart 1994 & Intervention was not dietary fat modification or low-fat diet. \\
\hline Lysikova 2003 & Intervention and randomised follow-up less than 6 months \\
\hline Macdonald 1972 & Intervention and randomised follow-up less than 6 months \\
\hline Mansel 1990 & Intervention was not dietary fat modification or low-fat diet \\
\hline MARGARIN 2002 & No appropriate control group (and not low fat vs modified fat) \\
\hline
\end{tabular}




\begin{tabular}{|c|c|}
\hline Study & Reason for exclusion \\
\hline Marniemi 1990 & Both intervention groups aimed to lose weight, while the control group did not. \\
\hline Mattson 1985 & Intervention and randomised follow-up less than 6 months \\
\hline McAuley 2005 & Follow-up less than 24 months \\
\hline McCarron 1997 & Intervention and randomised follow-up less than 6 months \\
\hline McCarron 2001 & Intervention was not dietary fat modification or low-fat diet. \\
\hline McKeown-Eyssen 1994 & $\begin{array}{l}\text { Intervention aim was to reduce total fat and increase dietary fibre (saturated fat not mentioned), } \\
\text { and no saturated fat intakes during trial reported. }\end{array}$ \\
\hline McManus 2001 & $\begin{array}{l}\text { Neither mortality nor cardiovascular morbidity data available (only decided after contact with at } \\
\text { least } 1 \text { author) }\end{array}$ \\
\hline McNamara 1981 & Intervention and randomised follow-up less than 6 months \\
\hline Medi-RIVAGE 2004 & $\begin{array}{l}\text { Weight reduction for some low-fat diet participants (those with } \mathrm{BMI}>25 \text { ) but not in Mediterranean } \\
\text { group }\end{array}$ \\
\hline MeDiet 2002 & Follow-up less than 24 months \\
\hline MEDINA & Less than 24 months duration \\
\hline Mensink 1987 & Intervention and randomised follow-up less than 6 months \\
\hline Mensink 1989 & Intervention and randomised follow-up less than 6 months \\
\hline Mensink 1990a & Intervention and randomised follow-up less than 6 months \\
\hline Mensink 1990b & Intervention and randomised follow-up less than 6 months \\
\hline Metroville Health 2003 & Unable to establish contact with authors to assess whether any relevant events occurred \\
\hline Michalsen 2006 & Diet plus stress management vs no intervention \\
\hline Miettinen 1994 & Intervention and randomised follow-up less than 6 months \\
\hline Millar 1973 & No appropriate control group (and not low fat vs modified fat) \\
\hline Miller 1998 & Intervention and randomised follow-up less than 6 months \\
\hline Miller 2001 & $\begin{array}{l}\text { Neither mortality nor cardiovascular morbidity data available (only decided after contact with at } \\
\text { least } 1 \text { author) }\end{array}$ \\
\hline Milne 1994 & $\begin{array}{l}\text { No appropriate control group (and not low fat vs modified fat) - the high } \mathrm{CHO} \text { diet was neither 'usu- } \\
\text { al' or 'low fat' to compare with the modified fat diet. }\end{array}$ \\
\hline Minnesota Coronary 1989 & $\begin{array}{l}\text { Although the study proceeded for over } 4 \text { years, participants (patients) came and went and mean } \\
\text { follow-up was only } 1 \text { year. }\end{array}$ \\
\hline Minnesota HHP 1990 & No appropriate control group (and not low fat vs modified fat) \\
\hline Mojonnier 1980 & Unable to establish contact with authors to assess whether any relevant events occurred \\
\hline
\end{tabular}




\begin{tabular}{|c|c|}
\hline Study & Reason for exclusion \\
\hline Mokuno 1988 & Intervention and randomised follow-up less than 6 months \\
\hline Mortensen 1983 & Intervention and randomised follow-up less than 6 months \\
\hline Mottalib 2018 & Less than 24 months duration \\
\hline MRFIT substudy 1986 & Intervention and randomised follow-up less than 6 months \\
\hline MSDELTA 1995 & Intervention and randomised follow-up less than 6 months \\
\hline MSFAT 1997 & Follow-up less than 24 months \\
\hline Mujeres Felices 2003 & Diet and breast self-examination vs no intervention \\
\hline Mutanen 1997 & Intervention and randomised follow-up less than 6 months \\
\hline Muzio 2007 & Intervention and randomised follow-up less than 6 months \\
\hline Naglak 2000 & Unable to establish contact with authors to assess whether any relevant events occurred \\
\hline NAS 1987 & Intervention and randomised follow-up less than 6 months \\
\hline National Diet Heart 1968 & Follow-up less than 24 months \\
\hline NCEP weight 1991 & $\begin{array}{l}\text { Neither mortality nor cardiovascular morbidity data available (only decided after contact with at } \\
\text { least } 1 \text { author) }\end{array}$ \\
\hline NCT01954472 & Study withdrawn (not completed) \\
\hline NCT03068078 & Less than 24 months duration \\
\hline Neil 1995 & No appropriate control group (and not low fat vs modified fat) \\
\hline Neverov 1997 & Multifactorial intervention \\
\hline Next Step 1995 & $\begin{array}{l}\text { Neither mortality nor cardiovascular morbidity data available (only decided after contact with at } \\
\text { least } 1 \text { author) }\end{array}$ \\
\hline Nordoy 1971 & Intervention and randomised follow-up less than 6 months \\
\hline Norway Veg Oil 1968 & No appropriate control group (and not low fat vs modified fat) \\
\hline Nutri-AGEs 2015 & Less than 24 months duration \\
\hline Nutrition Breast Health & Follow-up less than 24 months \\
\hline O'Brien 1976 & Intervention and randomised follow-up less than 6 months \\
\hline ODES 2006 & The study aimed for weight loss in 1 arm and not in the other arm. \\
\hline Oldroyd 2001 & Multifactorial intervention \\
\hline Ole Study 2002 & Follow-up less than 24 months \\
\hline OLIVE 1997 & Unable to establish contact with authors to assess whether any relevant events occurred \\
\hline
\end{tabular}




\begin{tabular}{|c|c|}
\hline Study & Reason for exclusion \\
\hline ORIGIN 2008 & Intervention was not dietary fat modification or low-fat diet. \\
\hline Oslo Study 2004 & Multifactorial intervention \\
\hline Pascale 1995 & Multifactorial intervention \\
\hline PEP 2001 & Multifactorial intervention \\
\hline PHYLLIS 1993 & No appropriate control group (and not low fat vs modified fat) \\
\hline Pilkington 1960 & $\begin{array}{l}\text { Neither mortality nor cardiovascular morbidity data available (only decided after contact with at } \\
\text { least } 1 \text { author) }\end{array}$ \\
\hline Polyp Prevention 1996 & $\begin{array}{l}\text { Intervention aim was to reduce total fat and increase dietary fibre, fruit and vegetables (saturated } \\
\text { fat not mentioned), and no saturated fat intakes during trial reported. }\end{array}$ \\
\hline POUNDS LOST 2009 & $\begin{array}{l}\text { All study arms (low or high total fat) prescribed low saturated fat intake }(8 \% \mathrm{E}) \text {; no usual fat com- } \\
\text { parator. }\end{array}$ \\
\hline PREDIMED 2008 & $\begin{array}{l}\text { Total fat goals in the low-fat arm were unclear and authors confirmed that aims were nonspecific (if } \\
\text { aims }<30 \% \text {, this study would be included). }\end{array}$ \\
\hline PREMIER 2003 & Follow-up less than 24 months \\
\hline Pritchard 2002 & The study aimed for weight loss in 1 arm and not in the comparison arm. \\
\hline Puget Sound EP 2000 & $\begin{array}{l}\text { Neither mortality nor cardiovascular morbidity data available (only decided after contact with at } \\
\text { least } 1 \text { author) }\end{array}$ \\
\hline Rabast 1979 & Intervention and randomised follow-up less than 6 months \\
\hline Rabkin 1981 & Intervention and randomised follow-up less than 6 months \\
\hline Radack 1990 & Intervention and randomised follow-up less than 6 months \\
\hline Rasmussen 1995 & Intervention and randomised follow-up less than 6 months \\
\hline Reaven 2001 & Intervention and randomised follow-up less than 6 months \\
\hline Reid 2002 & No appropriate control group (and not low fat vs modified fat) \\
\hline Renaud 1986 & Not randomised \\
\hline Rivellese 1994 & Follow-up less than 24 months \\
\hline Rivellese 2003 & Intervention and randomised follow-up less than 6 months \\
\hline Roderick 1997 & $\begin{array}{l}\text { Neither mortality nor cardiovascular morbidity data available (only decided after contact with at } \\
\text { least } 1 \text { author) }\end{array}$ \\
\hline Roman CHD prev 1986 & Multifactorial intervention \\
\hline Rose 1987 & No appropriate control group (and not low fat vs modified fat) \\
\hline Sarkkinen 1995 & Follow-up less than 24 months \\
\hline
\end{tabular}




\begin{tabular}{|c|c|}
\hline Study & Reason for exclusion \\
\hline Schaefer 1995a & Intervention and randomised follow-up less than 6 months \\
\hline Schaefer 1995b & Intervention and randomised follow-up less than 6 months \\
\hline Schectman 1996 & Multifactorial intervention \\
\hline Schlierf 1995 & Multifactorial intervention \\
\hline Seppanen-Laakso 1992 & Intervention and randomised follow-up less than 6 months \\
\hline Seppelt 1996 & Follow-up less than 24 months \\
\hline Singh 1991 & Multifactorial intervention \\
\hline Singh 1992 & No appropriate control group (and not low fat vs modified fat) \\
\hline Sirtori 1992 & Intervention and randomised follow-up less than 6 months \\
\hline SLIM 2008 & Multifactorial intervention \\
\hline Sopotsinskaia 1992 & The study aimed for weight loss in 1 arm and not in the comparison arm. \\
\hline Soul Food Light & Less than 24 months duration \\
\hline Stanford NAP 1997 & Intervention and randomised follow-up less than 6 months \\
\hline Stanford Weight 1994 & The study aimed for weight loss in 1 arm and not in the comparison arm. \\
\hline Starmans 1995 & Intervention and randomised follow-up less than 6 months \\
\hline Steinbach 1996 & Multifactorial intervention \\
\hline Steptoe 2001 & No appropriate control group (and not low fat vs modified fat) \\
\hline Stevens 2002 & Diet plus breast self examination vs no intervention \\
\hline Stevenson 1988 & No appropriate control group (and not low fat vs modified fat) \\
\hline Strychar 2009 & Follow-up less than 24 months \\
\hline Sweeney 2004 & Intervention was not dietary fat modification or low fat diet. \\
\hline Søndergaard 2003 & Follow-up less than 24 months \\
\hline TAIM 1992 & Intervention was not dietary fat modification or low fat diet. \\
\hline Tapsell 2004 & Unable to establish contact with authors to assess whether any relevant events occurred \\
\hline THIS DIET 2008 & All study arms prescribed low saturated fat intake, no usual fat comparator \\
\hline TOHP I 1992 & Multifactorial intervention \\
\hline TONE 1997 & Intervention was not dietary fat modification or low-fat diet. \\
\hline Toobert 2003 & Multifactorial intervention \\
\hline
\end{tabular}




\begin{tabular}{|c|c|}
\hline Study & Reason for exclusion \\
\hline Towle 1994 & Intervention and randomised follow-up less than 6 months \\
\hline TRANSFACT 2006 & Intervention and randomised follow-up less than 6 months \\
\hline Treatwell 1992 & $\begin{array}{l}\text { Neither mortality nor cardiovascular morbidity data available (only decided after contact with at } \\
\text { least } 1 \text { author) }\end{array}$ \\
\hline Tromsø Heart 1989 & Multifactorial intervention \\
\hline Troyer 2010 & Longest duration only 12 months \\
\hline UK PDS 1996 & No appropriate control group (and not low fat vs modified fat) \\
\hline Urbach 1952 & No appropriate control group (and not low fat vs modified fat) \\
\hline Uusitupa 1993 & Multifactorial intervention \\
\hline VASTKOST 2012 & Publications reported than no participants died or experienced CVD during the trial. \\
\hline Vavrikova 1958 & Intervention and randomised follow-up less than 6 months \\
\hline Verheiden 2003 & Unable to establish contact with authors to assess whether any relevant events occurred \\
\hline WAHA 2016 & $15 \% \mathrm{E}$ from walnuts vs usual diet (neither arm aimed to reduce saturated fat intake) \\
\hline Wass 1981 & Intervention and randomised follow-up less than 6 months \\
\hline Wassertheil 1985 & Intervention was not dietary fat modification or low fat diet. \\
\hline WATCH 1999 & $\begin{array}{l}\text { Neither mortality nor cardiovascular morbidity data available (only decided after contact with at } \\
\text { least } 1 \text { author) }\end{array}$ \\
\hline Watts 1988 & Intervention and randomised follow-up less than 6 months \\
\hline Weintraub 1992 & No appropriate control group (and not low fat vs modified fat) \\
\hline Westman 2006 & Intervention was not dietary fat modification or low fat diet. \\
\hline Weststrate 1998 & Intervention and randomised follow-up less than 6 months \\
\hline WHEL 2007 & $\begin{array}{l}\text { Study aimed to reduce total fat, but saturated fat goals were not mentioned, and saturated fat in- } \\
\text { take in the intervention group was more than } 80 \% \text { of that in the control }(81 \%) \text {. }\end{array}$ \\
\hline WHO primary prev 1979 & Multifactorial intervention \\
\hline WHT 1990 & $\begin{array}{l}\text { Neither mortality nor cardiovascular morbidity data available as such data were not collected in } \\
\text { the study }\end{array}$ \\
\hline WHT Feasibility 2003 & $\begin{array}{l}\text { Neither mortality nor cardiovascular morbidity data available (only decided after contact with at } \\
\text { least } 1 \text { author) }\end{array}$ \\
\hline Wilke 1974 & Intervention and randomised follow-up less than 6 months \\
\hline Williams 1990 & Intervention was not dietary fat modification or low-fat diet. \\
\hline
\end{tabular}




\begin{tabular}{ll}
\hline Study & Reason for exclusion \\
\hline Williams 1992 & Intervention was not dietary fat modification or low-fat diet. \\
\hline Williams 1994 & Intervention was not dietary fat modification or low-fat diet. \\
\hline Wilmot 1952 & No appropriate control group (and not low fat vs modified fat) \\
\hline Wing 1998 & No appropriate control group (and not low fat vs modified fat) \\
\hline WINS UK 2011 & Stated aim was to reduce total fat by 50\%; no saturated fat aims \\
\hline WOMAN 2007 & Lifestyle intervention included exercise and weight as well as diet. \\
\hline Wood 1988 & Intervention was not dietary fat modification or low-fat diet. \\
\hline Woollard 2003 & Multifactorial intervention including smoking, weight, exercise and alcohol components \\
\hline Working Well 1996 & Multifactorial intervention \\
\hline Zock 1995 & Intervention and randomised follow-up less than 6 months \\
\hline
\end{tabular}

CHO: carbohydrate

$\mathrm{CV}$ : cardiovascular

E: energy

vs: versus

Characteristics of studies awaiting classification [ordered by study ID]

\section{ICFAMED}

\begin{tabular}{ll}
\hline Methods & $\begin{array}{l}\text { A Mediterranean diet for preventing heart failure and atrial fibrillation in hypertensive patients (IC- } \\
\text { FAMED) } \\
\text { RCT, } 24 \text { months }\end{array}$ \\
\hline Participants & People with hypertension aged 55 to 75 years at high cardiovascular risk, but without existing CVD \\
\hline Interventions & $\begin{array}{l}\text { MedDiet: Mediterranean-style diet, dietary advice (individual and group) every three months } \\
\text { LFD: Low-fat diet according to American Heart Association guidelines, dietary advice (individual } \\
\text { and group) every three months }\end{array}$ \\
\hline
\end{tabular}

Outcomes

Primary: heart failure and/or atrial fibrillation

Secondary: echocardiographic variables \& BP variables

Actual outcomes from abstracts: MedDiet: 5 CVD events (atrial fibrillation (AF) 2; ischaemic heart disease (IHD) 2; stroke 1), LFD: 11 CVD events (AF 6, IHD 2, stroke 3). The crude rate for the occurrence of events per 1000 patient-months of follow-up was 197 (95\% Cl: 06-46) for MedDiet, 451 (95\% Cl: 3-8.1) for LFD. The HR for patients with MedDiet compared to LFD was 044 ( $95 \% \mathrm{Cl}$ : 015$126, \mathrm{P}>005)$.

Notes

Enrollment began in 2012; appears to have completed in 2017; abstract and poster publications only to date

Awaiting assessment because: Unclear whether one arm was higher in saturated fat than the other, awaiting fuller publication to assess 
AF: atrial fibrillation

CVD: cardiovascular disease

HR: hazard ratio

ICFAMED: A Mediterranean diet for preventing heart failure and atrial fibrillation in hypertensive patients

IHD: Ischaemic heart disease

LFD: low fat diet

MedDiet: Mediterranean style diet

$\mathrm{RCT}$ : randomised controlled trial

Characteristics of ongoing studies [ordered by study ID]

ENAbLE due unclear

\begin{tabular}{|c|c|}
\hline Study name & ENAbLE \\
\hline Methods & $\mathrm{RCT}, 2 \times 2$ diet and physical activity interventions, duration unclear \\
\hline Participants & Stroke survivors able to walk independently \\
\hline \multirow[t]{3}{*}{ Interventions } & $\begin{array}{l}\text { AusMed diet, adaptation of the Mediterranean diet to the Australian context, including provision of } \\
\text { starter foods, menu plans and regular counselling }\end{array}$ \\
\hline & Comparator unclear \\
\hline & Telehealth-delivered physical activity and diet interventions in both arms \\
\hline Outcomes & Primary: sBP \\
\hline Starting date & Mid 2019, planned completion date unclear \\
\hline Contact information & Coralie English, University of Western Australia (first author of abstract) \\
\hline \multirow[t]{2}{*}{ Notes } & Trial registration not found \\
\hline & $\begin{array}{l}\text { Unclear whether the intervention was truly lower vs higher saturated fat as saturated fat goals not } \\
\text { provided, and duration unclear }\end{array}$ \\
\hline
\end{tabular}

NCT02481466 due 2020

\begin{tabular}{|c|c|}
\hline Study name & Combined Portfolio diet and Exercise study (PortfolioEx) \\
\hline Methods & $\mathrm{RCT}, 2 \times 2$ factorial design with exercise intervention, 36 months \\
\hline Participants & Men and postmenopausal women with BMI up to $40 \mathrm{~kg} / \mathrm{m}^{2}$ with measurable arterial thickening \\
\hline \multirow[t]{3}{*}{ Interventions } & $\begin{array}{l}\text { Lower saturated fat: advice on a therapeutic diet appropriate for hypercholesterolemia (ie }<7 \% \\
\text { of energy from saturated fat, }<200 \mathrm{mg} / \mathrm{d} \text { cholesterol) PLUS the combination of viscous fibres, soy } \\
\text { protein, plant sterols and nuts, } 5 \% \text { extra monounsaturated fat, and selection of low glycemic index } \\
\text { foods }\end{array}$ \\
\hline & $\begin{array}{l}\text { Higher saturated fat: advice to follow a DASH-like diet of whole grains, and low-fat dairy products } \\
\text { with fruits and vegetables }\end{array}$ \\
\hline & $\begin{array}{l}\text { Both arms with or without instruction on the Laval exercise program - a standardised physical ac- } \\
\text { tivity/exercise component supervised by trained kinesiologists (exercise physiologists) }\end{array}$ \\
\hline
\end{tabular}


NCT02481466 due 2020 (Continued)

Outcomes

Primary: maximum vessel wall volume of the carotid arteries

Secondary: composite end point of myocardial infarction, revascularization, cardiovascular hospitalisation, cardiovascular mortality and stroke; atrial fibrillation; BP; and vessel outcomes

\begin{tabular}{ll}
\hline Starting date & Nov 2016, planned completion Dec 2022 \\
\hline Contact information & $\begin{array}{l}\text { Pl: David J Jenkins, MD, NutritionProject@smh.ca, Risk Factor Modification Centre, St. Michael's } \\
\text { Hospital }\end{array}$ \\
\hline Notes & Trials registration: NCT02481466 \\
& $\begin{array}{l}\text { Unclear whether the intervention was truly lower vs higher saturated fat as saturated fat goals not } \\
\text { provided for both arms }\end{array}$ \\
\hline
\end{tabular}

\section{NCT02938832 due 2023}

\begin{tabular}{ll}
\hline Study name & $\begin{array}{l}\text { Does the advice to eat a mediterranean diet with low carbohydrate intake, compared with a low-fat } \\
\text { diet, reduce diabetes and cardiovascular disease (CardioDiet) }\end{array}$ \\
\hline Methods & RCT, 36 months \\
\hline Participants & Adults with ischaemic heart disease followed up at cardiac rehabilitation units \\
\hline Interventions & Mediterranean diet with an energy content (E\%) from carbohydrates between 25-30\% \\
& Traditional low-fat diet with 45-60 E\% from carbohydrates \\
\hline Outcomes & $\begin{array}{l}\text { Primary: diabetes incidence } \\
\text { Secondary: CVD disease, quality of life }\end{array}$ \\
\hline Starting date & Oct 2016, planned completion Oct 2023 \\
\hline Contact information & $\begin{array}{l}\text { Pl: Fredrik H Nystrom, Professor, MD, University Hospital, Linkoeping, fredrik.nystrom@regionos- } \\
\text { tergotland.se }\end{array}$ \\
\hline Notes & $\begin{array}{l}\text { Trials registration NCT02938832 } \\
\text { provided. }\end{array}$ \\
\hline
\end{tabular}

NEW Soul Study due 2022

\begin{tabular}{ll}
\hline Study name & Nutritious Eating With Soul (NEW Soul) study \\
\hline Methods & RCT, 24 months \\
\hline Participants & African-American adults aged $18-65$ years with BMI $25-49.9 \mathrm{~kg} / \mathrm{m}^{2}$ \\
\hline Interventions & $\begin{array}{l}\text { Lower saturated fat: plant-based vegan diet, instructing participants to favour a diet built around } \\
\text { whole grains, fruits, vegetables, and legumes, supplemented by the Oldways African Heritage and } \\
\text { Health programme, which includes a food pyramid guide. A Taste of African Heritage (ATAH) six-les- } \\
\text { son nutrition and cooking programme has an online course for health professionals and cooking }\end{array}$
\end{tabular}


NEW Soul Study due 2022 (Continued)

instructors (all research and restaurant team members will complete this course). Interventions include intervention meetings, physical activity, and podcasts/mailings.

Higher saturated fat: low-fat omnivorous diet, supplemented by the Oldways African Heritage and Health programme, which includes a food pyramid guide. A Taste of African Heritage (ATAH) six-lesson nutrition and cooking programme has an online course for health professionals and cooking instructors (all research and restaurant team members will complete this course). Interventions include intervention meetings, physical activity, and podcasts/mailings.

\begin{tabular}{ll}
\hline Outcomes & $\begin{array}{l}\text { Primary: CVD events } \\
\text { Secondary: CVD risk factors (including LDL \& BP), body weight }\end{array}$ \\
\hline Starting date & May 2018, planned completion June 2022 \\
\hline Contact information & PI: Brie Turner-McGrievy, Associate Professor, University of South Carolina \\
& Trial website: https://newsoul.org/ \\
\hline Notes & Trials registration NCT03354377 \\
& $\begin{array}{l}\text { Unclear whether the intervention was truly lower vs higher saturated fat as saturated fat goals not } \\
\text { provided }\end{array}$
\end{tabular}

ATAH: A Taste of African Heritage

AusMed: Australian style Mediterranean diet

BMI: body mass index

BP: blood pressure

CVD: cardiovascular disease

DASH: Dietary Approaches to Stop Hypertension

E: energy

LDL: low density lipoprotein

PorffolioEx: Combined Portfolio diet and Exercise study

$\mathrm{RCT}$ : randomised controlled trial

SBP: systolic blood pressure

\section{DATA AND ANALYSES}

\section{Comparison 1. SFA reduction vs usual diet - primary outcomes}

\begin{tabular}{|c|c|c|c|c|}
\hline Outcome or subgroup title & $\begin{array}{l}\text { No. of } \\
\text { studies }\end{array}$ & $\begin{array}{l}\text { No. of } \\
\text { partici- } \\
\text { pants }\end{array}$ & Statistical method & Effect size \\
\hline 1.1 ALL-CAUSE MORTALITY & 12 & 55858 & $\begin{array}{l}\text { Risk Ratio (M-H, Random, 95\% } \\
\mathrm{Cl})\end{array}$ & $0.96[0.90,1.03]$ \\
\hline $\begin{array}{l}1.2 \text { All-cause mortality, SA low summary risk } \\
\text { of bias }\end{array}$ & 7 & 53219 & $\begin{array}{l}\text { Risk Ratio (M-H, Random, 95\% } \\
\mathrm{Cl})\end{array}$ & $0.95[0.84,1.08]$ \\
\hline 1.3 All-cause mortality, SA aim to reduce SFA & 9 & 53112 & $\begin{array}{l}\text { Risk Ratio (M-H, Random, 95\% } \\
\mathrm{Cl} \text { ) }\end{array}$ & $0.97[0.89,1.06]$ \\
\hline $\begin{array}{l}\text { 1.4 All-cause mortality, SA statistically signif- } \\
\text { icant SFA reduction }\end{array}$ & 8 & 54973 & $\begin{array}{l}\text { Risk Ratio (M-H, Random, 95\% } \\
\text { Cl) }\end{array}$ & $0.98[0.92,1.04]$ \\
\hline
\end{tabular}




\begin{tabular}{|c|c|c|c|c|}
\hline Outcome or subgroup title & $\begin{array}{l}\text { No. of } \\
\text { studies }\end{array}$ & $\begin{array}{l}\text { No. of } \\
\text { partici- } \\
\text { pants }\end{array}$ & Statistical method & Effect size \\
\hline 1.5 All-cause mortality, SA TC reduction & 8 & 53073 & $\begin{array}{l}\text { Risk Ratio (M-H, Random, 95\% } \\
\mathrm{Cl})\end{array}$ & $0.97[0.88,1.07]$ \\
\hline 1.6 All-cause mortality, SA excluding WHI & 11 & 7023 & $\begin{array}{l}\text { Risk Ratio (M-H, Random, 95\% } \\
\mathrm{Cl})\end{array}$ & $0.95[0.83,1.07]$ \\
\hline $\begin{array}{l}\text { 1.7 All-cause mortality, SA Mantel-Haenszel } \\
\text { fixed-effect }\end{array}$ & 12 & 55858 & Risk Ratio (M-H, Fixed, 95\% Cl) & $0.97[0.91,1.03]$ \\
\hline 1.8 All-cause mortality, SA Peto fixed-effect & 12 & 55858 & $\begin{array}{l}\text { Peto Odds Ratio (Peto, Fixed, } \\
95 \% \mathrm{Cl} \text { ) }\end{array}$ & $0.96[0.90,1.04]$ \\
\hline $\begin{array}{l}1.9 \text { All-cause mortality, subgroup by any } \\
\text { substitution }\end{array}$ & 12 & & $\begin{array}{l}\text { Risk Ratio (M-H, Random, 95\% } \\
\mathrm{Cl})\end{array}$ & Subtotals only \\
\hline 1.9.1 replaced by PUFA & 7 & 4238 & $\begin{array}{l}\text { Risk Ratio (M-H, Random, 95\% } \\
\mathrm{Cl})\end{array}$ & $0.96[0.82,1.13]$ \\
\hline 1.9.2 replaced by MUFA & 1 & 52 & $\begin{array}{l}\text { Risk Ratio (M-H, Random, 95\% } \\
\mathrm{Cl})\end{array}$ & $3.00[0.33,26.99]$ \\
\hline 1.9.3 replaced by $\mathrm{CHO}$ & 6 & 53669 & $\begin{array}{l}\text { Risk Ratio (M-H, Random, 95\% } \\
\mathrm{Cl})\end{array}$ & $0.97[0.90,1.04]$ \\
\hline 1.9.4 replaced by protein & 5 & 53614 & $\begin{array}{l}\text { Risk Ratio (M-H, Random, 95\% } \\
\mathrm{Cl})\end{array}$ & $0.97[0.90,1.04]$ \\
\hline 1.9.5 replacement unclear & 0 & 0 & $\begin{array}{l}\text { Risk Ratio (M-H, Random, 95\% } \\
\mathrm{Cl})\end{array}$ & Not estimable \\
\hline $\begin{array}{l}1.10 \text { All-cause mortality, subgroup by main } \\
\text { substitution }\end{array}$ & 12 & & $\begin{array}{l}\text { Risk Ratio (M-H, Random, 95\% } \\
\mathrm{Cl})\end{array}$ & Subtotals only \\
\hline 1.10.1 replaced by PUFA & 6 & 4183 & $\begin{array}{l}\text { Risk Ratio (M-H, Random, 95\% } \\
\mathrm{CI})\end{array}$ & $0.97[0.82,1.14]$ \\
\hline 1.10 .2 replaced by MUFA & 1 & 52 & $\begin{array}{l}\text { Risk Ratio (M-H, Random, 95\% } \\
\mathrm{Cl})\end{array}$ & $3.00[0.33,26.99]$ \\
\hline 1.10.3 replaced by $\mathrm{CHO}$ & 5 & 51636 & $\begin{array}{l}\text { Risk Ratio (M-H, Random, 95\% } \\
\mathrm{Cl})\end{array}$ & $0.97[0.90,1.04]$ \\
\hline 1.10.4 replaced by protein & 0 & 0 & $\begin{array}{l}\text { Risk Ratio (M-H, Random, 95\% } \\
\text { CI) }\end{array}$ & Not estimable \\
\hline 1.10.5 replacement unclear & 0 & 0 & $\begin{array}{l}\text { Risk Ratio (M-H, Random, 95\% } \\
\text { CI) }\end{array}$ & Not estimable \\
\hline $\begin{array}{l}1.11 \text { All-cause mortality, subgroup by dura- } \\
\text { tion }\end{array}$ & 12 & 55858 & $\begin{array}{l}\text { Risk Ratio (M-H, Random, 95\% } \\
\mathrm{Cl})\end{array}$ & $0.96[0.90,1.03]$ \\
\hline 1.11.1 up to $24 \mathrm{mo}$ & 4 & 2246 & $\begin{array}{l}\text { Risk Ratio (M-H, Random, 95\% } \\
\text { Cl) }\end{array}$ & $0.99[0.78,1.26]$ \\
\hline
\end{tabular}




\begin{tabular}{|c|c|c|c|c|}
\hline Outcome or subgroup title & $\begin{array}{l}\text { No. of } \\
\text { studies }\end{array}$ & $\begin{array}{l}\text { No. of } \\
\text { partici- } \\
\text { pants }\end{array}$ & Statistical method & Effect size \\
\hline $1.11 .2>24$ to $48 \mathrm{mo}$ & 3 & 1294 & $\begin{array}{l}\text { Risk Ratio (M-H, Random, 95\% } \\
\mathrm{Cl})\end{array}$ & $0.96[0.83,1.12]$ \\
\hline $1.11 .3>48 \mathrm{mo}$ & 4 & 52142 & $\begin{array}{l}\text { Risk Ratio (M-H, Random, 95\% } \\
\mathrm{Cl})\end{array}$ & $0.96[0.79,1.16]$ \\
\hline 1.11.4 unclear duration & 1 & 176 & $\begin{array}{l}\text { Risk Ratio (M-H, Random, 95\% } \\
\text { Cl) }\end{array}$ & $0.33[0.07,1.61]$ \\
\hline $\begin{array}{l}\text { 1.12 All-cause mortality, subgroup by base- } \\
\text { line SFA }\end{array}$ & 12 & 55858 & $\begin{array}{l}\text { Risk Ratio (M-H, Random, 95\% } \\
\mathrm{Cl})\end{array}$ & $0.96[0.90,1.03]$ \\
\hline 1.12.1 up to $12 \%$ E SFA baseline & 1 & 2437 & $\begin{array}{l}\text { Risk Ratio (M-H, Random, 95\% } \\
\mathrm{Cl})\end{array}$ & $0.90[0.67,1.21]$ \\
\hline $1.12 .2>12$ to $15 \% E$ SFA baseline & 5 & 51635 & $\begin{array}{l}\text { Risk Ratio (M-H, Random, 95\% } \\
\text { CI) }\end{array}$ & $1.01[0.86,1.19]$ \\
\hline $1.12 .3>15$ to $18 \%$ E SFA baseline & 1 & 55 & $\begin{array}{l}\text { Risk Ratio (M-H, Random, 95\% } \\
\mathrm{Cl})\end{array}$ & $0.35[0.04,3.12]$ \\
\hline 1.12.4 >18\%E SFA baseline & 1 & 846 & $\begin{array}{l}\text { Risk Ratio (M-H, Random, 95\% } \\
\text { CI) }\end{array}$ & $0.98[0.83,1.15]$ \\
\hline 1.12.5 unclear & 4 & 885 & $\begin{array}{l}\text { Risk Ratio (M-H, Random, 95\% } \\
\text { CI) }\end{array}$ & $0.80[0.62,1.04]$ \\
\hline $\begin{array}{l}1.13 \text { All-cause mortality, subgroup by SFA } \\
\text { change }\end{array}$ & 12 & 55858 & $\begin{array}{l}\text { Risk Ratio (M-H, Random, 95\% } \\
\text { Cl) }\end{array}$ & $0.96[0.90,1.03]$ \\
\hline 1.13.1 up to $4 \% \mathrm{E}$ difference & 5 & 53939 & $\begin{array}{l}\text { Risk Ratio (M-H, Random, 95\% } \\
\text { Cl) }\end{array}$ & $0.99[0.86,1.13]$ \\
\hline 1.13.2 $>4$ to $8 \%$ E difference & 2 & 188 & $\begin{array}{l}\text { Risk Ratio (M-H, Random, 95\% } \\
\text { CI) }\end{array}$ & $0.41[0.08,2.07]$ \\
\hline 1.13.3 $>8 \%$ E difference & 1 & 846 & $\begin{array}{l}\text { Risk Ratio (M-H, Random, 95\% } \\
\mathrm{Cl})\end{array}$ & $0.98[0.83,1.15]$ \\
\hline 1.13.4 unclear & 4 & 885 & $\begin{array}{l}\text { Risk Ratio (M-H, Random, 95\% } \\
\mathrm{Cl})\end{array}$ & $0.80[0.62,1.04]$ \\
\hline 1.14 All-cause mortality, subgroup by sex & 12 & 55858 & $\begin{array}{l}\text { Risk Ratio (M-H, Random, 95\% } \\
\mathrm{Cl})\end{array}$ & $0.96[0.90,1.03]$ \\
\hline 1.14.1 Men & 9 & 4410 & $\begin{array}{l}\text { Risk Ratio (M-H, Random, 95\% } \\
\mathrm{Cl})\end{array}$ & $0.96[0.83,1.11]$ \\
\hline 1.14.2 Women & 2 & 51272 & $\begin{array}{l}\text { Risk Ratio (M-H, Random, 95\% } \\
\mathrm{Cl})\end{array}$ & $0.97[0.90,1.05]$ \\
\hline 1.14.3 Mixed, men and women & 1 & 176 & $\begin{array}{l}\text { Risk Ratio (M-H, Random, 95\% } \\
\text { Cl) }\end{array}$ & $0.33[0.07,1.61]$ \\
\hline
\end{tabular}




\begin{tabular}{|c|c|c|c|c|}
\hline Outcome or subgroup title & $\begin{array}{l}\text { No. of } \\
\text { studies }\end{array}$ & $\begin{array}{l}\text { No. of } \\
\text { partici- } \\
\text { pants }\end{array}$ & Statistical method & Effect size \\
\hline $\begin{array}{l}1.15 \text { All-cause mortality, subgroup by CVD } \\
\text { risk }\end{array}$ & 12 & 55858 & $\begin{array}{l}\text { Risk Ratio (M-H, Random, 95\% } \\
\mathrm{Cl})\end{array}$ & $0.96[0.90,1.03]$ \\
\hline 1.15.1 Low CVD risk & 4 & 52251 & $\begin{array}{l}\text { Risk Ratio (M-H, Random, 95\% } \\
\mathrm{Cl})\end{array}$ & $0.97[0.91,1.04]$ \\
\hline 1.15.2 Moderate CVD risk & 1 & 176 & $\begin{array}{l}\text { Risk Ratio (M-H, Random, 95\% } \\
\mathrm{Cl})\end{array}$ & $0.33[0.07,1.61]$ \\
\hline 1.15.3 Existing CVD disease & 7 & 3431 & $\begin{array}{l}\text { Risk Ratio (M-H, Random, 95\% } \\
\mathrm{Cl})\end{array}$ & $0.97[0.76,1.24]$ \\
\hline $\begin{array}{l}\text { 1.16 All-cause mortality, subgroup by TC re- } \\
\text { duction }\end{array}$ & 12 & & $\begin{array}{l}\text { Risk Ratio (M-H, Random, 95\% } \\
\mathrm{Cl})\end{array}$ & Subtotals only \\
\hline $\begin{array}{l}\text { 1.16.1 serum chol reduced by at least } \\
0.2 \mathrm{mmol} / \mathrm{L}\end{array}$ & 7 & 4238 & $\begin{array}{l}\text { Risk Ratio (M-H, Random, 95\% } \\
\mathrm{Cl})\end{array}$ & $0.96[0.81,1.14]$ \\
\hline 1.16 .2 serum chol reduced by $<0.2 \mathrm{mmol} / \mathrm{L}$ & 4 & 51487 & $\begin{array}{l}\text { Risk Ratio (M-H, Random, 95\% } \\
\mathrm{Cl})\end{array}$ & $0.97[0.90,1.04]$ \\
\hline 1.16.3 serum chol reduction unclear & 1 & 133 & $\begin{array}{l}\text { Risk Ratio (M-H, Random, 95\% } \\
\mathrm{CI})\end{array}$ & $0.51[0.05,5.46]$ \\
\hline $\begin{array}{l}1.17 \text { All-cause mortality, subgroup decade of } \\
\text { publication }\end{array}$ & 12 & 55858 & $\begin{array}{l}\text { Risk Ratio (M-H, Random, 95\% } \\
\text { CI) }\end{array}$ & $0.96[0.90,1.03]$ \\
\hline $1.17 .11960 \mathrm{~s}$ & 5 & 1731 & $\begin{array}{l}\text { Risk Ratio (M-H, Random, 95\% } \\
\mathrm{Cl})\end{array}$ & $0.92[0.80,1.07]$ \\
\hline $1.17 .21970 \mathrm{~s}$ & 1 & 458 & $\begin{array}{l}\text { Risk Ratio (M-H, Random, 95\% } \\
\mathrm{Cl})\end{array}$ & $1.49[0.95,2.34]$ \\
\hline $1.17 .31980 \mathrm{~s}$ & 1 & 2033 & $\begin{array}{l}\text { Risk Ratio (M-H, Random, 95\% } \\
\mathrm{CI})\end{array}$ & $0.98[0.76,1.25]$ \\
\hline 1.17.4 1990s & 2 & 188 & $\begin{array}{l}\text { Risk Ratio (M-H, Random, 95\% } \\
\mathrm{Cl} \text { ) }\end{array}$ & $0.41[0.08,2.07]$ \\
\hline $1.17 .52000 \mathrm{~s}$ & 3 & 51448 & $\begin{array}{l}\text { Risk Ratio (M-H, Random, 95\% } \\
\mathrm{Cl})\end{array}$ & $0.97[0.88,1.05]$ \\
\hline 1.18 CARDIOVASCULAR MORTALITY & 11 & 53421 & $\begin{array}{l}\text { Risk Ratio (M-H, Random, 95\% } \\
\text { CI) }\end{array}$ & $0.94[0.78,1.13]$ \\
\hline $\begin{array}{l}1.19 \text { CVD mortality, SA low summary risk of } \\
\text { bias }\end{array}$ & 4 & 50315 & $\begin{array}{l}\text { Risk Ratio (M-H, Random, 95\% } \\
\text { CI) }\end{array}$ & $0.96[0.67,1.38]$ \\
\hline 1.20 CVD mortality, SA aim to reduce SFA & 9 & 53112 & $\begin{array}{l}\text { Risk Ratio (M-H, Random, 95\% } \\
\mathrm{Cl})\end{array}$ & $0.95[0.79,1.14]$ \\
\hline $\begin{array}{l}1.21 \text { CVD mortality, SA statistically signifi- } \\
\text { cant SFA reduction }\end{array}$ & 7 & 52536 & $\begin{array}{l}\text { Risk Ratio (M-H, Random, 95\% } \\
\text { Cl) }\end{array}$ & $0.95[0.75,1.21]$ \\
\hline
\end{tabular}




\begin{tabular}{|c|c|c|c|c|}
\hline Outcome or subgroup title & $\begin{array}{l}\text { No. of } \\
\text { studies }\end{array}$ & $\begin{array}{l}\text { No. of } \\
\text { partici- } \\
\text { pants }\end{array}$ & Statistical method & Effect size \\
\hline 1.22 CVD mortality, SA TC reduction & 8 & 53073 & $\begin{array}{l}\text { Risk Ratio (M-H, Random, 95\% } \\
\mathrm{CI})\end{array}$ & $0.95[0.78,1.15]$ \\
\hline 1.23 CVD mortality, SA excluding WHI & 10 & 4586 & $\begin{array}{l}\text { Risk Ratio (M-H, Random, 95\% } \\
\mathrm{CI})\end{array}$ & $0.92[0.72,1.18]$ \\
\hline $\begin{array}{l}\text { 1.24 CVD mortality, SA Mantel-Haenszel } \\
\text { fixed-effect }\end{array}$ & 11 & 53421 & Risk Ratio (M-H, Fixed, 95\% Cl) & $0.95[0.85,1.07]$ \\
\hline 1.25 CVD mortality, SA Peto fixed-effect & 11 & 53421 & $\begin{array}{l}\text { Peto Odds Ratio (Peto, Fixed, } \\
95 \% \mathrm{Cl} \text { ) }\end{array}$ & $0.95[0.84,1.08]$ \\
\hline $\begin{array}{l}1.26 \text { CVD mortality, subgroup by any substi- } \\
\text { tution }\end{array}$ & 11 & & $\begin{array}{l}\text { Risk Ratio (M-H, Random, 95\% } \\
\mathrm{CI} \text { ) }\end{array}$ & Subtotals only \\
\hline 1.26.1 replaced by PUFA & 7 & 4251 & $\begin{array}{l}\text { Risk Ratio (M-H, Random, 95\% } \\
\mathrm{Cl} \text { ) }\end{array}$ & $0.95[0.73,1.25]$ \\
\hline 1.26.2 replaced by MUFA & 1 & 52 & $\begin{array}{l}\text { Risk Ratio (M-H, Random, 95\% } \\
\mathrm{Cl} \text { ) }\end{array}$ & $3.00[0.33,26.99]$ \\
\hline 1.26.3 replace by $\mathrm{CHO}$ & 5 & 51232 & $\begin{array}{l}\text { Risk Ratio (M-H, Random, 95\% } \\
\mathrm{CI})\end{array}$ & $0.99[0.85,1.14]$ \\
\hline 1.26 .4 replaced by protein & 4 & 51177 & $\begin{array}{l}\text { Risk Ratio (M-H, Random, 95\% } \\
\text { Cl) }\end{array}$ & $0.99[0.86,1.14]$ \\
\hline 1.26.5 replacement unclear & 0 & 0 & $\begin{array}{l}\text { Risk Ratio (M-H, Random, 95\% } \\
\text { Cl) }\end{array}$ & Not estimable \\
\hline $\begin{array}{l}1.27 \text { CVD mortality, subgroup by main sub- } \\
\text { stitution }\end{array}$ & 11 & & $\begin{array}{l}\text { Risk Ratio (M-H, Random, 95\% } \\
\mathrm{CI})\end{array}$ & Subtotals only \\
\hline 1.27.1 replaced by PUFA & 6 & 4196 & $\begin{array}{l}\text { Risk Ratio (M-H, Random, 95\% } \\
\text { Cl) }\end{array}$ & $0.97[0.73,1.28]$ \\
\hline 1.27.2 replaced by MUFA & 1 & 52 & $\begin{array}{l}\text { Risk Ratio (M-H, Random, 95\% } \\
\text { Cl) }\end{array}$ & $3.00[0.33,26.99]$ \\
\hline 1.27.3 replace by $\mathrm{CHO}$ & 4 & 49199 & $\begin{array}{l}\text { Risk Ratio (M-H, Random, 95\% } \\
\mathrm{Cl} \text { ) }\end{array}$ & $0.78[0.42,1.46]$ \\
\hline 1.27.4 replaced by protein & 0 & 0 & $\begin{array}{l}\text { Risk Ratio (M-H, Random, 95\% } \\
\mathrm{CI})\end{array}$ & Not estimable \\
\hline 1.27.5 replacement unclear & 0 & 0 & $\begin{array}{l}\text { Risk Ratio (M-H, Random, 95\% } \\
\text { Cl) }\end{array}$ & Not estimable \\
\hline 1.28 CVD mortality, subgroup by duration & 11 & 53447 & $\begin{array}{l}\text { Risk Ratio (M-H, Random, 95\% } \\
\mathrm{CI})\end{array}$ & $0.95[0.78,1.16]$ \\
\hline 1.28 .1 up to $24 \mathrm{mo}$ & 4 & 2272 & $\begin{array}{l}\text { Risk Ratio (M-H, Random, 95\% } \\
\text { Cl) }\end{array}$ & $1.26[0.54,2.94]$ \\
\hline
\end{tabular}




\begin{tabular}{|c|c|c|c|c|}
\hline Outcome or subgroup title & $\begin{array}{l}\text { No. of } \\
\text { studies }\end{array}$ & $\begin{array}{l}\text { No. of } \\
\text { partici- } \\
\text { pants }\end{array}$ & Statistical method & Effect size \\
\hline $1.28 .2>24$ to $48 \mathrm{mo}$ & 3 & 1294 & $\begin{array}{l}\text { Risk Ratio (M-H, Random, 95\% } \\
\mathrm{Cl})\end{array}$ & $0.79[0.57,1.08]$ \\
\hline $1.28 .3>48 \mathrm{mo}$ & 3 & 49705 & $\begin{array}{l}\text { Risk Ratio (M-H, Random, 95\% } \\
\mathrm{Cl})\end{array}$ & $1.02[0.73,1.43]$ \\
\hline 1.28.4 unclear duration & 1 & 176 & $\begin{array}{l}\text { Risk Ratio (M-H, Random, 95\% } \\
\text { Cl) }\end{array}$ & $0.25[0.03,2.19]$ \\
\hline $\begin{array}{l}1.29 \text { CVD mortality, subgroup by baseline } \\
\text { SFA }\end{array}$ & 11 & 53447 & $\begin{array}{l}\text { Risk Ratio (M-H, Random, 95\% } \\
\text { Cl) }\end{array}$ & $0.95[0.78,1.16]$ \\
\hline 1.29.1 up to $12 \%$ E SFA baseline & 0 & 0 & $\begin{array}{l}\text { Risk Ratio (M-H, Random, 95\% } \\
\mathrm{Cl})\end{array}$ & Not estimable \\
\hline 1.29.2 $>12$ to $15 \%$ E SFA baseline & 5 & 51635 & $\begin{array}{l}\text { Risk Ratio (M-H, Random, 95\% } \\
\text { Cl) }\end{array}$ & $1.06[0.84,1.32]$ \\
\hline $1.29 .3>15$ to $18 \%$ E SFA baseline & 1 & 55 & $\begin{array}{l}\text { Risk Ratio (M-H, Random, 95\% } \\
\mathrm{Cl})\end{array}$ & $0.35[0.04,3.12]$ \\
\hline 1.29.4 >18\%E SFA baseline & 1 & 846 & $\begin{array}{l}\text { Risk Ratio (M-H, Random, 95\% } \\
\mathrm{Cl} \text { ) }\end{array}$ & $0.70[0.51,0.96]$ \\
\hline 1.29.5 unclear & 4 & 911 & $\begin{array}{l}\text { Risk Ratio (M-H, Random, 95\% } \\
\text { Cl) }\end{array}$ & $1.00[0.61,1.66]$ \\
\hline 1.30 CVD mortality, subgroup by SFA change & 11 & 53447 & $\begin{array}{l}\text { Risk Ratio (M-H, Random, 95\% } \\
\text { Cl) }\end{array}$ & $0.95[0.78,1.16]$ \\
\hline 1.30.1 up to $4 \% \mathrm{E}$ difference & 4 & 51502 & $\begin{array}{l}\text { Risk Ratio (M-H, Random, 95\% } \\
\mathrm{Cl})\end{array}$ & $1.07[0.85,1.33]$ \\
\hline 1.30.2 $>4$ to $8 \% \mathrm{E}$ difference & 2 & 188 & $\begin{array}{l}\text { Risk Ratio (M-H, Random, 95\% } \\
\text { Cl) }\end{array}$ & $0.29[0.05,1.70]$ \\
\hline 1.30.3 >8\%E difference & 1 & 846 & $\begin{array}{l}\text { Risk Ratio (M-H, Random, 95\% } \\
\mathrm{Cl})\end{array}$ & $0.70[0.51,0.96]$ \\
\hline 1.30.4 unclear & 4 & 911 & $\begin{array}{l}\text { Risk Ratio (M-H, Random, 95\% } \\
\mathrm{Cl})\end{array}$ & $1.00[0.61,1.66]$ \\
\hline 1.31 CVD mortality, subgroup by sex & 11 & 53447 & $\begin{array}{l}\text { Risk Ratio (M-H, Random, 95\% } \\
\mathrm{Cl})\end{array}$ & $0.95[0.78,1.16]$ \\
\hline 1.31.1 Men & 9 & 4436 & $\begin{array}{l}\text { Risk Ratio (M-H, Random, 95\% } \\
\mathrm{Cl})\end{array}$ & $0.96[0.73,1.25]$ \\
\hline 1.31.2 Women & 1 & 48835 & $\begin{array}{l}\text { Risk Ratio (M-H, Random, 95\% } \\
\mathrm{Cl})\end{array}$ & $1.00[0.84,1.19]$ \\
\hline 1.31.3 Mixed, men and women & 1 & 176 & $\begin{array}{l}\text { Risk Ratio (M-H, Random, 95\% } \\
\text { Cl) }\end{array}$ & $0.25[0.03,2.19]$ \\
\hline
\end{tabular}




\begin{tabular}{|c|c|c|c|c|}
\hline Outcome or subgroup title & $\begin{array}{l}\text { No. of } \\
\text { studies }\end{array}$ & $\begin{array}{l}\text { No. of } \\
\text { partici- } \\
\text { pants }\end{array}$ & Statistical method & Effect size \\
\hline 1.32 CVD mortality, subgroup by CVD risk & 11 & 53447 & $\begin{array}{l}\text { Risk Ratio (M-H, Random, 95\% } \\
\mathrm{Cl})\end{array}$ & $0.96[0.80,1.14]$ \\
\hline 1.32.1 Low CVD risk & 3 & 47537 & $\begin{array}{l}\text { Risk Ratio (M-H, Random, 95\% } \\
\text { Cl) }\end{array}$ & $0.84[0.60,1.16]$ \\
\hline 1.32.2 Moderate CVD risk & 1 & 176 & $\begin{array}{l}\text { Risk Ratio (M-H, Random, 95\% } \\
\text { Cl) }\end{array}$ & $0.25[0.03,2.19]$ \\
\hline 1.32.3 Existing CVD disease & 8 & 5734 & $\begin{array}{l}\text { Risk Ratio (M-H, Random, 95\% } \\
\text { Cl) }\end{array}$ & $1.04[0.83,1.31]$ \\
\hline $\begin{array}{l}1.33 \text { CVD mortality, subgroup by TC reduc- } \\
\text { tion }\end{array}$ & 11 & & $\begin{array}{l}\text { Risk Ratio (M-H, Random, 95\% } \\
\text { Cl) }\end{array}$ & Subtotals only \\
\hline $\begin{array}{l}1.33 .1 \text { serum chol reduced by at least } \\
0.2 \mathrm{mmol} / \mathrm{L}\end{array}$ & 7 & 4251 & $\begin{array}{l}\text { Risk Ratio (M-H, Random, 95\% } \\
\text { Cl) }\end{array}$ & $0.95[0.73,1.25]$ \\
\hline 1.33 .2 serum chol reduced by $<0.2 \mathrm{mmol} / \mathrm{L}$ & 3 & 49063 & $\begin{array}{l}\text { Risk Ratio (M-H, Random, 95\% } \\
\mathrm{Cl})\end{array}$ & $0.97[0.47,2.01]$ \\
\hline 1.33.3 serum chol reduction unclear & 1 & 133 & $\begin{array}{l}\text { Risk Ratio (M-H, Random, 95\% } \\
\mathrm{Cl})\end{array}$ & $0.20[0.01,4.15]$ \\
\hline $\begin{array}{l}\text { 1.34 CVD mortality, subgroup decade of } \\
\text { publication }\end{array}$ & 11 & 53421 & $\begin{array}{l}\text { Risk Ratio (M-H, Random, 95\% } \\
\mathrm{Cl})\end{array}$ & $0.94[0.78,1.13]$ \\
\hline $1.34 .11960 \mathrm{~s}$ & 5 & 1731 & $\begin{array}{l}\text { Risk Ratio (M-H, Random, 95\% } \\
\mathrm{Cl})\end{array}$ & $0.78[0.63,0.97]$ \\
\hline $1.34 .21970 \mathrm{~s}$ & 1 & 458 & $\begin{array}{l}\text { Risk Ratio (M-H, Random, 95\% } \\
\mathrm{Cl})\end{array}$ & $1.59[0.99,2.55]$ \\
\hline $1.34 .31980 \mathrm{~s}$ & 1 & 2033 & $\begin{array}{l}\text { Risk Ratio (M-H, Random, 95\% } \\
\mathrm{Cl})\end{array}$ & $1.01[0.77,1.31]$ \\
\hline $1.34 .41990 \mathrm{~s}$ & 2 & 188 & $\begin{array}{l}\text { Risk Ratio (M-H, Random, 95\% } \\
\mathrm{Cl})\end{array}$ & $0.29[0.05,1.70]$ \\
\hline $1.34 .52000 \mathrm{~s}$ & 2 & 49011 & $\begin{array}{l}\text { Risk Ratio (M-H, Random, 95\% } \\
\mathrm{Cl})\end{array}$ & $0.78[0.27,2.21]$ \\
\hline 1.35 COMBINED CARDIOVASCULAR EVENTS & 12 & 53300 & $\begin{array}{l}\text { Risk Ratio (M-H, Random, 95\% } \\
\text { Cl) }\end{array}$ & $0.79[0.66,0.93]$ \\
\hline $\begin{array}{l}1.36 \text { CVD events, SA low summary risk of } \\
\text { bias }\end{array}$ & 3 & 49857 & $\begin{array}{l}\text { Risk Ratio (M-H, Random, 95\% } \\
\text { Cl) }\end{array}$ & $0.89[0.75,1.07]$ \\
\hline 1.37 CVD events, SA aim to reduce SFA & 10 & 52991 & $\begin{array}{l}\text { Risk Ratio (M-H, Random, 95\% } \\
\mathrm{Cl})\end{array}$ & $0.79[0.66,0.95]$ \\
\hline $\begin{array}{l}1.38 \text { CVD events, SA statistically significant } \\
\text { SFA reduction }\end{array}$ & 7 & 52313 & $\begin{array}{l}\text { Risk Ratio (M-H, Random, 95\% } \\
\mathrm{Cl} \text { ) }\end{array}$ & $0.85[0.71,1.02]$ \\
\hline
\end{tabular}




\begin{tabular}{|c|c|c|c|c|}
\hline Outcome or subgroup title & $\begin{array}{l}\text { No. of } \\
\text { studies }\end{array}$ & $\begin{array}{l}\text { No. of } \\
\text { partici- } \\
\text { pants }\end{array}$ & Statistical method & Effect size \\
\hline 1.39 CVD events, SA TC reduction & 9 & 52952 & $\begin{array}{l}\text { Risk Ratio (M-H, Random, 95\% } \\
\mathrm{Cl})\end{array}$ & $0.78[0.65,0.94]$ \\
\hline 1.40 CVD events, SA excluding WHI & 11 & 4465 & $\begin{array}{l}\text { Risk Ratio (M-H, Random, 95\% } \\
\mathrm{Cl})\end{array}$ & $0.75[0.61,0.91]$ \\
\hline $\begin{array}{l}\text { 1.41 CVD events, SA Mantel-Haenszel fixed- } \\
\text { effect }\end{array}$ & 12 & 53300 & Risk Ratio (M-H, Fixed, 95\% Cl) & $0.93[0.88,0.99]$ \\
\hline 1.42 CVD events, SA Peto fixed-effect & 12 & 53300 & $\begin{array}{l}\text { Peto Odds Ratio (Peto, Fixed, } \\
95 \% \mathrm{Cl} \text { ) }\end{array}$ & $0.92[0.87,0.98]$ \\
\hline $\begin{array}{l}\text { 1.43 CVD events, SA excluding trials with ad- } \\
\text { ditional interventions }\end{array}$ & 9 & 3998 & $\begin{array}{l}\text { Risk Ratio (M-H, Random, 95\% } \\
\text { Cl) }\end{array}$ & $0.80[0.64,0.99]$ \\
\hline $\begin{array}{l}1.44 \text { CVD events, subgroup by any substitu- } \\
\text { tion }\end{array}$ & 12 & & $\begin{array}{l}\text { Risk Ratio (M-H, Random, 95\% } \\
\text { Cl) }\end{array}$ & Subtotals only \\
\hline 1.44.1 replaced by PUFA & 7 & 3895 & $\begin{array}{l}\text { Risk Ratio (M-H, Random, 95\% } \\
\text { Cl) }\end{array}$ & $0.73[0.58,0.92]$ \\
\hline 1.44.2 replaced by MUFA & 1 & 52 & $\begin{array}{l}\text { Risk Ratio (M-H, Random, 95\% } \\
\mathrm{Cl})\end{array}$ & $1.00[0.53,1.89]$ \\
\hline 1.44.3 replace by $\mathrm{CHO}$ & 5 & 51232 & $\begin{array}{l}\text { Risk Ratio (M-H, Random, 95\% } \\
\mathrm{Cl})\end{array}$ & $0.84[0.67,1.06]$ \\
\hline 1.44.4 replaced by protein & 4 & 51177 & $\begin{array}{l}\text { Risk Ratio (M-H, Random, 95\% } \\
\mathrm{Cl})\end{array}$ & $0.97[0.91,1.03]$ \\
\hline 1.44.5 replacement unclear & 1 & 235 & $\begin{array}{l}\text { Risk Ratio (M-H, Random, 95\% } \\
\mathrm{Cl})\end{array}$ & $1.68[0.41,6.87]$ \\
\hline $\begin{array}{l}1.45 \text { CVD events, subgroup by main substitu- } \\
\text { tion }\end{array}$ & 12 & & $\begin{array}{l}\text { Risk Ratio (M-H, Random, 95\% } \\
\mathrm{Cl})\end{array}$ & Subtotals only \\
\hline 1.45.1 replaced by PUFA & 6 & 3840 & $\begin{array}{l}\text { Risk Ratio (M-H, Random, 95\% } \\
\mathrm{Cl})\end{array}$ & $0.78[0.62,0.97]$ \\
\hline 1.45.2 replaced by MUFA & 1 & 52 & $\begin{array}{l}\text { Risk Ratio (M-H, Random, 95\% } \\
\mathrm{Cl})\end{array}$ & $1.00[0.53,1.89]$ \\
\hline 1.45.3 replace by $\mathrm{CHO}$ & 4 & 49199 & $\begin{array}{l}\text { Risk Ratio (M-H, Random, 95\% } \\
\mathrm{Cl})\end{array}$ & $0.67[0.39,1.16]$ \\
\hline 1.45.4 replaced by protein & 0 & 0 & $\begin{array}{l}\text { Risk Ratio (M-H, Random, 95\% } \\
\text { Cl) }\end{array}$ & Not estimable \\
\hline 1.45.5 replacement unclear & 1 & 235 & $\begin{array}{l}\text { Risk Ratio (M-H, Random, 95\% } \\
\mathrm{Cl})\end{array}$ & $1.68[0.41,6.87]$ \\
\hline 1.46 CVD events, subgroup by duration & 12 & 53300 & $\begin{array}{l}\text { Risk Ratio (M-H, Random, 95\% } \\
\text { CI) }\end{array}$ & $0.79[0.66,0.93]$ \\
\hline
\end{tabular}




\begin{tabular}{|c|c|c|c|c|}
\hline Outcome or subgroup title & $\begin{array}{l}\text { No. of } \\
\text { studies }\end{array}$ & $\begin{array}{l}\text { No. of } \\
\text { partici- } \\
\text { pants }\end{array}$ & Statistical method & Effect size \\
\hline 1.46 .1 up to $24 \mathrm{mo}$ & 5 & 2481 & $\begin{array}{l}\text { Risk Ratio (M-H, Random, 95\% } \\
\mathrm{Cl})\end{array}$ & $0.96[0.78,1.16]$ \\
\hline $1.46 .2>24$ to $48 \mathrm{mo}$ & 3 & 1294 & $\begin{array}{l}\text { Risk Ratio (M-H, Random, 95\% } \\
\mathrm{Cl})\end{array}$ & $0.73[0.56,0.95]$ \\
\hline $1.46 .3>48 \mathrm{mo}$ & 2 & 49247 & $\begin{array}{l}\text { Risk Ratio (M-H, Random, 95\% } \\
\text { Cl) }\end{array}$ & $0.85[0.63,1.16]$ \\
\hline 1.46.4 unclear duration & 2 & 278 & $\begin{array}{l}\text { Risk Ratio (M-H, Random, 95\% } \\
\text { Cl) }\end{array}$ & $0.43[0.17,1.08]$ \\
\hline 1.47 CVD events, subgroup by baseline SFA & 12 & 53300 & $\begin{array}{l}\text { Risk Ratio (M-H, Random, 95\% } \\
\mathrm{Cl})\end{array}$ & $0.79[0.66,0.93]$ \\
\hline 1.47.1 up to $12 \%$ E SFA baseline & 0 & 0 & $\begin{array}{l}\text { Risk Ratio (M-H, Random, 95\% } \\
\mathrm{Cl})\end{array}$ & Not estimable \\
\hline 1.47.2 $>12$ to $15 \%$ E SFA baseline & 5 & 51412 & $\begin{array}{l}\text { Risk Ratio (M-H, Random, 95\% } \\
\mathrm{Cl})\end{array}$ & $0.97[0.91,1.03]$ \\
\hline $1.47 .3>15$ to $18 \%$ E SFA baseline & 1 & 55 & $\begin{array}{l}\text { Risk Ratio (M-H, Random, 95\% } \\
\mathrm{Cl} \text { ) }\end{array}$ & $0.41[0.22,0.78]$ \\
\hline 1.47.4 >18\%E SFA baseline & 1 & 846 & $\begin{array}{l}\text { Risk Ratio (M-H, Random, 95\% } \\
\text { Cl) }\end{array}$ & $0.79[0.63,1.00]$ \\
\hline 1.47.5 unclear & 5 & 987 & $\begin{array}{l}\text { Risk Ratio (M-H, Random, 95\% } \\
\text { Cl) }\end{array}$ & $0.72[0.51,1.03]$ \\
\hline 1.48 CVD events, subgroup by SFA change & 12 & 53300 & $\begin{array}{l}\text { Risk Ratio (M-H, Random, 95\% } \\
\mathrm{Cl} \text { ) }\end{array}$ & $0.79[0.66,0.93]$ \\
\hline 1.48.1 up to $4 \% \mathrm{E}$ difference & 4 & 51279 & $\begin{array}{l}\text { Risk Ratio (M-H, Random, 95\% } \\
\text { Cl) }\end{array}$ & $0.97[0.91,1.03]$ \\
\hline 1.48.2 $>4$ to $8 \%$ E difference & 2 & 188 & $\begin{array}{l}\text { Risk Ratio (M-H, Random, 95\% } \\
\mathrm{Cl})\end{array}$ & $0.40[0.22,0.74]$ \\
\hline $1.48 .3>8 \% \mathrm{E}$ difference & 1 & 846 & $\begin{array}{l}\text { Risk Ratio (M-H, Random, 95\% } \\
\mathrm{Cl})\end{array}$ & $0.79[0.63,1.00]$ \\
\hline 1.48.4 unclear & 5 & 987 & $\begin{array}{l}\text { Risk Ratio (M-H, Random, 95\% } \\
\mathrm{Cl})\end{array}$ & $0.72[0.51,1.03]$ \\
\hline 1.49 CVD events, subgroup by sex & 12 & 53300 & $\begin{array}{l}\text { Risk Ratio (M-H, Random, 95\% } \\
\mathrm{Cl})\end{array}$ & $0.79[0.66,0.93]$ \\
\hline 1.49.1 Men & 8 & 3952 & $\begin{array}{l}\text { Risk Ratio (M-H, Random, 95\% } \\
\mathrm{Cl})\end{array}$ & $0.80[0.69,0.93]$ \\
\hline 1.49.2 Women & 1 & 48835 & $\begin{array}{l}\text { Risk Ratio (M-H, Random, 95\% } \\
\text { Cl) }\end{array}$ & $0.98[0.92,1.04]$ \\
\hline
\end{tabular}




\begin{tabular}{|c|c|c|c|c|}
\hline Outcome or subgroup title & $\begin{array}{l}\text { No. of } \\
\text { studies }\end{array}$ & $\begin{array}{l}\text { No. of } \\
\text { partici- } \\
\text { pants }\end{array}$ & Statistical method & Effect size \\
\hline 1.49.3 Mixed, men and women & 3 & 513 & $\begin{array}{l}\text { Risk Ratio (M-H, Random, 95\% } \\
\text { Cl) }\end{array}$ & $0.59[0.23,1.49]$ \\
\hline 1.50 CVD events, subgroup by CVD risk & 12 & 53300 & $\begin{array}{l}\text { Risk Ratio (M-H, Random, 95\% } \\
\mathrm{Cl})\end{array}$ & $0.83[0.72,0.96]$ \\
\hline 1.50.1 Low CVD risk & 3 & 47537 & $\begin{array}{l}\text { Risk Ratio (M-H, Random, 95\% } \\
\text { Cl) }\end{array}$ & $0.89[0.75,1.06]$ \\
\hline 1.50.2 Moderate CVD risk & 3 & 513 & $\begin{array}{l}\text { Risk Ratio (M-H, Random, 95\% } \\
\mathrm{Cl})\end{array}$ & $0.59[0.23,1.49]$ \\
\hline 1.50.3 Existing CVD disease & 7 & 5250 & $\begin{array}{l}\text { Risk Ratio (M-H, Random, 95\% } \\
\text { Cl) }\end{array}$ & $0.86[0.71,1.05]$ \\
\hline 1.51 CVD events, subgroup by TC reduction & 12 & & $\begin{array}{l}\text { Risk Ratio (M-H, Random, 95\% } \\
\mathrm{Cl})\end{array}$ & Subtotals only \\
\hline $\begin{array}{l}\text { 1.51.1 serum chol reduced by at least } \\
0.2 \mathrm{mmol} / \mathrm{L}\end{array}$ & 8 & 4117 & $\begin{array}{l}\text { Risk Ratio (M-H, Random, 95\% } \\
\mathrm{Cl})\end{array}$ & $0.74[0.59,0.92]$ \\
\hline 1.51 .2 serum chol reduced by $<0.2 \mathrm{mmol} / \mathrm{L}$ & 3 & 49050 & $\begin{array}{l}\text { Risk Ratio (M-H, Random, 95\% } \\
\mathrm{Cl})\end{array}$ & $0.98[0.91,1.04]$ \\
\hline 1.51.3 serum chol reduction unclear & 1 & 133 & $\begin{array}{l}\text { Risk Ratio (M-H, Random, 95\% } \\
\mathrm{Cl})\end{array}$ & $0.20[0.01,4.15]$ \\
\hline $\begin{array}{l}1.52 \text { CVD events, subgroup decade of publi- } \\
\text { cation }\end{array}$ & 12 & 53300 & $\begin{array}{l}\text { Risk Ratio (M-H, Random, 95\% } \\
\mathrm{Cl})\end{array}$ & $0.79[0.66,0.93]$ \\
\hline $1.52 .11960 \mathrm{~s}$ & 5 & 1731 & $\begin{array}{l}\text { Risk Ratio (M-H, Random, 95\% } \\
\mathrm{Cl})\end{array}$ & $0.79[0.69,0.91]$ \\
\hline $1.52 .21970 \mathrm{~s}$ & 1 & 102 & $\begin{array}{l}\text { Risk Ratio (M-H, Random, 95\% } \\
\text { Cl) }\end{array}$ & $0.27[0.14,0.52]$ \\
\hline $1.52 .31980 \mathrm{~s}$ & 1 & 2033 & $\begin{array}{l}\text { Risk Ratio (M-H, Random, 95\% } \\
\text { CI) }\end{array}$ & $0.92[0.74,1.15]$ \\
\hline $1.52 .41990 \mathrm{~s}$ & 2 & 188 & $\begin{array}{l}\text { Risk Ratio (M-H, Random, 95\% } \\
\mathrm{Cl})\end{array}$ & $0.40[0.22,0.74]$ \\
\hline $1.52 .52000 \mathrm{~s}$ & 3 & 49246 & $\begin{array}{l}\text { Risk Ratio (M-H, Random, 95\% } \\
\text { Cl) }\end{array}$ & $0.98[0.91,1.04]$ \\
\hline
\end{tabular}


Analysis 1.1. Comparison 1: SFA reduction vs usual diet - primary outcomes, Outcome 1: ALL-CAUSE MORTALITY

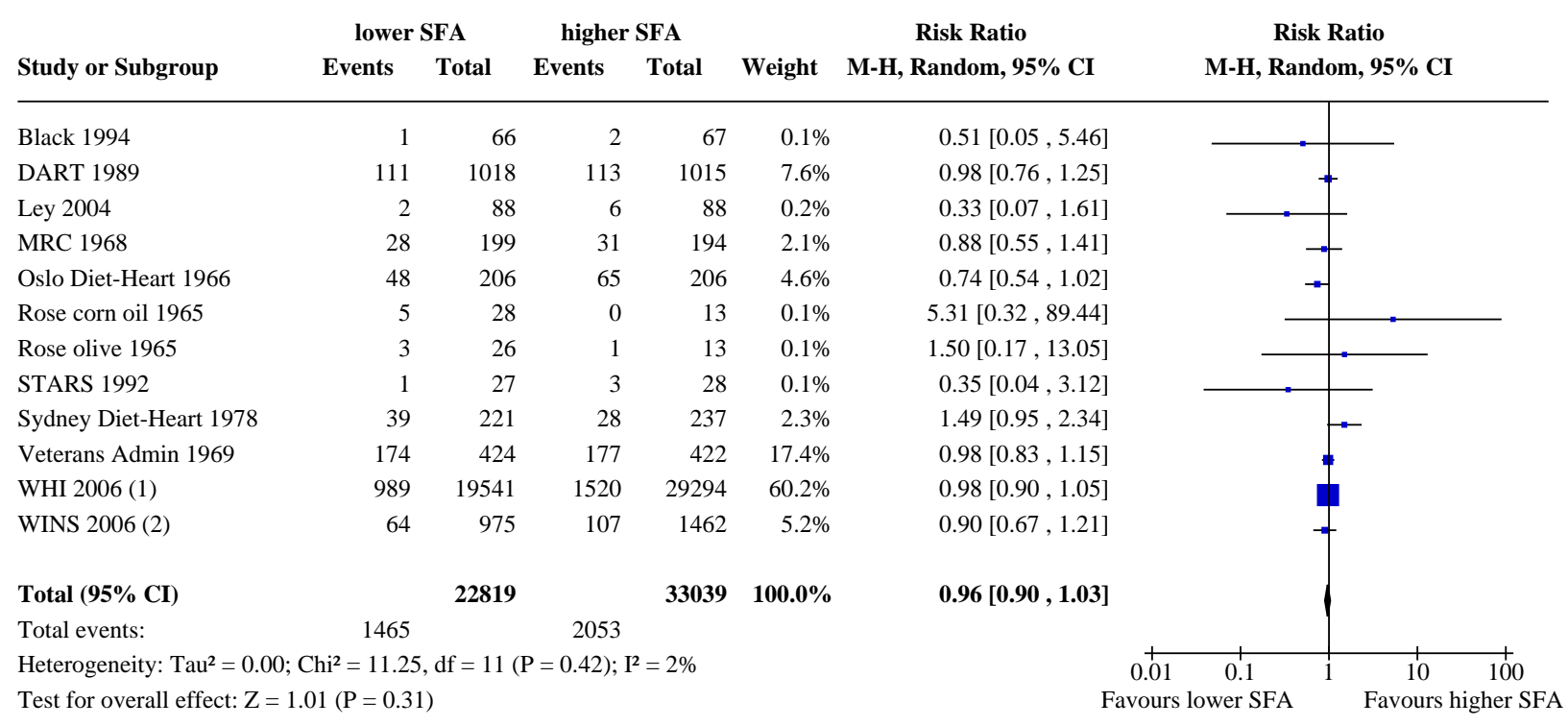

Footnotes

(1) All-cause death during study, Prentice 2017

(2) All-cause mortality during trial, Chlebowski 2015

\section{Analysis 1.2. Comparison 1: SFA reduction vs usual diet - primary outcomes, Outcome 2: All-cause mortality, SA low summary risk of bias}

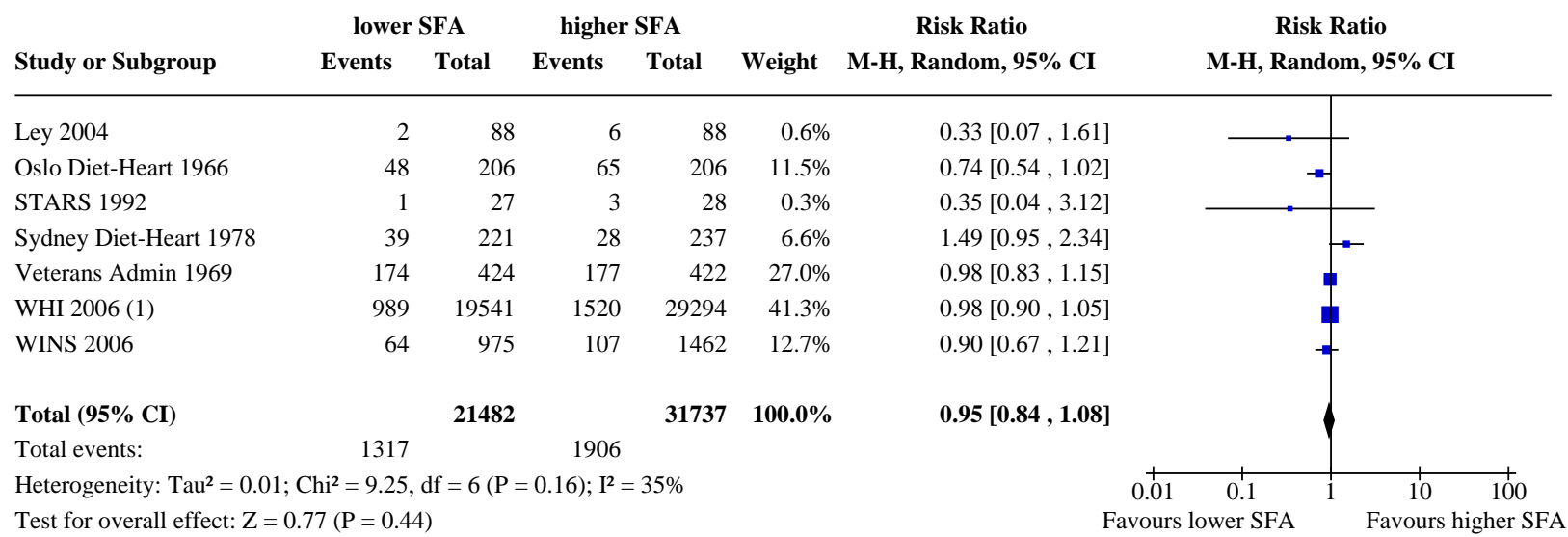

Footnotes

(1) All-cause death during study, Prentice 2017 


\section{Analysis 1.3. Comparison 1: SFA reduction vs usual diet - primary outcomes, Outcome 3: All-cause mortality, SA aim to reduce SFA}

\begin{tabular}{|c|c|c|c|c|c|c|c|}
\hline \multirow[b]{2}{*}{ Study or Subgroup } & \multicolumn{2}{|c|}{ lower SFA } & \multicolumn{2}{|c|}{ higher SFA } & \multirow[b]{2}{*}{ Weight } & \multirow{2}{*}{$\begin{array}{c}\text { Risk Ratio } \\
\text { M-H, Random, } 95 \% \text { CI }\end{array}$} & \multirow{2}{*}{$\begin{array}{c}\text { Risk Ratio } \\
\text { M-H, Random, } 95 \% \text { CI }\end{array}$} \\
\hline & Events & Total & Events & Total & & & \\
\hline DART 1989 & 111 & 1018 & 113 & 1015 & $10.8 \%$ & $0.98[0.76,1.25]$ & \\
\hline MRC 1968 & 28 & 199 & 31 & 194 & $3.2 \%$ & $0.88[0.55,1.41]$ & \\
\hline Oslo Diet-Heart 1966 & 48 & 206 & 65 & 206 & $6.8 \%$ & $0.74[0.54,1.02]$ & - \\
\hline Rose corn oil 1965 & 5 & 28 & 0 & 13 & $0.1 \%$ & $5.31[0.32,89.44]$ & \\
\hline Rose olive 1965 & 3 & 26 & 1 & 13 & $0.2 \%$ & $1.50[0.17,13.05]$ & \\
\hline STARS 1992 & 1 & 27 & 3 & 28 & $0.2 \%$ & $0.35[0.04,3.12]$ & \\
\hline Sydney Diet-Heart 1978 & 39 & 221 & 28 & 237 & $3.5 \%$ & $1.49[0.95,2.34]$ & - \\
\hline Veterans Admin 1969 & 174 & 424 & 177 & 422 & $22.2 \%$ & $0.98[0.83,1.15]$ & \\
\hline WHI $2006(1)$ & 989 & 19541 & 1520 & 29294 & $53.1 \%$ & $0.98[0.90,1.05]$ & \\
\hline Total $(95 \%$ CI $)$ & & 21690 & & 31422 & $100.0 \%$ & $0.97[0.89,1.06]$ & \\
\hline Total events: & 1398 & & 1938 & & & & \\
\hline \multicolumn{7}{|c|}{ Heterogeneity: $\mathrm{Tau}^{2}=0.00 ; \mathrm{Chi}^{2}=8.94, \mathrm{df}=8(\mathrm{P}=0.35) ; \mathrm{I}^{2}=11 \%$} & $0.01 \quad 0.1$ \\
\hline \multicolumn{6}{|c|}{ Test for overall effect: $\mathrm{Z}=0.69(\mathrm{P}=0.49)$} & & Favours lower SFA \\
\hline
\end{tabular}

Test for subgroup differences: Not applicable

Footnotes

(1) All-cause death during study, Prentice 2017

\section{Analysis 1.4. Comparison 1: SFA reduction vs usual diet - primary outcomes, Outcome 4: All-cause mortality, SA statistically significant SFA reduction}

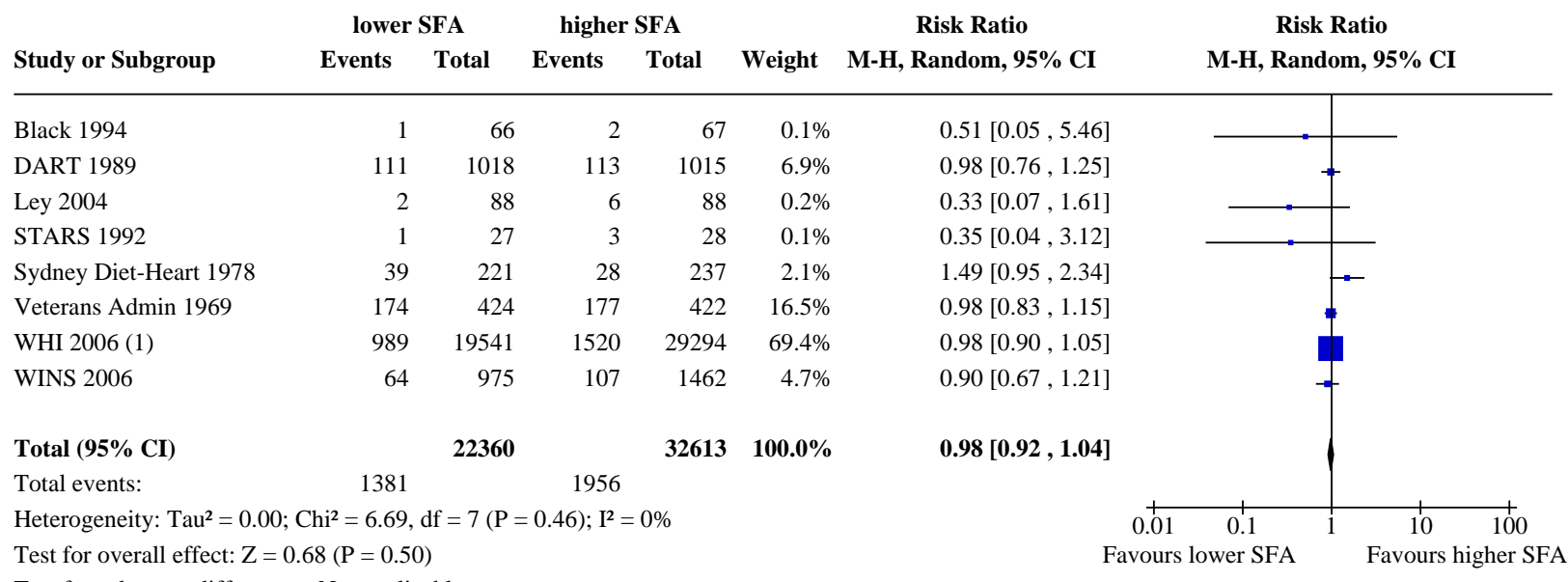

Test for subgroup differences: Not applicable

Footnotes

(1) All-cause death during study, Prentice 2017 


\section{Analysis 1.5. Comparison 1: SFA reduction vs usual diet - primary outcomes, Outcome 5: All-cause mortality, SA TC reduction}

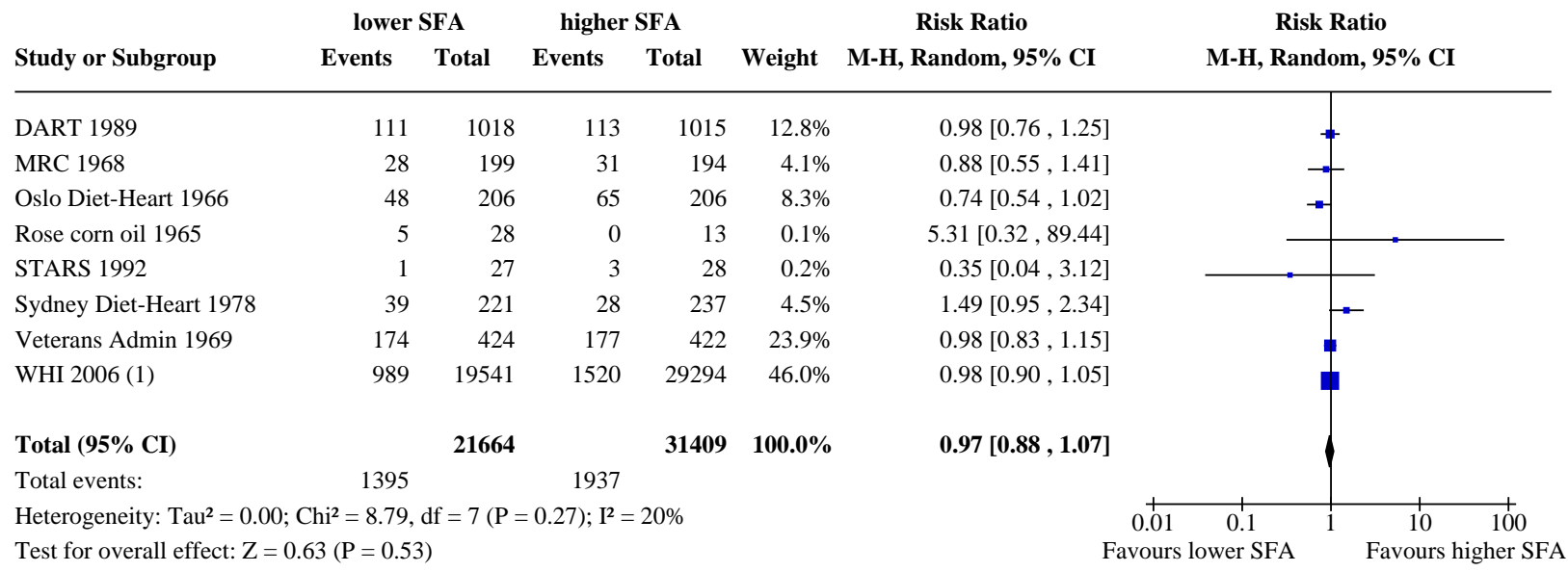

Test for subgroup differences: Not applicable

Footnotes

(1) All-cause death during study, Prentice 2017

\section{Analysis 1.6. Comparison 1: SFA reduction vs usual diet - primary outcomes, Outcome 6: All-cause mortality, SA excluding WHI}

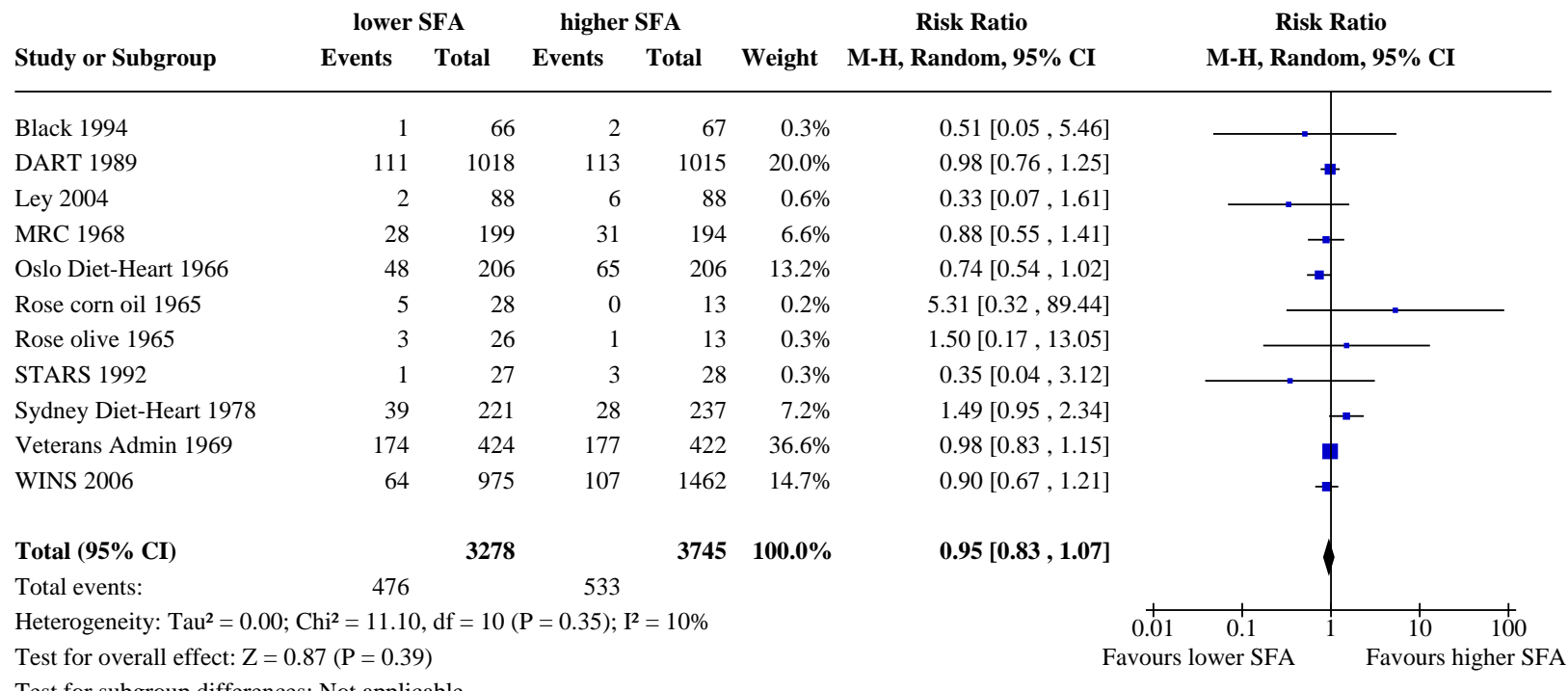


Analysis 1.7. Comparison 1: SFA reduction vs usual diet - primary outcomes, Outcome 7: All-cause mortality, SA Mantel-Haenszel fixed-effect

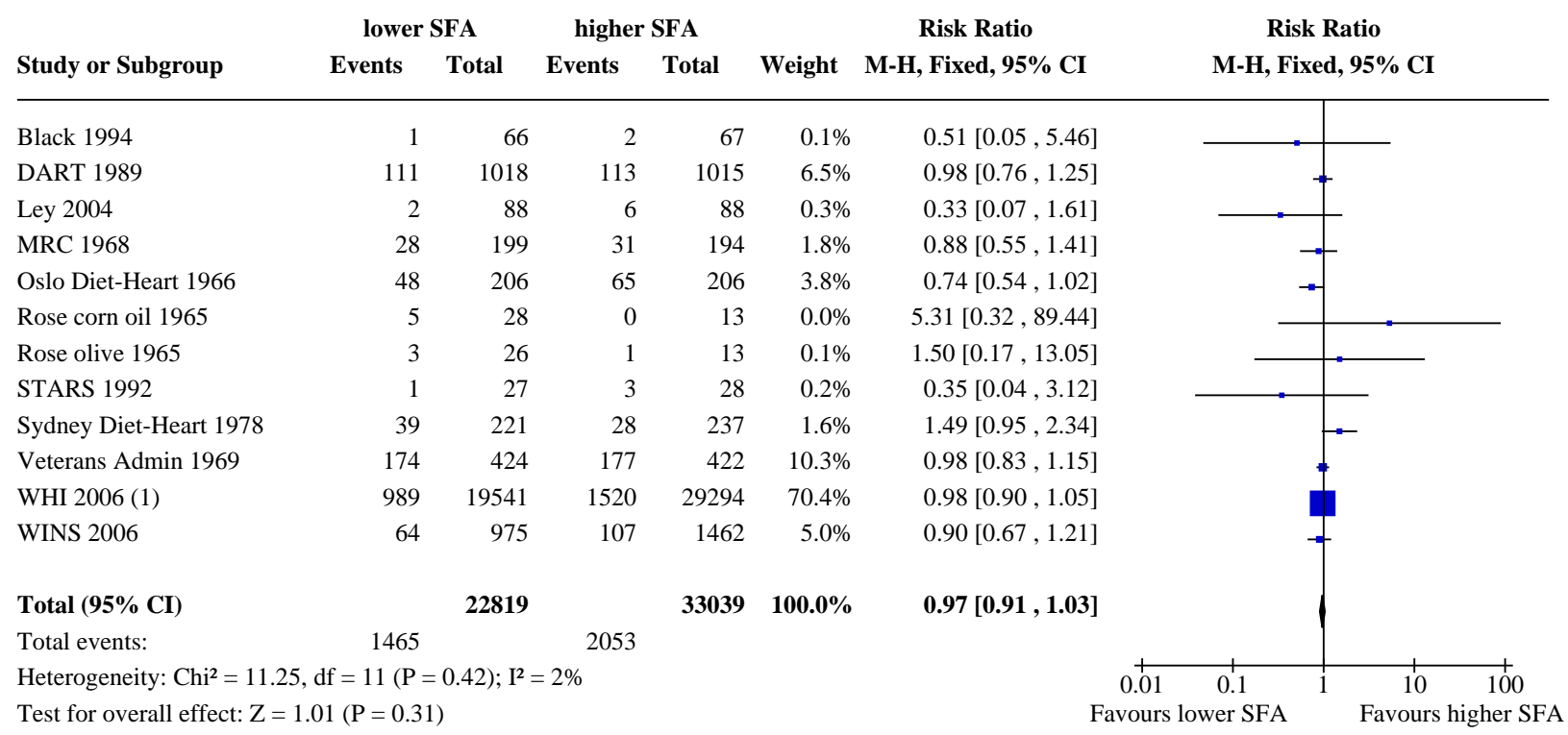

Test for subgroup differences: Not applicable

Footnotes

(1) All-cause death during study, Prentice 2017

\section{Analysis 1.8. Comparison 1: SFA reduction vs usual diet - primary outcomes, Outcome 8: All-cause mortality, SA Peto fixed-effect}

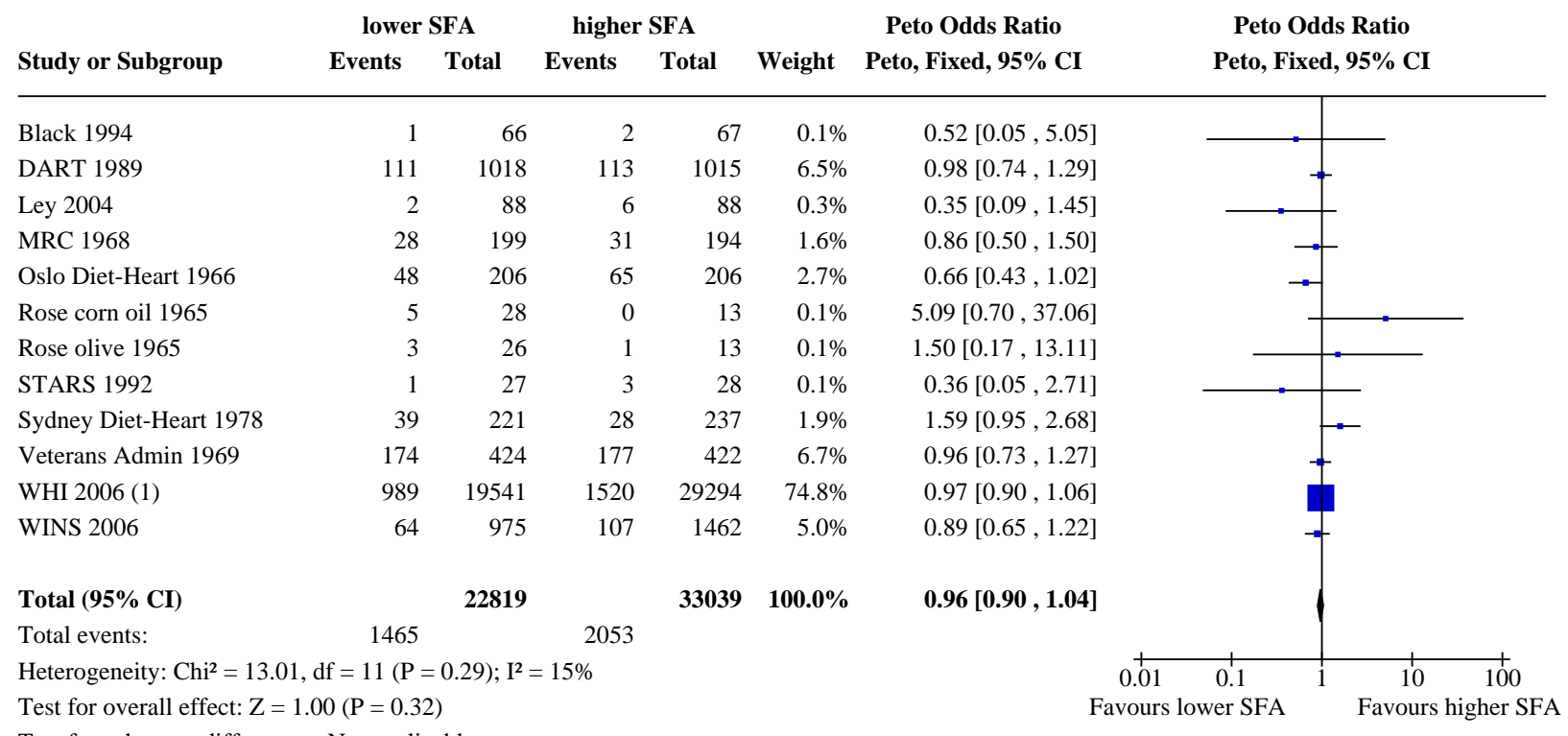

Footnotes

(1) All-cause death during study, Prentice 2017 
Analysis 1.9. Comparison 1: SFA reduction vs usual diet - primary outcomes, Outcome 9: All-cause mortality, subgroup by any substitution

\begin{tabular}{|c|c|c|c|c|c|c|c|}
\hline & low & SFA & high & SFA & & Risk Ratio & Risk Ratio \\
\hline Study or Subgroup & Events & Total & Events & Total & Weight & M-H, Random, 95\% CI & M-H, Random, 95\% CI \\
\hline
\end{tabular}

1.9.1 replaced by PUFA

DART 1989

MRC 1968

Oslo Diet-Heart 1966

Rose corn oil 1965

STARS 1992

Sydney Diet-Heart 1978

Veterans Admin 1969

Subtotal (95\% CI)

Total events:

Heterogeneity: $\mathrm{Tau}^{2}=0.01 ; \mathrm{Chi}^{2}=8.06, \mathrm{df}=6(\mathrm{P}=0.23) ; \mathrm{I}^{2}=26 \%$

Test for overall effect: $\mathrm{Z}=0.46(\mathrm{P}=0.64)$

\subsection{2 replaced by MUFA}

Rose olive 1965

Subtotal $(95 \%$ CI $)$

Total events:

26

26

$100.0 \%$

$26 \quad \mathbf{1 0 0 . 0} \%$

3

1

Heterogeneity: Not applicable

Test for overall effect: $\mathrm{Z}=0.98(\mathrm{P}=0.33)$

\subsection{3 replaced by $\mathrm{CHO}$}

Black 1994

DART 1989

Ley 2004

STARS 1992

WHI 2006 (1)

WINS 2006 (2)

Subtotal (95\% CI)

Total events:

$\begin{array}{rr}1 & 66 \\ 111 & 1018 \\ 2 & 88 \\ 1 & 27 \\ 989 & 19541 \\ 64 & 975 \\ & \mathbf{2 1 7 1 5}\end{array}$

2

67

$0.1 \%$

$8.5 \%$

$0.2 \%$

$0.1 \%$

$\begin{array}{rrr}3 & 28 & 0.1 \% \\ 1520 & 29294 & 85.3 \% \\ 107 & 1462 & 5.8 \%\end{array}$

$31954 \quad 100.0 \%$ 1751

Heterogeneity: $\mathrm{Tau}^{2}=0.00 ; \mathrm{Chi}^{2}=3.19, \mathrm{df}=5(\mathrm{P}=0.67) ; \mathrm{I}^{2}=0 \%$

Test for overall effect: $\mathrm{Z}=0.91(\mathrm{P}=0.36)$

1.9.4 replaced by protein

Black 1994

DART 1989

Ley 2004

WHI 2006 (1)

WINS 2006 (2)

Subtotal (95\% CI)

Total events:

$\begin{array}{rr}1 & 66 \\ 111 & 1018 \\ 2 & 88 \\ 989 & 19541 \\ 64 & 975 \\ & \mathbf{2 1 6 8 8}\end{array}$

1167
2

113

6
1520

$1520-29294$

$31926100.0 \%$

1748
$107 \quad 1462$

$0.51[0.05,5.46]$

$0.98[0.76,1.25]$

$0.33[0.07,1.61]$

$0.35[0.04,3.12]$

$0.98[0.90,1.05]$

$0.90[0.67,1.21]$

$0.97[0.90,1.04]$

$0.51[0.05,5.46]$

$0.98[0.76,1.25]$

$0.33[0.07,1.61]$

$0.98[0.90,1.05]$

$0.90[0.67,1.21]$

$0.97[0.90,1.04]$

Heterogeneity: $\mathrm{Tau}^{2}=0.00 ; \mathrm{Chi}^{2}=2.34, \mathrm{df}=4(\mathrm{P}=0.67) ; \mathrm{I}^{2}=0 \%$

Test for overall effect: $\mathrm{Z}=0.88(\mathrm{P}=0.38)$

1.9.5 replacement unclear

Subtotal (95\% CI)

$\mathbf{0}$

Total events:

0

Heterogeneity: Not applicable

Test for overall effect: Not applicable

Test for subgroup differences: $\mathrm{Chi}^{2}=1.02, \mathrm{df}=3(\mathrm{P}=0.80), \mathrm{I}^{2}=0 \%$

\section{Footnotes}

Not estimable

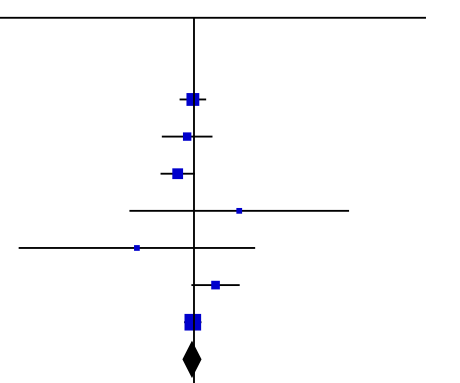

(1) All-cause death during study, Prentice 2017

(2) All-cause mortality during trial, Chlebowski 2015 
Analysis 1.10. Comparison 1: SFA reduction vs usual diet - primary outcomes, Outcome 10: All-cause mortality, subgroup by main substitution

Study or Subgroup

lower SFA

higher SFA

Risk Ratio

Risk Ratio

Events Total Events Total Weight M-H, Random, 95\% CI

424
2096

405

Subtotal $(95 \%$ CI)

Heterogeneity: $\mathrm{Tau}^{2}=0.01 ; \mathrm{Chi}^{2}=7.23$,

Test for overall effect: $\mathrm{Z}=0.39(\mathrm{P}=0.70)$

\subsection{2 replaced by MUFA}

Rose olive 1965

\section{Subtotal $(95 \%$ CI $)$}

3

26

26
26

1

$26 \quad 100.0 \%$

Total events:

3

Heterogeneity: Not applicable

Test for overall effect: $\mathrm{Z}=0.98(\mathrm{P}=0.33)$

\subsection{3 replaced by CHO}

Black 1994

Ley 2004

STARS 1992

WHI 2006 (1)

WINS 2006 (2)

Subtotal (95\% CI)

Total events:

$\begin{array}{rr}1 & 66 \\ 2 & 88 \\ 1 & 27 \\ 989 & 19541 \\ 64 & 975 \\ & \mathbf{2 0 6 9 7}\end{array}$

$\begin{array}{rrr}2 & 67 & 0.1 \% \\ 6 & 88 & 0.2 \% \\ 3 & 28 & 0.1 \% \\ 1520 & 29294 & 93.2 \% \\ 107 & 1462 & 6.3 \% \\ & \mathbf{3 0 9 3 9} & \mathbf{1 0 0 . 0 \%}\end{array}$
1638

Heterogeneity: $\mathrm{Tau}^{2}=0.00 ; \mathrm{Chi}^{2}=3.17, \mathrm{df}=4(\mathrm{P}=0.53) ; \mathrm{I}^{2}=0 \%$

Test for overall effect: $\mathrm{Z}=0.90(\mathrm{P}=0.37)$

1.10.4 replaced by protein

Subtotal (95\% CI)

$\mathbf{0}$

0

Total events:

0

0

Heterogeneity: Not applicable

Test for overall effect: Not applicable

1.10.5 replacement unclear

Subtotal (95\% CI)

0

0

Total events:

Heterogeneity: Not applicable

Test for overall effect: Not applicable

Test for subgroup differences: $\mathrm{Chi}^{2}=1.02, \mathrm{df}=2(\mathrm{P}=0.60), \mathrm{I}^{2}=0 \%$

\section{Footnotes}

(1) All-cause death during study, Prentice 2017

(2) All-cause mortality during trial, Chlebowski 2015
$0.98[0.76,1.25]$

$0.88[0.55,1.41]$

$0.74[0.54,1.02]$

$2.32[0.30,17.92]$

$1.49[0.95,2.34]$

$0.98[0.83,1.15]$

$0.97[0.82,1.14]$
$3.00[0.33,26.99]$

$3.00[0.33,26.99]$

$0.51[0.05,5.46]$

$0.33[0.07,1.61]$

$0.35[0.04,3.12]$

$0.98[0.90,1.05]$

$0.90[0.67,1.21]$

$0.97[0.90,1.04]$

M-H, Random, 95\% CI 


\section{Analysis 1.11. Comparison 1: SFA reduction vs usual diet - primary} outcomes, Outcome 11: All-cause mortality, subgroup by duration higher SFA

Events Total Events Total

Risk Ratio

Weight M-H, Random, 95\% CI
Risk Ratio

M-H, Random, 95\% CI

\subsection{1 up to $24 \mathrm{mo}$}

Black 1994

DART 1989

Rose corn oil 1965

Rose olive 1965

Subtotal $(95 \%$ CI)

Total events:

$\begin{array}{rr}1 & 66 \\ 111 & 1018 \\ 5 & 28 \\ 3 & 26 \\ & \mathbf{1 1 3 8}\end{array}$

120

Heterogeneity: $\mathrm{Tau}^{2}=0.00 ; \mathrm{Chi}^{2}=1.82, \mathrm{df}=3(\mathrm{P}=0.61) ; \mathrm{I}^{2}=0 \%$

Test for overall effect: $\mathrm{Z}=0.08(\mathrm{P}=0.94)$

\subsection{2 >24 to $48 \mathrm{mo}$}

MRC 1968

STARS 1992

Veterans Admin 1969

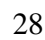

199

27

31
3
177

Subtotal (95\% CI)

$$
650
$$

422

$17.4 \%$

Total events:

203

211

Heterogeneity: $\mathrm{Tau}^{2}=0.00 ; \mathrm{Chi}^{2}=1.02, \mathrm{df}=2(\mathrm{P}=0.60) ; \mathrm{I}^{2}=0 \%$

Test for overall effect: $\mathrm{Z}=0.49(\mathrm{P}=0.63)$

\subsection{3 >48mo}

Oslo Diet-Heart 1966

Sydney Diet-Heart 1978

WHI 2006 (1)

WINS 2006 (2)

Subtotal (95\% CI)

Total events:

\section{8}

206

221

65

206

237

$4.6 \%$

$2.3 \%$

$989 \quad 19541$

1520

29294

$60.2 \%$

$64 \quad 975$

107

$1462 \quad 5.2 \%$

20943

1140

31199

$\mathbf{7 2 . 4 \%}$

1720
Heterogeneity: $\mathrm{Tau}^{2}=0.02 ; \mathrm{Chi}^{2}=6.63, \mathrm{df}=3(\mathrm{P}=0.08) ; \mathrm{I}^{2}=55 \%$

Test for overall effect: $\mathrm{Z}=0.45(\mathrm{P}=0.65)$

\subsection{4 unclear duration}

Ley 2004

Subtotal $(95 \%$ CI)

Total events:

$\begin{array}{lllll}2 & 88 & 6 & 88 & 0.2 \% \\ & \mathbf{8 8} & & \mathbf{8 8} & \mathbf{0 . 2 \%} \\ 2 & & 6 & & \end{array}$

Heterogeneity: Not applicable

Test for overall effect: $\mathrm{Z}=1.37(\mathrm{P}=0.17)$

\section{Total $(95 \%$ CI $)$}

Total events:

1465

2053

Heterogeneity: $\mathrm{Tau}^{2}=0.00 ; \mathrm{Chi}^{2}=11.25, \mathrm{df}=11(\mathrm{P}=0.42) ; \mathrm{I}^{2}=2 \%$

Test for overall effect: $\mathrm{Z}=1.01(\mathrm{P}=0.31)$

Test for subgroup differences: $\mathrm{Chi}^{2}=1.80, \mathrm{df}=3(\mathrm{P}=0.61), \mathrm{I}^{2}=0 \%$

\section{Footnotes}

(1) All-cause death during study, Prentice 2017

(2) All-cause mortality during trial, Chlebowski 2015
$0.51[0.05,5.46]$ $0.98[0.76,1.25]$

$5.31[0.32,89.44]$

$1.50[0.17,13.05]$

$0.99[0.78,1.26]$

$0.88[0.55,1.41]$

$0.35[0.04,3.12]$

$0.98[0.83,1.15]$

$0.96[0.83,1.12]$

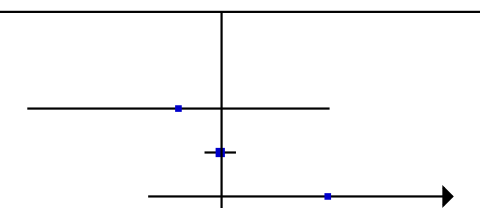

$0.74[0.54,1.02]$

$1.49[0.95,2.34]$

$0.98[0.90,1.05]$

$0.90[0.67,1.21]$

$0.96[0.79,1.16]$

$0.33[0.07,1.61]$

$0.33[0.07,1.61]$

$0.96[0.90,1.03]$

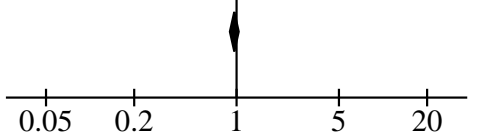

Favours lower SFA 
Analysis 1.13. Comparison 1: SFA reduction vs usual diet - primary outcomes, Outcome 13: All-cause mortality, subgroup by SFA change
Risk Ratio

Events Total Events Total Weight M-H, Random, 95\% CI
Risk Ratio

M-H, Random, $95 \%$ CI

\subsection{1 up to $4 \% \mathrm{E}$ difference}

DART 1989

Ley 2004

Sydney Diet-Heart 1978

WHI 2006 (1)

WINS 2006 (2)

Subtotal $(95 \%$ CI)

Total events:

$\begin{array}{rr}111 & 1018 \\ 2 & 88 \\ 39 & 221 \\ 989 & 19541 \\ 64 & 975 \\ & \mathbf{2 1 8 4 3}\end{array}$

113
6
28

$1015 \quad 7.6 \%$

$88 \quad 0.2 \%$

$29294 \quad 60.2 \%$

107

$1462 \quad 5.2 \%$

$32096 \quad 75.5 \%$ 1774

Heterogeneity: $\mathrm{Tau}^{2}=0.01 ; \mathrm{Chi}^{2}=5.53, \mathrm{df}=4(\mathrm{P}=0.24) ; \mathrm{I}^{2}=28 \%$

Test for overall effect: $\mathrm{Z}=0.17(\mathrm{P}=0.87)$

\subsection{2 $>4$ to $8 \% \mathrm{E}$ difference}

Black 1994

STARS 1992

Subtotal (95\% CI)

$\begin{array}{ll}1 & 66 \\ 1 & 27 \\ & 93\end{array}$

66
27
93

2
3

67

$0.1 \%$

$\mathbf{0 . 2 \%}$

25

Heterogeneity: $\mathrm{Tau}^{2}=0.00 ; \mathrm{Chi}^{2}=0.05, \mathrm{df}=1(\mathrm{P}=0.82) ; \mathrm{I}^{2}=0 \%$

Test for overall effect: $\mathrm{Z}=1.07(\mathrm{P}=0.28)$

\subsection{3 $>8 \% \mathrm{E}$ difference}

Veterans Admin 1969

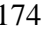

Subtotal (95\% CI)

Total events:

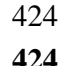

174

Heterogeneity: Not applicable

Test for overall effect: $\mathrm{Z}=0.27(\mathrm{P}=0.79)$

\subsection{4 unclear}

MRC 1968

Oslo Diet-Heart 1966

Rose corn oil 1965

Rose olive 1965

Subtotal (95\% CI)

Total events:

84

$\begin{array}{rr}28 & 199 \\ 48 & 206 \\ 5 & 28 \\ 3 & 26 \\ & \mathbf{4 5 9}\end{array}$

Heterogeneity: $\mathrm{Tau}^{2}=0.00 ; \mathrm{Chi}^{2}=2.50, \mathrm{df}=3(\mathrm{P}=0.47) ; \mathrm{I}^{2}=0 \%$

Test for overall effect: $\mathrm{Z}=1.67(\mathrm{P}=0.10)$

\section{Total $(95 \%$ CI $)$}

$33039 \quad 100.0 \%$

Total events:

1465

2053

Heterogeneity: $\mathrm{Tau}^{2}=0.00 ; \mathrm{Chi}^{2}=11.25, \mathrm{df}=11(\mathrm{P}=0.42) ; \mathrm{I}^{2}=2 \%$

Test for overall effect: $\mathrm{Z}=1.01(\mathrm{P}=0.31)$

Test for subgroup differences: $\mathrm{Chi}^{2}=3.13, \mathrm{df}=3(\mathrm{P}=0.37), \mathrm{I}^{2}=4.0 \%$
$0.98[0.76,1.25]$

$0.33[0.07,1.61]$

$1.49[0.95,2.34]$

$0.98[0.90,1.05]$

$0.90[0.67,1.21]$

$0.99[0.86,1.13]$

$0.51[0.05,5.46]$

$0.35[0.04,3.12]$

$0.41[0.08,2.07]$
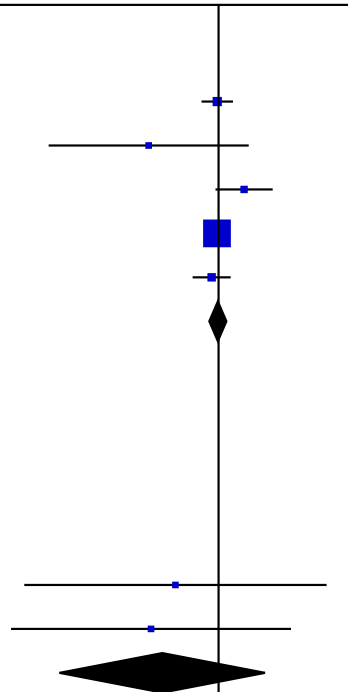

$0.98[0.83,1.15]$

$0.98[0.83,1.15]$

\section{Footnotes}

(1) All-cause death during study, Prentice 2017

(2) All-cause mortality during trial, Chlebowski 2015 


\section{Analysis 1.14. Comparison 1: SFA reduction vs usual diet - primary outcomes, Outcome 14: All-cause mortality, subgroup by sex}

\begin{tabular}{|c|c|c|c|c|c|c|c|c|c|}
\hline \multirow[b]{2}{*}{ Study or Subgroup } & \multicolumn{2}{|c|}{ lower SFA } & \multicolumn{2}{|c|}{ higher SFA } & \multirow[b]{2}{*}{ Weight } & \multirow{2}{*}{$\begin{array}{c}\text { Risk Ratio } \\
\text { M-H, Random, } 95 \% \text { CI }\end{array}$} & \multirow{2}{*}{\multicolumn{3}{|c|}{$\begin{array}{c}\text { Risk Ratio } \\
\text { M-H, Random, } 95 \% \text { CI }\end{array}$}} \\
\hline & Events & Total & Events & Total & & & & & \\
\hline \multicolumn{10}{|l|}{ 1.14.1 Men } \\
\hline Black 1994 & 1 & 66 & 2 & 67 & $0.1 \%$ & $0.51[0.05,5.46]$ & & & \\
\hline DART 1989 & 111 & 1018 & 113 & 1015 & $7.6 \%$ & $0.98[0.76,1.25]$ & & 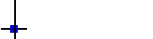 & \\
\hline MRC 1968 & 28 & 199 & 31 & 194 & $2.1 \%$ & $0.88[0.55,1.41]$ & & - & \\
\hline Oslo Diet-Heart 1966 & 48 & 206 & 65 & 206 & $4.6 \%$ & $0.74[0.54,1.02]$ & - & & \\
\hline Rose corn oil 1965 & 5 & 28 & 0 & 13 & $0.1 \%$ & $5.31[0.32,89.44]$ & & & $\rightarrow$ \\
\hline Rose olive 1965 & 3 & 26 & 1 & 13 & $0.1 \%$ & $1.50[0.17,13.05]$ & & & \\
\hline STARS 1992 & 1 & 27 & 3 & 28 & $0.1 \%$ & $0.35[0.04,3.12]$ & & & \\
\hline Sydney Diet-Heart 1978 & 39 & 221 & 28 & 237 & $2.3 \%$ & $1.49[0.95,2.34]$ & & $=$ & \\
\hline Veterans Admin 1969 & 174 & 424 & 177 & 422 & $17.4 \%$ & $0.98[0.83,1.15]$ & & & \\
\hline Subtotal (95\% CI) & & 2215 & & 2195 & $34.4 \%$ & $0.96[0.83,1.11]$ & & & \\
\hline Total events: & 410 & & 420 & & & & & & \\
\hline \multicolumn{10}{|c|}{ Heterogeneity: $\mathrm{Tau}^{2}=0.01 ; \mathrm{Chi}^{2}=9.20, \mathrm{df}=8(\mathrm{P}=0.33) ; \mathrm{I}^{2}=13 \%$} \\
\hline \multicolumn{10}{|c|}{ Test for overall effect: $\mathrm{Z}=0.53(\mathrm{P}=0.60)$} \\
\hline \multicolumn{10}{|l|}{ 1.14.2 Women } \\
\hline WHI 2006 (1) & 989 & 19541 & 1520 & 29294 & $60.2 \%$ & $0.98[0.90,1.05]$ & & & \\
\hline WINS 2006 (2) & 64 & 975 & 107 & 1462 & $5.2 \%$ & $0.90[0.67,1.21]$ & & & \\
\hline Subtotal $(95 \%$ CI $)$ & & 20516 & & 30756 & $65.4 \%$ & $0.97[0.90,1.05]$ & & & \\
\hline Total events: & 1053 & & 1627 & & & & & & \\
\hline \multicolumn{10}{|c|}{ Heterogeneity: $\mathrm{Tau}^{2}=0.00 ; \mathrm{Chi}^{2}=0.28, \mathrm{df}=1(\mathrm{P}=0.59) ; \mathrm{I}^{2}=0 \%$} \\
\hline \multicolumn{10}{|c|}{ Test for overall effect: $\mathrm{Z}=0.79(\mathrm{P}=0.43)$} \\
\hline \multicolumn{10}{|c|}{ 1.14.3 Mixed, men and women } \\
\hline Ley 2004 & 2 & 88 & 6 & 88 & $0.2 \%$ & $0.33[0.07,1.61]$ & & - & \\
\hline Subtotal (95\% CI) & & 88 & & 88 & $0.2 \%$ & $0.33[0.07,1.61]$ & & & \\
\hline Total events: & 2 & & 6 & & & & & & \\
\hline \multicolumn{10}{|c|}{ Heterogeneity: Not applicable } \\
\hline \multicolumn{10}{|c|}{ Test for overall effect: $\mathrm{Z}=1.37(\mathrm{P}=0.17)$} \\
\hline Total $(95 \%$ CI $)$ & & 22819 & & 33039 & $100.0 \%$ & $0.96[0.90,1.03]$ & & & \\
\hline Total events: & 1465 & & 2053 & & & & & & \\
\hline \multirow{2}{*}{\multicolumn{7}{|c|}{ Heterogeneity: $\mathrm{Tau}^{2}=0.00 ; \mathrm{Chi}^{2}=11.25, \mathrm{df}=11(\mathrm{P}=0.42) ; \mathrm{I}^{2}=2 \%$}} & $\begin{array}{ll}1 & 1 \\
0.05 & 0.2\end{array}$ & 1 & 20 \\
\hline & & & & & & & ours lower SFA & Favours $h$ & igher \\
\hline
\end{tabular}

Fotnotes

(1) All-cause death during study, Prentice 2017

(2) All-cause mortality during trial, Chlebowski 2015 


\section{Analysis 1.15. Comparison 1: SFA reduction vs usual diet - primary} outcomes, Outcome 15: All-cause mortality, subgroup by CVD risk

\begin{tabular}{|c|c|c|c|c|c|c|c|}
\hline \multirow[b]{2}{*}{ Study or Subgroup } & \multicolumn{2}{|c|}{ lower SFA } & \multicolumn{2}{|c|}{ higher SFA } & \multicolumn{2}{|r|}{ Risk Ratio } & Risk Ratio \\
\hline & Events & Total & Events & Total & Weight & M-H, Random, $95 \%$ CI & M-H, Random, $95 \%$ CI \\
\hline
\end{tabular}

\subsubsection{Low CVD risk}

Black 1994

Veterans Admin 1969

WHI 2006 (1)

WINS 2006 (2)

Subtotal (95\% CI)

Total events:

Heterogeneity: $\mathrm{Tau}^{2}=0.00 ; \mathrm{Chi}^{2}=0.58, \mathrm{df}=3(\mathrm{P}=0.90) ; \mathrm{I}^{2}=0 \%$

Test for overall effect: $\mathrm{Z}=0.84(\mathrm{P}=0.40)$

\subsubsection{Moderate CVD risk}

Ley 2004

Subtotal (95\% CI)

Total events:

2

88

88
$\mathbf{8 8}$

88

Heterogeneity: Not applicable

Test for overall effect: $\mathrm{Z}=1.37(\mathrm{P}=0.17)$

\subsubsection{Existing CVD disease}

DART 1989

MRC 1968

Oslo Diet-Heart 1966

Rose corn oil 1965

Rose olive 1965

STARS 1992

Sydney Diet-Heart 1978

Subtotal (95\% CI)

Total events:

$\begin{array}{rr}111 & 1018 \\ 28 & 199 \\ 48 & 206 \\ 5 & 28 \\ 3 & 26 \\ 1 & 27 \\ 39 & 221 \\ & \mathbf{1 7 2 5}\end{array}$

Heterogeneity: $\mathrm{Tau}^{2}=0.03 ; \mathrm{Chi}^{2}=8.89, \mathrm{df}=6(\mathrm{P}=0.18) ; \mathrm{I}^{2}=33 \%$

Test for overall effect: $\mathrm{Z}=0.26(\mathrm{P}=0.79)$

Total $(95 \%$ CI $)$

22819 2053

Total events:

1465

018
199
206
28
26
27
221
725

113

31

1015
194

$7.6 \%$

$2.1 \%$

$4.6 \%$

$0.1 \%$

$0.1 \%$

$0.1 \%$

$2.3 \%$

$16.9 \%$$$
1706
$$

$0.33[0.07,1.61]$

$0.33[0.07,1.61]$

$0.51[0.05,5.46]$

$0.98[0.83,1.15]$

$0.98[0.90,1.05]$

$0.90[0.67,1.21]$

$0.97[0.91,1.04]$

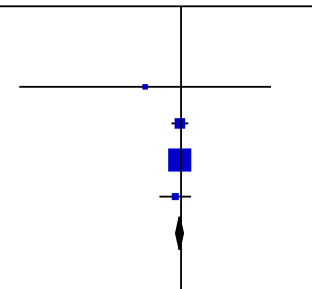

$0.98[0.76,1.25]$

$0.88[0.55,1.41]$

$0.74[0.54,1.02]$

$5.31[0.32,89.44]$

$1.50[0.17,13.05]$

$0.35[0.04,3.12]$

$1.49[0.95,2.34]$

$0.97[0.76,1.24]$

Heterogeneity: $\mathrm{Tau}^{2}=0.00 ; \mathrm{Chi}^{2}=11.25, \mathrm{df}=11(\mathrm{P}=0.42) ; \mathrm{I}^{2}=2 \%$

Test for overall effect: $\mathrm{Z}=1.01(\mathrm{P}=0.31)$

Test for subgroup differences: $\mathrm{Chi}^{2}=1.77, \mathrm{df}=2(\mathrm{P}=0.41), \mathrm{I}^{2}=0 \%$

$33039100.0 \%$

$0.96[0.90,1.03]$

\section{Footnotes}

(1) All-cause death during study, Prentice 2017

(2) All-cause mortality during trial, Chlebowski 2015 


\section{Analysis 1.16. Comparison 1: SFA reduction vs usual diet - primary outcomes, Outcome 16: All-cause mortality, subgroup by TC reduction}

\begin{tabular}{|c|c|c|c|c|c|c|c|}
\hline \multirow[b]{2}{*}{ Study or Subgroup } & \multicolumn{2}{|c|}{ lower SFA } & \multicolumn{2}{|c|}{ higher SFA } & \multicolumn{2}{|r|}{ Risk Ratio } & Risk Ratio \\
\hline & Events & Total & Events & Total & Weight & M-H, Random, $95 \%$ CI & M-H, Random, $95 \%$ CI \\
\hline
\end{tabular}

1.16.1 serum chol reduced by at least $0.2 \mathrm{mmol} / \mathrm{L}$

MRC $1968 \quad 28 \quad 199$

Oslo Diet-Heart $1966 \quad 48 \quad 206$

Rose corn oil $1965 \quad 5 \quad 28$

STARS 1992

Sydney Diet-Heart 1978

1

Veterans Admin 1969

Subtotal $(95 \%$ CI $)$

Total events:

$\begin{array}{rrr}113 & 1015 & 24.5 \% \\ 31 & 194 & 10.3 \% \\ 65 & 206 & 18.1 \% \\ 0 & 13 & 0.4 \% \\ 3 & 28 & 0.6 \% \\ 28 & 237 & 11.1 \% \\ 177 & 422 & 35.1 \% \\ & \mathbf{2 1 1 5} & \mathbf{1 0 0 . 0 \%}\end{array}$
417

Heterogeneity: $\mathrm{Tau}^{2}=0.01 ; \mathrm{Chi}^{2}=8.76, \mathrm{df}=6(\mathrm{P}=0.19) ; \mathrm{I}^{2}=32 \%$

Test for overall effect: $\mathrm{Z}=0.42(\mathrm{P}=0.67)$

1.16.2 serum chol reduced by $<0.2 \mathrm{mmol} / \mathrm{L}$

\section{Ley 2004}

Rose olive 1965

WHI 2006 (1)

WINS 2006 (2)

Subtotal (95\% CI)

Total events:

$$
\begin{array}{rr}
2 & \\
3 & \\
989 & 19 \\
64 &
\end{array}
$$

1058

88
26
19541
975
$\mathbf{2 0 6 3 0}$

6
1
1520

$\begin{array}{rr}88 & 0.2 \% \\ 13 & 0.1 \% \\ 29294 & 93.3 \% \\ 1462 & 6.4 \% \\ \mathbf{3 0 8 5 7} & \mathbf{1 0 0 . 0 \%}\end{array}$
1634
Heterogeneity: $\mathrm{Tau}^{2}=0.00 ; \mathrm{Chi}^{2}=2.21, \mathrm{df}=3(\mathrm{P}=0.53) ; \mathrm{I}^{2}=0 \%$

Test for overall effect: $\mathrm{Z}=0.84(\mathrm{P}=0.40)$

\subsection{3 serum chol reduction unclear}

Black 1994

Subtotal (95\% CI)

Total events:

Heterogeneity: Not applicable

Test for overall effect: $\mathrm{Z}=0.56(\mathrm{P}=0.58)$

Test for subgroup differences: $\mathrm{Chi}^{2}=0.29, \mathrm{df}=2(\mathrm{P}=0.87), \mathrm{I}^{2}=0 \%$

Footnotes

(1) All-cause death during study, Prentice 2017

(2) All-cause mortality during trial, Chlebowski 2015
$0.98[0.76,1.25]$

$0.88[0.55,1.41]$

$0.74[0.54,1.02]$

$5.31[0.32,89.44]$

$0.35[0.04,3.12]$

$1.49[0.95,2.34]$

$0.98[0.83,1.15]$

$0.96[0.81,1.14]$

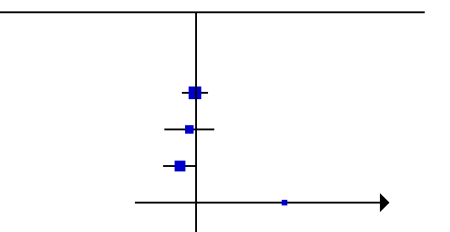

$0.33[0.07,1.61]$

$1.50[0.17,13.05]$

$0.98[0.90,1.05]$

$0.90[0.67,1.21]$

$0.97[0.90,1.04]$

$0.51[0.05,5.46]$

$0.51[0.05,5.46]$

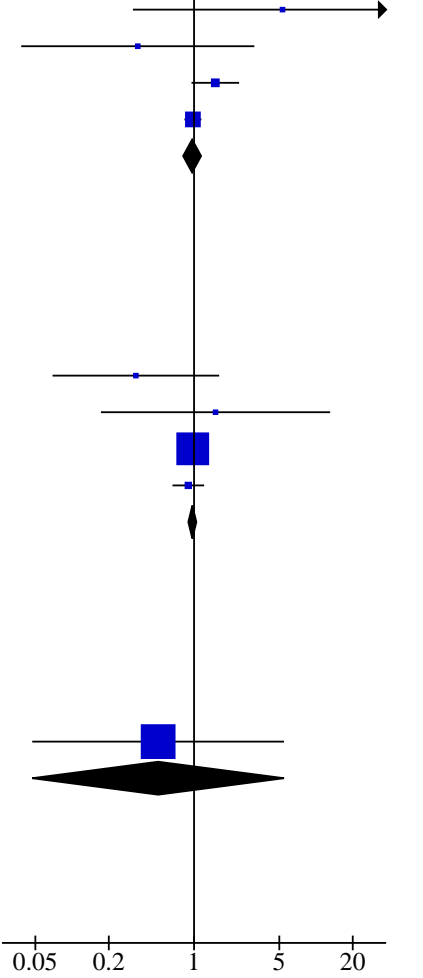

Favours lower SFA 
Analysis 1.17. Comparison 1: SFA reduction vs usual diet - primary outcomes, Outcome 17: All-cause mortality, subgroup decade of publication

\begin{tabular}{|c|c|c|c|c|c|c|}
\hline \multirow[b]{2}{*}{ Study or Subgroup } & \multicolumn{2}{|c|}{ lower SFA } & \multicolumn{2}{|c|}{ higher SFA } & \multirow[b]{2}{*}{ Weight } & \multirow{2}{*}{$\begin{array}{c}\text { Risk Ratio } \\
\text { M-H, Random, 95\% CI }\end{array}$} \\
\hline & Events & Total & Events & Total & & \\
\hline \multicolumn{7}{|l|}{ 1.17.1 1960s } \\
\hline MRC 1968 & 28 & 199 & 31 & 194 & $2.1 \%$ & $0.88[0.55,1.4$ \\
\hline Oslo Diet-Heart 1966 & 48 & 206 & 65 & 206 & $4.6 \%$ & $0.74[0.54,1.02$ \\
\hline Rose corn oil 1965 & 5 & 28 & 0 & 13 & $0.1 \%$ & $5.31[0.32,89.44$ \\
\hline Rose olive 1965 & 3 & 26 & 1 & 13 & $0.1 \%$ & $1.50[0.17,13.05]$ \\
\hline Veterans Admin 1969 & 174 & 424 & 177 & 422 & $17.4 \%$ & $0.98[0.83,1.15$ \\
\hline Subtotal $(95 \%$ CI $)$ & & 883 & & 848 & $24.3 \%$ & $0.92[0.80,1.07]$ \\
\hline
\end{tabular}

Heterogeneity: $\mathrm{Tau}^{2}=0.00 ; \mathrm{Chi}^{2}=4.09, \mathrm{df}=4(\mathrm{P}=0.39) ; \mathrm{I}^{2}=2 \%$

Test for overall effect: $\mathrm{Z}=1.09(\mathrm{P}=0.27)$

\subsubsection{0s}

Sydney Diet-Heart 1978

Subtotal (95\% CI)

39

221
221

28

237

$2.3 \%$

Total events:

39

28

Heterogeneity: Not applicable

Test for overall effect: $\mathrm{Z}=1.75(\mathrm{P}=0.08)$

\subsubsection{0s}

DART 1989

Subtotal (95\% CI)

Total events:

1018

113

1015

$7.6 \%$

111

113

Heterogeneity: Not applicable

Test for overall effect: $\mathrm{Z}=0.17(\mathrm{P}=0.87)$

\subsubsection{0s}

Black 1994

STARS 1992

Subtotal (95\% CI)

Total events:

Heterogeneity: $\mathrm{Tau}^{2}=0.00 ; \mathrm{Chi}^{2}=0.05, \mathrm{df}=1(\mathrm{P}=0.82) ; \mathrm{I}^{2}=0 \%$

Test for overall effect: $\mathrm{Z}=1.07(\mathrm{P}=0.28)$

\subsubsection{0s}

Ley 2004

WHI 2006 (1)

WINS 2006 (2)

Subtotal (95\% CI)

Total events:

2
989
64
1055

88
19541
975
$\mathbf{2 0 6 0 4}$

$\begin{array}{lll}6 & 88 & 0.2 \%\end{array}$

$1520 \quad 29294 \quad 60.2 \%$

$107 \quad 1462 \quad 5.2 \%$

$30844 \quad 65.6 \%$

Heterogeneity: $\mathrm{Tau}^{2}=0.00 ; \mathrm{Chi}^{2}=2.05, \mathrm{df}=2(\mathrm{P}=0.36) ; \mathrm{I}^{2}=3 \%$

Test for overall effect: $\mathrm{Z}=0.79(\mathrm{P}=0.43)$
Total $(95 \%$ CI)

Total events:

1465

22819 1465

2053

Heterogeneity: $\mathrm{Tau}^{2}=0.00 ; \mathrm{Chi}^{2}=11.25, \mathrm{df}=11(\mathrm{P}=0.42) ; \mathrm{I}^{2}=2 \%$

Test for overall effect: $\mathrm{Z}=1.01(\mathrm{P}=0.31)$

Test for subgroup differences: $\mathrm{Chi}^{2}=5.08, \mathrm{df}=4(\mathrm{P}=0.28), \mathrm{I}^{2}=21.2 \%$
Risk Ratio
M-H, Random, 95\% CI

Footnotes

(1) All-cause death during study, Prentice 2017

(2) All-cause mortality during trial, Chlebowski 2015
$1.49[0.95,2.34]$

$1.49[0.95,2.34]$

$0.98[0.76,1.25]$

$0.98[0.76,1.25]$

$0.51[0.05,5.46]$

$0.35[0.04,3.12]$

$0.41[0.08,2.07]$
$0.33[0.07,1.61]$

$0.98[0.90,1.05]$

$0.90[0.67,1.21]$

$0.97[0.88,1.05]$

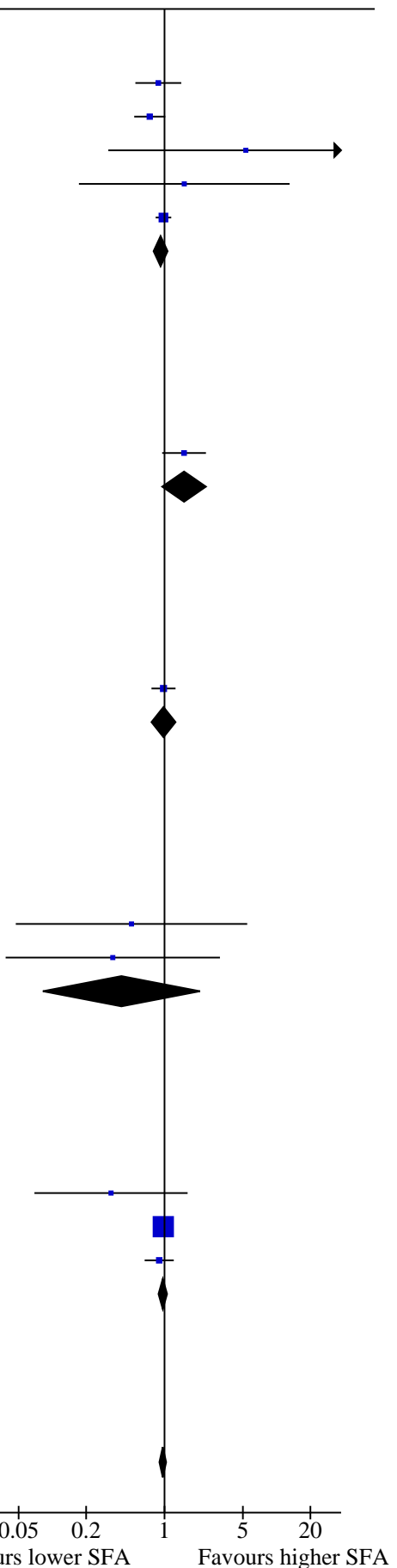


Analysis 1.18. Comparison 1: SFA reduction vs usual diet primary outcomes, Outcome 18: CARDIOVASCULAR MORTALITY

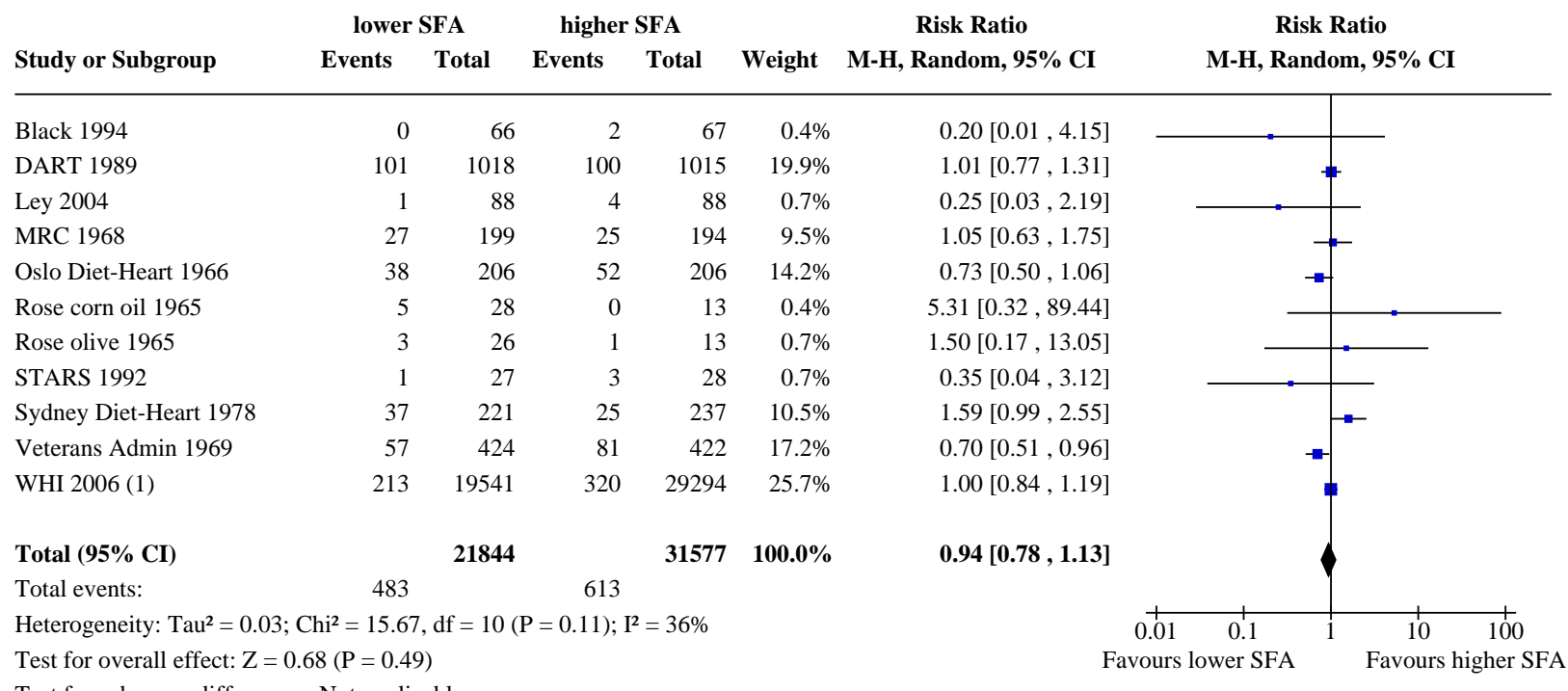

Test for subgroup differences: Not applicable

Footnotes

(1) In participants with and without CVD at baseline

Analysis 1.19. Comparison 1: SFA reduction vs usual diet - primary outcomes, Outcome 19: CVD mortality, SA low summary risk of bias

\begin{tabular}{|c|c|c|c|c|c|c|c|}
\hline \multirow[b]{2}{*}{ Study or Subgroup } & \multicolumn{2}{|c|}{ lower SFA } & \multicolumn{2}{|c|}{ higher SFA } & \multirow[b]{2}{*}{ Weight } & \multirow{2}{*}{$\begin{array}{c}\text { Risk Ratio } \\
\text { M-H, Random, 95\% CI }\end{array}$} & \multirow{2}{*}{$\begin{array}{c}\text { Risk Ratio } \\
\text { M-H, Random, 95\% CI }\end{array}$} \\
\hline & Events & Total & Events & Total & & & \\
\hline Ley 2004 & 1 & 88 & 4 & 88 & $2.6 \%$ & $0.25[0.03,2.19]$ & - \\
\hline Sydney Diet-Heart 1978 & 37 & 221 & 25 & 237 & $25.0 \%$ & $1.59[0.99,2.55]$ & - \\
\hline Veterans Admin 1969 & 57 & 424 & 81 & 422 & $32.9 \%$ & $0.70[0.51,0.96]$ & $t$ \\
\hline WHI 2006 (1) & 213 & 19541 & 320 & 29294 & $39.5 \%$ & $1.00[0.84,1.19]$ & \\
\hline Total $(95 \%$ CI $)$ & & 20274 & & 30041 & $100.0 \%$ & $0.96[0.67,1.38]$ & \\
\hline Total events: & 308 & & 430 & & & & \\
\hline \multicolumn{7}{|c|}{ Heterogeneity: $\mathrm{Tau}^{2}=0.08 ; \mathrm{Chi}^{2}=9.96, \mathrm{df}=3(\mathrm{P}=0.02) ; \mathrm{I}^{2}=70 \%$} & $\stackrel{+}{0.01}$ \\
\hline \multicolumn{6}{|c|}{ Test for overall effect: $\mathrm{Z}=0.21(\mathrm{P}=0.83)$} & & avours lower SFA \\
\hline
\end{tabular}

Test for subgroup differences: Not applicable

Footnotes

(1) In participants with and without CVD at baseline 


\section{Analysis 1.20. Comparison 1: SFA reduction vs usual diet - primary} outcomes, Outcome 20: CVD mortality, SA aim to reduce SFA

\begin{tabular}{|c|c|c|c|c|c|c|c|}
\hline \multirow[b]{2}{*}{ Study or Subgroup } & \multicolumn{2}{|c|}{ lower SFA } & \multicolumn{2}{|c|}{ higher SFA } & \multicolumn{2}{|r|}{ Risk Ratio } & Risk Ratio \\
\hline & Events & Total & Events & Total & Weight & M-H, Random, $95 \%$ CI & M-H, Random, $95 \%$ CI \\
\hline
\end{tabular}

\begin{tabular}{lrrrrrr}
\hline DART 1989 & 101 & 1018 & 100 & 1015 & $20.2 \%$ & $1.01[0.77,1.31]$ \\
MRC 1968 & 27 & 199 & 25 & 194 & $9.5 \%$ & $1.05[0.63,1.75]$ \\
Oslo Diet-Heart 1966 & 38 & 206 & 52 & 206 & $14.3 \%$ & $0.73[0.50,1.06]$ \\
Rose corn oil 1965 & 5 & 28 & 0 & 13 & $0.4 \%$ & $5.31[0.32,89.44]$ \\
Rose olive 1965 & 3 & 26 & 1 & 13 & $0.7 \%$ & $1.50[0.17,13.05]$ \\
STARS 1992 & 1 & 27 & 3 & 28 & $0.7 \%$ & $0.35[0.04,3.12]$ \\
Sydney Diet-Heart 1978 & 37 & 221 & 25 & 237 & $10.4 \%$ & $1.59[0.99,2.55]$ \\
Veterans Admin 1969 & 57 & 424 & 81 & 422 & $17.3 \%$ & $0.70[0.51,0.96]$ \\
WHI 2006(1) & 213 & 19541 & 320 & 29294 & $26.5 \%$ & $1.00[0.84,1.19]$ \\
& & & & & & \\
Total (95\% CI) & & $\mathbf{2 1 6 9 0}$ & & $\mathbf{3 1 4 2 2}$ & $\mathbf{1 0 0 . 0 \%}$ & $\mathbf{0 . 9 5}[\mathbf{0 . 7 9}, \mathbf{1 . 1 4}]$ \\
Total & 482 & & 607 & & &
\end{tabular}

Heterogeneity: $\mathrm{Tau}^{2}=0.03 ; \mathrm{Chi}^{2}=13.22, \mathrm{df}=8(\mathrm{P}=0.10) ; \mathrm{I}^{2}=39 \%$

Test for overall effect: $\mathrm{Z}=0.54(\mathrm{P}=0.59)$

Test for subgroup differences: Not applicable

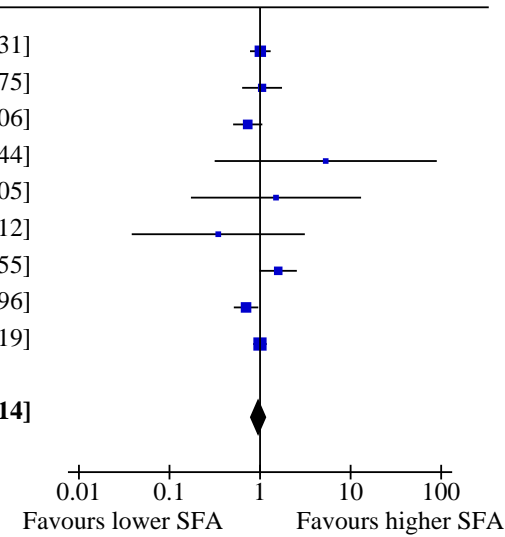

Footnotes

(1) In participants with and without CVD at baseline

\section{Analysis 1.21. Comparison 1: SFA reduction vs usual diet - primary outcomes, Outcome 21: CVD mortality, SA statistically significant SFA reduction}

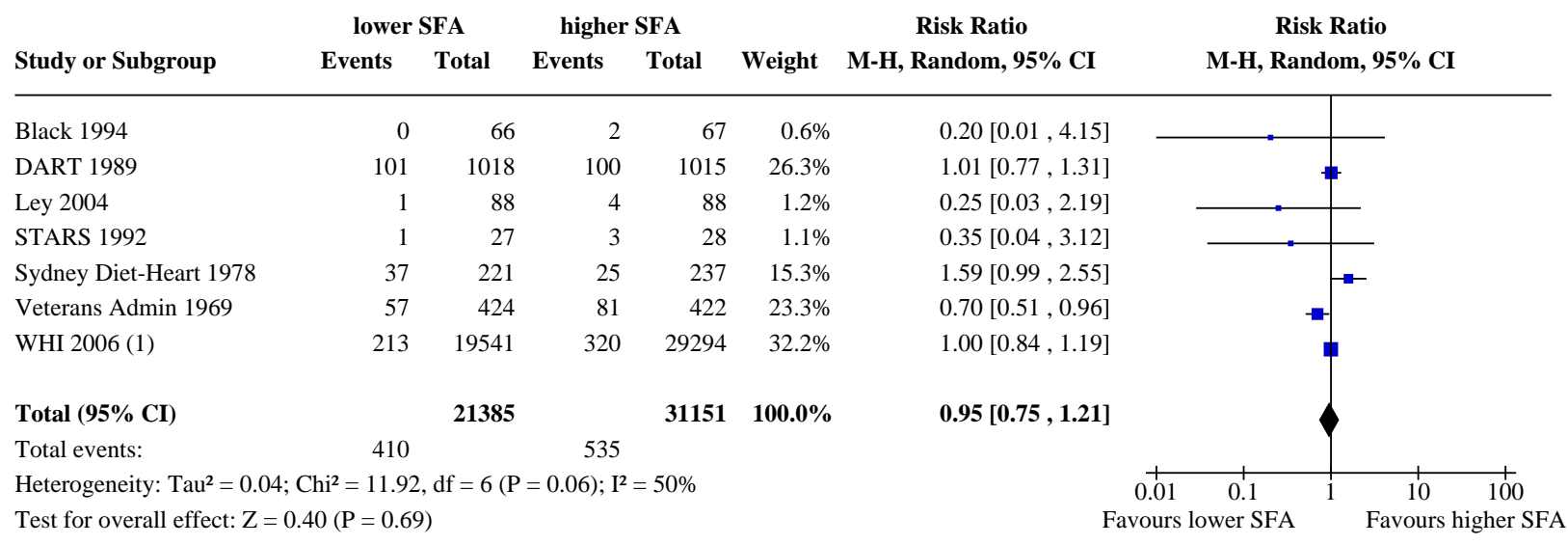

Test for subgroup differences: Not applicable

Footnotes

(1) In participants with and without CVD at baseline 
Analysis 1.22. Comparison 1: SFA reduction vs usual diet primary outcomes, Outcome 22: CVD mortality, SA TC reduction

\begin{tabular}{|c|c|c|c|c|c|c|c|}
\hline \multirow[b]{2}{*}{ Study or Subgroup } & \multicolumn{2}{|c|}{ lower SFA } & \multicolumn{2}{|c|}{ higher SFA } & \multirow[b]{2}{*}{ Weight } & \multirow{2}{*}{$\begin{array}{c}\text { Risk Ratio } \\
\text { M-H, Random, } 95 \% \text { CI }\end{array}$} & \multirow{2}{*}{$\begin{array}{c}\text { Risk Ratio } \\
\text { M-H, Random, 95\% CI }\end{array}$} \\
\hline & Events & Total & Events & Total & & & \\
\hline DART 1989 & 101 & 1018 & 100 & 1015 & $20.2 \%$ & $1.01[0.77,1.31]$ & \\
\hline MRC 1968 & 27 & 199 & 25 & 194 & $9.9 \%$ & $1.05[0.63,1.75]$ & \\
\hline Oslo Diet-Heart 1966 & 38 & 206 & 52 & 206 & $14.6 \%$ & $0.73[0.50,1.06]$ & $\rightarrow$ \\
\hline Rose corn oil 1965 & 5 & 28 & 0 & 13 & $0.5 \%$ & $5.31[0.32,89.44]$ & \\
\hline STARS 1992 & 1 & 27 & 3 & 28 & $0.7 \%$ & $0.35[0.04,3.12]$ & \\
\hline Sydney Diet-Heart 1978 & 37 & 221 & 25 & 237 & $10.9 \%$ & $1.59[0.99,2.55]$ & - \\
\hline Veterans Admin 1969 & 57 & 424 & 81 & 422 & $17.5 \%$ & $0.70[0.51,0.96]$ & $\rightarrow$ \\
\hline WHI 2006 (1) & 213 & 19541 & 320 & 29294 & $25.7 \%$ & $1.00[0.84,1.19]$ & $\phi$ \\
\hline Total $(95 \%$ CI $)$ & & 21664 & & 31409 & $100.0 \%$ & $0.95[0.78,1.15]$ & \\
\hline Total events: & 479 & & 606 & & & & \\
\hline Heterogeneity: $\mathrm{Tau}^{2}=0.0$ & $\mathrm{hi}^{2}=13.0$ & $\mathrm{df}=7(\mathrm{P}$ & $=0.07) ; \mathrm{I}^{2}$ & $46 \%$ & & & $\begin{array}{cc}+01 & 0.1 \\
0.01 & \end{array}$ \\
\hline
\end{tabular}

Test for subgroup differences: Not applicable

Footnotes

(1) In participants with and without CVD at baseline

Analysis 1.23. Comparison 1: SFA reduction vs usual diet primary outcomes, Outcome 23: CVD mortality, SA excluding WHI

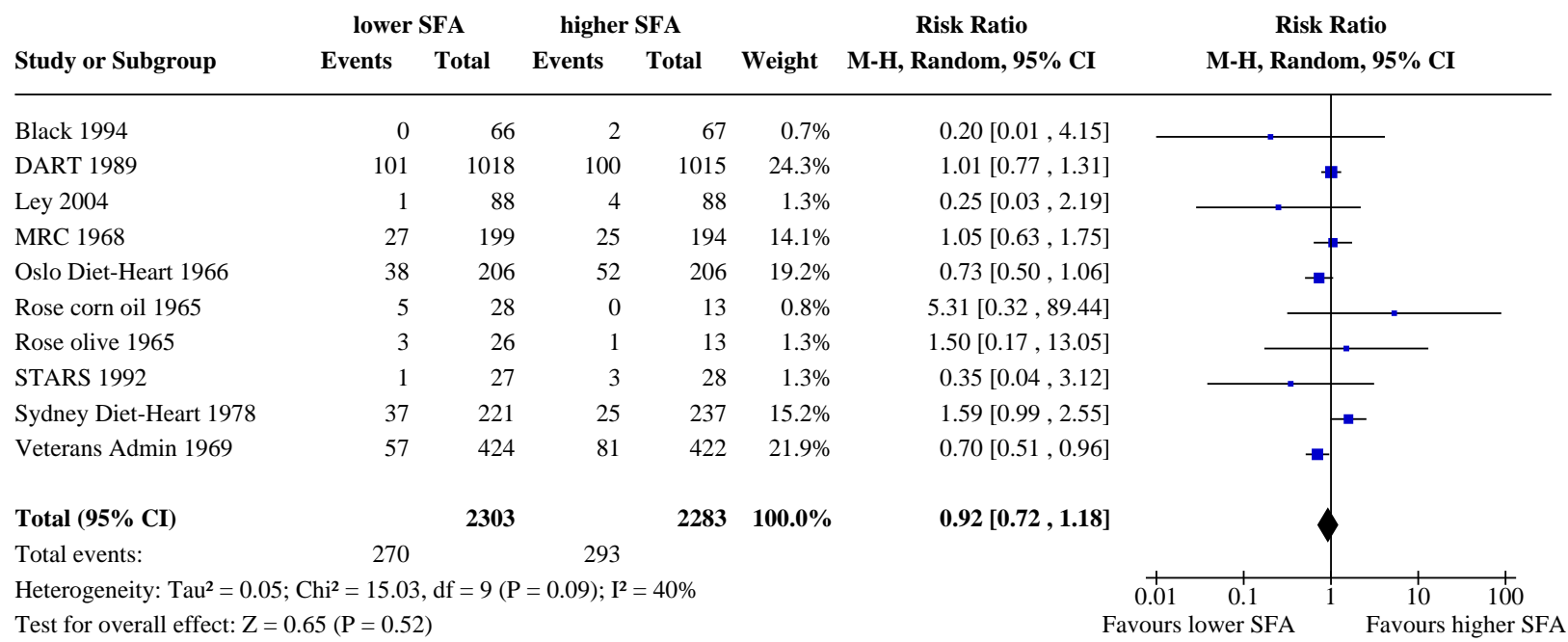




\section{Analysis 1.24. Comparison 1: SFA reduction vs usual diet - primary outcomes, Outcome 24: CVD mortality, SA Mantel-Haenszel fixed-effect}

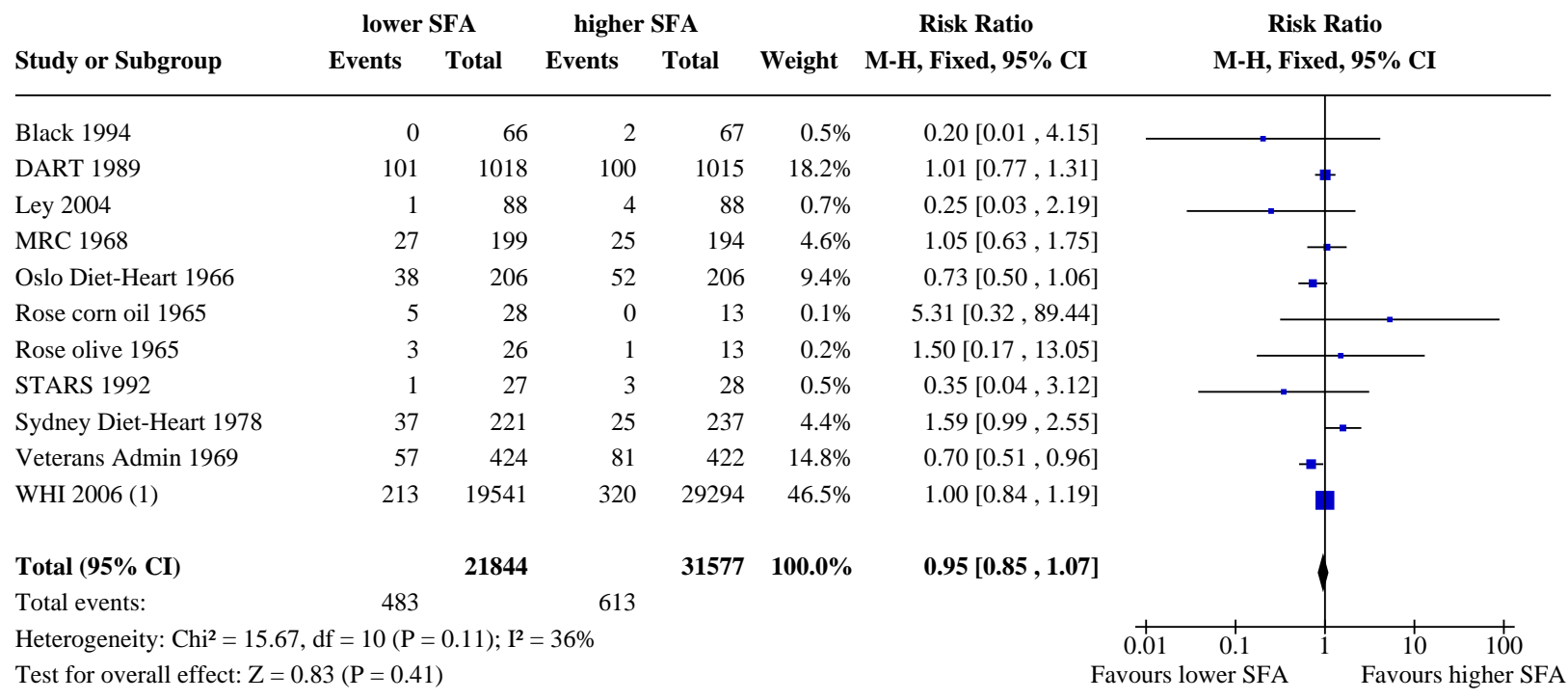

Footnotes

(1) In participants with and without CVD at baseline

Analysis 1.25. Comparison 1: SFA reduction vs usual diet - primary outcomes, Outcome 25: CVD mortality, SA Peto fixed-effect

\begin{tabular}{|c|c|c|c|c|c|c|c|}
\hline & low & $\mathbf{F A}$ & high & SFA & & Peto Odds Ratio & Peto Odds Ratio \\
\hline Study or Subgroup & Events & Total & Events & Total & Weight & Peto, Fixed, $95 \% \mathrm{Cl}$ & Peto, Fixed, 95\% CI \\
\hline
\end{tabular}

\begin{tabular}{|c|c|c|c|c|c|c|}
\hline Black 1994 & 0 & 66 & 2 & 67 & $0.2 \%$ & $0.14[0.01,2.19]$ \\
\hline DART 1989 & 101 & 1018 & 100 & 1015 & $18.3 \%$ & $1.01[0.75,1.35]$ \\
\hline Ley 2004 & 1 & 88 & 4 & 88 & $0.5 \%$ & $0.29[0.05,1.73]$ \\
\hline MRC 1968 & 27 & 199 & 25 & 194 & $4.6 \%$ & $1.06[0.59,1.90]$ \\
\hline Oslo Diet-Heart 1966 & 38 & 206 & 52 & 206 & $7.1 \%$ & $0.67[0.42,1.07]$ \\
\hline Rose corn oil 1965 & 5 & 28 & 0 & 13 & $0.4 \%$ & $5.09[0.70,37.06]$ \\
\hline Rose olive 1965 & 3 & 26 & 1 & 13 & $0.3 \%$ & $1.50[0.17,13.11]$ \\
\hline STARS 1992 & 1 & 27 & 3 & 28 & $0.4 \%$ & $0.36[0.05,2.71]$ \\
\hline Sydney Diet-Heart 1978 & 37 & 221 & 25 & 237 & $5.4 \%$ & $1.70[0.99,2.90]$ \\
\hline Veterans Admin 1969 & 57 & 424 & 81 & 422 & $11.7 \%$ & $0.66[0.46,0.95]$ \\
\hline WHI 2006 (1) & 213 & 19541 & 320 & 29294 & $51.1 \%$ & $1.00[0.84,1.19]$ \\
\hline Total $(95 \%$ CI $)$ & & 21844 & & 31577 & $100.0 \%$ & $0.95[0.84,1.08]$ \\
\hline Total events: & 483 & & 613 & & & \\
\hline
\end{tabular}

Heterogeneity: $\mathrm{Chi}^{2}=18.54, \mathrm{df}=10(\mathrm{P}=0.05) ; \mathrm{I}^{2}=46 \%$

Test for overall effect: $\mathrm{Z}=0.82(\mathrm{P}=0.41)$

Test for subgroup differences: Not applicable

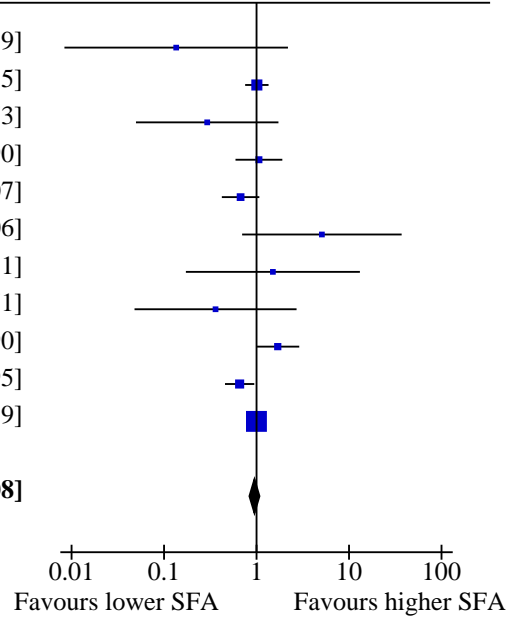

Footnotes

(1) In participants with and without CVD at baseline 


\section{Analysis 1.26. Comparison 1: SFA reduction vs usual diet - primary outcomes, Outcome 26: CVD mortality, subgroup by any substitution}

\begin{tabular}{lrrrrr} 
& \multicolumn{2}{c}{ lower SFA } & \multicolumn{2}{c}{ higher SFA } \\
Study or Subgroup & Events & Total & Events & Total & Weight \\
\hline 1.26.1 replaced by PUFA & & & & & \\
DART 1989 & 101 & 1018 & 100 & 1015 & $24.1 \%$ \\
MRC 1968 & 27 & 199 & 25 & 194 & $15.0 \%$ \\
Oslo Diet-Heart 1966 & 38 & 206 & 52 & 206 & $19.7 \%$ \\
Rose corn oil 1965 & 5 & 28 & 1 & 26 & $1.6 \%$ \\
STARS 1992 & 1 & 27 & 3 & 28 & $1.5 \%$ \\
Sydney Diet-Heart 1978 & 37 & 221 & 25 & 237 & $16.0 \%$ \\
Veterans Admin 1969 & 57 & 424 & 81 & 422 & $22.1 \%$ \\
Subtotal (95\% CI) & & $\mathbf{2 1 2 3}$ & & $\mathbf{2 1 2 8}$ & $\mathbf{1 0 0 . 0 \%}$ \\
Total events: & 266 & & 287 & &
\end{tabular}

Risk Ratio

Risk Ratio

M-H, Random, 95\% CI

M-H, Random, $95 \%$ CI

Heterogeneity: $\mathrm{Tau}^{2}=0.06 ; \mathrm{Chi}^{2}=13.37, \mathrm{df}=6(\mathrm{P}=0.04) ; \mathrm{I}^{2}=55 \%$

Test for overall effect: $\mathrm{Z}=0.34(\mathrm{P}=0.73)$

\subsection{2 replaced by MUFA}

Rose olive 1965

Subtotal (95\% CI)

Total events:

326

26

3

Heterogeneity: Not applicable

Test for overall effect: $\mathrm{Z}=0.98(\mathrm{P}=0.33)$

\subsection{3 replace by $\mathrm{CHO}$}

\section{Black 1994}

DART 1989

Ley 2004

STARS 1992

WHI 2006 (1)

Subtotal (95\% CI)

Total events:

Heterogeneity: $\mathrm{Tau}^{2}=0.00 ; \mathrm{Chi}^{2}=3.51, \mathrm{df}=4(\mathrm{P}=0.48) ; \mathrm{I}^{2}=0 \%$

Test for overall effect: $\mathrm{Z}=0.19(\mathrm{P}=0.85)$

\subsection{4 replaced by protein}

Black 1994

DART 1989

Ley 2004

WHI 2006 (1)

Subtotal (95\% CI)

$\begin{array}{rr}0 & 66 \\ 101 & 1018 \\ 1 & 88 \\ 1 & 27 \\ 213 & 19541 \\ & \mathbf{2 0 7 4 0}\end{array}$

$\begin{array}{rrr}2 & 67 & 0.2 \% \\ 100 & 1015 & 29.8 \% \\ 4 & 88 & 0.4 \% \\ 3 & 28 & 0.4 \% \\ 320 & 29294 & 69.1 \% \\ & \mathbf{3 0 4 9 2} & \mathbf{1 0 0 . 0 \%}\end{array}$

429

Total events:

$\begin{array}{rrrrr}0 & 66 & 2 & 67 & 0.2 \% \\ 101 & 1018 & 100 & 1015 & 29.9 \% \\ 1 & 88 & 4 & 88 & 0.4 \% \\ 213 & 19541 & 320 & 29294 & 69.4 \% \\ & \mathbf{2 0 7 1 3} & & \mathbf{3 0 4 6 4} & \mathbf{1 0 0 . 0 \%} \\ 315 & & 426 & & \end{array}$

Heterogeneity: $\mathrm{Tau}^{2}=0.00 ; \mathrm{Chi}^{2}=2.63, \mathrm{df}=3(\mathrm{P}=0.45) ; \mathrm{I}^{2}=0 \%$

Test for overall effect: $\mathrm{Z}=0.12(\mathrm{P}=0.90)$

1.26.5 replacement unclear

\section{Subtotal (95\% CI)}

$\mathbf{0}$

Total events:

0

Heterogeneity: Not applicable

Test for overall effect: Not applicable

Test for subgroup differences: $\mathrm{Chi}^{2}=1.05, \mathrm{df}=3(\mathrm{P}=0.79), \mathrm{I}^{2}=0 \%$

Footnotes

(1) Women with and without CVD at baseline
$1.01[0.77,1.31]$

$1.05[0.63,1.75]$

$0.73[0.50,1.06]$

$4.64[0.58,37.15]$

$0.35[0.04,3.12]$

$1.59[0.99,2.55]$

$0.70[0.51,0.96]$

$0.95[0.73,1.25]$

$3.00[0.33,26.99]$

$3.00[0.33,26.99]$

$0.20[0.01,4.15]$

$1.01[0.77,1.31]$

$0.25[0.03,2.19]$

$0.35[0.04,3.12]$

$1.00[0.84,1.19]$

$0.99[0.85,1.14]$

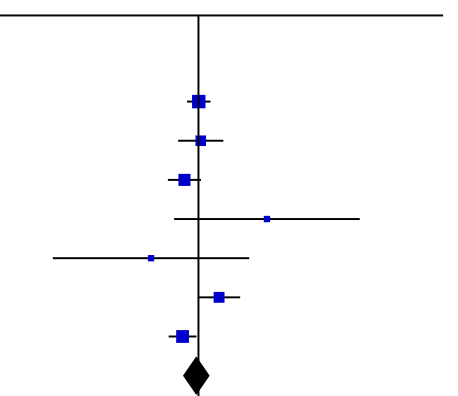

$0.20[0.01,4.15]$

$1.01[0.77,1.31]$

$0.25[0.03,2.19]$

$1.00[0.84,1.19]$

$0.99[0.86,1.14]$

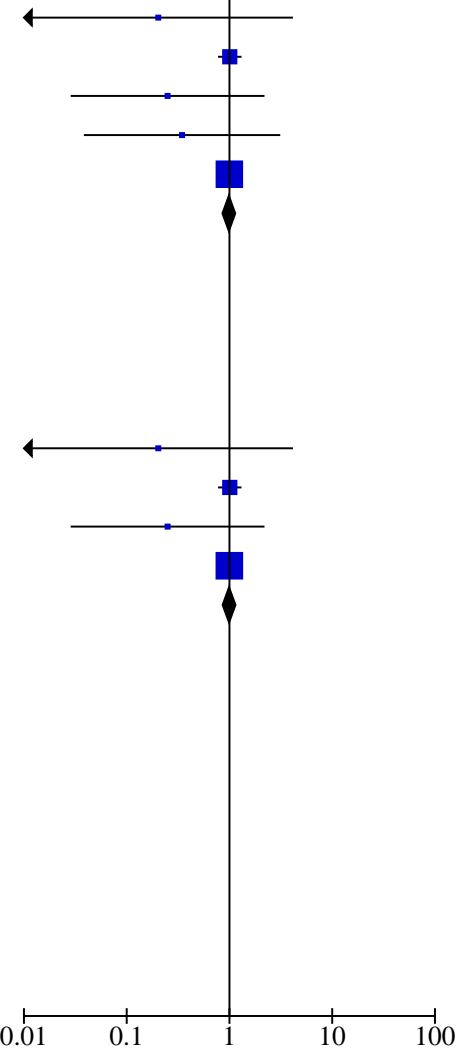

Favours lower SFA Favours higher SFA 
Analysis 1.27. Comparison 1: SFA reduction vs usual diet - primary outcomes, Outcome 27: CVD mortality, subgroup by main substitution

\begin{tabular}{lrrrrr} 
& \multicolumn{6}{c}{ lower SFA } & \multicolumn{2}{c}{ higher SFA } \\
Study or Subgroup & Events & Total & Events & Total & Weight \\
\hline 1.27.1 replaced by PUFA & & & & & \\
DART 1989 & 101 & 1018 & 100 & 1015 & $24.3 \%$ \\
MRC 1968 & 27 & 199 & 25 & 194 & $15.3 \%$ \\
Oslo Diet-Heart 1966 & 38 & 206 & 52 & 206 & $20.0 \%$ \\
Rose corn oil 1965 & 5 & 28 & 1 & 26 & $1.7 \%$ \\
Sydney Diet-Heart 1978 & 37 & 221 & 25 & 237 & $16.3 \%$ \\
Veterans Admin 1969 & 57 & 424 & 81 & 422 & $22.4 \%$ \\
Subtotal (95\% CI) & & $\mathbf{2 0 9 6}$ & & $\mathbf{2 1 0 0}$ & $\mathbf{1 0 0 . 0 \%}$ \\
Total events: & 265 & & 284 & &
\end{tabular}

Heterogeneity: $\mathrm{Tau}^{2}=0.06 ; \mathrm{Chi}^{2}=12.62, \mathrm{df}=5(\mathrm{P}=0.03) ; \mathrm{I}^{2}=60 \%$

Test for overall effect: $\mathrm{Z}=0.22(\mathrm{P}=0.82)$

\subsection{2 replaced by MUFA}

Rose olive 1965

Subtotal (95\% CI)

3

$$
26
$$

1

$26 \quad 100.0 \%$

Risk Ratio

Risk Ratio

Total events:

3

26

$26 \quad 100.0 \%$

1

Heterogeneity: Not applicable

Test for overall effect: $\mathrm{Z}=0.98(\mathrm{P}=0.33)$

\subsection{3 replace by $\mathrm{CHO}$}

Black 1994

Ley 2004

STARS 1992

WHI 2006 (1)

Subtotal (95\% CI)

Total events:

$\begin{array}{rr}0 & 66 \\ 1 & 88 \\ 1 & 27 \\ 213 & 19541 \\ & \mathbf{1 9 7 2 2}\end{array}$

$\begin{array}{rrr}2 & 67 & 4.1 \% \\ 4 & 88 & 7.6 \% \\ 3 & 28 & 7.4 \% \\ 320 & 29294 & 81.0 \% \\ & \mathbf{2 9 4 7 7} & \mathbf{1 0 0 . 0 \%}\end{array}$

$215 \quad 329$

Heterogeneity: $\mathrm{Tau}^{2}=0.12 ; \mathrm{Chi}^{2}=3.47, \mathrm{df}=3(\mathrm{P}=0.32) ; \mathrm{I}^{2}=14 \%$

Test for overall effect: $\mathrm{Z}=0.78(\mathrm{P}=0.43)$

1.27.4 replaced by protein

Subtotal (95\% CI)

0

Total events:

Heterogeneity: Not applicable

Test for overall effect: Not applicable

1.27.5 replacement unclear

Subtotal $(95 \%$ CI)

0

Total events:

0

Heterogeneity: Not applicable

Test for overall effect: Not applicable

Test for subgroup differences: $\mathrm{Chi}^{2}=1.46, \mathrm{df}=2(\mathrm{P}=0.48), \mathrm{I}^{2}=0 \%$

\section{Footnotes}

(1) Women with and without CVD at baseline
$1.01[0.77,1.31]$

$1.05[0.63,1.75]$

$0.73[0.50,1.06]$

$4.64[0.58,37.15]$

$1.59[0.99,2.55]$

$0.70[0.51,0.96]$

$0.97[0.73,1.28]$

$3.00[0.33,26.99]$

3.00 [0.33, 26.99]

$0.20[0.01,4.15]$

$0.25[0.03,2.19]$

0.35 [0.04, 3.12]

$1.00[0.84,1.19]$

$0.78[0.42,1.46]$

M-H, Random, 95\% CI 


\section{Analysis 1.28. Comparison 1: SFA reduction vs usual diet - primary outcomes, Outcome 28: CVD mortality, subgroup by duration}

lower SFA
Risk Ratio

M-H, Random, $95 \%$ CI
Risk Ratio

M-H, Random, $95 \%$ CI

\subsection{1 up to $24 \mathrm{mo}$}

Black 1994

DART 1989

Rose corn oil 1965

Rose olive 1965

Subtotal (95\% CI)

Total events:

Heterogeneity: $\mathrm{Tau}^{2}=0.26 ; \mathrm{Chi}^{2}=4.07, \mathrm{df}=3(\mathrm{P}=0.25) ; \mathrm{I}^{2}=26 \%$

Test for overall effect: $\mathrm{Z}=0.54(\mathrm{P}=0.59)$

\section{$1.28 .2>24$ to $48 \mathrm{mo}$}

MRC 1968

STARS 1992

Veterans Admin 1969

$\begin{array}{rr}27 & 199 \\ 1 & 27 \\ 57 & 424 \\ & \mathbf{6 5}\end{array}$

85

Subtotal (95\% CI)

Total events:
Heterogeneity: $\mathrm{Tau}^{2}=0.01 ; \mathrm{Chi}^{2}=2.33, \mathrm{df}=2(\mathrm{P}=0.31) ; \mathrm{I}^{2}=14 \%$

Test for overall effect: $\mathrm{Z}=1.49(\mathrm{P}=0.14)$

\subsection{3 $>48 \mathrm{mo}$}

Oslo Diet-Heart 1966

Sydney Diet-Heart 1978

WHI 2006 (1)

Subtotal (95\% CI)

Total events:

38
37
213

$\begin{array}{rr}206 & 52 \\ 221 & 25 \\ 19541 & 320 \\ \mathbf{1 9 9 6 8} & \end{array}$

$\begin{array}{rr}206 & 14.5 \% \\ 237 & 11.0 \% \\ 29294 & 24.1 \% \\ \mathbf{2 9 7 3 7} & \mathbf{4 9 . 6 \%}\end{array}$

397

Heterogeneity: $\mathrm{Tau}^{2}=0.06 ; \mathrm{Chi}^{2}=6.40, \mathrm{df}=2(\mathrm{P}=0.04) ; \mathrm{I}^{2}=69 \%$

Test for overall effect: $\mathrm{Z}=0.10(\mathrm{P}=0.92)$

\subsection{4 unclear duration}

Ley 2004

\section{Subtotal (95\% CI)}

Total events:

Heterogeneity: Not applicable

Test for overall effect: $\mathrm{Z}=1.25(\mathrm{P}=0.21)$

\section{Total $(95 \%$ CI)}

Total events:

21844

$31603100.0 \%$

483

$\begin{array}{llll}88 & 4 & 88 & 0.8 \% \\ \mathbf{8 8} & & \mathbf{8 8} & \mathbf{0 . 8 \%}\end{array}$

Heterogeneity: $\mathrm{Tau}^{2}=0.04 ; \mathrm{Chi}^{2}=17.36, \mathrm{df}=10(\mathrm{P}=0.07) ; \mathrm{I}^{2}=42 \%$

Test for overall effect: $\mathrm{Z}=0.53(\mathrm{P}=0.60)$

Test for subgroup differences: $\mathrm{Chi}^{2}=3.15, \mathrm{df}=3(\mathrm{P}=0.37), \mathrm{I}^{2}=4.9 \%$

\section{Footnotes}

(1) Women with and without CVD at baseline
$0.20[0.01,4.15]$

$1.01[0.77,1.31]$

$4.64[0.58,37.15]$

$3.00[0.33,26.99]$

$1.26[0.54,2.94]$
$1.05[0.63,1.75]$

$0.35[0.04,3.12]$

$0.70[0.51,0.96]$

$0.79[0.57,1.08]$

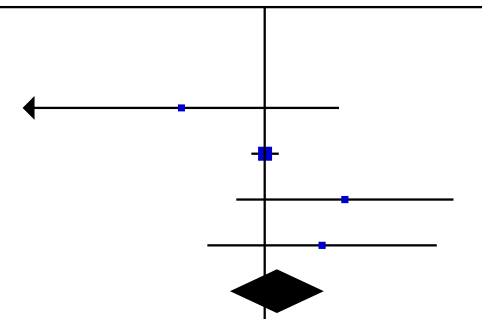

$0.73[0.50,1.06]$

$1.59[0.99,2.55]$

$1.00[0.84,1.19]$

$1.02[0.73,1.43]$
$0.25[0.03,2.19]$

$0.25[0.03,2.19]$ 


\section{Analysis 1.29. Comparison 1: SFA reduction vs usual diet - primary outcomes, Outcome 29: CVD mortality, subgroup by baseline SFA}

Study or Subgroup

lower SFA higher SFA

Risk Ratio

Risk Ratio

Events Total Events Total Weight M-H, Random, 95\% CI

M-H, Random, 95\% CI

1.29.1 up to $12 \%$ E SFA baseline Subtotal (95\% CI)

Total events:

0

0

Heterogeneity: Not applicable

Test for overall effect: Not applicable

1.29.2 $>12$ to $15 \%$ E SFA baseline

Black 1994

DART 1989

Ley 2004

Sydney Diet-Heart 1978

WHI 2006 (1)

Subtotal (95\% CI)

Total events:

$\begin{array}{rr}0 & 66 \\ 101 & 1018 \\ 1 & 88 \\ 37 & 221 \\ 213 & 19541 \\ & \mathbf{2 0 9 3 4} \\ 352 & \end{array}$

66
1018
88
221
9541
$\mathbf{0 9 3 4}$

$\begin{array}{rrr}2 & 67 & 0.4 \% \\ 100 & 1015 & 19.4 \% \\ 4 & 88 & 0.8 \% \\ 25 & 237 & 11.0 \% \\ 320 & 29294 & 24.1 \% \\ & \mathbf{3 0 7 0 1} & \mathbf{5 5 . 8 \%}\end{array}$

$0.20[0.01,4.15]$

$1.01[0.77,1.31]$

$0.25[0.03,2.19]$

$1.59[0.99,2.55]$

$1.00[0.84,1.19]$

$1.06[0.84,1.32]$

451

Heterogeneity: $\mathrm{Tau}^{2}=0.02 ; \mathrm{Chi}^{2}=6.12, \mathrm{df}=4(\mathrm{P}=0.19) ; \mathrm{I}^{2}=35 \%$

Test for overall effect: $\mathrm{Z}=0.48(\mathrm{P}=0.63)$

1.29.3 $>15$ to $18 \%$ E SFA baseline

STARS 1992

Subtotal $(95 \% \mathrm{CI})$

127

Total events:

27
27

3

28

$0.8 \%$

$0.35[0.04,3.12]$

$0.35[0.04,3.12]$

3

Heterogeneity: Not applicable

Test for overall effect: $\mathrm{Z}=0.95(\mathrm{P}=0.34)$

\subsection{4 $>18 \%$ E SFA baseline}

\section{Veterans Admin 1969}

$57 \quad 424$
$\mathbf{4 2 4}$

424
$\mathbf{4 2 4}$

81

422

$17.1 \%$

Subtotal (95\% CI)

57

Heterogeneity: Not applicable

Test for overall effect: $\mathrm{Z}=2.25(\mathrm{P}=0.02)$

1.29.5 unclear

MRC 1968

Oslo Diet-Heart 1966

Rose corn oil 1965

Rose olive 1965

Subtotal (95\% CI)

Total events:

$\begin{array}{rr}27 & 199 \\ 38 & 206 \\ 5 & 28 \\ 3 & 26 \\ & \mathbf{4 5 9}\end{array}$

25
52
1
1

194

$10.1 \%$

$1.05[0.63,1.75]$

$0.73[0.50,1.06]$

$4.64[0.58,37.15]$

$3.00[0.33,26.99]$

$1.00[0.61,1.66]$

$0.70[0.51,0.96]$

$0.70[0.51,0.96]$ 79

Heterogeneity: $\mathrm{Tau}^{2}=0.10 ; \mathrm{Chi}^{2}=5.16, \mathrm{df}=3(\mathrm{P}=0.16) ; \mathrm{I}^{2}=42 \%$

Test for overall effect: $\mathrm{Z}=0.01(\mathrm{P}=0.99)$

Total (95\% CI)

Total events:

21844

483

Heterogeneity: $\mathrm{Tau}^{2}=0.04 ; \mathrm{Chi}^{2}=17.36, \mathrm{df}=10(\mathrm{P}=0.07) ; \mathrm{I}^{2}=42 \%$

Test for overall effect: $\mathrm{Z}=0.53(\mathrm{P}=0.60)$

Test for subgroup differences: $\mathrm{Chi}^{2}=5.29, \mathrm{df}=3(\mathrm{P}=0.15), \mathrm{I}^{2}=43.3 \%$
$31603100.0 \%$

Footnotes

(1) Women with and without CVD at baseline 


\section{Analysis 1.30. Comparison 1: SFA reduction vs usual diet - primary outcomes, Outcome 30: CVD mortality, subgroup by SFA change}

lower SFA
Risk Ratio

Total Weight M-H, Random, 95\% CI
Risk Ratio

M-H, Random, 95\% CI

1.30.1 up to $4 \% \mathrm{E}$ difference

\section{DART 1989}

Ley 2004

Sydney Diet-Heart 1978

WHI 2006 (1)

Subtotal $(95 \%$ CI)

Total events:

Heterogeneity: $\mathrm{Tau}^{2}=0.02 ; \mathrm{Chi}^{2}=5.00, \mathrm{df}=3(\mathrm{P}=0.17) ; \mathrm{I}^{2}=40 \%$

Test for overall effect: $\mathrm{Z}=0.56(\mathrm{P}=0.57)$

\subsection{2 $>4$ to $8 \% \mathrm{E}$ difference}

Black 1994

STARS 1992

Subtotal (95\% CI)

Total events:

$\begin{array}{rrrrr}101 & 1018 & 100 & 1015 & 19.4 \% \\ 1 & 88 & 4 & 88 & 0.8 \% \\ 37 & 221 & 25 & 237 & 11.0 \% \\ 213 & 19541 & 320 & 29294 & 24.1 \% \\ & \mathbf{2 0 8 6 8} & & \mathbf{3 0 6 3 4} & \mathbf{5 5 . 4 \%}\end{array}$

449

Heterogeneity: $\mathrm{Tau}^{2}=0.00 ; \mathrm{Chi}^{2}=0.08, \mathrm{df}=1(\mathrm{P}=0.78) ; \mathrm{I}^{2}=0 \%$

Test for overall effect: $\mathrm{Z}=1.37(\mathrm{P}=0.17)$

\subsection{3 $>8 \% \mathrm{E}$ difference}

Veterans Admin 1969

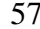

$$
424
$$$$
424
$$

57

Total events:

Heterogeneity: Not applicable

Test for overall effect: $\mathrm{Z}=2.25(\mathrm{P}=0.02)$

\subsection{4 unclear}

MRC 1968

Oslo Diet-Heart 1966

Rose corn oil 1965

Rose olive 1965

Subtotal $(95 \%$ CI)

Total events:

Heterogeneity: $\mathrm{Tau}^{2}=0.10 ; \mathrm{Chi}^{2}=5.16, \mathrm{df}=3(\mathrm{P}=0.16) ; \mathrm{I}^{2}=42 \%$

Test for overall effect: $\mathrm{Z}=0.01(\mathrm{P}=0.99)$

Total $(95 \%$ CI $)$

Total events:

21844

$31603100.0 \%$

483
614

Heterogeneity: $\mathrm{Tau}^{2}=0.04 ; \mathrm{Chi}^{2}=17.36, \mathrm{df}=10(\mathrm{P}=0.07) ; \mathrm{I}^{2}=42 \%$

Test for overall effect: $\mathrm{Z}=0.53(\mathrm{P}=0.60)$

Test for subgroup differences: $\mathrm{Chi}^{2}=6.39, \mathrm{df}=3(\mathrm{P}=0.09), \mathrm{I}^{2}=53.1 \%$

Footnotes

(1) Women with and without CVD at baseline

$0.8 \%$
$1.01[0.77,1.31]$

$0.25[0.03,2.19]$

$1.59[0.99,2.55]$

$1.00[0.84,1.19]$

$1.07[0.85,1.33]$

$0.20[0.01,4.15]$

$0.35[0.04,3.12]$

$0.29[0.05,1.70]$

$0.70[0.51,0.96]$

$0.70[0.51,0.96]$
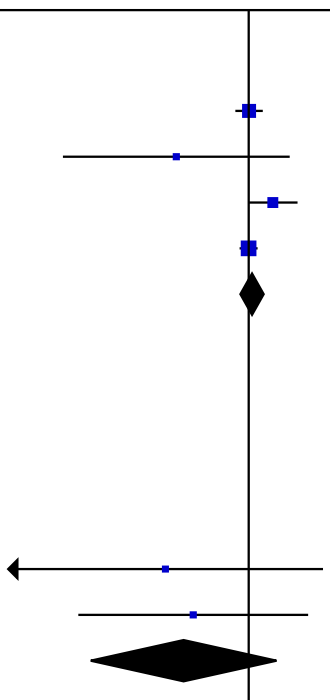

$1.05[0.63,1.75]$

$0.73[0.50,1.06]$

$4.64[0.58,37.15]$

$3.00[0.33,26.99]$

$1.00[0.61,1.66]$

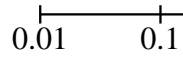

Favours lower SFA
Favours higher $\mathrm{SF}$ 
Analysis 1.31. Comparison 1: SFA reduction vs usual diet primary outcomes, Outcome 31: CVD mortality, subgroup by sex

\begin{tabular}{lccccccc} 
& \multicolumn{2}{c}{ lower SFA } & \multicolumn{2}{c}{ higher SFA } & \multicolumn{2}{c}{ Risk Ratio } & Risk Ratio \\
Study or Subgroup & Events & Total & Events & Total & Weight & M-H, Random, 95\% CI & M-H, Random, 95\% CI
\end{tabular}

\subsubsection{Men}

Black 1994

DART 1989

MRC 1968

Oslo Diet-Heart 1966

Rose corn oil 1965

Rose olive 1965

STARS 1992

Sydney Diet-Heart 1978

Veterans Admin 1969

Subtotal (95\% CI)

Total events:

M-H, Random, 95\% CI

$0.20[0.01,4.15]$

$1.01[0.77,1.31$

$1.05[0.63,1.75]$

$0.73[0.50,1.06]$

$4.64[0.58,37.15]$

$3.00[0.33,26.99]$

$0.35[0.04,3.12]$

$1.59[0.99,2.55]$

$0.70[0.51,0.96]$

$0.96[0.73,1.25]$

290

Heterogeneity: $\mathrm{Tau}^{2}=0.06 ; \mathrm{Chi}^{2}=15.45, \mathrm{df}=8(\mathrm{P}=0.05) ; \mathrm{I}^{2}=48 \%$

Test for overall effect: $\mathrm{Z}=0.32(\mathrm{P}=0.75)$

\subsubsection{Women}

WHI 2006 (1)

Subtotal (95\% CI)

213

19541

320

29294

$24.1 \%$

$1.00[0.84,1.19]$

Total events:

213

320

Heterogeneity: Not applicable

Test for overall effect: $\mathrm{Z}=0.02(\mathrm{P}=0.98)$

1.31.3 Mixed, men and women

Ley 2004

Subtotal (95\% CI)

Total events:

1

Heterogeneity: Not applicable

Test for overall effect: $\mathrm{Z}=1.25(\mathrm{P}=0.21)$

Total (95\% CI)

483

21844

$88 \quad 4$

$\begin{array}{ll}88 & 0.8 \% \\ \mathbf{8 8} & \mathbf{0 . 8 \%}\end{array}$

$0.25[0.03,2.19]$

88

$0.25[0.03,2.19]$

Total events

$31603100.0 \%$

Heterogeneity: $\mathrm{Tau}^{2}=0.04 ; \mathrm{Chi}^{2}=17.36, \mathrm{df}=10(\mathrm{P}=0.07) ; \mathrm{I}^{2}=42 \%$

Test for overall effect: $\mathrm{Z}=0.53(\mathrm{P}=0.60)$

Test for subgroup differences: $\mathrm{Chi}^{2}=1.59, \mathrm{df}=2(\mathrm{P}=0.45), \mathrm{I}^{2}=0 \%$

Footnotes

(1) Women with and without CVD at baseline
M-H, Random, 95\% CI

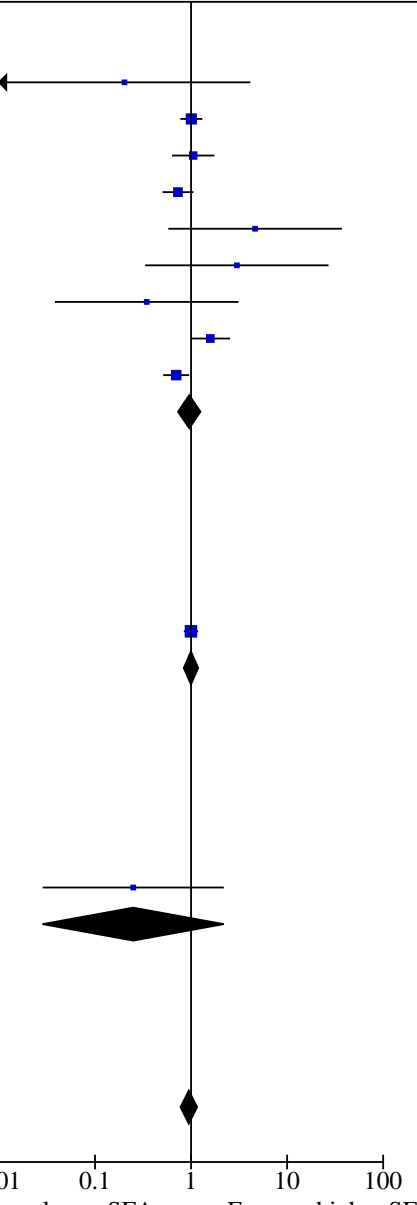

Favours lower SFA Favours higher SFA 


\section{Analysis 1.32. Comparison 1: SFA reduction vs usual diet - primary outcomes, Outcome 32: CVD mortality, subgroup by CVD risk}

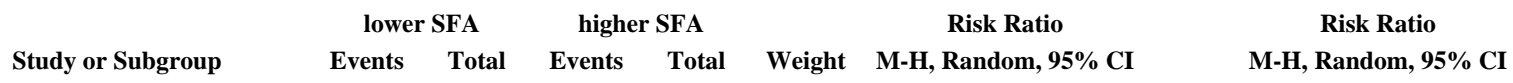

\subsubsection{Low CVD risk}

Black 1994

Veterans Admin 1969

WHI $2006(1)$

Subtotal $(95 \%$ CI $)$

Total events:

Heterogeneity: $\mathrm{Tau}^{2}=0.04 ; \mathrm{Chi}^{2}=4.33, \mathrm{df}=2(\mathrm{P}=0.11) ; \mathrm{I}^{2}=54 \%$

Test for overall effect: $\mathrm{Z}=1.06(\mathrm{P}=0.29)$

1.32.2 Moderate CVD risk

Ley 2004

Subtotal (95\% CI)

Total events:

Heterogeneity: Not applicable

Test for overall effect: $\mathrm{Z}=1.25(\mathrm{P}=0.21)$

1.32.3 Existing CVD disease

DART 1989

MRC 1968

Oslo Diet-Heart 1966

Rose corn oil 1965

Rose olive 1965

STARS 1992

Sydney Diet-Heart 1978

WHI 2006 (2)

Subtotal (95\% CI)

Total events:

$\begin{array}{rr}101 & 1018 \\ 27 & 199 \\ 38 & 206 \\ 5 & 28 \\ 3 & 26 \\ 1 & 27 \\ 37 & 221 \\ 43 & 908 \\ & \mathbf{2 6 3 3}\end{array}$

100

25

1015

194

$17.6 \%$

$8.5 \%$

$12.7 \%$

$0.7 \%$

$0.6 \%$

$0.6 \%$

$9.4 \%$

$12.3 \%$

$3101 \quad 62.4 \%$

255

269

Heterogeneity: $\mathrm{Tau}^{2}=0.03 ; \mathrm{Chi}^{2}=10.41, \mathrm{df}=7(\mathrm{P}=0.17) ; \mathrm{I}^{2}=33 \%$

Test for overall effect: $\mathrm{Z}=0.37(\mathrm{P}=0.71)$

Total $(95 \%$ CI $)$

483

21844

Total events: 614

Heterogeneity: $\mathrm{Tau}^{2}=0.03 ; \mathrm{Chi}^{2}=17.45, \mathrm{df}=11(\mathrm{P}=0.10) ; \mathrm{I}^{2}=37 \%$

Test for overall effect: $\mathrm{Z}=0.50(\mathrm{P}=0.61)$

Test for subgroup differences: $\mathrm{Chi}^{2}=2.66, \mathrm{df}=2(\mathrm{P}=0.26), \mathrm{I}^{2}=24.9 \%$

Footnotes

(1) Women without CVD at baseline

(2) Women with CVD at baseline
$0.20[0.01,4.15]$

$0.70[0.51,0.96]$

$0.99[0.81,1.20]$

$0.84[0.60,1.16]$

$\stackrel{+}{\longleftarrow}$

$0.25[0.03,2.19]$

0.25 [0.03, 2.19]

$1.01[0.77,1.31]$

$1.05[0.63,1.75]$

$0.73[0.50,1.06]$

$4.64[0.58,37.15]$

$3.00[0.33,26.99]$

$0.35[0.04,3.12]$

$1.59[0.99,2.55]$

$1.05[0.72,1.53]$

$1.04[0.83,1.31]$

$0.96[0.80,1.14]$

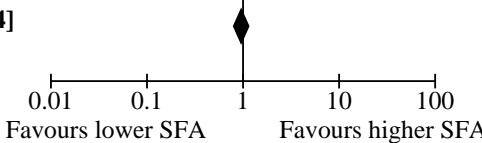




\section{Analysis 1.33. Comparison 1: SFA reduction vs usual diet - primary outcomes, Outcome 33: CVD mortality, subgroup by TC reduction}

\begin{tabular}{|c|c|c|c|c|c|c|c|}
\hline & lowe & FA & high & SFA & & Risk Ratio & Risk Ratio \\
\hline Study or Subgroup & Events & Total & Events & Total & Weight & M-H, Random, $95 \%$ CI & M-H, Random, 95\% CI \\
\hline
\end{tabular}

1.33.1 serum chol reduced by at least $0.2 \mathrm{mmol} / \mathrm{L}$

MRC $1968 \quad 27 \quad 199$

Oslo Diet-Heart $1966 \quad 38 \quad 206$

Rose corn oil 1965

STARS 1992

Sydney Diet-Heart 1978

$5 \quad 28$

1

Veterans Admin 1969

Subtotal $(95 \%$ CI $)$

\section{$100 \quad 1015 \quad 24.1 \%$}

$25 \quad 194 \quad 15.0 \%$

$52 \quad 206 \quad 19.7 \%$

$1 \quad 26 \quad 1.6 \%$

$3-28 \quad 1.5 \%$

$25 \quad 237 \quad 16.0 \%$

81

422

$2128100.0 \%$

287

Heterogeneity: $\mathrm{Tau}^{2}=0.06 ; \mathrm{Chi}^{2}=13.37, \mathrm{df}=6(\mathrm{P}=0.04) ; \mathrm{I}^{2}=55 \%$

Test for overall effect: $\mathrm{Z}=0.34(\mathrm{P}=0.73)$

1.33.2 serum chol reduced by $<0.2 \mathrm{mmol} / \mathrm{L}$

$\begin{array}{lrrrrr}\text { Ley 2004 } & 1 & 88 & 4 & 88 & 9.9 \% \\ \text { Rose olive 1965 } & 3 & 26 & 1 & 26 & 9.7 \% \\ \text { WHI 2006 (1) } & 213 & 19541 & 320 & 29294 & 80.3 \% \\ \text { Subtotal (95\% CI) } & & \mathbf{1 9 6 5 5} & & \mathbf{2 9 4 0 8} & \mathbf{1 0 0 . 0 \%} \\ \text { Total events: } & 217 & & 325 & & \end{array}$

Heterogeneity: $\mathrm{Tau}^{2}=0.16 ; \mathrm{Chi}^{2}=2.53, \mathrm{df}=2(\mathrm{P}=0.28) ; \mathrm{I}^{2}=21 \%$

Test for overall effect: $\mathrm{Z}=0.09(\mathrm{P}=0.93)$

1.33.3 serum chol reduction unclear

$\begin{array}{llllll}\text { Black } 1994 & 0 & 66 & 2 & 67 & 100.0 \% \\ \text { Subtotal (95\% CI) } & & \mathbf{6 6} & & \mathbf{6 7} & \mathbf{1 0 0 . 0 \%} \\ \text { Total events: } & 0 & & 2 & & \end{array}$

Heterogeneity: Not applicable

Test for overall effect: $\mathrm{Z}=1.04(\mathrm{P}=0.30)$

Test for subgroup differences: $\mathrm{Chi}^{2}=1.01, \mathrm{df}=2(\mathrm{P}=0.60), \mathrm{I}^{2}=0 \%$

Footnotes

(1) Women with and without CVD at baseline
$1.01[0.77,1.31]$

$1.05[0.63,1.75]$

$0.73[0.50,1.06]$

$4.64[0.58,37.15]$

$0.35[0.04,3.12]$

$1.59[0.99,2.55]$

$0.70[0.51,0.96]$

$0.95[0.73,1.25]$

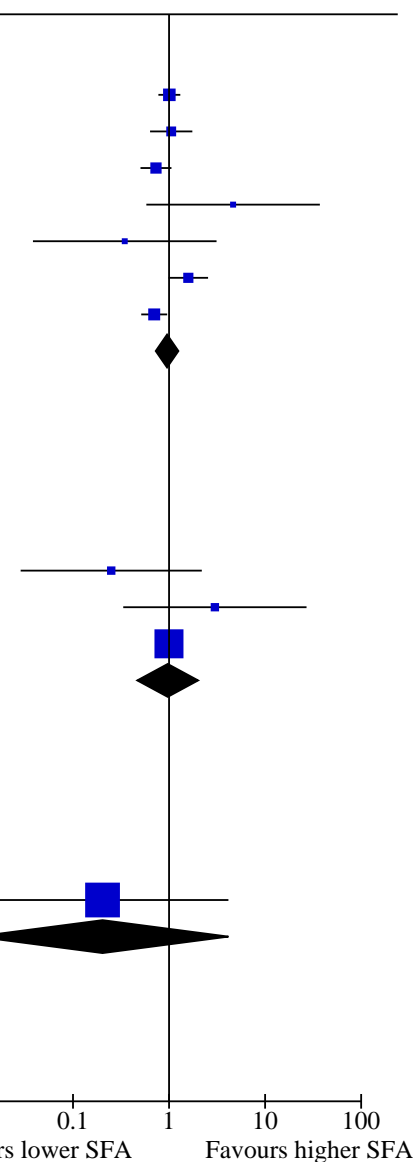




\section{Analysis 1.34. Comparison 1: SFA reduction vs usual diet - primary outcomes, Outcome 34: CVD mortality, subgroup decade of publication}

Study or Subgroup lower SFA higher SFA

Events Total Events Total Weight M-H, Random, 95\% CI
Risk Ratio

M-H, Random, $95 \%$ CI

\subsubsection{0s}

\section{MRC 1968}

Oslo Diet-Heart 1966

Rose corn oil 1965

Rose olive 1965

Veterans Admin 1969

Subtotal (95\% CI)

Total events:

$\begin{array}{rr}27 & 199 \\ 38 & 206 \\ 5 & 28 \\ 3 & 26 \\ 57 & 424 \\ & \mathbf{8 8 3}\end{array}$

Heterogeneity: $\mathrm{Tau}^{2}=0.00 ; \mathrm{Chi}^{2}=4.07, \mathrm{df}=4(\mathrm{P}=0.40) ; \mathrm{I}^{2}=2 \%$

Test for overall effect: $\mathrm{Z}=2.22(\mathrm{P}=0.03)$

\subsubsection{0s}

Sydney Diet-Heart 1978

Subtotal $(95 \%$ CI)

$\begin{array}{ll}37 & 221 \\ & \mathbf{2 2 1} \\ 37 & \end{array}$

25

$10.5 \%$

Total events:

Heterogeneity: Not applicable

Test for overall effect: $\mathrm{Z}=1.91(\mathrm{P}=0.06)$

\subsubsection{0s}

DART 1989

Subtotal (95\% CI)

$\begin{aligned} & 101 \quad 1018 \\ & \mathbf{1 0 1 8}\end{aligned}$

100

1015

101

1015

$19.9 \%$

100

Heterogeneity: Not applicable

Test for overall effect: $\mathrm{Z}=0.05(\mathrm{P}=0.96)$

\subsubsection{0s}

Black 1994

STARS 1992

Subtotal (95\% CI)

Total events:

$0 \quad 66$

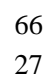

66
27

2
3

67

$0.4 \%$

$0.7 \%$

93

1

5

Heterogeneity: $\mathrm{Tau}^{2}=0.00 ; \mathrm{Chi}^{2}=0.08, \mathrm{df}=1(\mathrm{P}=0.78) ; \mathrm{I}^{2}=0 \%$

Test for overall effect: $\mathrm{Z}=1.37(\mathrm{P}=0.17)$

\subsubsection{0s}

Ley 2004

WHI 2006 (1)

$\begin{array}{rr}1 & 88 \\ 213 & 19541 \\ & \mathbf{1 9 6 2 9}\end{array}$

214
Subtotal $(95 \% \mathrm{CI})$

Total events:

Heterogeneity: $\mathrm{Tau}^{2}=0.34 ; \mathrm{Chi}^{2}=1.55, \mathrm{df}=1(\mathrm{P}=0.21) ; \mathrm{I}^{2}=36 \%$

Test for overall effect: $\mathrm{Z}=0.48(\mathrm{P}=0.63)$

\section{Total $(95 \%$ CI $)$}

Total events:

21844 483

$31577 \quad 100.0 \%$

Heterogeneity: $\mathrm{Tau}^{2}=0.03 ; \mathrm{Chi}^{2}=15.67, \mathrm{df}=10(\mathrm{P}=0.11) ; \mathrm{I}^{2}=36 \%$

Test for overall effect: $\mathrm{Z}=0.68(\mathrm{P}=0.49)$

Test for subgroup differences: $\mathrm{Chi}^{2}=9.48, \mathrm{df}=4(\mathrm{P}=0.05), \mathrm{I}^{2}=57.8 \%$
$1.05[0.63,1.75]$

$0.73[0.50,1.06]$

$5.31[0.32,89.44]$

$1.50[0.17,13.05]$

$0.70[0.51,0.96]$

$0.78[0.63,0.97]$
$1.59[0.99,2.55]$

$1.59[0.99,2.55]$

$1.01[0.77,1.31]$

$1.01[0.77,1.31]$
$0.20[0.01,4.15]$

$0.35[0.04,3.12]$

$0.29[0.05,1.70]$

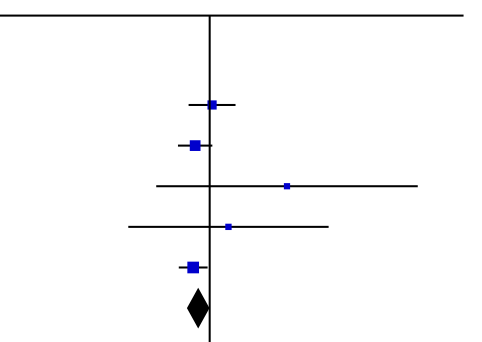

Footnotes

(1) Women with and without CVD at baseline 


\section{Analysis 1.35. Comparison 1: SFA reduction vs usual diet - primary outcomes, Outcome 35: COMBINED CARDIOVASCULAR EVENTS}

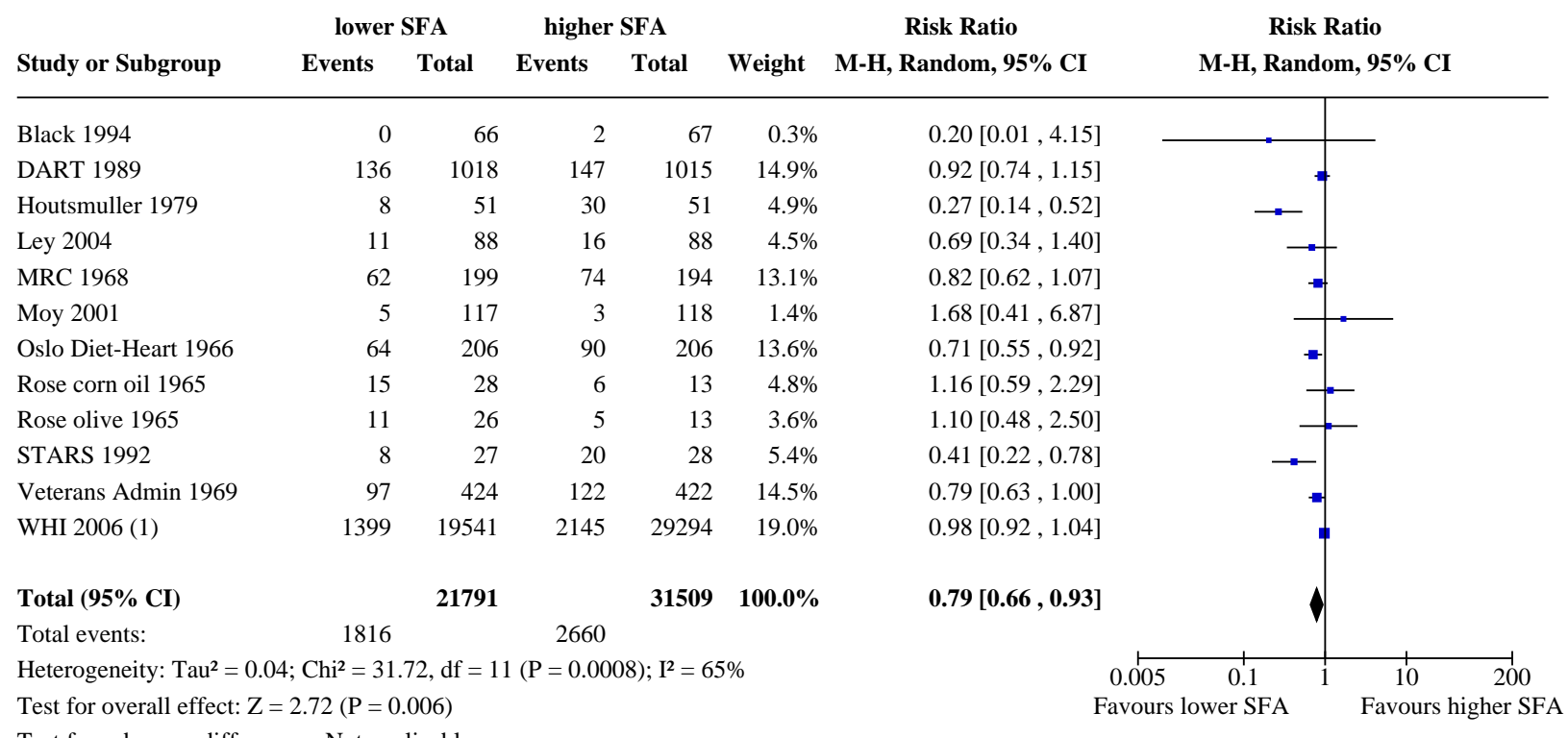

Footnotes

(1) Total CVD during study period, Prentice 2017

\section{Analysis 1.36. Comparison 1: SFA reduction vs usual diet - primary outcomes, Outcome 36: CVD events, SA low summary risk of bias}

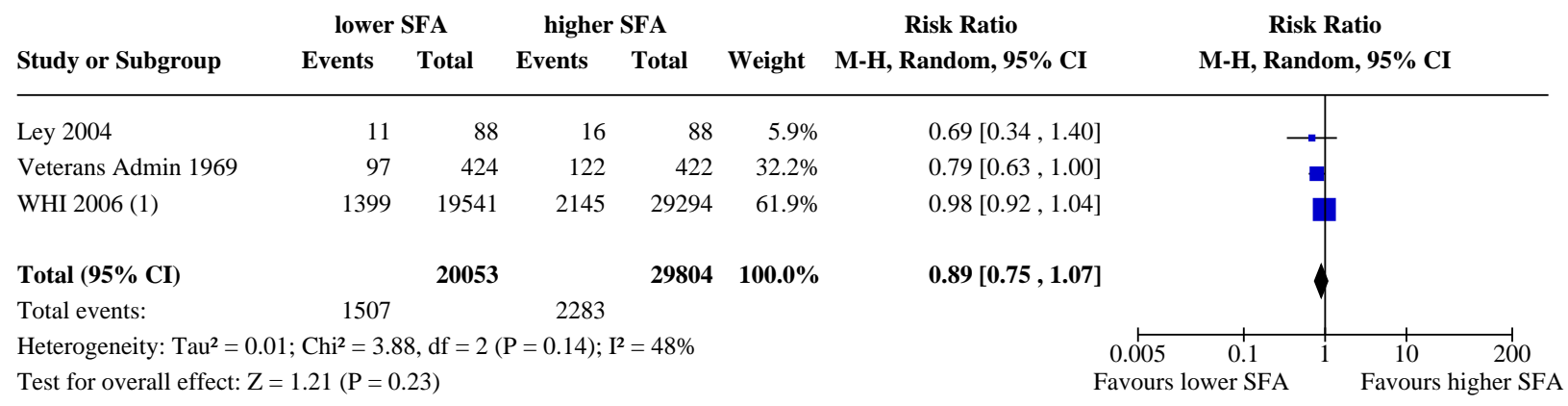

Test for subgroup differences: Not applicable

Footnotes

(1) Total CVD during study period, Prentice 2017 


\section{Analysis 1.37. Comparison 1: SFA reduction vs usual diet - primary outcomes, Outcome 37: CVD events, SA aim to reduce SFA}

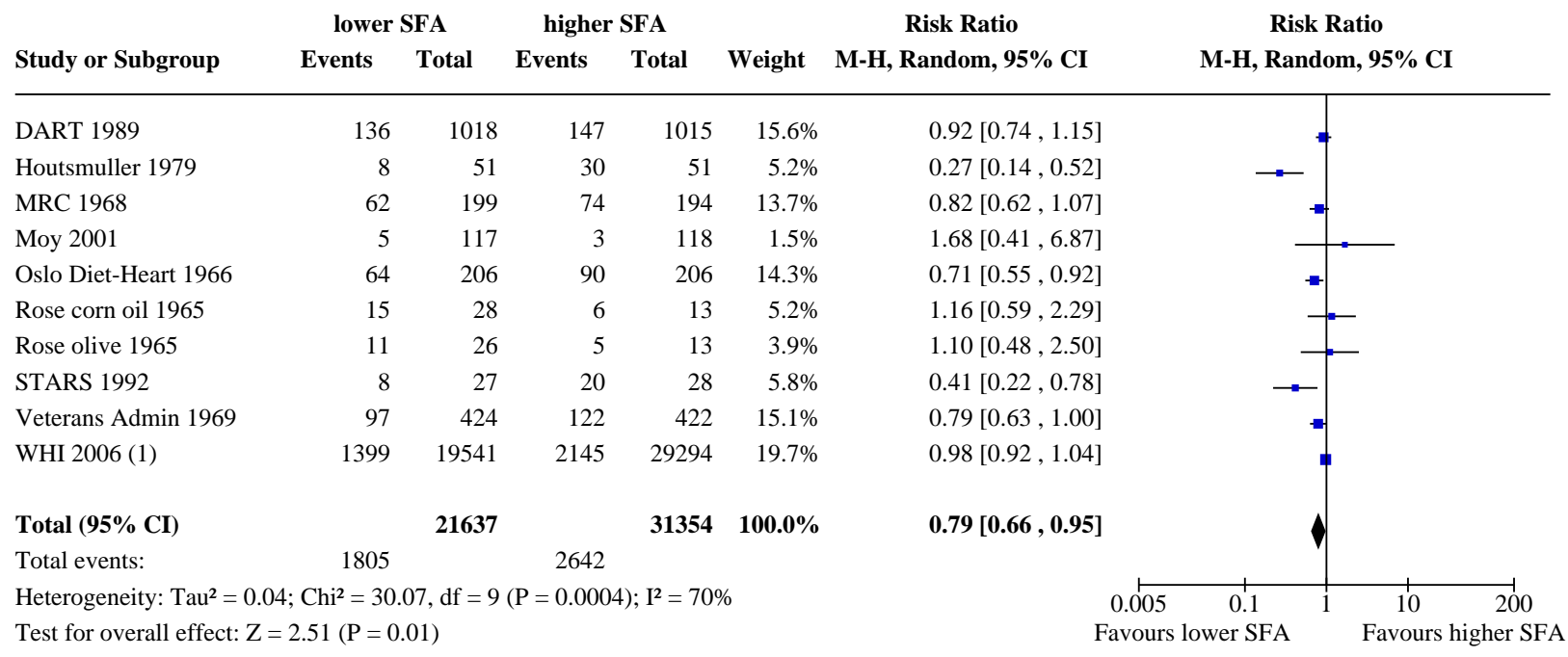

Test for subgroup differences: Not applicable

Footnotes

(1) Total CVD during study period, Prentice 2017

Analysis 1.38. Comparison 1: SFA reduction vs usual diet - primary outcomes, Outcome 38: CVD events, SA statistically significant SFA reduction

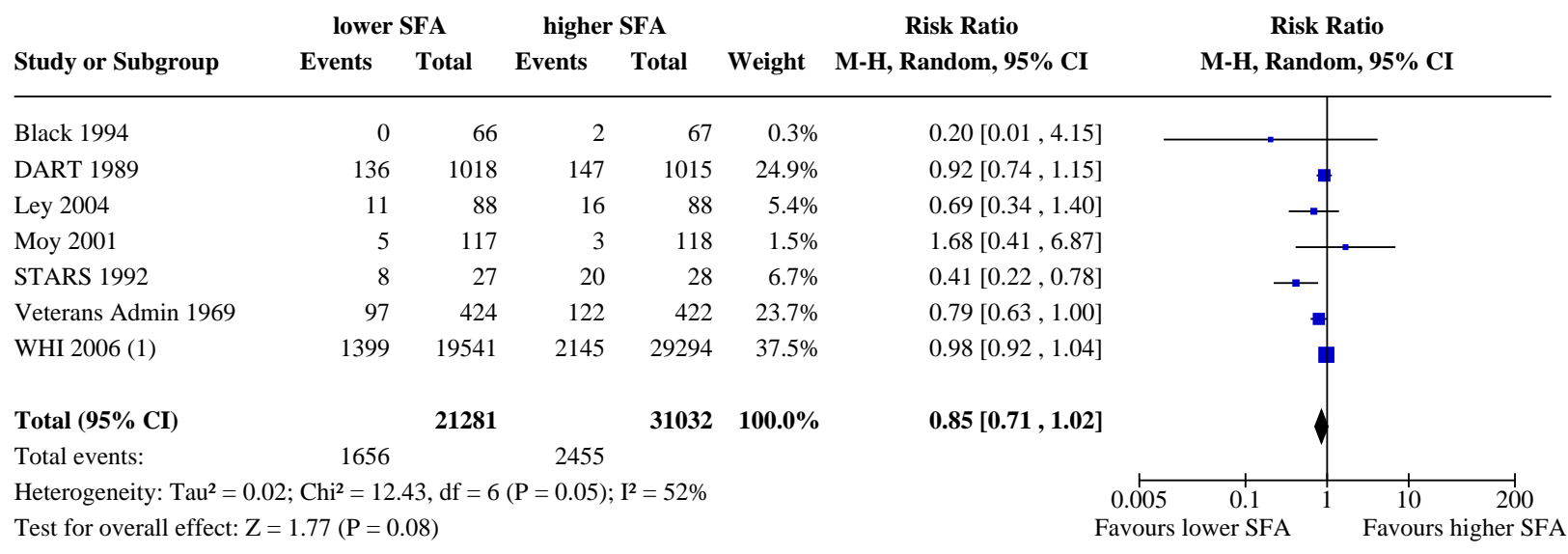

Test for subgroup differences: Not applicable

Footnotes

(1) Total CVD during study period, Prentice 2017 
Analysis 1.39. Comparison 1: SFA reduction vs usual diet primary outcomes, Outcome 39: CVD events, SA TC reduction

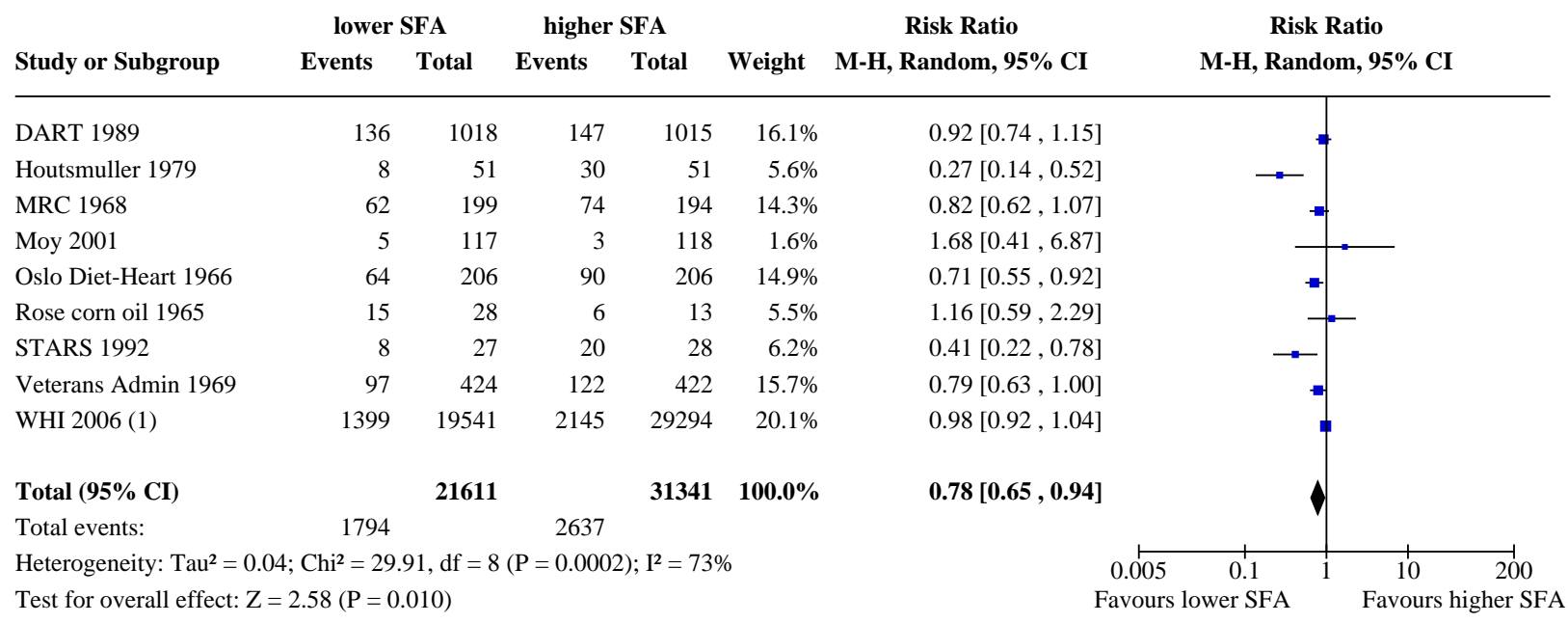

Test for subgroup differences: Not applicable

Footnotes

(1) Total CVD during study period, Prentice 2017

Analysis 1.40. Comparison 1: SFA reduction vs usual diet primary outcomes, Outcome 40: CVD events, SA excluding WHI

\begin{tabular}{|c|c|c|c|c|c|c|c|c|c|}
\hline \multirow{3}{*}{$\begin{array}{l}\text { Study or Subgroup } \\
\text { Black } 1994\end{array}$} & \multicolumn{2}{|c|}{ lower SFA } & \multicolumn{2}{|c|}{ higher SFA } & \multirow[b]{2}{*}{ Weight } & \multirow{2}{*}{$\begin{array}{c}\text { Risk Ratio } \\
\text { M-H, Random, 95\% CI }\end{array}$} & \multirow{2}{*}{\multicolumn{2}{|c|}{$\begin{array}{c}\text { Risk Ratio } \\
\text { M-H, Random, 95\% CI }\end{array}$}} & \\
\hline & \multirow{2}{*}{$\begin{array}{r}\text { Events } \\
0\end{array}$} & \multirow{2}{*}{$\begin{array}{r}\text { Total } \\
66\end{array}$} & \multirow{2}{*}{$\begin{array}{r}\text { Events } \\
2\end{array}$} & \multirow{2}{*}{$\begin{array}{r}\text { Total } \\
67\end{array}$} & & & & & \\
\hline & & & & & $0.4 \%$ & $0.20[0.01,4.15]$ & & & \\
\hline DART 1989 & 136 & 1018 & 147 & 1015 & $18.1 \%$ & $0.92[0.74,1.15]$ & & & \\
\hline Houtsmuller 1979 & 8 & 51 & 30 & 51 & $6.1 \%$ & $0.27[0.14,0.52]$ & $\longrightarrow$ & & \\
\hline Ley 2004 & 11 & 88 & 16 & 88 & $5.7 \%$ & $0.69[0.34,1.40]$ & $\rightarrow$ & & \\
\hline MRC 1968 & 62 & 199 & 74 & 194 & $16.0 \%$ & $0.82[0.62,1.07]$ & & & \\
\hline Moy 2001 & 5 & 117 & 3 & 118 & $1.8 \%$ & $1.68[0.41,6.87]$ & - & & \\
\hline Oslo Diet-Heart 1966 & 64 & 206 & 90 & 206 & $16.7 \%$ & $0.71[0.55,0.92]$ & $\Rightarrow$ & & \\
\hline Rose corn oil 1965 & 15 & 28 & 6 & 13 & $6.1 \%$ & $1.16[0.59,2.29]$ & - & & \\
\hline Rose olive 1965 & 11 & 26 & 5 & 13 & $4.6 \%$ & $1.10[0.48,2.50]$ & - & - & \\
\hline STARS 1992 & 8 & 27 & 20 & 28 & $6.9 \%$ & $0.41[0.22,0.78]$ & -- & & \\
\hline Veterans Admin 1969 & 97 & 424 & 122 & 422 & $17.6 \%$ & $0.79[0.63,1.00]$ & $=$ & & \\
\hline Total $(95 \%$ CI) & & 2250 & & 2215 & $100.0 \%$ & $0.75[0.61,0.91]$ & $\gamma$ & & \\
\hline Total events: & 417 & & 515 & & & & & & \\
\hline Heterogeneity: $\mathrm{Tau}^{2}=$ & ; $\mathrm{Chi}^{2}=20$ & $51, \mathrm{df}=10$ & $0(\mathrm{P}=0.02)$ & $\mathrm{I}^{2}=51 \%$ & & 0.005 & 0.1 & 10 & 200 \\
\hline Test for overall effect: & $2.93(\mathrm{P}=0$ & 003) & & & & Favours & ower SFA & Favours $\mathrm{h}$ & her SFA \\
\hline
\end{tabular}


Analysis 1.41. Comparison 1: SFA reduction vs usual diet - primary outcomes, Outcome 41: CVD events, SA Mantel-Haenszel fixed-effect

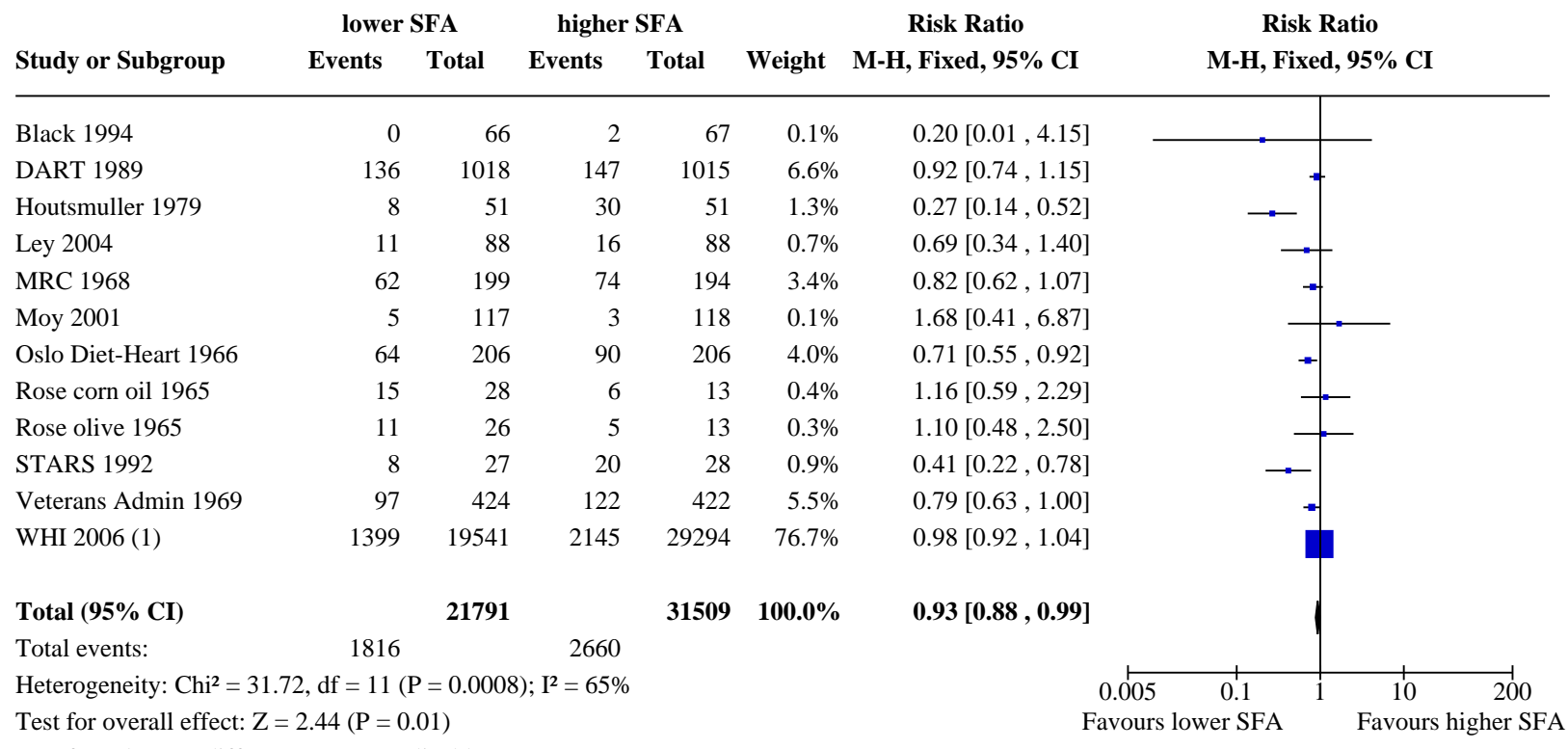

Footnotes

(1) Total CVD during study period, Prentice 2017

Analysis 1.42. Comparison 1: SFA reduction vs usual diet primary outcomes, Outcome 42: CVD events, SA Peto fixed-effect

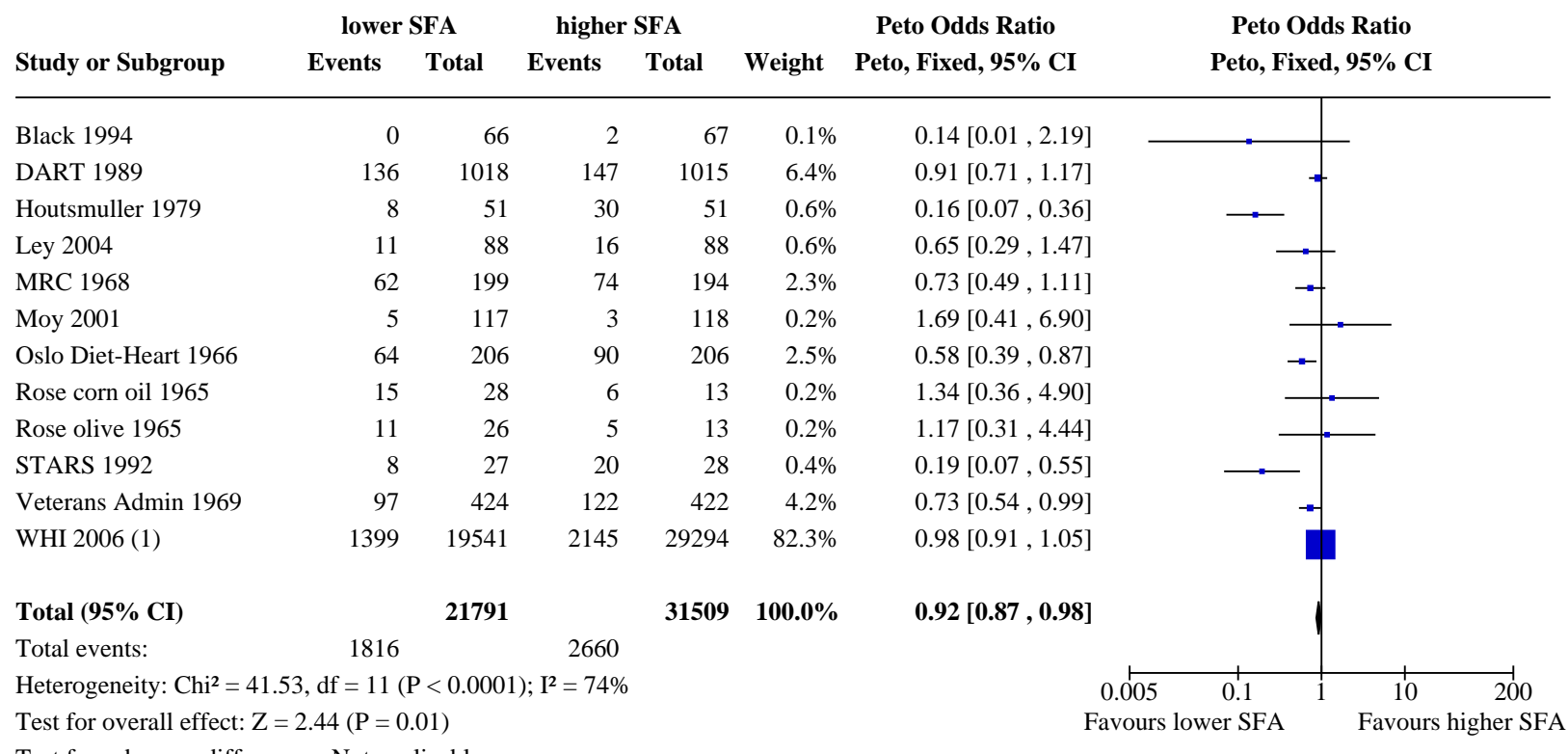

Footnotes

(1) Total CVD during study period, Prentice 2017 
Analysis 1.43. Comparison 1: SFA reduction vs usual diet - primary outcomes, Outcome 43: CVD events, SA excluding trials with additional interventions

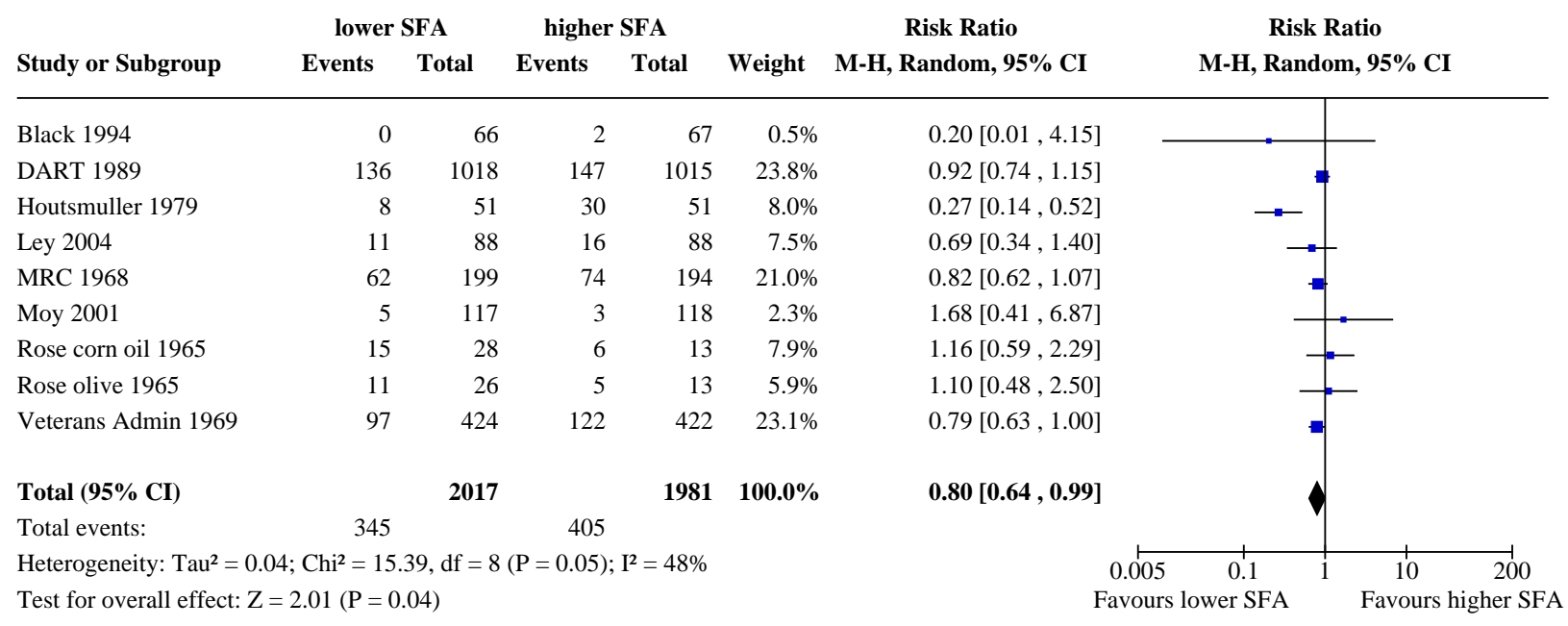




\section{Analysis 1.44. Comparison 1: SFA reduction vs usual diet - primary outcomes, Outcome 44: CVD events, subgroup by any substitution}

\begin{tabular}{|c|c|c|c|c|c|c|c|}
\hline \multirow[b]{2}{*}{ p } & \multicolumn{2}{|c|}{ lower SFA } & \multicolumn{2}{|c|}{ higher SFA } & \multicolumn{2}{|r|}{ Risk Ratio } & Risk Ratio \\
\hline & Events & Total & Events & Total & Weight & M-H, Random, 95\% CI & M-H, Random, 95\% C \\
\hline
\end{tabular}

1.44.1 replaced by PUFA

DART 1989

Houtsmuller 1979

MRC 1968

Oslo Diet-Heart 1966

Rose corn oil 1965

STARS 1992

Veterans Admin 1969

Subtotal (95\% CI)

Total events:

$\begin{array}{rr}136 & 1018 \\ 8 & 51 \\ 62 & 199 \\ 64 & 206 \\ 15 & 28 \\ 8 & 27 \\ 97 & 424 \\ & \mathbf{1 9 5 3}\end{array}$

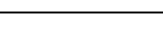

$\begin{array}{rr} & \\ 1015 & 19.5 \% \\ 51 & 7.7 \% \\ 194 & 17.6 \% \\ 206 & 18.2 \% \\ 26 & 9.6 \% \\ 28 & 8.5 \% \\ 422 & 19.0 \% \\ \mathbf{1 9 4 2} & \mathbf{1 0 0 . 0 \%}\end{array}$

390

Heterogeneity: $\mathrm{Tau}^{2}=0.06 ; \mathrm{Chi}^{2}=19.30, \mathrm{df}=6(\mathrm{P}=0.004) ; \mathrm{I}^{2}=69 \%$

Test for overall effect: $\mathrm{Z}=2.68(\mathrm{P}=0.007)$

\subsection{2 replaced by MUFA}

Rose olive 1965

Subtotal (95\% CI)

11

26
26

11

26

$100.0 \%$

Total events:

11

11

Heterogeneity: Not applicable

Test for overall effect: $\mathrm{Z}=0.00(\mathrm{P}=1.00)$

\subsection{3 replace by $\mathrm{CHO}$}

$\begin{array}{lrrrrr}\text { Black 1994 } & 0 & 66 & 2 & 67 & 0.6 \% \\ \text { DART 1989 } & 136 & 1018 & 147 & 1015 & 33.8 \% \\ \text { Ley 2004 } & 11 & 88 & 16 & 88 & 8.6 \% \\ \text { STARS 1992 } & 8 & 27 & 20 & 28 & 10.5 \% \\ \text { WHI 2006 (1) } & 1399 & 19541 & 2145 & 29294 & 46.5 \% \\ \text { Subtotal (95\% CI) } & & \mathbf{2 0 7 4 0} & & \mathbf{3 0 4 9 2} & \mathbf{1 0 0 . 0 \%} \\ \text { Total events: } & 1554 & & 2330 & & \end{array}$

Heterogeneity: $\mathrm{Tau}^{2}=0.03 ; \mathrm{Chi}^{2}=9.20, \mathrm{df}=4(\mathrm{P}=0.06) ; \mathrm{I}^{2}=57 \%$

Test for overall effect: $\mathrm{Z}=1.46(\mathrm{P}=0.14)$

1.44.4 replaced by protein

Black 1994

DART 1989

Ley 2004

WHI 2006 (1)

Subtotal $(95 \%$ CI)

Total events:

$\begin{array}{rr}0 & 66 \\ 136 & 1018 \\ 11 & 88 \\ 1399 & 19541 \\ & \mathbf{2 0 7 1 3}\end{array}$

$\begin{array}{rrr}2 & 67 & 0.0 \% \\ 147 & 1015 & 8.2 \% \\ 16 & 88 & 0.8 \% \\ 2145 & 29294 & 91.0 \% \\ & \mathbf{3 0 4 6 4} & \mathbf{1 0 0 . 0 \%}\end{array}$

$1546 \quad 2310$

Heterogeneity: $\mathrm{Tau}^{2}=0.00 ; \mathrm{Chi}^{2}=2.20, \mathrm{df}=3(\mathrm{P}=0.53) ; \mathrm{I}^{2}=0 \%$

Test for overall effect: $\mathrm{Z}=0.97(\mathrm{P}=0.33)$

1.44.5 replacement unclear

$\begin{array}{llllll}\text { Moy } 2001 & 5 & 117 & 3 & 118 & 100.0 \% \\ \text { Subtotal (95\% CI) } & & \mathbf{1 1 7} & & \mathbf{1 1 8} & \mathbf{1 0 0 . 0 \%} \\ \text { Total events: } & 5 & & 3 & & \end{array}$

Heterogeneity: Not applicable

Test for overall effect: $Z=0.72(P=0.47)$

Test for subgroup differences: $\mathrm{Chi}^{2}=7.10, \mathrm{df}=4(\mathrm{P}=0.13), \mathrm{I}^{2}=43.7 \%$

Footnotes

(1) Total CVD during study period, Prentice 2017
$0.92[0.74,1.15]$

$0.27[0.14,0.52]$

$0.82[0.62,1.07]$

$0.71[0.55,0.92]$

1.27 [0.72, 2.23]

$0.41[0.22,0.78]$

$0.79[0.63,1.00]$

0.73 [0.58, 0.92$]$

$1.00[0.53,1.89]$

$1.00[0.53,1.89]$

$0.20[0.01,4.15]$

$0.92[0.74,1.15]$

$0.69[0.34,1.40]$

$0.41[0.22,0.78]$

$0.98[0.92,1.04]$

0.84 [0.67, 1.06] 
Analysis 1.45. Comparison 1: SFA reduction vs usual diet - primary outcomes, Outcome 45: CVD events, subgroup by main substitution

\begin{tabular}{lrrrrr} 
& \multicolumn{2}{c}{ lower SFA } & \multicolumn{2}{c}{ higher SFA } \\
Study or Subgroup & Events & Total & Events & Total & Weight \\
\hline 1.45.1 replaced by PUFA & & & & & \\
DART 1989 & 136 & 1018 & 147 & 1015 & $21.8 \%$ \\
Houtsmuller 1979 & 8 & 51 & 30 & 51 & $7.7 \%$ \\
MRC 1968 & 62 & 199 & 74 & 194 & $19.4 \%$ \\
Oslo Diet-Heart 1966 & 64 & 206 & 90 & 206 & $20.1 \%$ \\
Rose corn oil 1965 & 15 & 28 & 11 & 26 & $9.8 \%$ \\
Veterans Admin 1969 & 97 & 424 & 122 & 422 & $21.2 \%$ \\
Subtotal (95\% CI) & & $\mathbf{1 9 2 6}$ & & $\mathbf{1 9 1 4}$ & $\mathbf{1 0 0 . 0 \%}$ \\
Total events: & 382 & & 474 & & \\
\end{tabular}

Heterogeneity: $\mathrm{Tau}^{2}=0.05 ; \mathrm{Chi}^{2}=15.17, \mathrm{df}=5(\mathrm{P}=0.010) ; \mathrm{I}^{2}=67 \%$

Test for overall effect: $\mathrm{Z}=2.25(\mathrm{P}=0.02)$

\subsection{2 replaced by MUFA}

Rose olive 1965 11

$$
26
$$

26

$100.0 \%$

Subtotal $(95 \%$ CI $)$

11

$$
26
$$

26

$100.0 \%$

11

Heterogeneity: Not applicable

Test for overall effect: $\mathrm{Z}=0.00(\mathrm{P}=1.00)$

\subsection{3 replace by $\mathrm{CHO}$}

Black 1994

Ley 2004

STARS 1992

WHI 2006 (1)

Subtotal (95\% CI)

Total events:

Heterogeneity: $\mathrm{Tau}^{2}=0.17 ; \mathrm{Chi}^{2}=9.04, \mathrm{df}=3(\mathrm{P}=0.03) ; \mathrm{I}^{2}=67 \%$

Test for overall effect: $\mathrm{Z}=1.44(\mathrm{P}=0.15)$

1.45.4 replaced by protein Subtotal (95\% CI)

0

0

0

Total events:

0

Heterogeneity: Not applicable

Test for overall effect: Not applicable

\subsection{5 replacement unclear}

Moy 2001

\section{Subtotal $(95 \%$ CI $)$}

Total events:

5

117

117

5

Heterogeneity: Not applicable

Test for overall effect: $\mathrm{Z}=0.72(\mathrm{P}=0.47)$

Test for subgroup differences: $\mathrm{Chi}^{2}=2.00, \mathrm{df}=3(\mathrm{P}=0.57), \mathrm{I}^{2}=0 \%$

\section{Footnotes}

(1) Total CVD during study period, Prentice 2017
Risk Ratio

Risk Ratio

M-H, Random, $95 \%$ CI
$1.00[0.53,1.89]$

$1.00[0.53,1.89]$
$0.20[0.01,4.15]$

$0.69[0.34,1.40]$

$0.41[0.22,0.78]$

$0.98[0.92,1.04]$

$0.67[0.39,1.16]$

Not estimable

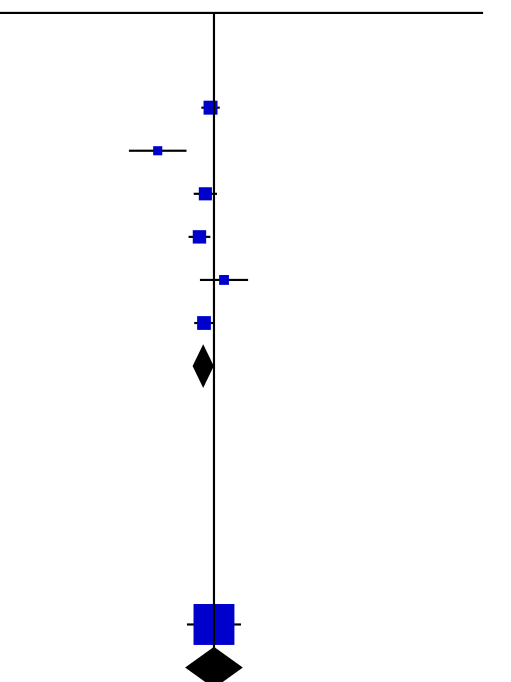


Analysis 1.46. Comparison 1: SFA reduction vs usual diet primary outcomes, Outcome 46: CVD events, subgroup by duration

\section{lower SFA}

higher SFA

Events Total Events Total Weight M-H, Random, 95\% CI
Risk Ratio

M-H, Random, 95\% CI

\subsection{1 up to $24 \mathrm{mo}$}

Black 1994

DART 1989

Moy 2001

Rose corn oil 1965

Rose olive 1965

Subtotal (95\% CI)

Total events:

$\begin{array}{rrrrr}0 & 66 & 2 & 67 & 0.3 \% \\ 136 & 1018 & 147 & 1015 & 14.9 \% \\ 5 & 117 & 3 & 118 & 1.4 \% \\ 15 & 28 & 6 & 13 & 4.8 \% \\ 11 & 26 & 5 & 13 & 3.6 \% \\ & \mathbf{1 2 5 5} & & \mathbf{1 2 2 6} & \mathbf{2 5 . 0 \%}\end{array}$

167

163

Test for overall effect: $\mathrm{Z}=0.45(\mathrm{P}=0.65)$

\subsection{2 >24 to $48 \mathrm{mo}$}

MRC 1968

STARS 1992

Veterans Admin 1969

Subtotal (95\% CI)

Total events:

$\begin{array}{rr}62 & 199 \\ 8 & 27 \\ 97 & 424 \\ & \mathbf{6 5 0}\end{array}$

74

$13.1 \%$

$20 \quad 28 \quad 5.4 \%$

$422 \quad 14.5 \%$

$64433.0 \%$

216

Heterogeneity: $\mathrm{Tau}^{2}=0.03 ; \mathrm{Chi}^{2}=3.97, \mathrm{df}=2(\mathrm{P}=0.14) ; \mathrm{I}^{2}=50 \%$

Test for overall effect: $\mathrm{Z}=2.31(\mathrm{P}=0.02)$

\subsection{3 >48mo}

Oslo Diet-Heart 1966

WHI 2006 (1)

Subtotal $(95 \% \mathrm{CI})$

Total events:

$\begin{array}{rr}64 & 206 \\ 1399 & 19541 \\ & \mathbf{1 9 7 4 7}\end{array}$

1463
90

2145

206

29294

29500 2235
$13.6 \%$

$19.0 \%$

$32.6 \%$

Heterogeneity: $\mathrm{Tau}^{2}=0.04 ; \mathrm{Chi}^{2}=5.62, \mathrm{df}=1(\mathrm{P}=0.02) ; \mathrm{I}^{2}=82 \%$

Test for overall effect: $\mathrm{Z}=0.99(\mathrm{P}=0.32)$

\subsection{4 unclear duration}

Houtsmuller 1979

Ley 2004

Subtotal (95\% CI)

$\begin{array}{rrrrr}8 & 51 & 30 & 51 & 4.9 \% \\ 11 & 88 & 16 & 88 & 4.5 \% \\ & \mathbf{1 3 9} & & \mathbf{1 3 9} & \mathbf{9 . 4 \%}\end{array}$

Total events:

19

46

Heterogeneity: $\mathrm{Tau}^{2}=0.32 ; \mathrm{Chi}^{2}=3.59, \mathrm{df}=1(\mathrm{P}=0.06) ; \mathrm{I}^{2}=72 \%$

Test for overall effect: $\mathrm{Z}=1.80(\mathrm{P}=0.07)$

Total $(95 \%$ CI)

21791

$31509 \quad 100.0 \%$

Total events:

1816

2660

Heterogeneity: $\mathrm{Tau}^{2}=0.04 ; \mathrm{Chi}^{2}=31.72, \mathrm{df}=11(\mathrm{P}=0.0008) ; \mathrm{I}^{2}=65 \%$

Test for overall effect: $\mathrm{Z}=2.72(\mathrm{P}=0.006)$

Test for subgroup differences: $\mathrm{Chi}^{2}=4.71, \mathrm{df}=3(\mathrm{P}=0.19), \mathrm{I}^{2}=36.3 \%$
$0.20[0.01,4.15]$

$0.92[0.74,1.15]$

$1.68[0.41,6.87]$

$1.16[0.59,2.29]$

$1.10[0.48,2.50]$

$0.96[0.78,1.16]$

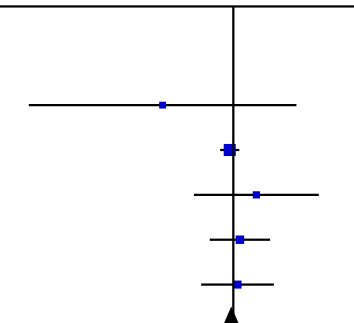

$0.82[0.62,1.07]$

$0.41[0.22,0.78]$

$0.79[0.63,1.00]$

$0.73[0.56,0.95]$

$0.71[0.55,0.92]$

$0.98[0.92,1.04]$

$0.85[0.63,1.16]$

$0.27[0.14,0.52]$

$0.69[0.34,1.40]$

$0.43[0.17,1.08]$

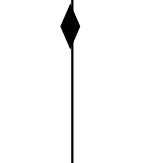

\section{Footnotes}

(1) Total CVD during study period, Prentice 2017 


\section{Analysis 1.47. Comparison 1: SFA reduction vs usual diet - primary outcomes, Outcome 47: CVD events, subgroup by baseline SFA}

\begin{tabular}{|c|c|c|c|c|c|c|c|}
\hline & lowe & SFA & hight & SFA & & Risk Ratio & Risk Ratio \\
\hline Study or Subgroup & Events & Total & Events & Total & Weight & M-H, Random, $95 \%$ CI & M-H, Random, $95 \%$ CI \\
\hline
\end{tabular}

1.47.1 up to $12 \%$ E SFA baseline

Subtotal $(95 \%$ CI)

Total events:

0

Heterogeneity: Not applicable

Test for overall effect: Not applicable

1.47.2 >12 to $15 \%$ E SFA baseline

Black 1994

DART 1989

Ley 2004

Moy 2001

WHI 2006 (1)

Subtotal (95\% CI)

0
136
11
5

66
1018
88
117
19541

2

$67 \quad 0.3 \%$

$1015 \quad 14.9 \%$

$88 \quad 4.5 \%$

$118 \quad 1.4 \%$

$1399 \quad 19541 \quad 2145 \quad 29294 \quad 19.0 \%$

$20830 \quad 30582 \quad 40.1 \%$

Total events:

1551

2313

Heterogeneity: $\mathrm{Tau}^{2}=0.00 ; \mathrm{Chi}^{2}=2.79, \mathrm{df}=4(\mathrm{P}=0.59) ; \mathrm{I}^{2}=0 \%$

Test for overall effect: $\mathrm{Z}=0.94(\mathrm{P}=0.35)$

\subsection{3 $>15$ to $18 \%$ E SFA baseline}

\section{STARS 1992}

Subtotal $(95 \%$ CI)

\section{8}

Total events:

8

$$
27
$$

27

8

Heterogeneity: Not applicable

Test for overall effect: $\mathrm{Z}=2.75(\mathrm{P}=0.006)$

\subsection{4 >18\% E SFA baseline}

Veterans Admin 1969

Subtotal (95\% CI)

97

$$
\begin{aligned}
& 424 \\
& 424
\end{aligned}
$$

97

Heterogeneity: Not applicable

Test for overall effect: $Z=1.99(P=0.05)$

\subsection{5 unclear}

Houtsmuller 1979

MRC 1968

Oslo Diet-Heart 1966

Rose corn oil 1965

Rose olive 1965

Subtotal (95\% CI)

Total events:

$\begin{array}{rr}8 & 5 \\ 62 & 199 \\ 64 & 206 \\ 15 & 28 \\ 11 & 26 \\ & \mathbf{5 1 0}\end{array}$

160

Heterogeneity: $\mathrm{Tau}^{2}=0.09 ; \mathrm{Chi}^{2}=12.08, \mathrm{df}=4(\mathrm{P}=0.02) ; \mathrm{I}^{2}=67 \%$

Test for overall effect: $\mathrm{Z}=1.80(\mathrm{P}=0.07)$

\section{Total $(95 \%$ CI $)$}

$31509100.0 \%$

Total events:

1816

2660

Heterogeneity: $\mathrm{Tau}^{2}=0.04 ; \mathrm{Chi}^{2}=31.72, \mathrm{df}=11(\mathrm{P}=0.0008) ; \mathrm{I}^{2}=65 \%$

Test for overall effect: $\mathrm{Z}=2.72(\mathrm{P}=0.006)$

Test for subgroup differences: $\mathrm{Chi}^{2}=11.84, \mathrm{df}=3(\mathrm{P}=0.008), \mathrm{I}^{2}=74.7 \%$
Not estimable

$0.20[0.01,4.15]$

$0.92[0.74,1.15]$

$0.69[0.34,1.40]$

$1.68[0.41,6.87]$

$0.98[0.92,1.04]$

$0.97[0.91,1.03]$

$0.41[0.22,0.78]$

$0.41[0.22,0.78]$

$\mathbf{5 . 4 \%}$

$0.79[0.63,1.00]$

$0.79[0.63,1.00]$
$0.27[0.14,0.52]$

$0.82[0.62,1.07]$

$0.71[0.55,0.92]$

$1.16[0.59,2.29]$

$1.10[0.48,2.50]$

$0.72[0.51,1.03]$

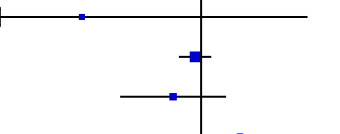

Footnotes

(1) Total CVD during study period, Prentice 2017

Reduction in saturated fat intake for cardiovascular disease (Review) 


\section{Analysis 1.48. Comparison 1: SFA reduction vs usual diet - primary outcomes, Outcome 48: CVD events, subgroup by SFA change}

lower SFA

higher SFA

Events Total Events Total
Risk Ratio

Weight M-H, Random, 95\% CI
Risk Ratio

M-H, Random, $95 \%$ CI

1.48.1 up to $4 \% \mathrm{E}$ difference

DART 1989

Ley 2004

Moy 2001

$\begin{array}{rrrrr}136 & 1018 & 147 & 1015 & 14.9 \% \\ 11 & 88 & 16 & 88 & 4.5 \% \\ 5 & 117 & 3 & 118 & 1.4 \% \\ 1399 & 19541 & 2145 & 29294 & 19.0 \% \\ & \mathbf{2 0 7 6 4} & & \mathbf{3 0 5 1 5} & \mathbf{3 9 . 8 \%} \\ 1551 & & 2311 & & \end{array}$

$0.92[0.74,1.15]$

$0.69[0.34,1.40]$

$1.68[0.41,6.87]$

WHI 2006 (1)

Subtotal $(95 \%$ CI $)$

1551

2311

$0.98[0.92,1.04]$

$0.97[0.91,1.03]$

Total events:

Heterogeneity: $\mathrm{Tau}^{2}=0.00 ; \mathrm{Chi}^{2}=1.76, \mathrm{df}=3(\mathrm{P}=0.62) ; \mathrm{I}^{2}=0 \%$

Test for overall effect: $\mathrm{Z}=0.92(\mathrm{P}=0.36)$

\subsection{2 $>4$ to $8 \% \mathrm{E}$ difference}

Black 1994

STARS 1992

Subtotal (95\% CI)

Total events:

$0 \quad 66$

\section{6}

27

93

Heterogeneity: $\mathrm{Tau}^{2}=0.00 ; \mathrm{Chi}^{2}=0.22, \mathrm{df}=1(\mathrm{P}=0.64) ; \mathrm{I}^{2}=0 \%$

Test for overall effect: $\mathrm{Z}=2.90(\mathrm{P}=0.004)$

\subsection{3 >8\% $\mathrm{E}$ difference}

Veterans Admin 1969

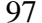

424
$\mathbf{4 2 4}$

97

424
Total events:

Heterogeneity: Not applicable

Test for overall effect: $\mathrm{Z}=1.99(\mathrm{P}=0.05)$

\subsection{4 unclear}

Houtsmuller 1979

MRC 1968

Oslo Diet-Heart 1966

Rose corn oil 1965

Rose olive 1965

Subtotal $(95 \%$ CI $)$

Total events:

Heterogeneity: $\mathrm{Tau}^{2}=0.09 ; \mathrm{Chi}^{2}=12.08, \mathrm{df}=4(\mathrm{P}=0.02) ; \mathrm{I}^{2}=67 \%$

Test for overall effect: $\mathrm{Z}=1.80(\mathrm{P}=0.07)$

Total $(95 \%$ CI $)$

21791

$31509 \quad 100.0 \%$

Total events:

1816

2660

Heterogeneity: $\mathrm{Tau}^{2}=0.04 ; \mathrm{Chi}^{2}=31.72, \mathrm{df}=11(\mathrm{P}=0.0008) ; \mathrm{I}^{2}=65 \%$

Test for overall effect: $\mathrm{Z}=2.72(\mathrm{P}=0.006)$

Test for subgroup differences: $\mathrm{Chi}^{2}=12.67, \mathrm{df}=3(\mathrm{P}=0.005), \mathrm{I}^{2}=76.3 \%$
$0.20[0.01,4.15]$

$0.41[0.22,0.78]$

$0.40[0.22,0.74]$
$0.79[0.63,1.00]$

$0.79[0.63,1.00]$

\section{Footnotes}

(1) Total CVD during study period, Prentice 2017

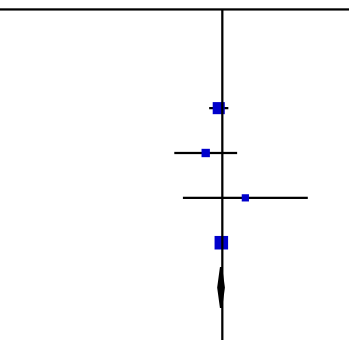


Analysis 1.49. Comparison 1: SFA reduction vs usual diet primary outcomes, Outcome 49: CVD events, subgroup by sex

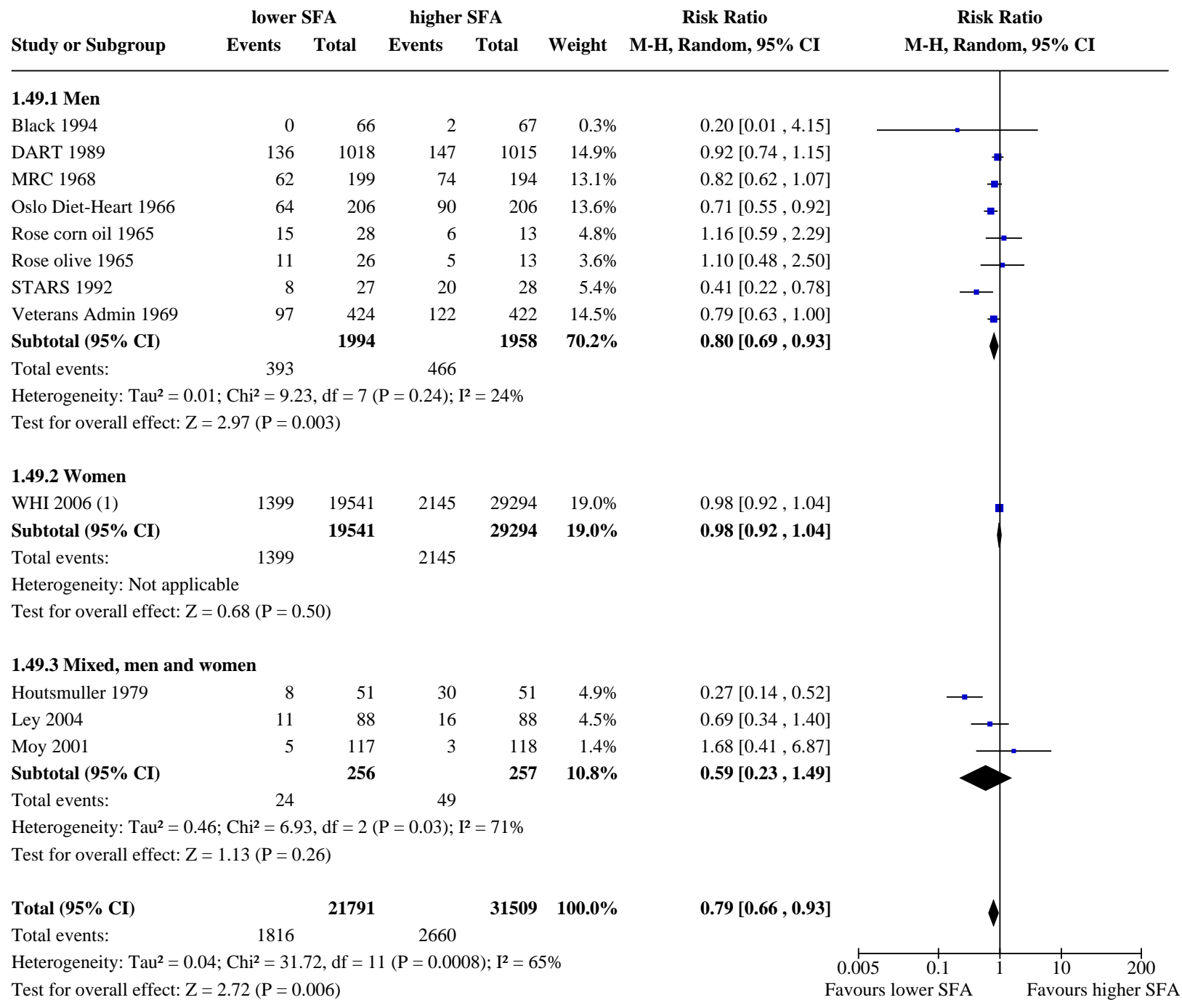

(1) Total CVD during study period, Prentice 2017 


\section{Analysis 1.50. Comparison 1: SFA reduction vs usual diet - primary outcomes, Outcome 50: CVD events, subgroup by CVD risk}

\begin{tabular}{lrrrrr} 
& \multicolumn{2}{c}{ lower SFA } & \multicolumn{2}{c}{ higher SFA } \\
Study or Subgroup & Events & Total & Events & Total & Weight \\
\hline 1.50.1 Low CVD risk & & & & & \\
Black 1994 & 0 & 66 & 2 & 67 & $0.2 \%$ \\
Veterans Admin 1969 & 97 & 424 & 122 & 422 & $12.5 \%$ \\
WHI 2006 (1) & 1132 & 18633 & 1777 & 27925 & $17.4 \%$ \\
Subtotal (95\% CI) & & $\mathbf{1 9 1 2 3}$ & & $\mathbf{2 8 4 1 4}$ & $\mathbf{3 0 . 2 \%}$ \\
Total events: & 1229 & & 1901 & &
\end{tabular}

Risk Ratio

Risk Ratio

M-H, Random, $95 \%$ CI

M-H, Random, $95 \%$ CI

Heterogeneity: $\mathrm{Tau}^{2}=0.01 ; \mathrm{Chi}^{2}=3.32, \mathrm{df}=2(\mathrm{P}=0.19) ; \mathrm{I}^{2}=40 \%$

Test for overall effect: $\mathrm{Z}=1.28(\mathrm{P}=0.20)$

1.50.2 Moderate CVD risk

Houtsmuller 1979

Ley 2004

Moy 2001

Subtotal (95\% CI)

$8 \quad 51$

$11 \quad 88$

$5 \quad 117$

Total events:

24

\section{0}

16

3

49

49

Heterogeneity: $\mathrm{Tau}^{2}=0.46 ; \mathrm{Chi}^{2}=6.93, \mathrm{df}=2(\mathrm{P}=0.03) ; \mathrm{I}^{2}=71 \%$

Test for overall effect: $\mathrm{Z}=1.13(\mathrm{P}=0.26)$

\subsubsection{Existing CVD disease}

DART 1989

MRC 1968

Oslo Diet-Heart 1966

Rose corn oil 1965

Rose olive 1965

STARS 1992

WHI 2006 (2)

Subtotal (95\% CI)

Total events:

$136 \quad 1018$

$\begin{array}{rr}136 & 1018 \\ 62 & 199\end{array}$

147
74

1015

194

206

13

13
13

$13 \quad 2.7 \%$

$11 \quad 26$

$8 \quad 27$

$225 \quad 908$

2412

521

653

terogeneity: $\mathrm{Tau}^{2}=0.04 ; \mathrm{Chi}^{2}=16.43, \mathrm{df}=6(\mathrm{P}=0.01) ; \mathrm{I}^{2}=63 \%$

Test for overall effect: $\mathrm{Z}=1.45(\mathrm{P}=0.15)$

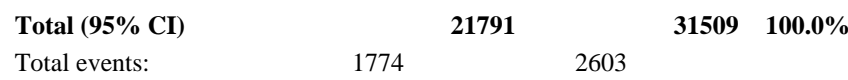

Heterogeneity: $\mathrm{Tau}^{2}=0.03 ; \mathrm{Chi}^{2}=34.25, \mathrm{df}=12(\mathrm{P}=0.0006) ; \mathrm{I}^{2}=65 \%$

Test for overall effect: $\mathrm{Z}=2.50(\mathrm{P}=0.01)$

Test for subgroup differences: $\mathrm{Chi}^{2}=0.79, \mathrm{df}=2(\mathrm{P}=0.67), \mathrm{I}^{2}=0 \%$

Footnotes

(1) Women without CVD at baseline

(2) Women with CVD at baseline
$0.20[0.01,4.15]$

$0.79[0.63,1.00]$

$0.95[0.89,1.03]$

$0.89[0.75,1.06]$

$0.27[0.14,0.52]$

$0.69[0.34,1.40]$

$1.68[0.41,6.87]$

0.59 [0.23, 1.49]

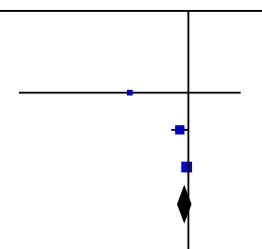

$0.92[0.74,1.15]$

$0.82[0.62,1.07]$

$0.71[0.55,0.92]$

$1.16[0.59,2.29]$

$1.10[0.48,2.50]$

$0.41[0.22,0.78]$

$1.09[0.94,1.27]$

$0.86[0.71,1.05]$

$0.83[0.72,0.96]$

Favours lower SFA
$10 \quad 200$ Favours higher SFA 


\section{Analysis 1.51. Comparison 1: SFA reduction vs usual diet - primary outcomes, Outcome 51: CVD events, subgroup by TC reduction}

\begin{tabular}{|c|c|c|c|c|c|c|c|}
\hline & lowe & & highe & SFA & & Risk Ratio & Risk Ratio \\
\hline t & Events & Total & Events & Total & Weigh & M-H, Random, 95\% CI & M-H, Random, 95\% \\
\hline
\end{tabular}

1.51.1 serum chol reduced by at least $0.2 \mathrm{mmol} / \mathrm{L}$

$\begin{array}{lrr}\text { DART 1989 } & 136 & 1018 \\ \text { Houtsmuller 1979 } & 8 & 51 \\ \text { MRC 1968 } & 62 & 199 \\ \text { Moy 2001 } & 5 & 117 \\ \text { Oslo Diet-Heart 1966 } & 64 & 206 \\ \text { Rose corn oil 1965 } & 15 & 28 \\ \text { STARS 1992 } & 8 & 27 \\ \text { Veterans Admin 1969 } & 97 & 424 \\ \text { Subtotal (95\% CI) } & & \mathbf{2 0 7 0}\end{array}$

$147 \quad 1015 \quad 19.7 \%$

$30 \quad 51 \quad 7.4 \%$

$74 \quad 194 \quad 17.7 \%$

$3 \quad 118 \quad 2.2 \%$

$90 \quad 206 \quad 18.3 \%$

$6 \quad 13 \quad 7.3 \%$

$20 \quad 28 \quad 8.2 \%$

$122 \quad 422 \quad 19.2 \%$

$2047 \quad 100.0 \%$

Total events:

395

492

Heterogeneity: $\mathrm{Tau}^{2}=0.05 ; \mathrm{Chi}^{2}=18.95, \mathrm{df}=7(\mathrm{P}=0.008) ; \mathrm{I}^{2}=63 \%$

Test for overall effect: $\mathrm{Z}=2.73(\mathrm{P}=0.006)$

1.51.2 serum chol reduced by $<0.2 \mathrm{mmol} / \mathrm{L}$

$\begin{array}{lrrrrr}\text { Ley 2004 } & 11 & 88 & 16 & 88 & 0.8 \% \\ \text { Rose olive 1965 } & 11 & 26 & 5 & 13 & 0.6 \% \\ \text { WHI 2006 (1) } & 1399 & 19541 & 2145 & 29294 & 98.6 \% \\ \text { Subtotal (95\% CI) } & & \mathbf{1 9 6 5 5} & & \mathbf{2 9 3 9 5} & \mathbf{1 0 0 . 0 \%} \\ \text { Total events: } & 1421 & & 2166 & & \end{array}$

Heterogeneity: $\mathrm{Tau}^{2}=0.00 ; \mathrm{Chi}^{2}=1.02, \mathrm{df}=2(\mathrm{P}=0.60) ; \mathrm{I}^{2}=0 \%$

Test for overall effect: $\mathrm{Z}=0.75(\mathrm{P}=0.45)$

1.51.3 serum chol reduction unclear

Black 1994

Subtotal (95\% CI)

$0 \quad 66$

\section{6}

2

$67 \quad 100.0 \%$

Total events:

0

$67 \quad 100.0 \%$

Heterogeneity: Not applicable

Test for overall effect: $\mathrm{Z}=1.04(\mathrm{P}=0.30)$

Test for subgroup differences: $\mathrm{Chi}^{2}=6.80, \mathrm{df}=2(\mathrm{P}=0.03), \mathrm{I}^{2}=70.6 \%$

Footnotes

(1) Total CVD during study period, Prentice 2017
$0.92[0.74,1.15]$

$0.27[0.14,0.52]$

$0.82[0.62,1.07]$

$1.68[0.41,6.87]$

$0.71[0.55,0.92]$

$1.16[0.59,2.29]$

$0.41[0.22,0.78]$

$0.79[0.63,1.00]$

$0.74[0.59,0.92]$
$0.69[0.34,1.40]$

$1.10[0.48,2.50]$

$0.98[0.92,1.04]$

$0.98[0.91,1.04]$
$0.20[0.01,4.15]$

$0.20[0.01,4.15]$
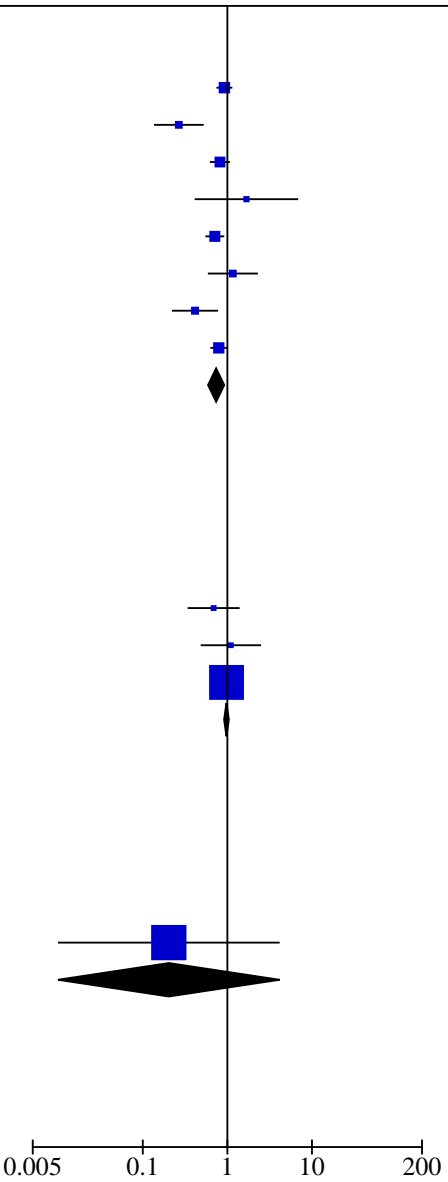

Favours lower SFA 


\section{Analysis 1.52. Comparison 1: SFA reduction vs usual diet - primary outcomes, Outcome 52: CVD events, subgroup decade of publication}

\begin{tabular}{|c|c|c|c|c|c|c|c|}
\hline \multirow[b]{2}{*}{ Study or Subgroup } & \multicolumn{2}{|c|}{ lower SFA } & \multicolumn{2}{|c|}{ higher SFA } & \multicolumn{2}{|r|}{ Risk Ratio } & Risk Ratio \\
\hline & Events & Total & Events & Total & Weight & M-H, Random, 95\% CI & M-H, Random, 95\% CI \\
\hline
\end{tabular}

\begin{tabular}{|c|c|c|c|}
\hline \multicolumn{4}{|l|}{ 1.52.1 1960s } \\
\hline MRC 1968 & 62 & 199 & 74 \\
\hline Oslo Diet-Heart 1966 & 64 & 206 & 90 \\
\hline Rose corn oil 1965 & 15 & 28 & 6 \\
\hline Rose olive 1965 & 11 & 26 & 5 \\
\hline Veterans Admin 1969 & 97 & 424 & 122 \\
\hline Subtotal $(95 \%$ CI $)$ & & 883 & \\
\hline Total events: & 249 & & 297 \\
\hline
\end{tabular}

\subsubsection{0s}

Houtsmuller 1979

Subtotal (95\% CI)

51

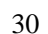

51

$4.9 \%$

$\mathbf{4 . 9 \%}$

30

Total events:

8

Heterogeneity: Not applicable

Test for overall effect: $\mathrm{Z}=3.83(\mathrm{P}=0.0001)$

\subsubsection{0s}

DART 1989

Subtotal (95\% CI)

136

1018

147

1015

$14.9 \%$

Total events:

136

1018

147

Heterogeneity: Not applicable

Test for overall effect: $\mathrm{Z}=0.73(\mathrm{P}=0.46)$

\subsubsection{0s}

Black 1994

STARS 1992

Subtotal $(95 \%$ CI)

Total events:

Heterogeneity: $\mathrm{Tau}^{2}=0.00 ; \mathrm{Chi}^{2}=0.22, \mathrm{df}=1(\mathrm{P}=0.64) ; \mathrm{I}^{2}=0 \%$

Test for overall effect: $\mathrm{Z}=2.90(\mathrm{P}=0.004)$

\subsubsection{0s}

Ley 2004

Moy 2001

WHI 2006 (1)

Subtotal $(95 \%$ CI $)$

827

2

67

$0.3 \%$

$5.4 \%$

Total events:

Heterogeneity: $\mathrm{Tau}^{2}=0.00 ; \mathrm{Chi}^{2}=1.51, \mathrm{df}=2(\mathrm{P}=0.47) ; \mathrm{I}^{2}=0 \%$

Test for overall effect: $\mathrm{Z}=0.74(\mathrm{P}=0.46)$

\section{Total $(95 \%$ CI $)$}

21791

$31509100.0 \%$

Total events:

1816 2660

Heterogeneity: $\mathrm{Tau}^{2}=0.04 ; \mathrm{Chi}^{2}=31.72, \mathrm{df}=11(\mathrm{P}=0.0008) ; \mathrm{I}^{2}=65 \%$

Test for overall effect: $\mathrm{Z}=2.72(\mathrm{P}=0.006)$

Test for subgroup differences: $\mathrm{Chi}^{2}=27.36, \mathrm{df}=4(\mathrm{P}<0.0001), \mathrm{I}^{2}=85.4 \%$

Footnotes

(1) Total CVD during study period, Prentice 2017
$0.82[0.62,1.07]$

$0.71[0.55,0.92]$

$1.16[0.59,2.29]$

$1.10[0.48,2.50]$

$0.79[0.63,1.00]$

$0.79[0.69,0.91]$

$0.27[0.14,0.52]$

$0.27[0.14,0.52]$

$0.92[0.74,1.15]$

$0.92[0.74,1.15]$

$0.20[0.01,4.15]$

$0.41[0.22,0.78]$

$0.40[0.22,0.74]$ 
Comparison 2. SFA reduction vs usual diet - secondary health events

\begin{tabular}{|c|c|c|c|c|}
\hline Outcome or subgroup title & $\begin{array}{l}\text { No. of } \\
\text { studies }\end{array}$ & $\begin{array}{l}\text { No. of } \\
\text { partici- } \\
\text { pants }\end{array}$ & Statistical method & Effect size \\
\hline 2.1 MYOCARDIAL INFARCTION & 11 & 53167 & $\begin{array}{l}\text { Risk Ratio (M-H, Random, 95\% } \\
\mathrm{Cl})\end{array}$ & $0.90[0.80,1.01]$ \\
\hline $2.2 \mathrm{MI}, \mathrm{SA}$ by low summary risk of bias & 3 & 49857 & $\begin{array}{l}\text { Risk Ratio (M-H, Random, 95\% } \\
\mathrm{Cl})\end{array}$ & $0.93[0.81,1.08]$ \\
\hline $2.3 \mathrm{MI}, \mathrm{SA}$ aim to reduce SFA & 10 & 52991 & $\begin{array}{l}\text { Risk Ratio (M-H, Random, 95\% } \\
\mathrm{Cl})\end{array}$ & $0.89[0.78,1.02]$ \\
\hline $\begin{array}{l}\text { 2.4 MI, SA statistically significant SFA re- } \\
\text { duction }\end{array}$ & 6 & 52180 & $\begin{array}{l}\text { Risk Ratio (M-H, Random, 95\% } \\
\mathrm{Cl})\end{array}$ & $0.94[0.85,1.04]$ \\
\hline 2.5 MI, SA by TC reduction & 9 & 52952 & $\begin{array}{l}\text { Risk Ratio (M-H, Random, 95\% } \\
\mathrm{Cl})\end{array}$ & $0.88[0.77,1.01]$ \\
\hline 2.6 MI, SA excluding WHI & 10 & 4332 & $\begin{array}{l}\text { Risk Ratio (M-H, Random, 95\% } \\
\mathrm{Cl})\end{array}$ & $0.85[0.73,0.98]$ \\
\hline 2.7 MI, SA Mantel-Haenszel fixed-effect & 11 & 53167 & Risk Ratio (M-H, Fixed, 95\% Cl) & $0.92[0.84,1.01]$ \\
\hline 2.8 MI, SA Peto fixed-effect & 11 & 53167 & $\begin{array}{l}\text { Peto Odds Ratio (Peto, Fixed, } \\
95 \% \mathrm{Cl} \text { ) }\end{array}$ & $0.92[0.83,1.01]$ \\
\hline 2.9 MI, subgroup by any substitution & 11 & & $\begin{array}{l}\text { Risk Ratio (M-H, Random, 95\% } \\
\mathrm{Cl})\end{array}$ & Subtotals only \\
\hline 2.9.1 replaced by PUFA & 7 & 3895 & $\begin{array}{l}\text { Risk Ratio (M-H, Random, 95\% } \\
\mathrm{Cl})\end{array}$ & $0.83[0.67,1.02]$ \\
\hline 2.9.2 replaced by MUFA & 1 & 52 & $\begin{array}{l}\text { Risk Ratio (M-H, Random, 95\% } \\
\mathrm{Cl})\end{array}$ & $1.40[0.51,3.85]$ \\
\hline 2.9 .3 replace by $\mathrm{CHO}$ & 4 & 51099 & $\begin{array}{l}\text { Risk Ratio (M-H, Random, 95\% } \\
\mathrm{Cl})\end{array}$ & $0.96[0.86,1.06]$ \\
\hline 2.9.4 replaced by protein & 3 & 51044 & $\begin{array}{l}\text { Risk Ratio (M-H, Random, 95\% } \\
\mathrm{Cl})\end{array}$ & $0.96[0.86,1.07]$ \\
\hline 2.9.5 replacement unclear & 1 & 235 & $\begin{array}{l}\text { Risk Ratio (M-H, Random, 95\% } \\
\mathrm{Cl})\end{array}$ & $2.02[0.19,21.94]$ \\
\hline 2.10 MI, subgroup by main substitution & 11 & & $\begin{array}{l}\text { Risk Ratio (M-H, Random, 95\% } \\
\mathrm{Cl})\end{array}$ & Subtotals only \\
\hline 2.10 .1 replaced by PUFA & 6 & 3840 & $\begin{array}{l}\text { Risk Ratio (M-H, Random, 95\% } \\
\mathrm{Cl})\end{array}$ & $0.83[0.67,1.04]$ \\
\hline 2.10 .2 replaced by MUFA & 1 & 52 & $\begin{array}{l}\text { Risk Ratio (M-H, Random, 95\% } \\
\mathrm{Cl})\end{array}$ & $1.40[0.51,3.85]$ \\
\hline 2.10 .3 replace by $\mathrm{CHO}$ & 3 & 49066 & $\begin{array}{l}\text { Risk Ratio (M-H, Random, 95\% } \\
\mathrm{Cl})\end{array}$ & $0.97[0.86,1.09]$ \\
\hline
\end{tabular}




\begin{tabular}{|c|c|c|c|c|}
\hline Outcome or subgroup title & $\begin{array}{l}\text { No. of } \\
\text { studies }\end{array}$ & $\begin{array}{l}\text { No. of } \\
\text { partici- } \\
\text { pants }\end{array}$ & Statistical method & Effect size \\
\hline 2.10.4 replaced by protein & 0 & 0 & $\begin{array}{l}\text { Risk Ratio (M-H, Random, 95\% } \\
\mathrm{Cl})\end{array}$ & Not estimable \\
\hline 2.10.5 replacement unclear & 1 & 235 & $\begin{array}{l}\text { Risk Ratio (M-H, Random, 95\% } \\
\mathrm{Cl} \text { ) }\end{array}$ & $2.02[0.19,21.94]$ \\
\hline 2.11 MI, subgroup by duration & 11 & 53167 & $\begin{array}{l}\text { Risk Ratio (M-H, Random, 95\% } \\
\mathrm{CI})\end{array}$ & $0.90[0.80,1.01]$ \\
\hline 2.11 .1 up to $24 \mathrm{mo}$ & 4 & 2348 & $\begin{array}{l}\text { Risk Ratio (M-H, Random, 95\% } \\
\mathrm{CI})\end{array}$ & $0.95[0.77,1.17]$ \\
\hline $2.11 .2>24$ to $48 \mathrm{mo}$ & 3 & 1294 & $\begin{array}{l}\text { Risk Ratio (M-H, Random, 95\% } \\
\mathrm{Cl})\end{array}$ & $0.83[0.64,1.06]$ \\
\hline $2.11 .3>48 \mathrm{mo}$ & 2 & 49247 & $\begin{array}{l}\text { Risk Ratio (M-H, Random, 95\% } \\
\mathrm{Cl})\end{array}$ & $0.81[0.54,1.24]$ \\
\hline 2.11.4 unclear & 2 & 278 & $\begin{array}{l}\text { Risk Ratio (M-H, Random, 95\% } \\
\mathrm{Cl})\end{array}$ & $0.41[0.02,7.73]$ \\
\hline 2.12 MI, subgroup by baseline SFA & 11 & 53167 & $\begin{array}{l}\text { Risk Ratio (M-H, Random, 95\% } \\
\mathrm{Cl})\end{array}$ & $0.90[0.80,1.01]$ \\
\hline 2.12.1 up to $12 \%$ E SFA baseline & 0 & 0 & $\begin{array}{l}\text { Risk Ratio (M-H, Random, 95\% } \\
\mathrm{Cl} \text { ) }\end{array}$ & Not estimable \\
\hline $2.12 .2>12$ to $15 \% \mathrm{E}$ SFA baseline & 4 & 51279 & $\begin{array}{l}\text { Risk Ratio (M-H, Random, 95\% } \\
\mathrm{CI})\end{array}$ & $0.96[0.87,1.07]$ \\
\hline $2.12 .3>15$ to $18 \%$ E SFA baseline & 1 & 55 & $\begin{array}{l}\text { Risk Ratio (M-H, Random, 95\% } \\
\mathrm{CI})\end{array}$ & $0.52[0.05,5.39]$ \\
\hline 2.12.4 >18\%E SFA baseline & 1 & 846 & $\begin{array}{l}\text { Risk Ratio (M-H, Random, 95\% } \\
\mathrm{CI})\end{array}$ & $0.76[0.55,1.05]$ \\
\hline 2.12.5 unclear & 5 & 987 & $\begin{array}{l}\text { Risk Ratio (M-H, Random, 95\% } \\
\text { Cl) }\end{array}$ & $0.84[0.54,1.30]$ \\
\hline 2.13 MI, subgroup by SFA change & 11 & 53167 & $\begin{array}{l}\text { Risk Ratio (M-H, Random, 95\% } \\
\mathrm{Cl} \text { ) }\end{array}$ & $0.90[0.80,1.01]$ \\
\hline 2.13 .1 up to $4 \% \mathrm{E}$ difference & 4 & 51279 & $\begin{array}{l}\text { Risk Ratio (M-H, Random, 95\% } \\
\text { Cl) }\end{array}$ & $0.96[0.87,1.07]$ \\
\hline 2.13.2 $>4$ to $8 \% \mathrm{E}$ difference & 1 & 55 & $\begin{array}{l}\text { Risk Ratio (M-H, Random, 95\% } \\
\mathrm{Cl} \text { ) }\end{array}$ & $0.52[0.05,5.39]$ \\
\hline $2.13 .3>8 \% \mathrm{E}$ difference & 1 & 846 & $\begin{array}{l}\text { Risk Ratio (M-H, Random, 95\% } \\
\mathrm{Cl} \text { ) }\end{array}$ & $0.76[0.55,1.05]$ \\
\hline 2.13.4 unclear & 5 & 987 & $\begin{array}{l}\text { Risk Ratio (M-H, Random, 95\% } \\
\text { Cl) }\end{array}$ & $0.84[0.54,1.30]$ \\
\hline
\end{tabular}




\begin{tabular}{|c|c|c|c|c|}
\hline Outcome or subgroup title & $\begin{array}{l}\text { No. of } \\
\text { studies }\end{array}$ & $\begin{array}{l}\text { No. of } \\
\text { partici- } \\
\text { pants }\end{array}$ & Statistical method & Effect size \\
\hline $2.14 \mathrm{MI}$, subgroup by sex & 11 & 53167 & $\begin{array}{l}\text { Risk Ratio (M-H, Random, 95\% } \\
\mathrm{Cl})\end{array}$ & $0.90[0.80,1.01]$ \\
\hline 2.14.1 Men & 7 & 3819 & $\begin{array}{l}\text { Risk Ratio (M-H, Random, 95\% } \\
\mathrm{Cl})\end{array}$ & $0.85[0.73,0.98]$ \\
\hline 2.14.2 Women & 1 & 48835 & $\begin{array}{l}\text { Risk Ratio (M-H, Random, 95\% } \\
\mathrm{Cl} \text { ) }\end{array}$ & $0.97[0.86,1.09]$ \\
\hline 2.14.3 Mixed, men and women & 3 & 513 & $\begin{array}{l}\text { Risk Ratio (M-H, Random, 95\% } \\
\mathrm{Cl})\end{array}$ & $0.75[0.13,4.47]$ \\
\hline 2.15 MI, subgroup by CVD risk & 11 & 53167 & $\begin{array}{l}\text { Risk Ratio (M-H, Random, 95\% } \\
\mathrm{Cl})\end{array}$ & $0.90[0.80,1.01]$ \\
\hline 2.15.1 Low CVD risk & 2 & 49681 & $\begin{array}{l}\text { Risk Ratio (M-H, Random, 95\% } \\
\text { Cl) }\end{array}$ & $0.90[0.72,1.13]$ \\
\hline 2.15.2 Moderate CVD risk & 3 & 513 & $\begin{array}{l}\text { Risk Ratio (M-H, Random, 95\% } \\
\mathrm{Cl} \text { ) }\end{array}$ & $0.75[0.13,4.47]$ \\
\hline 2.15.3 Existing CVD disease & 6 & 2973 & $\begin{array}{l}\text { Risk Ratio (M-H, Random, 95\% } \\
\mathrm{Cl})\end{array}$ & $0.87[0.74,1.03]$ \\
\hline 2.16 MI, subgroup by TC reduction & 11 & & $\begin{array}{l}\text { Risk Ratio (M-H, Random, 95\% } \\
\mathrm{CI})\end{array}$ & Subtotals only \\
\hline $\begin{array}{l}2.16 .1 \text { serum chol reduced by at least } \\
0.2 \mathrm{mmol} / \mathrm{L}\end{array}$ & 8 & 4117 & $\begin{array}{l}\text { Risk Ratio (M-H, Random, 95\% } \\
\mathrm{Cl} \text { ) }\end{array}$ & $0.83[0.70,0.98]$ \\
\hline 2.16 .2 serum chol reduced by $<0.2 \mathrm{mmol} / \mathrm{L}$ & 3 & 49050 & $\begin{array}{l}\text { Risk Ratio (M-H, Random, 95\% } \\
\mathrm{Cl} \text { ) }\end{array}$ & $0.98[0.87,1.10]$ \\
\hline 2.16.3 serum chol reduction unclear & 0 & 0 & $\begin{array}{l}\text { Risk Ratio (M-H, Random, 95\% } \\
\text { Cl) }\end{array}$ & Not estimable \\
\hline 2.17 MI, subgroup decade of publication & 11 & 53167 & $\begin{array}{l}\text { Risk Ratio (M-H, Random, 95\% } \\
\mathrm{Cl} \text { ) }\end{array}$ & $0.90[0.80,1.01]$ \\
\hline $2.17 .11960 \mathrm{~s}$ & 5 & 1731 & $\begin{array}{l}\text { Risk Ratio (M-H, Random, 95\% } \\
\text { Cl) }\end{array}$ & $0.80[0.64,1.00]$ \\
\hline $2.17 .21970 \mathrm{~s}$ & 1 & 102 & $\begin{array}{l}\text { Risk Ratio (M-H, Random, 95\% } \\
\mathrm{Cl} \text { ) }\end{array}$ & $0.08[0.00,1.33]$ \\
\hline $2.17 .31980 \mathrm{~s}$ & 1 & 2033 & $\begin{array}{l}\text { Risk Ratio (M-H, Random, 95\% } \\
\mathrm{Cl} \text { ) }\end{array}$ & $0.91[0.73,1.14]$ \\
\hline $2.17 .41990 \mathrm{~s}$ & 1 & 55 & $\begin{array}{l}\text { Risk Ratio (M-H, Random, 95\% } \\
\mathrm{Cl})\end{array}$ & $0.52[0.05,5.39]$ \\
\hline $2.17 .52000 \mathrm{~s}$ & 3 & 49246 & $\begin{array}{l}\text { Risk Ratio (M-H, Random, 95\% } \\
\text { Cl) }\end{array}$ & $0.98[0.87,1.10]$ \\
\hline
\end{tabular}




\begin{tabular}{|c|c|c|c|c|}
\hline Outcome or subgroup title & $\begin{array}{l}\text { No. of } \\
\text { studies }\end{array}$ & $\begin{array}{l}\text { No. of } \\
\text { partici- } \\
\text { pants }\end{array}$ & Statistical method & Effect size \\
\hline 2.18 NON-FATAL MYOCARDIAL INFARCTION & 8 & 52834 & $\begin{array}{l}\text { Risk Ratio (M-H, Random, 95\% } \\
\text { Cl) }\end{array}$ & $0.97[0.87,1.07]$ \\
\hline $\begin{array}{l}2.19 \text { Non-fatal MI, SA by low summary risk } \\
\text { of bias }\end{array}$ & 2 & 49681 & $\begin{array}{l}\text { Risk Ratio (M-H, Random, 95\% } \\
\mathrm{Cl})\end{array}$ & $0.89[0.58,1.35]$ \\
\hline 2.20 Non-fatal MI, SA aim to reduce SFA & 8 & 52834 & $\begin{array}{l}\text { Risk Ratio (M-H, Random, 95\% } \\
\mathrm{Cl})\end{array}$ & $0.97[0.87,1.07]$ \\
\hline $\begin{array}{l}2.21 \text { Non-fatal MI, SA statistically signifi- } \\
\text { cant SFA reduction }\end{array}$ & 4 & 51949 & $\begin{array}{l}\text { Risk Ratio (M-H, Random, 95\% } \\
\text { Cl) }\end{array}$ & $0.90[0.72,1.14]$ \\
\hline 2.22 Non-fatal MI, SA by TC reduction & 7 & 52795 & $\begin{array}{l}\text { Risk Ratio (M-H, Random, 95\% } \\
\text { Cl) }\end{array}$ & $0.97[0.87,1.07]$ \\
\hline 2.23 Non-fatal MI, SA excluding WHI & 7 & 3999 & $\begin{array}{l}\text { Risk Ratio (M-H, Random, 95\% } \\
\text { Cl) }\end{array}$ & $0.81[0.64,1.04]$ \\
\hline $\begin{array}{l}\text { 2.24 Non-fatal MI, SA Mantel-Haenszel } \\
\text { fixed-effect }\end{array}$ & 8 & 52834 & Risk Ratio (M-H, Fixed, 95\% Cl) & $0.97[0.87,1.08]$ \\
\hline 2.25 Non-fatal MI, SA Peto fixed-effect & 8 & 52834 & $\begin{array}{l}\text { Peto Odds Ratio (Peto, Fixed, } \\
95 \% \mathrm{Cl} \text { ) }\end{array}$ & $0.97[0.87,1.08]$ \\
\hline $\begin{array}{l}2.26 \text { Non-fatal MI, subgroup by any substi- } \\
\text { tution }\end{array}$ & 8 & & $\begin{array}{l}\text { Risk Ratio (M-H, Random, 95\% } \\
\text { Cl) }\end{array}$ & Subtotals only \\
\hline 2.26.1 replaced by PUFA & 5 & 3738 & $\begin{array}{l}\text { Risk Ratio (M-H, Random, 95\% } \\
\mathrm{Cl})\end{array}$ & $0.80[0.63,1.03]$ \\
\hline 2.26.2 replaced by MUFA & 1 & 52 & $\begin{array}{l}\text { Risk Ratio (M-H, Random, 95\% } \\
\mathrm{Cl})\end{array}$ & $1.20[0.42,3.45]$ \\
\hline 2.26.3 replace by $\mathrm{CHO}$ & 2 & 50868 & $\begin{array}{l}\text { Risk Ratio (M-H, Random, 95\% } \\
\mathrm{Cl})\end{array}$ & $0.93[0.72,1.21]$ \\
\hline 2.26.4 replaced by protein & 2 & 50868 & $\begin{array}{l}\text { Risk Ratio (M-H, Random, 95\% } \\
\mathrm{Cl})\end{array}$ & $0.93[0.72,1.21]$ \\
\hline 2.26.5 replacement unclear & 1 & 235 & $\begin{array}{l}\text { Risk Ratio (M-H, Random, 95\% } \\
\mathrm{Cl})\end{array}$ & $2.02[0.19,21.94]$ \\
\hline $\begin{array}{l}2.27 \text { Non-fatal MI, subgroup by main sub- } \\
\text { stitution }\end{array}$ & 8 & & $\begin{array}{l}\text { Risk Ratio (M-H, Random, 95\% } \\
\text { Cl) }\end{array}$ & Subtotals only \\
\hline 2.27.1 replaced by PUFA & 5 & 3738 & $\begin{array}{l}\text { Risk Ratio (M-H, Random, 95\% } \\
\text { Cl) }\end{array}$ & $0.80[0.63,1.03]$ \\
\hline 2.27.2 replaced by MUFA & 1 & 52 & $\begin{array}{l}\text { Risk Ratio (M-H, Random, 95\% } \\
\text { Cl) }\end{array}$ & $1.20[0.42,3.45]$ \\
\hline 2.27.3 replace by $\mathrm{CHO}$ & 1 & 48835 & $\begin{array}{l}\text { Risk Ratio (M-H, Random, 95\% } \\
\text { Cl) }\end{array}$ & $1.01[0.90,1.13]$ \\
\hline
\end{tabular}




\begin{tabular}{|c|c|c|c|c|}
\hline Outcome or subgroup title & $\begin{array}{l}\text { No. of } \\
\text { studies }\end{array}$ & $\begin{array}{l}\text { No. of } \\
\text { partici- } \\
\text { pants }\end{array}$ & Statistical method & Effect size \\
\hline 2.27.4 replaced by protein & 0 & 0 & $\begin{array}{l}\text { Risk Ratio (M-H, Random, 95\% } \\
\mathrm{Cl})\end{array}$ & Not estimable \\
\hline 2.27.5 replacement unclear & 1 & 235 & $\begin{array}{l}\text { Risk Ratio (M-H, Random, 95\% } \\
\mathrm{Cl})\end{array}$ & $2.02[0.19,21.94]$ \\
\hline 2.28 Non-fatal MI, subgroup by duration & 8 & 52834 & $\begin{array}{l}\text { Risk Ratio (M-H, Random, 95\% } \\
\mathrm{CI})\end{array}$ & $0.97[0.87,1.07]$ \\
\hline 2.28 .1 up to $24 \mathrm{mo}$ & 4 & 2348 & $\begin{array}{l}\text { Risk Ratio (M-H, Random, 95\% } \\
\mathrm{CI})\end{array}$ & $0.83[0.57,1.22]$ \\
\hline $2.28 .2>24$ to $48 \mathrm{mo}$ & 2 & 1239 & $\begin{array}{l}\text { Risk Ratio (M-H, Random, 95\% } \\
\mathrm{Cl})\end{array}$ & $0.82[0.53,1.27]$ \\
\hline $2.28 .3>48 \mathrm{mo}$ & 2 & 49247 & $\begin{array}{l}\text { Risk Ratio (M-H, Random, 95\% } \\
\mathrm{Cl})\end{array}$ & $0.99[0.88,1.12]$ \\
\hline 2.28.4 unclear & 0 & 0 & $\begin{array}{l}\text { Risk Ratio (M-H, Random, 95\% } \\
\mathrm{CI})\end{array}$ & Not estimable \\
\hline $\begin{array}{l}\text { 2.29 Non-fatal MI, subgroup by baseline } \\
\text { SFA }\end{array}$ & 8 & 52834 & $\begin{array}{l}\text { Risk Ratio (M-H, Random, 95\% } \\
\mathrm{Cl})\end{array}$ & $0.97[0.87,1.07]$ \\
\hline 2.29 .1 up to $12 \%$ E SFA baseline & 0 & 0 & $\begin{array}{l}\text { Risk Ratio (M-H, Random, 95\% } \\
\text { Cl) }\end{array}$ & Not estimable \\
\hline 2.29.2 $>12$ to $15 \%$ E SFA baseline & 3 & 51103 & $\begin{array}{l}\text { Risk Ratio (M-H, Random, 95\% } \\
\mathrm{CI})\end{array}$ & $0.97[0.83,1.13]$ \\
\hline $2.29 .3>15$ to $18 \%$ E SFA baseline & 0 & 0 & $\begin{array}{l}\text { Risk Ratio (M-H, Random, 95\% } \\
\mathrm{Cl})\end{array}$ & Not estimable \\
\hline 2.29.4 >18\%E SFA baseline & 1 & 846 & $\begin{array}{l}\text { Risk Ratio (M-H, Random, 95\% } \\
\mathrm{Cl})\end{array}$ & $0.62[0.31,1.21]$ \\
\hline 2.29.5 unclear & 4 & 885 & $\begin{array}{l}\text { Risk Ratio (M-H, Random, 95\% } \\
\text { Cl) }\end{array}$ & $0.91[0.65,1.27]$ \\
\hline 2.30 Non-fatal MI, subgroup by SFA change & 8 & 52834 & $\begin{array}{l}\text { Risk Ratio (M-H, Random, 95\% } \\
\mathrm{Cl} \text { ) }\end{array}$ & $0.97[0.87,1.07]$ \\
\hline 2.30 .1 up to $4 \% \mathrm{E}$ difference & 3 & 51103 & $\begin{array}{l}\text { Risk Ratio (M-H, Random, 95\% } \\
\text { Cl) }\end{array}$ & $0.97[0.83,1.13]$ \\
\hline 2.30.2 $>4$ to $8 \% \mathrm{E}$ difference & 0 & 0 & $\begin{array}{l}\text { Risk Ratio (M-H, Random, 95\% } \\
\mathrm{Cl} \text { ) }\end{array}$ & Not estimable \\
\hline $2.30 .3>8 \% \mathrm{E}$ difference & 1 & 846 & $\begin{array}{l}\text { Risk Ratio (M-H, Random, 95\% } \\
\mathrm{Cl})\end{array}$ & $0.62[0.31,1.21]$ \\
\hline 2.30.4 unclear & 4 & 885 & $\begin{array}{l}\text { Risk Ratio (M-H, Random, 95\% } \\
\text { Cl) }\end{array}$ & $0.91[0.65,1.27]$ \\
\hline
\end{tabular}




\begin{tabular}{|c|c|c|c|c|}
\hline Outcome or subgroup title & $\begin{array}{l}\text { No. of } \\
\text { studies }\end{array}$ & $\begin{array}{l}\text { No. of } \\
\text { partici- } \\
\text { pants }\end{array}$ & Statistical method & Effect size \\
\hline 2.31 Non-fatal MI, subgroup by sex & 8 & 52834 & $\begin{array}{l}\text { Risk Ratio (M-H, Random, 95\% } \\
\mathrm{Cl})\end{array}$ & $0.97[0.87,1.07]$ \\
\hline 2.31.1 Men & 6 & 3764 & $\begin{array}{l}\text { Risk Ratio (M-H, Random, 95\% } \\
\mathrm{Cl})\end{array}$ & $0.81[0.63,1.03]$ \\
\hline 2.31.2 Women & 1 & 48835 & $\begin{array}{l}\text { Risk Ratio (M-H, Random, 95\% } \\
\mathrm{Cl})\end{array}$ & $1.01[0.90,1.13]$ \\
\hline 2.31.3 Mixed, men and women & 1 & 235 & $\begin{array}{l}\text { Risk Ratio (M-H, Random, 95\% } \\
\mathrm{Cl})\end{array}$ & $2.02[0.19,21.94]$ \\
\hline 2.32 Non-fatal MI, subgroup by CVD risk & 8 & 52834 & $\begin{array}{l}\text { Risk Ratio (M-H, Random, 95\% } \\
\mathrm{Cl})\end{array}$ & $0.95[0.80,1.13]$ \\
\hline 2.32.1 Low CVD risk & 2 & 47404 & $\begin{array}{l}\text { Risk Ratio (M-H, Random, 95\% } \\
\mathrm{Cl} \text { ) }\end{array}$ & $0.87[0.68,1.12]$ \\
\hline 2.32.2 Moderate CVD risk & 1 & 235 & $\begin{array}{l}\text { Risk Ratio (M-H, Random, 95\% } \\
\mathrm{Cl} \text { ) }\end{array}$ & $2.02[0.19,21.94]$ \\
\hline 2.32.3 Existing CVD disease & 6 & 5195 & $\begin{array}{l}\text { Risk Ratio (M-H, Random, 95\% } \\
\mathrm{Cl})\end{array}$ & $1.00[0.76,1.31]$ \\
\hline $\begin{array}{l}2.33 \text { Non-fatal MI, subgroup by } \mathrm{TC} \text { reduc- } \\
\text { tion }\end{array}$ & 8 & & $\begin{array}{l}\text { Risk Ratio (M-H, Random, 95\% } \\
\mathrm{Cl})\end{array}$ & Subtotals only \\
\hline $\begin{array}{l}2.33 .1 \text { serum chol reduced by at least } \\
0.2 \mathrm{mmol} / \mathrm{L}\end{array}$ & 6 & 3960 & $\begin{array}{l}\text { Risk Ratio (M-H, Random, 95\% } \\
\mathrm{Cl} \text { ) }\end{array}$ & $0.80[0.62,1.03]$ \\
\hline 2.33.2 serum chol reduced by $<0.2 \mathrm{mmol} / \mathrm{L}$ & 2 & 48874 & $\begin{array}{l}\text { Risk Ratio (M-H, Random, 95\% } \\
\mathrm{Cl})\end{array}$ & $1.01[0.90,1.13]$ \\
\hline 2.33.3 serum chol reduction unclear & 0 & 0 & $\begin{array}{l}\text { Risk Ratio (M-H, Random, 95\% } \\
\text { Cl) }\end{array}$ & Not estimable \\
\hline $\begin{array}{l}\text { 2.34 Non-fatal MI, subgroup decade of pub- } \\
\text { lication }\end{array}$ & 8 & 52834 & $\begin{array}{l}\text { Risk Ratio (M-H, Random, 95\% } \\
\mathrm{Cl} \text { ) }\end{array}$ & $0.97[0.87,1.07]$ \\
\hline $2.34 .11960 \mathrm{~s}$ & 5 & 1731 & $\begin{array}{l}\text { Risk Ratio (M-H, Random, 95\% } \\
\mathrm{Cl} \text { ) }\end{array}$ & $0.84[0.62,1.13]$ \\
\hline $2.34 .21970 \mathrm{~s}$ & 0 & 0 & $\begin{array}{l}\text { Risk Ratio (M-H, Random, 95\% } \\
\mathrm{Cl} \text { ) }\end{array}$ & Not estimable \\
\hline $2.34 .31980 \mathrm{~s}$ & 1 & 2033 & $\begin{array}{l}\text { Risk Ratio (M-H, Random, 95\% } \\
\mathrm{Cl} \text { ) }\end{array}$ & $0.74[0.48,1.14]$ \\
\hline $2.34 .41990 \mathrm{~s}$ & 0 & 0 & $\begin{array}{l}\text { Risk Ratio (M-H, Random, 95\% } \\
\mathrm{Cl})\end{array}$ & Not estimable \\
\hline $2.34 .52000 \mathrm{~s}$ & 2 & 49070 & $\begin{array}{l}\text { Risk Ratio (M-H, Random, 95\% } \\
\text { Cl) }\end{array}$ & $1.01[0.90,1.13]$ \\
\hline
\end{tabular}




\begin{tabular}{|c|c|c|c|c|}
\hline Outcome or subgroup title & $\begin{array}{l}\text { No. of } \\
\text { studies }\end{array}$ & $\begin{array}{l}\text { No. of } \\
\text { partici- } \\
\text { pants }\end{array}$ & Statistical method & Effect size \\
\hline 2.35 STROKE & 7 & 50952 & $\begin{array}{l}\text { Risk Ratio (M-H, Random, 95\% } \\
\mathrm{Cl})\end{array}$ & $0.92[0.68,1.25]$ \\
\hline 2.36 Stroke, SA by low summary risk of bias & 3 & 49857 & $\begin{array}{l}\text { Risk Ratio (M-H, Random, 95\% } \\
\mathrm{Cl})\end{array}$ & $0.76[0.42,1.38]$ \\
\hline 2.37 Stroke, SA aim to reduce SFA & 6 & 50776 & $\begin{array}{l}\text { Risk Ratio (M-H, Random, 95\% } \\
\mathrm{Cl})\end{array}$ & $1.01[0.90,1.14]$ \\
\hline $\begin{array}{l}2.38 \text { Stroke, SA statistically significant SFA } \\
\text { reduction }\end{array}$ & 5 & 50147 & $\begin{array}{l}\text { Risk Ratio (M-H, Random, 95\% } \\
\mathrm{Cl})\end{array}$ & $0.83[0.55,1.25]$ \\
\hline 2.39 Stroke, SA by TC reduction & 6 & 50776 & $\begin{array}{l}\text { Risk Ratio (M-H, Random, 95\% } \\
\mathrm{Cl})\end{array}$ & $1.01[0.90,1.14]$ \\
\hline 2.40 Stroke, SA excluding WHI & 6 & 2117 & $\begin{array}{l}\text { Risk Ratio (M-H, Random, 95\% } \\
\mathrm{Cl} \text { ) }\end{array}$ & $0.63[0.35,1.14]$ \\
\hline $\begin{array}{l}2.41 \text { Stroke, SA Mantel-Haenszel fixed-ef- } \\
\text { fect }\end{array}$ & 7 & 50952 & Risk Ratio (M-H, Fixed, 95\% Cl) & $1.01[0.89,1.13]$ \\
\hline 2.42 Stroke, SA Peto fixed-effect & 7 & 50952 & $\begin{array}{l}\text { Peto Odds Ratio (Peto, Fixed, } \\
95 \% \mathrm{Cl} \text { ) }\end{array}$ & $1.01[0.89,1.14]$ \\
\hline 2.43 Stroke, subgroup by any substitution & 7 & & $\begin{array}{l}\text { Risk Ratio (M-H, Random, 95\% } \\
\mathrm{Cl})\end{array}$ & Subtotals only \\
\hline 2.43.1 replaced by PUFA & 4 & 1706 & $\begin{array}{l}\text { Risk Ratio (M-H, Random, 95\% } \\
\mathrm{Cl})\end{array}$ & $0.68[0.37,1.27]$ \\
\hline 2.43.2 replaced by MUFA & 0 & 0 & $\begin{array}{l}\text { Risk Ratio (M-H, Random, 95\% } \\
\mathrm{Cl})\end{array}$ & Not estimable \\
\hline 2.43.3 replace by $\mathrm{CHO}$ & 3 & 49066 & $\begin{array}{l}\text { Risk Ratio (M-H, Random, 95\% } \\
\text { Cl) }\end{array}$ & $0.73[0.29,1.87]$ \\
\hline 2.43.4 replaced by protein & 2 & 49011 & $\begin{array}{l}\text { Risk Ratio (M-H, Random, 95\% } \\
\mathrm{Cl} \text { ) }\end{array}$ & $0.65[0.15,2.75]$ \\
\hline 2.43.5 replacement unclear & 1 & 235 & $\begin{array}{l}\text { Risk Ratio (M-H, Random, 95\% } \\
\mathrm{Cl} \text { ) }\end{array}$ & $1.01[0.06,15.93]$ \\
\hline 2.44 Stroke, subgroup by main substitution & 7 & & $\begin{array}{l}\text { Risk Ratio (M-H, Random, 95\% } \\
\mathrm{Cl} \text { ) }\end{array}$ & Subtotals only \\
\hline 2.44.1 replaced by PUFA & 3 & 1651 & $\begin{array}{l}\text { Risk Ratio (M-H, Random, 95\% } \\
\mathrm{Cl})\end{array}$ & $0.92[0.31,2.69]$ \\
\hline 2.44.2 replaced by MUFA & 0 & 0 & $\begin{array}{l}\text { Risk Ratio (M-H, Random, 95\% } \\
\mathrm{Cl})\end{array}$ & Not estimable \\
\hline 2.44.3 replace by $\mathrm{CHO}$ & 3 & 49066 & $\begin{array}{l}\text { Risk Ratio (M-H, Random, 95\% } \\
\mathrm{Cl} \text { ) }\end{array}$ & $0.73[0.29,1.87]$ \\
\hline
\end{tabular}




\begin{tabular}{|c|c|c|c|c|}
\hline Outcome or subgroup title & $\begin{array}{l}\text { No. of } \\
\text { studies }\end{array}$ & $\begin{array}{l}\text { No. of } \\
\text { partici- } \\
\text { pants }\end{array}$ & Statistical method & Effect size \\
\hline 2.44.4 replaced by protein & 0 & 0 & $\begin{array}{l}\text { Risk Ratio (M-H, Random, 95\% } \\
\mathrm{Cl})\end{array}$ & Not estimable \\
\hline 2.44.5 replacement unclear & 1 & 235 & $\begin{array}{l}\text { Risk Ratio (M-H, Random, 95\% } \\
\mathrm{Cl} \text { ) }\end{array}$ & $1.01[0.06,15.93]$ \\
\hline 2.45 Stroke, subgroup by duration & 6 & 50559 & $\begin{array}{l}\text { Risk Ratio (M-H, Random, 95\% } \\
\mathrm{CI})\end{array}$ & $0.91[0.67,1.23]$ \\
\hline 2.45 .1 up to $24 \mathrm{mo}$ & 1 & 235 & $\begin{array}{l}\text { Risk Ratio (M-H, Random, 95\% } \\
\mathrm{CI})\end{array}$ & $1.01[0.06,15.93]$ \\
\hline $2.45 .2>24$ to $48 \mathrm{mo}$ & 2 & 901 & $\begin{array}{l}\text { Risk Ratio (M-H, Random, 95\% } \\
\mathrm{Cl})\end{array}$ & $0.57[0.30,1.11]$ \\
\hline $2.45 .3>48 \mathrm{mo}$ & 2 & 49247 & $\begin{array}{l}\text { Risk Ratio (M-H, Random, 95\% } \\
\mathrm{Cl})\end{array}$ & $1.03[0.91,1.16]$ \\
\hline 2.45.4 unclear duration & 1 & 176 & $\begin{array}{l}\text { Risk Ratio (M-H, Random, 95\% } \\
\mathrm{Cl})\end{array}$ & $0.20[0.02,1.68]$ \\
\hline 2.46 Stroke, subgroup by baseline SFA & 6 & 50559 & $\begin{array}{l}\text { Risk Ratio (M-H, Random, 95\% } \\
\mathrm{Cl})\end{array}$ & $0.91[0.67,1.23]$ \\
\hline 2.46.1 up to $12 \%$ E SFA baseline & 0 & 0 & $\begin{array}{l}\text { Risk Ratio (M-H, Random, 95\% } \\
\mathrm{Cl} \text { ) }\end{array}$ & Not estimable \\
\hline $2.46 .2>12$ to $15 \%$ E SFA baseline & 3 & 49246 & $\begin{array}{l}\text { Risk Ratio (M-H, Random, 95\% } \\
\mathrm{CI})\end{array}$ & $0.91[0.50,1.66]$ \\
\hline $2.46 .3>15$ to $18 \%$ E SFA baseline & 1 & 55 & $\begin{array}{l}\text { Risk Ratio (M-H, Random, 95\% } \\
\mathrm{CI})\end{array}$ & $0.35[0.01,8.12]$ \\
\hline 2.46.4 >18\%E SFA baseline & 1 & 846 & $\begin{array}{l}\text { Risk Ratio (M-H, Random, 95\% } \\
\mathrm{CI})\end{array}$ & $0.59[0.30,1.15]$ \\
\hline 2.46.5 unclear & 1 & 412 & $\begin{array}{l}\text { Risk Ratio (M-H, Random, 95\% } \\
\text { Cl) }\end{array}$ & $2.00[0.18,21.89]$ \\
\hline 2.47 Stroke, subgroup by SFA change & 6 & 50559 & $\begin{array}{l}\text { Risk Ratio (M-H, Random, 95\% } \\
\mathrm{Cl} \text { ) }\end{array}$ & $0.91[0.67,1.23]$ \\
\hline 2.47 .1 up to $4 \% \mathrm{E}$ difference & 3 & 49246 & $\begin{array}{l}\text { Risk Ratio (M-H, Random, 95\% } \\
\text { Cl) }\end{array}$ & $0.91[0.50,1.66]$ \\
\hline $2.47 .2>4$ to $8 \% \mathrm{E}$ difference & 1 & 55 & $\begin{array}{l}\text { Risk Ratio (M-H, Random, 95\% } \\
\mathrm{Cl} \text { ) }\end{array}$ & $0.35[0.01,8.12]$ \\
\hline $2.47 .3>8 \% \mathrm{E}$ difference & 1 & 846 & $\begin{array}{l}\text { Risk Ratio (M-H, Random, 95\% } \\
\mathrm{Cl} \text { ) }\end{array}$ & $0.59[0.30,1.15]$ \\
\hline 2.47.4 unclear & 1 & 412 & $\begin{array}{l}\text { Risk Ratio (M-H, Random, 95\% } \\
\text { Cl) }\end{array}$ & $2.00[0.18,21.89]$ \\
\hline
\end{tabular}




\begin{tabular}{|c|c|c|c|c|}
\hline Outcome or subgroup title & $\begin{array}{l}\text { No. of } \\
\text { studies }\end{array}$ & $\begin{array}{l}\text { No. of } \\
\text { partici- } \\
\text { pants }\end{array}$ & Statistical method & Effect size \\
\hline 2.48 Stroke, subgroup by sex & 6 & 50559 & $\begin{array}{l}\text { Risk Ratio (M-H, Random, 95\% } \\
\mathrm{Cl})\end{array}$ & $0.91[0.67,1.23]$ \\
\hline 2.48.1 Men & 3 & 1313 & $\begin{array}{l}\text { Risk Ratio (M-H, Random, 95\% } \\
\mathrm{Cl})\end{array}$ & $0.63[0.33,1.18]$ \\
\hline 2.48.2 Women & 1 & 48835 & $\begin{array}{l}\text { Risk Ratio (M-H, Random, 95\% } \\
\mathrm{Cl} \text { ) }\end{array}$ & $1.03[0.91,1.16]$ \\
\hline 2.48.3 Mixed, men and women & 2 & 411 & $\begin{array}{l}\text { Risk Ratio (M-H, Random, 95\% } \\
\mathrm{Cl})\end{array}$ & $0.37[0.07,1.97]$ \\
\hline 2.49 Stroke, subgroup by CVD risk & 6 & 50559 & $\begin{array}{l}\text { Risk Ratio (M-H, Random, 95\% } \\
\mathrm{Cl})\end{array}$ & $1.00[0.89,1.11]$ \\
\hline 2.49.1 Low CVD risk & 2 & 47404 & $\begin{array}{l}\text { Risk Ratio (M-H, Random, 95\% } \\
\mathrm{Cl} \text { ) }\end{array}$ & $0.86[0.52,1.42]$ \\
\hline 2.49.2 Moderate CVD risk & 2 & 411 & $\begin{array}{l}\text { Risk Ratio (M-H, Random, 95\% } \\
\mathrm{Cl} \text { ) }\end{array}$ & $0.37[0.07,1.97]$ \\
\hline 2.49.3 Existing CVD disease & 3 & 2744 & $\begin{array}{l}\text { Risk Ratio (M-H, Random, 95\% } \\
\mathrm{Cl} \text { ) }\end{array}$ & $1.01[0.86,1.18]$ \\
\hline 2.50 Stroke, subgroup by TC reduction & 7 & & $\begin{array}{l}\text { Risk Ratio (M-H, Random, 95\% } \\
\mathrm{CI})\end{array}$ & Subtotals only \\
\hline $\begin{array}{l}2.50 .1 \text { serum chol reduced by at least } \\
0.2 \mathrm{mmol} / \mathrm{L}\end{array}$ & 5 & 1941 & $\begin{array}{l}\text { Risk Ratio (M-H, Random, 95\% } \\
\mathrm{Cl} \text { ) }\end{array}$ & $0.70[0.38,1.28]$ \\
\hline 2.50 .2 serum chol reduced by $<0.2 \mathrm{mmol} / \mathrm{L}$ & 2 & 49011 & $\begin{array}{l}\text { Risk Ratio (M-H, Random, 95\% } \\
\mathrm{Cl} \text { ) }\end{array}$ & $0.65[0.15,2.75]$ \\
\hline 2.50 .3 serum chol reduction unclear & 0 & 0 & $\begin{array}{l}\text { Risk Ratio (M-H, Random, 95\% } \\
\text { Cl) }\end{array}$ & Not estimable \\
\hline $\begin{array}{l}2.51 \text { Stroke, subgroup decade of publica- } \\
\text { tion }\end{array}$ & 7 & 50952 & $\begin{array}{l}\text { Risk Ratio (M-H, Random, 95\% } \\
\mathrm{Cl} \text { ) }\end{array}$ & $0.92[0.68,1.25]$ \\
\hline $2.51 .11960 \mathrm{~s}$ & 3 & 1651 & $\begin{array}{l}\text { Risk Ratio (M-H, Random, 95\% } \\
\mathrm{Cl} \text { ) }\end{array}$ & $0.92[0.31,2.69]$ \\
\hline $2.51 .21970 \mathrm{~s}$ & 0 & 0 & $\begin{array}{l}\text { Risk Ratio (M-H, Random, 95\% } \\
\mathrm{Cl} \text { ) }\end{array}$ & Not estimable \\
\hline $2.51 .31980 \mathrm{~s}$ & 0 & 0 & $\begin{array}{l}\text { Risk Ratio (M-H, Random, 95\% } \\
\mathrm{Cl} \text { ) }\end{array}$ & Not estimable \\
\hline $2.51 .41990 \mathrm{~s}$ & 1 & 55 & $\begin{array}{l}\text { Risk Ratio (M-H, Random, 95\% } \\
\mathrm{Cl})\end{array}$ & $0.35[0.01,8.12]$ \\
\hline $2.51 .52000 \mathrm{~s}$ & 3 & 49246 & $\begin{array}{l}\text { Risk Ratio (M-H, Random, 95\% } \\
\mathrm{Cl} \text { ) }\end{array}$ & $0.91[0.50,1.66]$ \\
\hline
\end{tabular}




\begin{tabular}{|c|c|c|c|c|}
\hline Outcome or subgroup title & $\begin{array}{l}\text { No. of } \\
\text { studies }\end{array}$ & $\begin{array}{l}\text { No. of } \\
\text { partici- } \\
\text { pants }\end{array}$ & Statistical method & Effect size \\
\hline $\begin{array}{l}2.52 \text { CORONARY HEART DISEASE MORTALI- } \\
\text { TY }\end{array}$ & 9 & 53159 & $\begin{array}{l}\text { Risk Ratio (M-H, Random, 95\% } \\
\mathrm{Cl} \text { ) }\end{array}$ & $0.97[0.82,1.16]$ \\
\hline $\begin{array}{l}2.53 \text { CHD mortality, SA by low summary } \\
\text { risk of bias }\end{array}$ & 3 & 50139 & $\begin{array}{l}\text { Risk Ratio (M-H, Random, 95\% } \\
\mathrm{Cl})\end{array}$ & $1.05[0.77,1.43]$ \\
\hline 2.54 CHD mortality, SA aim to reduce SFA & 9 & 53159 & $\begin{array}{l}\text { Risk Ratio (M-H, Random, 95\% } \\
\mathrm{Cl})\end{array}$ & $0.97[0.82,1.16]$ \\
\hline $\begin{array}{l}\text { 2.55 CHD mortality, SA statistically signifi- } \\
\text { cant SFA reduction }\end{array}$ & 4 & 52172 & $\begin{array}{l}\text { Risk Ratio (M-H, Random, 95\% } \\
\mathrm{Cl})\end{array}$ & $1.02[0.84,1.24]$ \\
\hline 2.56 CHD mortality, SA by TC reduction & 8 & 53120 & $\begin{array}{l}\text { Risk Ratio (M-H, Random, 95\% } \\
\mathrm{Cl})\end{array}$ & $0.97[0.81,1.16]$ \\
\hline 2.57 CHD mortality, SA excluding WHI & 8 & 4324 & $\begin{array}{l}\text { Risk Ratio (M-H, Random, 95\% } \\
\mathrm{Cl} \text { ) }\end{array}$ & $0.97[0.76,1.24]$ \\
\hline $\begin{array}{l}\text { 2.58 CHD mortality, SA Mantel-Haenszel } \\
\text { fixed-effect }\end{array}$ & 9 & 53159 & Risk Ratio (M-H, Fixed, 95\% Cl) & $0.97[0.86,1.10]$ \\
\hline 2.59 CHD mortality, SA Peto fixed-effect & 9 & 53159 & $\begin{array}{l}\text { Peto Odds Ratio (Peto, Fixed, } \\
95 \% \mathrm{Cl} \text { ) }\end{array}$ & $0.97[0.85,1.11]$ \\
\hline $\begin{array}{l}2.60 \text { CHD mortality, subgroup by any sub- } \\
\text { stitution }\end{array}$ & 9 & & $\begin{array}{l}\text { Risk Ratio (M-H, Random, 95\% } \\
\mathrm{Cl} \text { ) }\end{array}$ & Subtotals only \\
\hline 2.60.1 replaced by PUFA & 7 & 4298 & $\begin{array}{l}\text { Risk Ratio (M-H, Random, 95\% } \\
\mathrm{Cl})\end{array}$ & $0.98[0.74,1.28]$ \\
\hline 2.60 .2 replaced by MUFA & 1 & 52 & $\begin{array}{l}\text { Risk Ratio (M-H, Random, 95\% } \\
\mathrm{Cl})\end{array}$ & $3.00[0.33,26.99]$ \\
\hline 2.60.3 replaced by $\mathrm{CHO}$ & 2 & 50868 & $\begin{array}{l}\text { Risk Ratio (M-H, Random, 95\% } \\
\mathrm{Cl})\end{array}$ & $0.99[0.85,1.16]$ \\
\hline 2.60.4 replaced by protein & 2 & 50868 & $\begin{array}{l}\text { Risk Ratio (M-H, Random, 95\% } \\
\mathrm{Cl})\end{array}$ & $0.99[0.85,1.16]$ \\
\hline 2.60.5 replacement unclear & 0 & 0 & $\begin{array}{l}\text { Risk Ratio (M-H, Random, 95\% } \\
\text { Cl) }\end{array}$ & Not estimable \\
\hline $\begin{array}{l}2.61 \text { CHD mortality, subgroup by main sub- } \\
\text { stitution }\end{array}$ & 9 & & $\begin{array}{l}\text { Risk Ratio (M-H, Random, 95\% } \\
\text { Cl) }\end{array}$ & Subtotals only \\
\hline 2.61.1 replaced by PUFA & 7 & 4298 & $\begin{array}{l}\text { Risk Ratio (M-H, Random, 95\% } \\
\text { Cl) }\end{array}$ & $0.98[0.74,1.28]$ \\
\hline 2.61.2 replaced by MUFA & 1 & 52 & $\begin{array}{l}\text { Risk Ratio (M-H, Random, 95\% } \\
\mathrm{Cl} \text { ) }\end{array}$ & $3.00[0.33,26.99]$ \\
\hline 2.61.3 replaced by $\mathrm{CHO}$ & 1 & 48835 & $\begin{array}{l}\text { Risk Ratio (M-H, Random, 95\% } \\
\text { Cl) }\end{array}$ & $0.99[0.82,1.20]$ \\
\hline
\end{tabular}




\begin{tabular}{|c|c|c|c|c|}
\hline Outcome or subgroup title & $\begin{array}{l}\text { No. of } \\
\text { studies }\end{array}$ & $\begin{array}{l}\text { No. of } \\
\text { partici- } \\
\text { pants }\end{array}$ & Statistical method & Effect size \\
\hline 2.61.4 replaced by protein & 0 & 0 & $\begin{array}{l}\text { Risk Ratio (M-H, Random, 95\% } \\
\mathrm{Cl} \text { ) }\end{array}$ & Not estimable \\
\hline 2.61 .5 replacement unclear & 0 & 0 & $\begin{array}{l}\text { Risk Ratio (M-H, Random, 95\% } \\
\mathrm{Cl} \text { ) }\end{array}$ & Not estimable \\
\hline $2.62 \mathrm{CHD}$ mortality, subgroup by duration & 9 & & $\begin{array}{l}\text { Risk Ratio (M-H, Random, 95\% } \\
\mathrm{CI} \text { ) }\end{array}$ & Subtotals only \\
\hline 2.62 .1 up to $24 \mathrm{mo}$ & 3 & 2113 & $\begin{array}{l}\text { Risk Ratio (M-H, Random, 95\% } \\
\mathrm{Cl} \text { ) }\end{array}$ & $1.02[0.78,1.33]$ \\
\hline $2.62 .2>24$ to 48 months & 2 & 1239 & $\begin{array}{l}\text { Risk Ratio (M-H, Random, 95\% } \\
\mathrm{Cl} \text { ) }\end{array}$ & $0.87[0.64,1.19]$ \\
\hline $2.62 .3>48$ months & 3 & 49705 & $\begin{array}{l}\text { Risk Ratio (M-H, Random, 95\% } \\
\mathrm{Cl} \text { ) }\end{array}$ & $1.02[0.72,1.45]$ \\
\hline 2.62.4 unclear duration & 1 & 102 & $\begin{array}{l}\text { Risk Ratio (M-H, Random, 95\% } \\
\mathrm{Cl})\end{array}$ & $0.09[0.01,1.60]$ \\
\hline $\begin{array}{l}2.63 \text { CHD mortality, subgroup by baseline } \\
\text { SFA }\end{array}$ & 9 & & $\begin{array}{l}\text { Risk Ratio (M-H, Random, 95\% } \\
\text { Cl) }\end{array}$ & Subtotals only \\
\hline 2.63.1 up to $12 \%$ E SFA baseline & 0 & 0 & $\begin{array}{l}\text { Risk Ratio (M-H, Random, 95\% } \\
\text { Cl) }\end{array}$ & Not estimable \\
\hline $2.63 .2>12 \%$ to $15 \%$ E SFA baseline & 3 & 51326 & $\begin{array}{l}\text { Risk Ratio (M-H, Random, 95\% } \\
\text { Cl) }\end{array}$ & $1.07[0.86,1.34]$ \\
\hline $2.63 .3>15$ to $18 \%$ E SFA baseline & 0 & 0 & $\begin{array}{l}\text { Risk Ratio (M-H, Random, 95\% } \\
\mathrm{Cl} \text { ) }\end{array}$ & Not estimable \\
\hline 2.63.4 $>18 \%$ E SFA baseline & 1 & 846 & $\begin{array}{l}\text { Risk Ratio (M-H, Random, 95\% } \\
\text { Cl) }\end{array}$ & $0.82[0.55,1.21]$ \\
\hline 2.63.5 unclear & 5 & 987 & $\begin{array}{l}\text { Risk Ratio (M-H, Random, 95\% } \\
\text { Cl) }\end{array}$ & $0.85[0.56,1.29]$ \\
\hline $\begin{array}{l}\text { 2.64 CHD mortality, subgroup by SFA } \\
\text { change }\end{array}$ & 9 & & $\begin{array}{l}\text { Risk Ratio (M-H, Random, 95\% } \\
\mathrm{Cl})\end{array}$ & Subtotals only \\
\hline 2.64 .1 up to $4 \% \mathrm{E}$ difference & 3 & 51326 & $\begin{array}{l}\text { Risk Ratio (M-H, Random, 95\% } \\
\mathrm{Cl})\end{array}$ & $1.07[0.86,1.34]$ \\
\hline 2.64.2 $>4$ to $8 \% \mathrm{E}$ difference & 0 & 0 & $\begin{array}{l}\text { Risk Ratio (M-H, Random, 95\% } \\
\mathrm{CI})\end{array}$ & Not estimable \\
\hline 2.64.3>8\%E difference & 1 & 846 & $\begin{array}{l}\text { Risk Ratio (M-H, Random, 95\% } \\
\mathrm{Cl} \text { ) }\end{array}$ & $0.82[0.55,1.21]$ \\
\hline 2.64.4 unclear & 5 & 987 & $\begin{array}{l}\text { Risk Ratio (M-H, Random, 95\% } \\
\text { Cl) }\end{array}$ & $0.85[0.56,1.29]$ \\
\hline
\end{tabular}




\begin{tabular}{|c|c|c|c|c|}
\hline Outcome or subgroup title & $\begin{array}{l}\text { No. of } \\
\text { studies }\end{array}$ & $\begin{array}{l}\text { No. of } \\
\text { partici- } \\
\text { pants }\end{array}$ & Statistical method & Effect size \\
\hline 2.65 CHD mortality, subgroup by sex & 9 & & $\begin{array}{l}\text { Risk Ratio (M-H, Random, 95\% } \\
\mathrm{Cl})\end{array}$ & Subtotals only \\
\hline 2.65.1 Men & 7 & 4222 & $\begin{array}{l}\text { Risk Ratio (M-H, Random, 95\% } \\
\mathrm{Cl})\end{array}$ & $0.98[0.79,1.23]$ \\
\hline 2.65.2 Women & 1 & 48835 & $\begin{array}{l}\text { Risk Ratio (M-H, Random, 95\% } \\
\mathrm{Cl})\end{array}$ & $0.99[0.82,1.20]$ \\
\hline 2.65.3 Mixed, men and women & 1 & 102 & $\begin{array}{l}\text { Risk Ratio (M-H, Random, 95\% } \\
\mathrm{Cl})\end{array}$ & $0.09[0.01,1.60]$ \\
\hline 2.66 CHD mortality, subgroup by CVD risk & 9 & & $\begin{array}{l}\text { Risk Ratio (M-H, Random, 95\% } \\
\mathrm{Cl})\end{array}$ & Subtotals only \\
\hline 2.66.1 Low CVD risk & 2 & 47404 & $\begin{array}{l}\text { Risk Ratio (M-H, Random, 95\% } \\
\mathrm{Cl} \text { ) }\end{array}$ & $0.95[0.78,1.16]$ \\
\hline 2.66.2 Moderate CVD risk & 1 & 102 & $\begin{array}{l}\text { Risk Ratio (M-H, Random, 95\% } \\
\mathrm{Cl} \text { ) }\end{array}$ & $0.09[0.01,1.60]$ \\
\hline 2.66.3 Existing CVD disease & 7 & 5653 & $\begin{array}{l}\text { Risk Ratio (M-H, Random, 95\% } \\
\mathrm{Cl})\end{array}$ & $1.03[0.83,1.27]$ \\
\hline $\begin{array}{l}\text { 2.67 CHD mortality, subgroup by TC reduc- } \\
\text { tion }\end{array}$ & 9 & & $\begin{array}{l}\text { Risk Ratio (M-H, Random, 95\% } \\
\mathrm{Cl})\end{array}$ & Subtotals only \\
\hline $\begin{array}{l}2.67 .1 \text { serum chol reduced by at least } \\
0.2 \mathrm{mmol} / \mathrm{L}\end{array}$ & 7 & 4285 & $\begin{array}{l}\text { Risk Ratio (M-H, Random, 95\% } \\
\mathrm{Cl})\end{array}$ & $0.96[0.75,1.24]$ \\
\hline 2.67.2 serum chol reduced by $<0.2 \mathrm{mmol} / \mathrm{L}$ & 2 & 48874 & $\begin{array}{l}\text { Risk Ratio (M-H, Random, 95\% } \\
\mathrm{Cl})\end{array}$ & $0.99[0.82,1.20]$ \\
\hline 2.67.3 serum chol reduction unclear & 0 & 0 & $\begin{array}{l}\text { Risk Ratio (M-H, Random, 95\% } \\
\text { Cl) }\end{array}$ & Not estimable \\
\hline $\begin{array}{l}2.68 \mathrm{CHD} \text { mortality, subgroup decade of } \\
\text { publication }\end{array}$ & 9 & 53159 & $\begin{array}{l}\text { Risk Ratio (M-H, Random, 95\% } \\
\mathrm{Cl} \text { ) }\end{array}$ & $0.97[0.82,1.16]$ \\
\hline $2.68 .11960 \mathrm{~s}$ & 5 & 1731 & $\begin{array}{l}\text { Risk Ratio (M-H, Random, 95\% } \\
\mathrm{Cl} \text { ) }\end{array}$ & $0.84[0.66,1.06]$ \\
\hline $2.68 .21970 \mathrm{~s}$ & 2 & 560 & $\begin{array}{l}\text { Risk Ratio (M-H, Random, 95\% } \\
\mathrm{Cl} \text { ) }\end{array}$ & $0.54[0.03,9.26]$ \\
\hline $2.68 .31980 \mathrm{~s}$ & 1 & 2033 & $\begin{array}{l}\text { Risk Ratio (M-H, Random, 95\% } \\
\mathrm{Cl} \text { ) }\end{array}$ & $1.00[0.76,1.30]$ \\
\hline $2.68 .41990 \mathrm{~s}$ & 0 & 0 & $\begin{array}{l}\text { Risk Ratio (M-H, Random, 95\% } \\
\mathrm{Cl})\end{array}$ & Not estimable \\
\hline $2.68 .52000 \mathrm{~s}$ & 1 & 48835 & $\begin{array}{l}\text { Risk Ratio (M-H, Random, 95\% } \\
\text { Cl) }\end{array}$ & $0.99[0.82,1.20]$ \\
\hline
\end{tabular}




\begin{tabular}{|c|c|c|c|c|}
\hline Outcome or subgroup title & $\begin{array}{l}\text { No. of } \\
\text { studies }\end{array}$ & $\begin{array}{l}\text { No. of } \\
\text { partici- } \\
\text { pants }\end{array}$ & Statistical method & Effect size \\
\hline 2.69 CORONARY HEART DISEASE EVENTS & 11 & 53199 & $\begin{array}{l}\text { Risk Ratio (M-H, Random, 95\% } \\
\mathrm{Cl})\end{array}$ & $0.83[0.68,1.01]$ \\
\hline $\begin{array}{l}\text { 2.70 CHD events, SA by low summary risk } \\
\text { of bias }\end{array}$ & 3 & 49857 & $\begin{array}{l}\text { Risk Ratio (M-H, Random, 95\% } \\
\mathrm{Cl})\end{array}$ & $0.92[0.77,1.10]$ \\
\hline 2.71 CHD events, SA excluding WHI & 10 & 4364 & $\begin{array}{l}\text { Risk Ratio (M-H, Random, 95\% } \\
\mathrm{Cl} \text { ) }\end{array}$ & $0.80[0.62,1.03]$ \\
\hline $\begin{array}{l}\text { 2.72 CHD events, SA statistically significant } \\
\text { SFA reduction }\end{array}$ & 6 & 52212 & $\begin{array}{l}\text { Risk Ratio (M-H, Random, 95\% } \\
\text { Cl) }\end{array}$ & $0.91[0.77,1.06]$ \\
\hline 2.73 CHD events, SA by TC reduction & 9 & 52984 & $\begin{array}{l}\text { Risk Ratio (M-H, Random, 95\% } \\
\mathrm{Cl})\end{array}$ & $0.80[0.65,0.99]$ \\
\hline 2.74 CHD events, SA aim to reduce SFA & 10 & 53023 & $\begin{array}{l}\text { Risk Ratio (M-H, Random, 95\% } \\
\mathrm{Cl} \text { ) }\end{array}$ & $0.82[0.67,1.00]$ \\
\hline $\begin{array}{l}\text { 2.75 CHD events, SA Mantel-Haenszel fixed- } \\
\text { effect }\end{array}$ & 11 & 53199 & Risk Ratio (M-H, Fixed, 95\% Cl) & $0.91[0.84,0.99]$ \\
\hline 2.76 CHD events, SA Peto fixed-effect & 11 & 53199 & $\begin{array}{l}\text { Peto Odds Ratio (Peto, Fixed, } \\
95 \% \mathrm{Cl} \text { ) }\end{array}$ & $0.90[0.83,0.99]$ \\
\hline $\begin{array}{l}2.77 \text { CHD events, subgroup by any substi- } \\
\text { tution }\end{array}$ & 11 & & $\begin{array}{l}\text { Risk Ratio (M-H, Random, 95\% } \\
\text { Cl) }\end{array}$ & Subtotals only \\
\hline 2.77.1 replaced by PUFA & 7 & 3895 & $\begin{array}{l}\text { Risk Ratio (M-H, Random, 95\% } \\
\mathrm{Cl} \text { ) }\end{array}$ & $0.76[0.57,1.00]$ \\
\hline 2.77.2 replaced by MUFA & 1 & 52 & $\begin{array}{l}\text { Risk Ratio (M-H, Random, 95\% } \\
\mathrm{Cl} \text { ) }\end{array}$ & $1.50[0.62,3.61]$ \\
\hline 2.77.3 replaced by $\mathrm{CHO}$ & 4 & 51104 & $\begin{array}{l}\text { Risk Ratio (M-H, Random, 95\% } \\
\mathrm{Cl} \text { ) }\end{array}$ & $0.93[0.78,1.11]$ \\
\hline 2.77.4 replaced by protein & 3 & 51044 & $\begin{array}{l}\text { Risk Ratio (M-H, Random, 95\% } \\
\mathrm{Cl})\end{array}$ & $0.96[0.88,1.05]$ \\
\hline 2.77.5 replacement unclear & 1 & 267 & $\begin{array}{l}\text { Risk Ratio (M-H, Random, 95\% } \\
\mathrm{Cl})\end{array}$ & $2.93[0.31,27.84]$ \\
\hline $\begin{array}{l}2.78 \mathrm{CHD} \text { events, subgroup by main substi- } \\
\text { tution }\end{array}$ & 11 & & $\begin{array}{l}\text { Risk Ratio (M-H, Random, 95\% } \\
\mathrm{Cl} \text { ) }\end{array}$ & Subtotals only \\
\hline 2.78.1 replaced by PUFA & 6 & 3840 & $\begin{array}{l}\text { Risk Ratio (M-H, Random, 95\% } \\
\mathrm{Cl} \text { ) }\end{array}$ & $0.79[0.60,1.04]$ \\
\hline 2.78.2 replaced by MUFA & 1 & 52 & $\begin{array}{l}\text { Risk Ratio (M-H, Random, 95\% } \\
\mathrm{Cl})\end{array}$ & $1.50[0.62,3.61]$ \\
\hline 2.78 .3 replaced by $\mathrm{CHO}$ & 3 & 49071 & $\begin{array}{l}\text { Risk Ratio (M-H, Random, 95\% } \\
\text { Cl) }\end{array}$ & $0.82[0.39,1.72]$ \\
\hline
\end{tabular}




\begin{tabular}{|c|c|c|c|c|}
\hline Outcome or subgroup title & $\begin{array}{l}\text { No. of } \\
\text { studies }\end{array}$ & $\begin{array}{l}\text { No. of } \\
\text { partici- } \\
\text { pants }\end{array}$ & Statistical method & Effect size \\
\hline 2.78 .4 replaced by protein & 0 & 0 & $\begin{array}{l}\text { Risk Ratio (M-H, Random, 95\% } \\
\mathrm{Cl})\end{array}$ & Not estimable \\
\hline 2.78.5 replacement unclear & 1 & 267 & $\begin{array}{l}\text { Risk Ratio (M-H, Random, 95\% } \\
\mathrm{Cl})\end{array}$ & $2.93[0.31,27.84]$ \\
\hline 2.79 CHD events, subgroup by duration & 11 & & $\begin{array}{l}\text { Risk Ratio (M-H, Random, 95\% } \\
\mathrm{Cl} \text { ) }\end{array}$ & Subtotals only \\
\hline 2.79 .1 up to 24 months & 4 & 2380 & $\begin{array}{l}\text { Risk Ratio (M-H, Random, 95\% } \\
\mathrm{Cl})\end{array}$ & $1.01[0.76,1.35]$ \\
\hline $2.79 .2>24$ to 48 months & 3 & 1294 & $\begin{array}{l}\text { Risk Ratio (M-H, Random, 95\% } \\
\mathrm{Cl})\end{array}$ & $0.79[0.55,1.13]$ \\
\hline $2.79 .3>48$ months & 2 & 49247 & $\begin{array}{l}\text { Risk Ratio (M-H, Random, 95\% } \\
\text { Cl) }\end{array}$ & $0.85[0.63,1.15]$ \\
\hline 2.79.4 unclear duration & 2 & 278 & $\begin{array}{l}\text { Risk Ratio (M-H, Random, 95\% } \\
\text { Cl) }\end{array}$ & $0.60[0.10,3.58]$ \\
\hline 2.80 CHD events, subgroup by baseline SFA & 11 & & $\begin{array}{l}\text { Risk Ratio (M-H, Random, 95\% } \\
\mathrm{Cl})\end{array}$ & Subtotals only \\
\hline 2.80.1 up to $12 \%$ E SFA baseline & 0 & 0 & $\begin{array}{l}\text { Risk Ratio (M-H, Random, 95\% } \\
\mathrm{CI})\end{array}$ & Not estimable \\
\hline $2.80 .2>12$ to $15 \% E$ SFA baseline & 4 & 51311 & $\begin{array}{l}\text { Risk Ratio (M-H, Random, 95\% } \\
\mathrm{Cl} \text { ) }\end{array}$ & $0.96[0.88,1.06]$ \\
\hline $2.80 .3>15$ to $18 \% E$ SFA baseline & 1 & 55 & $\begin{array}{l}\text { Risk Ratio (M-H, Random, 95\% } \\
\mathrm{Cl})\end{array}$ & $0.31[0.10,1.01]$ \\
\hline 2.80.4 $>18 \%$ E SFA baseline & 1 & 846 & $\begin{array}{l}\text { Risk Ratio (M-H, Random, 95\% } \\
\text { Cl) }\end{array}$ & $0.77[0.56,1.04]$ \\
\hline 2.80 .5 unclear & 5 & 987 & $\begin{array}{l}\text { Risk Ratio (M-H, Random, 95\% } \\
\mathrm{Cl} \text { ) }\end{array}$ & $0.78[0.49,1.26]$ \\
\hline 2.81 CHD events, subgroup by SFA change & 11 & & $\begin{array}{l}\text { Risk Ratio (M-H, Random, 95\% } \\
\text { Cl) }\end{array}$ & Subtotals only \\
\hline 2.81.1 up to $4 \% \mathrm{E}$ difference & 4 & 51311 & $\begin{array}{l}\text { Risk Ratio (M-H, Random, 95\% } \\
\mathrm{Cl} \text { ) }\end{array}$ & $0.96[0.88,1.06]$ \\
\hline 2.81.2 $>4$ to $8 \% \mathrm{E}$ difference & 1 & 55 & $\begin{array}{l}\text { Risk Ratio (M-H, Random, 95\% } \\
\mathrm{Cl})\end{array}$ & $0.31[0.10,1.01]$ \\
\hline $2.81 .3>8 \% \mathrm{E}$ difference & 1 & 846 & $\begin{array}{l}\text { Risk Ratio (M-H, Random, 95\% } \\
\mathrm{Cl})\end{array}$ & $0.77[0.56,1.04]$ \\
\hline 2.81.4 unclear & 5 & 987 & $\begin{array}{l}\text { Risk Ratio (M-H, Random, 95\% } \\
\mathrm{Cl} \text { ) }\end{array}$ & $0.78[0.49,1.26]$ \\
\hline
\end{tabular}




\begin{tabular}{|c|c|c|c|c|}
\hline Outcome or subgroup title & $\begin{array}{l}\text { No. of } \\
\text { studies }\end{array}$ & $\begin{array}{l}\text { No. of } \\
\text { partici- } \\
\text { pants }\end{array}$ & Statistical method & Effect size \\
\hline 2.82 CHD events, subgroup by sex & 11 & & $\begin{array}{l}\text { Risk Ratio (M-H, Random, 95\% } \\
\mathrm{Cl})\end{array}$ & Subtotals only \\
\hline 2.82.1 Men & 7 & 3819 & $\begin{array}{l}\text { Risk Ratio (M-H, Random, 95\% } \\
\mathrm{Cl})\end{array}$ & $0.84[0.70,1.02]$ \\
\hline 2.82.2 Women & 1 & 48835 & $\begin{array}{l}\text { Risk Ratio (M-H, Random, 95\% } \\
\mathrm{CI})\end{array}$ & $0.97[0.87,1.07]$ \\
\hline 2.82.3 Mixed, men and women & 3 & 545 & $\begin{array}{l}\text { Risk Ratio (M-H, Random, 95\% } \\
\mathrm{CI})\end{array}$ & $0.88[0.18,4.36]$ \\
\hline 2.83 CHD events, subgroup by CVD risk & 11 & & $\begin{array}{l}\text { Risk Ratio (M-H, Random, 95\% } \\
\mathrm{Cl})\end{array}$ & Subtotals only \\
\hline 2.83.1 Low CVD risk & 2 & 47404 & $\begin{array}{l}\text { Risk Ratio (M-H, Random, 95\% } \\
\mathrm{CI})\end{array}$ & $0.90[0.76,1.05]$ \\
\hline 2.83.2 Moderate CVD risk & 3 & 545 & $\begin{array}{l}\text { Risk Ratio (M-H, Random, 95\% } \\
\mathrm{Cl})\end{array}$ & $0.88[0.18,4.36]$ \\
\hline 2.83.3 Existing CVD disease & 7 & 5250 & $\begin{array}{l}\text { Risk Ratio (M-H, Random, 95\% } \\
\mathrm{CI})\end{array}$ & $0.94[0.75,1.16]$ \\
\hline 2.84 CHD events, subgroup by TC reduction & 11 & & $\begin{array}{l}\text { Risk Ratio (M-H, Random, 95\% } \\
\text { Cl) }\end{array}$ & Subtotals only \\
\hline $\begin{array}{l}2.84 .1 \text { serum chol reduced by at least } \\
0.2 \mathrm{mmol} / \mathrm{L}\end{array}$ & 8 & 4149 & $\begin{array}{l}\text { Risk Ratio (M-H, Random, 95\% } \\
\mathrm{CI})\end{array}$ & $0.76[0.58,0.99]$ \\
\hline 2.84 .2 serum chol reduced by $<0.2 \mathrm{mmol} / \mathrm{L}$ & 3 & 49050 & $\begin{array}{l}\text { Risk Ratio (M-H, Random, 95\% } \\
\mathrm{Cl})\end{array}$ & $0.97[0.88,1.08]$ \\
\hline 2.84.3 serum chol reduction unclear & 0 & 0 & $\begin{array}{l}\text { Risk Ratio (M-H, Random, 95\% } \\
\mathrm{Cl})\end{array}$ & Not estimable \\
\hline $\begin{array}{l}\text { 2.85 CHD events, subgroup decade of pub- } \\
\text { lication }\end{array}$ & 11 & 53201 & $\begin{array}{l}\text { Risk Ratio (M-H, Random, 95\% } \\
\mathrm{CI})\end{array}$ & $0.83[0.68,1.01]$ \\
\hline $2.85 .11960 \mathrm{~s}$ & 5 & 1731 & $\begin{array}{l}\text { Risk Ratio (M-H, Random, 95\% } \\
\mathrm{Cl})\end{array}$ & $0.84[0.68,1.05]$ \\
\hline $2.85 .21970 \mathrm{~s}$ & 1 & 102 & $\begin{array}{l}\text { Risk Ratio (M-H, Random, 95\% } \\
\text { Cl) }\end{array}$ & $0.27[0.14,0.52]$ \\
\hline $2.85 .31980 \mathrm{~s}$ & 1 & 2033 & $\begin{array}{l}\text { Risk Ratio (M-H, Random, 95\% } \\
\mathrm{Cl} \text { ) }\end{array}$ & $0.91[0.73,1.14]$ \\
\hline $2.85 .41990 \mathrm{~s}$ & 1 & 57 & $\begin{array}{l}\text { Risk Ratio (M-H, Random, 95\% } \\
\mathrm{Cl})\end{array}$ & $0.33[0.10,1.09]$ \\
\hline $2.85 .52000 \mathrm{~s}$ & 3 & 49278 & $\begin{array}{l}\text { Risk Ratio (M-H, Random, 95\% } \\
\text { Cl) }\end{array}$ & $0.97[0.88,1.08]$ \\
\hline
\end{tabular}




\begin{tabular}{lllll}
\hline Outcome or subgroup title & $\begin{array}{l}\text { No. of } \\
\text { studies }\end{array}$ & $\begin{array}{l}\text { No. of } \\
\text { partici- } \\
\text { pants }\end{array}$ & Statistical method & Effect size \\
\hline 2.86 DIABETES DIAGNOSES & 1 & $\begin{array}{l}\text { Risk Ratio (M-H, Random, 95\% } \\
\text { Cl) }\end{array}$ & Subtotals only \\
\hline
\end{tabular}

Analysis 2.1. Comparison 2: SFA reduction vs usual diet secondary health events, Outcome 1: MYOCARDIAL INFARCTION

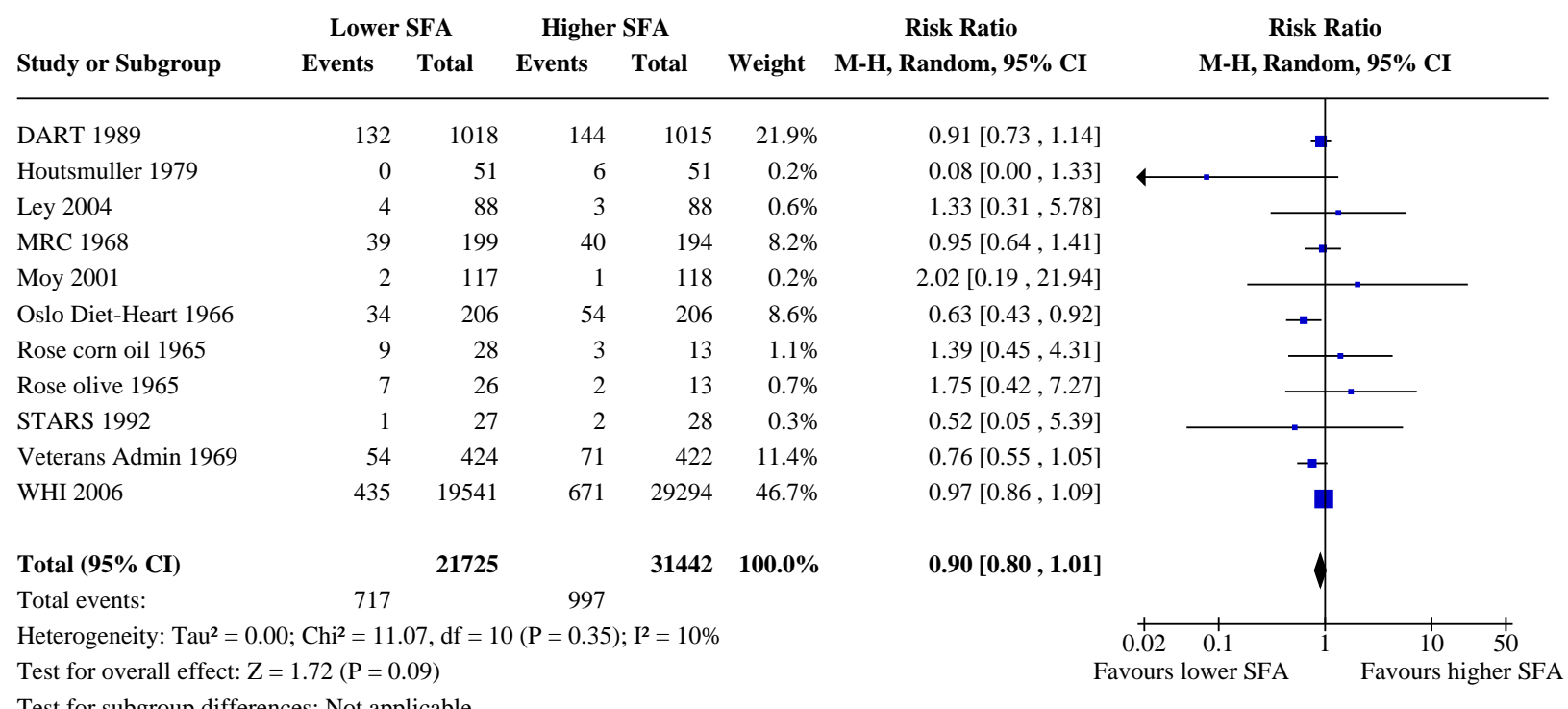

Test for subgroup differences: Not applicable

Analysis 2.2. Comparison 2: SFA reduction vs usual diet - secondary health events, Outcome 2: MI, SA by low summary risk of bias

\begin{tabular}{|c|c|c|c|c|c|c|c|}
\hline \multirow[b]{2}{*}{ Study or Subgroup } & \multicolumn{2}{|c|}{ Lower SFA } & \multicolumn{2}{|c|}{ Higher SFA } & \multirow[b]{2}{*}{ Weight } & \multirow{2}{*}{$\begin{array}{c}\text { Risk Ratio } \\
\text { M-H, Random, } 95 \% \text { CI }\end{array}$} & \multirow{2}{*}{$\begin{array}{c}\text { Risk Ratio } \\
\text { M-H, Random, } 95 \% \text { CI }\end{array}$} \\
\hline & Events & Total & Events & Total & & & \\
\hline Ley 2004 & 4 & 88 & 3 & 88 & $1.0 \%$ & $1.33[0.31,5.78]$ & \\
\hline Veterans Admin 1969 & 54 & 424 & 71 & 422 & $17.5 \%$ & $0.76[0.55,1.05]$ & \\
\hline WHI 2006 & 435 & 19541 & 671 & 29294 & $81.6 \%$ & $0.97[0.86,1.09]$ & \\
\hline Total $(95 \%$ CI) & & 20053 & & 29804 & $100.0 \%$ & $0.93[0.81,1.08]$ & \\
\hline Total events: & 493 & & 745 & & & & \\
\hline \multicolumn{7}{|c|}{ Heterogeneity: $\mathrm{Tau}^{2}=0.00 ; \mathrm{Chi}^{2}=2.19, \mathrm{df}=2(\mathrm{P}=0.33) ; \mathrm{I}^{2}=9 \%$} & $0.5 \quad 0.7$ \\
\hline \multicolumn{5}{|c|}{ Test for overall effect: $\mathrm{Z}=0.94(\mathrm{P}=0.35)$} & & & Favours lower SFA \\
\hline
\end{tabular}


Analysis 2.3. Comparison 2: SFA reduction vs usual diet secondary health events, Outcome 3: MI, SA aim to reduce SFA

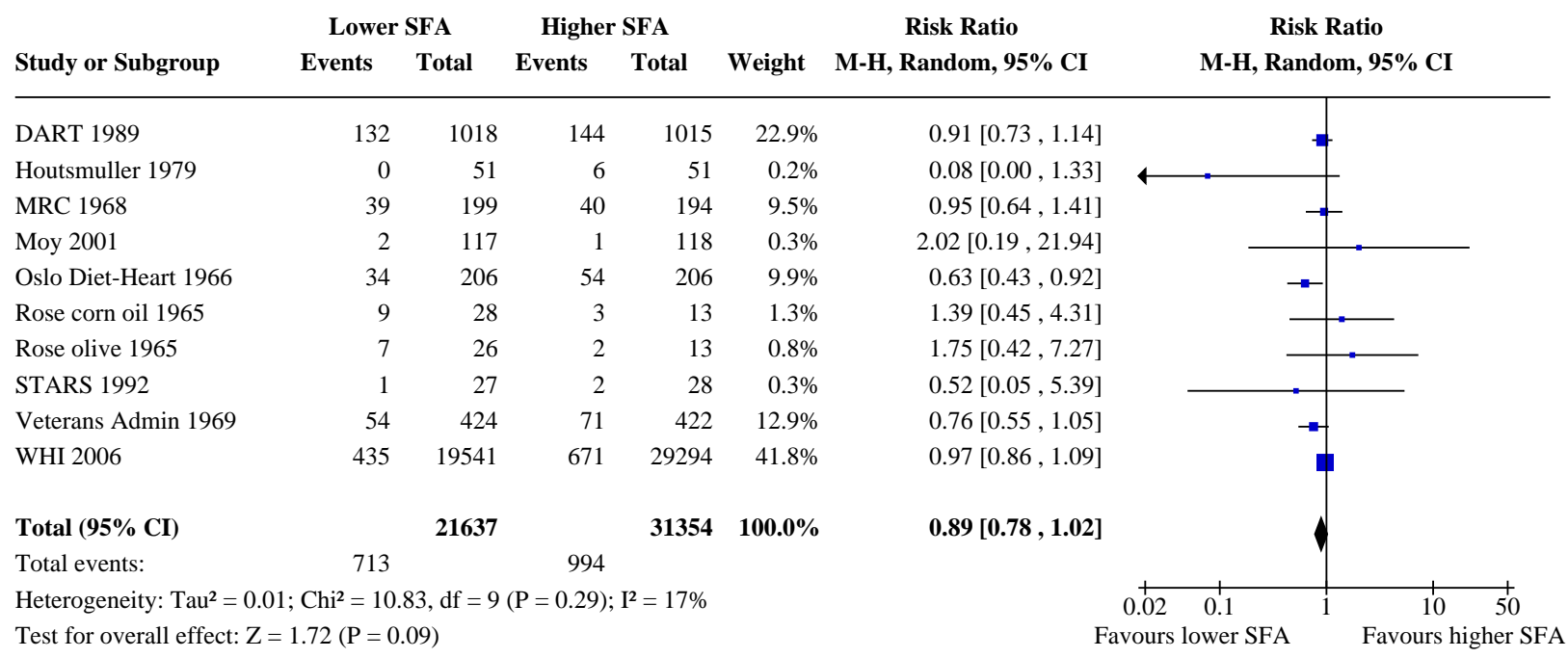

Test for subgroup differences: Not applicable

Analysis 2.4. Comparison 2: SFA reduction vs usual diet - secondary health events, Outcome 4: MI, SA statistically significant SFA reduction

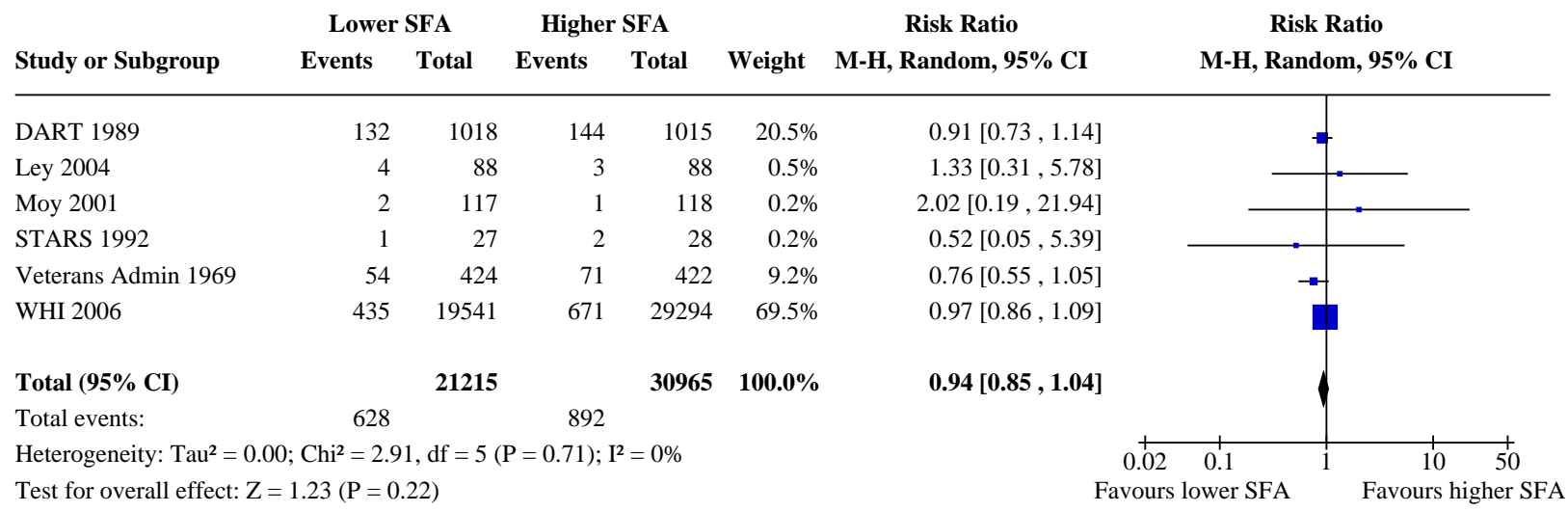


Analysis 2.5. Comparison 2: SFA reduction vs usual diet secondary health events, Outcome 5: MI, SA by TC reduction

\begin{tabular}{|c|c|c|c|c|c|c|c|}
\hline \multirow[b]{2}{*}{ Study or Subgroup } & \multicolumn{2}{|c|}{ Lower SFA } & \multicolumn{2}{|c|}{ Higher SFA } & \multirow[b]{2}{*}{ Weight } & \multirow{2}{*}{$\begin{array}{c}\text { Risk Ratio } \\
\text { M-H, Random, } 95 \% \text { CI }\end{array}$} & \multirow{2}{*}{$\begin{array}{c}\text { Risk Ratio } \\
\text { M-H, Random, 95\% CI }\end{array}$} \\
\hline & Events & Total & Events & Total & & & \\
\hline DART 1989 & 132 & 1018 & 144 & 1015 & $23.3 \%$ & $0.91[0.73,1.14]$ & \\
\hline Houtsmuller 1979 & 0 & 51 & 6 & 51 & $0.2 \%$ & $0.08[0.00,1.33]$ & 4 \\
\hline MRC 1968 & 39 & 199 & 40 & 194 & $9.9 \%$ & $0.95[0.64,1.41]$ & | \\
\hline Moy 2001 & 2 & 117 & 1 & 118 & $0.3 \%$ & $2.02[0.19,21.94]$ & \\
\hline Oslo Diet-Heart 1966 & 34 & 206 & 54 & 206 & $10.3 \%$ & $0.63[0.43,0.92]$ & $\rightarrow-$ \\
\hline Rose corn oil 1965 & 9 & 28 & 3 & 13 & $1.4 \%$ & $1.39[0.45,4.31]$ & \\
\hline STARS 1992 & 1 & 27 & 2 & 28 & $0.3 \%$ & $0.52[0.05,5.39]$ & \\
\hline Veterans Admin 1969 & 54 & 424 & 71 & 422 & $13.3 \%$ & $0.76[0.55,1.05]$ & - \\
\hline WHI 2006 & 435 & 19541 & 671 & 29294 & $40.9 \%$ & $0.97[0.86,1.09]$ & \\
\hline Total $(95 \%$ CI) & & 21611 & & 31341 & $100.0 \%$ & $0.88[0.77,1.01]$ & 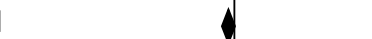 \\
\hline Total events: & 706 & & 992 & & & & \\
\hline Heterogeneity: $\mathrm{Tau}^{2}=$ & $\begin{array}{c}\mathrm{Chi}^{2}=10 \\
79(\mathrm{P}=0\end{array}$ & $4, \mathrm{df}=8$ & $(\mathrm{P}=0.26)$ & $I^{2}=20 \%$ & & & $0.02 \quad 0.1$ \\
\hline
\end{tabular}

Analysis 2.6. Comparison 2: SFA reduction vs usual diet - secondary health events, Outcome 6: MI, SA excluding WHI

\begin{tabular}{|c|c|c|c|c|c|c|c|c|}
\hline \multirow[b]{2}{*}{ Study or Subgroup } & \multicolumn{2}{|c|}{ Lower SFA } & \multicolumn{2}{|c|}{ Higher SFA } & \multirow[b]{2}{*}{ Weight } & \multirow{2}{*}{$\begin{array}{c}\text { Risk Ratio } \\
\text { M-H, Random, } 95 \% \text { CI }\end{array}$} & \multirow{2}{*}{\multicolumn{2}{|c|}{$\begin{array}{c}\text { Risk Ratio } \\
\text { M-H, Random, 95\% CI }\end{array}$}} \\
\hline & Events & Total & Events & Total & & & & \\
\hline DART 1989 & 132 & 1018 & 144 & 1015 & $44.9 \%$ & $0.91[0.73,1.14]$ & & \\
\hline Houtsmuller 1979 & 0 & 51 & 6 & 51 & $0.3 \%$ & $0.08[0.00,1.33]$ & $\longleftarrow$ & \\
\hline Ley 2004 & 4 & 88 & 3 & 88 & $1.0 \%$ & $1.33[0.31,5.78]$ & & \\
\hline MRC 1968 & 39 & 199 & 40 & 194 & $14.3 \%$ & $0.95[0.64,1.41]$ & & 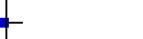 \\
\hline Moy 2001 & 2 & 117 & 1 & 118 & $0.4 \%$ & $2.02[0.19,21.94]$ & & \\
\hline Oslo Diet-Heart 1966 & 34 & 206 & 54 & 206 & $15.1 \%$ & $0.63[0.43,0.92]$ & 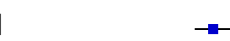 & \\
\hline Rose corn oil 1965 & 9 & 28 & 3 & 13 & $1.8 \%$ & $1.39[0.45,4.31]$ & & \\
\hline Rose olive 1965 & 7 & 26 & 2 & 13 & $1.1 \%$ & $1.75[0.42,7.27]$ & & \\
\hline STARS 1992 & 1 & 27 & 2 & 28 & $0.4 \%$ & $0.52[0.05,5.39]$ & & \\
\hline Veterans Admin 1969 & 54 & 424 & 71 & 422 & $20.7 \%$ & $0.76[0.55,1.05]$ & 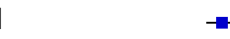 & \\
\hline Total $(95 \%$ CI $)$ & & 2184 & & 2148 & $100.0 \%$ & $0.85[0.73,0.98]$ & & \\
\hline Total events: & 282 & & 326 & & & & & \\
\hline Heterogeneity: $\mathrm{Tau}^{2}=$ & ; $\mathrm{Chi}^{2}=9$. & $7, \mathrm{df}=9$ & $P=0.43) ; I$ & $=1 \%$ & & & 0.02 & 10 \\
\hline
\end{tabular}




\section{Analysis 2.7. Comparison 2: SFA reduction vs usual diet - secondary health events, Outcome 7: MI, SA Mantel-Haenszel fixed-effect}

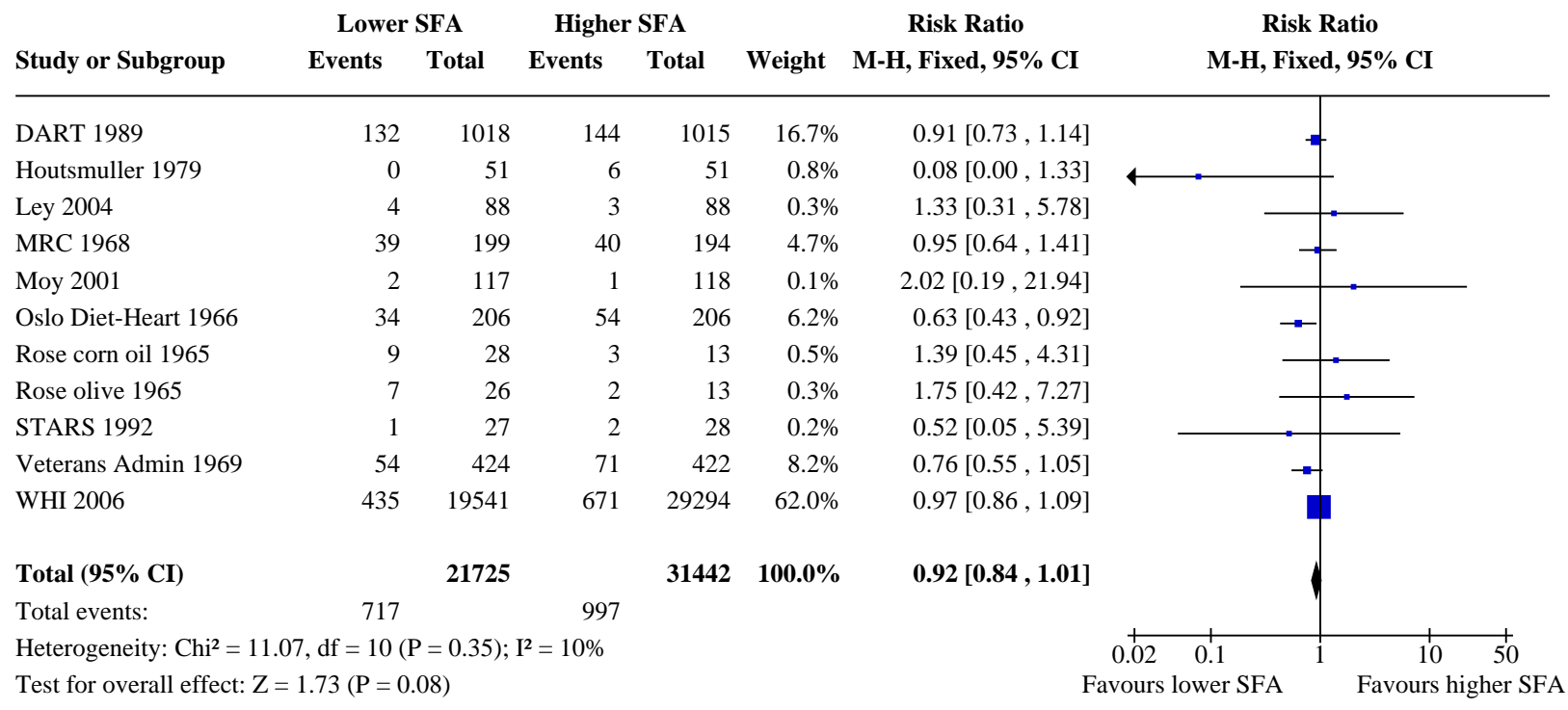

Test for subgroup differences: Not applicable

Analysis 2.8. Comparison 2: SFA reduction vs usual diet secondary health events, Outcome 8: MI, SA Peto fixed-effect

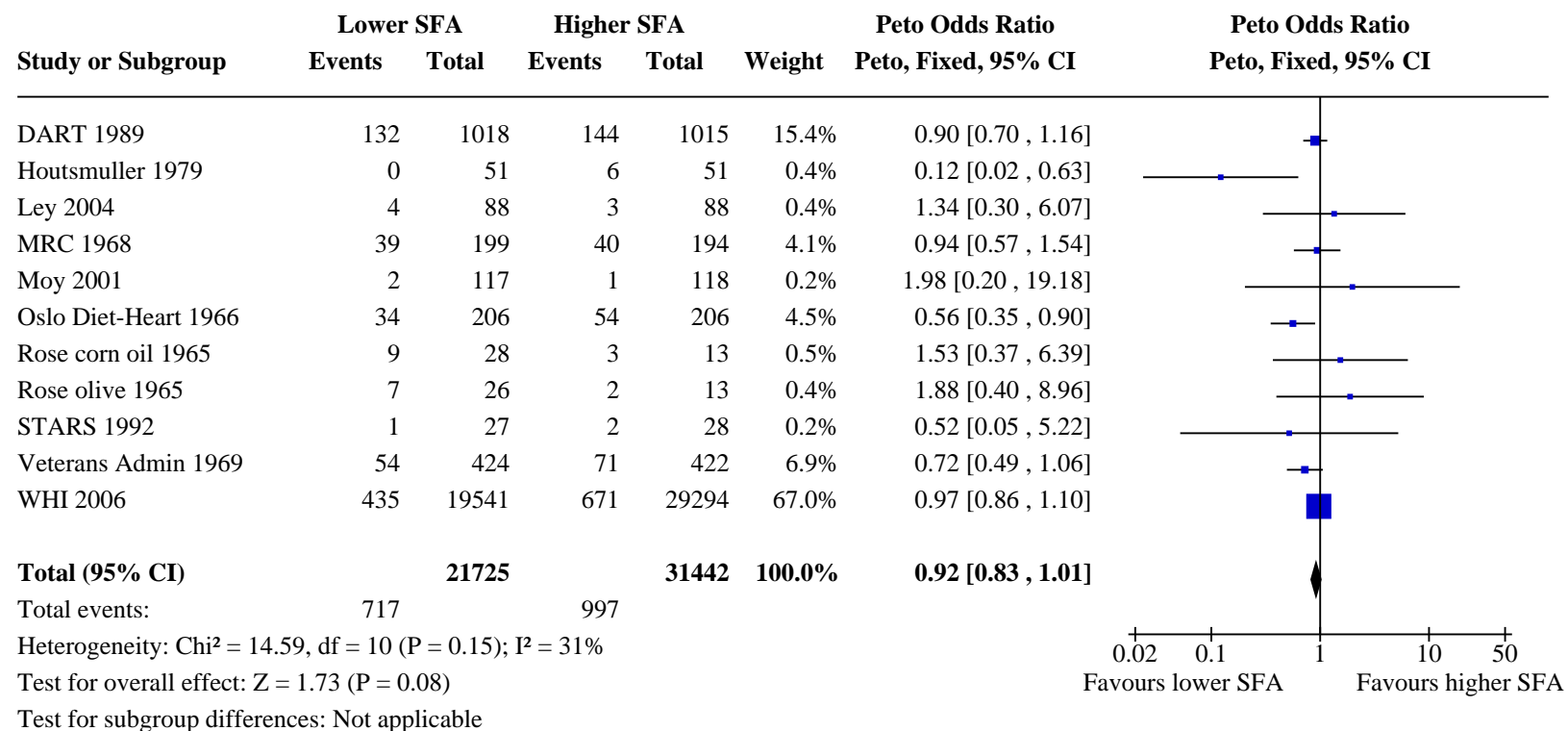




\section{Analysis 2.9. Comparison 2: SFA reduction vs usual diet - secondary health events, Outcome 9: MI, subgroup by any substitution}

\begin{tabular}{lrrrrr} 
Study or Subgroup & $\begin{array}{c}\text { Lower SFA } \\
\text { Events }\end{array}$ & $\begin{array}{c}\text { Total } \\
\text { Events }\end{array}$ & $\begin{array}{l}\text { Total } \\
\text { Weight }\end{array}$ & Wer SFA \\
\hline 2.9.1 replaced by PUFA & & & & & \\
DART 1989 & 132 & 1018 & 144 & 1015 & $34.0 \%$ \\
Houtsmuller 1979 & 0 & 51 & 6 & 51 & $0.5 \%$ \\
MRC 1968 & 39 & 199 & 40 & 194 & $18.3 \%$ \\
Oslo Diet-Heart 1966 & 34 & 206 & 54 & 206 & $19.0 \%$ \\
Rose corn oil 1965 & 9 & 28 & 5 & 26 & $4.3 \%$ \\
STARS 1992 & 1 & 27 & 2 & 28 & $0.8 \%$ \\
Veterans Admin 1969 & 54 & 424 & 71 & 422 & $23.1 \%$ \\
Subtotal (95\% CI) & & $\mathbf{1 9 5 3}$ & & $\mathbf{1 9 4 2}$ & $\mathbf{1 0 0 . 0 \%}$ \\
Total events: & 269 & & 322 & &
\end{tabular}

Heterogeneity: $\mathrm{Tau}^{2}=0.02 ; \mathrm{Chi}^{2}=8.40, \mathrm{df}=6(\mathrm{P}=0.21) ; \mathrm{I}^{2}=29 \%$

Test for overall effect: $\mathrm{Z}=1.79(\mathrm{P}=0.07)$

\subsection{2 replaced by MUFA}

Rose olive 1965

Subtotal (95\% CI)

Total events:

$7-26$

26
26

5

26

$100.0 \%$

$26 \quad 100.0 \%$

7

Heterogeneity: Not applicable

Test for overall effect: $\mathrm{Z}=0.65(\mathrm{P}=0.51)$

\subsection{3 replace by $\mathrm{CHO}$}

DART 1989

Ley 2004

STARS 1992

WHI 2006

Subtotal (95\% CI)

Total events:

Heterogeneity: $\mathrm{Tau}^{2}=0.00 ; \mathrm{Chi}^{2}=0.69, \mathrm{df}=3(\mathrm{P}=0.88) ; \mathrm{I}^{2}=0 \%$

Test for overall effect: $\mathrm{Z}=0.79(\mathrm{P}=0.43)$

2.9.4 replaced by protein

$\begin{array}{lrrrrr}\text { DART 1989 } & 132 & 1018 & 144 & 1015 & 22.7 \% \\ \text { Ley 2004 } & 4 & 88 & 3 & 88 & 0.5 \% \\ \text { WHI 2006 } & 435 & 19541 & 671 & 29294 & 76.8 \% \\ \text { Subtotal (95\% CI) } & & \mathbf{2 0 6 4 7} & & \mathbf{3 0 3 9 7} & \mathbf{1 0 0 . 0 \%} \\ \text { Total events: } & 571 & & 818 & & \end{array}$

Heterogeneity: $\mathrm{Tau}^{2}=0.00 ; \mathrm{Chi}^{2}=0.43, \mathrm{df}=2(\mathrm{P}=0.81) ; \mathrm{I}^{2}=0 \%$

Test for overall effect: $\mathrm{Z}=0.77(\mathrm{P}=0.44)$

2.9.5 replacement unclear

Moy 2001

$2 \quad 117$

117
117

1

$118 \quad 100.0 \%$

$2.02[0.19,21.94]$

Subtotal $(95 \%$ CI)

2

Total events:

$118 \quad \mathbf{1 0 0 . 0 \%}$

$2.02[0.19,21.94]$

$0.91[0.73,1.14]$

$1.33[0.31,5.78]$

$0.97[0.86,1.09]$

$0.96[0.86,1.07]$

$0.96[0.86,1.07]$
Risk Ratio

M-H, Random, $95 \%$ CI

Heterogeneity: Not applicable

Test for overall effect: $\mathrm{Z}=0.58(\mathrm{P}=0.56)$

Test for subgroup differences: $\mathrm{Chi}^{2}=2.70, \mathrm{df}=4(\mathrm{P}=0.61), \mathrm{I}^{2}=0 \%$

$1.40[0.51,3.85]$

$1.40[0.51,3.85]$

$0.91[0.73,1.14]$

$1.33[0.31,5.78]$

$0.52[0.05,5.39]$

$0.97[0.86,1.09]$

$0.96[0.86,1.06]$

(

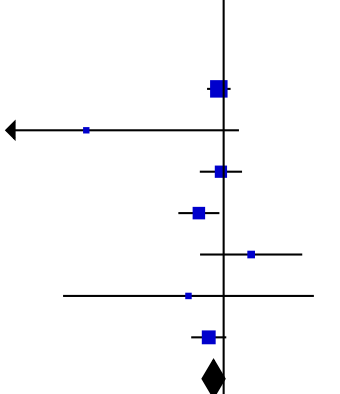

$0.91[0.73,1.14]$

$0.95[0.64,1.41]$

$1.67[0.64,4.34]$

$[0.05,5.39]$

$0.83[0.67,1.02]$

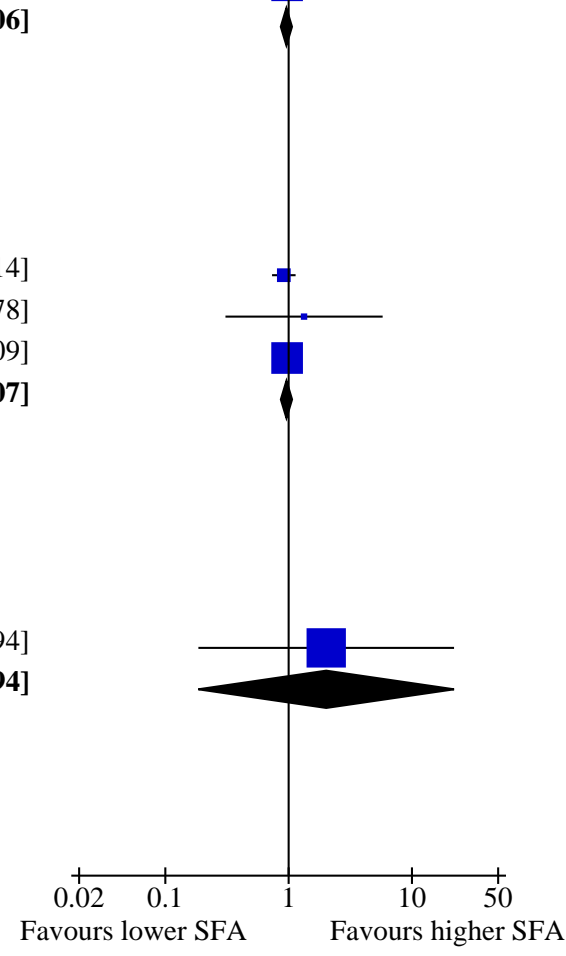

Reduction in saturated fat intake for cardiovascular disease (Review) 


\section{Analysis 2.10. Comparison 2: SFA reduction vs usual diet - secondary health events, Outcome 10: MI, subgroup by main substitution}

Weight M-H, Random, 95\% CI

M-H, Random, 95\% CI

\subsection{1 replaced by PUFA}

DART 1989

Houtsmuller 1979

MRC 1968

Oslo Diet-Heart 1966

Rose corn oil 1965

Veterans Admin 1969

Subtotal (95\% CI)

Total events:

Heterogeneity: $\mathrm{Tau}^{2}=0.03 ; \mathrm{Chi}^{2}=8.23, \mathrm{df}=5(\mathrm{P}=0.14) ; \mathrm{I}^{2}=39 \%$

Test for overall effect: $\mathrm{Z}=1.63(\mathrm{P}=0.10)$

\subsection{2 replaced by MUFA}

Rose olive 1965

Subtotal $(95 \%$ CI)

$7 \quad 26$

\section{5}

$26 \quad 100.0 \%$

$1.40[0.51,3.85]$

$1.40[0.51,3.85]$

$0.91[0.73,1.14]$

$0.08[0.00,1.33]$

$0.95[0.64,1.41]$

$0.63[0.43,0.92]$

$1.67[0.64,4.34]$

$0.76[0.55,1.05]$

$0.83[0.67,1.04]$

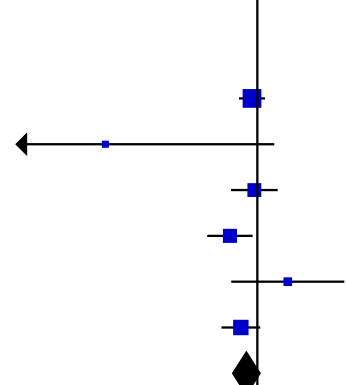

Total events:

Heterogeneity: Not applicable

Test for overall effect: $\mathrm{Z}=0.65(\mathrm{P}=0.51)$

\subsection{3 replace by $\mathrm{CHO}$}

Ley 2004

STARS 1992

WHI 2006

Subtotal (95\% CI)

Total events:

Heterogeneity: $\mathrm{Tau}^{2}=0.00 ; \mathrm{Chi}^{2}=0.45, \mathrm{df}=2(\mathrm{P}=0.80) ; \mathrm{I}^{2}=0 \%$

Test for overall effect: $\mathrm{Z}=0.46(\mathrm{P}=0.64)$

\subsection{4 replaced by protein}

Subtotal (95\% CI)

0

0

Total events:

0

0

Heterogeneity: Not applicable

Test for overall effect: Not applicable

\subsection{5 replacement unclear}

Moy 2001

Subtotal (95\% CI)

Total events:

Heterogeneity: Not applicable

Test for overall effect: $\mathrm{Z}=0.58(\mathrm{P}=0.56)$

Test for subgroup differences: $\mathrm{Chi}^{2}=2.48, \mathrm{df}=3(\mathrm{P}=0.48), \mathrm{I}^{2}=0 \%$

\section{$1.33[0.31,5.78]$ \\ $0.52[0.05,5.39]$ \\ $0.97[0.86,1.09]$ \\ $0.97[0.86,1.09]$}

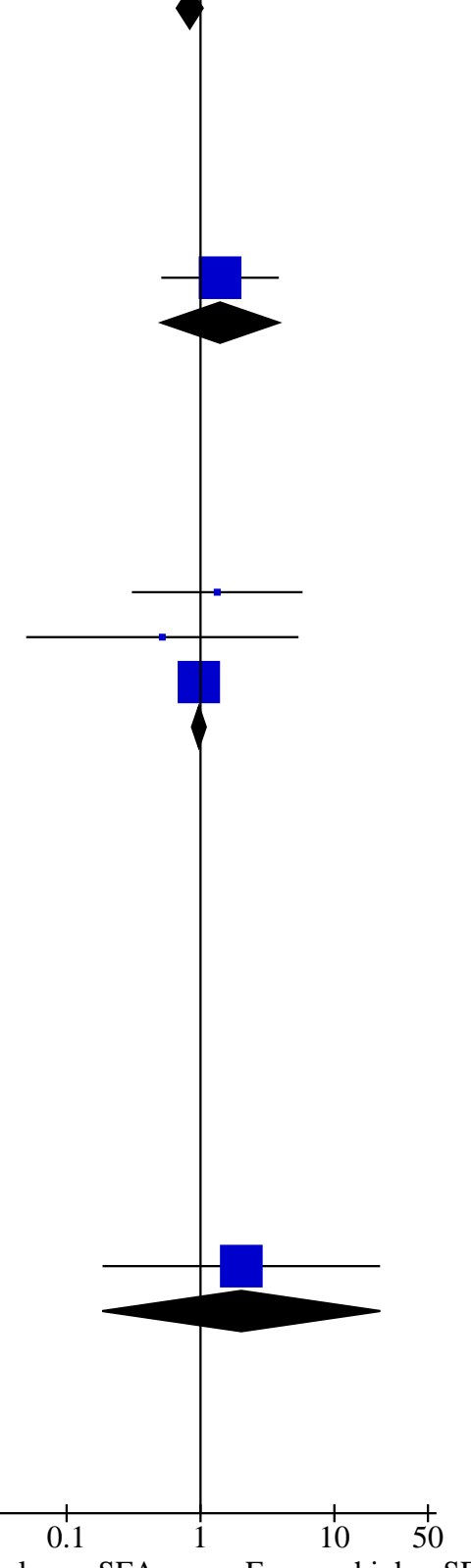

$2.02[0.19,21.94]$

$2.02[0.19,21.94]$

Not estimable

0.02

Favours lower SFA
Favours higher SFA 
Analysis 2.11. Comparison 2: SFA reduction vs usual diet secondary health events, Outcome 11: MI, subgroup by duration

\begin{tabular}{lrrrrr} 
& \multicolumn{2}{c}{ Lower SFA } & \multicolumn{2}{c}{ Higher SFA } & \\
Study or Subgroup & Events & Total & Events & Total & Weight \\
\hline 2.11.1 up to 24mo & & & & & \\
DART 1989 & 132 & 1018 & 144 & 1015 & $21.9 \%$ \\
Moy 2001 & 2 & 117 & 1 & 118 & $0.2 \%$ \\
Rose corn oil 1965 & 9 & 28 & 3 & 13 & $1.1 \%$ \\
Rose olive 1965 & 7 & 26 & 2 & 13 & $0.7 \%$ \\
Subtotal (95\% CI) & & $\mathbf{1 1 8 9}$ & & $\mathbf{1 1 5 9}$ & $\mathbf{2 3 . 9 \%}$ \\
Total events: & 150 & & 150 & &
\end{tabular}

Risk Ratio

H, Random, $95 \%$ CI
Risk Ratio

M-H, Random, $95 \%$ CI

Heterogeneity: $\mathrm{Tau}^{2}=0.00 ; \mathrm{Chi}^{2}=1.65, \mathrm{df}=3(\mathrm{P}=0.65) ; \mathrm{I}^{2}=0 \%$

Test for overall effect: $\mathrm{Z}=0.50(\mathrm{P}=0.62)$

\subsection{2 >24 to $48 \mathrm{mo}$}

\section{MRC 1968}

STARS 1992

Veterans Admin 1969

Subtotal (95\% CI)

Total events:

Heterogeneity: $\mathrm{Tau}^{2}=0.00 ; \mathrm{Chi}^{2}=0.91, \mathrm{df}=2(\mathrm{P}=0.63) ; \mathrm{I}^{2}=0 \%$

Test for overall effect: $\mathrm{Z}=1.49(\mathrm{P}=0.13)$

\subsection{3 >48mo}

Oslo Diet-Heart 1966

WHI 2006

Subtotal (95\% CI)

Total events:

$\begin{array}{rr}34 & 206 \\ 435 & 1954 \\ & \mathbf{1 9 7 4}\end{array}$

469

19747

54
671

206

29294

29500

$8.6 \%$

$46.7 \%$ 725

Heterogeneity: $\mathrm{Tau}^{2}=0.07 ; \mathrm{Chi}^{2}=4.50, \mathrm{df}=1(\mathrm{P}=0.03) ; \mathrm{I}^{2}=78 \%$

Test for overall effect: $\mathrm{Z}=0.96(\mathrm{P}=0.34)$

\subsection{4 unclear}

Houtsmuller 1979

Ley 2004

Subtotal (95\% CI)

Total events:

Heterogeneity: $\mathrm{Tau}^{2}=3.31 ; \mathrm{Chi}^{2}=3.47, \mathrm{df}=1(\mathrm{P}=0.06) ; \mathrm{I}^{2}=71 \%$

Test for overall effect: $\mathrm{Z}=0.60(\mathrm{P}=0.55)$

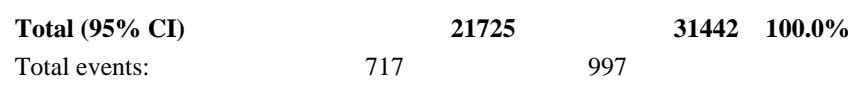

Heterogeneity: $\mathrm{Tau}^{2}=0.00 ; \mathrm{Chi}^{2}=11.07, \mathrm{df}=10(\mathrm{P}=0.35) ; \mathrm{I}^{2}=10 \%$

Test for overall effect: $\mathrm{Z}=1.72(\mathrm{P}=0.09)$

Test for subgroup differences: $\mathrm{Chi}^{2}=1.10, \mathrm{df}=3(\mathrm{P}=0.78), \mathrm{I}^{2}=0 \%$
$0.91[0.73,1.14]$ $2.02[0.19,21.94]$

$1.39[0.45,4.31]$

$1.75[0.42,7.27]$

$0.95[0.77,1.17]$

$0.95[0.64,1.41]$ $0.52[0.05,5.39]$ $0.76[0.55,1.05]$ $0.83[0.64,1.06]$

$0.63[0.43,0.92]$ $0.97[0.86,1.09]$ $0.81[0.54,1.24]$

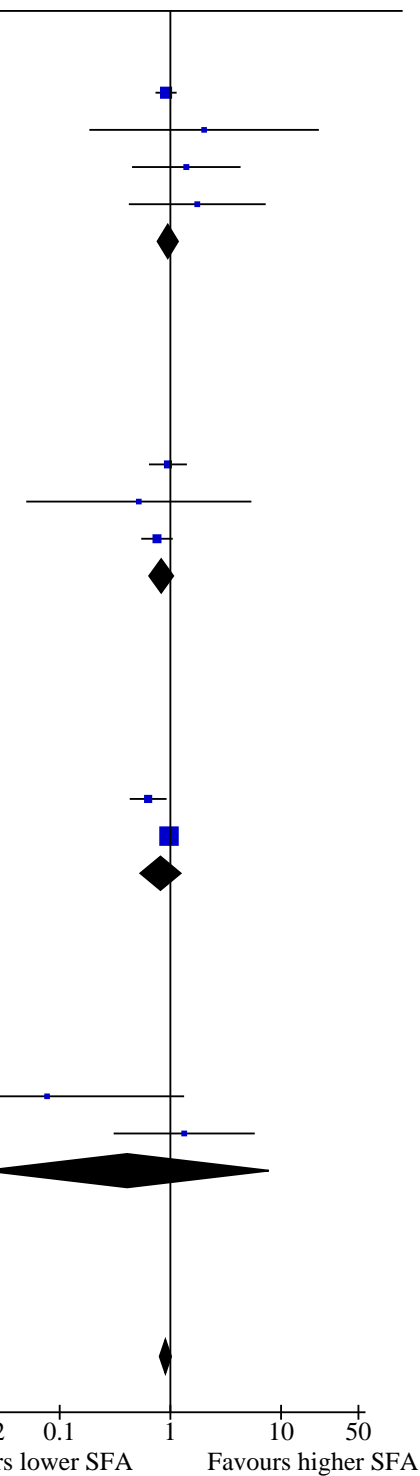


Analysis 2.12. Comparison 2: SFA reduction vs usual diet secondary health events, Outcome 12: MI, subgroup by baseline SFA

Lower SFA

Higher SFA

Events Tota
Risk Ratio

Weight M-H, Random, 95\% CI
Risk Ratio

M-H, Random, $95 \%$ CI

\subsection{1 up to $12 \%$ E SFA baseline}

Subtotal (95\% CI)

Total events:

0

Heterogeneity: Not applicable

Test for overall effect: Not applicable

2.12.2 $>12$ to $15 \%$ E SFA baseline

DART 1989

Ley 2004

Moy 2001

WHI 2006

Subtotal $(95 \%$ CI $)$

Total events:

132
4
2
435

1018

88

117

19541

20764

\author{
Events
}

573
Heterogeneity: $\mathrm{Tau}^{2}=0.00 ; \mathrm{Chi}^{2}=0.80, \mathrm{df}=3(\mathrm{P}=0.85) ; \mathrm{I}^{2}=0 \%$

Test for overall effect: $\mathrm{Z}=0.74(\mathrm{P}=0.46)$

\subsection{3 >15 to $18 \%$ E SFA baseline}

STARS 1992

Subtotal $(95 \%$ CI)

Not estimable

Total events:

Heterogeneity: Not applicable

Test for overall effect: $\mathrm{Z}=0.55(\mathrm{P}=0.58)$

\subsection{4 $>18 \%$ E SFA baseline}

Subtotal (95\% CI)

Total events:

Heterogeneity: Not applicable

Test for overall effect: $\mathrm{Z}=1.67(\mathrm{P}=0.10)$

\subsection{5 unclear}

Houtsmuller 1979

MRC 1968

Oslo Diet-Heart 1966

Rose corn oil 1965

Rose olive 1965

Subtotal $(95 \%$ CI)

Total events:

$\begin{array}{rr}0 & 51 \\ 39 & 199 \\ 34 & 206 \\ 9 & 28 \\ 7 & 26 \\ & \mathbf{5 1 0}\end{array}$

$\begin{array}{rrr}6 & 51 & 0.2 \% \\ 40 & 194 & 8.2 \% \\ 54 & 206 & 8.6 \% \\ 3 & 13 & 1.1 \% \\ 2 & 13 & 0.7 \% \\ & \mathbf{4 7 7} & \mathbf{1 8 . 8 \%}\end{array}$

105

Heterogeneity: $\mathrm{Tau}^{2}=0.09 ; \mathrm{Chi}^{2}=6.98, \mathrm{df}=4(\mathrm{P}=0.14) ; \mathrm{I}^{2}=43 \%$

Test for overall effect: $\mathrm{Z}=0.78(\mathrm{P}=0.44)$

Total $(95 \%$ CI)

$31442100.0 \%$

Total events:

717 997

Heterogeneity: $\mathrm{Tau}^{2}=0.00 ; \mathrm{Chi}^{2}=11.07, \mathrm{df}=10(\mathrm{P}=0.35) ; \mathrm{I}^{2}=10 \%$

Test for overall effect: $\mathrm{Z}=1.72(\mathrm{P}=0.09)$

Test for subgroup differences: $\mathrm{Chi}^{2}=2.34, \mathrm{df}=3(\mathrm{P}=0.50), \mathrm{I}^{2}=0 \%$
$0.91[0.73,1.14]$

$2.02[0.19,21.94]$

$0.97[0.86,1.09]$

$0.96[0.87,1.07]$

$0.52[0.05,5.39]$

$0.52[0.05,5.39]$

$0.76[0.55,1.05]$

$0.76[0.55,1.05]$
$1.33[0.31,5.78]$
$0.08[0.00,1.33]$

$0.95[0.64,1.41]$

$0.63[0.43,0.92]$

$1.39[0.45,4.31]$

$1.75[0.42,7.27]$

$0.84[0.54,1.30]$

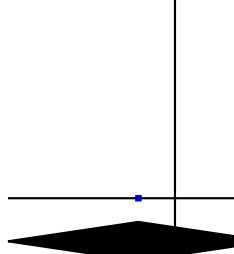


Analysis 2.13. Comparison 2: SFA reduction vs usual diet secondary health events, Outcome 13: MI, subgroup by SFA change
Lower SFA
Higher SFA
Risk Ratio
Risk Ratio

Study or Subgroup Events Total

Events Tota

Weight M-H, Random, 95\% CI

2.13.1 up to $4 \% \mathrm{E}$ difference DART 1989

Ley 2004

Moy 2001

WHI 2006

Subtotal $(95 \%$ CI $)$

Total events:

132

\section{8}

144

1015

$21.9 \%$

$0.6 \%$

$118 \quad 0.2 \%$

$2 \quad 117$

$435 \quad 19541$

20764

573

$671 \quad 29294 \quad 46.7 \%$

$30515 \quad 69.5 \%$ 819

Heterogeneity: $\mathrm{Tau}^{2}=0.00 ; \mathrm{Chi}^{2}=0.80, \mathrm{df}=3(\mathrm{P}=0.85) ; \mathrm{I}^{2}=0 \%$

Test for overall effect: $\mathrm{Z}=0.74(\mathrm{P}=0.46)$

2.13.2 $>4$ to $8 \% \mathrm{E}$ difference

STARS 1992

Subtotal (95\% CI)

1

Total events:

27

Heterogeneity:

: Not applicable

\subsection{3 $>8 \% \mathrm{E}$ difference}

Veterans Admin 1969

Subtotal (95\% CI)

$54 \quad 424$

$424 \quad 71$

71

422

$11.4 \%$

Total events:

54

71

$0.3 \%$

$\mathbf{0 . 3 \%}$

Heterogeneity: Not applicable

Test for overall effect: $\mathrm{Z}=1.67(\mathrm{P}=0.10)$

\subsection{4 unclear}

Houtsmuller 1979

MRC 1968

Oslo Diet-Heart 1966

Rose corn oil 1965

Rose olive 1965

Subtotal (95\% CI)

Total events:

Heterogeneity: $\mathrm{Tau}^{2}=0.09 ; \mathrm{Chi}^{2}=6.98, \mathrm{df}=4(\mathrm{P}=0.14) ; \mathrm{I}^{2}=43 \%$

Test for overall effect: $\mathrm{Z}=0.78(\mathrm{P}=0.44)$

Total $(95 \%$ CI $)$

21725

$31442100.0 \%$

Total events:

717 997

Heterogeneity: $\mathrm{Tau}^{2}=0.00 ; \mathrm{Chi}^{2}=11.07, \mathrm{df}=10(\mathrm{P}=0.35) ; \mathrm{I}^{2}=10 \%$

Test for overall effect: $\mathrm{Z}=1.72(\mathrm{P}=0.09)$

Test for subgroup differences: $\mathrm{Chi}^{2}=2.34, \mathrm{df}=3(\mathrm{P}=0.50), \mathrm{I}^{2}=0 \%$
M-H, Random, $95 \%$ CI

$0.91[0.73,1.14]$

$1.33[0.31,5.78]$

$2.02[0.19,21.94]$

$0.97[0.86,1.09]$

0.96 [0.87, 1.07]

$0.52[0.05,5.39]$

$0.52[0.05,5.39]$

$0.76[0.55,1.05]$

$0.76[0.55,1.05]$

$0.08[0.00,1.33]$

$0.95[0.64,1.41]$

$0.63[0.43,0.92]$

$1.39[0.45,4.31]$

$1.75[0.42,7.27]$

$0.84[0.54,1.30]$ 
Analysis 2.14. Comparison 2: SFA reduction vs usual diet secondary health events, Outcome 14: MI, subgroup by sex

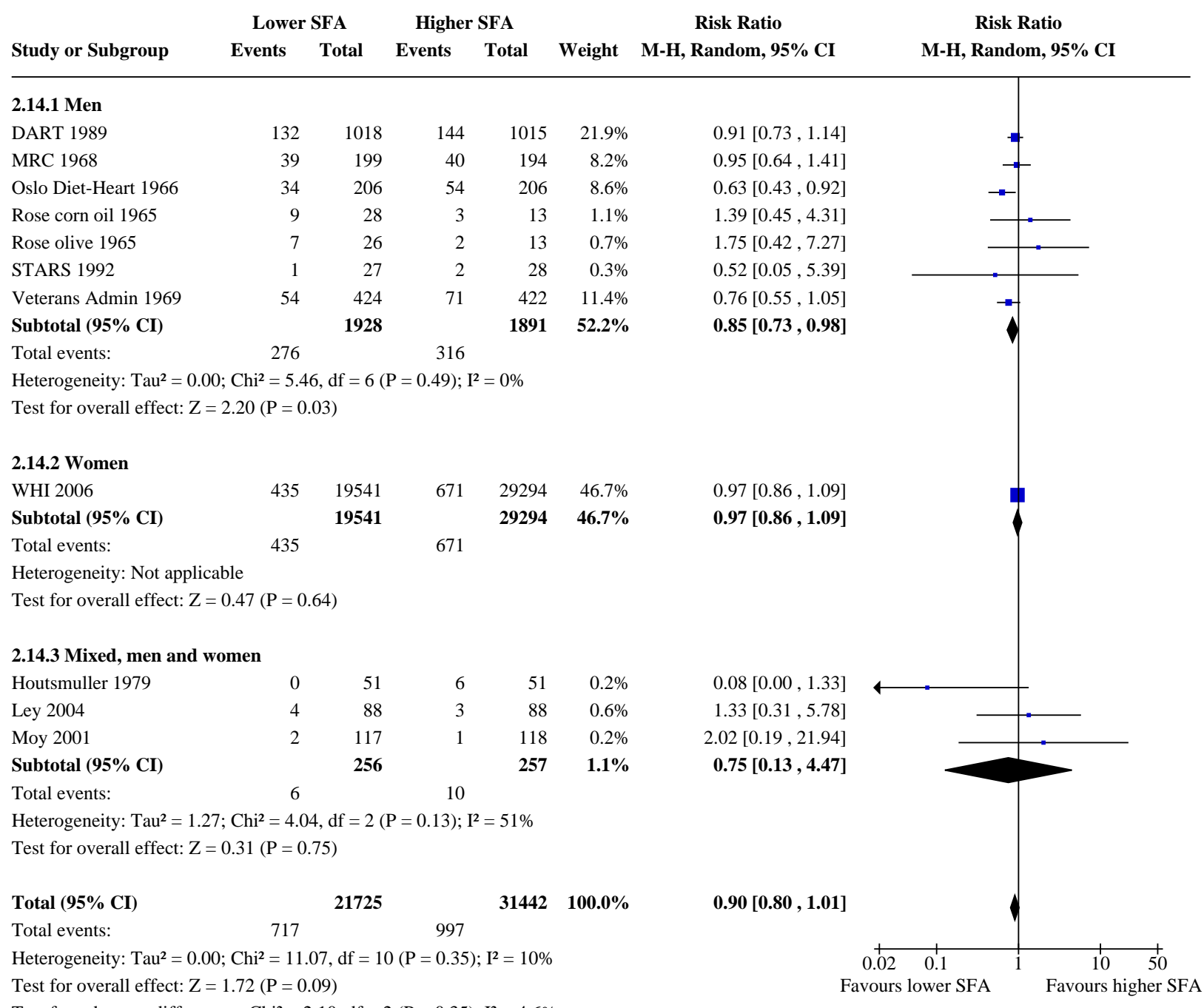




\section{Analysis 2.15. Comparison 2: SFA reduction vs usual diet - secondary health events, Outcome 15: MI, subgroup by CVD risk}

\begin{tabular}{|c|c|c|c|c|c|c|c|}
\hline \multirow[b]{2}{*}{ Study or Subgroup } & \multicolumn{2}{|c|}{ Lower SFA } & \multicolumn{2}{|c|}{ Higher SFA } & \multirow[b]{2}{*}{ Weight } & \multirow{2}{*}{$\begin{array}{c}\text { Risk Ratio } \\
\text { M-H, Random, } 95 \% \text { CI }\end{array}$} & \multirow{2}{*}{$\begin{array}{c}\text { Risk Ratio } \\
\text { M-H, Random, } 95 \% \text { CI }\end{array}$} \\
\hline & Events & Total & Events & Total & & & \\
\hline \multicolumn{8}{|l|}{ 2.15.1 Low CVD risk } \\
\hline Veterans Admin 1969 & 54 & 424 & 71 & 422 & $11.4 \%$ & $0.76[0.55,1.05]$ & $\rightarrow$ \\
\hline WHI 2006 & 435 & 19541 & 671 & 29294 & $46.7 \%$ & $0.97[0.86,1.09]$ & \\
\hline Subtotal (95\% CI) & & 19965 & & 29716 & $58.2 \%$ & $0.90[0.72,1.13]$ & \\
\hline Total events: & 489 & & 742 & & & & \\
\hline \multicolumn{8}{|c|}{ Heterogeneity: $\mathrm{Tau}^{2}=0.02 ; \mathrm{Chi}^{2}=1.98, \mathrm{df}=1(\mathrm{P}=0.16) ; \mathrm{I}^{2}=49 \%$} \\
\hline \multicolumn{8}{|c|}{ Test for overall effect: $\mathrm{Z}=0.91(\mathrm{P}=0.36)$} \\
\hline \multicolumn{8}{|c|}{ 2.15.2 Moderate CVD risk } \\
\hline Houtsmuller 1979 & 0 & 51 & 6 & 51 & $0.2 \%$ & $0.08[0.00,1.33]$ & $\longleftarrow$ \\
\hline Ley 2004 & 4 & 88 & 3 & 88 & $0.6 \%$ & $1.33[0.31,5.78]$ & \\
\hline Moy 2001 & 2 & 117 & 1 & 118 & $0.2 \%$ & $2.02[0.19,21.94]$ & \\
\hline Subtotal $(95 \%$ CI $)$ & & 256 & & 257 & $1.1 \%$ & $0.75[0.13,4.47]$ & \\
\hline Total events: & 6 & & 10 & & & & \\
\hline \multicolumn{8}{|c|}{ Heterogeneity: $\mathrm{Tau}^{2}=1.27 ; \mathrm{Chi}^{2}=4.04, \mathrm{df}=2(\mathrm{P}=0.13) ; \mathrm{I}^{2}=51 \%$} \\
\hline \multicolumn{8}{|c|}{ Test for overall effect: $Z=0.31(P=0.75)$} \\
\hline \multicolumn{8}{|c|}{ 2.15.3 Existing CVD disease } \\
\hline DART 1989 & 132 & 1018 & 144 & 1015 & $21.9 \%$ & $0.91[0.73,1.14]$ & \\
\hline MRC 1968 & 39 & 199 & 40 & 194 & $8.2 \%$ & $0.95[0.64,1.41]$ & \\
\hline Oslo Diet-Heart 1966 & 34 & 206 & 54 & 206 & $8.6 \%$ & $0.63[0.43,0.92]$ & - \\
\hline Rose corn oil 1965 & 9 & 28 & 3 & 13 & $1.1 \%$ & $1.39[0.45,4.31]$ & 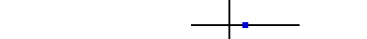 \\
\hline Rose olive 1965 & 7 & 26 & 2 & 13 & $0.7 \%$ & $1.75[0.42,7.27]$ & \\
\hline STARS 1992 & 1 & 27 & 2 & 28 & $0.3 \%$ & $0.52[0.05,5.39]$ & - \\
\hline Subtotal $(95 \%$ CI $)$ & & 1504 & & 1469 & $40.8 \%$ & $0.87[0.74,1.03]$ & \\
\hline Total events: & 222 & & 245 & & & & \\
\hline \multicolumn{8}{|c|}{ Heterogeneity: $\mathrm{Tau}^{2}=0.00 ; \mathrm{Chi}^{2}=4.90, \mathrm{df}=5(\mathrm{P}=0.43) ; \mathrm{I}^{2}=0 \%$} \\
\hline \multicolumn{8}{|c|}{ Test for overall effect: $\mathrm{Z}=1.62(\mathrm{P}=0.10)$} \\
\hline Total $(95 \% \mathrm{CI})$ & & 21725 & & 31442 & $100.0 \%$ & $0.90[0.80,1.01]$ & 1 \\
\hline Total events: & 717 & & 997 & & & & \\
\hline \multirow{2}{*}{\multicolumn{5}{|c|}{$\begin{array}{l}\text { Heterogeneity: } \mathrm{Tau}^{2}=0.00 ; \mathrm{Chi}^{2}=11.07, \mathrm{df}=10(\mathrm{P}=0.35) ; \mathrm{I}^{2}=10 \% \\
\text { Test for overall effect: } \mathrm{Z}=1.72(\mathrm{P}=0.09)\end{array}$}} & & & $0.02 \quad 0.1$ \\
\hline & & & & & & & avours lower SFA \\
\hline
\end{tabular}


Analysis 2.16. Comparison 2: SFA reduction vs usual diet secondary health events, Outcome 16: MI, subgroup by TC reduction

\begin{tabular}{|c|c|c|c|c|c|c|c|}
\hline \multirow[b]{2}{*}{ Subgr } & \multicolumn{2}{|c|}{ Lower SFA } & \multicolumn{2}{|c|}{ Higher SFA } & \multicolumn{2}{|r|}{ Risk Ratio } & Risk Ratio \\
\hline & Events & Total & Events & Total & Weight & M-H, Random, $95 \%$ CI & M-H, Random, 95\% \\
\hline
\end{tabular}

2.16.1 serum chol reduced by at least $0.2 \mathrm{mmol} / \mathrm{L}$

Houtsmuller 1979

$\begin{array}{lllll}132 & 1018 & 144 & 1015 & 41.0 \%\end{array}$

MRC 1968

51

$\begin{array}{rrr}144 & 1015 & 41.0 \% \\ 6 & 51 & 0.4 \%\end{array}$

MRC 1968

$39 \quad 199$

$\begin{array}{rrr}6 & 51 & 0.4 \% \\ 40 & 194 & 16.2 \%\end{array}$

Oslo Diet-Heart 1966

117

1

$\begin{array}{rr}194 & 16.2 \% \\ 118 & 0.5 \%\end{array}$

Rose corn oil 1965

STARS 1992

$34 \quad 206$

54

$206 \quad 17.0 \%$

Veterans Admin 1969

28

$13 \quad 2.2 \%$

Subtotal (95\% CI)

$1 \quad 27$

Total events:

2070

Heterogeneity: $\mathrm{Tau}^{2}=0.01 ; \mathrm{Chi}^{2}=7.68, \mathrm{df}=7(\mathrm{P}=0.36) ; \mathrm{I}^{2}=9 \%$

Test for overall effect: $\mathrm{Z}=2.17(\mathrm{P}=0.03)$

2.16.2 serum chol reduced by $<0.2 \mathrm{mmol} / \mathrm{L}$

$\begin{array}{lrrrrr}\text { Ley 2004 } & 4 & 88 & 3 & 88 & 0.7 \% \\ \text { Rose olive 1965 } & 7 & 26 & 2 & 13 & 0.7 \% \\ \text { WHI 2006 } & 435 & 19541 & 671 & 29294 & 98.7 \% \\ \text { Subtotal (95\% CI) } & & \mathbf{1 9 6 5 5} & & \mathbf{2 9 3 9 5} & \mathbf{1 0 0 . 0 \%} \\ \text { Total events: } & 446 & & 676 & & \end{array}$

$1.33[0.31,5.78]$

$1.75[0.42,7.27]$

$0.97[0.86,1.09]$

$0.98[0.87,1.10]$

Heterogeneity: $\mathrm{Tau}^{2}=0.00 ; \mathrm{Chi}^{2}=0.82, \mathrm{df}=2(\mathrm{P}=0.66) ; \mathrm{I}^{2}=0 \%$

Test for overall effect: $\mathrm{Z}=0.37(\mathrm{P}=0.71)$

2.16.3 serum chol reduction unclear

Subtotal (95\% CI)

0

Total events:

0

(1)

Heterogeneity: Not applicable

Test for overall effect: Not applicable

Test for subgroup differences: $\mathrm{Chi}^{2}=2.45, \mathrm{df}=1(\mathrm{P}=0.12), \mathrm{I}^{2}=59.1 \%$

$0.91[0.73,1.14]$

$[0.00,1.33]$

$02[0.19,21.94]$

$0.63[0.43,0.92]$

$1.39[0.45,4.31]$

$0.52[0.05,5.39]$

$0.76[0.55,1.05]$

$0.83[0.70,0.98]$
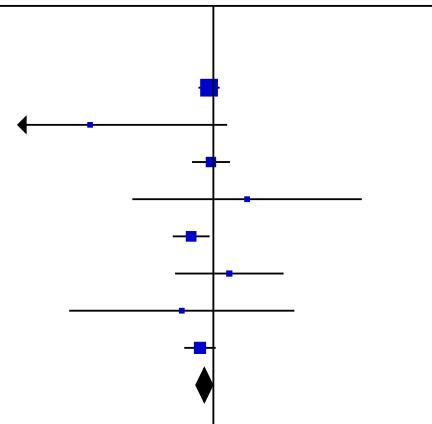


\section{Analysis 2.17. Comparison 2: SFA reduction vs usual diet - secondary health events, Outcome 17: MI, subgroup decade of publication}

Lower SFA Events Total
Higher SFA

Study or Subgroup

\author{
Events Tota
}

Risk Ratio

Weight M-H, Random, 95\% CI
Risk Ratio

M-H, Random, 95\% CI

\subsubsection{0s}

MRC 1968

Oslo Diet-Heart 1966

Rose corn oil 1965

Rose olive 1965

Veterans Admin 1969

Subtotal (95\% CI)

Total events:

$\begin{array}{rr}39 & 199 \\ 34 & 206 \\ 9 & 28 \\ 7 & 26 \\ 54 & 424 \\ & \mathbf{8 8 3}\end{array}$

$\begin{array}{rrr}40 & 194 & 8.2 \% \\ 54 & 206 & 8.6 \% \\ 3 & 13 & 1.1 \% \\ 2 & 13 & 0.7 \% \\ 71 & 422 & 11.4 \% \\ & \mathbf{8 4 8} & \mathbf{3 0 . 0 \%}\end{array}$

Heterogeneity: $\mathrm{Tau}^{2}=0.01 ; \mathrm{Chi}^{2}=4.43, \mathrm{df}=4(\mathrm{P}=0.35) ; \mathrm{I}^{2}=10 \%$

Test for overall effect: $\mathrm{Z}=1.98(\mathrm{P}=0.05)$

\subsubsection{0s}

Houtsmuller 1979

\section{0}

$$
51
$$

6

$51 \quad 0.2 \%$

Subtotal $(95 \%$ CI)

0

Heterogeneity: Not applicable

Test for overall effect: $\mathrm{Z}=1.76(\mathrm{P}=0.08)$

\subsubsection{0s}

\section{DART 1989}

\section{Subtotal $(95 \%$ CI $)$}

Total events:

132

$\begin{array}{ll}1018 & 144 \\ 1018 & \end{array}$

$1015 \quad 21.9 \%$

$1015 \quad 21.9 \%$

Heterogeneity: Not applicable

Test for overall effect: $\mathrm{Z}=0.80(\mathrm{P}=0.42)$

\subsubsection{0s}

\section{STARS 1992}

Subtotal (95\% CI)

Total events:

Heterogeneity: Not applicable

Test for overall effect: $\mathrm{Z}=0.55(\mathrm{P}=0.58)$

\subsubsection{0s}

Ley 2004

Moy 2001

WHI 2006

Subtotal (95\% CI)

Total events:

Heterogeneity: $\mathrm{Tau}^{2}=0.00 ; \mathrm{Chi}^{2}=0.53, \mathrm{df}=2(\mathrm{P}=0.77) ; \mathrm{I}^{2}=0 \%$

Test for overall effect: $\mathrm{Z}=0.41(\mathrm{P}=0.68)$
Total (95\% CI)

Total events:

Heterogeneity: $\mathrm{Tau}^{2}=0.00 ; \mathrm{Chi}^{2}=11.07, \mathrm{df}=10(\mathrm{P}=0.35) ; \mathrm{I}^{2}=10 \%$

Test for overall effect: $\mathrm{Z}=1.72(\mathrm{P}=0.09)$

Test for subgroup differences: $\mathrm{Chi}^{2}=5.62, \mathrm{df}=4(\mathrm{P}=0.23), \mathrm{I}^{2}=28.8 \%$
$0.95[0.64,1.41]$

$0.63[0.43,0.92]$

$1.39[0.45,4.31]$

$1.75[0.42,7.27]$

$0.76[0.55,1.05]$

$0.80[0.64,1.00]$

$0.08[0.00,1.33]$

$0.08[0.00,1.33]$

$0.91[0.73,1.14]$

$0.91[0.73,1.14]$
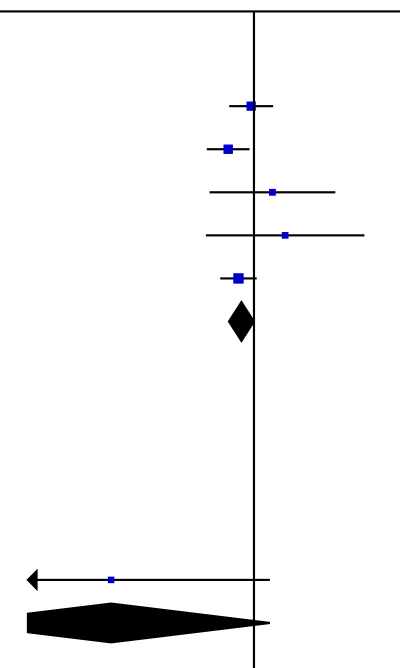

$0.52[0.05,5.39]$
$1.33[0.31,5.78]$

2.02 [0.19, 21.94]

$0.97[0.86,1.09]$
$0.98[0.87,1.10]$
$0.52[0.05,5.39]$
$0.3 \%$
0.3\%

$0.6 \%$

$0.2 \%$

$47.6 \%$

19541

29294

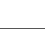




\section{Analysis 2.18. Comparison 2: SFA reduction vs usual diet - secondary health events, Outcome 18: NON-FATAL MYOCARDIAL INFARCTION}

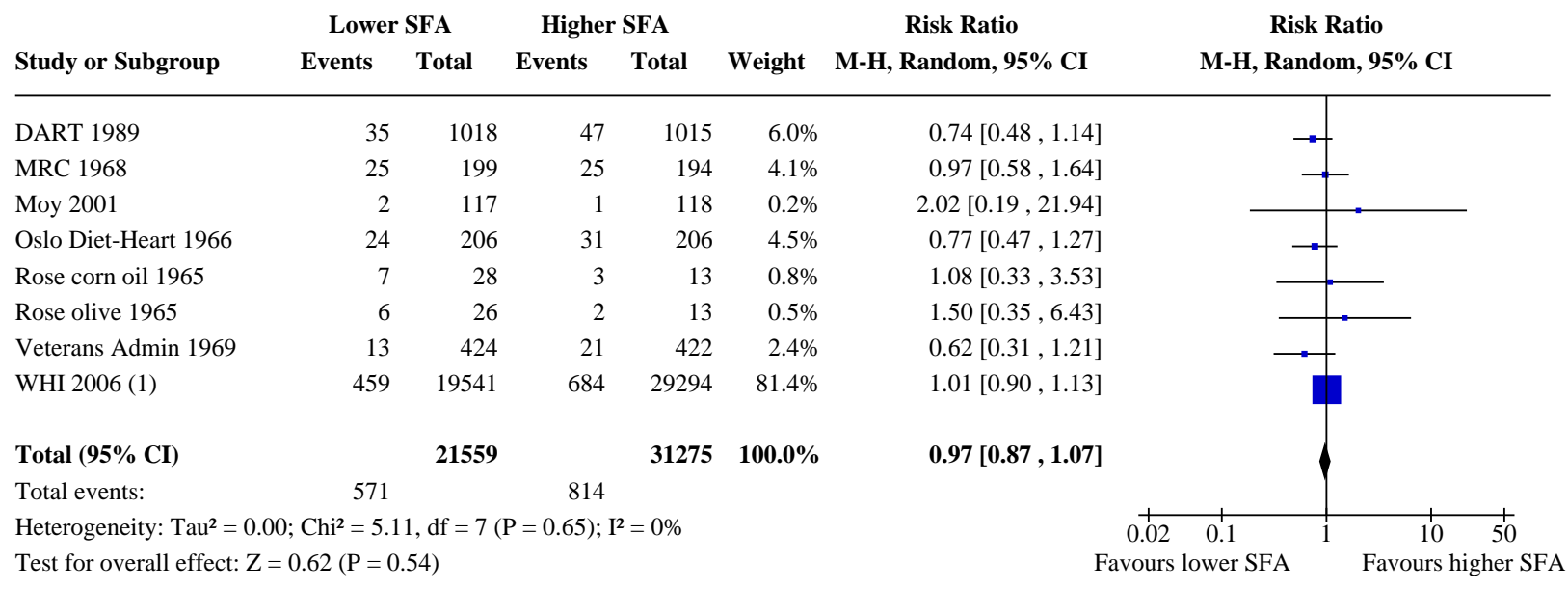

Test for subgroup differences: Not applicable

Footnotes

(1) Non-fatal MI during trial, Prentice 2017

Analysis 2.19. Comparison 2: SFA reduction vs usual diet - secondary health events, Outcome 19: Non-fatal MI, SA by low summary risk of bias

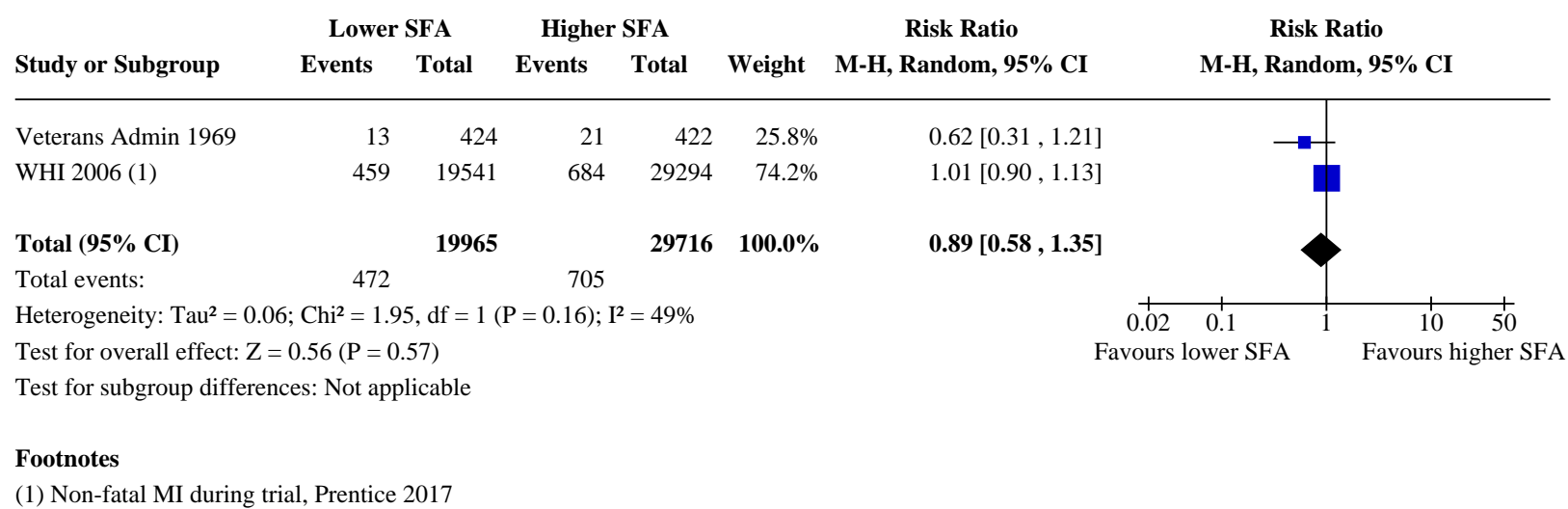




\section{Analysis 2.20. Comparison 2: SFA reduction vs usual diet - secondary} health events, Outcome 20: Non-fatal MI, SA aim to reduce SFA

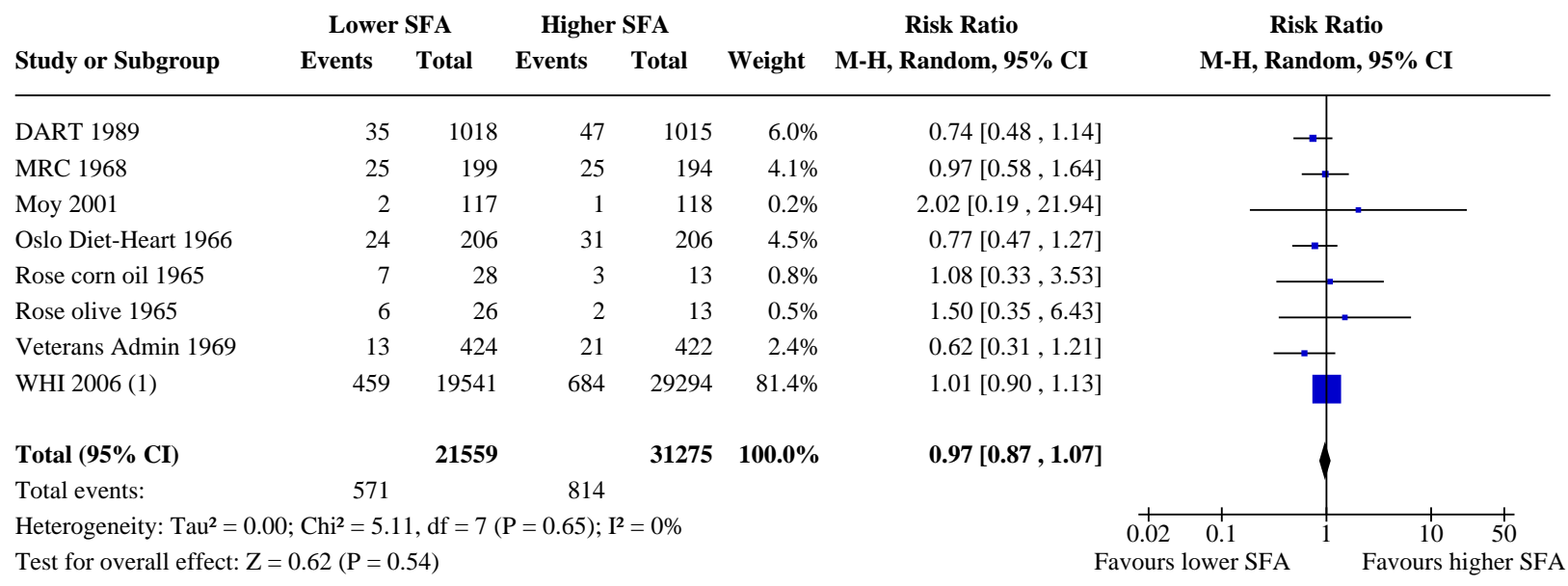

Test for subgroup differences: Not applicable

Footnotes

(1) Non-fatal MI during trial, Prentice 2017

Analysis 2.21. Comparison 2: SFA reduction vs usual diet - secondary health events, Outcome 21: Non-fatal MI, SA statistically significant SFA reduction

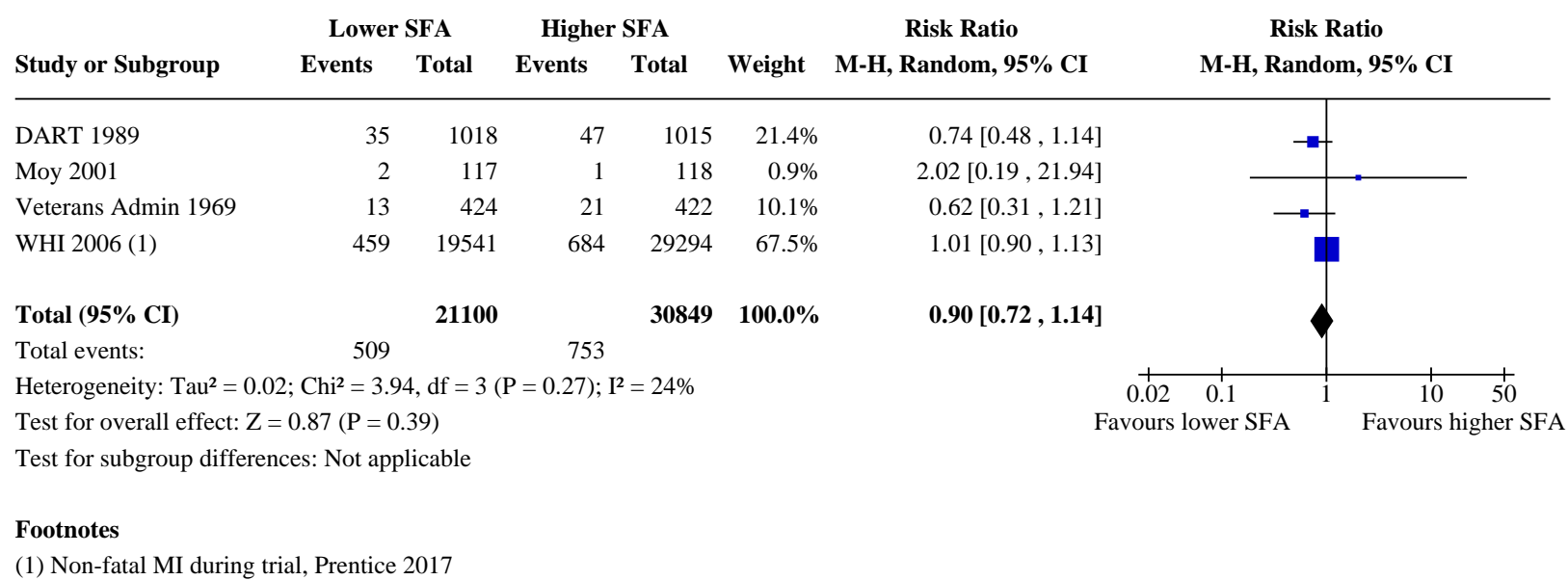




\section{Analysis 2.22. Comparison 2: SFA reduction vs usual diet - secondary} health events, Outcome 22: Non-fatal MI, SA by TC reduction

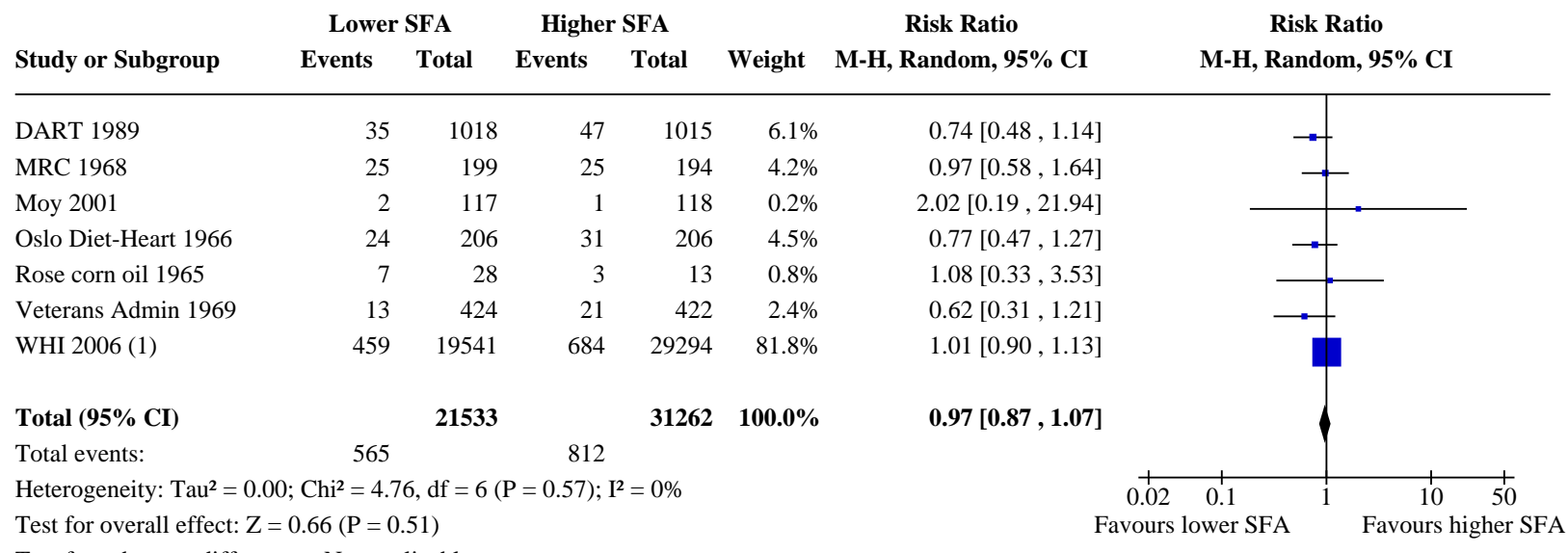

Test for subgroup differences: Not applicable

Footnotes

(1) Non-fatal MI during trial, Prentice 2017

\section{Analysis 2.23. Comparison 2: SFA reduction vs usual diet - secondary health events, Outcome 23: Non-fatal MI, SA excluding WHI}

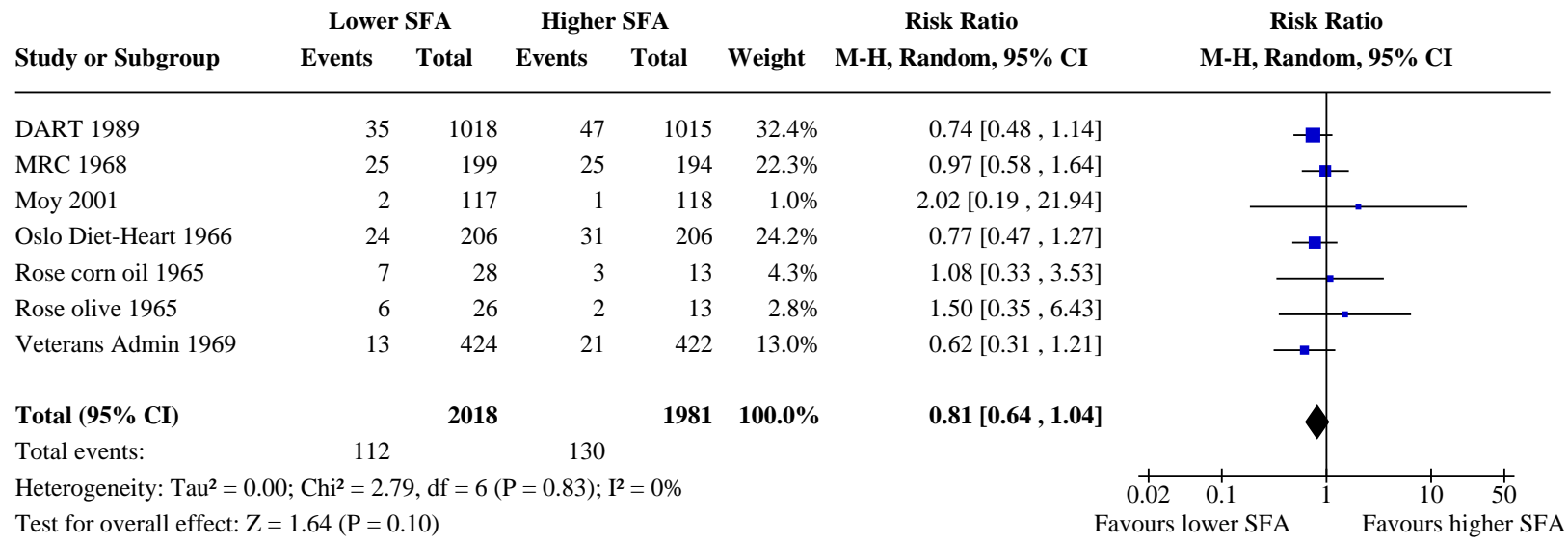


Analysis 2.24. Comparison 2: SFA reduction vs usual diet - secondary health events, Outcome 24: Non-fatal MI, SA Mantel-Haenszel fixed-effect

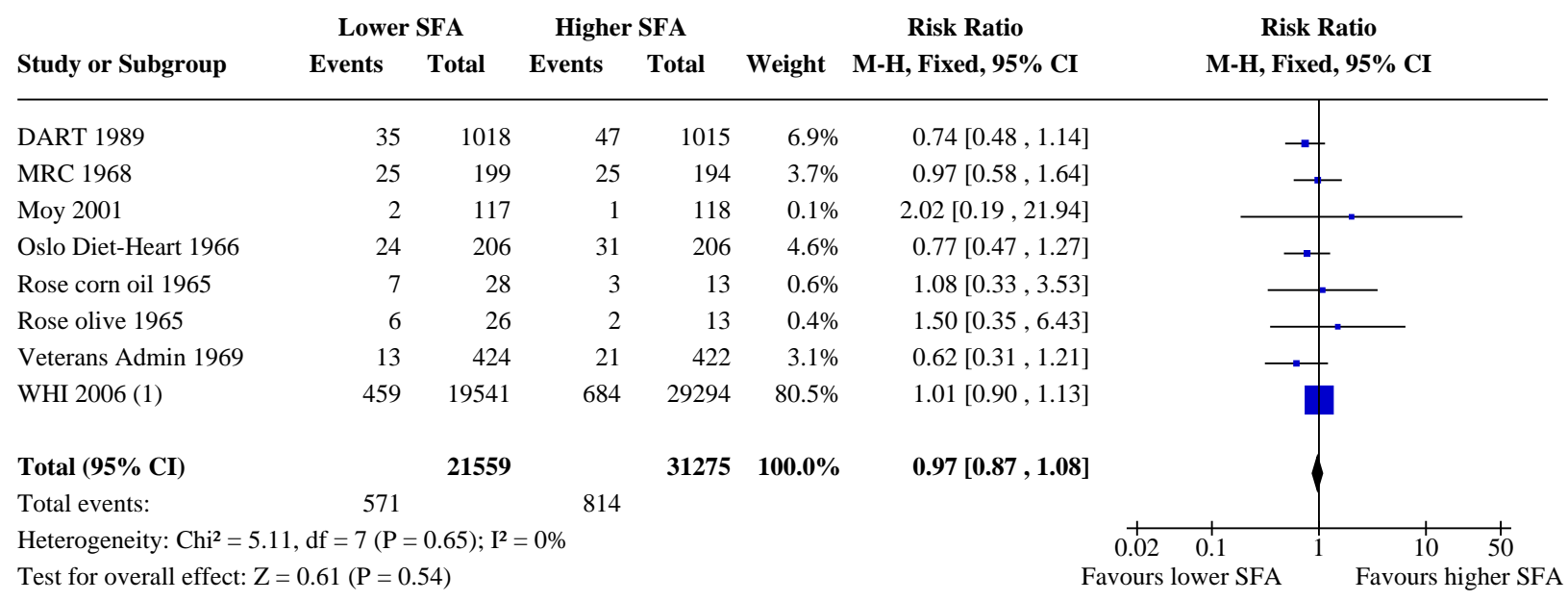

Test for subgroup differences: Not applicable

Footnotes

(1) Non-fatal MI during trial, Prentice 2017

\section{Analysis 2.25. Comparison 2: SFA reduction vs usual diet - secondary health events, Outcome 25: Non-fatal MI, SA Peto fixed-effect}

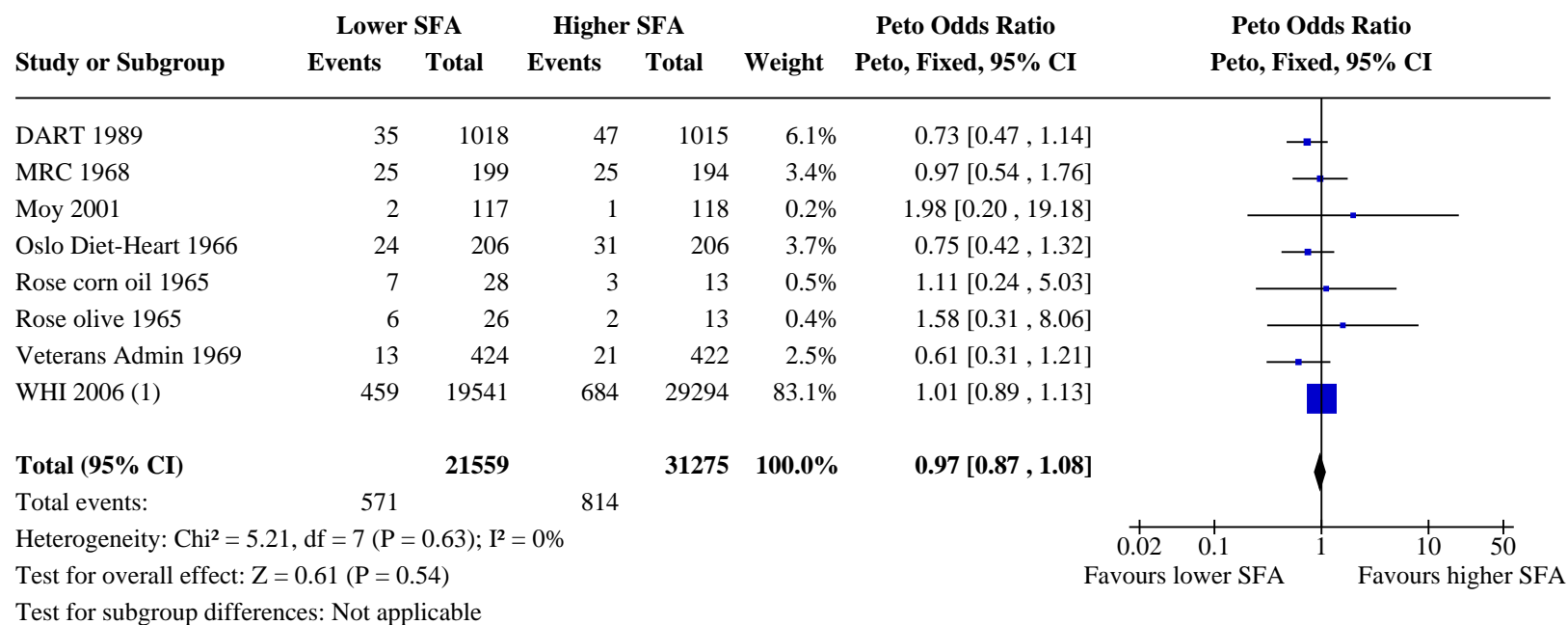

Footnotes

(1) Non-fatal MI during trial, Prentice 2017 
Analysis 2.26. Comparison 2: SFA reduction vs usual diet - secondary health events, Outcome 26: Non-fatal MI, subgroup by any substitution

\section{Study or Subgroup \\ 2.26.1 replaced by PUFA \\ DART 1989}

Lower SFA
Higher SFA

Events Total
Risk Ratio

Weight M-H, Random, 95\% CI
Risk Ratio

M-H, Random, 95\% CI

MRC 1968

Oslo Diet-Heart 1966

Rose corn oil 1965

Veterans Admin 1969

Subtotal (95\% CI)

Total events:

$\begin{array}{rr}35 & 1018 \\ 25 & 199 \\ 24 & 206 \\ 7 & 28 \\ 13 & 424 \\ & \mathbf{1 8 7 5}\end{array}$

47

1015

$33.2 \%$

$194 \quad 22.8 \%$

Heterogeneity: $\mathrm{Tau}^{2}=0.00 ; \mathrm{Chi}^{2}=2.14, \mathrm{df}=4(\mathrm{P}=0.71) ; \mathrm{I}^{2}=0 \%$

Test for overall effect: $\mathrm{Z}=1.72(\mathrm{P}=0.09)$

\subsection{2 replaced by MUFA}

Rose olive 1965

$6 \quad 26$

5

$26 \quad 5.9 \%$

$422 \quad 13.3 \%$

Subtotal (95\% CI)

26

6

Heterogeneity: Not applicable

Test for overall effect: $\mathrm{Z}=0.34(\mathrm{P}=0.73)$

\subsection{3 replace by CHO}

\section{DART 1989}

WHI 2006 (1)

Subtotal (95\% CI)

Total events:

Heterogeneity: $\mathrm{Tau}^{2}=0.02 ; \mathrm{Chi}^{2}=1.79, \mathrm{df}=1(\mathrm{P}=0.18) ; \mathrm{I}^{2}=44 \%$

Test for overall effect: $\mathrm{Z}=0.55(\mathrm{P}=0.58)$

\subsection{4 replaced by protein}

$\begin{array}{lrrrrr}\text { DART 1989 } & 35 & 1018 & 47 & 1015 & 26.0 \% \\ \text { WHI 2006 (1) } & 459 & 19541 & 684 & 29294 & 74.0 \% \\ \text { Subtotal (95\% CI) } & & \mathbf{2 0 5 5 9} & & \mathbf{3 0 3 0 9} & \mathbf{1 0 0 . 0 \%} \\ \text { Total events: } & 494 & & 731 & & \end{array}$

Heterogeneity: $\mathrm{Tau}^{2}=0.02 ; \mathrm{Chi}^{2}=1.79, \mathrm{df}=1(\mathrm{P}=0.18) ; \mathrm{I}^{2}=44 \%$

Test for overall effect: $\mathrm{Z}=0.55(\mathrm{P}=0.58)$

\subsection{5 replacement unclear}

$\begin{array}{llllll}\text { Moy } 2001 & 2 & 117 & 1 & 118 & 100.0 \% \\ \text { Subtotal (95\% CI) } & & \mathbf{1 1 7} & & \mathbf{1 1 8} & \mathbf{1 0 0 . 0 \%} \\ \text { Total events: } & 2 & & 1 & & \end{array}$

Heterogeneity: Not applicable

Test for overall effect: $\mathrm{Z}=0.58(\mathrm{P}=0.56)$

Test for subgroup differences: $\mathrm{Chi}^{2}=1.61, \mathrm{df}=4(\mathrm{P}=0.81), \mathrm{I}^{2}=0 \%$

\section{Footnotes}

(1) Non-fatal MI during trial, Prentice 2017
$0.74[0.48,1.14]$

$0.97[0.58,1.64]$

$0.77[0.47,1.27]$

$1.30[0.47,3.59]$

$0.62[0.31,1.21]$

$0.80[0.63,1.03]$

$1.20[0.42,3.45]$
$\mathbf{1 . 2 0}[\mathbf{0 . 4 2}, \mathbf{3 . 4 5}]$

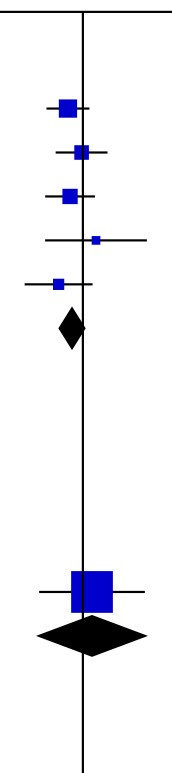

$0.74[0.48,1.14]$

$1.01[0.90,1.13]$

$0.93[0.72,1.21]$

$0.74[0.48,1.14]$

$1.01[0.90,1.13]$

$0.93[0.72,1.21]$

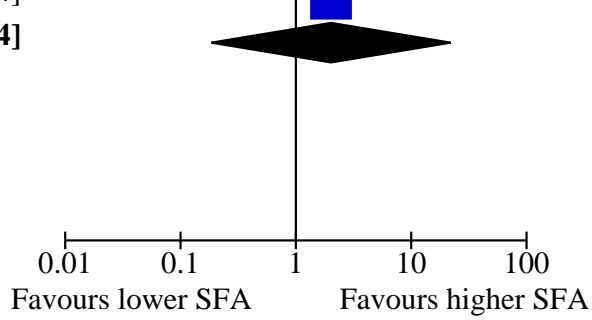


Analysis 2.27. Comparison 2: SFA reduction vs usual diet - secondary health events, Outcome 27: Non-fatal MI, subgroup by main substitution

Lower SFA

Higher SFA

Events Total
Risk Ratio

Risk Ratio

Study or Subgroup

\subsection{1 replaced by PUFA}

DART 1989

MRC 1968

$\begin{array}{rrrrr}35 & 1018 & 47 & 1015 & 33.2 \% \\ 25 & 199 & 25 & 194 & 22.8 \% \\ 24 & 206 & 31 & 206 & 24.8 \% \\ 7 & 28 & 5 & 26 & 5.9 \% \\ 13 & 424 & 21 & 422 & 13.3 \% \\ & \mathbf{1 8 7 5} & & \mathbf{1 8 6 3} & \mathbf{1 0 0 . 0 \%}\end{array}$

104

129

Total events:

Heterogeneity: $\mathrm{Tau}^{2}=0.00 ; \mathrm{Chi}^{2}=2.14, \mathrm{df}=4(\mathrm{P}=0.71) ; \mathrm{I}^{2}=0 \%$

Test for overall effect: $\mathrm{Z}=1.72(\mathrm{P}=0.09)$

\subsection{2 replaced by MUFA}

Rose olive 1965

Subtotal (95\% CI)

$6 \quad 26$

26

6

Total events:

Heterogeneity: Not applicable

Test for overall effect: $\mathrm{Z}=0.34(\mathrm{P}=0.73)$

\subsection{3 replace by $\mathrm{CHO}$}

WHI 2006 (1)

Subtotal (95\% CI)

Total events:

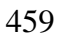

19541

684

$29294 \quad 100.0 \%$

$29294100.0 \%$

459

684

5
$0.74[0.48,1.14]$

$0.97[0.58,1.64]$

$0.77[0.47,1.27]$

$1.30[0.47,3.59]$

$0.62[0.31,1.21]$

$0.80[0.63,1.03]$
Heterogeneity: Not applicable

Test for overall effect: $\mathrm{Z}=0.10(\mathrm{P}=0.92)$

2.27.4 replaced by protein

Subtotal (95\% CI)

0

0

$1.20[0.42,3.45]$

$1.20[0.42,3.45]$

Total events:

0

Not estimable

Heterogeneity: Not applicable

Test for overall effect: Not applicable

2.27.5 replacement unclear

$\begin{array}{lcccccc}\text { Moy } 2001 & 2 & 117 & 1 & 118 & 100.0 \% & 2.02[0.19,21.94] \\ \text { Subtotal }(\mathbf{9 5 \%} \text { CI) } & & \mathbf{1 1 7} & & \mathbf{1 1 8} & \mathbf{1 0 0 . 0 \%} & \mathbf{2 . 0 2}[\mathbf{0 . 1 9}, \mathbf{2 1 . 9 4}] \\ \text { Total events: } & 2 & & 1 & & & \end{array}$

Heterogeneity: Not applicable

Test for overall effect: $\mathrm{Z}=0.58(\mathrm{P}=0.56)$

Test for subgroup differences: $\mathrm{Chi}^{2}=3.08, \mathrm{df}=3(\mathrm{P}=0.38), \mathrm{I}^{2}=2.5 \%$

0.01 M-H, Random, 95\% CI

\section{Footnotes}

(1) Non-fatal MI during trial, Prentice 2017 


\section{Analysis 2.28. Comparison 2: SFA reduction vs usual diet - secondary health events, Outcome 28: Non-fatal MI, subgroup by duration}

\begin{tabular}{|c|c|c|c|c|c|c|}
\hline \multirow[b]{2}{*}{ Study or Subgroup } & \multicolumn{2}{|c|}{ Lower SFA } & \multicolumn{2}{|c|}{ Higher SFA } & \multirow[b]{2}{*}{ Weight } & \multirow{2}{*}{$\begin{array}{c}\text { Risk Ratio } \\
\text { M-H, Random, } 95 \% \text { CI }\end{array}$} \\
\hline & Events & Total & Events & Total & & \\
\hline \multicolumn{7}{|l|}{ 2.28.1 up to $24 \mathrm{mo}$} \\
\hline DART 1989 & 35 & 1018 & 47 & 1015 & $6.0 \%$ & $0.74[0.48,1.14]$ \\
\hline Moy 2001 & 2 & 117 & 1 & 118 & $0.2 \%$ & $2.02[0.19,21.94]$ \\
\hline Rose corn oil 1965 & 7 & 28 & 3 & 13 & $0.8 \%$ & $1.08[0.33,3.53]$ \\
\hline Rose olive 1965 & 6 & 26 & 2 & 13 & $0.5 \%$ & $1.50[0.35,6.43]$ \\
\hline Subtotal $(95 \%$ CI $)$ & & 1189 & & 1159 & $7.6 \%$ & $0.83[0.57,1.22]$ \\
\hline Total events: & 50 & & 53 & & & \\
\hline
\end{tabular}

Heterogeneity: $\mathrm{Tau}^{2}=0.00 ; \mathrm{Chi}^{2}=1.62, \mathrm{df}=3(\mathrm{P}=0.65) ; \mathrm{I}^{2}=0 \%$

Test for overall effect: $\mathrm{Z}=0.94(\mathrm{P}=0.35)$

2.28.2 $>24$ to $48 \mathrm{mo}$

MRC 1968

Veterans Admin 1969

Subtotal (95\% CI)

$\begin{array}{ll}25 & 199 \\ 13 & 42 \\ & 62\end{array}$

199
424
$\mathbf{6 2 3}$

25
21

Total events:

38

46

Heterogeneity: $\mathrm{Tau}^{2}=0.01 ; \mathrm{Chi}^{2}=1.11, \mathrm{df}=1(\mathrm{P}=0.29) ; \mathrm{I}^{2}=10 \%$

Test for overall effect: $\mathrm{Z}=0.90(\mathrm{P}=0.37)$

\subsection{3 $>48 \mathrm{mo}$}

Oslo Diet-Heart 1966

WHI 2006 (1)

Subtotal (95\% CI)

Total events:

24
459

206
19541
$\mathbf{1 9 7 4 7}$

31

206

$4.5 \%$

$81.4 \%$

483

29500

$\mathbf{8 5 . 9 \%}$

715

Heterogeneity: $\mathrm{Tau}^{2}=0.00 ; \mathrm{Chi}^{2}=1.01, \mathrm{df}=1(\mathrm{P}=0.31) ; \mathrm{I}^{2}=1 \%$

Test for overall effect: $\mathrm{Z}=0.15(\mathrm{P}=0.88)$

\subsection{4 unclear}

Subtotal (95\% CI)

Total events:

$\mathbf{0}$

0

Heterogeneity: Not applicable

Test for overall effect: Not applicable

Total $(95 \%$ CI $)$

571

21559

$31275100.0 \%$

Total events: 571

814

Heterogeneity: $\mathrm{Tau}^{2}=0.00 ; \mathrm{Chi}^{2}=5.11, \mathrm{df}=7(\mathrm{P}=0.65) ; \mathrm{I}^{2}=0 \%$

Test for overall effect: $\mathrm{Z}=0.62(\mathrm{P}=0.54)$

Test for subgroup differences: $\mathrm{Chi}^{2}=1.30, \mathrm{df}=2(\mathrm{P}=0.52), \mathrm{I}^{2}=0 \%$

Footnotes

(1) Non-fatal MI during trial, Prentice 2017
$0.97[0.58,1.64]$ $0.62[0.31,1.21]$ $0.82[0.53,1.27]$

$0.77[0.47,1.27]$ $1.01[0.90,1.13]$ $0.99[0.88,1.12]$

Not estimable M-H, Random, $95 \%$ CI 


\section{Analysis 2.29. Comparison 2: SFA reduction vs usual diet - secondary health events, Outcome 29: Non-fatal MI, subgroup by baseline SFA}

Lower SFA
Risk Ratio

Risk Ratio

Study or Subgroup

Events Tota
Weight M-H, Random, 95\% CI
M-H, Random, 95\% CI

\subsection{1 up to $12 \%$ E SFA baseline}

Subtotal $(95 \%$ CI)

Total events:

0

Heterogeneity: Not applicable

Test for overall effect: Not applicable

\subsection{2 >12 to $15 \%$ E SFA baseline}

DART 1989

Moy 2001

WHI $2006(1)$

Subtotal $(95 \%$ CI $)$

Total events:

Heterogeneity: $\mathrm{Tau}^{2}=0.00 ; \mathrm{Chi}^{2}=2.14, \mathrm{df}=2(\mathrm{P}=0.34) ; \mathrm{I}^{2}=6 \%$

Test for overall effect: $\mathrm{Z}=0.35(\mathrm{P}=0.73)$

\subsection{3 $>15$ to $18 \%$ E SFA baseline}

Subtotal $(95 \%$ CI $)$

0

0

Total events:

0

Heterogeneity: Not applicable

Test for overall effect: Not applicable

2.29.4 >18\% E SFA baseline

Veterans Admin 1969

13

424

21

422

$2.4 \%$

Subtotal $(95 \%$ CI)

13

424

$422 \quad 2.4 \%$

21

Heterogeneity: Not applicable

Test for overall effect: $\mathrm{Z}=1.40(\mathrm{P}=0.16)$

\subsection{5 unclear}

MRC 1968

Oslo Diet-Heart 1966

Rose corn oil 1965

Rose olive 1965

Subtotal (95\% CI)

Total events:

$\begin{array}{rr}25 & 199 \\ 24 & 206 \\ 7 & 28 \\ 6 & 26 \\ & \end{array}$

Heterogeneity: $\mathrm{Tau}^{2}=0.00 ; \mathrm{Chi}^{2}=1.01, \mathrm{df}=3(\mathrm{P}=0.80) ; \mathrm{I}^{2}=0 \%$

Test for overall effect: $\mathrm{Z}=0.58(\mathrm{P}=0.56)$

Total (95\% CI)

21559

Total events:

571

$31275 \quad 100.0 \%$

Heterogeneity: $\mathrm{Tau}^{2}=0.00 ; \mathrm{Chi}^{2}=5.11, \mathrm{df}=7(\mathrm{P}=0.65) ; \mathrm{I}^{2}=0 \%$

Test for overall effect: $\mathrm{Z}=0.62(\mathrm{P}=0.54)$

Test for subgroup differences: $\mathrm{Chi}^{2}=1.72, \mathrm{df}=2(\mathrm{P}=0.42), \mathrm{I}^{2}=0 \%$
Not estimable

$0.74[0.48,1.14]$

$2.02[0.19,21.94]$

$1.01[0.90,1.13]$

$0.97[0.83,1.13]$

\section{Footnotes}

(1) Non-fatal MI during trial, Prentice 2017 


\section{Analysis 2.30. Comparison 2: SFA reduction vs usual diet - secondary health events, Outcome 30: Non-fatal MI, subgroup by SFA change}

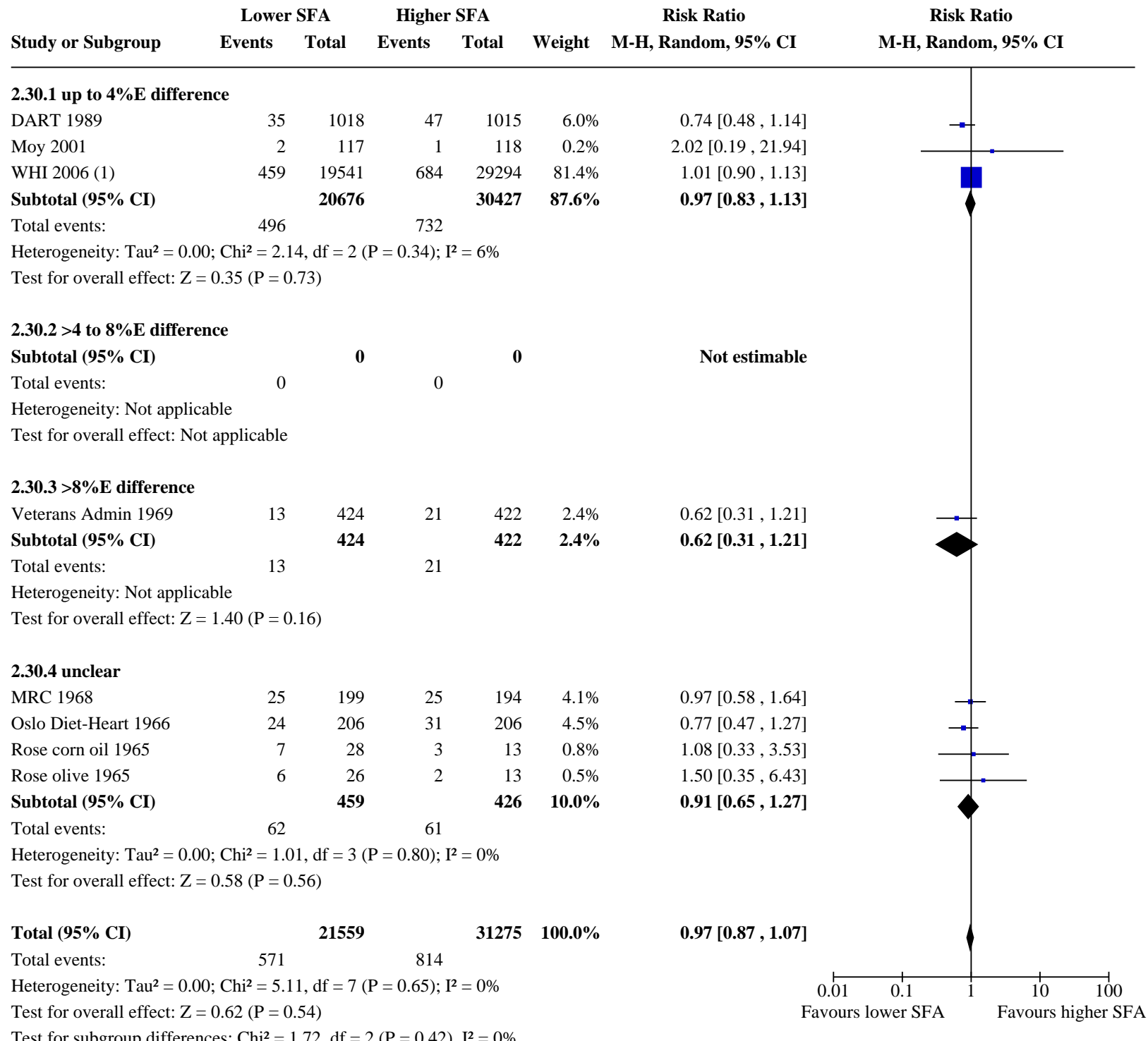

Footnotes

(1) Non-fatal MI during trial, Prentice 2017 
Analysis 2.31. Comparison 2: SFA reduction vs usual diet secondary health events, Outcome 31: Non-fatal MI, subgroup by sex

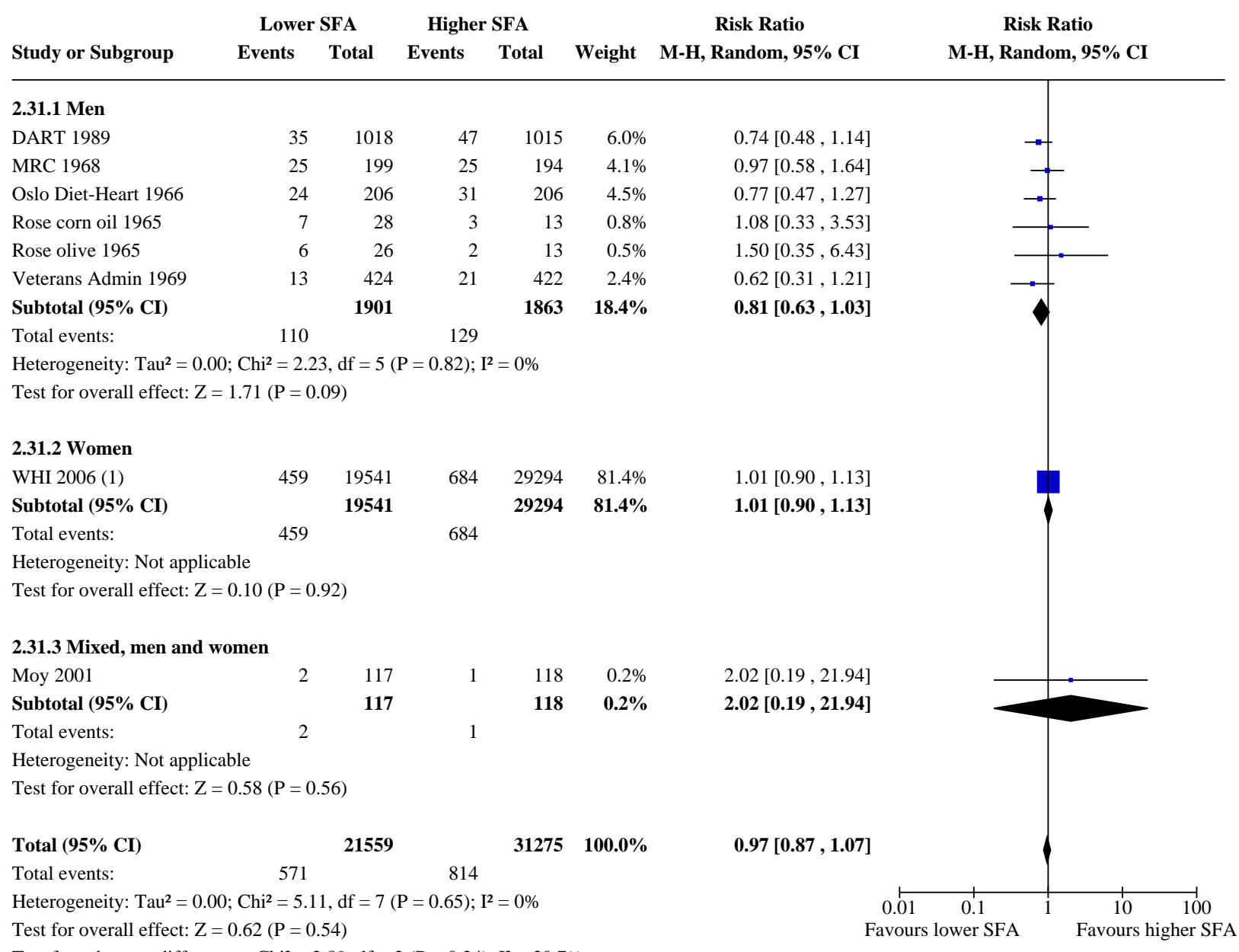

\section{Footnotes}

(1) Non-fatal MI during trial, Prentice 2017 


\section{Analysis 2.32. Comparison 2: SFA reduction vs usual diet - secondary health events, Outcome 32: Non-fatal MI, subgroup by CVD risk}

\begin{tabular}{|c|c|c|c|c|c|c|c|}
\hline \multirow[b]{2}{*}{ Study or Subgroup } & \multicolumn{2}{|c|}{ Lower SFA } & \multicolumn{2}{|c|}{ Higher SFA } & \multirow[b]{2}{*}{ Weight } & \multirow{2}{*}{$\begin{array}{c}\text { Risk Ratio } \\
\text { M-H, Random, } 95 \% \text { CI }\end{array}$} & \multirow{2}{*}{$\begin{array}{c}\text { Risk Ratio } \\
\text { M-H, Random, } 95 \% \text { CI }\end{array}$} \\
\hline & Events & Total & Events & Total & & & \\
\hline \multicolumn{8}{|l|}{ 2.32.1 Low CVD risk } \\
\hline Veterans Admin 1969 & 13 & 424 & 21 & 422 & $5.9 \%$ & $0.62[0.31,1.21]$ & $\rightarrow$ \\
\hline WHI 2006 & 353 & 18633 & 581 & 27925 & $37.3 \%$ & $0.91[0.80,1.04]$ & \\
\hline Subtotal (95\% CI) & & 19057 & & 28347 & $43.3 \%$ & $0.87[0.68,1.12]$ & \\
\hline Total events: & 366 & & 602 & & & & \\
\hline \multicolumn{8}{|c|}{ Heterogeneity: $\mathrm{Tau}^{2}=0.01 ; \mathrm{Chi}^{2}=1.23, \mathrm{df}=1(\mathrm{P}=0.27) ; \mathrm{I}^{2}=19 \%$} \\
\hline \multicolumn{8}{|c|}{ Test for overall effect: $\mathrm{Z}=1.11(\mathrm{P}=0.27)$} \\
\hline \multicolumn{8}{|c|}{ 2.32.2 Moderate CVD risk } \\
\hline Moy 2001 & 2 & 117 & 1 & 118 & $0.5 \%$ & $2.02[0.19,21.94]$ & \\
\hline Subtotal $(95 \%$ CI $)$ & & 117 & & 118 & $0.5 \%$ & $2.02[0.19,21.94]$ & \\
\hline Total events: & 2 & & 1 & & & & \\
\hline \multicolumn{8}{|c|}{ Heterogeneity: Not applicable } \\
\hline \multicolumn{8}{|c|}{ Test for overall effect: $\mathrm{Z}=0.58(\mathrm{P}=0.56)$} \\
\hline \multicolumn{8}{|c|}{ 2.32.3 Existing CVD disease } \\
\hline DART 1989 & 35 & 1018 & 47 & 1015 & $12.4 \%$ & $0.74[0.48,1.14]$ & \\
\hline MRC 1968 & 25 & 199 & 25 & 194 & $9.3 \%$ & $0.97[0.58,1.64]$ & \\
\hline Oslo Diet-Heart 1966 & 24 & 206 & 31 & 206 & $10.0 \%$ & $0.77[0.47,1.27]$ & \\
\hline Rose corn oil 1965 & 7 & 28 & 3 & 13 & $2.1 \%$ & $1.08[0.33,3.53]$ & \\
\hline Rose olive 1965 & 6 & 26 & 2 & 13 & $1.4 \%$ & $1.50[0.35,6.43]$ & \\
\hline WHI 2006 (1) & 82 & 908 & 90 & 1369 & $20.9 \%$ & $1.37[1.03,1.83]$ & 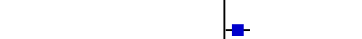 \\
\hline Subtotal (95\% CI) & & 2385 & & 2810 & $56.2 \%$ & $1.00[0.76,1.31]$ & \\
\hline Total events: & 179 & & 198 & & & & \\
\hline \multicolumn{8}{|c|}{ Heterogeneity: $\mathrm{Tau}^{2}=0.04 ; \mathrm{Chi}^{2}=7.63, \mathrm{df}=5(\mathrm{P}=0.18) ; \mathrm{I}^{2}=34 \%$} \\
\hline \multicolumn{8}{|c|}{ Test for overall effect: $\mathrm{Z}=0.01(\mathrm{P}=0.99)$} \\
\hline Total $(95 \% \mathrm{CI})$ & & 21559 & & 31275 & $100.0 \%$ & $0.95[0.80,1.13]$ & \\
\hline Total events: & 547 & & 801 & & & & \\
\hline \multicolumn{7}{|c|}{ Heterogeneity: $\mathrm{Tau}^{2}=0.02 ; \mathrm{Chi}^{2}=11.03, \mathrm{df}=8(\mathrm{P}=0.20) ; \mathrm{I}^{2}=27 \%$} & $0 . \stackrel{\prime}{\prime}^{\prime} \quad 0.1$ \\
\hline Test for overall effect: & $0.56(\mathrm{P}=0$ & & & & & & avours lower SFA \\
\hline
\end{tabular}

Footnotes

(1) Women with CVD at baseline 


\section{Analysis 2.33. Comparison 2: SFA reduction vs usual diet - secondary health events, Outcome 33: Non-fatal MI, subgroup by TC reduction}

\begin{tabular}{|c|c|c|c|c|c|c|c|}
\hline & Lowe & SFA & High & SFA & & Risk Ratio & Risk Ratio \\
\hline Study or Subgroup & Events & Total & Events & Total & Weight & M-H, Random, $95 \%$ CI & M-H, Random, $95 \%$ CI \\
\hline
\end{tabular}

2.33.1 serum chol reduced by at least $0.2 \mathrm{mmol} / \mathrm{L}$

$\begin{array}{lrrrrrr}\text { DART 1989 } & 35 & 1018 & 47 & 1015 & 33.4 \% & 0.74[0.48,1.14] \\ \text { MRC 1968 } & 25 & 199 & 25 & 194 & 22.9 \% & 0.97[0.58,1.64] \\ \text { Moy 2001 } & 2 & 117 & 1 & 118 & 1.1 \% & 2.02[0.19,21.94] \\ \text { Oslo Diet-Heart 1966 } & 24 & 206 & 31 & 206 & 24.9 \% & 0.77[0.47,1.27] \\ \text { Rose corn oil 1965 } & 7 & 28 & 3 & 13 & 4.4 \% & 1.08[0.33,3.53] \\ \text { Veterans Admin 1969 } & 13 & 424 & 21 & 422 & 13.3 \% & 0.62[0.31,1.21] \\ \text { Subtotal (95\% CI) } & & \mathbf{1 9 9 2} & & \mathbf{1 9 6 8} & \mathbf{1 0 0 . 0 \%} & \mathbf{0 . 8 0}[\mathbf{0 . 6 2}, \mathbf{1 . 0 3}]\end{array}$

Total events: $106 \quad 128$

Heterogeneity: $\mathrm{Tau}^{2}=0.00 ; \mathrm{Chi}^{2}=2.09, \mathrm{df}=5(\mathrm{P}=0.84) ; \mathrm{I}^{2}=0 \%$

Test for overall effect: $\mathrm{Z}=1.76(\mathrm{P}=0.08)$

2.33.2 serum chol reduced by $<0.2 \mathrm{mmol} / \mathrm{L}$

Rose olive 1965

WHI 2006 (1)

Subtotal (95\% CI)

Total events:

$\begin{array}{rr}6 & 26 \\ 459 & 19541 \\ & \mathbf{1 9 5 6 7}\end{array}$

465

19567

2

$\begin{array}{rr}13 & 0.6 \% \\ 29294 & 99.4 \%\end{array}$

$1.50[0.35,6.43]$

$1.01[0.90,1.13]$

$1.01[0.90,1.13]$ 686

Heterogeneity: $\mathrm{Tau}^{2}=0.00 ; \mathrm{Chi}^{2}=0.29, \mathrm{df}=1(\mathrm{P}=0.59) ; \mathrm{I}^{2}=0 \%$

Test for overall effect: $\mathrm{Z}=0.14(\mathrm{P}=0.89)$

2.33.3 serum chol reduction unclear Subtotal (95\% CI)

0

Total events:

0

Heterogeneity: Not applicable

Test for overall effect: Not applicable

Test for subgroup differences: $\mathrm{Chi}^{2}=2.73, \mathrm{df}=1(\mathrm{P}=0.10), \mathrm{I}^{2}=63.4 \%$

Footnotes

(1) Non-fatal MI during trial, Prentice 2017
Random, $95 \%$ CI

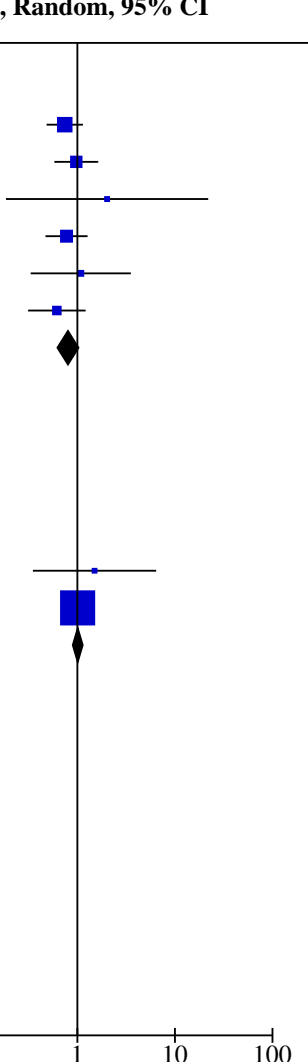


Analysis 2.34. Comparison 2: SFA reduction vs usual diet - secondary health events, Outcome 34: Non-fatal MI, subgroup decade of publication

Lower SFA Higher SFA

Study or Subgroup
Events Total

\author{
Events Total
}

Risk Ratio

Weight M-H, Random, 95\% CI
Risk Ratio

M-H, Random, $95 \%$ CI

\subsubsection{0s}

MRC 1968

Oslo Diet-Heart 1966

Rose corn oil 1965

Rose olive 1965

Veterans Admin 1969

Subtotal (95\% CI)

Total events:

$\begin{array}{rr}25 & 199 \\ 24 & 206 \\ 7 & 28 \\ 6 & 26 \\ 13 & 424 \\ & \mathbf{8 8 3}\end{array}$

$\begin{array}{rrr}25 & 194 & 4.1 \% \\ 31 & 206 & 4.5 \% \\ 3 & 13 & 0.8 \% \\ 2 & 13 & 0.5 \% \\ 21 & 422 & 2.4 \% \\ & \mathbf{8 4 8} & \mathbf{1 2 . 4 \%}\end{array}$

$75 \quad 82$

Heterogeneity: $\mathrm{Tau}^{2}=0.00 ; \mathrm{Chi}^{2}=2.01, \mathrm{df}=4(\mathrm{P}=0.73) ; \mathrm{I}^{2}=0 \%$

Test for overall effect: $\mathrm{Z}=1.14(\mathrm{P}=0.26)$

\subsubsection{0s}

Subtotal (95\% CI)

0

Total events:

0

0

Heterogeneity: Not applicable

Test for overall effect: Not applicable

\subsubsection{0s}

DART 1989

Subtotal (95\% CI)

35

$$
\begin{aligned}
& 1018 \\
& 1018
\end{aligned}
$$

47

1015

$6.0 \%$

$1015 \quad 6.0 \%$

35

47

Heterogeneity: Not applicable

Test for overall effect: $\mathrm{Z}=1.36(\mathrm{P}=0.17)$

\subsubsection{0s}

Subtotal (95\% CI)

0

Total events:

0

Heterogeneity: Not applicable

Test for overall effect: Not applicable

\subsubsection{0s}

\section{Moy 2001}

WHI 2006 (1)

Subtotal (95\% CI)

$\begin{array}{rr}2 & 117 \\ 459 & 19541 \\ & \mathbf{1 9 6 5 8}\end{array}$

$\begin{array}{rrr}1 & 118 & 0.2 \% \\ 684 & 29294 & 81.4 \% \\ & \mathbf{2 9 4 1 2} & \mathbf{8 1 . 6 \%}\end{array}$

$461 \quad 685$

Heterogeneity: $\mathrm{Tau}^{2}=0.00 ; \mathrm{Chi}^{2}=0.33, \mathrm{df}=1(\mathrm{P}=0.57) ; \mathrm{I}^{2}=0 \%$

Test for overall effect: $\mathrm{Z}=0.13(\mathrm{P}=0.90)$

\section{Total $(95 \%$ CI)}

21559

Total events:

571 814

Heterogeneity: $\mathrm{Tau}^{2}=0.00 ; \mathrm{Chi}^{2}=5.11, \mathrm{df}=7(\mathrm{P}=0.65) ; \mathrm{I}^{2}=0 \%$

Test for overall effect: $\mathrm{Z}=0.62(\mathrm{P}=0.54)$

Test for subgroup differences: $\mathrm{Chi}^{2}=2.78, \mathrm{df}=2(\mathrm{P}=0.25), \mathrm{I}^{2}=28.0 \%$
$0.97[0.58,1.64]$

$0.77[0.47,1.27]$

$1.08[0.33,3.53]$

$1.50[0.35,6.43]$

$0.62[0.31,1.21]$

$0.84[0.62,1.13]$

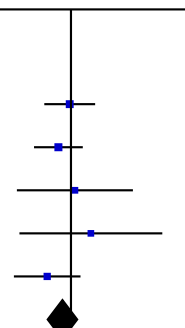

$0.74[0.48,1.14]$

$0.74[0.48,1.14]$

Not estimable

Not estimable

$2.02[0.19,21.94]$

$1.01[0.90,1.13]$

$1.01[0.90,1.13]$

\section{Footnotes}

(1) Non-fatal MI during trial, Prentice 2017 
Analysis 2.35. Comparison 2: SFA reduction vs usual diet - secondary health events, Outcome 35: STROKE

\begin{tabular}{lccccccc} 
& \multicolumn{2}{c}{ Lower SFA } & \multicolumn{2}{c}{ Higher SFA } & \multicolumn{2}{c}{ Risk Ratio } & Risk Ratio \\
Study or Subgroup & Events & Total & Events & Total & Weight & M-H, Random, 95\% CI & M-H, Random, 95\% CI
\end{tabular}

\begin{tabular}{lrrrrrr}
\hline Ley 2004 & 1 & 88 & 5 & 88 & $2.0 \%$ & $0.20[0.02,1.68]$ \\
MRC 1968 & 2 & 199 & 0 & 194 & $1.0 \%$ & $4.88[0.24,100.89]$ \\
Moy 2001 & 1 & 117 & 1 & 118 & $1.2 \%$ & $1.01[0.06,15.93]$ \\
Oslo Diet-Heart 1966 & 2 & 206 & 1 & 206 & $1.6 \%$ & $2.00[0.18,21.89]$ \\
STARS 1992 & 0 & 27 & 1 & 28 & $0.9 \%$ & $0.35[0.01,8.12]$ \\
Veterans Admin 1969 & 13 & 424 & 22 & 422 & $16.4 \%$ & $0.59[0.30,1.15]$ \\
WHI 2006(1) & 435 & 19541 & 634 & 29294 & $76.9 \%$ & $1.03[0.91,1.16]$ \\
& & & & & & \\
Total (95\% CI) & & $\mathbf{2 0 6 0 2}$ & & $\mathbf{3 0 3 5 0}$ & $\mathbf{1 0 0 . 0 \%}$ & $\mathbf{0 . 9 2}[\mathbf{0 . 6 8 , \mathbf { 1 . 2 5 }}]$ \\
Total events: & 454 & & 664 & & &
\end{tabular}

Heterogeneity: $\mathrm{Tau}^{2}=0.03 ; \mathrm{Chi}^{2}=6.59, \mathrm{df}=6(\mathrm{P}=0.36) ; \mathrm{I}^{2}=9 \%$

Test for overall effect: $\mathrm{Z}=0.52(\mathrm{P}=0.60)$

Test for subgroup differences: Not applicable

Random, 95\% CI

Footnotes

(1) During trial, Prentice 2017

\section{Analysis 2.36. Comparison 2: SFA reduction vs usual diet - secondary health events, Outcome 36: Stroke, SA by low summary risk of bias}

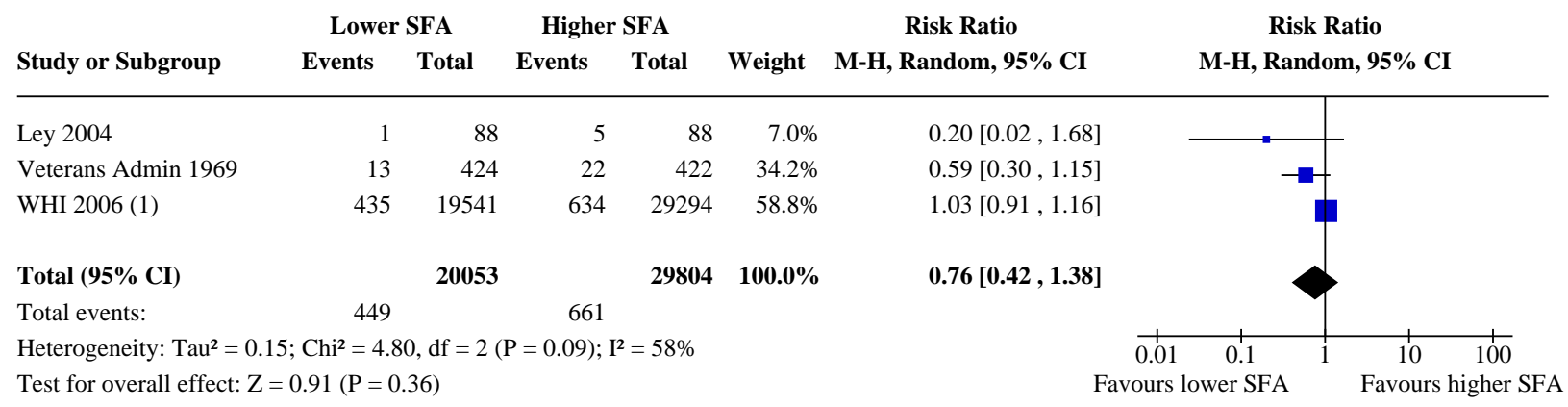

Test for subgroup differences: Not applicable

Footnotes

(1) During trial, Prentice 2017 
Analysis 2.37. Comparison 2: SFA reduction vs usual diet secondary health events, Outcome 37: Stroke, SA aim to reduce SFA

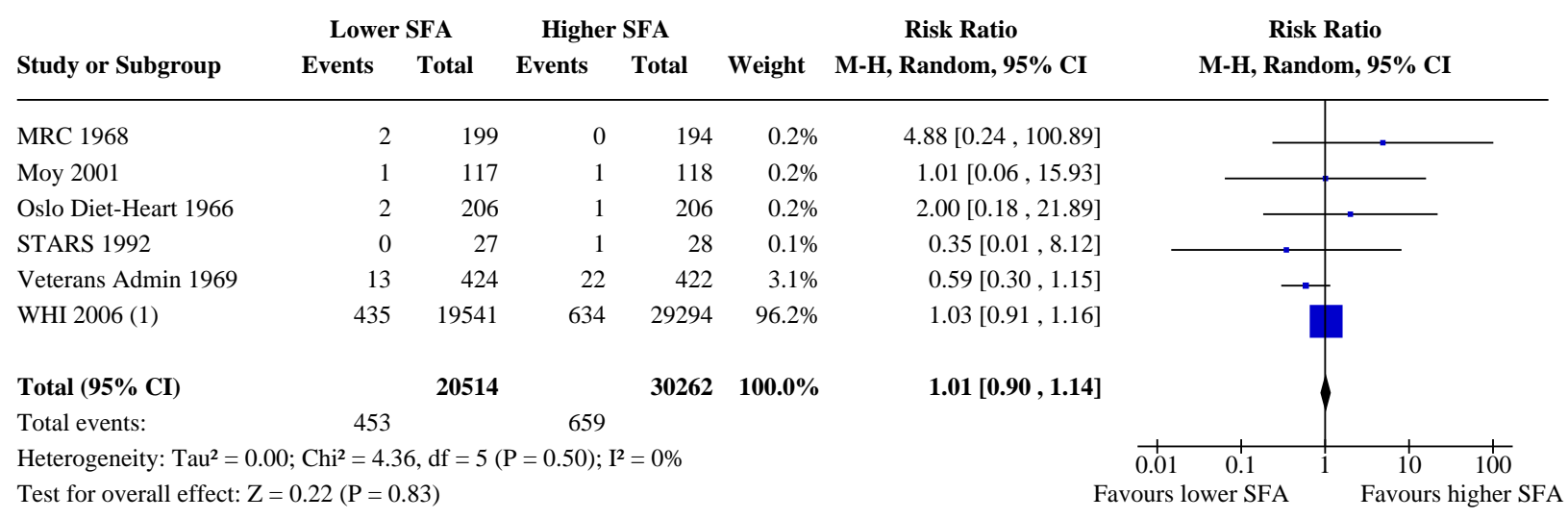

Test for subgroup differences: Not applicable

Footnotes

(1) During trial, Prentice 2017

Analysis 2.38. Comparison 2: SFA reduction vs usual diet - secondary health events, Outcome 38: Stroke, SA statistically significant SFA reduction

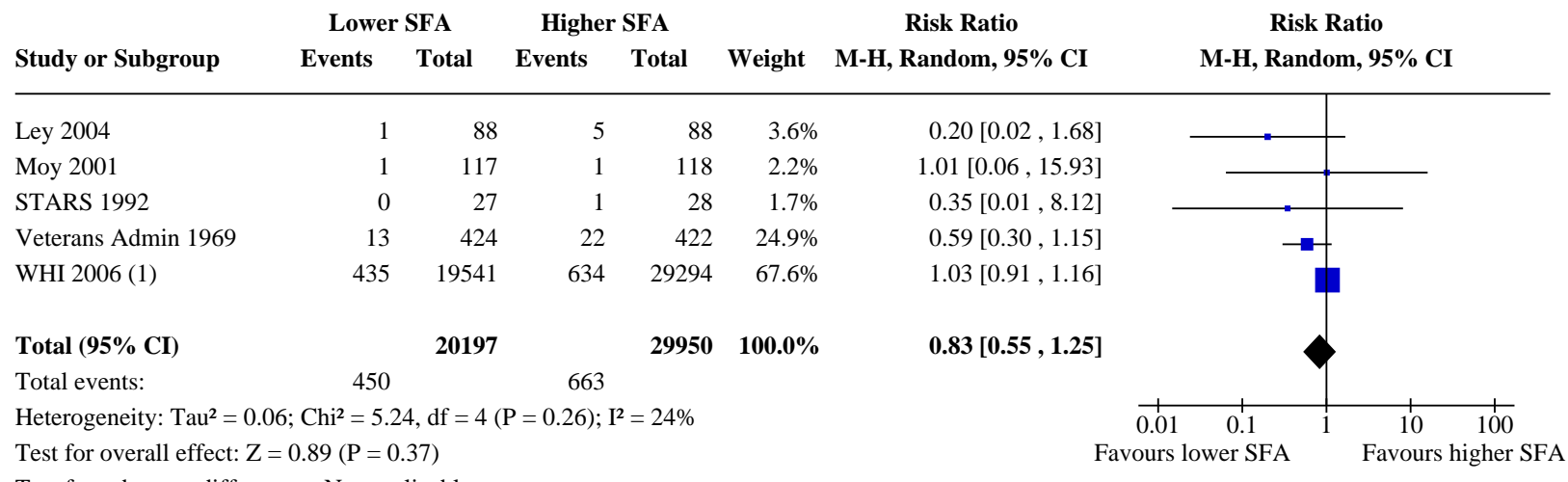

Test for subgroup differences: Not applicable

Footnotes

(1) During trial, Prentice 2017 
Analysis 2.39. Comparison 2: SFA reduction vs usual diet secondary health events, Outcome 39: Stroke, SA by TC reduction

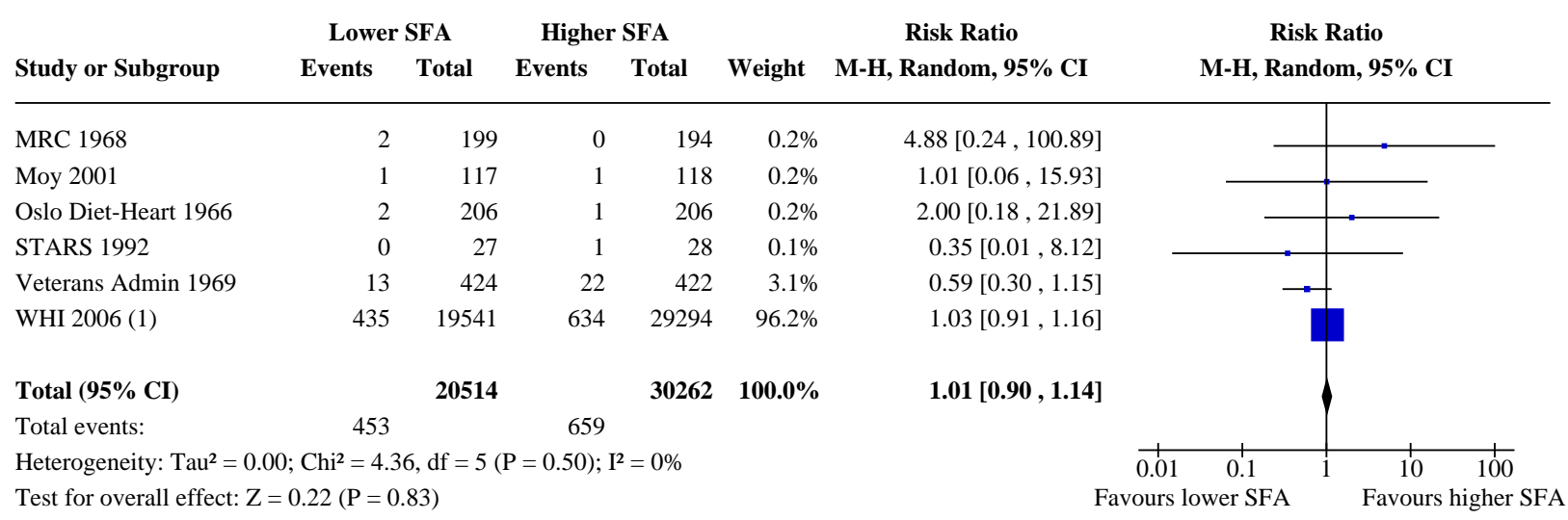

Test for subgroup differences: Not applicable

Footnotes

(1) During trial, Prentice 2017

Analysis 2.40. Comparison 2: SFA reduction vs usual diet secondary health events, Outcome 40: Stroke, SA excluding WHI

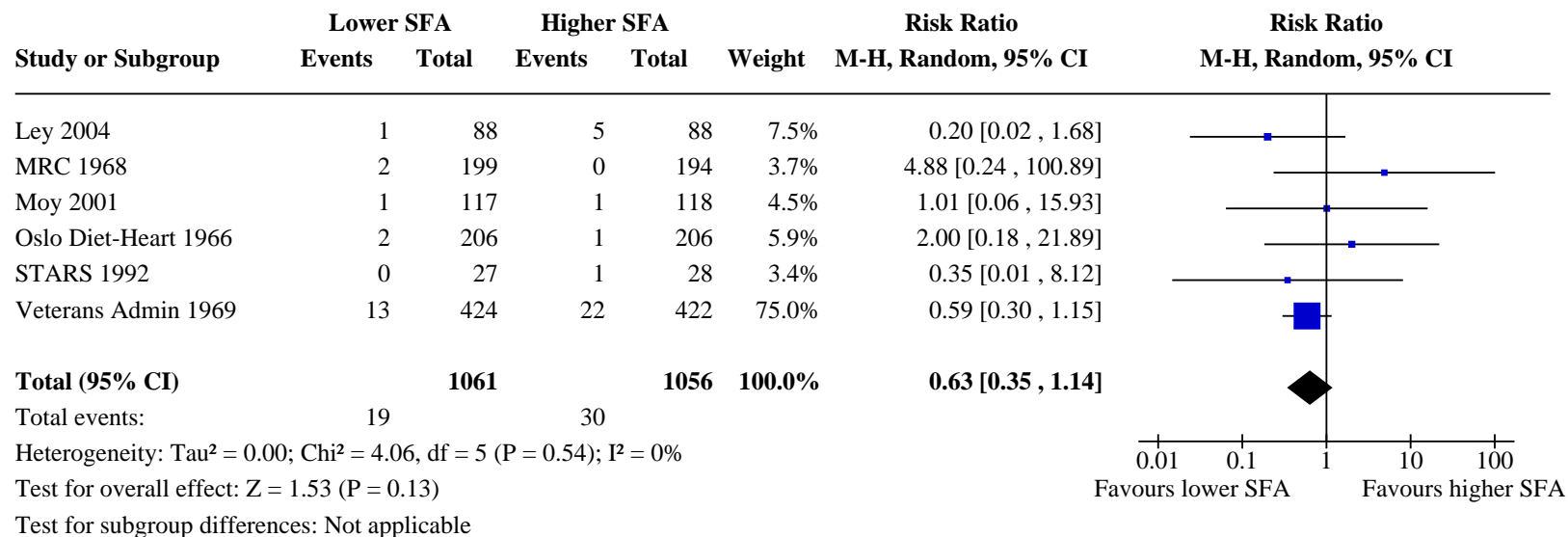


Analysis 2.41. Comparison 2: SFA reduction vs usual diet - secondary health events, Outcome 41: Stroke, SA Mantel-Haenszel fixed-effect

\begin{tabular}{cccccccc} 
& \multicolumn{2}{c}{ Lower SFA } & \multicolumn{2}{c}{ Higher SFA } & \multicolumn{2}{c}{ Risk Ratio } & \multicolumn{2}{c}{ Risk Ratio } \\
Study or Subgroup & Events & Total & Events & Total & Weight & M-H, Fixed, 95\% CI & M-H, Fixed, 95\% CI
\end{tabular}

$\begin{array}{lrrrrrr}\text { Ley 2004 } & 1 & 88 & 5 & 88 & 0.9 \% & 0.20[0.02,1.68] \\ \text { MRC 1968 } & 2 & 199 & 0 & 194 & 0.1 \% & 4.88[0.24,100.89] \\ \text { Moy 2001 } & 1 & 117 & 1 & 118 & 0.2 \% & 1.01[0.06,15.93] \\ \text { Oslo Diet-Heart 1966 } & 2 & 206 & 1 & 206 & 0.2 \% & 2.00[0.18,21.89] \\ \text { STARS 1992 } & 0 & 27 & 1 & 28 & 0.3 \% & 0.35[0.01,8.12] \\ \text { Veterans Admin 1969 } & 13 & 424 & 22 & 422 & 4.1 \% & 0.59[0.30,1.15] \\ \text { WHI 2006 (1) } & 435 & 19541 & 634 & 29294 & 94.2 \% & 1.03[0.91,1.16] \\ & & & & & & \\ \text { Total (95\% CI) } & & \mathbf{2 0 6 0 2} & & \mathbf{3 0 3 5 0} & \mathbf{1 0 0 . 0 \%} & \mathbf{1 . 0 1 [ 0 . 8 9 , \mathbf { 1 . 1 3 } ]}\end{array}$

Total events: $\quad 454 \quad 664$

Heterogeneity: $\mathrm{Chi}^{2}=6.59, \mathrm{df}=6(\mathrm{P}=0.36) ; \mathrm{I}^{2}=9 \%$

Test for overall effect: $\mathrm{Z}=0.11(\mathrm{P}=0.92)$

Test for subgroup differences: Not applicable

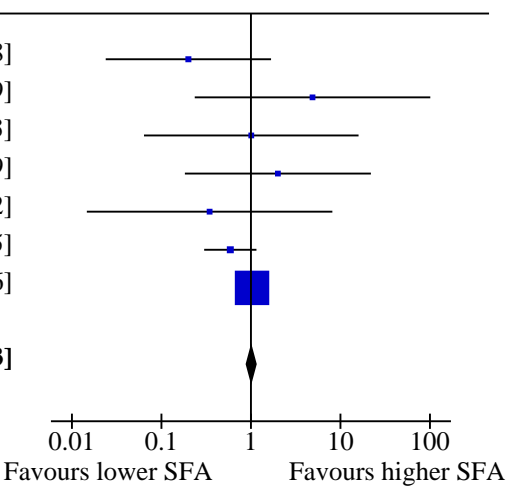

Footnotes

(1) During trial, Prentice 2017

Analysis 2.42. Comparison 2: SFA reduction vs usual diet secondary health events, Outcome 42: Stroke, SA Peto fixed-effect

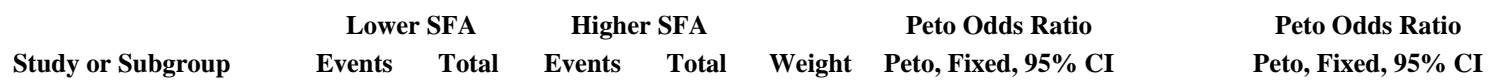

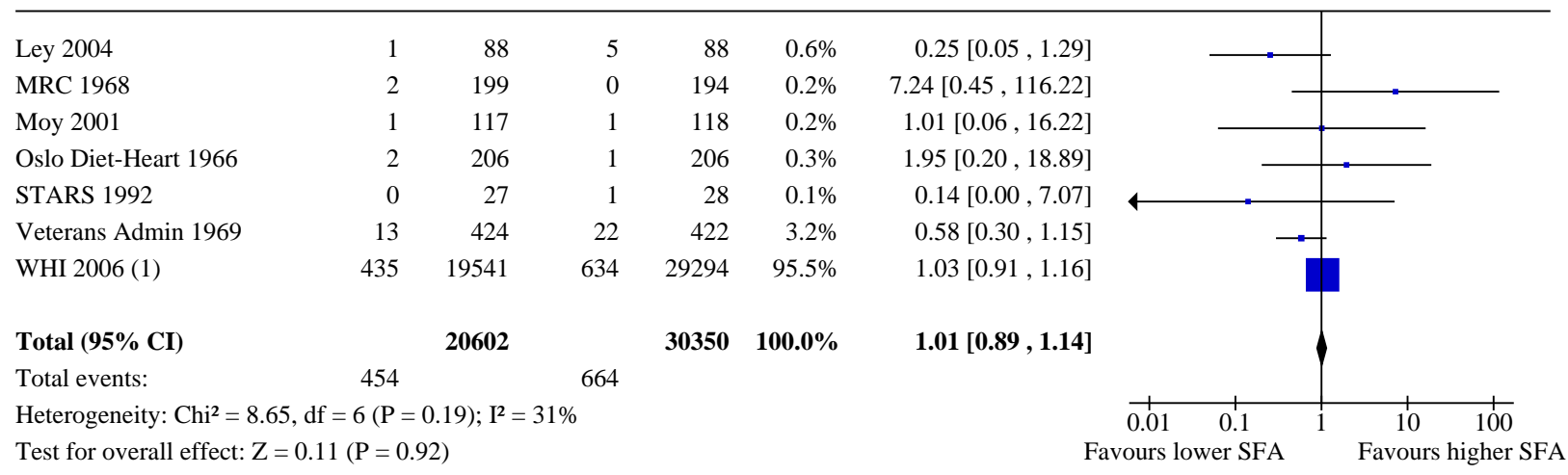

Test for subgroup differences: Not applicable

Footnotes

(1) During trial, Prentice 2017 


\section{Analysis 2.43. Comparison 2: SFA reduction vs usual diet - secondary} health events, Outcome 43: Stroke, subgroup by any substitution
Higher SFA
Risk Ratio

Events Total Weight M-H, Random, 95\% CI
Risk Ratio

M-H, Random, $95 \%$ CI

\subsection{1 replaced by PUFA}

MRC 1968

Oslo Diet-Heart 1966

STARS 1992

Veterans Admin 1969

Subtotal $(95 \%$ CI $)$

Total events:

Heterogeneity: $\mathrm{Tau}^{2}=0.00 ; \mathrm{Chi}^{2}=2.78, \mathrm{df}=3(\mathrm{P}=0.43) ; \mathrm{I}^{2}=0 \%$

Test for overall effect: $\mathrm{Z}=1.20(\mathrm{P}=0.23)$

\subsection{2 replaced by MUFA}

Subtotal $(95 \%$ CI $)$

0

Heterogeneity: Not applicable

Test for overall effect: Not applicable

\subsection{3 replace by $\mathrm{CHO}$}

Ley 2004

STARS 1992

WHI 2006 (1)

Subtotal $(95 \%$ CI $)$

Total events:

$\begin{array}{rrrrr}1 & 88 & 5 & 88 & 15.6 \% \\ 0 & 27 & 1 & 28 & 7.9 \% \\ 435 & 19541 & 634 & 29294 & 76.5 \% \\ & \mathbf{1 9 6 5 6} & & \mathbf{2 9 4 1 0} & \mathbf{1 0 0 . 0 \%} \\ 436 & & 640 & & \end{array}$

Heterogeneity: $\mathrm{Tau}^{2}=0.30 ; \mathrm{Chi}^{2}=2.73, \mathrm{df}=2(\mathrm{P}=0.26) ; \mathrm{I}^{2}=27 \%$

Test for overall effect: $\mathrm{Z}=0.65(\mathrm{P}=0.51)$

\subsection{4 replaced by protein}

$\begin{array}{lrrrrr}\text { Ley } 2004 & 1 & 88 & 5 & 88 & 28.1 \% \\ \text { WHI 2006 (1) } & 435 & 19541 & 634 & 29294 & 71.9 \% \\ \text { Subtotal (95\% CI) } & & \mathbf{1 9 6 2 9} & & \mathbf{2 9 3 8 2} & \mathbf{1 0 0 . 0 \%} \\ \text { Total events: } & 436 & & 639 & & \end{array}$

Heterogeneity: $\mathrm{Tau}^{2}=0.75 ; \mathrm{Chi}^{2}=2.27, \mathrm{df}=1(\mathrm{P}=0.13) ; \mathrm{I}^{2}=56 \%$

Test for overall effect: $\mathrm{Z}=0.59(\mathrm{P}=0.56)$

\subsection{5 replacement unclear}

Moy 2001

Subtotal (95\% CI)

117
$\mathbf{1 1 7}$
$1 \quad 118 \quad 100.0 \%$
$118 \quad 100.0 \%$

Total events:

1

Heterogeneity: Not applicable

Test for overall effect: $\mathrm{Z}=0.01(\mathrm{P}=1.00)$

Test for subgroup differences: $\mathrm{Chi}^{2}=0.09, \mathrm{df}=3(\mathrm{P}=0.99), \mathrm{I}^{2}=0 \%$

\section{Footnotes}

(1) During trial, Prentice 2017
$4.88[0.24,100.89]$

$2.00[0.18,21.89]$

$0.35[0.01,8.12]$

$0.59[0.30,1.15]$

$0.68[0.37,1.27]$

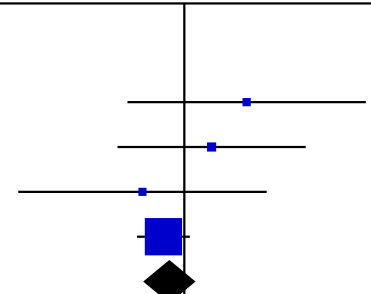

$0.20[0.02,1.68]$

$0.35[0.01,8.12]$

$1.03[0.91,1.16]$

$0.73[0.29,1.87]$

$0.20[0.02,1.68]$

$1.03[0.91,1.16]$

$0.65[0.15,2.75]$

$1.01[0.06,15.93]$

$1.01[0.06,15.93]$ 


\section{Analysis 2.44. Comparison 2: SFA reduction vs usual diet - secondary health events, Outcome 44: Stroke, subgroup by main substitution}

Lower SFA

Higher SFA
Risk Ratio

Events Total Events Total Weight M-H, Random, 95\% CI
Risk Ratio

M-H, Random, 95\% CI

.44 .1 replaced by PUFA

URC 1968

slo Diet-Heart 1966

Teterans Admin 1969

ubtotal $(95 \%$ CI $)$

otal events:

$\begin{array}{rrrrr}2 & 199 & 0 & 194 & 11.2 \% \\ 2 & 206 & 1 & 206 & 16.8 \% \\ 13 & 424 & 22 & 422 & 72.0 \% \\ & \mathbf{8 2 9} & & \mathbf{8 2 2} & \mathbf{1 0 0 . 0 \%}\end{array}$

17

23

Teterogeneity: $\mathrm{Tau}^{2}=0.30 ; \mathrm{Chi}^{2}=2.60, \mathrm{df}=2(\mathrm{P}=0.27) ; \mathrm{I}^{2}=23 \%$

est for overall effect: $\mathrm{Z}=0.16(\mathrm{P}=0.87)$

\section{2 replaced by MUFA}

ubtotal $(95 \% \mathrm{CI})$

0

0

otal events:

0

Ieterogeneity: Not applicable

est for overall effect: Not applicable

\section{.44 .3 replace by $\mathrm{CHO}$}

ey 2004

TARS 1992

VHI 2006 (1)

ubtotal $(95 \%$ CI $)$

otal events:

$\begin{array}{rrrrr}1 & 88 & 5 & 88 & 15.6 \% \\ 0 & 27 & 1 & 28 & 7.9 \% \\ 435 & 19541 & 634 & 29294 & 76.5 \% \\ & \mathbf{1 9 6 5 6} & & \mathbf{2 9 4 1 0} & \mathbf{1 0 0 . 0 \%} \\ 436 & & 640 & & \end{array}$

$0.20[0.02,1.68]$

$0.35[0.01,8.12]$

$1.03[0.91,1.16]$

$0.73[0.29,1.87]$

Teterogeneity: $\mathrm{Tau}^{2}=0.30 ; \mathrm{Chi}^{2}=2.73, \mathrm{df}=2(\mathrm{P}=0.26) ; \mathrm{I}^{2}=27 \%$

est for overall effect: $\mathrm{Z}=0.65(\mathrm{P}=0.51)$

.44.4 replaced by protein

ubtotal $(95 \%$ CI)

0

0

otal events:

0

0

Not estimable

Ieterogeneity: Not applicable

'est for overall effect: Not applicable

.44 .5 replacement unclear

Ioy 200

ubtotal $(95 \%$ CI $)$

otal events:

$\begin{array}{llll}117 & 1 & 118 & 100.0 \% \\ \mathbf{1 1 7} & & \mathbf{1 1 8} & \mathbf{1 0 0 . 0 \%}\end{array}$

$1.01[0.06,15.93]$

$1.01[0.06,15.93]$

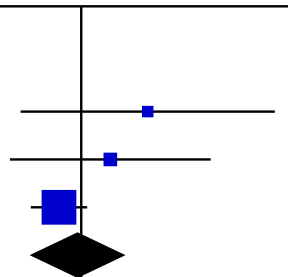

Ieterogeneity: Not applicable

est for overall effect: $\mathrm{Z}=0.01(\mathrm{P}=1.00)$

est for subgroup differences: $\mathrm{Chi}^{2}=0.12, \mathrm{df}=2(\mathrm{P}=0.94), \mathrm{I}^{2}=0 \%$

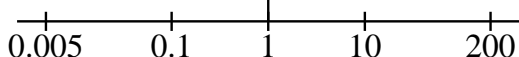

Favours lower SFA

Favours higher SFA

ootnotes

1) During trial, Prentice 2017 
Analysis 2.45. Comparison 2: SFA reduction vs usual diet secondary health events, Outcome 45: Stroke, subgroup by duration

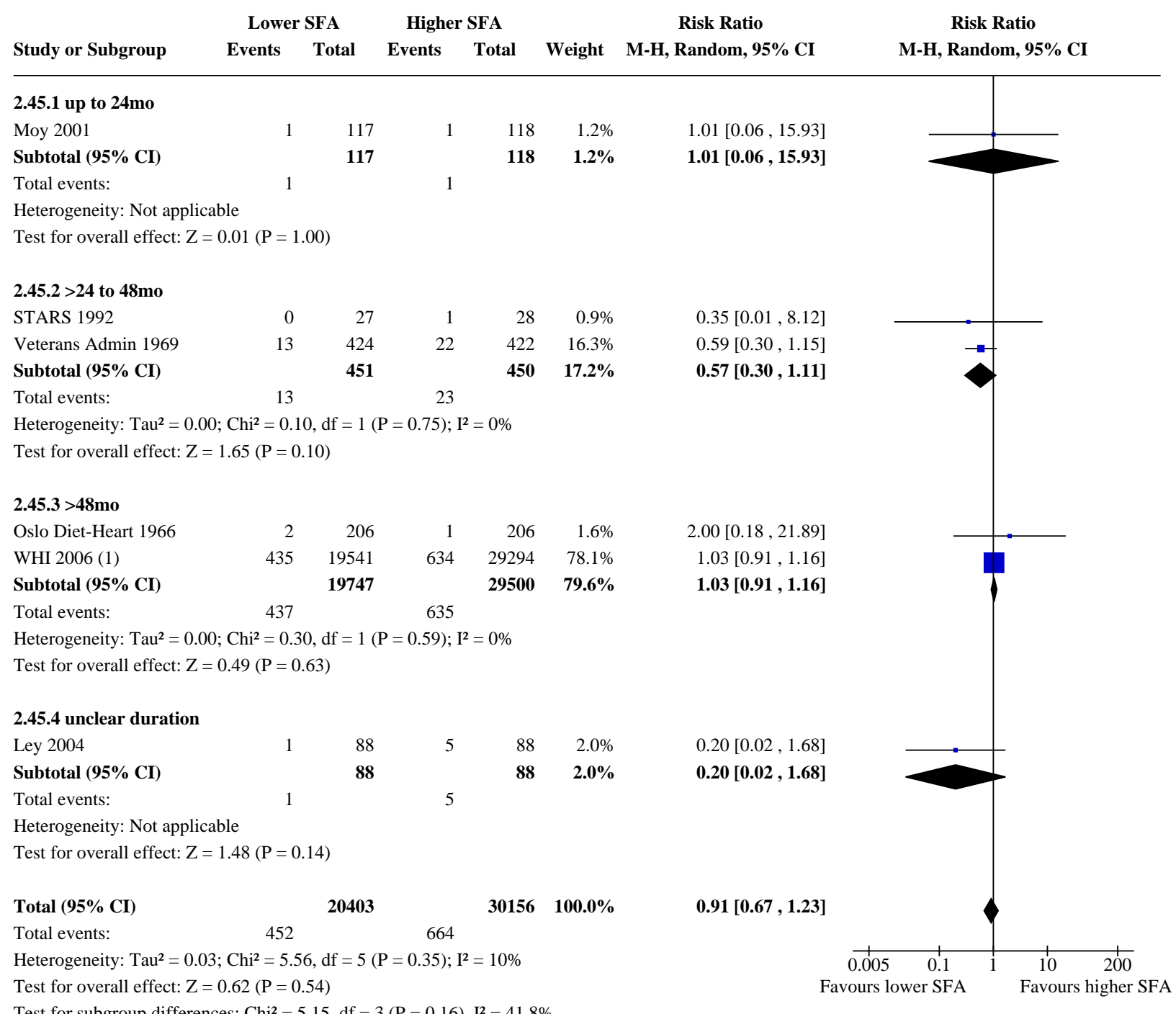

Footnotes

(1) During trial, Prentice 2017 
Analysis 2.46. Comparison 2: SFA reduction vs usual diet - secondary health events, Outcome 46: Stroke, subgroup by baseline SFA

Lower SFA

Higher SFA
Risk Ratio

Weight M-H, Random, 95\% CI
Risk Ratio

M-H, Random, 95\% CI

\subsection{1 up to $12 \%$ E SFA baseline} Subtotal (95\% CI)

Total events:

0
0

0
Not estimable

Heterogeneity: Not applicable

Test for overall effect: Not applicable

\subsection{2 $>12$ to $15 \%$ E SFA baseline}

Ley 2004

Moy 2001

WHI 2006 (1)

Subtotal $(95 \%$ CI)

Total events:

Heterogeneity: $\mathrm{Tau}^{2}=0.10 ; \mathrm{Chi}^{2}=2.27, \mathrm{df}=2(\mathrm{P}=0.32) ; \mathrm{I}^{2}=12 \%$

Test for overall effect: $\mathrm{Z}=0.30(\mathrm{P}=0.76)$

\subsection{3 >15 to 18\% E SFA baseline}

STARS 1992

Subtotal $(95 \%$ CI $)$

Total events:

Heterogeneity: Not applicable

Test for overall effect: $\mathrm{Z}=0.66(\mathrm{P}=0.51)$

\subsection{4 >18\% E SFA baseline}

Veterans Admin 1969

Subtotal (95\% CI)

Heterogeneity: Not applicable

Test for overall effect: $\mathrm{Z}=1.55(\mathrm{P}=0.12)$

\subsection{5 unclear}

Oslo Diet-Heart 1966

Subtotal (95\% CI)

$\begin{array}{llll}206 & 1 & 206 & 1.6 \% \\ \mathbf{2 0 6} & & \mathbf{2 0 6} & \mathbf{1 . 6 \%}\end{array}$

Total events:

2

Heterogeneity: Not applicable

Test for overall effect: $\mathrm{Z}=0.57(\mathrm{P}=0.57)$

Total $(95 \%$ CI $)$

20403

$30156 \quad 100.0 \%$

Total events:

452

664

Heterogeneity: $\mathrm{Tau}^{2}=0.03 ; \mathrm{Chi}^{2}=5.56, \mathrm{df}=5(\mathrm{P}=0.35) ; \mathrm{I}^{2}=10 \%$

Test for overall effect: $\mathrm{Z}=0.62(\mathrm{P}=0.54)$

Test for subgroup differences: $\mathrm{Chi}^{2}=1.79, \mathrm{df}=3(\mathrm{P}=0.62), \mathrm{I}^{2}=0 \%$
$0.20[0.02,1.68]$

$1.01[0.06,15.93]$

$1.03[0.91,1.16]$

$0.91[0.50,1.66]$
$0.35[0.01,8.12]$

$0.35[0.01,8.12]$

\section{Footnotes}

(1) During trial, Prentice 2017 


\section{Analysis 2.47. Comparison 2: SFA reduction vs usual diet - secondary health events, Outcome 47: Stroke, subgroup by SFA change}

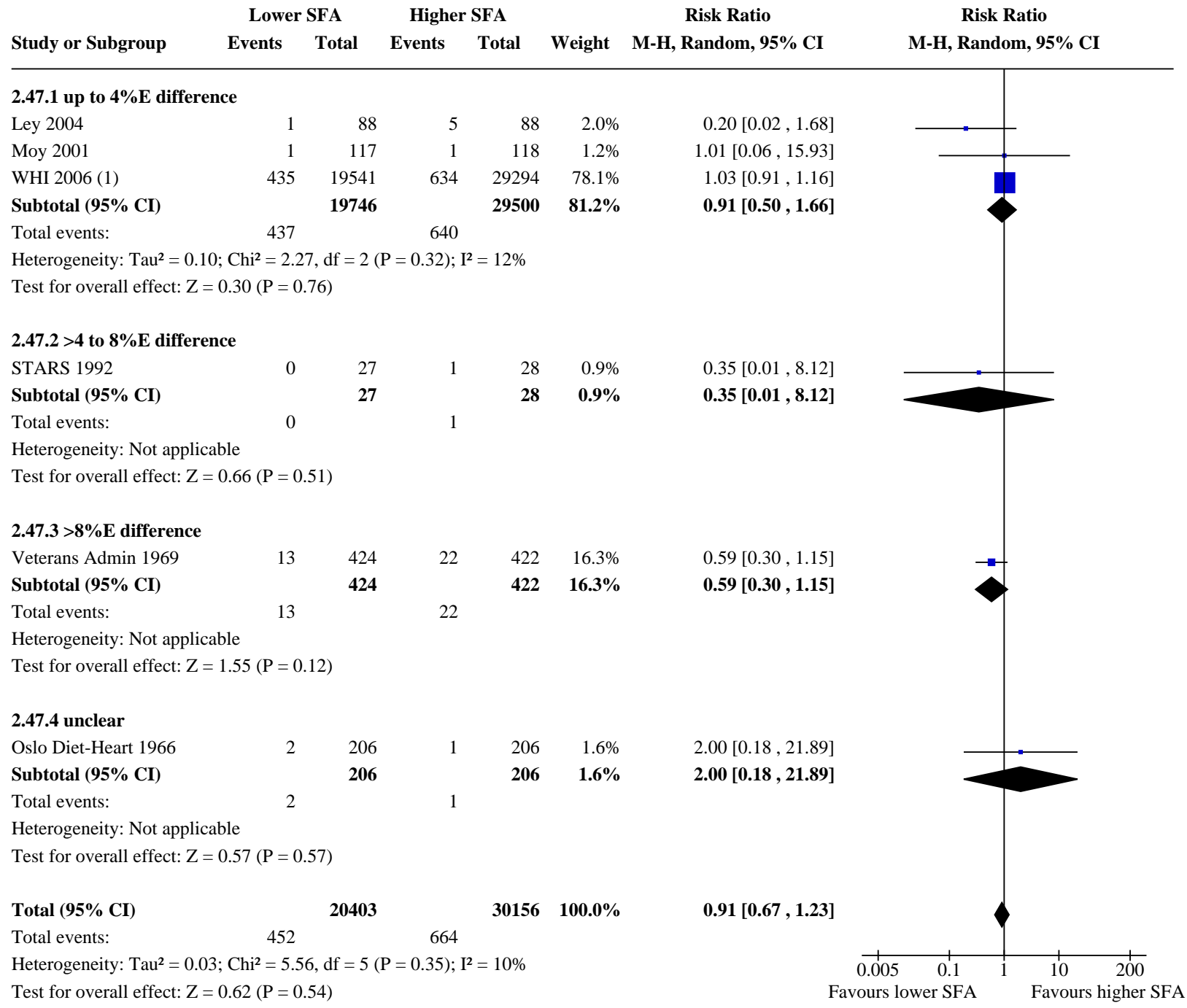

Footnotes

(1) During trial, Prentice 2017 


\section{Analysis 2.48. Comparison 2: SFA reduction vs usual diet - secondary health events, Outcome 48: Stroke, subgroup by sex}

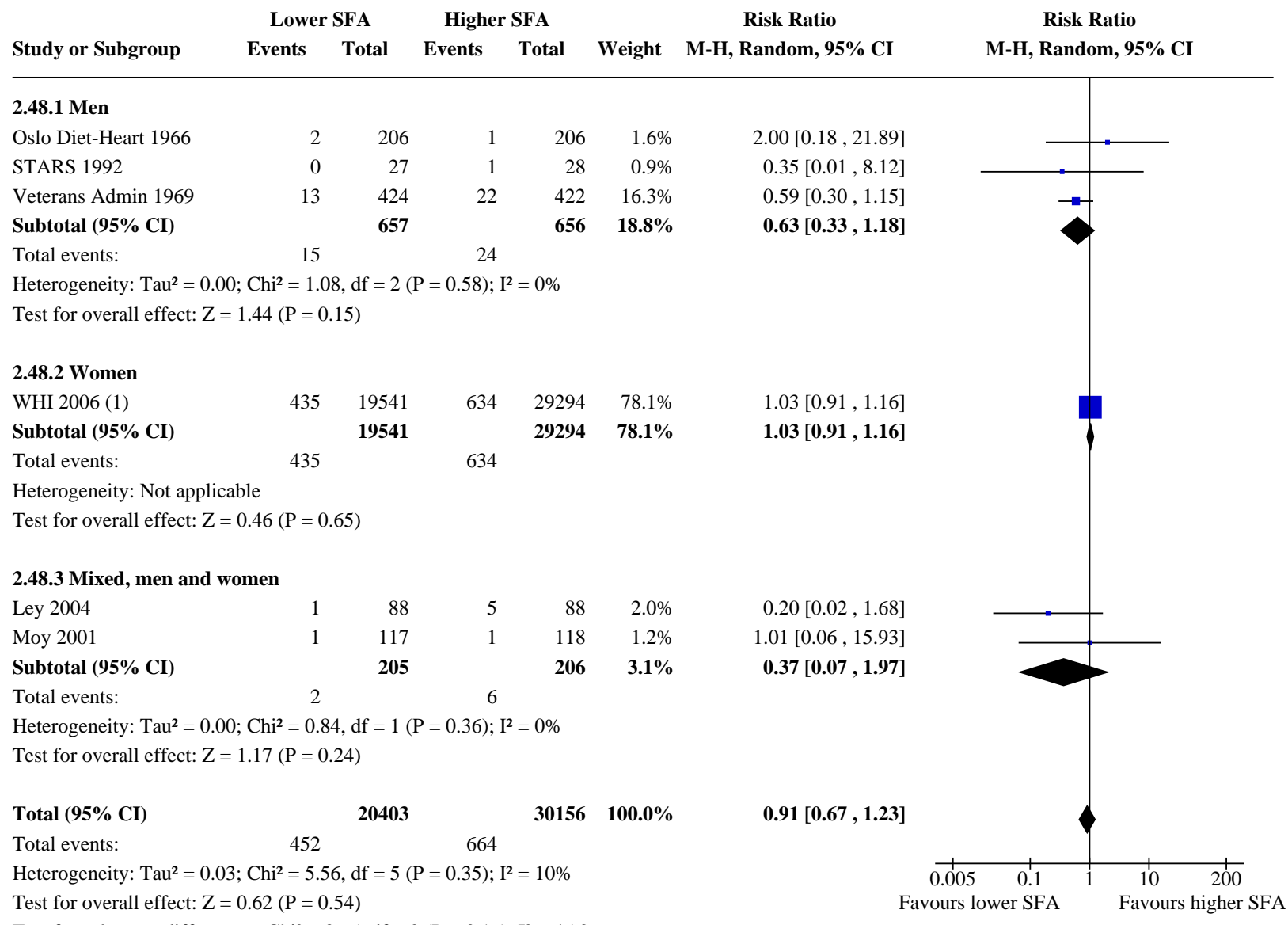

Footnotes

(1) During trial, Prentice 2017 
Analysis 2.49. Comparison 2: SFA reduction vs usual diet secondary health events, Outcome 49: Stroke, subgroup by CVD risk

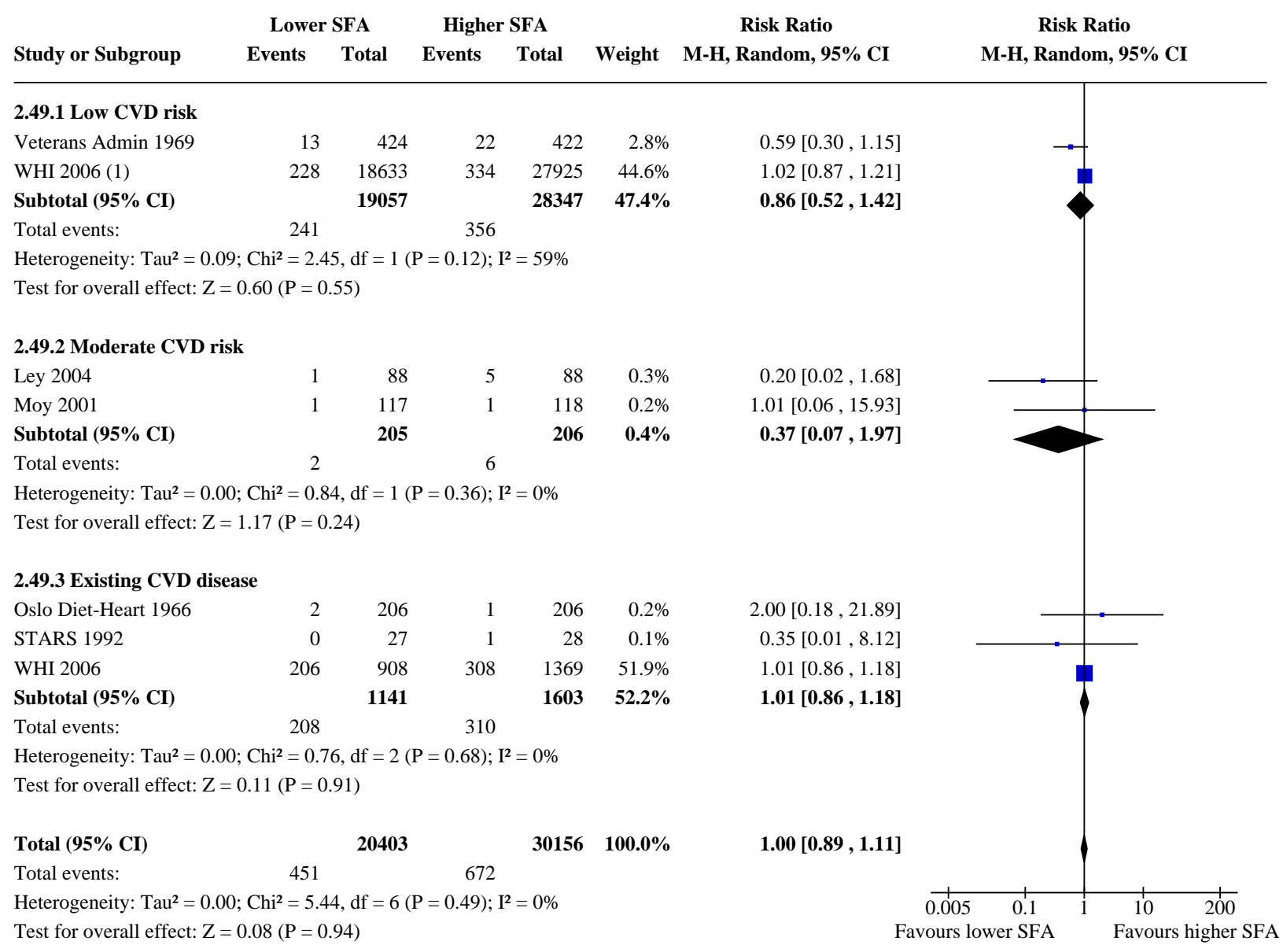

Footnotes

(1) Women without CVD at baseline 


\section{Analysis 2.50. Comparison 2: SFA reduction vs usual diet - secondary health events, Outcome 50: Stroke, subgroup by TC reduction}

\begin{tabular}{|c|c|c|c|c|c|}
\hline \multirow[b]{2}{*}{ or Subgi } & \multicolumn{2}{|c|}{ Lower SFA } & \multicolumn{2}{|c|}{ Higher SFA } & Risk Ratio \\
\hline & Events & Total & Events & Total & M-H, Random, $95 \%$ CI \\
\hline
\end{tabular}

2.50.1 serum chol reduced by at least $0.2 \mathrm{mmol} / \mathrm{L}$

MRC 1968

Moy 2001

Oslo Diet-Heart 1966

STARS 1992

Veterans Admin 1969

Subtotal $(95 \%$ CI)

Total events:

$2 \quad 199$

117

206

$2 \quad 206$

$0 \quad 27$

$13 \quad 424$

973

18

$0 \quad 194 \quad 4.0 \%$

$1 \quad 118 \quad 4.8 \%$

$1206 \quad 6.4 \%$

$1 \quad 28 \quad 3.7 \%$

$22 \quad 422 \quad 81.1 \%$

$968 \quad 100.0 \%$

Heterogeneity: $\mathrm{Tau}^{2}=0.00 ; \mathrm{Chi}^{2}=2.85, \mathrm{df}=4(\mathrm{P}=0.58) ; \mathrm{I}^{2}=0 \%$

Test for overall effect: $\mathrm{Z}=1.17(\mathrm{P}=0.24)$

2.50.2 serum chol reduced by $<0.2 \mathrm{mmol} / \mathrm{L}$

$\begin{array}{lrrrrr}\text { Ley 2004 } & 1 & 88 & 5 & 88 & 28.1 \% \\ \text { WHI 2006 (1) } & 435 & 19541 & 634 & 29294 & 71.9 \% \\ \text { Subtotal (95\% CI) } & & \mathbf{1 9 6 2 9} & & \mathbf{2 9 3 8 2} & \mathbf{1 0 0 . 0 \%} \\ \text { Total events: } & 436 & & 639 & & \end{array}$

Heterogeneity: $\mathrm{Tau}^{2}=0.75 ; \mathrm{Chi}^{2}=2.27, \mathrm{df}=1(\mathrm{P}=0.13) ; \mathrm{I}^{2}=56 \%$

Test for overall effect: $\mathrm{Z}=0.59(\mathrm{P}=0.56)$

2.50.3 serum chol reduction unclear

Subtotal (95\% CI)

0

Total events: $\quad 0$

0

0

Heterogeneity: Not applicable

Test for overall effect: Not applicable

Test for subgroup differences: $\mathrm{Chi}^{2}=0.01, \mathrm{df}=1(\mathrm{P}=0.93), \mathrm{I}^{2}=0 \%$

Footnotes

(1) During trial, Prentice 2017
$4.88[0.24,100.89]$

$1.01[0.06,15.93]$

$2.00[0.18,21.89]$

$0.35[0.01,8.12]$

$0.59[0.30,1.15]$

$0.70[0.38,1.28]$

$0.20[0.02,1.68]$

$1.03[0.91,1.16]$

$0.65[0.15,2.75]$

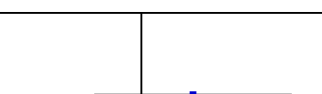

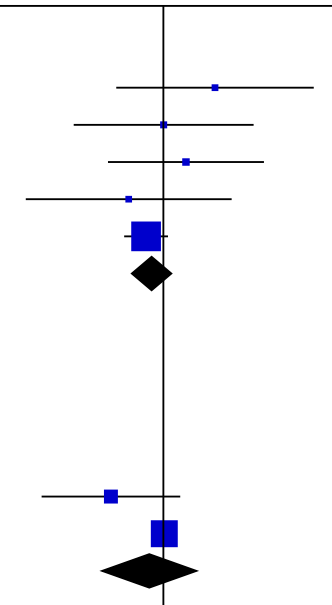

Favours lower SFA $\quad$ Favours higher SFA

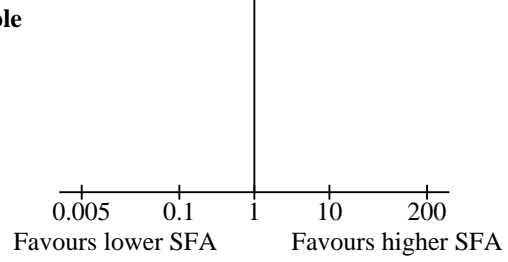

Not estimable 


\section{Analysis 2.51. Comparison 2: SFA reduction vs usual diet - secondary} health events, Outcome 51: Stroke, subgroup decade of publication

Lower SFA
Risk Ratio

Weight M-H, Random, 95\% CI
Risk Ratio

M-H, Random, 95\% CI

\subsubsection{0s}

MRC 1968

Oslo Diet-Heart 1966

vents Total

$4.88[0.24,100.89]$

$2.00[0.18,21.89]$

$0.59[0.30,1.15]$

$0.92[0.31,2.69]$

Subtotal $(95 \%$ CI)

$422 \quad 16.4 \%$

$822 \quad 19.0 \%$

Total events:

17

23

Heterogeneity: $\mathrm{Tau}^{2}=0.30 ; \mathrm{Chi}^{2}=2.60, \mathrm{df}=2(\mathrm{P}=0.27) ; \mathrm{I}^{2}=23 \%$

Test for overall effect: $\mathrm{Z}=0.16(\mathrm{P}=0.87)$

\subsubsection{0s}

\section{Subtotal (95\% CI)}

Total events:

0

0

Heterogeneity: Not applicable

Test for overall effect: Not applicable

\subsubsection{0s}

Subtotal (95\% CI)

Total events:

0

Heterogeneity: Not applicable

Test for overall effect: Not applicable

\subsubsection{0s}

STARS 1992

Subtotal (95\% CI)

Total events:

0

27

27

0

Not estimable

Heterogeneity: Not applicable

Test for overall effect: $\mathrm{Z}=0.66(\mathrm{P}=0.51)$

\subsubsection{0s}

Ley 2004

Moy 2001

WHI 2006 (1)

Subtotal (95\% CI)

Total events:

$\begin{array}{rr}1 & 88 \\ 1 & 117 \\ 435 & 1954 \\ & \mathbf{1 9 7 4 6}\end{array}$

$\begin{array}{llll}88 & 5 & 88 & 2.0 \%\end{array}$

$1.2 \%$

9541

118

$76.9 \%$

437

$29500 \quad 80.1 \%$ 640

Heterogeneity: $\mathrm{Tau}^{2}=0.10 ; \mathrm{Chi}^{2}=2.27, \mathrm{df}=2(\mathrm{P}=0.32) ; \mathrm{I}^{2}=12 \%$

Test for overall effect: $\mathrm{Z}=0.30(\mathrm{P}=0.76)$

Total $(95 \%$ CI $)$

Total events:

Heterogeneity: $\mathrm{Tau}^{2}=0.03 ; \mathrm{Chi}^{2}=6.59, \mathrm{df}=6(\mathrm{P}=0.36) ; \mathrm{I}^{2}=9 \%$

Test for overall effect: $\mathrm{Z}=0.52(\mathrm{P}=0.60)$

Test for subgroup differences: $\mathrm{Chi}^{2}=0.35, \mathrm{df}=2(\mathrm{P}=0.84), \mathrm{I}^{2}=0 \%$
$0.20[0.02,1.68]$

$1.01[0.06,15.93]$

$1.03[0.91,1.16]$

$0.91[0.50,1.66]$

$0.35[0.01,8.12]$

$0.35[0.01,8.12]$

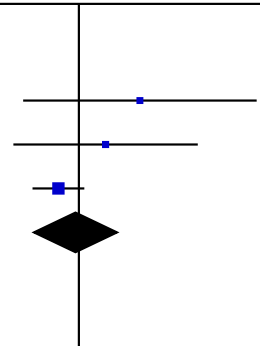

\section{Footnotes}

(1) During trial, Prentice 2017 


\section{Analysis 2.52. Comparison 2: SFA reduction vs usual diet - secondary health events, Outcome 52: CORONARY HEART DISEASE MORTALITY}

\begin{tabular}{|c|c|c|c|c|c|c|c|}
\hline \multirow[b]{2}{*}{ Study or Subgroup } & \multicolumn{2}{|c|}{ Lower SFA } & \multicolumn{2}{|c|}{ Higher SFA } & \multirow[b]{2}{*}{ Weight } & \multirow{2}{*}{$\begin{array}{c}\text { Risk Ratio } \\
\text { M-H, Random, } 95 \% \text { CI }\end{array}$} & \multirow{2}{*}{$\begin{array}{c}\text { Risk Ratio } \\
\text { M-H, Random, 95\% CI }\end{array}$} \\
\hline & Events & Total & Events & Total & & & \\
\hline DART 1989 & 97 & 1018 & 97 & 1015 & $21.9 \%$ & $1.00[0.76,1.30]$ & \\
\hline Houtsmuller 1979 & 0 & 51 & 5 & 51 & $0.4 \%$ & $0.09[0.01,1.60]$ & $\longleftarrow$ \\
\hline MRC 1968 & 25 & 199 & 25 & 194 & $9.1 \%$ & $0.97[0.58,1.64]$ & 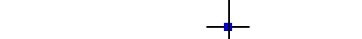 \\
\hline Oslo Diet-Heart 1966 & 37 & 206 & 50 & 206 & $14.5 \%$ & $0.74[0.51,1.08]$ & $\rightarrow$ \\
\hline Rose corn oil 1965 & 5 & 28 & 1 & 13 & $0.7 \%$ & $2.32[0.30,17.92]$ & \\
\hline Rose olive 1965 & 3 & 26 & 0 & 13 & $0.4 \%$ & $3.63[0.20,65.44]$ & \\
\hline Sydney Diet-Heart 1978 & 35 & 221 & 23 & 237 & $9.9 \%$ & $1.63[1.00,2.67]$ & - \\
\hline Veterans Admin 1969 & 41 & 424 & 50 & 422 & $13.9 \%$ & $0.82[0.55,1.21]$ & \\
\hline WHI 2006 (1) & 172 & 19541 & 261 & 29294 & $29.2 \%$ & $0.99[0.82,1.20]$ & \\
\hline Total $(95 \%$ CI $)$ & & 21714 & & 31445 & $100.0 \%$ & $0.97[0.82,1.16]$ & 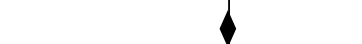 \\
\hline Total events: & 415 & & 512 & & & & \\
\hline \multicolumn{5}{|c|}{ Heterogeneity: $\mathrm{Tau}^{2}=0.02 ; \mathrm{Chi}^{2}=11.18, \mathrm{df}=8(\mathrm{P}=0.19) ; \mathrm{I}^{2}=28 \%$} & & & 0.01 \\
\hline Test for overall effect: $Z$ & $2(\mathrm{P}=0.75$ & & & & & & Gavours lower SFA \\
\hline
\end{tabular}

Footnotes

(1) CHD death during trial, Prentice 2017

Analysis 2.53. Comparison 2: SFA reduction vs usual diet - secondary health events, Outcome 53: CHD mortality, SA by low summary risk of bias

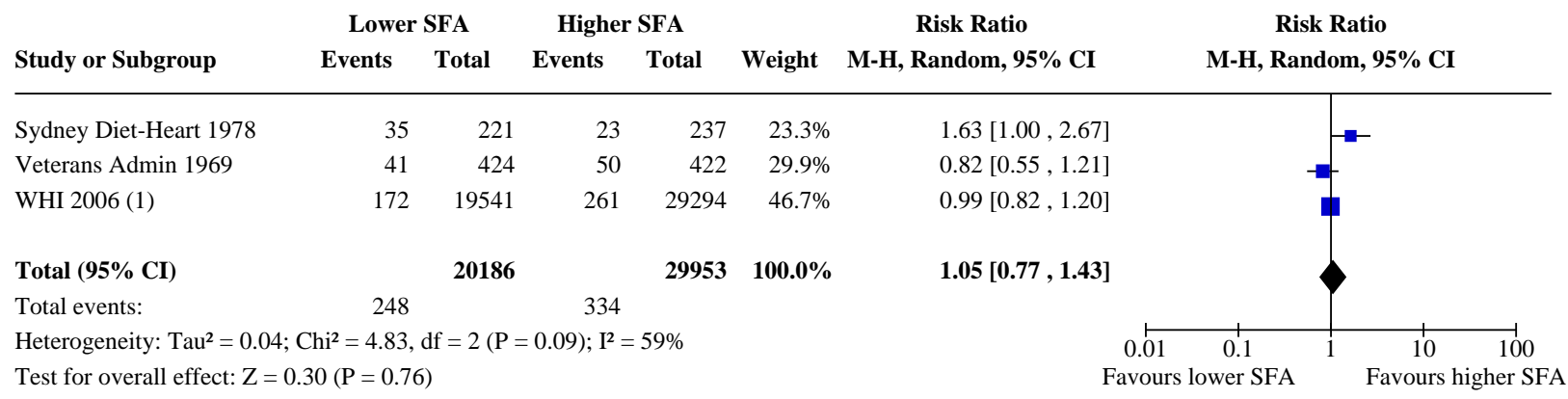

Test for subgroup differences: Not applicable

Footnotes

(1) CHD death during trial, Prentice 2017 
Analysis 2.54. Comparison 2: SFA reduction vs usual diet - secondary health events, Outcome 54: CHD mortality, SA aim to reduce SFA

\begin{tabular}{cccccccc} 
& \multicolumn{2}{c}{ Lower SFA } & \multicolumn{2}{c}{ Higher SFA } & \multicolumn{2}{c}{ Risk Ratio } & Risk Ratio \\
Study or Subgroup & Events & Total & Events & Total & Weight & M-H, Random, 95\% CI & M-H, Random, 95\% CI
\end{tabular}

\begin{tabular}{lrrrrrr}
\hline DART 1989 & 97 & 1018 & 97 & 1015 & $21.9 \%$ & $1.00[0.76,1.30]$ \\
Houtsmuller 1979 & 0 & 51 & 5 & 51 & $0.4 \%$ & $0.09[0.01,1.60]$ \\
MRC 1968 & 25 & 199 & 25 & 194 & $9.1 \%$ & $0.97[0.58,1.64]$ \\
Oslo Diet-Heart 1966 & 37 & 206 & 50 & 206 & $14.5 \%$ & $0.74[0.51,1.08]$ \\
Rose corn oil 1965 & 5 & 28 & 1 & 13 & $0.7 \%$ & $2.32[0.30,17.92]$ \\
Rose olive 1965 & 3 & 26 & 0 & 13 & $0.4 \%$ & $3.63[0.20,65.44]$ \\
Sydney Diet-Heart 1978 & 35 & 221 & 23 & 237 & $9.9 \%$ & $1.63[1.00,2.67]$ \\
Veterans Admin 1969 & 41 & 424 & 50 & 422 & $13.9 \%$ & $0.82[0.55,1.21]$ \\
WHI 2006(1) & 172 & 19541 & 261 & 29294 & $29.2 \%$ & $0.99[0.82,1.20]$ \\
& & & & & & \\
Total (95\% CI) & & $\mathbf{2 1 7 1 4}$ & & $\mathbf{3 1 4 4 5}$ & $\mathbf{1 0 0 . 0 \%}$ & $\mathbf{0 . 9 7}[\mathbf{0 . 8 2 , \mathbf { 1 . 1 6 }}]$ \\
Total events: & 415 & & 512 & & &
\end{tabular}

Heterogeneity: $\mathrm{Tau}^{2}=0.02 ; \mathrm{Chi}^{2}=11.18, \mathrm{df}=8(\mathrm{P}=0.19) ; \mathrm{I}^{2}=28 \%$

Test for overall effect: $\mathrm{Z}=0.32(\mathrm{P}=0.75)$

Test for subgroup differences: Not applicable

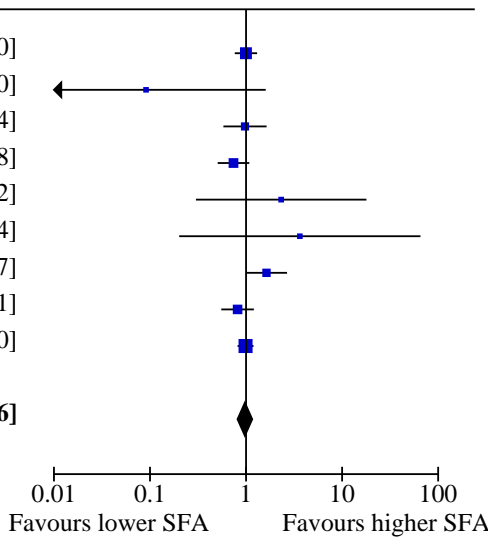

Footnotes

(1) CHD death during trial, Prentice 2017

Analysis 2.55. Comparison 2: SFA reduction vs usual diet - secondary health events, Outcome 55: CHD mortality, SA statistically significant SFA reduction

\begin{tabular}{|c|c|c|c|c|c|c|c|}
\hline \multirow[b]{2}{*}{ Study or Subgroup } & \multicolumn{2}{|c|}{ Lower SFA } & \multicolumn{2}{|c|}{ Higher SFA } & \multirow[b]{2}{*}{ Weight } & \multirow{2}{*}{$\begin{array}{c}\text { Risk Ratio } \\
\text { M-H, Random, } 95 \% \text { CI }\end{array}$} & \multirow{2}{*}{$\begin{array}{c}\text { Risk Ratio } \\
\text { M-H, Random, } 95 \% \text { CI }\end{array}$} \\
\hline & Events & Total & Events & Total & & & \\
\hline DART 1989 & 97 & 1018 & 97 & 1015 & $29.3 \%$ & $1.00[0.76,1.30]$ & \\
\hline Sydney Diet-Heart 1978 & 35 & 221 & 23 & 237 & $12.5 \%$ & $1.63[1.00,2.67]$ & (a) \\
\hline Veterans Admin 1969 & 41 & 424 & 50 & 422 & $18.0 \%$ & $0.82[0.55,1.21]$ & \\
\hline WHI 2006 (1) & 172 & 19541 & 261 & 29294 & $40.3 \%$ & $0.99[0.82,1.20]$ & \\
\hline Total $(95 \%$ CI $)$ & & 21204 & & 30968 & $100.0 \%$ & $1.02[0.84,1.24]$ & 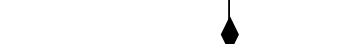 \\
\hline Total events: & 345 & & 431 & & & & \\
\hline $\begin{array}{l}\text { Heterogeneity: } \mathrm{Tau}^{2}=0.0 \\
\text { Test for overall effect: } \mathrm{Z}\end{array}$ & $\begin{array}{l}\mathrm{hi}^{2}=4.84 \\
19(\mathrm{P}=0.8\end{array}$ & $\mathrm{f}=3(\mathrm{P}=$ & $=0.18) ; \mathrm{I}^{2}$ & $38 \%$ & & & 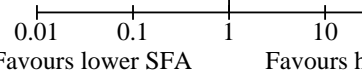 \\
\hline
\end{tabular}

Test for subgroup differences: Not applicable

Footnotes

(1) CHD death during trial, Prentice 2017 


\section{Analysis 2.56. Comparison 2: SFA reduction vs usual diet - secondary} health events, Outcome 56: CHD mortality, SA by TC reduction

\begin{tabular}{|c|c|c|c|c|c|c|c|}
\hline \multirow[b]{2}{*}{ Study or Subgroup } & \multicolumn{2}{|c|}{ Lower SFA } & \multicolumn{2}{|c|}{ Higher SFA } & \multirow[b]{2}{*}{ Weight } & \multirow{2}{*}{$\begin{array}{c}\text { Risk Ratio } \\
\text { M-H, Random, } 95 \% \text { CI }\end{array}$} & \multirow{2}{*}{$\begin{array}{c}\text { Risk Ratio } \\
\text { M-H, Random, 95\% CI }\end{array}$} \\
\hline & Events & Total & Events & Total & & & \\
\hline DART 1989 & 97 & 1018 & 97 & 1015 & $21.9 \%$ & $1.00[0.76,1.30]$ & \\
\hline Houtsmuller 1979 & 0 & 51 & 5 & 51 & $0.4 \%$ & $0.09[0.01,1.60]$ & $\longleftarrow$ \\
\hline MRC 1968 & 25 & 199 & 25 & 194 & $9.3 \%$ & $0.97[0.58,1.64]$ & - \\
\hline Oslo Diet-Heart 1966 & 37 & 206 & 50 & 206 & $14.7 \%$ & $0.74[0.51,1.08]$ & $\rightarrow$ \\
\hline Rose corn oil 1965 & 5 & 28 & 1 & 13 & $0.7 \%$ & $2.32[0.30,17.92]$ & \\
\hline Sydney Diet-Heart 1978 & 35 & 221 & 23 & 237 & $10.0 \%$ & $1.63[1.00,2.67]$ & - \\
\hline Veterans Admin 1969 & 41 & 424 & 50 & 422 & $14.1 \%$ & $0.82[0.55,1.21]$ & \\
\hline WHI 2006 (1) & 172 & 19541 & 261 & 29294 & $28.9 \%$ & $0.99[0.82,1.20]$ & 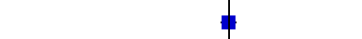 \\
\hline Total $(95 \%$ CI $)$ & & 21688 & & 31432 & $100.0 \%$ & $0.97[0.81,1.16]$ & 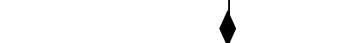 \\
\hline Total events: & 412 & & 512 & & & & \\
\hline $\begin{array}{l}\text { Heterogeneity: } \mathrm{Tau}^{2}=0 . \\
\text { Test for overall effect: } \mathrm{Z}\end{array}$ & $\begin{array}{l}\mathrm{hi}^{2}=10.3 \\
6(\mathrm{P}=0.7\end{array}$ & $d f=7(P$ & $=0.17) ; \mathrm{I}^{2}$ & $33 \%$ & & & $\begin{array}{cc}0.01 & 0.1 \\
\text { Favours lower SFA } & 1\end{array}$ \\
\hline
\end{tabular}

Test for subgroup differences: Not applicable

Footnotes

(1) CHD death during trial, Prentice 2017

\section{Analysis 2.57. Comparison 2: SFA reduction vs usual diet - secondary health events, Outcome 57: CHD mortality, SA excluding WHI}

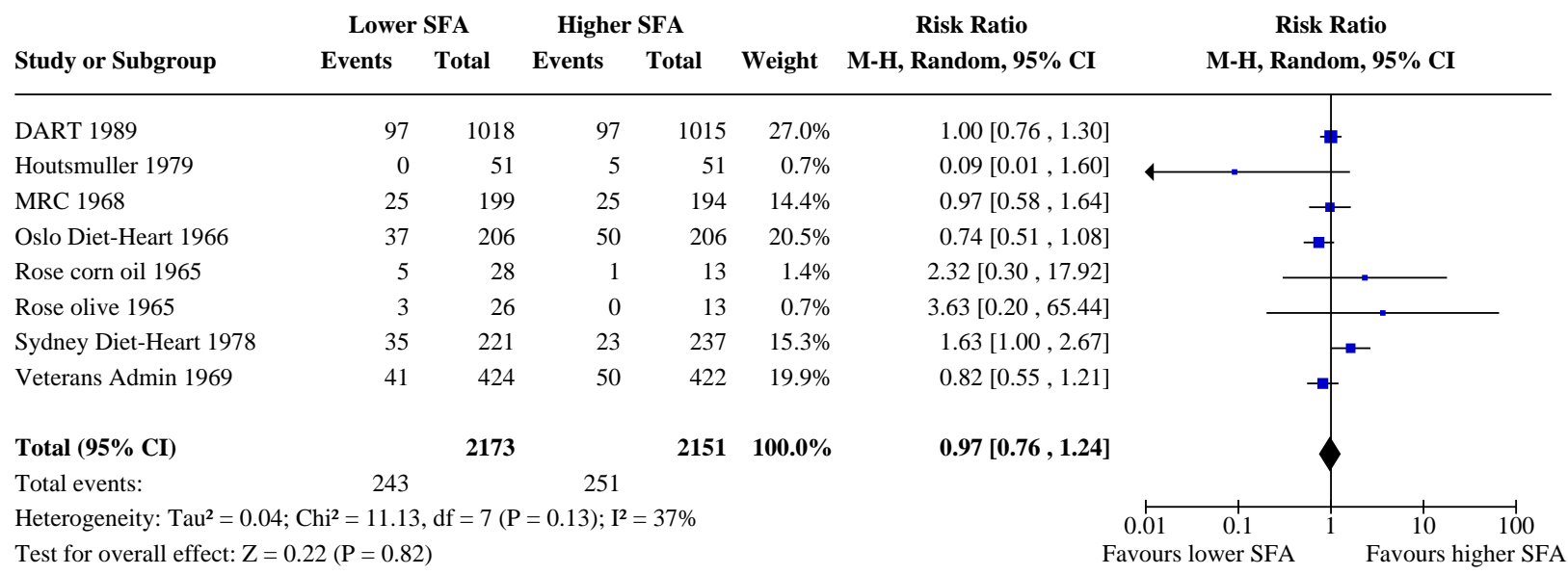


Analysis 2.58. Comparison 2: SFA reduction vs usual diet - secondary health events, Outcome 58: CHD mortality, SA Mantel-Haenszel fixed-effect

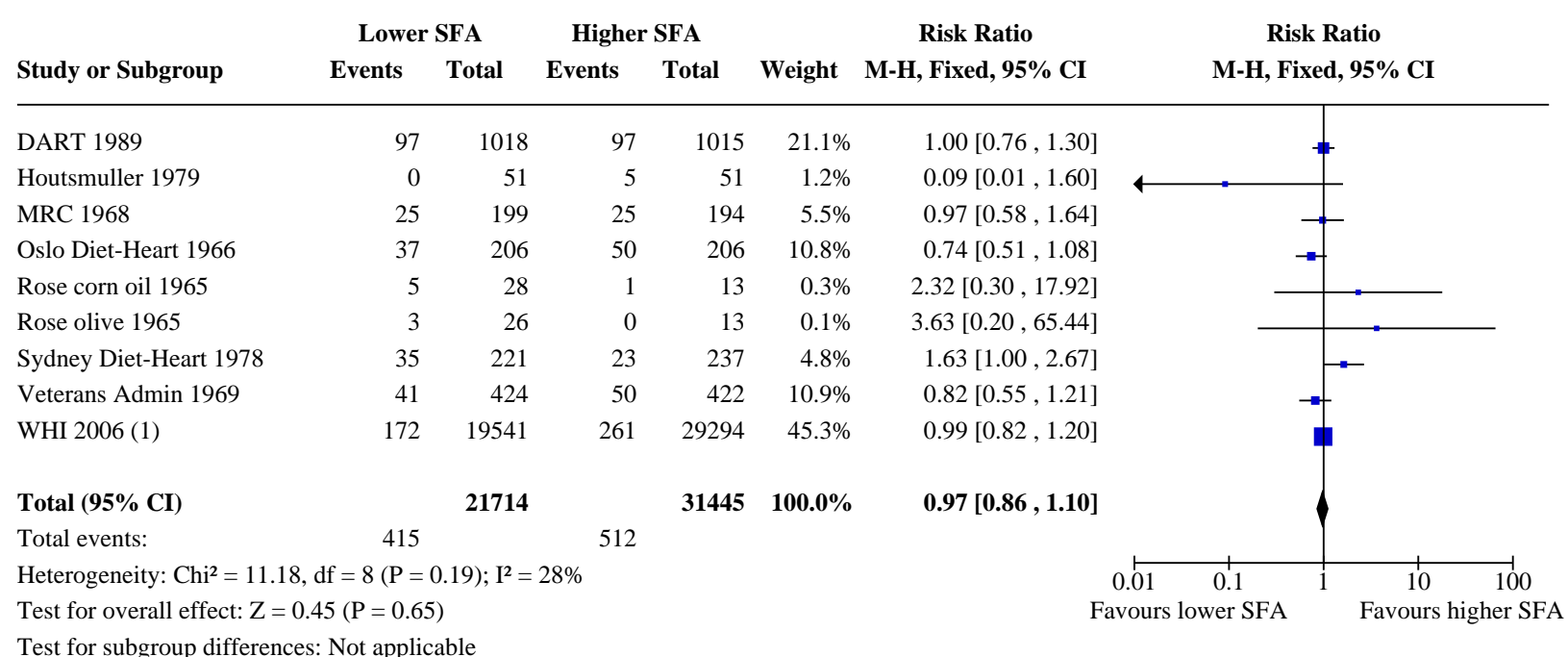

Footnotes

(1) CHD death during trial, Prentice 2017

\section{Analysis 2.59. Comparison 2: SFA reduction vs usual diet - secondary health events, Outcome 59: CHD mortality, SA Peto fixed-effect}

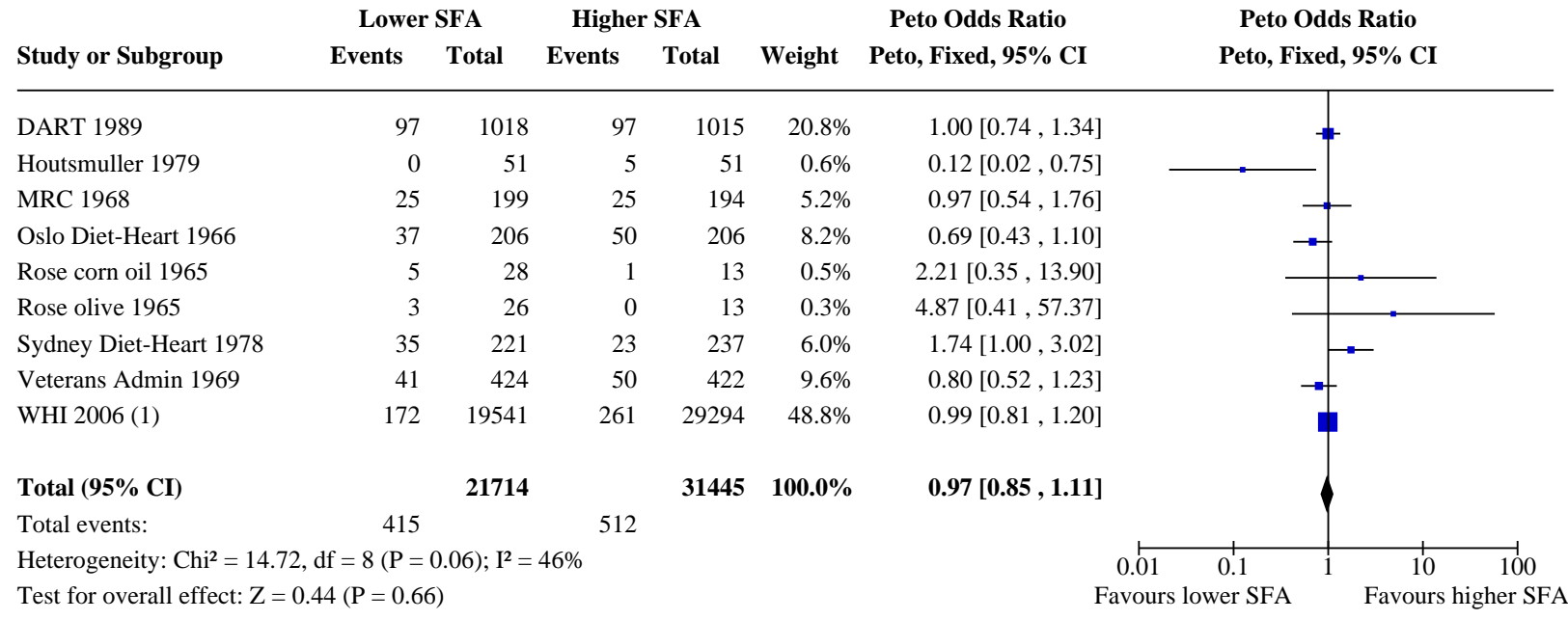

Footnotes

(1) CHD death during trial, Prentice 2017 
Analysis 2.60. Comparison 2: SFA reduction vs usual diet - secondary health events, Outcome 60: CHD mortality, subgroup by any substitution

Lower SFA Higher SFA Events Total Events Total
Risk Ratio

Weight M-H, Random, 95\% CI
Risk Ratio

M-H, Random, $95 \%$ CI

\subsection{1 replaced by PUFA}

DART 1989

Houtsmuller 1979

MRC 1968

Oslo Diet-Heart 1966

Rose corn oil 1965

Sydney Diet-Heart 1978

Veterans Admin 1969

Subtotal $(95 \%$ CI)

Total events:

\section{7}

0

1018

51

$25 \quad 199$

$\begin{array}{ll}25 & 199 \\ 37 & 206\end{array}$

$\begin{array}{rr}37 & 28\end{array}$

$35 \quad 221$

$41 \quad 424$

2147

240

97
5
25
50
1
23
50

$\begin{array}{rr}1015 & 25.6 \% \\ 51 & 0.9 \% \\ 194 & 15.2 \% \\ 206 & 20.5 \% \\ 26 & 1.6 \% \\ 237 & 16.1 \% \\ 422 & 20.0 \% \\ \mathbf{2 1 5 1} & \mathbf{1 0 0 . 0 \%}\end{array}$

251
Heterogeneity: $\mathrm{Tau}^{2}=0.06 ; \mathrm{Chi}^{2}=11.81, \mathrm{df}=6(\mathrm{P}=0.07) ; \mathrm{I}^{2}=49 \%$

Test for overall effect: $\mathrm{Z}=0.18(\mathrm{P}=0.86)$

\subsection{2 replaced by MUFA}

Rose olive 1965

Subtotal (95\% CI)

26

$$
1
$$

$26 \quad 100.0 \%$

$3.00[0.33,26.99]$

$3.00[0.33,26.99]$

$26 \quad \mathbf{1 0 0 . 0 \%}$
$1.00[0.76,1.30]$

$0.09[0.01,1.60]$

$0.97[0.58,1.64]$

$0.74[0.51,1.08]$

$4.64[0.58,37.15]$

$1.63[1.00,2.67]$

$0.82[0.55,1.21]$

$0.98[0.74,1.28]$

Total events: 3

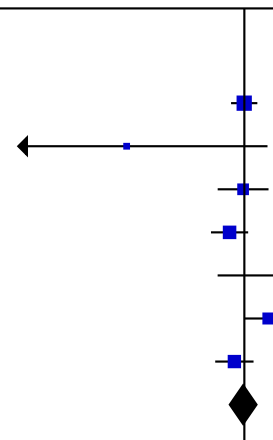

Heterogeneity: Not applicable

Test for overall effect: $\mathrm{Z}=0.98(\mathrm{P}=0.33)$

\subsection{3 replaced by $\mathrm{CHO}$}

$\begin{array}{lrrrrr}\text { DART 1989 } & 97 & 1018 & 97 & 1015 & 33.9 \% \\ \text { WHI 2006 (1) } & 172 & 19541 & 261 & 29294 & 66.1 \% \\ \text { Subtotal (95\% CI) } & & \mathbf{2 0 5 5 9} & & \mathbf{3 0 3 0 9} & \mathbf{1 0 0 . 0 \%} \\ \text { Total events: } & 269 & & 358 & & \end{array}$

$1.00[0.76,1.30]$

$0.99[0.82,1.20]$

$0.99[0.85,1.16]$

Heterogeneity: $\mathrm{Tau}^{2}=0.00 ; \mathrm{Chi}^{2}=0.00, \mathrm{df}=1(\mathrm{P}=0.96) ; \mathrm{I}^{2}=0 \%$

Test for overall effect: $\mathrm{Z}=0.11(\mathrm{P}=0.91)$

2.60.4 replaced by protein

$\begin{array}{lrrrrr}\text { DART 1989 } & 97 & 1018 & 97 & 1015 & 33.9 \% \\ \text { WHI 2006 (1) } & 172 & 19541 & 261 & 29294 & 66.1 \% \\ \text { Subtotal (95\% CI) } & & \mathbf{2 0 5 5 9} & & \mathbf{3 0 3 0 9} & \mathbf{1 0 0 . 0 \%} \\ \text { Total events: } & 269 & & 358 & & \end{array}$

$1.00[0.76,1.30]$

$0.99[0.82,1.20]$

$0.99[0.85,1.16]$

Heterogeneity: $\mathrm{Tau}^{2}=0.00 ; \mathrm{Chi}^{2}=0.00, \mathrm{df}=1(\mathrm{P}=0.96) ; \mathrm{I}^{2}=0 \%$

Test for overall effect: $\mathrm{Z}=0.11(\mathrm{P}=0.91)$

\subsection{5 replacement unclear}

Subtotal (95\% CI)

Total events:

0

0

0

Heterogeneity: Not applicable

Test for overall effect: Not applicable

Test for subgroup differences: $\mathrm{Chi}^{2}=0.99, \mathrm{df}=3(\mathrm{P}=0.80), \mathrm{I}^{2}=0 \%$

\section{Footnotes}

(1) CHD death during trial, Prentice 2017 o Not estimable 
Analysis 2.61. Comparison 2: SFA reduction vs usual diet - secondary health events, Outcome 61: CHD mortality, subgroup by main substitution

Lower SFA

Higher SFA

Events Total
Risk Ratio

Risk Ratio

Events Total Weight M-H, Random, 95\% CI
M-H, Random, $95 \%$ CI

\subsection{1 replaced by PUFA}

DART 1989

Houtsmuller 1979

MRC 1968

Oslo Diet-Heart 1966

Rose corn oil 1965

Sydney Diet-Heart 1978

Veterans Admin 1969

Subtotal (95\% CI)

Total events:

Heterogeneity: $\mathrm{Tau}^{2}=0.06 ; \mathrm{Chi}^{2}=11.81, \mathrm{df}=6(\mathrm{P}=0.07) ; \mathrm{I}^{2}=49 \%$

Test for overall effect: $\mathrm{Z}=0.18(\mathrm{P}=0.86)$

\subsection{2 replaced by MUFA}

Rose olive 1965

Subtotal (95\% CI)

326
26

1

\section{$26 \quad 100.0 \%$ \\ $26 \quad 100.0 \%$}

3

1

Heterogeneity: Not applicable

Test for overall effect: $\mathrm{Z}=0.98(\mathrm{P}=0.33)$

\subsection{3 replaced by CHO}

WHI 2006 (1)

Subtotal (95\% CI)

Heterogeneity: Not applicable

Test for overall effect: $\mathrm{Z}=0.12(\mathrm{P}=0.90)$

\subsection{4 replaced by protein}

\section{Subtotal (95\% CI)}

0

0

0

0

Heterogeneity: Not applicable

Test for overall effect: Not applicable

\subsection{5 replacement unclear}

\section{Subtotal (95\% CI)}

0

0

Total events:
$1.00[0.76,1.30]$

$0.97[0.58,1.64]$

$0.74[0.51,1.08]$

$4.64[0.58,37.15]$

$1.63[1.00,2.67]$

$0.82[0.55,1.21]$

$0.98[0.74,1.28]$
$0.09[0.01,1.60]$

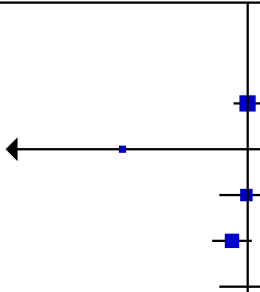

$3.00[0.33,26.99]$

3.00 [0.33 , 26.99]
$0.99[0.82,1.20]$

$0.99[0.82,1.20]$

Not estimable

Test for overall effect: Not applicable

Test for subgroup differences: $\mathrm{Chi}^{2}=0.99, \mathrm{df}=2(\mathrm{P}=0.61), \mathrm{I}^{2}=0 \%$

\section{Footnotes}

Not estimable

(1) CHD death during trial, Prentice 2017 


\section{Analysis 2.62. Comparison 2: SFA reduction vs usual diet - secondary health events, Outcome 62: CHD mortality, subgroup by duration}

\begin{tabular}{|c|c|c|c|c|c|c|}
\hline \multirow[b]{2}{*}{ Study or Subgroup } & \multicolumn{2}{|c|}{ Lower SFA } & \multicolumn{2}{|c|}{ Higher SFA } & \multirow[b]{2}{*}{ Weight } & \multirow{2}{*}{$\begin{array}{c}\text { Risk Ratio } \\
\text { M-H, Random, 95\% CI }\end{array}$} \\
\hline & Events & Total & Events & Total & & \\
\hline \multicolumn{7}{|l|}{ 2.62.1 up to $24 \mathrm{mo}$} \\
\hline DART 1989 & 97 & 1018 & 97 & 1015 & $97.5 \%$ & $1.00[0.76,1.30]$ \\
\hline Rose corn oil 1965 & 5 & 28 & 1 & 13 & $1.7 \%$ & $2.32[0.30,17.92]$ \\
\hline Rose olive 1965 & 3 & 26 & 0 & 13 & $0.8 \%$ & $3.63[0.20,65.44]$ \\
\hline Subtotal (95\% CI) & & 1072 & & 1041 & $100.0 \%$ & $1.02[0.78,1.33]$ \\
\hline Total events: & 105 & & 98 & & & \\
\hline
\end{tabular}

Heterogeneity: $\mathrm{Tau}^{2}=0.00 ; \mathrm{Chi}^{2}=1.40, \mathrm{df}=2(\mathrm{P}=0.50) ; \mathrm{I}^{2}=0 \%$

Test for overall effect: $\mathrm{Z}=0.16(\mathrm{P}=0.87)$

2.62.2 > 24 to 48 months

\section{MRC 1968}

Veterans Admin 1969

Subtotal (95\% CI)

Total events:

25

199

$41 \quad 424$

25

194

$36.2 \%$

623

422

$63.8 \%$

66 75

Heterogeneity: $\mathrm{Tau}^{2}=0.00 ; \mathrm{Chi}^{2}=0.29, \mathrm{df}=1(\mathrm{P}=0.59) ; \mathrm{I}^{2}=0 \%$

Test for overall effect: $\mathrm{Z}=0.87(\mathrm{P}=0.38)$

\subsection{3 >48 months}

Oslo Diet-Heart 1966

Sydney Diet-Heart 1978

WHI 2006 (1)

Subtotal (95\% CI)

Total events:

37
35
172

206
221
19541
$\mathbf{1 9 9 6 8}$

50
23

206

237

$31.5 \%$

$25.1 \%$

$261 \quad 29294 \quad 43.4 \%$

$29737 \mathbf{1 0 0 . 0} \%$

244 334

Heterogeneity: $\mathrm{Tau}^{2}=0.06 ; \mathrm{Chi}^{2}=6.22, \mathrm{df}=2(\mathrm{P}=0.04) ; \mathrm{I}^{2}=68 \%$

Test for overall effect: $\mathrm{Z}=0.13(\mathrm{P}=0.90)$

\subsection{4 unclear duration}

Houtsmuller 1979

Subtotal (95\% CI)

Total events:

0

51

51

$51 \quad 100.0 \%$

Heterogeneity: Not applicable

Test for overall effect: $\mathrm{Z}=1.64(\mathrm{P}=0.10)$

Test for subgroup differences: $\mathrm{Chi}^{2}=3.31, \mathrm{df}=3(\mathrm{P}=0.35), \mathrm{I}^{2}=9.4 \%$

Footnotes

(1) CHD death during trial, Prentice 2017
$0.97[0.58,1.64]$

$0.82[0.55,1.21]$

$0.87[0.64,1.19]$
Risk Ratio

M-H, Random, $95 \%$ CI 
Analysis 2.63. Comparison 2: SFA reduction vs usual diet - secondary health events, Outcome 63: CHD mortality, subgroup by baseline SFA

Lower SFA
Higher SFA

Events Total Events
Risk Ratio

Total Weight M-H, Random, 95\% CI
Risk Ratio

M-H, Random, $95 \%$ CI
2.63.1 up to $12 \%$ E SFA baseline Subtotal (95\% CI)

Total events:
0
0
Not estimable

Heterogeneity: Not applicable

Test for overall effect: Not applicable

2.63.2 $>12 \%$ to $15 \%$ E SFA baseline

$\begin{array}{lrrrrr}\text { DART 1989 } & 97 & 1018 & 97 & 1015 & 35.9 \% \\ \text { Sydney Diet-Heart 1978 } & 35 & 221 & 23 & 237 & 15.9 \% \\ \text { WHI 2006 (1) } & 172 & 19541 & 261 & 29294 & 48.2 \% \\ \text { Subtotal (95\% CI) } & & \mathbf{2 0 7 8 0} & & \mathbf{3 0 5 4 6} & \mathbf{1 0 0 . 0 \%} \\ \text { Total events: } & 304 & & 381 & & \end{array}$

Heterogeneity: $\mathrm{Tau}^{2}=0.02 ; \mathrm{Chi}^{2}=3.58, \mathrm{df}=2(\mathrm{P}=0.17) ; \mathrm{I}^{2}=44 \%$

Test for overall effect: $\mathrm{Z}=0.63(\mathrm{P}=0.53)$

\subsection{3 $>15$ to $18 \% \mathrm{E}$ SFA baseline}

\section{Subtotal (95\% CI)}

0

0

Heterogeneity: Not applicable

Test for overall effect: Not applicable

\subsection{4 >18\% E SFA baseline}

Subtotal (95\% CI)
Veterans Admin 1969

Total events:

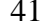

424

424

41

Heterogeneity: Not applicable

Test for overall effect: $\mathrm{Z}=1.02(\mathrm{P}=0.31)$

\subsection{5 unclear}

Houtsmuller 1979

MRC 1968

Oslo Diet-Heart 1966

Rose corn oil 1965

Rose olive 1965

Subtotal (95\% CI)

Total events:

$\begin{array}{rrrrr}0 & 51 & 5 & 51 & 2.0 \% \\ 25 & 199 & 25 & 194 & 38.5 \% \\ 37 & 206 & 50 & 206 & 53.5 \% \\ 5 & 28 & 1 & 13 & 3.9 \% \\ 3 & 26 & 0 & 13 & 2.0 \% \\ & \mathbf{5 1 0} & & \mathbf{4 7 7} & \mathbf{1 0 0 . 0 \%}\end{array}$

Heterogeneity: $\mathrm{Tau}^{2}=0.05 ; \mathrm{Chi}^{2}=4.98, \mathrm{df}=4(\mathrm{P}=0.29) ; \mathrm{I}^{2}=20 \%$

Test for overall effect: $\mathrm{Z}=0.76(\mathrm{P}=0.45)$

Test for subgroup differences: $\mathrm{Chi}^{2}=1.94, \mathrm{df}=2(\mathrm{P}=0.38), \mathrm{I}^{2}=0 \%$

Footnotes

(1) CHD death during trial, Prentice 2017
$1.00[0.76,1.30]$

$1.63[1.00,2.67]$

$0.99[0.82,1.20]$

$1.07[0.86,1.34]$

Not estimable

$0.82[0.55,1.21]$

$0.82[0.55,1.21]$
$0.09[0.01,1.60]$

$0.97[0.58,1.64]$

$0.74[0.51,1.08]$

$2.32[0.30,17.92]$

$3.63[0.20,65.44]$

$0.85[0.56,1.29]$ 


\section{Analysis 2.64. Comparison 2: SFA reduction vs usual diet - secondary health events, Outcome 64: CHD mortality, subgroup by SFA change}

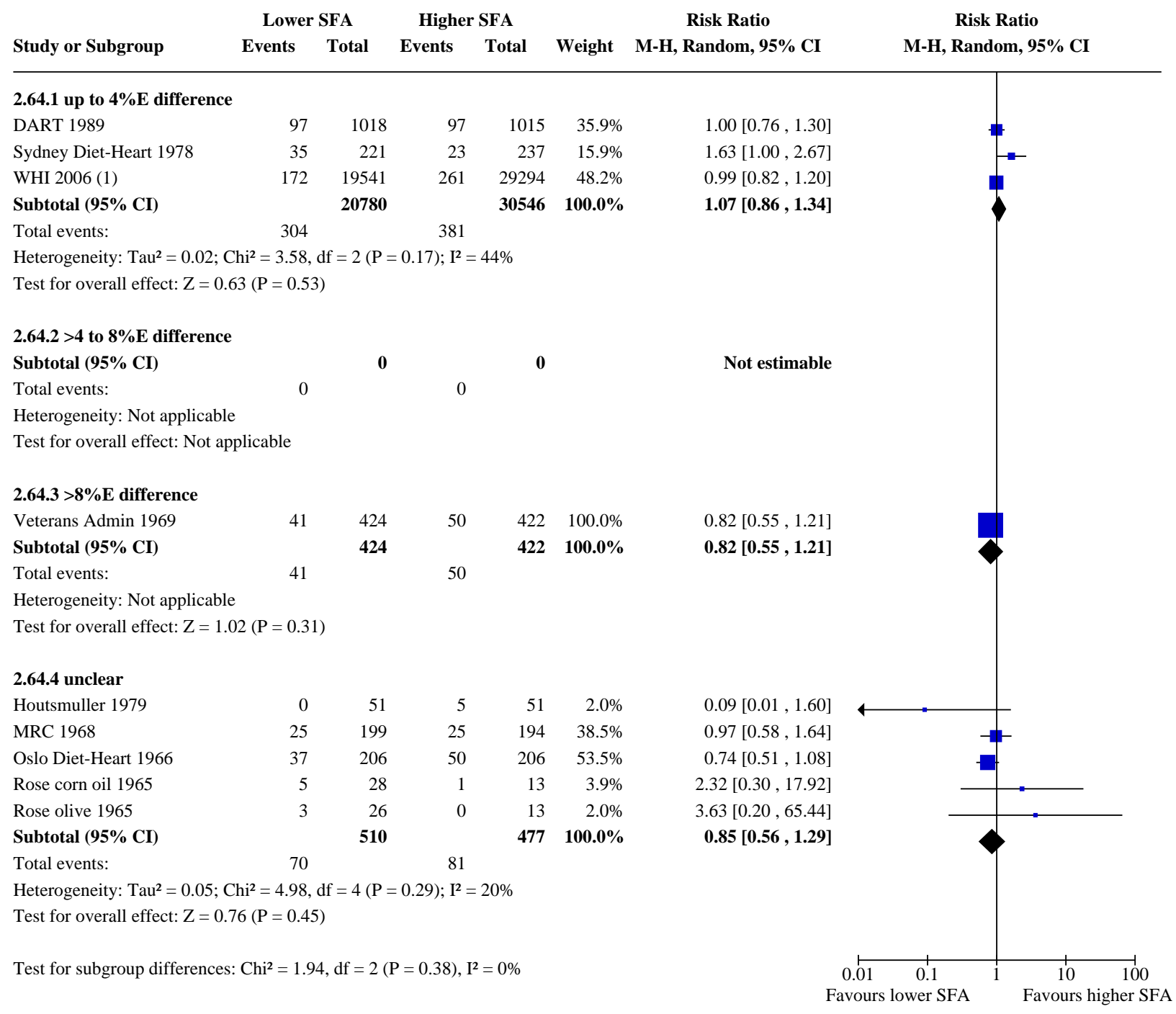

Footnotes

(1) CHD death during trial, Prentice 2017 


\section{Analysis 2.65. Comparison 2: SFA reduction vs usual diet - secondary} health events, Outcome 65: CHD mortality, subgroup by sex

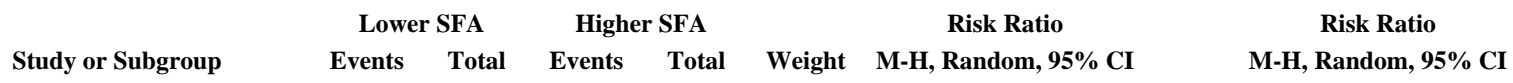

\begin{tabular}{lrrrrr}
\hline 2.65.1 Men & & & & & \\
DART 1989 & 97 & 1018 & 97 & 1015 & $29.6 \%$ \\
MRC 1968 & 25 & 199 & 25 & 194 & $13.6 \%$ \\
Oslo Diet-Heart 1966 & 37 & 206 & 50 & 206 & $20.7 \%$ \\
Rose corn oil 1965 & 5 & 28 & 1 & 13 & $1.2 \%$ \\
Rose olive 1965 & 3 & 26 & 0 & 13 & $0.6 \%$ \\
Sydney Diet-Heart 1978 & 35 & 221 & 23 & 237 & $14.6 \%$ \\
Veterans Admin 1969 & 41 & 424 & 50 & 422 & $19.9 \%$ \\
Subtotal (95\% CI) & & $\mathbf{2 1 2 2}$ & & $\mathbf{2 1 0 0}$ & $\mathbf{1 0 0 . 0 \%}$ \\
Total events: & 243 & & 246 & & \\
\end{tabular}

Heterogeneity: $\mathrm{Tau}^{2}=0.02 ; \mathrm{Chi}^{2}=8.55, \mathrm{df}=6(\mathrm{P}=0.20) ; \mathrm{I}^{2}=30 \%$

Test for overall effect: $\mathrm{Z}=0.16(\mathrm{P}=0.87)$

\subsubsection{Women}

WHI 2006 (1)

Subtotal $(95 \%$ CI)

$172 \quad 19541$

19541
$261 \quad 29294 \quad 100.0 \%$
$29294100.0 \%$

Total events:

172

261

Heterogeneity: Not applicable

Test for overall effect: $\mathrm{Z}=0.12(\mathrm{P}=0.90)$

2.65.3 Mixed, men and women

Houtsmuller 1979

Subtotal (95\% CI)

Total events:

0

Heterogeneity: Not applicable

Test for overall effect: $\mathrm{Z}=1.64(\mathrm{P}=0.10)$

Test for subgroup differences: $\mathrm{Chi}^{2}=2.64, \mathrm{df}=2(\mathrm{P}=0.27), \mathrm{I}^{2}=24.4 \%$

Footnotes

(1) CHD death during trial, Prentice 2017
$1.00[0.76,1.30]$

$0.97[0.58,1.64]$

$0.74[0.51,1.08]$

$2.32[0.30,17.92]$

$3.63[0.20,65.44]$

$1.63[1.00,2.67]$

$0.82[0.55,1.21]$

$0.98[0.79,1.23]$

$0.99[0.82,1.20]$

$0.99[0.82,1.20]$ 


\section{Analysis 2.66. Comparison 2: SFA reduction vs usual diet - secondary} health events, Outcome 66: CHD mortality, subgroup by CVD risk

\begin{tabular}{cccccccc} 
& \multicolumn{2}{c}{ Lower SFA } & \multicolumn{2}{c}{ Higher SFA } & \multicolumn{2}{c}{ Risk Ratio } & Risk Ratio \\
Study or Subgroup & Events & Total & Events & Total & Weight & M-H, Random, 95\% CI & M-H, Random, 95\% CI
\end{tabular}

\subsubsection{Low CVD risk}

Veterans Admin 1969

WHI 2006 (1)

Events Total

M-H, Random, 95\% CI

$0.82[0.55,1.21]$

$1.00[0.80,1.26]$

$0.95[0.78,1.16]$

$124 \quad 18633$

19057

$\begin{array}{rr}422 & 25.2 \% \\ 27925 & 74.8 \% \\ \mathbf{2 8 3 4 7} & \mathbf{1 0 0 . 0 \%}\end{array}$
235

Heterogeneity: $\mathrm{Tau}^{2}=0.00 ; \mathrm{Chi}^{2}=0.81, \mathrm{df}=1(\mathrm{P}=0.37) ; \mathrm{I}^{2}=0 \%$

Test for overall effect: $\mathrm{Z}=0.48(\mathrm{P}=0.63)$

\subsubsection{Moderate CVD risk}

Houtsmuller 1979

Subtotal (95\% CI)

Total events:

0

51

5

$51 \quad 100.0 \%$

Heterogeneity: Not applicable

Test for overall effect: $\mathrm{Z}=1.64(\mathrm{P}=0.10)$

2.66.3 Existing CVD disease

$\begin{array}{lrrrrr}\text { DART 1989 } & 97 & 1018 & 97 & 1015 & 32.2 \% \\ \text { MRC 1968 } & 25 & 199 & 25 & 194 & 13.2 \% \\ \text { Oslo Diet-Heart 1966 } & 37 & 206 & 50 & 206 & 21.1 \% \\ \text { Rose corn oil 1965 } & 5 & 28 & 1 & 13 & 1.0 \% \\ \text { Rose olive 1965 } & 3 & 26 & 0 & 13 & 0.5 \% \\ \text { Sydney Diet-Heart 1978 } & 35 & 221 & 23 & 237 & 14.3 \% \\ \text { WHI 2006 (2) } & 34 & 908 & 49 & 1369 & 17.7 \% \\ \text { Subtotal (95\% CI) } & & \mathbf{2 6 0 6} & & \mathbf{3 0 4 7} & \mathbf{1 0 0 . 0 \%} \\ \text { Total events: } & 236 & & 245 & & \end{array}$

Heterogeneity: $\mathrm{Tau}^{2}=0.02 ; \mathrm{Chi}^{2}=7.69, \mathrm{df}=6(\mathrm{P}=0.26) ; \mathrm{I}^{2}=22 \%$

Test for overall effect: $\mathrm{Z}=0.24(\mathrm{P}=0.81)$

Test for subgroup differences: $\mathrm{Chi}^{2}=2.90, \mathrm{df}=2(\mathrm{P}=0.23), \mathrm{I}^{2}=31.0 \%$

Footnotes

(1) Women without CVD at baseline

(2) Women with CVD at baseline

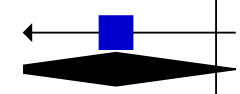


Analysis 2.67. Comparison 2: SFA reduction vs usual diet - secondary health events, Outcome 67: CHD mortality, subgroup by TC reduction

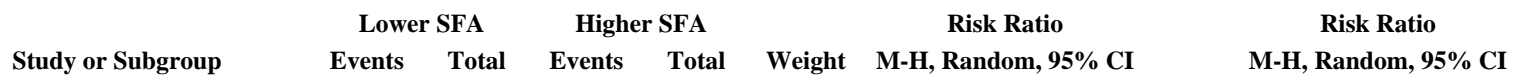

2.67.1 serum chol reduced by at least $0.2 \mathrm{mmol} / \mathrm{L}$

Houtsmuller 1979

MRC 1968

$\begin{array}{rr}97 & 1018 \\ 0 & 51\end{array}$

Oslo Diet-Heart 1966

$25 \quad 199$

Rose corn oil 1965

Sydney Diet-Heart 1978

$37 \quad 206$

5

28

Veterans Admin 1969

Subtotal $(95 \%$ CI)

2147

Total events:

$97 \quad 1015 \quad 27.0 \%$

$\begin{array}{lll}5 & 51 \quad 0.7 \%\end{array}$

$25 \quad 194 \quad 14.6 \%$

$50 \quad 206 \quad 20.6 \%$

$1 \quad 13 \quad 1.4 \%$

$23 \quad 237 \quad 15.5 \%$

$50 \quad 422 \quad 20.0 \%$

$2138100.0 \%$ 251

Heterogeneity: $\mathrm{Tau}^{2}=0.04 ; \mathrm{Chi}^{2}=10.31, \mathrm{df}=6(\mathrm{P}=0.11) ; \mathrm{I}^{2}=42 \%$

Test for overall effect: $\mathrm{Z}=0.29(\mathrm{P}=0.77)$

2.67.2 serum chol reduced by $<0.2 \mathrm{mmol} / \mathrm{L}$

Rose olive 1965

WHI 2006 (1)

3

Subtotal (95\% CI)

172

\begin{abstract}
26
9541
19567
\end{abstract}

0

$\begin{array}{rr}13 & 0.4 \% \\ 29294 & 99.6 \% \\ \mathbf{2 9 3 0 7} & \mathbf{1 0 0 . 0 \%}\end{array}$

$29307100.0 \%$

Total events:

175 261

Heterogeneity: $\mathrm{Tau}^{2}=0.00 ; \mathrm{Chi}^{2}=0.78, \mathrm{df}=1(\mathrm{P}=0.38) ; \mathrm{I}^{2}=0 \%$

Test for overall effect: $\mathrm{Z}=0.07(\mathrm{P}=0.95)$

2.67.3 serum chol reduction unclear

Subtotal (95\% CI)

$\mathbf{0}$

Total events:

0

$\mathbf{0}$

Heterogeneity: Not applicable

Test for overall effect: Not applicable

Test for subgroup differences: $\mathrm{Chi}^{2}=0.04, \mathrm{df}=1(\mathrm{P}=0.85), \mathrm{I}^{2}=0 \%$

Footnotes

(1) CHD death during trial, Prentice 2017
$1.00[0.76,1.30]$

$0.09[0.01,1.60]$

$0.97[0.58,1.64]$

$0.74[0.51,1.08]$

$2.32[0.30,17.92]$

$1.63[1.00,2.67]$

$0.82[0.55,1.21]$

$0.96[0.75,1.24]$

$\leftarrow$

$3.63[0.20,65.44]$ $0.99[0.82,1.20]$ $0.99[0.82,1.20]$

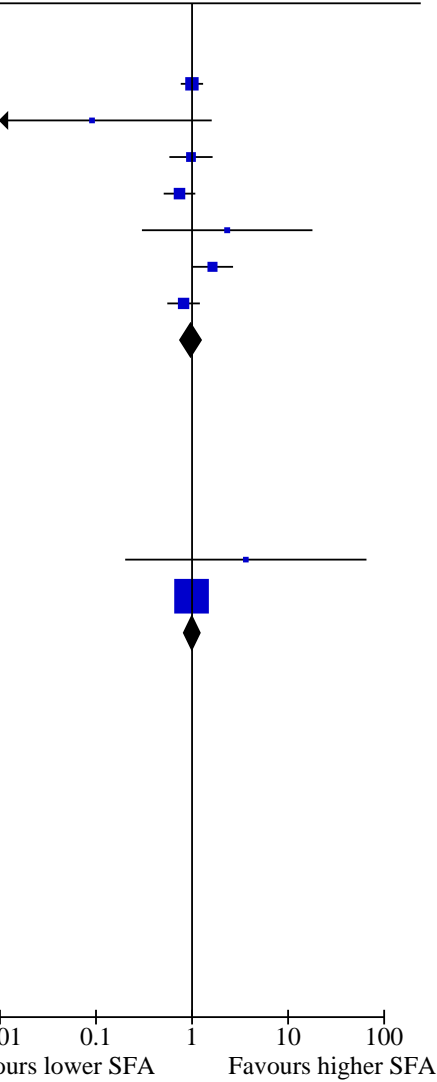


Analysis 2.68. Comparison 2: SFA reduction vs usual diet - secondary health events, Outcome 68: CHD mortality, subgroup decade of publication

Study or Subgroup

Lower SFA Higher SFA Events Total
Events Total
Risk Ratio

Weight M-H, Random, 95\% CI
Risk Ratio

M-H, Random, $95 \%$ CI

\subsubsection{0s}

MRC 1968

Oslo Diet-Heart 1966

Rose corn oil 1965

Rose olive 1965

Veterans Admin 1969

Subtotal (95\% CI)

Total events:

25

37

199
206
28
26
424
$\mathbf{8 8 3}$

25
50
1
0
50

194

$206 \quad 14.5 \%$

$13 \quad 0.7 \%$

$130.4 \%$

$422 \quad 13.9 \%$

$848 \quad 38.7 \%$

126

Heterogeneity: $\mathrm{Tau}^{2}=0.00 ; \mathrm{Chi}^{2}=2.72, \mathrm{df}=4(\mathrm{P}=0.61) ; \mathrm{I}^{2}=0 \%$

Test for overall effect: $\mathrm{Z}=1.48(\mathrm{P}=0.14)$

\subsubsection{0s}

Houtsmuller 1979

Sydney Diet-Heart 1978

Subtotal (95\% CI)

$0 \quad 51$

$35 \quad 221$

272

35

Total events:

Heterogeneity: $\mathrm{Tau}^{2}=3.35 ; \mathrm{Chi}^{2}=4.03, \mathrm{df}=1(\mathrm{P}=0.04) ; \mathrm{I}^{2}=75 \%$

Test for overall effect: $\mathrm{Z}=0.43(\mathrm{P}=0.67)$

\subsubsection{0s}

DART 1989

Subtotal (95\% CI)

97

1018

97

$1015 \quad 21.9 \%$

Total events:

97

1018

$1015 \quad 21.9 \%$

Heterogeneity: Not applicable

Test for overall effect: $\mathrm{Z}=0.02(\mathrm{P}=0.98)$

\subsubsection{0s}

Subtotal (95\% CI)

$\mathbf{0}$

97

Total events:

0

Heterogeneity: Not applicable

Test for overall effect: Not applicable

\subsubsection{0s}

WHI 2006 (1)

Subtotal (95\% CI)

Total events:

19541

261

$29294 \quad 29.2 \%$

$29294 \quad 29.2 \%$

261

Heterogeneity: Not applicable

Test for overall effect: $\mathrm{Z}=0.12(\mathrm{P}=0.90)$

Total $(95 \%$ CI $)$

21714

$31445100.0 \%$

Total events:

415 512

Heterogeneity: $\mathrm{Tau}^{2}=0.02 ; \mathrm{Chi}^{2}=11.18, \mathrm{df}=8(\mathrm{P}=0.19) ; \mathrm{I}^{2}=28 \%$

Test for overall effect: $\mathrm{Z}=0.32(\mathrm{P}=0.75)$

Test for subgroup differences: $\mathrm{Chi}^{2}=1.54, \mathrm{df}=3(\mathrm{P}=0.67), \mathrm{I}^{2}=0 \%$

\section{Footnotes}

(1) CHD death during trial, Prentice 2017
$0.97[0.58,1.64]$

$0.74[0.51,1.08]$

$2.32[0.30,17.92]$

$3.63[0.20,65.44]$

$0.82[0.55,1.21]$

$0.84[0.66,1.06]$

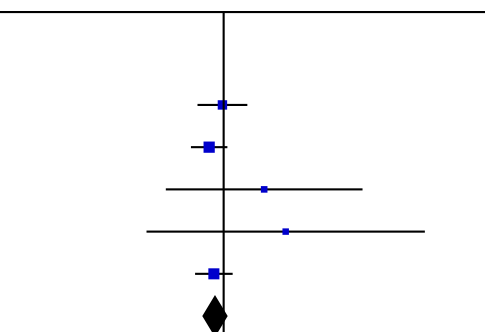

$0.09[0.01,1.60]$

$1.63[1.00,2.67]$

$0.54[0.03,9.26]$

$1.00[0.76,1.30]$

$1.00[0.76,1.30]$

Not estimable

$0.99[0.82,1.20]$

$0.99[0.82,1.20]$

$0.97[0.82,1.16]$

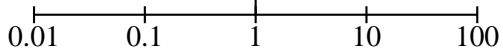

Favours lower SFA 


\section{Analysis 2.69. Comparison 2: SFA reduction vs usual diet - secondary health events, Outcome 69: CORONARY HEART DISEASE EVENTS}

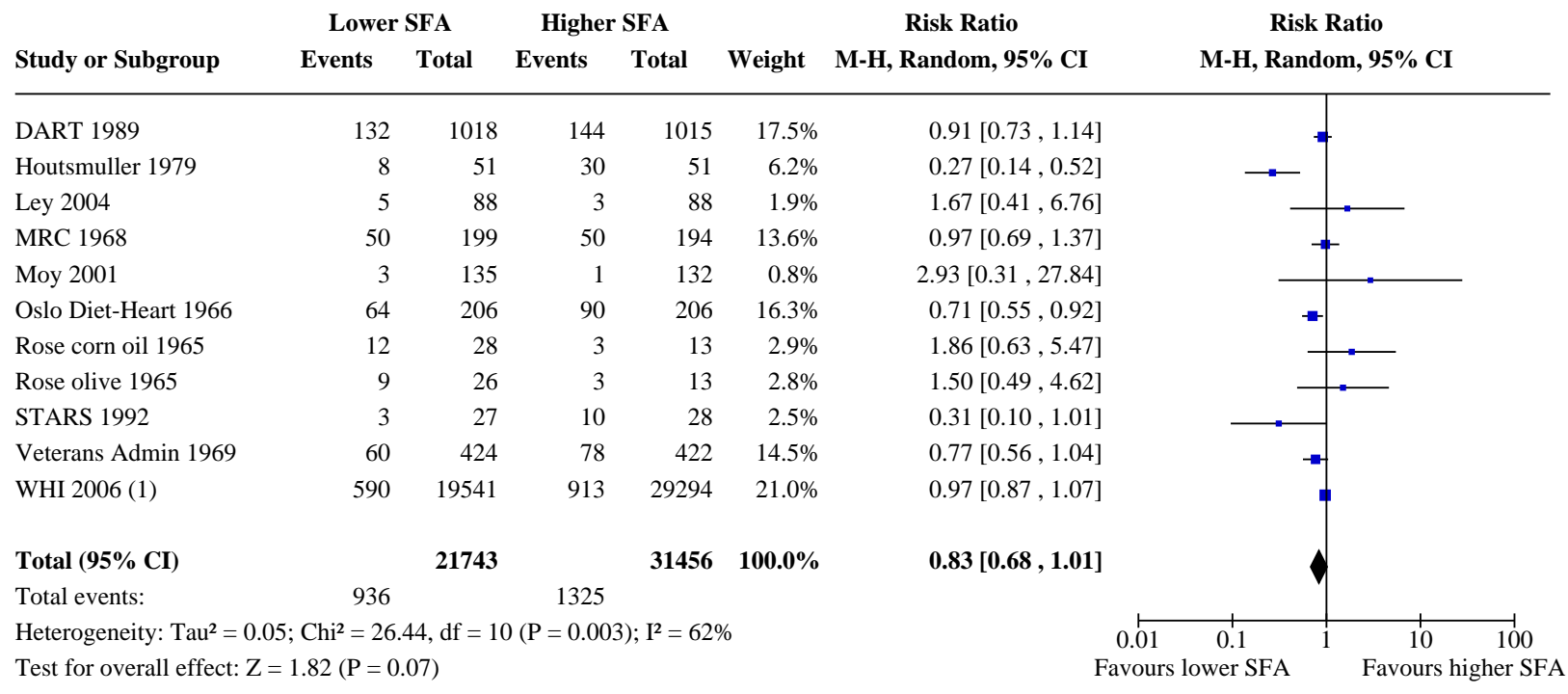

Footnotes

(1) CHD events including CHD death \& MI, Prentice 2017

Analysis 2.70. Comparison 2: SFA reduction vs usual diet - secondary health events, Outcome 70: CHD events, SA by low summary risk of bias

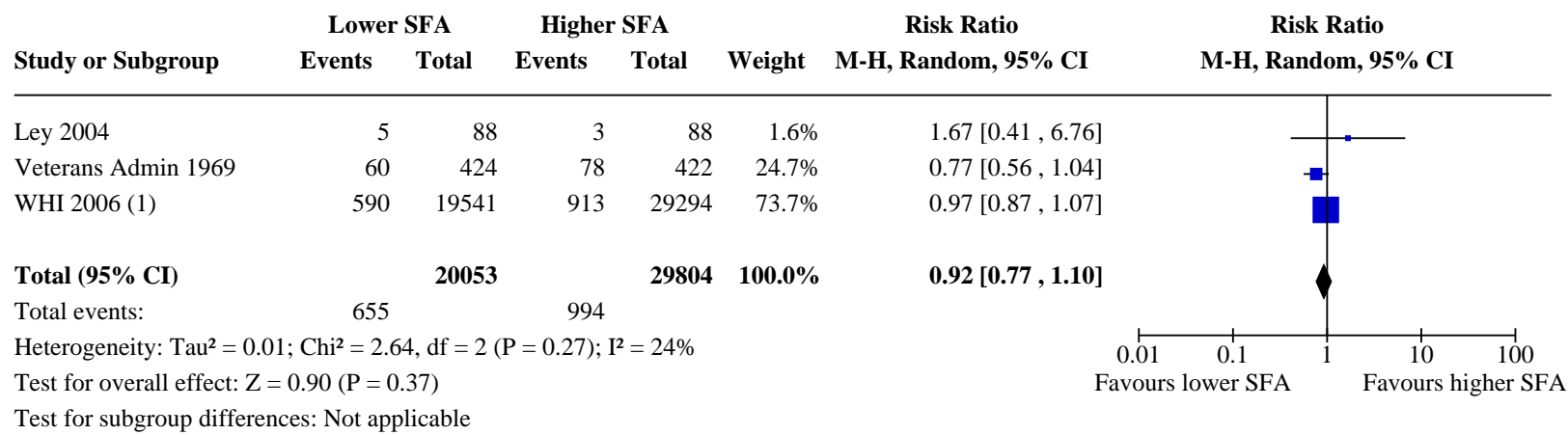

Footnotes

(1) CHD events including CHD death \& MI, Prentice 2017 
Analysis 2.71. Comparison 2: SFA reduction vs usual diet secondary health events, Outcome 71: CHD events, SA excluding WHI

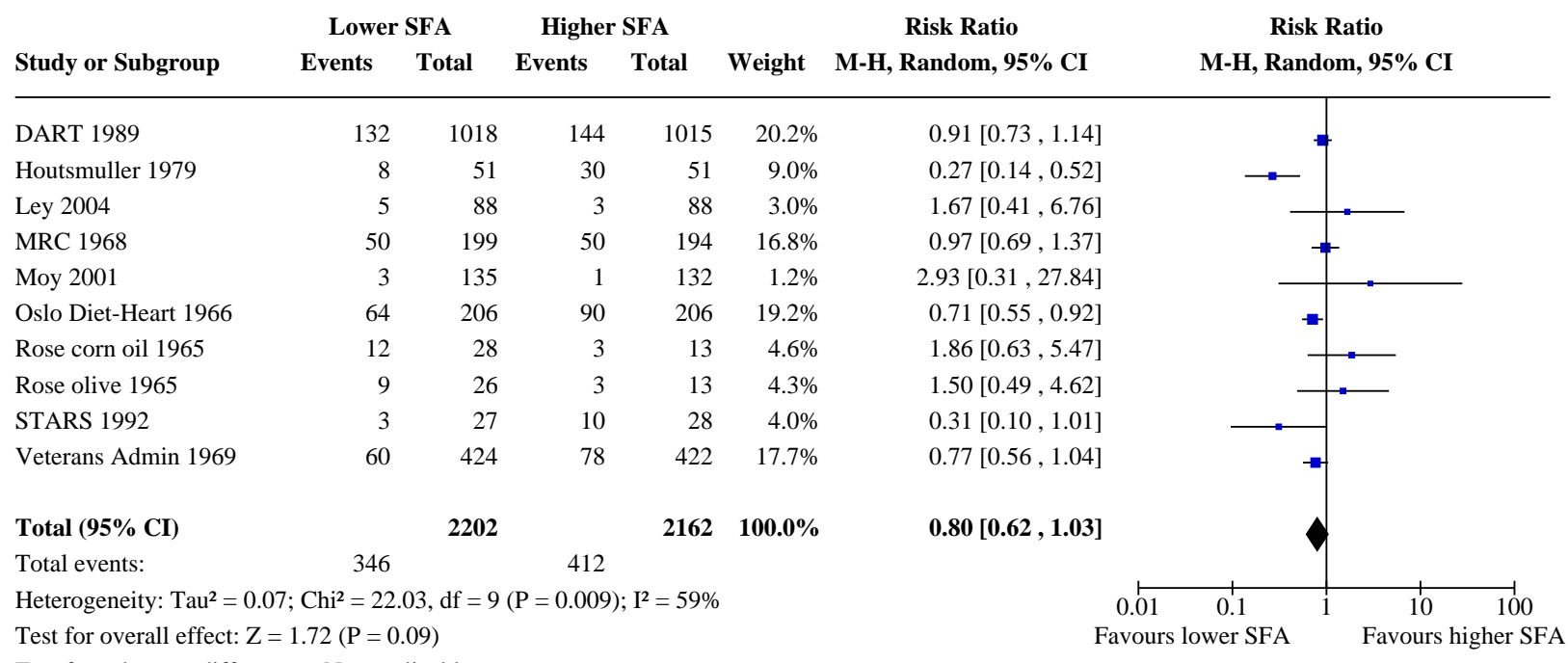

Test for subgroup differences: Not applicable

Analysis 2.72. Comparison 2: SFA reduction vs usual diet - secondary health events, Outcome 72: CHD events, SA statistically significant SFA reduction

\begin{tabular}{|c|c|c|c|c|c|c|c|}
\hline \multirow[b]{2}{*}{ Study or Subgroup } & \multicolumn{2}{|c|}{ Lower SFA } & \multicolumn{2}{|c|}{ Higher SFA } & \multirow[b]{2}{*}{ Weight } & \multirow{2}{*}{$\begin{array}{c}\text { Risk Ratio } \\
\text { M-H, Random, } 95 \% \text { CI }\end{array}$} & \multirow{2}{*}{$\begin{array}{c}\text { Risk Ratio } \\
\text { M-H, Random, 95\% CI }\end{array}$} \\
\hline & Events & Total & Events & Total & & & \\
\hline DART 1989 & 132 & 1018 & 144 & 1015 & $28.4 \%$ & $0.91[0.73,1.14]$ & \\
\hline Ley 2004 & 5 & 88 & 3 & 88 & $1.3 \%$ & $1.67[0.41,6.76]$ & \\
\hline Moy 2001 & 3 & 135 & 1 & 132 & $0.5 \%$ & $2.93[0.31,27.84]$ & \\
\hline STARS 1992 & 3 & 27 & 10 & 28 & $1.8 \%$ & $0.31[0.10,1.01]$ & - \\
\hline Veterans Admin 1969 & 60 & 424 & 78 & 422 & $18.5 \%$ & $0.77[0.56,1.04]$ & - \\
\hline WHI 2006 (1) & 590 & 19541 & 913 & 29294 & $49.6 \%$ & $0.97[0.87,1.07]$ & \\
\hline Total $(95 \%$ CI $)$ & & 21233 & & 30979 & $100.0 \%$ & $0.91[0.77,1.06]$ & \\
\hline Total events: & 793 & & 1149 & & & & \\
\hline Heterogeneity: $\mathrm{Tau}^{2}=$ & $\mathrm{Chi}^{2}=7.1$ & $2, \mathrm{df}=5(\mathrm{P}$ & $P=0.21) ; \mathrm{I}$ & $=30 \%$ & & & $0 . \stackrel{\vdash}{01}$ \\
\hline
\end{tabular}

Test for subgroup differences: Not applicable

Footnotes

(1) CHD events including CHD death \& MI, Prentice 2017 


\section{Analysis 2.73. Comparison 2: SFA reduction vs usual diet - secondary} health events, Outcome 73: CHD events, SA by TC reduction

\begin{tabular}{|c|c|c|c|c|c|}
\hline \multirow[b]{2}{*}{ Study or Subgroup } & \multicolumn{2}{|c|}{ Lower SFA } & \multicolumn{2}{|c|}{ Higher SFA } & \multirow[b]{2}{*}{ Weight } \\
\hline & Events & Total & Events & Total & \\
\hline DART 1989 & 132 & 1018 & 144 & 1015 & $18.3 \%$ \\
\hline Houtsmuller 1979 & 8 & 51 & 30 & 51 & $6.7 \%$ \\
\hline MRC 1968 & 50 & 199 & 50 & 194 & $14.3 \%$ \\
\hline Moy 2001 & 3 & 135 & 1 & 132 & $0.8 \%$ \\
\hline Oslo Diet-Heart 1966 & 64 & 206 & 90 & 206 & $17.0 \%$ \\
\hline Rose corn oil 1965 & 12 & 28 & 3 & 13 & $3.2 \%$ \\
\hline STARS 1992 & 3 & 27 & 10 & 28 & $2.7 \%$ \\
\hline Veterans Admin 1969 & 60 & 424 & 78 & 422 & $15.2 \%$ \\
\hline WHI 2006 (1) & 590 & 19541 & 913 & 29294 & $21.8 \%$ \\
\hline Total $(95 \%$ CI $)$ & & 21629 & & 31355 & $100.0 \%$ \\
\hline Total events: & 922 & & 1319 & & \\
\hline
\end{tabular}

Risk Ratio

Risk Ratio

Study or Subgroup

M-H, Random, $95 \%$ CI

M-H, Random, $95 \%$ CI

Heterogeneity: $\mathrm{Tau}^{2}=0.05 ; \mathrm{Chi}^{2}=24.92, \mathrm{df}=8(\mathrm{P}=0.002) ; \mathrm{I}^{2}=68 \%$

Test for overall effect: $\mathrm{Z}=2.05(\mathrm{P}=0.04)$

Test for subgroup differences: Not applicable

\section{$0.91[0.73,1.14]$}

$0.27[0.14,0.52]$

$0.97[0.69,1.37]$

$2.93[0.31,27.84]$

$0.71[0.55,0.92]$

$1.86[0.63,5.47]$

$0.31[0.10,1.01]$

$0.77[0.56,1.04]$

$0.97[0.87,1.07]$

$0.80[0.65,0.99]$

Footnotes

(1) CHD events including CHD death \& MI, Prentice 2017

\section{Analysis 2.74. Comparison 2: SFA reduction vs usual diet - secondary health events, Outcome 74: CHD events, SA aim to reduce SFA}

\begin{tabular}{lrrrrr} 
& \multicolumn{6}{c}{ Lower SFA } & \multicolumn{2}{c}{ Higher SFA } \\
Study or Subgroup & Events & Total & Events & Total & Weight \\
\hline DART 1989 & 132 & 1018 & 144 & 1015 & $17.8 \%$ \\
Houtsmuller 1979 & 8 & 51 & 30 & 51 & $6.4 \%$ \\
MRC 1968 & 50 & 199 & 50 & 194 & $13.8 \%$ \\
Moy 2001 & 3 & 135 & 1 & 132 & $0.8 \%$ \\
Oslo Diet-Heart 1966 & 64 & 206 & 90 & 206 & $16.6 \%$ \\
Rose corn oil 1965 & 12 & 28 & 3 & 13 & $3.0 \%$ \\
Rose olive 1965 & 9 & 26 & 3 & 13 & $2.8 \%$ \\
STARS 1992 & 3 & 27 & 10 & 28 & $2.6 \%$ \\
Veterans Admin 1969 & 60 & 424 & 78 & 422 & $14.8 \%$ \\
WHI 2006 (1) & 590 & 19541 & 913 & 29294 & $21.3 \%$ \\
& & & & & \\
Total (95\% CI) & & $\mathbf{2 1 6 5 5}$ & & $\mathbf{3 1 3 6 8}$ & $\mathbf{1 0 0 . 0 \%}$ \\
Total events: & 931 & & 1322 & &
\end{tabular}

Risk Ratio

M-H, Random, 95\% CI
Risk Ratio M-H, Random, $95 \%$ CI

Heterogeneity: $\mathrm{Tau}^{2}=0.05 ; \mathrm{Chi}^{2}=25.71, \mathrm{df}=9(\mathrm{P}=0.002) ; \mathrm{I}^{2}=65 \%$

Test for overall effect: $\mathrm{Z}=1.92(\mathrm{P}=0.05)$

Test for subgroup differences: Not applicable

$$
\begin{array}{r}
0.91[0.73,1.14] \\
0.27[0.14,0.52] \\
0.97[0.69,1.37] \\
2.93[0.31,27.84] \\
0.71[0.55,0.92] \\
1.86[0.63,5.47] \\
1.50[0.49,4.62] \\
0.31[0.10,1.01] \\
0.77[0.56,1.04] \\
0.97[0.87,1.07] \\
\\
\mathbf{0 . 8 2}[\mathbf{0 . 6 7}, \mathbf{1 . 0 0}]
\end{array}
$$

\section{Footnotes}

(1) CHD events including CHD death \& MI, Prentice 2017 
Analysis 2.75. Comparison 2: SFA reduction vs usual diet - secondary health events, Outcome 75: CHD events, SA Mantel-Haenszel fixed-effect

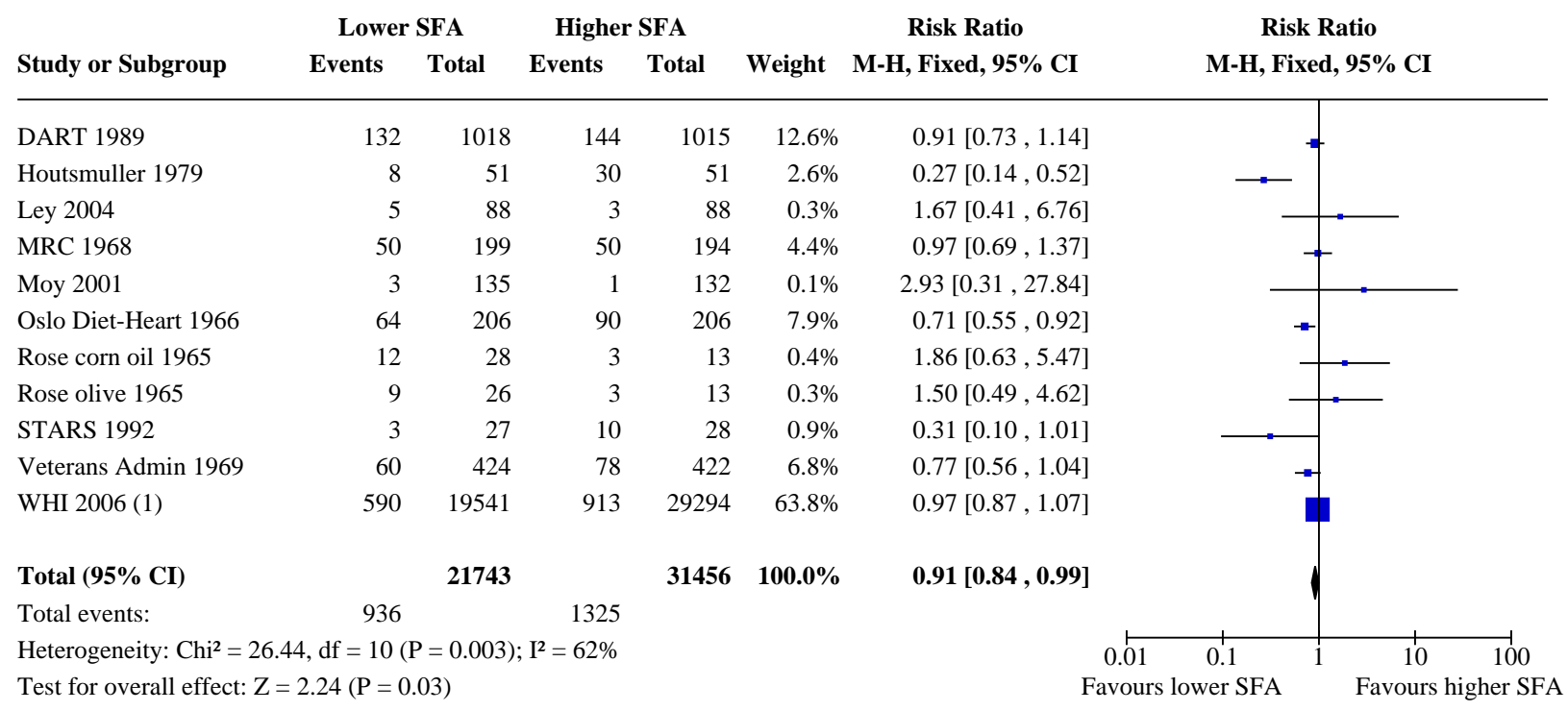

Test for subgroup differences: Not applicable

Footnotes

(1) CHD events including CHD death \& MI, Prentice 2017

\section{Analysis 2.76. Comparison 2: SFA reduction vs usual diet - secondary health events, Outcome 76: CHD events, SA Peto fixed-effect}

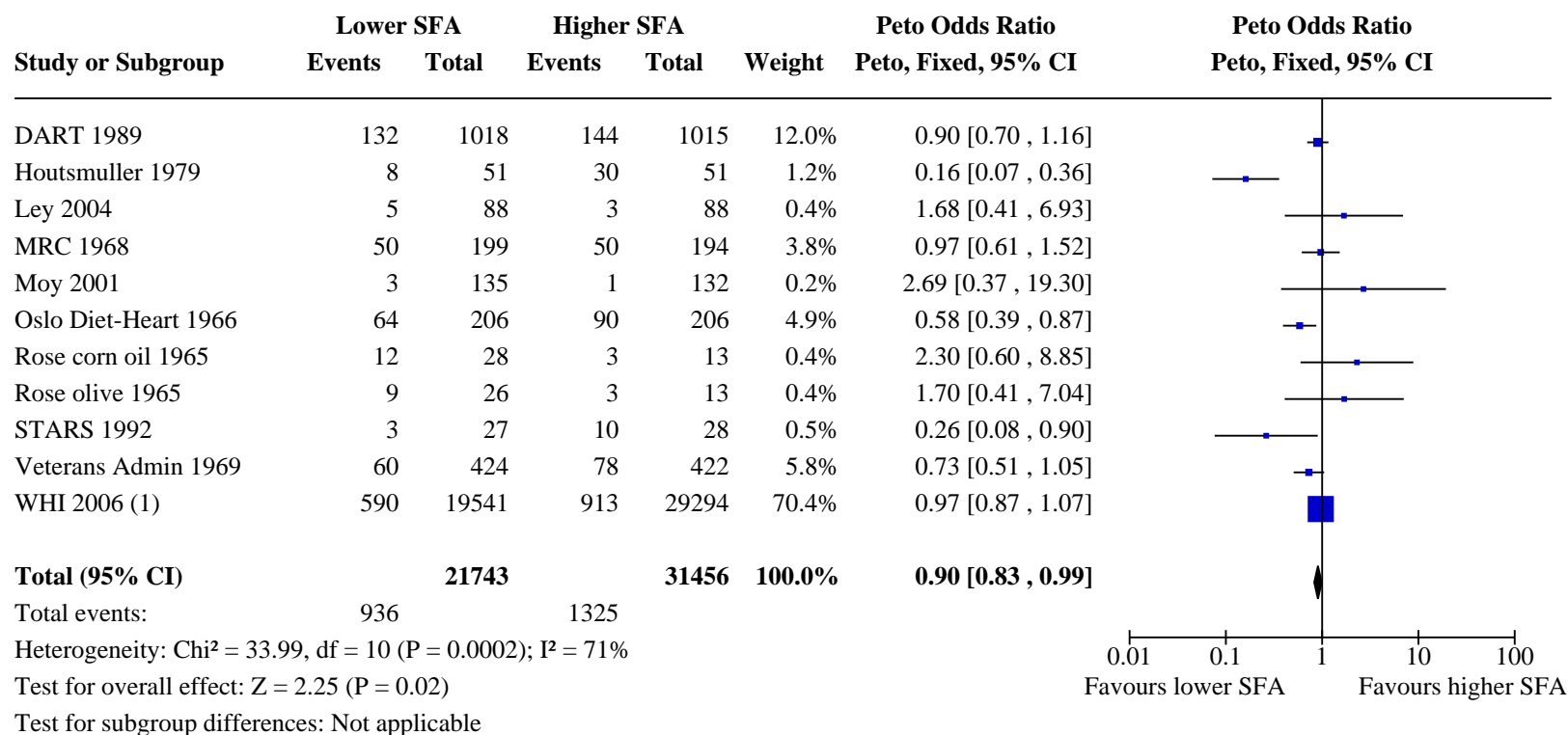

Footnotes

(1) CHD events including CHD death \& MI, Prentice 2017 


\section{Analysis 2.77. Comparison 2: SFA reduction vs usual diet - secondary} health events, Outcome 77: CHD events, subgroup by any substitution

Risk Ratio

Risk Ratio

M-H, Random, $95 \%$ CI

M-H, Random, $95 \%$ CI

\begin{tabular}{lrrrrr} 
& \multicolumn{2}{c}{ Lower SFA } & \multicolumn{2}{c}{ Higher SFA } & \\
Study or Subgroup & Events & Total & Events & Total & Weight \\
\hline 2.77.1 replaced by PUFA & & & & & \\
DART 1989 & 132 & 1018 & 144 & 1015 & $21.1 \%$ \\
Houtsmuller 1979 & 8 & 51 & 30 & 51 & $9.9 \%$ \\
MRC 1968 & 50 & 199 & 50 & 194 & $17.9 \%$ \\
Oslo Diet-Heart 1966 & 64 & 206 & 90 & 206 & $20.2 \%$ \\
Rose corn oil 1965 & 12 & 28 & 6 & 26 & $7.7 \%$ \\
STARS 1992 & 3 & 27 & 10 & 28 & $4.5 \%$ \\
Veterans Admin 1969 & 60 & 424 & 78 & 422 & $18.7 \%$ \\
Subtotal (95\% CI) & & $\mathbf{1 9 5 3}$ & & $\mathbf{1 9 4 2}$ & $\mathbf{1 0 0 . 0 \%}$ \\
Total events: & 329 & & 408 & & \\
\end{tabular}

Heterogeneity: $\mathrm{Tau}^{2}=0.08 ; \mathrm{Chi}^{2}=20.25, \mathrm{df}=6(\mathrm{P}=0.002) ; \mathrm{I}^{2}=70 \%$

Test for overall effect: $\mathrm{Z}=1.97(\mathrm{P}=0.05)$

\subsection{2 replaced by MUFA}

Rose olive 1965

Subtotal (95\% CI)

926

6

26

$100.0 \%$

$100.0 \%$

Total events:

9

Heterogeneity: Not applicable

Test for overall effect: $Z=0.90(P=0.37)$

\subsection{3 replaced by $\mathrm{CHO}$}

\section{DART 1989}

Ley 2004

STARS 1992

WHI $2006(1)$

Subtotal (95\% CI)

Total events:

Heterogeneity: $\mathrm{Tau}^{2}=0.01 ; \mathrm{Chi}^{2}=4.49, \mathrm{df}=3(\mathrm{P}=0.21) ; \mathrm{I}^{2}=33 \%$

Test for overall effect: $\mathrm{Z}=0.76(\mathrm{P}=0.44)$

\subsection{4 replaced by protein}

$\begin{array}{lrrrrr}\text { DART 1989 } & 132 & 1018 & 144 & 1015 & 17.7 \% \\ \text { Ley 2004 } & 5 & 88 & 3 & 88 & 0.4 \% \\ \text { WHI 2006 (1) } & 590 & 19541 & 913 & 29294 & 81.9 \% \\ \text { Subtotal (95\% CI) } & & \mathbf{2 0 6 4 7} & & \mathbf{3 0 3 9 7} & \mathbf{1 0 0 . 0 \%} \\ \text { Total events: } & 727 & & 1060 & & \end{array}$

Heterogeneity: $\mathrm{Tau}^{2}=0.00 ; \mathrm{Chi}^{2}=0.82, \mathrm{df}=2(\mathrm{P}=0.66) ; \mathrm{I}^{2}=0 \%$

Test for overall effect: $\mathrm{Z}=0.84(\mathrm{P}=0.40)$

\subsection{5 replacement unclear}

Moy 2001

Subtotal (95\% CI)

Total events:

Heterogeneity: Not applicable

Test for overall effect: $\mathrm{Z}=0.94(\mathrm{P}=0.35)$

Test for subgroup differences: $\mathrm{Chi}^{2}=4.64, \mathrm{df}=4(\mathrm{P}=0.33), \mathrm{I}^{2}=13.8 \%$

Footnotes

(1) CHD events including CHD death \& MI, Prentice 2017

$100.0 \%$
$0.91[0.73,1.14]$

$0.27[0.14,0.52]$

$0.97[0.69,1.37]$

$0.71[0.55,0.92]$

$1.86[0.82,4.22]$

$0.31[0.10,1.01]$

$0.77[0.56,1.04]$

$0.76[0.57,1.00]$

$1.50[0.62,3.61]$

$1.50[0.62,3.61]$

$0.91[0.73,1.14]$

$1.67[0.41,6.76]$

$0.30[0.09,0.98]$

$0.97[0.87,1.07]$

$0.93[0.78,1.11]$

$0.91[0.73,1.14]$

$1.67[0.41,6.76]$

$0.97[0.87,1.07]$

$0.96[0.88,1.05]$

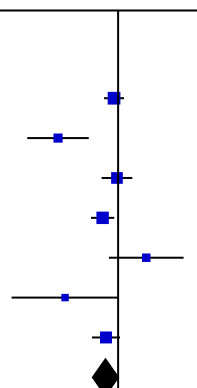

$2.93[0.31,27.84]$

$2.93[0.31,27.84]$ .

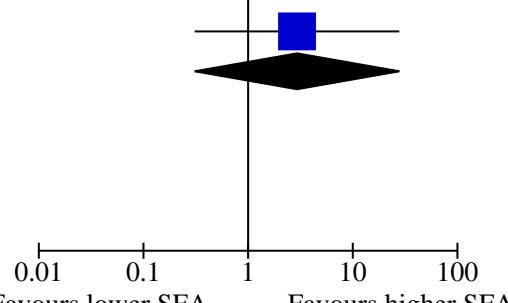

Favours lower SFA Favours higher SFA 
Analysis 2.78. Comparison 2: SFA reduction vs usual diet - secondary health events, Outcome 78: CHD events, subgroup by main substitution

Lower SFA Higher SFA Risk Ratio

Study or Subgroup Events Total

Events Total

Weigh
M-H, Random, 95\% CI
Risk Ratio

M-H, Random, 95\% CI

\subsection{1 replaced by PUFA}

DART 1989

Houtsmuller 1979

MRC 1968

Oslo Diet-Heart 1966

Rose corn oil 1965

Veterans Admin 1969

Subtotal (95\% CI)

$\begin{array}{rrrrr}132 & 1018 & 144 & 1015 & 22.4 \% \\ 8 & 51 & 30 & 51 & 10.1 \% \\ 50 & 199 & 50 & 194 & 18.7 \% \\ 64 & 206 & 90 & 206 & 21.3 \% \\ 12 & 28 & 6 & 26 & 7.8 \% \\ 60 & 424 & 78 & 422 & 19.7 \% \\ & \mathbf{1 9 2 6} & & \mathbf{1 9 1 4} & \mathbf{1 0 0 . 0 \%} \\ 326 & & 398 & & \end{array}$

$0.91[0.73,1.14]$

$0.27[0.14,0.52]$

$0.97[0.69,1.37]$

$0.71[0.55,0.92]$

$1.86[0.82,4.22]$

$0.77[0.56,1.04]$

$0.79[0.60,1.04]$

Total events:

$0.003) ; \mathrm{I}^{2}=72 \%$

Heterogeneity: $\mathrm{Tau}^{2}=0.07 ; \mathrm{Chi}^{2}=17.71$,
Test for overall effect: $\mathrm{Z}=1.68(\mathrm{P}=0.09)$

\subsection{2 replaced by MUFA}

Rose olive 1965

Subtotal (95\% CI)

Total events:

926

6

26

$100.0 \%$

Heterogeneity: Not applicable

Test for overall effect: $\mathrm{Z}=0.90(\mathrm{P}=0.37)$

\subsection{3 replaced by CHO}

Ley 2004

STARS 1992

WHI 2006 (1)

Subtotal (95\% CI)

Total events:

Heterogeneity: $\mathrm{Tau}^{2}=0.25 ; \mathrm{Chi}^{2}=4.31, \mathrm{df}=2(\mathrm{P}=0.12) ; \mathrm{I}^{2}=54 \%$

Test for overall effect: $\mathrm{Z}=0.54(\mathrm{P}=0.59)$

2.78.4 replaced by protein

Subtotal (95\% CI)

0

0

0

Heterogeneity: Not applicable

Test for overall effect: Not applicable

2.78.5 replacement unclear

Moy 2001

Subtotal (95\% CI)

Total events:

Heterogeneity: Not applicable

Test for overall effect: $\mathrm{Z}=0.94(\mathrm{P}=0.35)$

Test for subgroup differences: $\mathrm{Chi}^{2}=3.05, \mathrm{df}=3(\mathrm{P}=0.38), \mathrm{I}^{2}=1.7 \%$

Footnotes

(1) CHD events including CHD death \& MI, Prentice 2017
$1.50[0.62,3.61]$

$1.50[0.62,3.61]$
$1.67[0.41,6.76]$

$0.30[0.09,0.98]$

$0.97[0.87,1.07]$

$0.82[0.39,1.72]$

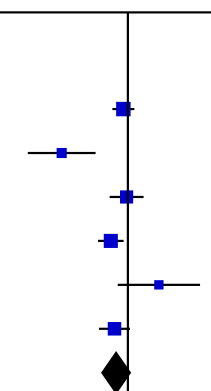

Not estimable 


\section{Analysis 2.79. Comparison 2: SFA reduction vs usual diet - secondary health events, Outcome 79: CHD events, subgroup by duration}

\begin{tabular}{lrrrrr} 
& \multicolumn{2}{c}{ Lower SFA } & \multicolumn{2}{c}{ Higher SFA } & \\
Study or Subgroup & Events & Total & Events & Total & Weight \\
\hline 2.79.1 up to 24 months & & & & & \\
DART 1989 & 132 & 1018 & 144 & 1015 & $85.1 \%$ \\
Moy 2001 & 3 & 135 & 1 & 132 & $1.6 \%$ \\
Rose corn oil 1965 & 12 & 28 & 3 & 13 & $6.9 \%$ \\
Rose olive 1965 & 9 & 26 & 3 & 13 & $6.4 \%$ \\
Subtotal (95\% CI) & & $\mathbf{1 2 0 7}$ & & $\mathbf{1 1 7 3}$ & $\mathbf{1 0 0 . 0 \%}$ \\
Total events: & 156 & & 151 & &
\end{tabular}

Risk Ratio

M-H, Random, $95 \%$ CI

$0.91[0.73,1.14]$

$2.93[0.31,27.84]$

$1.86[0.63,5.47]$

$1.50[0.49,4.62]$

$1.01[0.76,1.35]$

Heterogeneity: $\mathrm{Tau}^{2}=0.01 ; \mathrm{Chi}^{2}=3.17, \mathrm{df}=3(\mathrm{P}=0.37) ; \mathrm{I}^{2}=5 \%$

Test for overall effect: $\mathrm{Z}=0.06(\mathrm{P}=0.95)$

2.79.2 > 24 to 48 months

MRC 1968

STARS 1992

Veterans Admin 1969

Subtotal $(95 \%$ CI)

Total events:

$50 \quad 199$

$3 \quad 27$

$60 \quad 424$

650

113

Heterogeneity: $\mathrm{Tau}^{2}=0.04 ; \mathrm{Chi}^{2}=3.82, \mathrm{df}=2(\mathrm{P}=0.15) ; \mathrm{I}^{2}=48 \%$

Test for overall effect: $\mathrm{Z}=1.28(\mathrm{P}=0.20)$

\subsection{3 >48 months}

Oslo Diet-Heart 1966

WHI 2006 (1)

Subtotal (95\% CI)

Total events:

$\begin{array}{rr}64 & 206 \\ 590 & 19541 \\ & \mathbf{1 9 7 4 7}\end{array}$

90

206

29294

$42.6 \%$

29500

654 1003

Heterogeneity: $\mathrm{Tau}^{2}=0.04 ; \mathrm{Chi}^{2}=4.91, \mathrm{df}=1(\mathrm{P}=0.03) ; \mathrm{I}^{2}=80 \%$

Test for overall effect: $\mathrm{Z}=1.06(\mathrm{P}=0.29)$

\subsection{4 unclear duration}

Houtsmuller 1979

Ley 2004

Subtotal (95\% CI)

Total events:

$8 \quad 51$

$5 \quad 88$

30

$\begin{array}{rr}51 & 55.8 \% \\ 88 & 44.2 \% \\ \mathbf{1 3 9} & \mathbf{1 0 0 . 0 \%}\end{array}$

13

33

Heterogeneity: $\mathrm{Tau}^{2}=1.37 ; \mathrm{Chi}^{2}=5.35, \mathrm{df}=1(\mathrm{P}=0.02) ; \mathrm{I}^{2}=81 \%$

Test for overall effect: $\mathrm{Z}=0.56(\mathrm{P}=0.57)$

Test for subgroup differences: $\mathrm{Chi}^{2}=1.42, \mathrm{df}=3(\mathrm{P}=0.70), \mathrm{I}^{2}=0 \%$

Footnotes

(1) CHD events including CHD death \& MI, Prentice 2017

$0.71[0.55,0.92]$
Risk Ratio

M-H, Random, 95\% CI

(n)

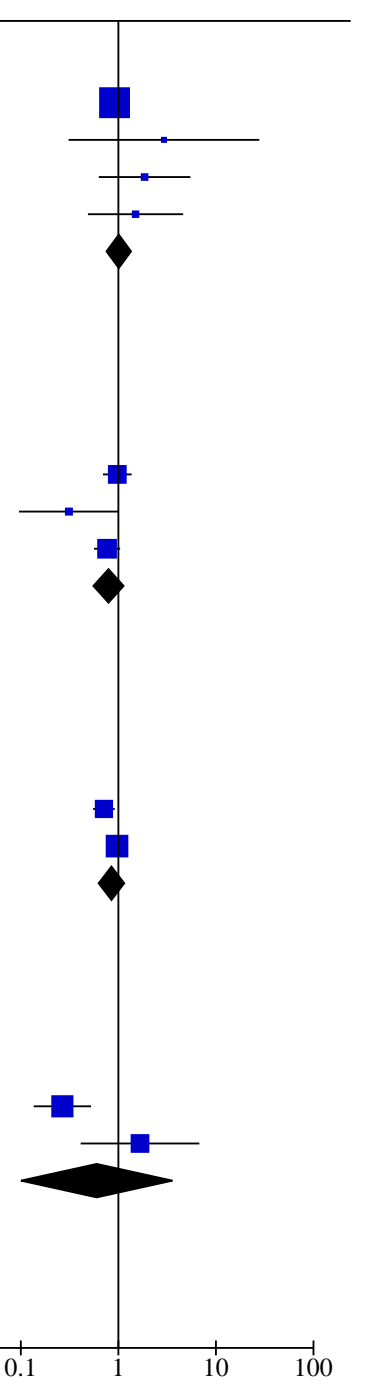




\section{Analysis 2.80. Comparison 2: SFA reduction vs usual diet - secondary \\ health events, Outcome 80: CHD events, subgroup by baseline SFA}

Lower SFA Higher SFA

Events Total Events Total
Risk Ratio

Weight M-H, Random, 95\% CI
Risk Ratio

M-H, Random, $95 \%$ CI

\subsection{1 up to $12 \%$ E SFA baseline}

Subtotal $(95 \%$ CI)

Total events:

Heterogeneity: Not applicable

Test for overall effect: Not applicable

\subsection{2 $>12$ to $15 \%$ E SFA baseline}

$\begin{array}{lrrrrr}\text { DART 1989 } & 132 & 1018 & 144 & 1015 & 17.6 \% \\ \text { Ley 2004 } & 5 & 88 & 3 & 88 & 0.4 \% \\ \text { Moy 2001 } & 3 & 135 & 1 & 132 & 0.2 \% \\ \text { WHI 2006 (1) } & 590 & 19541 & 913 & 29294 & 81.8 \% \\ \text { Subtotal (95\% CI) } & & \mathbf{2 0 7 8 2} & & \mathbf{3 0 5 2 9} & \mathbf{1 0 0 . 0 \%} \\ \text { Total events: } & 730 & & 1061 & & \end{array}$

Heterogeneity: $\mathrm{Tau}^{2}=0.00 ; \mathrm{Chi}^{2}=1.76, \mathrm{df}=3(\mathrm{P}=0.62) ; \mathrm{I}^{2}=0 \%$

Test for overall effect: $\mathrm{Z}=0.80(\mathrm{P}=0.42)$

\subsection{3 $>15$ to $18 \%$ E SFA baseline}

\section{STARS 1992 \\ Subtotal $(95 \%$ CI $)$}

327

Total events:

10

0

Heterogeneity: Not applicable

Test for overall effect: $\mathrm{Z}=1.94(\mathrm{P}=0.05)$

\subsection{4 >18\% E SFA baseline}

Veterans Admin 1969

60

Subtotal (95\% CI)

60

78

Heterogeneity: Not applicable

Test for overall effect: $\mathrm{Z}=1.70(\mathrm{P}=0.09)$

\subsection{5 unclear}

Houtsmuller 1979

MRC 1968

Oslo Diet-Heart 1966

Rose corn oil 1965

Rose olive 1965

Subtotal (95\% CI)

Total events:

$\begin{array}{rrrrr}8 & 51 & 30 & 51 & 19.5 \% \\ 50 & 199 & 50 & 194 & 27.6 \% \\ 64 & 206 & 90 & 206 & 29.4 \% \\ 12 & 28 & 3 & 13 & 12.1 \% \\ 9 & 26 & 3 & 13 & 11.5 \% \\ & \mathbf{5 1 0} & & \mathbf{4 7 7} & \mathbf{1 0 0 . 0 \%}\end{array}$

$422 \quad 100.0 \%$
Not estimable
$0.91[0.73,1.14]$

$1.67[0.41,6.76]$

$2.93[0.31,27.84]$

$0.97[0.87,1.07]$

$0.96[0.88,1.06]$
Heterogeneity: $\mathrm{Tau}^{2}=0.18 ; \mathrm{Chi}^{2}=15.59, \mathrm{df}=4(\mathrm{P}=0.004) ; \mathrm{I}^{2}=74 \%$

Test for overall effect: $\mathrm{Z}=1.00(\mathrm{P}=0.32)$

Test for subgroup differences: $\mathrm{Chi}^{2}=5.89, \mathrm{df}=3(\mathrm{P}=0.12), \mathrm{I}^{2}=49.1 \%$

$0.27[0.14,0.52]$

$0.97[0.69,1.37]$

$0.71[0.55,0.92]$

$1.86[0.63,5.47]$

$1.50[0.49,4.62]$

$0.78[0.49,1.26]$

$0.77[0.56,1.04]$

$0.31[0.10,1.01]$

$0.31[0.10,1.01]$

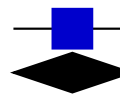

\section{Footnotes}

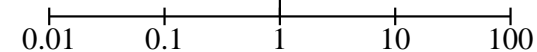

Favours lower SFA

(1) CHD events including CHD death \& MI, Prentice 2017 


\section{Analysis 2.81. Comparison 2: SFA reduction vs usual diet - secondary health events, Outcome 81: CHD events, subgroup by SFA change}

M-H, Random, $95 \%$ CI

Study or Subgroup

Weight M-H, Random, 95\% CI

2.81.1 up to $4 \% \mathrm{E}$ difference

DART 1989

Ley 2004

Moy 2001

WHI 2006 (1)

Subtotal $(95 \%$ CI $)$

Total events:

132

Total

Events Total

Heterogeneity: $\mathrm{Tau}^{2}=0.00 ; \mathrm{Chi}^{2}=1.76, \mathrm{df}=3(\mathrm{P}=0.62) ; \mathrm{I}^{2}=0 \%$

Test for overall effect: $\mathrm{Z}=0.80(\mathrm{P}=0.42)$

2.81.2 $>4$ to $8 \% \mathrm{E}$ difference

$\begin{array}{llllll}\text { STARS } 1992 & 3 & 27 & 10 & 28 & 100.0 \% \\ \text { Subtotal }(\mathbf{9 5 \%} \text { CI) } & & \mathbf{2 7} & & \mathbf{2 8} & \mathbf{1 0 0 . 0 \%} \\ \text { Total events: } & 3 & & 10 & & \end{array}$

Heterogeneity: Not applicable

Test for overall effect: $\mathrm{Z}=1.94(\mathrm{P}=0.05)$

\subsection{3 $>$ 8\% E difference}

Veterans Admin 1969

Subtotal (95\% CI)

$424 \quad 78$

$422 \quad 100.0 \%$

$422100.0 \%$

Total events:

60

78

Heterogeneity: Not applicable

Test for overall effect: $\mathrm{Z}=1.70(\mathrm{P}=0.09)$

\subsection{4 unclear}

Houtsmuller 1979

MRC 1968

Oslo Diet-Heart 1966

Rose corn oil 1965

Rose olive 1965

Subtotal (95\% CI)

Total events:

Heterogeneity: $\mathrm{Tau}^{2}=0.18 ; \mathrm{Chi}^{2}=15.59, \mathrm{df}=4(\mathrm{P}=0.004) ; \mathrm{I}^{2}=74 \%$

Test for overall effect: $\mathrm{Z}=1.00(\mathrm{P}=0.32)$

Test for subgroup differences: $\mathrm{Chi}^{2}=5.89, \mathrm{df}=3(\mathrm{P}=0.12), \mathrm{I}^{2}=49.1 \%$

\section{Footnotes}

(1) CHD events including CHD death \& MI, Prentice 2017
$0.31[0.10,1.01]$

$0.31[0.10,1.01]$

$0.91[0.73,1.14]$

$1.67[0.41,6.76]$

$2.93[0.31,27.84]$

$0.97[0.87,1.07]$

$0.96[0.88,1.06]$

$0.77[0.56,1.04]$

$0.77[0.56,1.04]$ 
Analysis 2.82. Comparison 2: SFA reduction vs usual diet secondary health events, Outcome 82: CHD events, subgroup by sex

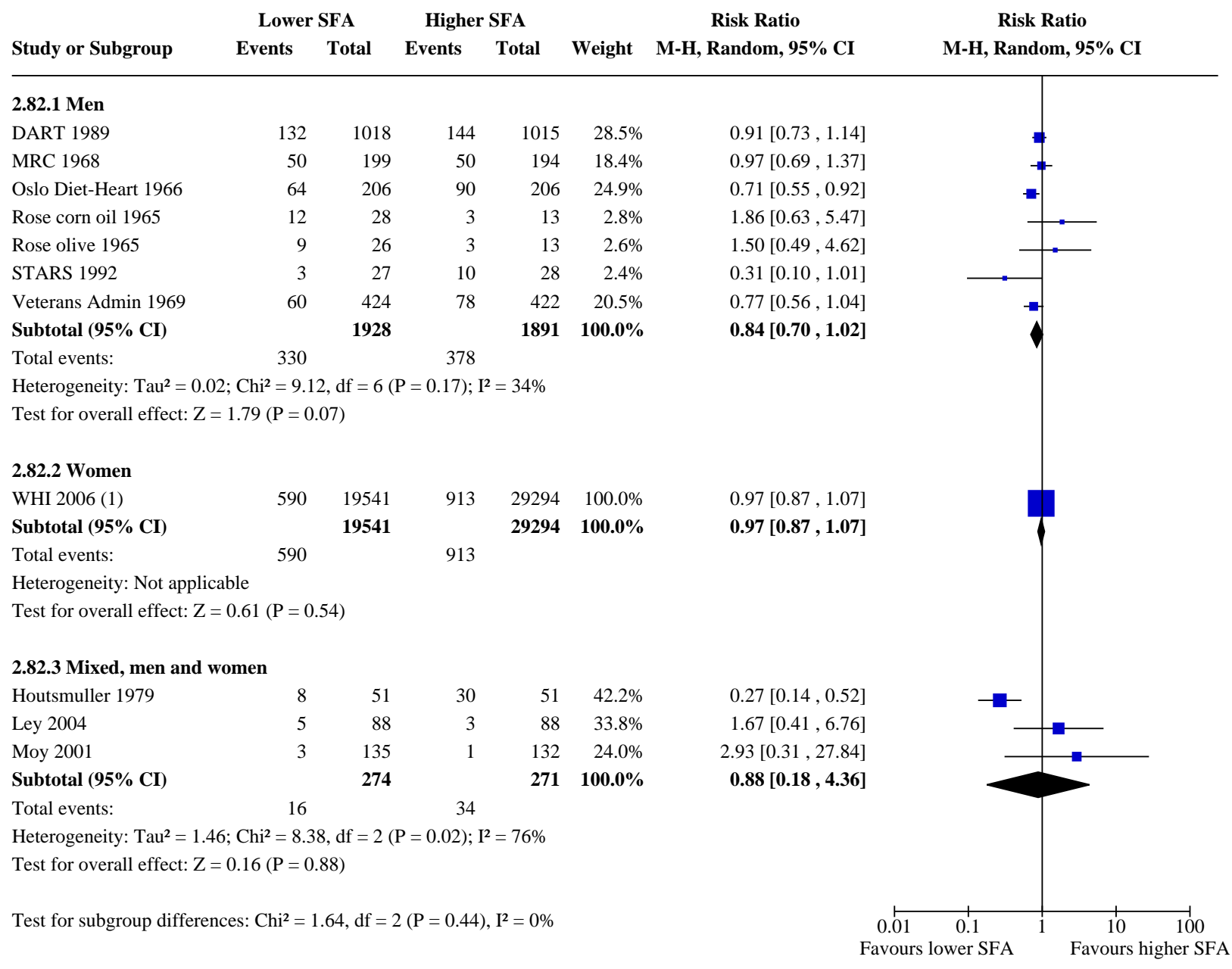

\section{Footnotes}

(1) CHD events including CHD death \& MI, Prentice 2017 


\section{Analysis 2.83. Comparison 2: SFA reduction vs usual diet - secondary} health events, Outcome 83: CHD events, subgroup by CVD risk

\begin{tabular}{|c|c|c|c|c|c|c|c|}
\hline \multirow[b]{2}{*}{ Study or Subgroup } & \multicolumn{2}{|c|}{ Lower SFA } & \multicolumn{2}{|c|}{ Higher SFA } & \multirow[b]{2}{*}{ Weight } & \multirow{2}{*}{$\begin{array}{c}\text { Risk Ratio } \\
\text { M-H, Random, } 95 \% \text { CI }\end{array}$} & \multirow{2}{*}{$\begin{array}{c}\text { Risk Ratio } \\
\text { M-H, Random, 95\% CI }\end{array}$} \\
\hline & Events & Total & Events & Total & & & \\
\hline \multicolumn{8}{|l|}{ 2.83.1 Low CVD risk } \\
\hline Veterans Admin 1969 & 60 & 424 & 78 & 422 & $21.4 \%$ & $0.77[0.56,1.04]$ & - \\
\hline WHI 2006 (1) & 806 & 18633 & 1292 & 27925 & $78.6 \%$ & $0.93[0.86,1.02]$ & \\
\hline Subtotal (95\% CI) & & 19057 & & 28347 & $100.0 \%$ & $0.90[0.76,1.05]$ & \\
\hline Total events: & 866 & & 1370 & & & & \\
\hline \multicolumn{8}{|c|}{ Heterogeneity: $\mathrm{Tau}^{2}=0.01 ; \mathrm{Chi}^{2}=1.50, \mathrm{df}=1(\mathrm{P}=0.22) ; \mathrm{I}^{2}=33 \%$} \\
\hline \multicolumn{8}{|c|}{ Test for overall effect: $\mathrm{Z}=1.34(\mathrm{P}=0.18)$} \\
\hline \multicolumn{8}{|c|}{ 2.83.2 Moderate CVD risk } \\
\hline Houtsmuller 1979 & 8 & 51 & 30 & 51 & $42.2 \%$ & $0.27[0.14,0.52]$ & \\
\hline Ley 2004 & 5 & 88 & 3 & 88 & $33.8 \%$ & $1.67[0.41,6.76]$ & \\
\hline Moy 2001 & 3 & 135 & 1 & 132 & $24.0 \%$ & $2.93[0.31,27.84]$ & 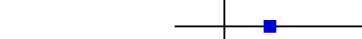 \\
\hline Subtotal (95\% CI) & & 274 & & 271 & $100.0 \%$ & $0.88[0.18,4.36]$ & \\
\hline Total events: & 16 & & 34 & & & & \\
\hline \multicolumn{8}{|c|}{ Heterogeneity: $\mathrm{Tau}^{2}=1.46 ; \mathrm{Chi}^{2}=8.38, \mathrm{df}=2(\mathrm{P}=0.02) ; \mathrm{I}^{2}=76 \%$} \\
\hline \multicolumn{8}{|c|}{ Test for overall effect: $\mathrm{Z}=0.16(\mathrm{P}=0.88)$} \\
\hline \multicolumn{8}{|c|}{ 2.83.3 Existing CVD disease } \\
\hline DART 1989 & 132 & 1018 & 144 & 1015 & $23.7 \%$ & $0.91[0.73,1.14]$ & \\
\hline MRC 1968 & 50 & 199 & 50 & 194 & $17.9 \%$ & $0.97[0.69,1.37]$ & \\
\hline Oslo Diet-Heart 1966 & 64 & 206 & 90 & 206 & $21.9 \%$ & $0.71[0.55,0.92]$ & $\rightarrow$ \\
\hline Rose corn oil 1965 & 12 & 28 & 3 & 13 & $3.6 \%$ & $1.86[0.63,5.47]$ & \\
\hline Rose olive 1965 & 9 & 26 & 3 & 13 & $3.4 \%$ & $1.50[0.49,4.62]$ & 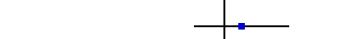 \\
\hline STARS 1992 & 3 & 27 & 10 & 28 & $3.1 \%$ & $0.31[0.10,1.01]$ & \\
\hline WHI 2006 (2) & 194 & 908 & 257 & 1369 & $26.4 \%$ & $1.14[0.96,1.34]$ & 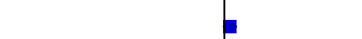 \\
\hline Subtotal (95\% CI) & & 2412 & & 2838 & $100.0 \%$ & $0.94[0.75,1.16]$ & \\
\hline Total events: & 464 & & 557 & & & & \\
\hline \multicolumn{8}{|c|}{ Heterogeneity: $\mathrm{Tau}^{2}=0.04 ; \mathrm{Chi}^{2}=15.05, \mathrm{df}=6(\mathrm{P}=0.02) ; \mathrm{I}^{2}=60 \%$} \\
\hline \multicolumn{8}{|c|}{ Test for overall effect: $\mathrm{Z}=0.59(\mathrm{P}=0.55)$} \\
\hline Test for subgroup diffe & es: $\mathrm{Chi}^{2}=0$ & $.10, \mathrm{df}=2$ & $(\mathrm{P}=0.95)$ & $\mathrm{I}^{2}=0 \%$ & & & $0 . \stackrel{1}{01}^{\prime} 0.1$ \\
\hline
\end{tabular}

\section{Footnotes}

(1) Women without CVD at baseline

(2) Women with CVD at baseline 


\section{Analysis 2.84. Comparison 2: SFA reduction vs usual diet - secondary health events, Outcome 84: CHD events, subgroup by TC reduction}

\begin{tabular}{cccccccc} 
& \multicolumn{2}{c}{ Lower SFA } & \multicolumn{2}{c}{ Higher SFA } & \multicolumn{2}{c}{ Risk Ratio } & Risk Ratio \\
Study or Subgroup & Events & Total & Events & Total & Weight & M-H, Random, 95\% CI & M-H, Random, 95\% CI
\end{tabular}

2.84.1 serum chol reduced by at least $0.2 \mathrm{mmol} / \mathrm{L}$

DART 1989

Houtsmuller 1979

MRC 1968

Moy 2001

Oslo Diet-Heart 1966

Rose corn oil 1965

STARS 1992

Veterans Admin 1969

Subtotal (95\% CI)

Total events:

$132 \quad 1018$

1018
51

144

$1015 \quad 21.7 \%$

$50 \quad 199$

30

$51 \quad 9.8 \%$

$3 \quad 135$

$64 \quad 206$

$12 \quad 28$

$3 \quad 27$

60

424

332

2088

Heterogeneity: $\mathrm{Tau}^{2}=0.07 ; \mathrm{Chi}^{2}=19.84, \mathrm{df}=7(\mathrm{P}=0.006) ; \mathrm{I}^{2}=65 \%$

Test for overall effect: $\mathrm{Z}=2.03(\mathrm{P}=0.04)$

2.84.2 serum chol reduced by $<0.2 \mathrm{mmol} / \mathrm{L}$

$\begin{array}{lrrrrr}\text { Ley 2004 } & 5 & 88 & 3 & 88 & 0.5 \% \\ \text { Rose olive 1965 } & 9 & 26 & 3 & 13 & 0.8 \% \\ \text { WHI 2006 (1) } & 590 & 19541 & 913 & 29294 & 98.7 \% \\ \text { Subtotal (95\% CI) } & & \mathbf{1 9 6 5 5} & & \mathbf{2 9 3 9 5} & \mathbf{1 0 0 . 0 \%} \\ \text { Total events: } & 604 & & 919 & & \end{array}$

Heterogeneity: $\mathrm{Tau}^{2}=0.00 ; \mathrm{Chi}^{2}=1.14, \mathrm{df}=2(\mathrm{P}=0.56) ; \mathrm{I}^{2}=0 \%$

Test for overall effect: $\mathrm{Z}=0.49(\mathrm{P}=0.62)$

2.84.3 serum chol reduction unclear

Subtotal (95\% CI)

0

Total events:

0

Heterogeneity: Not applicable

Test for overall effect: Not applicable

Test for subgroup differences: $\mathrm{Chi}^{2}=3.00, \mathrm{df}=1(\mathrm{P}=0.08), \mathrm{I}^{2}=66.6 \%$

Footnotes

(1) CHD events including CHD death \& MI, Prentice 2017
$0.91[0.73,1.14]$

$0.27[0.14,0.52]$

$0.97[0.69,1.37]$

$2.93[0.31,27.84]$

$0.71[0.55,0.92]$

$1.86[0.63,5.47]$

$0.31[0.10,1.01]$

$0.77[0.56,1.04]$

$0.76[0.58,0.99]$

$1.67[0.41,6.76]$

$1.50[0.49,4.62]$

$0.97[0.87,1.07]$

0.97 [0.88, 1.08] 
Analysis 2.85. Comparison 2: SFA reduction vs usual diet - secondary health events, Outcome 85: CHD events, subgroup decade of publication

\begin{tabular}{|c|c|c|c|c|c|}
\hline \multirow[b]{2}{*}{ Study or Subgroup } & \multicolumn{2}{|c|}{ Lower SFA } & \multicolumn{2}{|c|}{ Higher SFA } & \multirow[b]{2}{*}{ Weight } \\
\hline & Events & Total & Events & Total & \\
\hline \multicolumn{6}{|l|}{$2.85 .11960 \mathrm{~s}$} \\
\hline MRC 1968 & 50 & 199 & 50 & 194 & $13.5 \%$ \\
\hline Oslo Diet-Heart 1966 & 64 & 206 & 90 & 206 & 16.3 \\
\hline Rose corn oil 1965 & 12 & 28 & 3 & 13 & 2.9 \\
\hline Rose olive 1965 & 9 & 26 & 3 & 13 & $2.7 \%$ \\
\hline Veterans Admin 1969 & 60 & 424 & 78 & 422 & $14.5 \%$ \\
\hline Subtotal (95\% CI) & & 883 & & 848 & $50.0 \%$ \\
\hline Total events: & 195 & & 224 & & \\
\hline
\end{tabular}

\section{Risk Ratio}

M-H, Random, $95 \%$ CI
Risk Ratio

M-H, Random, $95 \%$ CI

Heterogeneity: $\mathrm{Tau}^{2}=0.02 ; \mathrm{Chi}^{2}=5.70, \mathrm{df}=4(\mathrm{P}=0.22) ; \mathrm{I}^{2}=30 \%$

Test for overall effect: $\mathrm{Z}=1.51(\mathrm{P}=0.13)$

\subsubsection{0s}

Houtsmuller 1979

Subtotal $(95 \%$ CI $)$

Total events:

8

$$
51
$$

30

8

Heterogeneity: Not applicable

Test for overall effect: $\mathrm{Z}=3.83(\mathrm{P}=0.0001)$

\subsubsection{0s}

DART 1989

Subtotal (95\% CI)

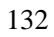

1018

144

1015

$17.6 \%$

Total events:

132

1018

$1015 \quad 17.6 \%$

Heterogeneity: Not applicable

Test for overall effect: $\mathrm{Z}=0.80(\mathrm{P}=0.42)$

\subsubsection{0s}

\section{STARS 1992}

Subtotal (95\% CI)

Total events:

3

3

27

10

30

$2.5 \%$

$2.5 \%$

10

Heterogeneity: Not applicable

Test for overall effect: $\mathrm{Z}=1.82(\mathrm{P}=0.07)$

2.85.5 2000s

Ley 2004

Moy 2001

WHI 2006 (1)

Subtotal (95\% CI)

Total events:

$5 \quad 88$

$3 \quad 135$

$590-19541$

19764

598

Heterogeneity: $\mathrm{Tau}^{2}=0.00 ; \mathrm{Chi}^{2}=1.50, \mathrm{df}=2(\mathrm{P}=0.47) ; \mathrm{I}^{2}=0 \%$

Test for overall effect: $\mathrm{Z}=0.51(\mathrm{P}=0.61)$

\section{Total $(95 \%$ CI $)$}

21743

$31458 \quad 100.0 \%$

Total events:

936

1325

Heterogeneity: $\mathrm{Tau}^{2}=0.05 ; \mathrm{Chi}^{2}=26.03, \mathrm{df}=10(\mathrm{P}=0.004) ; \mathrm{I}^{2}=62 \%$

Test for overall effect: $\mathrm{Z}=1.82(\mathrm{P}=0.07)$

Test for subgroup differences: $\mathrm{Chi}^{2}=17.51, \mathrm{df}=4(\mathrm{P}=0.002), \mathrm{I}^{2}=77.2 \%$

Footnotes

(1) CHD events including CHD death \& MI, Prentice 2017

$23.8 \%$
$0.97[0.69,1.37]$

$0.71[0.55,0.92]$

$1.86[0.63,5.47]$

$1.50[0.49,4.62]$

$0.77[0.56,1.04]$

$0.84[0.68,1.05]$
$0.91[0.73,1.14]$

$0.91[0.73,1.14]$

$0.27[0.14,0.52]$

$0.27[0.14,0.52]$

$0.33[0.10,1.09]$

$0.33[0.10,1.09]$

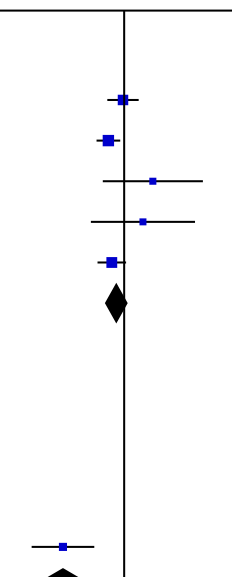

$1.67[0.41,6.76]$

$2.93[0.31,27.84]$

$0.97[0.87,1.07]$

$0.97[0.88,1.08]$

\section{年}


Analysis 2.86. Comparison 2: SFA reduction vs usual diet secondary health events, Outcome 86: DIABETES DIAGNOSES

\begin{tabular}{|c|c|c|c|c|c|c|c|}
\hline \multirow[b]{2}{*}{ Study or Subgroup } & \multicolumn{2}{|c|}{ Lower SFA } & \multicolumn{2}{|c|}{ Higher SFA } & \multirow{2}{*}{$\begin{array}{c}\text { Risk Ratio } \\
\text { M-H, Random, 95\% CI }\end{array}$} & \multirow{2}{*}{\multicolumn{2}{|c|}{$\begin{array}{c}\text { Risk Ratio } \\
\text { M-H, Random, 95\% CI }\end{array}$}} \\
\hline & Events & Total & Events & Total & & & \\
\hline WHI 2006 & 1303 & 19541 & 2039 & 29294 & $0.96[0.90,1.02]$ & $\longrightarrow$ & \\
\hline \multicolumn{6}{|c|}{ Test for subgroup differences: Not applicable } & $\begin{array}{c}1 \\
0.850 .9 \\
\text { s lower SFA }\end{array}$ & $\begin{array}{c}1.1 \\
\text { Favours h }\end{array}$ \\
\hline
\end{tabular}

Comparison 3. SFA reduction vs usual diet - secondary blood outcomes

\begin{tabular}{|c|c|c|c|c|}
\hline Outcome or subgroup title & $\begin{array}{l}\text { No. of } \\
\text { studies }\end{array}$ & $\begin{array}{l}\text { No. of } \\
\text { partici- } \\
\text { pants }\end{array}$ & Statistical method & Effect size \\
\hline 3.1 Total cholesterol, $\mathrm{mmol} / \mathrm{L}$ & 14 & 7115 & $\begin{array}{l}\text { Mean Difference (IV, Random, } \\
95 \% \mathrm{Cl} \text { ) }\end{array}$ & $-0.24[-0.36,-0.13]$ \\
\hline $\begin{array}{l}\text { 3.2 TC, mmol/L, subgroup by any replace- } \\
\text { ment }\end{array}$ & 14 & & $\begin{array}{l}\text { Mean Difference (IV, Random, } \\
95 \% \mathrm{CI})\end{array}$ & Subtotals only \\
\hline 3.2.1 replaced by PUFA & 9 & 3888 & $\begin{array}{l}\text { Mean Difference (IV, Random, } \\
95 \% \mathrm{CI} \text { ) }\end{array}$ & $-0.33[-0.47,-0.19]$ \\
\hline 3.2.2 replace by MUFA & 1 & 24 & $\begin{array}{l}\text { Mean Difference (IV, Random, } \\
95 \% \mathrm{CI} \text { ) }\end{array}$ & $0.30[-0.93,1.53]$ \\
\hline 3.2.3 replace by $\mathrm{CHO}$ & 6 & 5094 & $\begin{array}{l}\text { Mean Difference (IV, Random, } \\
95 \% \mathrm{CI} \text { ) }\end{array}$ & $-0.18[-0.32,-0.04]$ \\
\hline 3.2.4 replace by protein & 4 & 4986 & $\begin{array}{l}\text { Mean Difference (IV, Random, } \\
95 \% \mathrm{CI})\end{array}$ & $-0.15[-0.27,-0.04]$ \\
\hline 3.2.5 replacement unclear & 1 & 72 & $\begin{array}{l}\text { Mean Difference (IV, Random, } \\
95 \% \mathrm{CI} \text { ) }\end{array}$ & $-0.34[-0.64,-0.04]$ \\
\hline $\begin{array}{l}\text { 3.3 TC, mmol/L, subgroup by main re- } \\
\text { placement }\end{array}$ & 14 & & $\begin{array}{l}\text { Mean Difference (IV, Random, } \\
95 \% \mathrm{CI} \text { ) }\end{array}$ & Subtotals only \\
\hline 3.3.1 replaced by PUFA & 8 & 3838 & $\begin{array}{l}\text { Mean Difference (IV, Random, } \\
95 \% \mathrm{Cl} \text { ) }\end{array}$ & $-0.28[-0.37,-0.19]$ \\
\hline 3.3.2 replace by MUFA & 1 & 24 & $\begin{array}{l}\text { Mean Difference (IV, Random, } \\
95 \% \mathrm{CI} \text { ) }\end{array}$ & $0.30[-0.93,1.53]$ \\
\hline 3.3.3 replace by $\mathrm{CHO}$ & 4 & 3181 & $\begin{array}{l}\text { Mean Difference (IV, Random, } \\
95 \% \mathrm{CI} \text { ) }\end{array}$ & $-0.19[-0.40,0.01]$ \\
\hline 3.3.4 replace by protein & 0 & 0 & $\begin{array}{l}\text { Mean Difference (IV, Random, } \\
95 \% \mathrm{CI} \text { ) }\end{array}$ & Not estimable \\
\hline 3.3.5 replacement unclear & 1 & 72 & $\begin{array}{l}\text { Mean Difference (IV, Random, } \\
95 \% \mathrm{CI})\end{array}$ & $-0.34[-0.64,-0.04]$ \\
\hline
\end{tabular}




\begin{tabular}{|c|c|c|c|c|}
\hline Outcome or subgroup title & $\begin{array}{l}\text { No. of } \\
\text { studies }\end{array}$ & $\begin{array}{l}\text { No. of } \\
\text { partici- } \\
\text { pants }\end{array}$ & Statistical method & Effect size \\
\hline 3.4 LDL cholesterol, $\mathrm{mmol} / \mathrm{L}$ & 5 & 3291 & $\begin{array}{l}\text { Mean Difference (IV, Random, } \\
95 \% \mathrm{CI})\end{array}$ & $-0.19[-0.33,-0.05]$ \\
\hline $\begin{array}{l}3.5 \mathrm{LDL}, \mathrm{mmol} / \mathrm{L} \text {, subgroup by any re- } \\
\text { placement }\end{array}$ & 5 & & $\begin{array}{l}\text { Mean Difference (IV, Random, } \\
95 \% \mathrm{CI})\end{array}$ & Subtotals only \\
\hline 3.5.1 replaced by PUFA & 1 & 50 & $\begin{array}{l}\text { Mean Difference (IV, Random, } \\
95 \% \mathrm{CI})\end{array}$ & $-0.48[-0.90,-0.06]$ \\
\hline 3.5.2 replace by MUFA & 0 & 0 & $\begin{array}{l}\text { Mean Difference (IV, Random, } \\
95 \% \mathrm{CI})\end{array}$ & Not estimable \\
\hline 3.5.3 replace by $\mathrm{CHO}$ & 3 & 2985 & $\begin{array}{l}\text { Mean Difference (IV, Random, } \\
95 \% \mathrm{CI})\end{array}$ & $-0.16[-0.35,0.02]$ \\
\hline 3.5.4 replace by protein & 2 & 2935 & $\begin{array}{l}\text { Mean Difference (IV, Random, } \\
95 \% \mathrm{CI})\end{array}$ & $-0.09[-0.15,-0.04]$ \\
\hline 3.5.5 replacement unclear & 2 & 306 & $\begin{array}{l}\text { Mean Difference (IV, Random, } \\
95 \% \mathrm{CI})\end{array}$ & $-0.29[-0.51,-0.08]$ \\
\hline $\begin{array}{l}\text { 3.6 LDL, mmol/L, subgroup by main re- } \\
\text { placement }\end{array}$ & 5 & & $\begin{array}{l}\text { Mean Difference (IV, Random, } \\
95 \% \mathrm{CI})\end{array}$ & Subtotals only \\
\hline 3.6.1 replaced by PUFA & 0 & 0 & $\begin{array}{l}\text { Mean Difference (IV, Random, } \\
95 \% \mathrm{CI} \text { ) }\end{array}$ & Not estimable \\
\hline 3.6.2 replace by MUFA & 0 & 0 & $\begin{array}{l}\text { Mean Difference (IV, Random, } \\
95 \% \mathrm{CI})\end{array}$ & Not estimable \\
\hline 3.6.3 replace by $\mathrm{CHO}$ & 3 & 2985 & $\begin{array}{l}\text { Mean Difference (IV, Random, } \\
95 \% \mathrm{CI})\end{array}$ & $-0.16[-0.35,0.02]$ \\
\hline 3.6.4 replace by protein & 0 & 0 & $\begin{array}{l}\text { Mean Difference (IV, Random, } \\
95 \% \mathrm{CI})\end{array}$ & Not estimable \\
\hline 3.6.5 replacement unclear & 2 & 306 & $\begin{array}{l}\text { Mean Difference (IV, Random, } \\
95 \% \mathrm{CI})\end{array}$ & $-0.29[-0.51,-0.08]$ \\
\hline 3.7 HDL cholesterol, $\mathrm{mmol} / \mathrm{L}$ & 6 & 5147 & $\begin{array}{l}\text { Mean Difference (IV, Random, } \\
95 \% \mathrm{CI})\end{array}$ & $-0.01[-0.02,0.01]$ \\
\hline $\begin{array}{l}\text { 3.8 HDL, } \mathrm{mmol} / \mathrm{L} \text {, subgroup by any re- } \\
\text { placement }\end{array}$ & 6 & & $\begin{array}{l}\text { Mean Difference (IV, Random, } \\
95 \% \mathrm{CI} \text { ) }\end{array}$ & Subtotals only \\
\hline 3.8.1 replaced by PUFA & 2 & 1905 & $\begin{array}{l}\text { Mean Difference (IV, Random, } \\
95 \% \mathrm{CI} \text { ) }\end{array}$ & $-0.01[-0.04,0.01]$ \\
\hline 3.8.2 replace by MUFA & 0 & 0 & $\begin{array}{l}\text { Mean Difference (IV, Random, } \\
95 \% \mathrm{CI})\end{array}$ & Not estimable \\
\hline 3.8.3 replace by $\mathrm{CHO}$ & 4 & 4840 & $\begin{array}{l}\text { Mean Difference (IV, Random, } \\
95 \% \mathrm{CI} \text { ) }\end{array}$ & $-0.01[-0.03,0.00]$ \\
\hline
\end{tabular}




\begin{tabular}{|c|c|c|c|c|}
\hline Outcome or subgroup title & $\begin{array}{l}\text { No. of } \\
\text { studies }\end{array}$ & $\begin{array}{l}\text { No. of } \\
\text { partici- } \\
\text { pants }\end{array}$ & Statistical method & Effect size \\
\hline 3.8.4 replace by protein & 3 & 4790 & $\begin{array}{l}\text { Mean Difference (IV, Random, } \\
95 \% \mathrm{CI})\end{array}$ & $-0.01[-0.03,0.00]$ \\
\hline 3.8.5 replacement unclear & 2 & 307 & $\begin{array}{l}\text { Mean Difference (IV, Random, } \\
95 \% \mathrm{CI} \text { ) }\end{array}$ & $0.01[-0.10,0.12]$ \\
\hline $\begin{array}{l}\text { 3.9 HDL, mmol/L, subgroup by main re- } \\
\text { placement }\end{array}$ & 6 & & $\begin{array}{l}\text { Mean Difference (IV, Random, } \\
95 \% \mathrm{CI} \text { ) }\end{array}$ & Subtotals only \\
\hline 3.9.1 replaced by PUFA & 1 & 1855 & $\begin{array}{l}\text { Mean Difference (IV, Random, } \\
95 \% \mathrm{CI})\end{array}$ & $-0.01[-0.04,0.02]$ \\
\hline 3.9.2 replace by MUFA & 0 & 0 & $\begin{array}{l}\text { Mean Difference (IV, Random, } \\
95 \% \mathrm{CI} \text { ) }\end{array}$ & Not estimable \\
\hline 3.9.3 replace by $\mathrm{CHO}$ & 3 & 2985 & $\begin{array}{l}\text { Mean Difference (IV, Random, } \\
95 \% \mathrm{CI} \text { ) }\end{array}$ & $-0.01[-0.03,0.01]$ \\
\hline 3.9.4 replace by protein & 0 & 0 & $\begin{array}{l}\text { Mean Difference (IV, Random, } \\
95 \% \mathrm{CI})\end{array}$ & Not estimable \\
\hline 3.9.5 replacement unclear & 2 & 307 & $\begin{array}{l}\text { Mean Difference (IV, Random, } \\
95 \% \mathrm{CI} \text { ) }\end{array}$ & $0.01[-0.10,0.12]$ \\
\hline 3.10 Triglycerides, mmol/L & 7 & 3845 & $\begin{array}{l}\text { Mean Difference (IV, Random, } \\
95 \% \mathrm{CI} \text { ) }\end{array}$ & $-0.08[-0.21,0.04]$ \\
\hline $\begin{array}{l}\text { 3.11 TG, mmol/L, subgroup by any re- } \\
\text { placement }\end{array}$ & 7 & & $\begin{array}{l}\text { Mean Difference (IV, Random, } \\
95 \% \mathrm{CI})\end{array}$ & Subtotals only \\
\hline 3.11.1 replaced by PUFA & 3 & 604 & $\begin{array}{l}\text { Mean Difference (IV, Random, } \\
95 \% \mathrm{CI} \text { ) }\end{array}$ & $-0.19[-0.35,-0.02]$ \\
\hline 3.11 .2 replace by MUFA & 0 & 0 & $\begin{array}{l}\text { Mean Difference (IV, Random, } \\
95 \% \mathrm{CI} \text { ) }\end{array}$ & Not estimable \\
\hline 3.11.3 replace by $\mathrm{CHO}$ & 3 & 2985 & $\begin{array}{l}\text { Mean Difference (IV, Random, } \\
95 \% \mathrm{CI})\end{array}$ & $-0.04[-0.32,0.25]$ \\
\hline 3.11.4 replace by protein & 2 & 2935 & $\begin{array}{l}\text { Mean Difference (IV, Random, } \\
95 \% \mathrm{CI})\end{array}$ & $0.01[-0.08,0.09]$ \\
\hline 3.11.5 replacement unclear & 2 & 306 & $\begin{array}{l}\text { Mean Difference (IV, Random, } \\
95 \% \mathrm{CI} \text { ) }\end{array}$ & $-0.09[-0.52,0.33]$ \\
\hline $\begin{array}{l}3.12 \mathrm{TG}, \mathrm{mmol} / \mathrm{L} \text {, subgroup by main re- } \\
\text { placement }\end{array}$ & 7 & & $\begin{array}{l}\text { Mean Difference (IV, Random, } \\
95 \% \mathrm{CI})\end{array}$ & Subtotals only \\
\hline 3.12.1 replaced by PUFA & 2 & 554 & $\begin{array}{l}\text { Mean Difference (IV, Random, } \\
95 \% \mathrm{CI} \text { ) }\end{array}$ & $-0.16[-0.30,-0.01]$ \\
\hline 3.12 .2 replace by MUFA & 0 & 0 & $\begin{array}{l}\text { Mean Difference (IV, Random, } \\
95 \% \mathrm{CI})\end{array}$ & Not estimable \\
\hline
\end{tabular}




\begin{tabular}{|c|c|c|c|c|}
\hline Outcome or subgroup title & $\begin{array}{l}\text { No. of } \\
\text { studies }\end{array}$ & $\begin{array}{l}\text { No. of } \\
\text { partici- } \\
\text { pants }\end{array}$ & Statistical method & Effect size \\
\hline 3.12.3 replace by $\mathrm{CHO}$ & 3 & 2985 & $\begin{array}{l}\text { Mean Difference (IV, Random, } \\
95 \% \mathrm{Cl} \text { ) }\end{array}$ & $-0.04[-0.32,0.25]$ \\
\hline 3.12.4 replace by protein & 0 & 0 & $\begin{array}{l}\text { Mean Difference (IV, Random, } \\
95 \% \mathrm{CI})\end{array}$ & Not estimable \\
\hline 3.12.5 replacement unclear & 2 & 306 & $\begin{array}{l}\text { Mean Difference (IV, Random, } \\
95 \% \mathrm{Cl})\end{array}$ & $-0.09[-0.52,0.33]$ \\
\hline 3.13 total cholesterol /HDL ratio & 3 & 2985 & $\begin{array}{l}\text { Mean Difference (IV, Random, } \\
95 \% \mathrm{Cl})\end{array}$ & $-0.10[-0.33,0.13]$ \\
\hline $\begin{array}{l}\text { 3.14 TC / HDL ratio, subgroup by any re- } \\
\text { placement }\end{array}$ & 3 & & $\begin{array}{l}\text { Mean Difference (IV, Random, } \\
95 \% \mathrm{Cl})\end{array}$ & Subtotals only \\
\hline 3.14.1 replaced by PUFA & 1 & 50 & $\begin{array}{l}\text { Mean Difference (IV, Random, } \\
95 \% \mathrm{Cl})\end{array}$ & $-0.58[-1.33,0.17]$ \\
\hline 3.14 .2 replace by MUFA & 0 & 0 & $\begin{array}{l}\text { Mean Difference (IV, Random, } \\
95 \% \mathrm{Cl})\end{array}$ & Not estimable \\
\hline 3.14.3 replace by $\mathrm{CHO}$ & 3 & 2985 & $\begin{array}{l}\text { Mean Difference (IV, Random, } \\
95 \% \mathrm{Cl} \text { ) }\end{array}$ & $-0.10[-0.33,0.13]$ \\
\hline 3.14.4 replace by protein & 2 & 2935 & $\begin{array}{l}\text { Mean Difference (IV, Random, } \\
95 \% \mathrm{CI} \text { ) }\end{array}$ & $-0.09[-0.21,0.04]$ \\
\hline 3.14.5 replacement unclear & 0 & 0 & $\begin{array}{l}\text { Mean Difference (IV, Random, } \\
95 \% \mathrm{CI})\end{array}$ & Not estimable \\
\hline $\begin{array}{l}\text { 3.15 TC / HDL ratio, subgroup by main re- } \\
\text { placement }\end{array}$ & 3 & & $\begin{array}{l}\text { Mean Difference (IV, Random, } \\
95 \% \mathrm{CI})\end{array}$ & Subtotals only \\
\hline 3.15.1 replaced by PUFA & 0 & 0 & $\begin{array}{l}\text { Mean Difference (IV, Random, } \\
95 \% \mathrm{CI} \text { ) }\end{array}$ & Not estimable \\
\hline 3.15.2 replace by MUFA & 0 & 0 & $\begin{array}{l}\text { Mean Difference (IV, Random, } \\
95 \% \mathrm{CI} \text { ) }\end{array}$ & Not estimable \\
\hline 3.15.3 replace by $\mathrm{CHO}$ & 3 & 2985 & $\begin{array}{l}\text { Mean Difference (IV, Random, } \\
95 \% \mathrm{Cl})\end{array}$ & $-0.10[-0.33,0.13]$ \\
\hline 3.15.4 replace by protein & 0 & 0 & $\begin{array}{l}\text { Mean Difference (IV, Random, } \\
95 \% \mathrm{CI})\end{array}$ & Not estimable \\
\hline 3.15.5 replacement unclear & 0 & 0 & $\begin{array}{l}\text { Mean Difference (IV, Random, } \\
95 \% \mathrm{Cl} \text { ) }\end{array}$ & Not estimable \\
\hline 3.16 LDL / HDL ratio & 1 & & $\begin{array}{l}\text { Mean Difference (IV, Random, } \\
95 \% \mathrm{CI})\end{array}$ & Subtotals only \\
\hline 3.17 Lp(a), mmol/L & 2 & 2882 & $\begin{array}{l}\text { Mean Difference (IV, Random, } \\
95 \% \mathrm{CI} \text { ) }\end{array}$ & $0.00[-0.00,0.00]$ \\
\hline
\end{tabular}




\begin{tabular}{|c|c|c|c|c|}
\hline Outcome or subgroup title & $\begin{array}{l}\text { No. of } \\
\text { studies }\end{array}$ & $\begin{array}{l}\text { No. of } \\
\text { partici- } \\
\text { pants }\end{array}$ & Statistical method & Effect size \\
\hline $\begin{array}{l}3.18 \mathrm{Lp}(\mathrm{a}), \mathrm{mmol} / \mathrm{L} \text {, subgroup by any re- } \\
\text { placement }\end{array}$ & 2 & & $\begin{array}{l}\text { Mean Difference (IV, Random, } \\
95 \% \mathrm{CI})\end{array}$ & Subtotals only \\
\hline 3.18.1 replaced by PUFA & 1 & 50 & $\begin{array}{l}\text { Mean Difference (IV, Random, } \\
95 \% \mathrm{CI})\end{array}$ & $0.00[-1.37,1.37]$ \\
\hline 3.18 .2 replace by MUFA & 0 & 0 & $\begin{array}{l}\text { Mean Difference (IV, Random, } \\
95 \% \mathrm{CI})\end{array}$ & Not estimable \\
\hline 3.18.3 replace by $\mathrm{CHO}$ & 2 & 2882 & $\begin{array}{l}\text { Mean Difference (IV, Random, } \\
95 \% \mathrm{CI})\end{array}$ & $0.00[-0.00,0.00]$ \\
\hline 3.18.4 replace by protein & 1 & 2832 & $\begin{array}{l}\text { Mean Difference (IV, Random, } \\
95 \% \mathrm{CI})\end{array}$ & $0.00[-0.00,0.00]$ \\
\hline 3.18.5 replacement unclear & 0 & 0 & $\begin{array}{l}\text { Mean Difference (IV, Random, } \\
95 \% \mathrm{CI})\end{array}$ & Not estimable \\
\hline $\begin{array}{l}\text { 3.19 Lp(a), mmol/L, subgroup by main re- } \\
\text { placement }\end{array}$ & 2 & & $\begin{array}{l}\text { Mean Difference (IV, Random, } \\
95 \% \mathrm{CI})\end{array}$ & Subtotals only \\
\hline 3.19.1 replaced by PUFA & 0 & 0 & $\begin{array}{l}\text { Mean Difference (IV, Random, } \\
95 \% \mathrm{CI})\end{array}$ & Not estimable \\
\hline 3.19.2 replace by MUFA & 0 & 0 & $\begin{array}{l}\text { Mean Difference (IV, Random, } \\
95 \% \mathrm{CI})\end{array}$ & Not estimable \\
\hline 3.19.3 replace by $\mathrm{CHO}$ & 2 & 2882 & $\begin{array}{l}\text { Mean Difference (IV, Random, } \\
95 \% \mathrm{CI} \text { ) }\end{array}$ & $0.00[-0.00,0.00]$ \\
\hline 3.19.4 replace by protein & 0 & 0 & $\begin{array}{l}\text { Mean Difference (IV, Random, } \\
95 \% \mathrm{CI} \text { ) }\end{array}$ & Not estimable \\
\hline 3.19.5 replacement unclear & 0 & 0 & $\begin{array}{l}\text { Mean Difference (IV, Random, } \\
95 \% \mathrm{CI} \text { ) }\end{array}$ & Not estimable \\
\hline 3.20 Insulin sensitivity & 4 & & $\begin{array}{l}\text { Mean Difference (IV, Random, } \\
95 \% \mathrm{CI} \text { ) }\end{array}$ & Subtotals only \\
\hline $\begin{array}{l}\text { 3.20.1 HbA1c (glycosylated haemoglo- } \\
\text { bin), } \%\end{array}$ & 0 & 0 & $\begin{array}{l}\text { Mean Difference (IV, Random, } \\
95 \% \mathrm{CI})\end{array}$ & Not estimable \\
\hline $\begin{array}{l}\text { 3.20.2 GTT (glucose tolerance test), glu- } \\
\text { cose at } 2 \text { hours, mmol/L }\end{array}$ & 3 & 249 & $\begin{array}{l}\text { Mean Difference (IV, Random, } \\
95 \% \mathrm{CI} \text { ) }\end{array}$ & $-1.69[-2.55,-0.82]$ \\
\hline 3.20.3 HOMA & 1 & 2832 & $\begin{array}{l}\text { Mean Difference (IV, Random, } \\
95 \% \mathrm{Cl})\end{array}$ & $0.00[-0.04,0.04]$ \\
\hline
\end{tabular}


Analysis 3.1. Comparison 3: SFA reduction vs usual diet secondary blood outcomes, Outcome 1: Total cholesterol, $\mathrm{mmol} / \mathrm{L}$

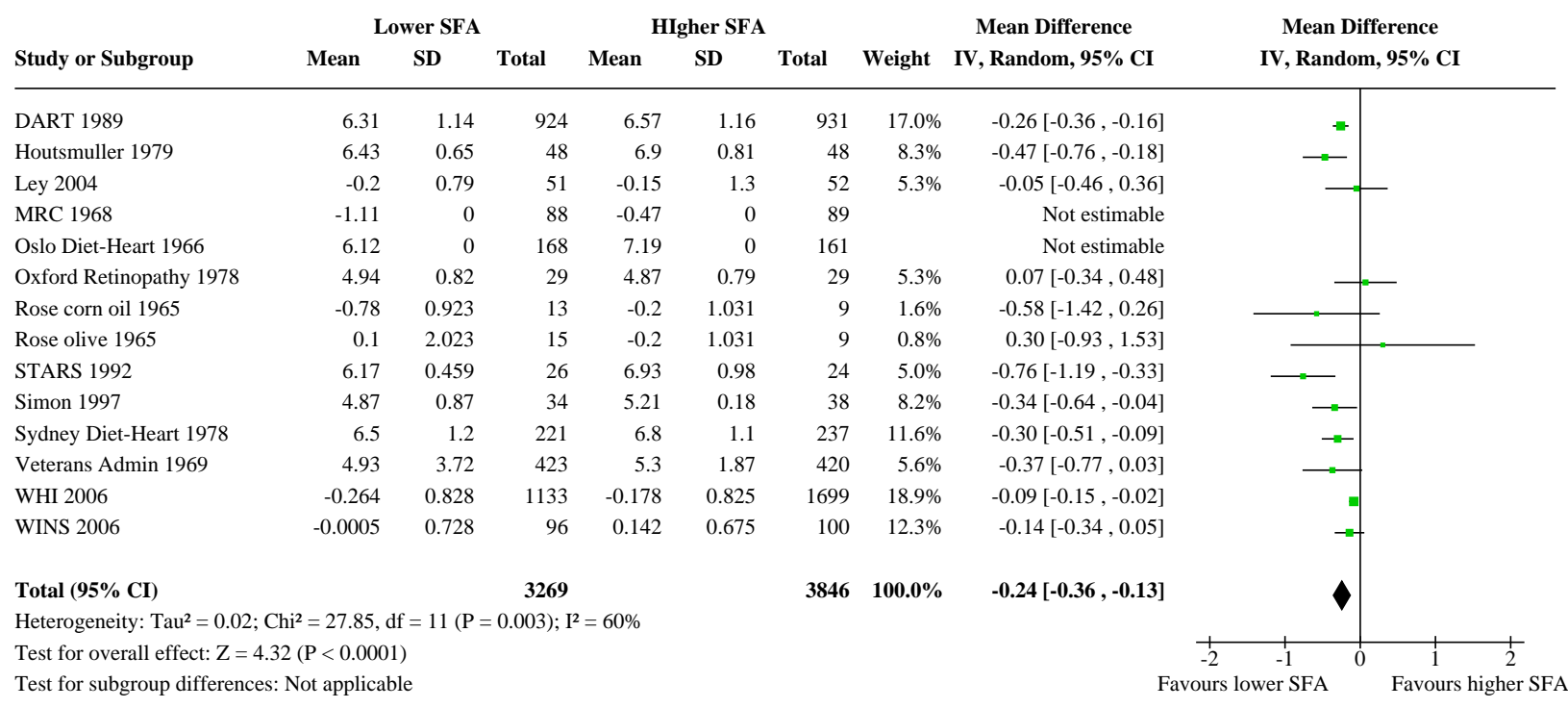


Analysis 3.3. Comparison 3: SFA reduction vs usual diet - secondary blood outcomes, Outcome 3: TC, mmol/L, subgroup by main replacement

\begin{tabular}{|c|c|c|c|c|c|c|c|c|c|}
\hline \multirow[b]{2}{*}{ Study or Subgroup } & \multicolumn{3}{|c|}{ Lower SFA } & \multicolumn{3}{|c|}{ HIgher SFA } & \multicolumn{2}{|r|}{ Mean Difference } & Mean Difference \\
\hline & Mean & SD & Total & Mean & SD & Total & Weight & IV, Random, 95\% CI & IV, Random, 95\% CI \\
\hline
\end{tabular}

3.3.1 replaced by PUFA

$\begin{array}{llllllll}6.31 & 1.14 & 924 & 6.57 & 1.16 & 931 & 61.4 \% & -0.26[-0.36,-0.16]\end{array}$

Houtsmuller 1979

MRC 1968

Oslo Diet-Heart 1966

Oxford Retinopathy 1978

Rose corn oil 1965

Sydney Diet-Heart 1978

Veterans Admin 1969

\begin{tabular}{|c|c|c|}
\hline 6.43 & 0.65 & 48 \\
\hline-1.11 & 0 & 88 \\
\hline 6.12 & 0 & 168 \\
\hline 4.94 & 0.82 & 29 \\
\hline-0.78 & 0.923 & 13 \\
\hline 6.5 & 1.2 & 221 \\
\hline \multirow[t]{2}{*}{4.93} & 3.72 & 423 \\
\hline & & 1914 \\
\hline
\end{tabular}

0.81

48

$9.5 \%$

$-0.47[-0.76$

Not estimable

Not estimable

$0.07[-0.34,0.48]$

$0.79 \quad 29 \quad 4.8 \%$

1.031

$1.2 \%$

$-0.58[-1.42,0.26]$

$-0.30[-0.51,-0.09]$

$-0.37[-0.77,0.03]$

Subtotal $(95 \%$ CI)

1914

$237 \quad 17.8 \%$

$420 \quad 5.3 \%$

$-0.28[-0.37,-0.19]$

Heterogeneity: $\mathrm{Tau}^{2}=0.00 ; \mathrm{Chi}^{2}=5.21, \mathrm{df}=5(\mathrm{P}=0.39) ; \mathrm{I}^{2}=4 \%$

$1924100.0 \%$

Test for overall effect: $\mathrm{Z}=5.98(\mathrm{P}<0.00001)$

\subsection{2 replace by MUFA}

Rose olive 1965

$0.1 \quad 2.023$

$\begin{array}{lll}15 & -0.2 & 1.031 \\ \mathbf{1 5} & & \end{array}$

$9 \quad 100.0 \%$

Subtotal (95\% CI)

$\begin{array}{rrrr}15 & 9 & 100.0 \%\end{array}$

$0.30[-0.93,1.53]$

Heterogeneity: Not applicable

Test for overall effect: $\mathrm{Z}=0.48(\mathrm{P}=0.63)$

3.3.3 replace by $\mathrm{CHO}$

Ley 2004

STARS 1992

WHI 2006

WINS 2006

Subtotal (95\% CI)

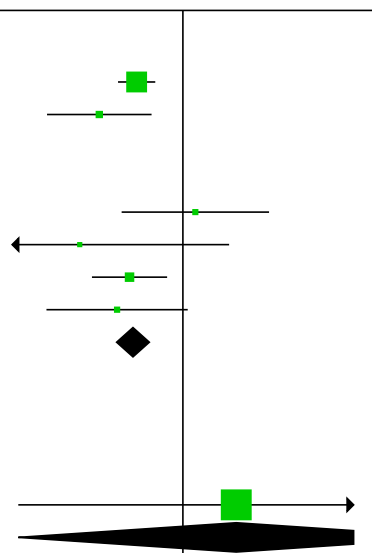

Heterogeneity: $\mathrm{Tau}^{2}=0.03 ; \mathrm{Chi}^{2}=9.48, \mathrm{df}=3(\mathrm{P}=0.02) ; \mathrm{I}^{2}=68 \%$

Test for overall effect: $\mathrm{Z}=1.89(\mathrm{P}=0.06)$

\subsection{4 replace by protein}

Subtotal (95\% CI)

o

0

Not estimable

eneity: Not applicable

Test for overall effect: Not applicable

\subsection{5 replacement unclear}

Simon 1997

Subtotal (95\% CI)

$38 \quad 100.0 \%$

$-0.05[-0.46,0.36]$ $-0.76[-1.19,-0.33]$ $-0.09[-0.15,-0.02]$ $-0.14[-0.34,0.05]$ $-0.19[-0.40,0.01]$

Heterogeneity: Not applicable

Test for overall effect: $\mathrm{Z}=2.24(\mathrm{P}=0.03)$

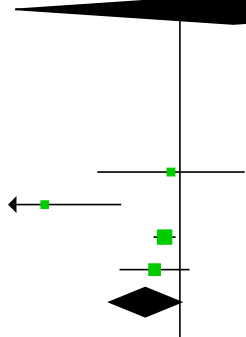

$\begin{array}{llll}\text { Lower SFA } & \text { HIgher SFA } & \text { Mean Difference } & \text { Mean Difference }\end{array}$

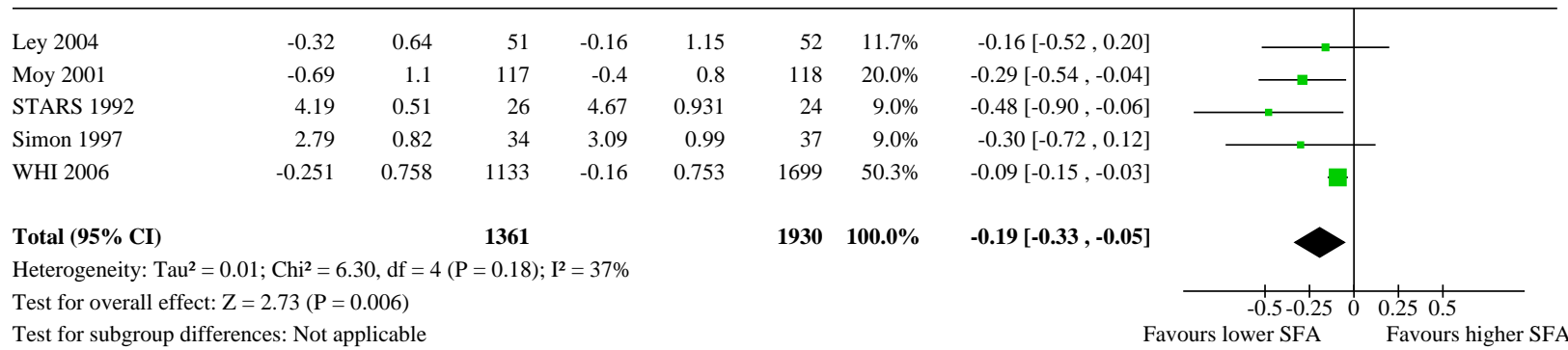


Analysis 3.5. Comparison 3: SFA reduction vs usual diet - secondary blood outcomes, Outcome 5: LDL, $\mathrm{mmol} / \mathrm{L}$, subgroup by any replacement

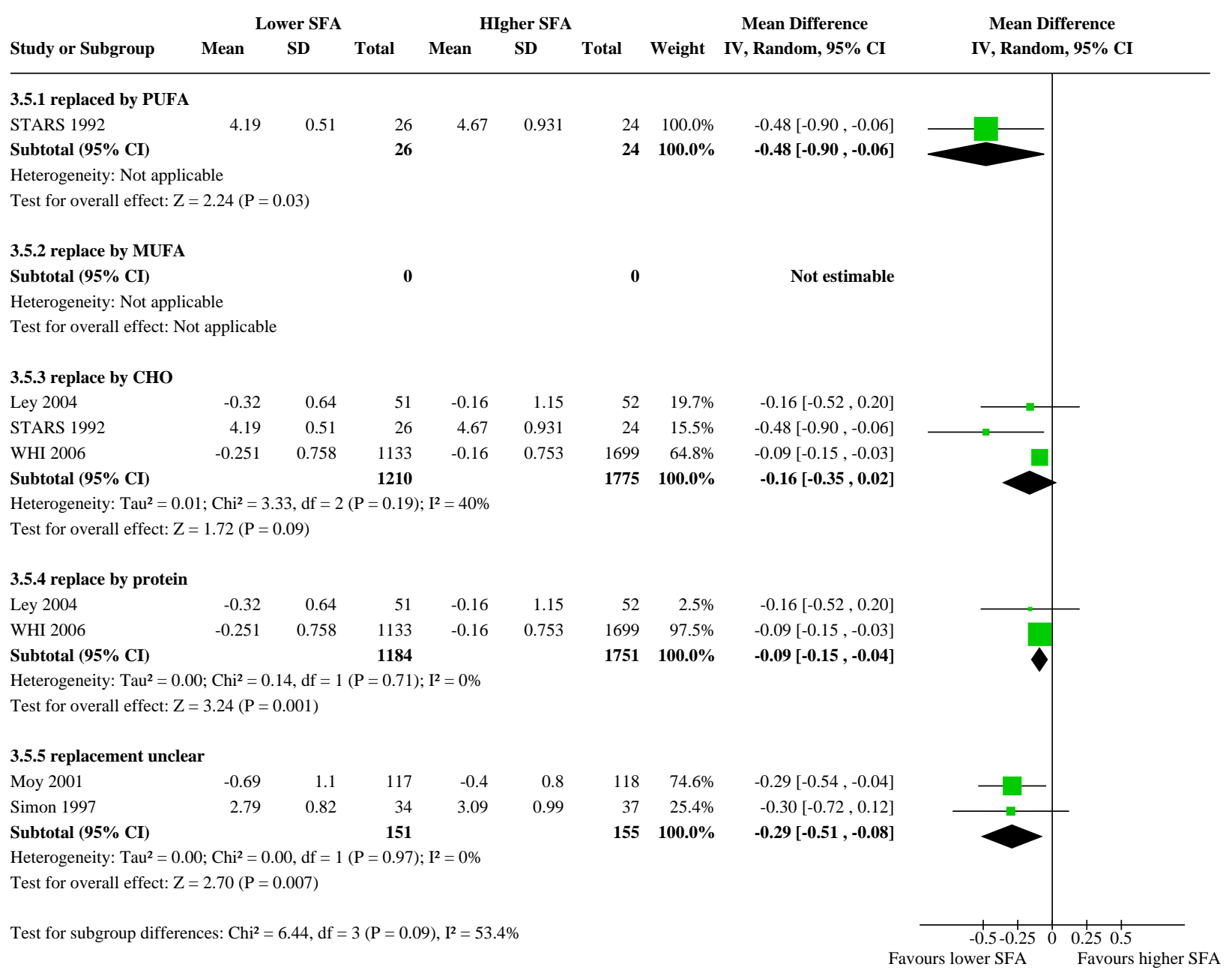


Analysis 3.6. Comparison 3: SFA reduction vs usual diet - secondary blood outcomes, Outcome 6: LDL, mmol/L, subgroup by main replacement

\begin{tabular}{|c|c|c|c|c|c|c|c|c|c|}
\hline & \multicolumn{3}{|c|}{ Lower SFA } & \multicolumn{3}{|c|}{ HIgher SFA } & \multirow{2}{*}{\multicolumn{2}{|c|}{$\begin{array}{cc} & \text { Mean Difference } \\
\text { Weight } & \text { IV, Random, 95\% CI }\end{array}$}} & \multirow{2}{*}{$\begin{array}{c}\text { Mean Difference } \\
\text { IV, Random, 95\% CI }\end{array}$} \\
\hline Study or Subgroup & Mean & SD & Total & Mean & SD & Total & & & \\
\hline
\end{tabular}

3.6.1 replaced by PUFA

Subtotal (95\% CI)

Heterogeneity: Not applicable

Test for overall effect: Not applicable

3.6.2 replace by MUFA

Subtotal (95\% CI)

0

Heterogeneity: Not applicable

Test for overall effect: Not applicable

3.6.3 replace by $\mathrm{CHO}$

$\begin{array}{lrrrrrrr}\text { Ley 2004 } & -0.32 & 0.64 & 51 & -0.16 & 1.15 & 52 & 19.7 \% \\ \text { STARS 1992 } & 4.19 & 0.51 & 26 & 4.67 & 0.931 & 24 & 15.5 \% \\ \text { WHI 2006 } & -0.251 & 0.758 & 1133 & -0.16 & 0.753 & 1699 & 64.8 \%\end{array}$

WHI 2006

Subtotal (95\% CI)

1210

0

Not estimable

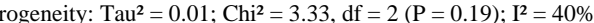

Test for overall effect: $\mathrm{Z}=1.72(\mathrm{P}=0.09)$

3.6.4 replace by protein

Subtotal (95\% CI)

$\mathbf{0}$

Heterogeneity: Not applicable

Test for overall effect: Not applicable

\subsection{5 replacement unclear}

$\begin{array}{lrrrrrrrr}\text { Moy 2001 } & -0.69 & 1.1 & 117 & -0.4 & 0.8 & 118 & 74.6 \% & -0.29[-0.54,-0.04] \\ \text { Simon } 1997 & 2.79 & 0.82 & 34 & 3.09 & 0.99 & 37 & 25.4 \% & -0.30[-0.72,0.12] \\ \text { Subtotal (95\% CI) } & & & \mathbf{1 5 1} & & & \mathbf{1 5 5} & \mathbf{1 0 0 . 0 \%} & \mathbf{- 0 . 2 9}[-\mathbf{0 . 5 1}, \mathbf{- 0 . 0 8}]\end{array}$

Heterogeneity: $\mathrm{Tau}^{2}=0.00 ; \mathrm{Chi}^{2}=0.00, \mathrm{df}=1(\mathrm{P}=0.97) ; \mathrm{I}^{2}=0 \%$

Test for overall effect: $\mathrm{Z}=2.70(\mathrm{P}=0.007)$

Test for subgroup differences: $\mathrm{Chi}^{2}=0.78, \mathrm{df}=1(\mathrm{P}=0.38), \mathrm{I}^{2}=0 \%$
$-0.16[-0.52,0.20]$

$-0.48[-0.90,-0.06]$

$-0.09[-0.15,-0.03]$

$\mathbf{- 0 . 1 6}[-0.35,0.02]$

Not estimable
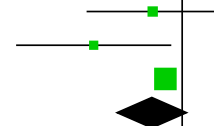

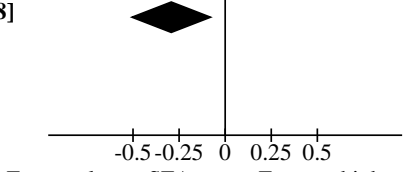

Favours lower SFA $\quad$ Favours higher SFA

Analysis 3.7. Comparison 3: SFA reduction vs usual diet secondary blood outcomes, Outcome 7: HDL cholesterol, mmol/L

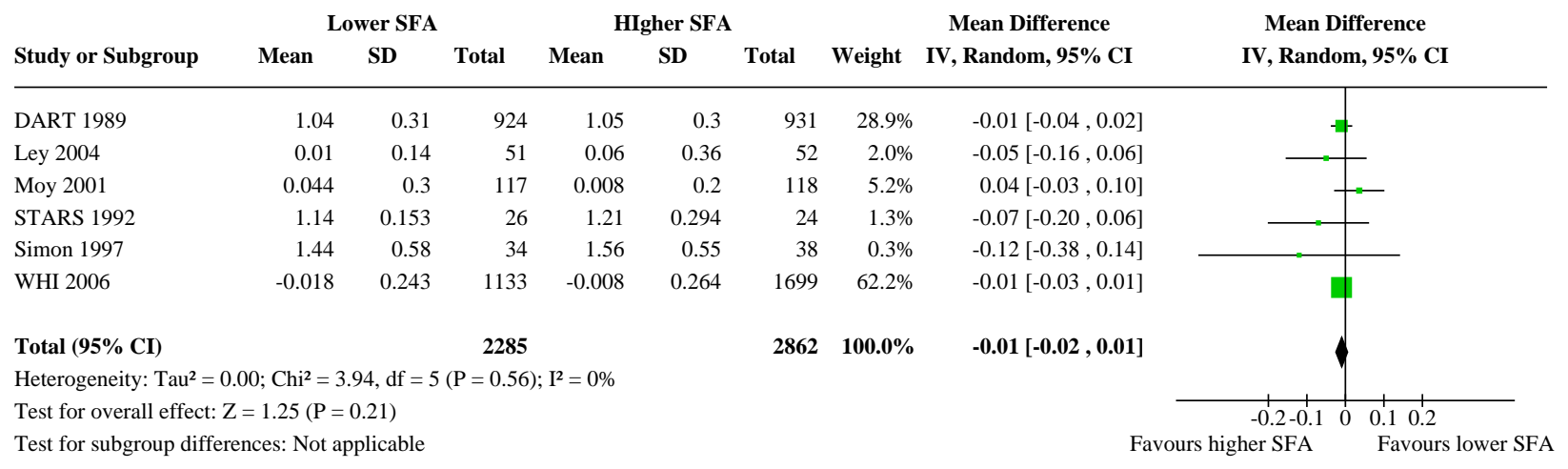


Analysis 3.8. Comparison 3: SFA reduction vs usual diet - secondary blood outcomes, Outcome 8: HDL, mmol/L, subgroup by any replacement

\begin{tabular}{|c|c|c|c|c|c|c|c|c|c|}
\hline \multirow[b]{2}{*}{ Study or Subgroup } & \multicolumn{3}{|c|}{ Lower SFA } & \multicolumn{3}{|c|}{ HIgher SFA } & \multirow[b]{2}{*}{ Weight } & \multirow{2}{*}{$\begin{array}{c}\text { Mean Difference } \\
\text { IV, Random, 95\% CI }\end{array}$} & \multirow{2}{*}{$\begin{array}{c}\text { Mean Difference } \\
\text { IV, Random, 95\% CI }\end{array}$} \\
\hline & Mean & SD & Total & Mean & SD & Total & & & \\
\hline \multicolumn{10}{|c|}{ 3.8.1 replaced by PUFA } \\
\hline DART 1989 & 1.04 & 0.31 & 924 & 1.05 & 0.3 & 931 & $95.7 \%$ & $-0.01[-0.04,0.02]$ & \\
\hline STARS 1992 & 1.14 & 0.153 & 26 & 1.21 & 0.294 & 24 & $4.3 \%$ & $-0.07[-0.20,0.06]$ & \\
\hline Subtotal $(95 \%$ CI $)$ & & & 950 & & & 955 & $100.0 \%$ & $-0.01[-0.04,0.01]$ & \\
\hline \multicolumn{10}{|c|}{ Heterogeneity: $\mathrm{Tau}^{2}=0.00 ; \mathrm{Chi}^{2}=0.77, \mathrm{df}=1(\mathrm{P}=0.38) ; \mathrm{I}^{2}=0 \%$} \\
\hline \multicolumn{10}{|c|}{ Test for overall effect: $\mathrm{Z}=0.91(\mathrm{P}=0.36)$} \\
\hline \multicolumn{10}{|c|}{ 3.8.2 replace by MUFA } \\
\hline Subtotal $(95 \%$ CI $)$ & & & $\mathbf{0}$ & & & $\mathbf{0}$ & & Not estimable & \\
\hline \multicolumn{10}{|c|}{ Heterogeneity: Not applicable } \\
\hline \multicolumn{10}{|c|}{ Test for overall effect: Not applicable } \\
\hline \multicolumn{10}{|l|}{ 3.8.3 replace by $\mathrm{CHO}$} \\
\hline DART 1989 & 1.04 & 0.31 & 924 & 1.05 & 0.3 & 931 & $30.6 \%$ & $-0.01[-0.04,0.02]$ & \\
\hline Ley 2004 & 0.01 & 0.14 & 51 & 0.06 & 0.36 & 52 & $2.1 \%$ & $-0.05[-0.16,0.06]$ & \\
\hline STARS 1992 & 1.14 & 0.153 & 26 & 1.21 & 0.294 & 24 & $1.4 \%$ & $-0.07[-0.20,0.06]$ & \\
\hline WHI 2006 & -0.018 & 0.243 & 1133 & -0.008 & 0.264 & 1699 & $65.9 \%$ & $-0.01[-0.03,0.01]$ & \\
\hline Subtotal $(95 \%$ CI $)$ & & & 2134 & & & 2706 & $100.0 \%$ & $-0.01[-0.03,0.00]$ & \\
\hline \multicolumn{10}{|c|}{ Heterogeneity: $\mathrm{Tau}^{2}=0.00 ; \mathrm{Chi}^{2}=1.31, \mathrm{df}=3(\mathrm{P}=0.73) ; \mathrm{I}^{2}=0 \%$} \\
\hline \multicolumn{10}{|c|}{ Test for overall effect: $\mathrm{Z}=1.49(\mathrm{P}=0.14)$} \\
\hline \multicolumn{10}{|c|}{ 3.8.4 replace by protein } \\
\hline DART 1989 & 1.04 & 0.31 & 924 & 1.05 & 0.3 & 931 & $31.0 \%$ & $-0.01[-0.04,0.02]$ & -7 \\
\hline Ley 2004 & 0.01 & 0.14 & 51 & 0.06 & 0.36 & 52 & $2.2 \%$ & $-0.05[-0.16,0.06]$ & \\
\hline WHI 2006 & -0.018 & 0.243 & 1133 & -0.008 & 0.264 & 1699 & $66.8 \%$ & $-0.01[-0.03,0.01]$ & \\
\hline Subtotal $(95 \%$ CI $)$ & & & 2108 & & & 2682 & $100.0 \%$ & $-0.01[-0.03,0.00]$ & \\
\hline \multicolumn{10}{|c|}{ Heterogeneity: $\mathrm{Tau}^{2}=0.00 ; \mathrm{Chi}^{2}=0.54, \mathrm{df}=2(\mathrm{P}=0.76) ; \mathrm{I}^{2}=0 \%$} \\
\hline \multicolumn{10}{|c|}{ Test for overall effect: $\mathrm{Z}=1.38(\mathrm{P}=0.17)$} \\
\hline \multicolumn{10}{|c|}{ 3.8.5 replacement unclear } \\
\hline Moy 2001 & 0.044 & 0.3 & 117 & 0.008 & 0.2 & 118 & $84.4 \%$ & $0.04[-0.03,0.10]$ & \\
\hline Simon 1997 & 1.44 & 0.58 & 34 & 1.56 & 0.55 & 38 & $15.6 \%$ & $-0.12[-0.38,0.14]$ & \\
\hline Subtotal $(95 \%$ CI $)$ & & & 151 & & & 156 & $100.0 \%$ & $0.01[-0.10,0.12]$ & \\
\hline \multicolumn{10}{|c|}{ Heterogeneity: $\mathrm{Tau}^{2}=0.00 ; \mathrm{Chi}^{2}=1.28, \mathrm{df}=1(\mathrm{P}=0.26) ; \mathrm{I}^{2}=22 \%$} \\
\hline \multicolumn{10}{|c|}{ Test for overall effect: $\mathrm{Z}=0.21(\mathrm{P}=0.84)$} \\
\hline Test for subgroup diff & cees: $\mathrm{Chi}^{2}=$ & $0.18, \mathrm{df}=$ & $3(\mathrm{P}=0$. & $2^{2}=0 \%$ & & & & & $\begin{array}{cccc}-0.2-0.1 & 0 & 0.1 & 0.2 \\
\text { gher SFA } & & \text { Favour }\end{array}$ \\
\hline
\end{tabular}


Analysis 3.9. Comparison 3: SFA reduction vs usual diet - secondary blood outcomes, Outcome 9: HDL, mmol/L, subgroup by main replacement

\begin{tabular}{|c|c|c|c|c|c|c|c|c|c|}
\hline & \multicolumn{3}{|c|}{ Lower SFA } & \multicolumn{3}{|c|}{ HIgher SFA } & \multicolumn{2}{|r|}{ Mean Difference } & \multirow{2}{*}{$\begin{array}{c}\text { Mean Difference } \\
\text { IV, Random, 95\% CI }\end{array}$} \\
\hline Study or Subgroup & Mean & SD & Total & Mean & SD & Total & Weight & IV, Random, $95 \%$ CI & \\
\hline
\end{tabular}

3.9.1 replaced by PUFA

$\begin{array}{lllllllll}\text { DART } 1989 & 1.04 & 0.31 & 924 & 1.05 & 0.3 & 931 & 100.0 \% & -0.01[-0.04,0.02] \\ \text { Subtotal }(\mathbf{9 5 \%} \mathbf{C I}) & & & \mathbf{9 2 4} & & & \mathbf{9 3 1} & \mathbf{1 0 0 . 0 \%} & \mathbf{- 0 . 0 1}[-\mathbf{0 . 0 4} \mathbf{0 . 0 2}]\end{array}$

Subtotal (95\% C1)

924

$931 \quad 100.0 \%$

$\mathbf{0 . 0 1}[-0.04,0.02]$

Heterogeneity: Not applicable

Test for overall effect: $\mathrm{Z}=0.71(\mathrm{P}=0.48)$

3.9.2 replace by MUFA

Subtotal $(95 \%$ CI $)$

Not estimable

Heterogeneity: Not applicable

Test for overall effect: Not applicable

3.9.3 replace by CHO

STARS 1992

WHI 2006

Subtotal $(95 \% \mathrm{CI})$

1210

$1775100.0 \%$

$-0.07[-0.20,0.06]$

$-0.01[-0.03,0.01]$

Heterogeneity: $\mathrm{Tau}^{2}=0.00 ; \mathrm{Chi}^{2}=1.29, \mathrm{df}=2(\mathrm{P}=0.52) ; \mathrm{I}^{2}=0 \%$

$\mathbf{- 0 . 0 1}[-0.03,0.01]$

Test for overall effect: $\mathrm{Z}=1.32(\mathrm{P}=0.19)$

3.9.4 replace by protein

Subtotal (95\% CI)

$\mathbf{0}$

0

Not estimable

Sulcable

Test for overall effect: Not applicable

3.9.5 replacement unclear

$\begin{array}{lrrrrrrrr}\text { Moy } 2001 & 0.044 & 0.3 & 117 & 0.008 & 0.2 & 118 & 84.4 \% & 0.04[-0.03,0.10] \\ \text { Simon 1997 } & 1.44 & 0.58 & 34 & 1.56 & 0.55 & 38 & 15.6 \% & -0.12[-0.38,0.14] \\ \text { Subtotal (95\% CI) } & & & \mathbf{1 5 1} & & & \mathbf{1 5 6} & \mathbf{1 0 0 . 0 \%} & \mathbf{0 . 0 1}[\mathbf{- 0 . 1 0 , 0 . 1 2}]\end{array}$

Heterogeneity: $\mathrm{Tau}^{2}=0.00 ; \mathrm{Chi}^{2}=1.28, \mathrm{df}=1(\mathrm{P}=0.26) ; \mathrm{I}^{2}=22 \%$

Test for overall effect: $\mathrm{Z}=0.21(\mathrm{P}=0.84)$

Test for subgroup differences: $\mathrm{Chi}^{2}=0.19, \mathrm{df}=2(\mathrm{P}=0.91), \mathrm{I}^{2}=0 \%$

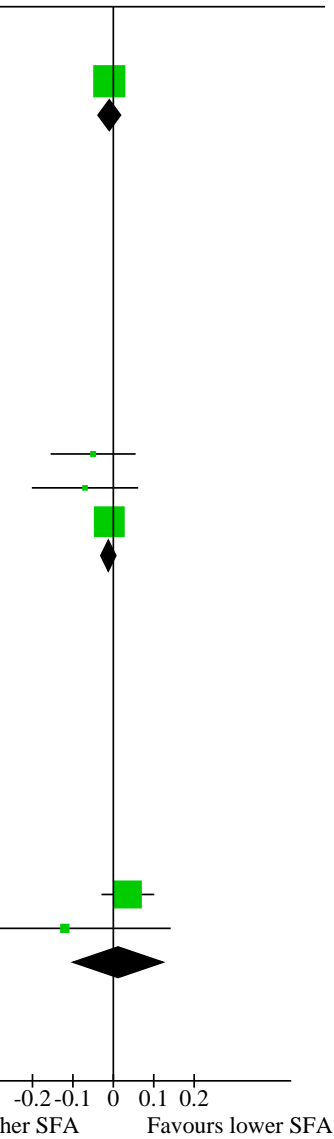

Analysis 3.10. Comparison 3: SFA reduction vs usual diet secondary blood outcomes, Outcome 10: Triglycerides, mmol/L

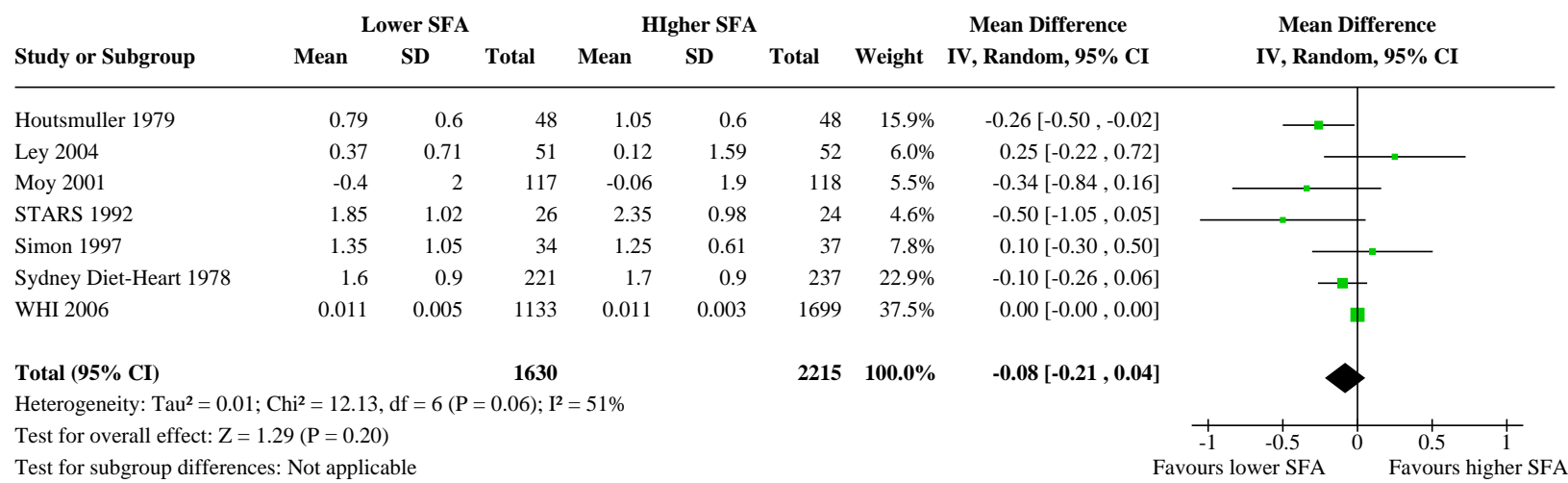


Analysis 3.11. Comparison 3: SFA reduction vs usual diet - secondary blood outcomes, Outcome 11: TG, $\mathrm{mmol} / \mathrm{L}$, subgroup by any replacement

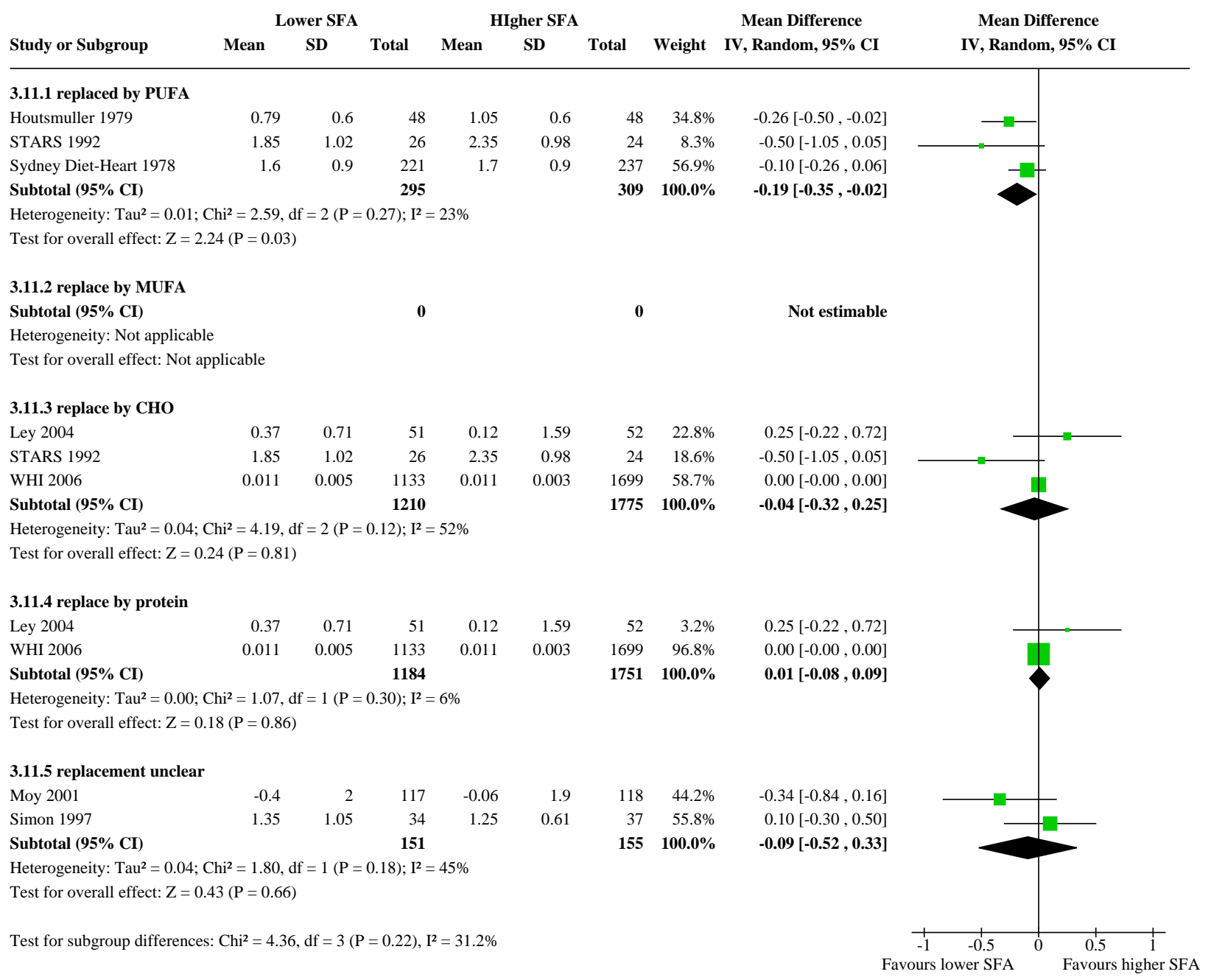


Analysis 3.12. Comparison 3: SFA reduction vs usual diet - secondary blood outcomes, Outcome 12: TG, $\mathrm{mmol} / \mathrm{L}$, subgroup by main replacement

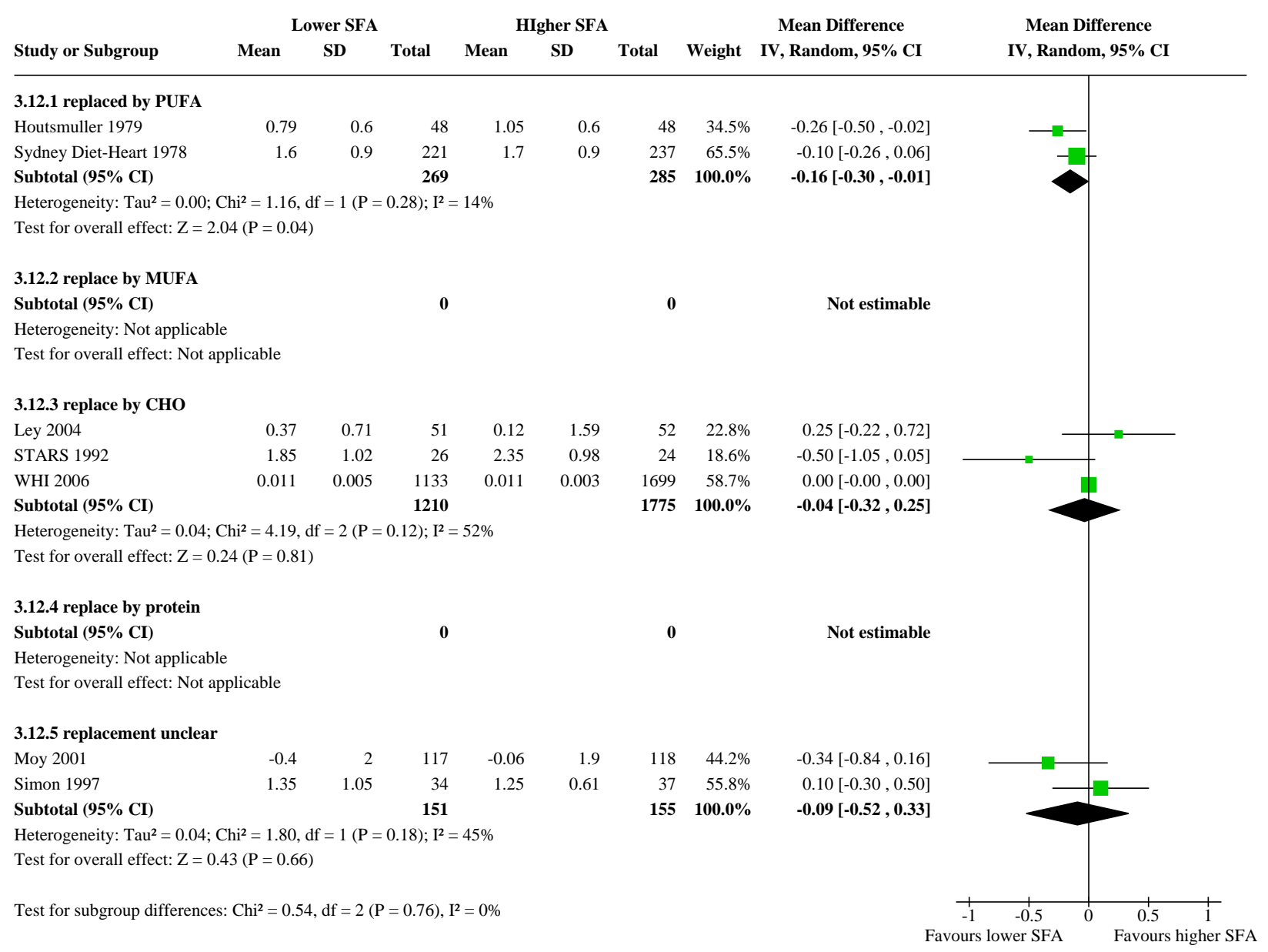

Analysis 3.13. Comparison 3: SFA reduction vs usual diet secondary blood outcomes, Outcome 13: total cholesterol /HDL ratio

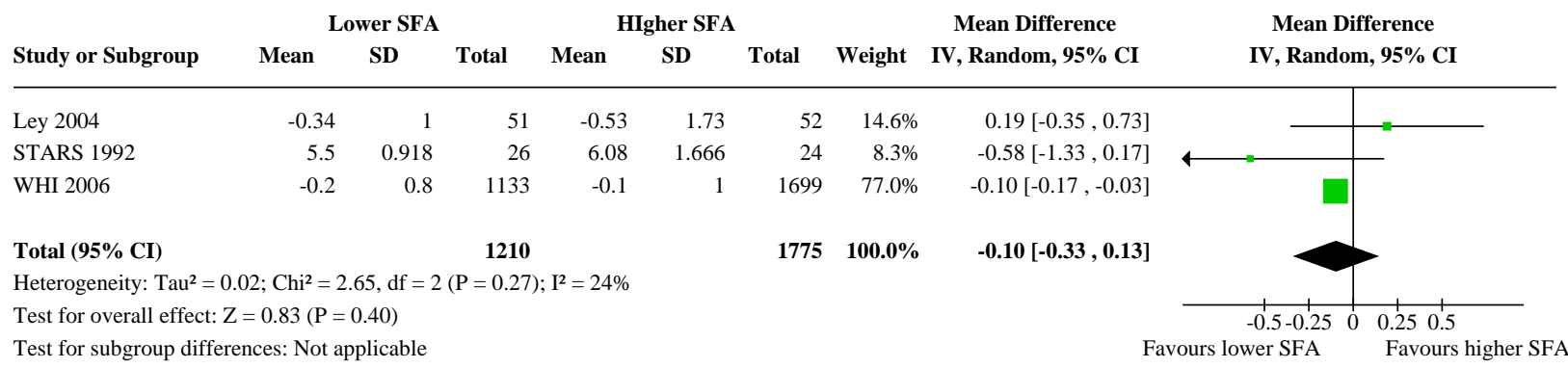


Analysis 3.14. Comparison 3: SFA reduction vs usual diet - secondary blood outcomes, Outcome 14: TC /HDL ratio, subgroup by any replacement

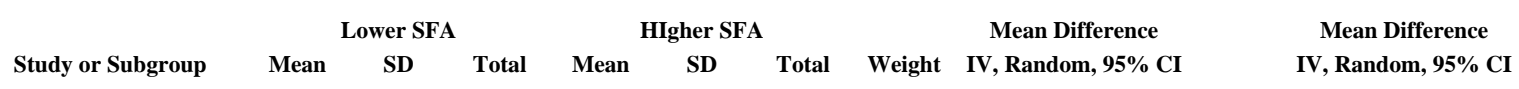

3.14.1 replaced by PUFA

STARS 1992

$\begin{array}{lllll}5.5 & 0.918 & 26 & 6.08 & 1.666\end{array}$

$24 \quad 100.0 \%$

Subtotal (95\% CI)

26

$24 \quad 100.0 \%$

Heterogeneity: Not applicable

Test for overall effect: $\mathrm{Z}=1.51(\mathrm{P}=0.13)$

\subsection{2 replace by MUFA}

Subtotal (95\% CI)

Not estimable

Test for overall effect: Not applicable

3.14.3 replace by $\mathrm{CHO}$

STARS 1992

WHI 2006

$\begin{array}{rr}-0.34 & 1 \\ 5.5 & 0.918\end{array}$

51
26

$-0.53 \quad 1.73$

6.08

1.666

Subtotal (95\% CI)

$\begin{array}{lll}-0.2 & 0.8 \quad 1133\end{array}$

$-0.1$

1210

Heterogeneity: $\mathrm{Tau}^{2}=0.02 ; \mathrm{Chi}^{2}=2.65, \mathrm{df}=2(\mathrm{P}=0.27) ; \mathrm{I}^{2}=24 \%$

Test for overall effect: $\mathrm{Z}=0.83(\mathrm{P}=0.40)$

3.14.4 replace by protein

$\begin{array}{lrrrrrrrr}\text { Ley } 2004 & -0.34 & 1 & 51 & -0.53 & 1.73 & 52 & 4.8 \% & 0.19[-0.35,0.73] \\ \text { WHI } 2006 & -0.2 & 0.8 & 1133 & -0.1 & 1 & 1699 & 95.2 \% & -0.10[-0.17,-0.03] \\ \text { Subtotal (95\% CI) } & & & \mathbf{1 1 8 4} & & & \mathbf{1 7 5 1} & \mathbf{1 0 0 . 0 \%} & \mathbf{- 0 . 0 9}[-\mathbf{0 . 2 1}, \mathbf{0 . 0 4}]\end{array}$

Heterogeneity: $\mathrm{Tau}^{2}=0.00 ; \mathrm{Chi}^{2}=1.07, \mathrm{df}=1(\mathrm{P}=0.30) ; \mathrm{I}^{2}=7 \%$

$-0.58[-1.33,0.17]$

$\mathbf{- 0 . 5 8}[-1.33,0.17]$

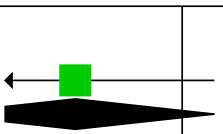

Test for overall effect: $\mathrm{Z}=1.39(\mathrm{P}=0.17)$

\subsection{5 replacement unclear}

Subtotal (95\% CI)

o

0

Not estimable

Heterogeneity: Not applicable

Test for overall effect: Not applicable

Test for subgroup differences: $\mathrm{Chi}^{2}=1.61, \mathrm{df}=2(\mathrm{P}=0.45), \mathrm{I}^{2}=0 \%$

$1751 \quad 100.0 \%$

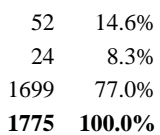

$0.19[-0.35,0.73]$

$-0.58[-1.33,0.17]$

$-0.10[-0.17,-0.03]$

$-0.10[-0.33,0.13]$

$-0.09[-0.21,0.04]$

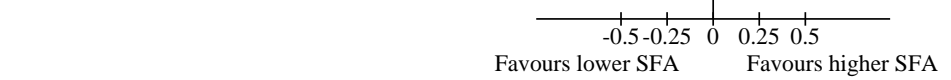




\section{Analysis 3.15. Comparison 3: SFA reduction vs usual diet - secondary blood outcomes, Outcome 15: TC /HDL ratio, subgroup by main replacement}

\begin{tabular}{|c|c|c|c|c|c|c|c|c|c|}
\hline & \multicolumn{3}{|c|}{ Lower SFA } & \multicolumn{3}{|c|}{ HIgher SFA } & \multirow{2}{*}{\multicolumn{2}{|c|}{$\begin{array}{cc} & \text { Mean Difference } \\
\text { Weight } & \text { IV, Random, 95\% CI }\end{array}$}} & \multirow{2}{*}{$\begin{array}{c}\text { Mean Difference } \\
\text { IV, Random, 95\% CI }\end{array}$} \\
\hline Study or Subgroup & Mean & SD & Total & Mean & SD & Total & & & \\
\hline
\end{tabular}

3.15.1 replaced by PUFA

Subtotal (95\% CI)

Heterogeneity: Not applicable

Test for overall effect: Not applicable

3.15.2 replace by MUFA

Subtotal (95\% CI)

0

Heterogeneity: Not applicable

Test for overall effect: Not applicable

3.15.3 replace by $\mathrm{CHO}$

Ley 2004

STARS 1992

WHI 2006

Subtotal (95\% CI)

$\begin{array}{rr}-0.34 & \\ 5.5 & 0.918 \\ -0.2 & 0.8\end{array}$

1
0.918

51
26
1133

$\begin{array}{rr}-0.53 & 1.73 \\ 6.08 & 1.666 \\ -0.1 & 1\end{array}$

1210

Heterogeneity: $\mathrm{Tau}^{2}=0.02 ; \mathrm{Chi}^{2}=2.65, \mathrm{df}=2(\mathrm{P}=0.27) ; \mathrm{I}^{2}=24 \%$

Test for overall effect: $\mathrm{Z}=0.83(\mathrm{P}=0.40)$

3.15.4 replace by protein

Subtotal (95\% CI)

0

Heterogeneity: Not applicable

Test for overall effect: Not applicable

3.15.5 replacement unclear

Subtotal (95\% CI)

o

Heterogeneity: Not applicable

Test for overall effect: Not applicable

Test for subgroup differences: Not applicable
Not estimable

Not estimable

IV, Random, 95\% C

$\begin{array}{rr}52 & 14.6 \% \\ 24 & 8.3 \% \\ 1699 & 77.0 \%\end{array}$

$1775100.0 \%$
$0.19[-0.35,0.73]$

$-0.58[-1.33,0.17]$

$-0.10[-0.17,-0.03]$

$-0.10[-0.33,0.13]$

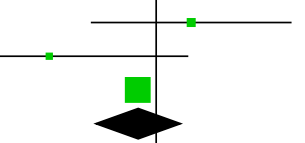

Analysis 3.16. Comparison 3: SFA reduction vs usual diet - secondary blood outcomes, Outcome 16: LDL /HDL ratio

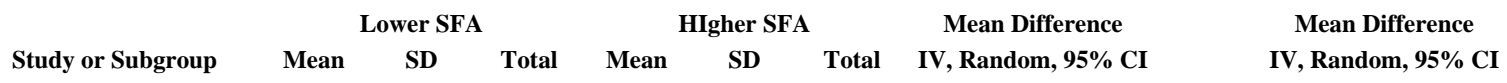

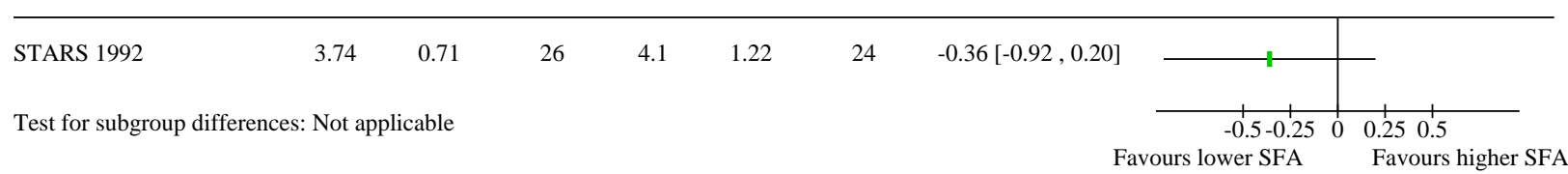

Analysis 3.17. Comparison 3: SFA reduction vs usual diet - secondary blood outcomes, Outcome 17: Lp(a), mmol/L

\begin{tabular}{|c|c|c|c|c|c|c|c|c|c|}
\hline \multirow[b]{2}{*}{ Study or Subgroup } & \multicolumn{3}{|c|}{ Lower SFA } & \multicolumn{3}{|c|}{ HIgher SFA } & \multirow[b]{2}{*}{ Weight } & \multirow{2}{*}{$\begin{array}{c}\text { Mean Difference } \\
\text { IV, Random, 95\% CI }\end{array}$} & \multirow{2}{*}{$\begin{array}{c}\text { Mean Difference } \\
\text { IV, Random, 95\% CI }\end{array}$} \\
\hline & Mean & SD & Total & Mean & SD & Total & & & \\
\hline STARS 1992 & 0.96 & 1.89 & 26 & 0.96 & 2.89 & 24 & $0.0 \%$ & $0.00[-1.37,1.37]$ & \\
\hline WHI 2006 & 0.03 & 0.02 & 1133 & 0.03 & 0.02 & 1699 & $100.0 \%$ & $0.00[-0.00,0.00]$ & \\
\hline Total $(95 \%$ CI $)$ & & & 1159 & & & 1723 & $100.0 \%$ & $0.00[-0.00,0.00]$ & \\
\hline \multicolumn{10}{|c|}{ Heterogeneity: $\mathrm{Tau}^{2}=0.00 ; \mathrm{Chi}^{2}=0.00, \mathrm{df}=1(\mathrm{P}=1.00) ; \mathrm{I}^{2}=0 \%$} \\
\hline \multicolumn{9}{|c|}{ Test for overall effect: $\mathrm{Z}=0.00(\mathrm{P}=1.00)$} & $\begin{array}{lll}+1 & + & + \\
-1 & -0.5 & 0\end{array}$ \\
\hline \multicolumn{9}{|c|}{ Test for subgroup differences: Not applicable } & wer SFA Favours \\
\hline
\end{tabular}




\section{Analysis 3.18. Comparison 3: SFA reduction vs usual diet - secondary blood outcomes, Outcome 18: Lp(a), mmol/L, subgroup by any replacement}

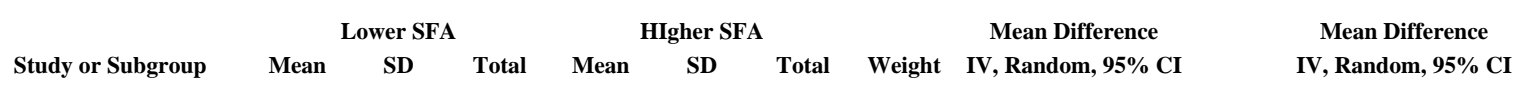

3.18.1 replaced by PUFA

$\begin{array}{lllllllll}\text { STARS } 1992 & 0.96 & 1.89 & 26 & 0.96 & 2.89 & 24 & 100.0 \% & 0.00[-1.37,1.37] \\ \text { Subtotal (95\% CI) } & & & \mathbf{2 6} & & & \mathbf{2 4} & \mathbf{1 0 0 . 0 \%} & \mathbf{0 . 0 0}[-\mathbf{1 . 3 7}, \mathbf{1 . 3 7}] \\ \text { Heterogeneity: Not applicable } & & & & & & & \end{array}$

Heterogeneity: Not applicable

Test for overall effect: $\mathrm{Z}=0.00(\mathrm{P}=1.00)$

\subsection{2 replace by MUFA}

Subtotal (95\% CI)

0

0

Not estimable

Test for overall effect: Not applicable

3.18.3 replace by CHO

STARS 1992

Heterogeneity: $\mathrm{Tau}^{2}=0.00 ; \mathrm{Chi}^{2}=0.00, \mathrm{df}=1(\mathrm{P}=1.00) ; \mathrm{I}^{2}=0 \%$

Test for overall effect: $\mathrm{Z}=0.00(\mathrm{P}=1.00)$

\subsection{4 replace by protein}

$\begin{array}{lllllllll}\text { WHI } 2006 & 0.03 & 0.02 & 1133 & 0.03 & 0.02 & 1699 & 100.0 \% & 0.00[-0.00,0.00] \\ \text { Subtotal (95\% CI) } & & & \mathbf{1 1 3 3} & & & \mathbf{1 6 9 9} & \mathbf{1 0 0 . 0 \%} & \mathbf{0 . 0 0}[-\mathbf{0 . 0 0 , 0 . 0 0}]\end{array}$

Heterogeneity: Not applicable

$1699 \quad 100.0 \%$

Test for overall effect: $\mathrm{Z}=0.00(\mathrm{P}=1.00)$

\subsection{5 replacement unclear}

Subtotal (95\% CI)

Heterogeneity: Not applicable

Test for overall effect: Not applicable

IV, Random,




\section{Analysis 3.19. Comparison 3: SFA reduction vs usual diet - secondary blood outcomes, Outcome 19: Lp(a), mmol/L, subgroup by main replacement}

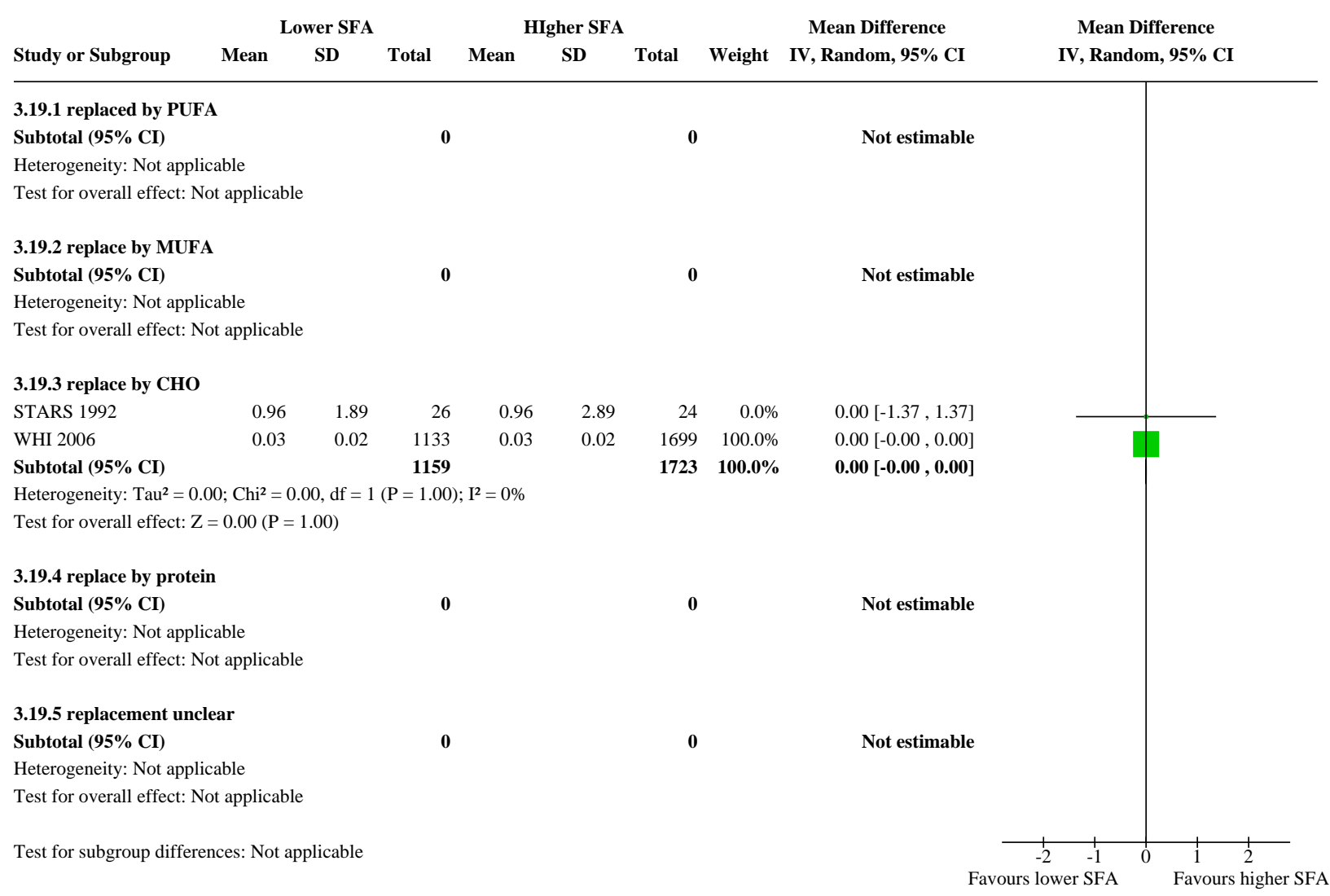

Analysis 3.20. Comparison 3: SFA reduction vs usual diet secondary blood outcomes, Outcome 20: Insulin sensitivity

\begin{tabular}{|c|c|c|c|c|c|c|c|c|c|}
\hline & \multicolumn{3}{|c|}{ Lower SFA } & \multicolumn{3}{|c|}{ HIgher SFA } & \multicolumn{2}{|r|}{ Mean Difference } & Mean Difference \\
\hline Study or Subgroup & Mean & SD & Total & Mean & SD & Total & Weight & IV, Random, $95 \%$ CI & IV, Random, $95 \%$ CI \\
\hline
\end{tabular}

3.20.1 HbA1c (glycosylated haemoglobin), \%

Subtotal (95\% CI) o

0

Not estimable

Heterogeneity: Not applicable

Test for overall effect: Not applicable

3.20.2 GTT (glucose tolerance test), glucose at 2 hours, $\mathrm{mmol} / \mathrm{L}$ $\begin{array}{lllll}\text { Houtsmuller } 1979 & 7.7 & 2.5 & 48 & 10.2\end{array}$

Ley 2004

$1.02 \quad 2.9$

$51 \quad 2.3$

STARS 1992

$4.6 \quad 1.7$

Subtotal $(95 \%$ CI $)$

125

Heterogeneity: $\mathrm{Tau}^{2}=0.26 ; \mathrm{Chi}^{2}=3.61, \mathrm{df}=2(\mathrm{P}=0.16) ; \mathrm{I}^{2}=45 \%$

Test for overall effect: $\mathrm{Z}=3.83(\mathrm{P}=0.0001)$

\subsubsection{HOMA}

\section{WHI 2006}

Subtotal (95\% CI)

Heterogeneity: Not applicable

$1699100.0 \%$

Test for overall effect: $\mathrm{Z}=0.00(\mathrm{P}=1.00)$ 
Comparison 4. SFA reduction vs usual diet - secondary outcomes including potential adverse effects

\begin{tabular}{|c|c|c|c|c|}
\hline Outcome or subgroup title & $\begin{array}{l}\text { No. of } \\
\text { studies }\end{array}$ & $\begin{array}{l}\text { No. of } \\
\text { partici- } \\
\text { pants }\end{array}$ & Statistical method & Effect size \\
\hline 4.1 Cancer diagnoses & 4 & 52294 & Risk Ratio (M-H, Random, 95\% Cl) & $0.94[0.83,1.07]$ \\
\hline 4.2 Cancer deaths & 5 & 52283 & Risk Ratio (M-H, Random, 95\% Cl) & $1.00[0.61,1.64]$ \\
\hline 4.3 Weight, kg & 6 & 43062 & Mean Difference (IV, Random, 95\% CI) & $-1.77[-3.54,-0.01]$ \\
\hline 4.4 BMI, kg/m2 & 6 & 43894 & Mean Difference (IV, Random, 95\% CI) & $-0.42[-0.72,-0.12]$ \\
\hline $\begin{array}{l}\text { 4.5 Systolic Blood Pressure, } \\
\mathrm{mmHg}\end{array}$ & 5 & 3812 & Mean Difference (IV, Random, 95\% CI) & $-0.19[-1.36,0.97]$ \\
\hline $\begin{array}{l}\text { 4.6 Diastolic Blood Pressure, } \\
\mathrm{mmHg}\end{array}$ & 5 & 3812 & Mean Difference (IV, Random, 95\% CI) & $-0.36[-1.03,0.32]$ \\
\hline 4.7 Quality of Life & 1 & 40130 & Mean Difference (IV, Random, 95\% CI) & $0.04[0.01,0.07]$ \\
\hline
\end{tabular}

Analysis 4.1. Comparison 4: SFA reduction vs usual diet - secondary outcomes including potential adverse effects, Outcome 1: Cancer diagnoses

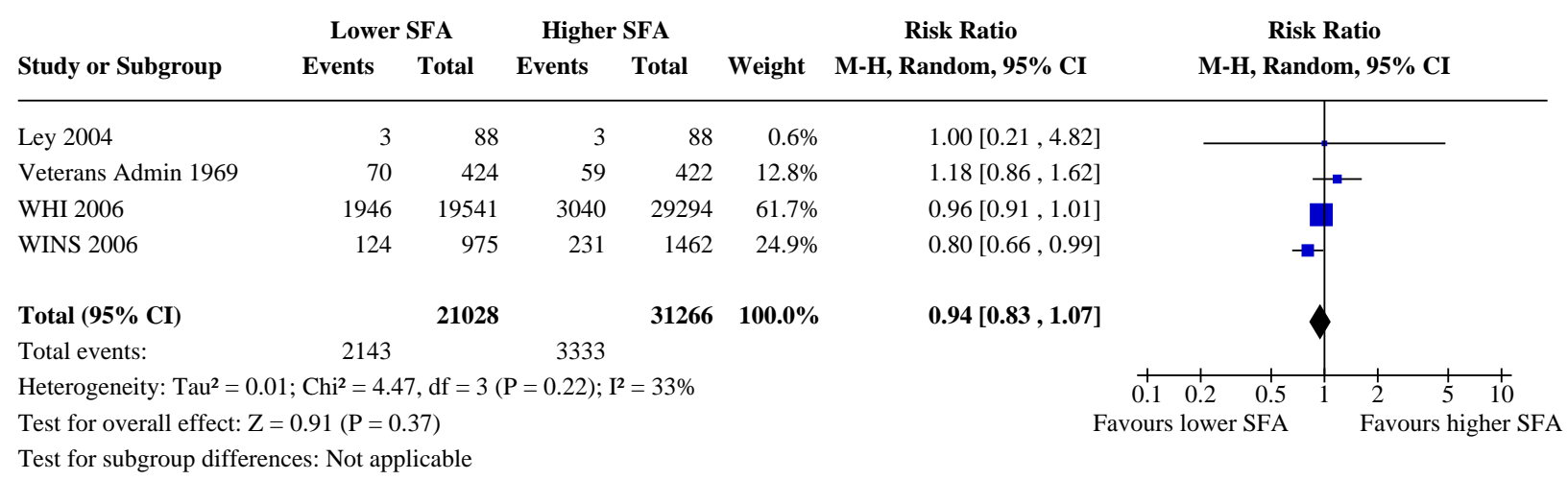


Analysis 4.2. Comparison 4: SFA reduction vs usual diet - secondary outcomes including potential adverse effects, Outcome 2: Cancer deaths

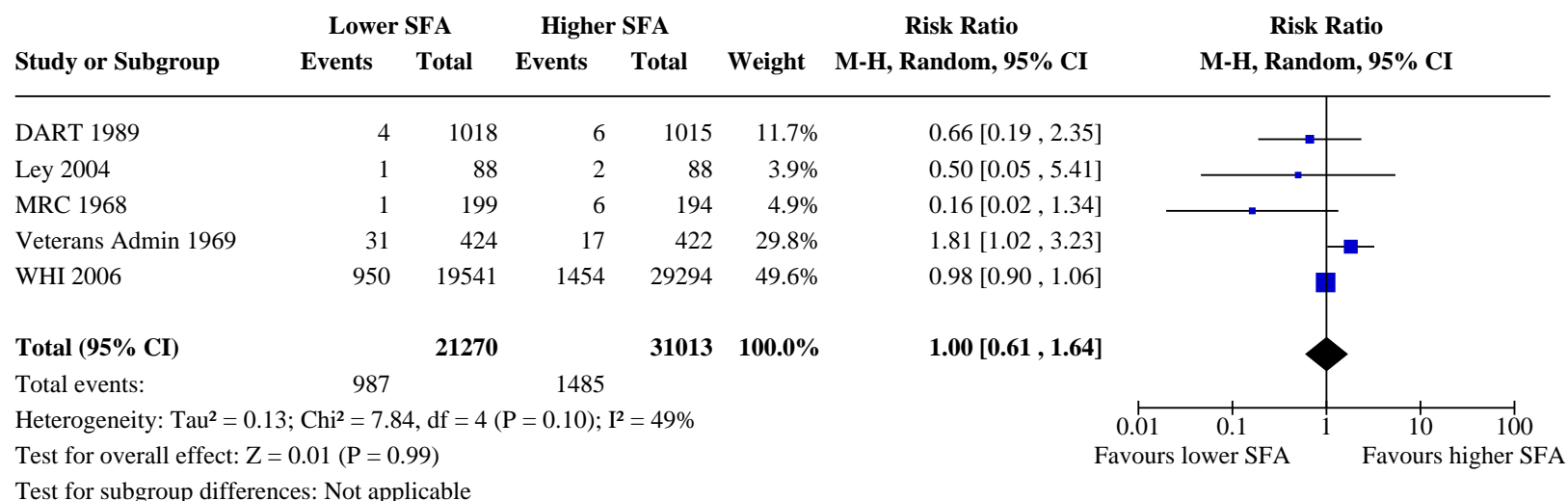

Analysis 4.3. Comparison 4: SFA reduction vs usual diet - secondary outcomes including potential adverse effects, Outcome 3: Weight, kg

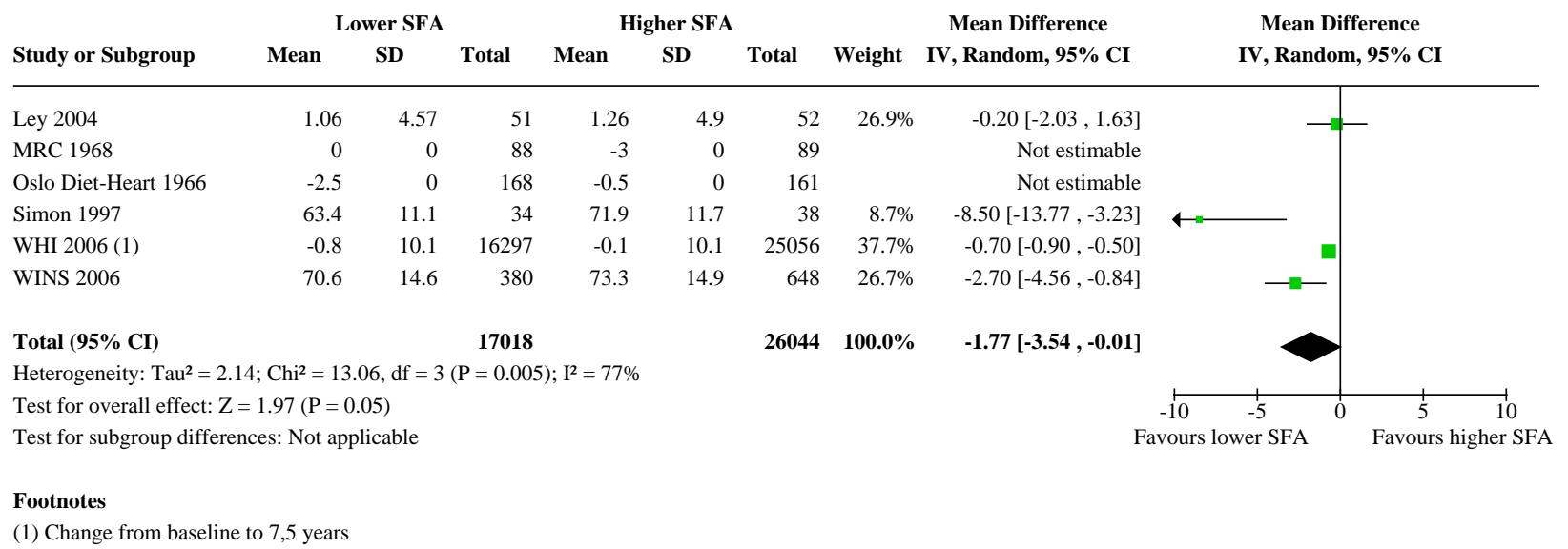

Analysis 4.4. Comparison 4: SFA reduction vs usual diet - secondary outcomes including potential adverse effects, Outcome 4: BMI, $\mathrm{kg} / \mathrm{m} 2$

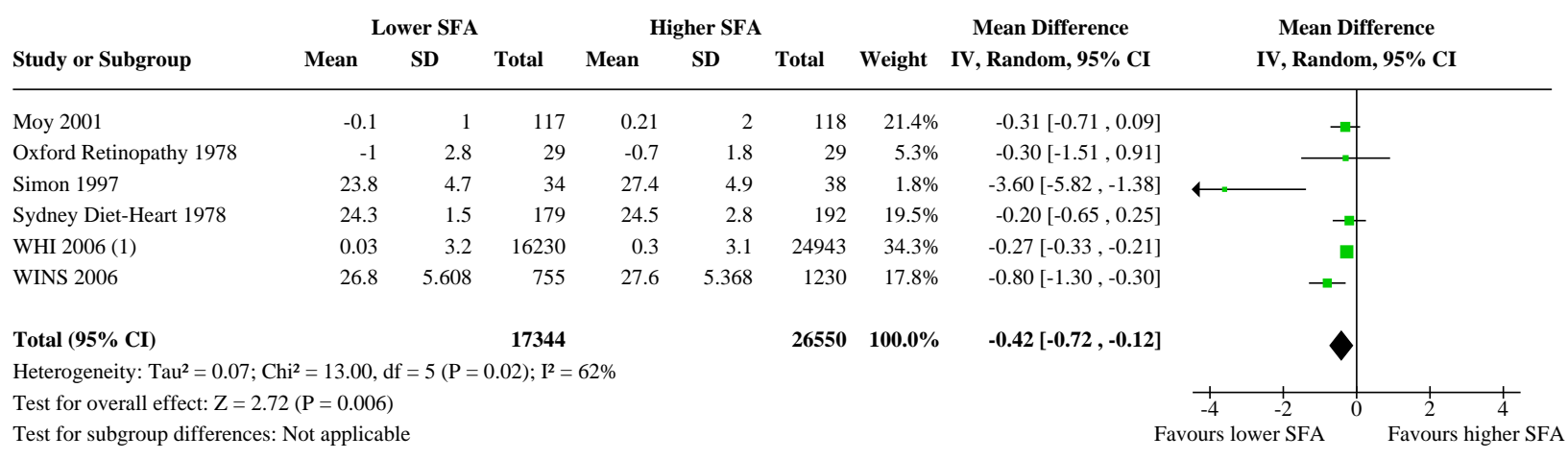

Footnotes

(1) Change to 7.5 years 
Analysis 4.5. Comparison 4: SFA reduction vs usual diet - secondary outcomes including potential adverse effects, Outcome 5: Systolic Blood Pressure, $\mathrm{mmHg}$

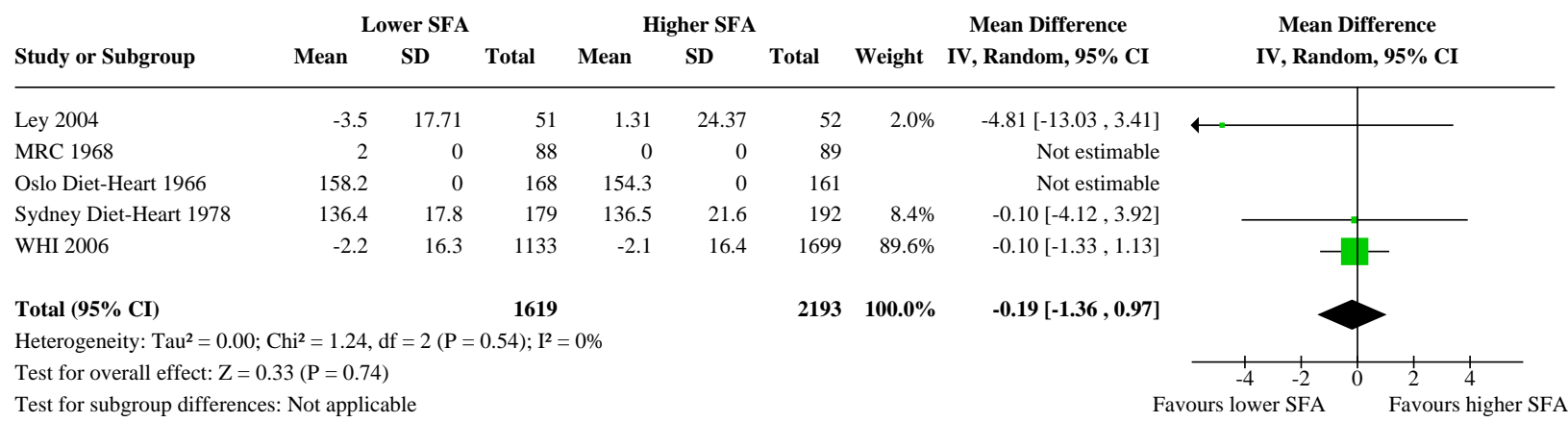

Analysis 4.6. Comparison 4: SFA reduction vs usual diet - secondary outcomes including potential adverse effects, Outcome 6: Diastolic Blood Pressure, $\mathrm{mmHg}$

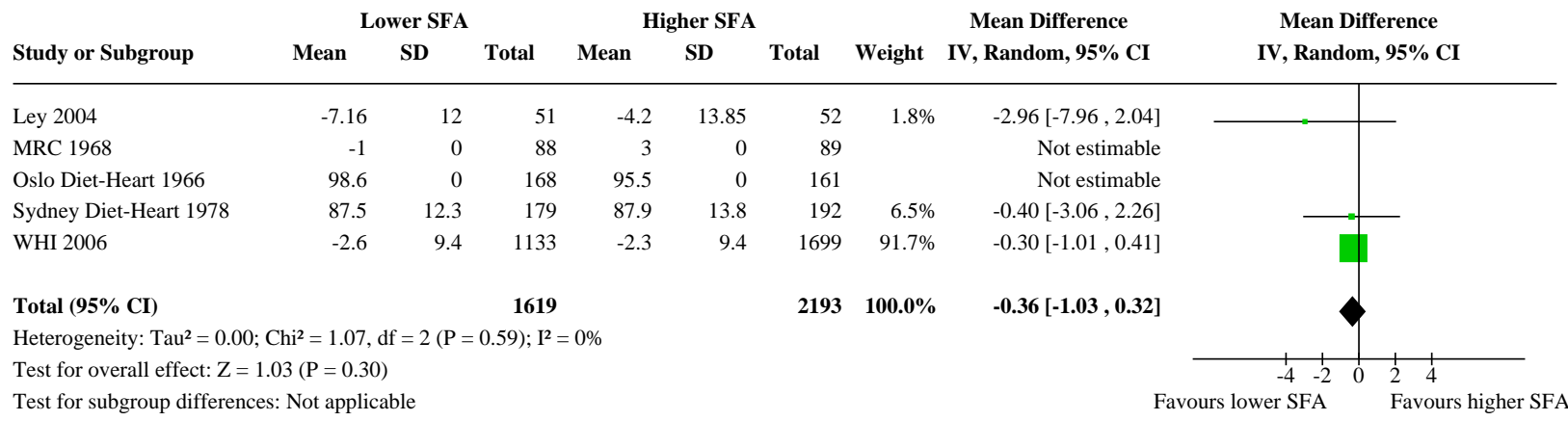

Analysis 4.7. Comparison 4: SFA reduction vs usual diet - secondary outcomes including potential adverse effects, Outcome 7: Quality of Life

\begin{tabular}{|c|c|c|c|c|c|c|c|c|c|c|}
\hline \multirow[b]{2}{*}{ Study or Subgroup } & \multicolumn{3}{|c|}{ Lower SFA } & \multicolumn{3}{|c|}{ HIgher SFA } & \multirow[b]{2}{*}{ Weight } & \multirow{2}{*}{$\begin{array}{c}\text { Mean Difference } \\
\text { IV, Random, 95\% CI }\end{array}$} & \multirow{2}{*}{\multicolumn{2}{|c|}{$\begin{array}{c}\text { Mean Difference } \\
\text { IV, Random, 95\% CI }\end{array}$}} \\
\hline & Mean & SD & Total & Mean & SD & Total & & & & \\
\hline WHI 2006 (1) & 0.07 & 1.41 & 15788 & 0.03 & 1.44 & 24342 & $100.0 \%$ & $0.04[0.01,0.07]$ & & - \\
\hline Total $(95 \% \mathrm{CI})$ & & & 15788 & & & 24342 & $100.0 \%$ & $0.04[0.01,0.07]$ & & \\
\hline \multicolumn{11}{|c|}{ Heterogeneity: Not applicable } \\
\hline \multicolumn{4}{|c|}{ Test for overall effect: $\mathrm{Z}=2.75(\mathrm{P}=0.006)$} & & & & & & $\begin{array}{ll}1 \\
-0.1\end{array}$ & $\frac{1}{0.2}$ \\
\hline \multicolumn{4}{|c|}{ Test for subgroup differences: Not applicable } & & & & & & Favours lower SFA & Favours higher SFA \\
\hline
\end{tabular}

ADDITIONAL TABLES

Reduction in saturated fat intake for cardiovascular disease (Review) 
Table 1. Comparison of study interventions for included RCTs

\begin{tabular}{|c|c|c|c|c|c|c|c|}
\hline Reference & Population & $\begin{array}{l}\text { CVD risk } \\
\text { category }\end{array}$ & $\begin{array}{l}\text { Is interven- } \\
\text { tion deliv- } \\
\text { ered to In- } \\
\text { dividual or } \\
\text { group? }\end{array}$ & $\begin{array}{l}\text { interven- } \\
\text { tion given } \\
\text { by? }\end{array}$ & $\begin{array}{l}\text { Face-to- } \\
\text { face or oth- } \\
\text { er? }\end{array}$ & Number of visits & $\begin{array}{l}\text { Is intervention advice } \\
\text { only or other interven- } \\
\text { tion? }\end{array}$ \\
\hline Black 1994 & $\begin{array}{l}\text { People with non-melanoma } \\
\text { skin cancer }\end{array}$ & Low & Unclear & Dietitian & Face-to-face & $\begin{array}{l}8 \times \text { weekly classes then monthly } \\
\text { follow-up sessions }\end{array}$ & $\begin{array}{l}\text { Advice (behaviour tech- } \\
\text { niques learning) }\end{array}$ \\
\hline $\begin{array}{l}\text { Houtsmuller } \\
1979\end{array}$ & $\begin{array}{l}\text { Adults with newly-diag- } \\
\text { nosed diabetes }\end{array}$ & Moderate & Unclear & Dietitian & Unclear & Unclear & Advice? \\
\hline Ley 2004 & $\begin{array}{l}\text { People with impaired glu- } \\
\text { cose intolerance or high } \\
\text { normal blood glucose }\end{array}$ & Moderate & Small group & Unclear & Face-to-face & Monthly meetings & $\begin{array}{l}\text { Advice (education, per- } \\
\text { sonal goal-setting, self- } \\
\text { monitoring) }\end{array}$ \\
\hline Moy 2001 & $\begin{array}{l}\text { Middle-aged siblings of peo- } \\
\text { ple with early CHD, with at } \\
\text { least } 1 \text { CVD risk factor }\end{array}$ & Moderate & Individual & $\begin{array}{l}\text { Trained } \\
\text { nurse }\end{array}$ & Face-to-face & 6 - 8 weekly for 2 years & $\begin{array}{l}\text { Advice (individualised } \\
\text { counselling sessions) }\end{array}$ \\
\hline MRC 1968 & $\begin{array}{l}\text { Free-living men who have } \\
\text { survived a 1st MI }\end{array}$ & High & Individual & Dietitian & Face-to-face & Unclear & $\begin{array}{l}\text { Advice and supplement } \\
\text { (soy oil) }\end{array}$ \\
\hline $\begin{array}{l}\text { Oslo Di- } \\
\text { et-Heart } \\
1966\end{array}$ & Men with previous MI & High & Individual & Dietitian & $\begin{array}{l}\text { Face-to-face } \\
\text { and other }\end{array}$ & Unclear & $\begin{array}{l}\text { Advice and supplement } \\
\text { (food) }\end{array}$ \\
\hline $\begin{array}{l}\text { Oxford } \\
\text { Retinopa- } \\
\text { thy } 1978\end{array}$ & $\begin{array}{l}\text { Newly-diagnosed non-in- } \\
\text { sulin-dependent diabetics }\end{array}$ & Moderate & Individual & $\begin{array}{l}\text { Diabetes di- } \\
\text { etitian }\end{array}$ & Face-to-face & $\begin{array}{l}\text { After } 1 \text { month then at 3-month } \\
\text { intervals }\end{array}$ & Advice \\
\hline $\begin{array}{l}\text { Rose corn } \\
\text { oil } 1965\end{array}$ & $\begin{array}{l}\text { Men (?) with angina or fol- } \\
\text { lowing MI }\end{array}$ & High & Unclear & Unclear & Unclear & $\begin{array}{l}\text { Follow-up clinic monthly, then } \\
\text { every } 2 \text { months }\end{array}$ & $\begin{array}{l}\text { Advice and supplement } \\
\text { (oil) }\end{array}$ \\
\hline Simon 1997 & $\begin{array}{l}\text { Women with a high risk of } \\
\text { breast cancer }\end{array}$ & Low & $\begin{array}{l}\text { Individual } \\
\text { followed by }\end{array}$ & Dietitian & Face-to-face & $\begin{array}{l}\text { Bi-weekly over } 3 \text { months fol- } \\
\text { lowed by monthly }\end{array}$ & $\begin{array}{l}\text { Advice (individualised eat } \\
\text { ing plan and counselling } \\
\text { sessions) }\end{array}$ \\
\hline
\end{tabular}


Table 1. Comparison of study interventions for included RCTs (Continued)

individual or

group

\begin{tabular}{|c|c|c|c|c|c|c|c|}
\hline STARS 1992 & $\begin{array}{l}\text { Men with angina referred for } \\
\text { angiography }\end{array}$ & High & Individual & Dietitian & Face-to-face & $\begin{array}{l}\text { Clinic visits at 3-month inter- } \\
\text { vals }\end{array}$ & Advice \\
\hline $\begin{array}{l}\text { Sydney Di- } \\
\text { et-Heart } \\
1978\end{array}$ & $\begin{array}{l}\text { Men with angina referred for } \\
\text { angiography }\end{array}$ & High & Individual & Unclear & Face-to-face & $\begin{array}{l}3 \text { times in 1st year and twice an- } \\
\text { nually thereafter }\end{array}$ & Advice \\
\hline $\begin{array}{l}\text { Veterans } \\
\text { Admin } 1969\end{array}$ & $\begin{array}{l}\text { Men living at the Veterans } \\
\text { Administration Center }\end{array}$ & Low & Individual & $\begin{array}{l}\text { Unclear } \\
\text { (whole diet } \\
\text { provided) }\end{array}$ & $\mathrm{N} / \mathrm{A}$ & $\mathrm{N} / \mathrm{A}$ & Diet provided \\
\hline WHI 2006 & $\begin{array}{l}\text { Postmenopausal women } \\
\text { aged } 50 \text { - } 79 \text { with or without } \\
\text { CVD at baseline }\end{array}$ & $\begin{array}{l}\text { Low and } \\
\text { High }\end{array}$ & Group & Nutritionists & Face-to-face & $\begin{array}{l}18 \text { sessions/1st yr and quarterly } \\
\text { maintenance sessions after }\end{array}$ & Advice \\
\hline WINS 2006 & $\begin{array}{l}\text { Women with localised re- } \\
\text { sected breast cancer }\end{array}$ & Low & $\begin{array}{l}\text { Individual } \\
\text { followed by } \\
\text { group }\end{array}$ & Dietitian & Face-to-face & $\begin{array}{l}8 \text { bi-weekly sessions, then } 3 \text { - } \\
\text { monthly contact and optional } \\
\text { monthly sessions }\end{array}$ & Advice \\
\hline
\end{tabular}

MI: myocardial infarction

N/A: not applicable

Table 2. Number of participants and number of outcomes for dichotomous variables (by intervention arm) $\begin{array}{lllllll}- & \text { Participants } & \text { All-cause CV mor- CVD } & \text { MI Non- Stroke CHD } & \text { CHD } & \text { Dia- }\end{array}$ All-cause CV mor- CVD mortality tality events

fatal
MI

mortal- events

ity

Diag-

noses

\begin{tabular}{|c|c|c|c|c|c|c|c|c|c|c|}
\hline Black 1994 & 133 & 133 & 133 & 133 & 0 & 0 & 0 & 0 & 0 & 0 \\
\hline DART 1989 & 2033 & 2033 & 2033 & 2033 & 2033 & 2033 & 0 & 2033 & 2033 & 0 \\
\hline Houtsmuller 1979 & 102 & 0 & 0 & 102 & 102 & 0 & 0 & 102 & 102 & 0 \\
\hline Ley 2004 & 176 & 176 & 176 & 176 & 176 & 0 & 176 & 0 & 176 & 0 \\
\hline Moy 2001 & 267 & 0 & 0 & 235 & 235 & 235 & 235 & 0 & 267 & 0 \\
\hline
\end{tabular}




\begin{tabular}{|c|c|c|c|c|c|c|c|c|c|c|}
\hline MRC 1968 & 393 & 393 & 393 & 393 & 393 & 393 & 393 & 393 & 393 & 0 \\
\hline Oslo Diet-Heart 1966 & 412 & 412 & 412 & 412 & 412 & 412 & 412 & 412 & 412 & 0 \\
\hline Oxford Retinopathy 1978 & $\begin{array}{l}249 \text { (data not provid- } \\
\text { ed by arm) }\end{array}$ & 0 & 0 & 0 & 0 & 0 & 0 & 0 & 0 & 0 \\
\hline Rose corn oil 1965 & 41 & 41 & 41 & 41 & 41 & 41 & 0 & 41 & 41 & 0 \\
\hline Rose olive 1965 & 39 & 39 & 39 & 39 & 39 & 39 & 0 & 39 & 39 & 0 \\
\hline Simon 1997 & $\begin{array}{l}194 \text { (data not provid- } \\
\text { ed by arm) }\end{array}$ & 0 & 0 & 0 & 0 & 0 & 0 & 0 & 0 & 0 \\
\hline STARS 1992 & 60 & 55 & 55 & 55 & 55 & 0 & 55 & 0 & 55 & 0 \\
\hline Sydney Diet-Heart 1978 & 458 & 458 & 458 & 0 & 0 & 0 & 0 & 458 & 0 & 0 \\
\hline Veterans Admin 1969 & 846 & 846 & 846 & 846 & 846 & 846 & 846 & 846 & 846 & 0 \\
\hline WHI with CVD 2006 & 2277 & 0 & 2277 & 2277 & 0 & 2277 & 2277 & 2277 & 2277 & 0 \\
\hline WHI 2006 & 48,835 & 48,835 & 46,558 & 46,558 & 48,835 & 46,558 & 46,558 & 46,558 & 46,558 & 48,835 \\
\hline WINS 2006 & 2437 & 2437 & 0 & 0 & 0 & 0 & 0 & 0 & 0 & 0 \\
\hline Total Participants & 58,509 & 55,858 & 53,421 & 53,300 & 53,167 & 52,834 & 50,952 & 53,159 & 53,204 & 48,835 \\
\hline Percent of participants for this outcome & $100 \%$ & $95 \%$ & $91 \%$ & $91 \%$ & $91 \%$ & $90 \%$ & $87 \%$ & $91 \%$ & $91 \%$ & $83 \%$ \\
\hline
\end{tabular}

These numbers are the numbers of participants in each study who were available for assessment of outcomes within meta-analysis (not necessarily the number of participants randomised within the trial).

CHD: coronary heart disease

CV: cardiovascular

CVD: cardiovascular disease

Table 3. Number of participants and number of participants with data for continuous outcomes (by intervention arm)

$\begin{array}{llllllllll}\text { Participants } & \begin{array}{l}\text { Total cho- } \\ \text { lesterol }\end{array} & \begin{array}{l}\text { LDL } \\ \text { cho- }\end{array} & \begin{array}{l}\text { HDL } \\ \text { cho- }\end{array} & \begin{array}{l}\text { Triglyc- } \\ \text { erides }\end{array} & \text { HDL } & \begin{array}{l}\text { Total } \\ \text { choles- }\end{array} & \begin{array}{l}\text { LDL/ } \\ \text { HDL } \\ \text { ratio }\end{array} & \begin{array}{l}\text { LP } \\ \text { (a) }\end{array} & \begin{array}{l}\text { Insulin } \\ \text { sensi- } \\ \text { tivity }\end{array}\end{array}$




\begin{tabular}{|c|c|c|c|c|c|c|c|c|c|c|}
\hline & & & $\begin{array}{l}\text { les- } \\
\text { terol }\end{array}$ & $\begin{array}{l}\text { les- } \\
\text { terol }\end{array}$ & & $\begin{array}{l}\text { ra- } \\
\text { tio }\end{array}$ & $\begin{array}{l}\text { terol/HDL } \\
\text { ratio }\end{array}$ & & & \\
\hline Black 1994 & 133 & 0 & 0 & 0 & 0 & 0 & 0 & 0 & 0 & 0 \\
\hline DART 1989 & 2033 & 1855 & 0 & 1855 & 0 & 0 & 0 & 0 & 0 & 0 \\
\hline Houtsmuller 1979 & 102 & 96 & 0 & 0 & 96 & 0 & 0 & 0 & 0 & 96 \\
\hline Ley 2004 & 176 & 103 & 103 & 103 & 103 & 0 & 103 & 0 & 0 & 103 \\
\hline Moy 2001 & 267 & 0 & 235 & 235 & 235 & 0 & 0 & 0 & 0 & 0 \\
\hline MRC 1968 & 393 & 177 & 0 & 0 & 0 & 0 & 0 & 0 & 0 & 0 \\
\hline Oslo Diet-Heart 1966 & 412 & 329 & 0 & 0 & 0 & 0 & 0 & 0 & 0 & 0 \\
\hline Oxford Retinopathy 1978 & 249 & 58 & 0 & 0 & 0 & 0 & 0 & 0 & 0 & 0 \\
\hline Rose corn oil 1965 & 41 & 22 & 0 & 0 & 0 & 0 & 0 & 0 & 0 & 0 \\
\hline Rose olive 1965 & 39 & 24 & 0 & 0 & 0 & 0 & 0 & 0 & 0 & 0 \\
\hline Simon 1997 & 194 & 72 & 71 & 72 & 71 & 0 & 0 & 0 & 0 & 0 \\
\hline STARS 1992 & 55 & 50 & 50 & 50 & 50 & 0 & 50 & 50 & 50 & 50 \\
\hline Sydney Diet-Heart 1978 & 458 & 458 & 0 & 0 & 458 & 0 & 0 & 0 & 0 & 0 \\
\hline Veterans Admin 1969 & 846 & 843 & 0 & 0 & 0 & 0 & 0 & 0 & 0 & 0 \\
\hline WHI with CVD 2006 & 2277 & 0 & 0 & 0 & 0 & 0 & 0 & 0 & 0 & 0 \\
\hline WHI 2006 & 48,835 & 2832 & 2832 & 2832 & 2832 & 0 & 2832 & 0 & 2832 & 2832 \\
\hline WINS 2006 & 2437 & 196 & 0 & 0 & 0 & 0 & 0 & 0 & 0 & 0 \\
\hline Total Participants & 58952 & 7115 & 3291 & 5147 & 3845 & 0 & 2985 & 50 & 2882 & 3081 \\
\hline Percent of participants for this outcome & $100 \%$ & $12 \%$ & $6 \%$ & $9 \%$ & $7 \%$ & $0 \%$ & $5 \%$ & $0.1 \%$ & $5 \%$ & $5 \%$ \\
\hline
\end{tabular}


These numbers are the numbers of participants in each study who were available for assessment of outcomes within meta-analysis (not necessarily the number of participants randomised within the trial).

HDL: high density lipoprotein

$p$ (a): lipoprotein (a)

TG: triglyceride 
Table 4. Meta-regression of effects of SFA reduction on cardiovascular events

\begin{tabular}{|c|c|c|c|c|c|}
\hline Regression factor & $\begin{array}{l}\text { No. of } \\
\text { studies }\end{array}$ & Constant & Coefficient $(95 \% \mathrm{CI})$ & $P$ value & $\begin{array}{l}\text { Proportion } \\
\text { of between } \\
\text { study vari- } \\
\text { ation ex- } \\
\text { plained }\end{array}$ \\
\hline Change in SFA as \%E & 8 & 0.01 & $0.05(-0.03$ to 0.13$)$ & 0.16 & $89 \%$ \\
\hline Change in SFA as \% of control & 8 & 0.26 & $0.01(-0.01$ to 0.03$)$ & 0.14 & $89 \%$ \\
\hline Baseline SFA as \%E & 8 & 0.68 & $-0.06(-0.15$ to 0.04$)$ & 0.19 & $81 \%$ \\
\hline Change in TC, $\mathrm{mmol} / \mathrm{L}$ & 12 & 0.03 & 0.69 (0.05 to 1.33$)$ & 0.04 & $99 \%$ \\
\hline Change in PUFA as $\% E$ & 5 & -0.01 & $-0.02(-0.08$ to 0.03$)$ & 0.25 & $100 \%$ \\
\hline Change in MUFA as \%E & 5 & -0.26 & $-0.03(-0.14$ to 0.09$)$ & 0.50 & $-87 \%$ \\
\hline Change in $\mathrm{CHO}$ as $\% \mathrm{E}$ & 7 & -0.11 & $-0.00(-0.05$ to 0.05$)$ & 0.92 & $-273 \%$ \\
\hline Change in total fat intake as $\% E$ & 9 & -0.17 & $-0.01(-0.03$ to 0.01$)$ & 0.28 & $100 \%$ \\
\hline Gender* & 13 & -0.17 & $-0.14(-0.63$ to 0.35$)$ & 0.55 & $-13 \%$ \\
\hline Study duration & 13 & -0.47 & $0.00(-0.01$ to 0.02$)$ & 0.76 & $-24.8 \%$ \\
\hline CVD risk at baseline ${ }^{\star \star}$ & 13 & -0.44 & $0.03(-0.48$ to 0.55$)$ & 0.89 & $-39 \%$ \\
\hline
\end{tabular}

*Gender was coded as follows: 0 = women, $1=$ mixed, 2 = men

${ }^{\star \star} \mathrm{CVD}$ risk at baseline was coded as follows: $1=$ Low CVD risk, $2=$ Moderate CVD risk, $3=$ existing CVD

CHO: carbohydrate

$\mathrm{Cl}$ : confidence interval

CVD: cardiovascular disease

E: energy

MUFA: monounsaturated fatty acid

PUFA: polyunsaturated fatty fat

SFA: saturated fatty acid

TC: total cholesterol 


\begin{tabular}{|c|c|c|c|c|c|c|c|c|}
\hline Cut- off & $\begin{array}{l}\text { RR of all- } \\
\text { cause mor- } \\
\text { tality }\end{array}$ & RR of CVD mortality & RR of CVD events & RR of MI & $\begin{array}{l}\text { RR of non-fatal } \\
\text { MI }\end{array}$ & RR of stroke & $\begin{array}{l}\text { RR of CHD } \\
\text { mortality }\end{array}$ & $\begin{array}{l}\text { RR of CHD } \\
\text { events }\end{array}$ \\
\hline $7 \% \mathrm{E}$ & $\begin{array}{l}0.89 \text { ( } 0.66 \text { to } \\
1.20)\end{array}$ & 0.20 (0.01 to 4.15$)$ & 0.20 (0.01 to 4.15$)$ & $\mathrm{N} / \mathrm{A}$ & $\mathrm{N} / \mathrm{A}$ & $\mathrm{N} / \mathrm{A}$ & N/A & $\mathrm{N} / \mathrm{A}$ \\
\hline $8 \% E$ & $\begin{array}{l}0.89 \text { ( } 0.66 \text { to } \\
1.20)\end{array}$ & 0.20 (0.01 to 4.15$)$ & $0.20(0.01$ to 4.15$)$ & $\mathrm{N} / \mathrm{A}$ & $\mathrm{N} / \mathrm{A}$ & $\mathrm{N} / \mathrm{A}$ & $\mathrm{N} / \mathrm{A}$ & $\mathrm{N} / \mathrm{A}$ \\
\hline $9 \% \mathrm{E}$ & $\begin{array}{l}0.96 \text { ( } 0.83 \text { to } \\
1.10)\end{array}$ & 0.69 (0.51 to 0.94$)$ & 0.79 (0.62 to 0.99$)$ & $\begin{array}{l}0.76 \text { ( } 0.55 \text { to } \\
1.05)\end{array}$ & $\begin{array}{l}0.62 \text { ( } 0.31 \text { to } \\
1.21)\end{array}$ & $\begin{array}{l}0.59 \text { ( } 0.30 \text { to } \\
1.15)\end{array}$ & $\begin{array}{l}0.82 \text { ( } 0.55 \text { to } \\
1.21)\end{array}$ & $\begin{array}{l}0.77 \text { (0.56 to } \\
1.04)\end{array}$ \\
\hline $10 \% \mathrm{E}$ & $\begin{array}{l}0.99 \text { ( } 0.90 \text { to } \\
1.09)\end{array}$ & 0.95 (0.67 to 1.35$)$ & 0.78 (0.57 to 1.05$)$ & $\begin{array}{l}0.93 \text { ( } 0.80 \text { to } \\
1.08)\end{array}$ & $\begin{array}{l}0.89 \text { (0.58 to } \\
1.35)\end{array}$ & $\begin{array}{l}0.87(0.58 \text { to } \\
1.33)\end{array}$ & $\begin{array}{l}1.05(0.77 \text { to } \\
1.43)\end{array}$ & $\begin{array}{l}0.82 \text { (0.60 to } \\
1.13)\end{array}$ \\
\hline $11 \% \mathrm{E}$ & $\begin{array}{l}0.99 \text { (0.88 to } \\
1.12)\end{array}$ & 0.92 (0.65 to 1.31 ) & 0.77 (0.59 to 1.02$)$ & $\begin{array}{l}0.94 \text { (0.84 to } \\
1.06)\end{array}$ & $\begin{array}{l}0.89 \text { ( } 0.58 \text { to } \\
1.35)\end{array}$ & $\begin{array}{l}0.76 \text { ( } 0.45 \text { to } \\
1.30)\end{array}$ & $\begin{array}{l}1.02 \text { ( } 0.84 \text { to } \\
1.24)\end{array}$ & $\begin{array}{l}0.85 \text { (0.63 to } \\
1.15)\end{array}$ \\
\hline $12 \% \mathrm{E}$ & $\begin{array}{l}0.98 \text { (0.91 to } \\
1.07)\end{array}$ & 0.95 (0.75 to 1.21$)$ & 0.85 (0.71 to 1.02$)$ & $\begin{array}{l}0.94 \text { (0.85 to } \\
1.04)\end{array}$ & $\begin{array}{l}0.90(0.72 \text { to } \\
1.14)\end{array}$ & $\begin{array}{l}0.93 \text { ( } 0.55 \text { to } \\
1.25)\end{array}$ & $\begin{array}{l}1.02 \text { ( } 0.84 \text { to } \\
1.24)\end{array}$ & $\begin{array}{l}0.90 \text { (0.77 to } \\
1.06)\end{array}$ \\
\hline $13 \% \mathrm{E}$ & $\begin{array}{l}1.02 \text { ( } 0.83 \text { to } \\
1.25)\end{array}$ & 0.93 (0.63 to 1.38 ) & $0.78(0.61$ to 1.00$)$ & $\begin{array}{l}0.87 \text { ( } 0.73 \text { to } \\
1.04)\end{array}$ & $\begin{array}{l}0.72 \text { ( } 0.50 \text { to } \\
1.03)\end{array}$ & $\begin{array}{l}0.54 \text { ( } 0.29 \text { to } \\
1.00)\end{array}$ & $\begin{array}{l}1.06 \text { ( } 0.76 \text { to } \\
1.48)\end{array}$ & $\begin{array}{l}0.84 \text { (0.63 to } \\
1.12)\end{array}$ \\
\hline $\begin{array}{l}\text { CHD: coro } \\
\text { CVD: cardi } \\
\text { E: energy } \\
\text { MI: myoca } \\
\text { N/A: not a } \\
\text { RR: risk ra } \\
\text { SFA: satur }\end{array}$ & $\begin{array}{l}\text { heart disease } \\
\text { cular disease } \\
\text { infarction } \\
\text { fable (no relev } \\
\text { fat, as percent }\end{array}$ & studies) & & & & & & \\
\hline
\end{tabular}




\section{APPENDICES}

\section{Appendix 1. Search strategies 2019}

\section{CENTRAL}

\#1 lipid near (low* or reduc ${ }^{\star}$ or modifi ${ }^{\star}$ )

\#2 cholesterol ${ }^{\star}$ near (low* or modifi ${ }^{\star}$ or reduc ${ }^{\star}$ )

\#3 (\#1 or \#2)

\#4 MeSH descriptor: [Nutrition Therapy] explode all trees

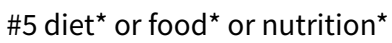

\#6 (\#4 or \#5)

\#7 (\#3 and \#6)

\#8 fat ${ }^{\star}$ near (low ${ }^{\star}$ or reduc ${ }^{\star}$ or modifi ${ }^{\star}$ or animal ${ }^{\star}$ or saturat ${ }^{\star}$ or unsaturat ${ }^{\star}$ )

\#9 MeSH descriptor: [Diet, Atherogenic] explode all trees

\#10 MeSH descriptor: [Diet Therapy] explode all trees

$\# 11$ (\#7 or \#8 or \#9 or \#10)

\#12 MeSH descriptor: [Cardiovascular Diseases] this term only

\#13 MeSH descriptor: [Heart Diseases] explode all trees

\#14 MeSH descriptor: [Vascular Diseases] explode all trees

\#15 MeSH descriptor: [Cerebrovascular Disorders] this term only

\#16 MeSH descriptor: [Brain Ischemia] explode all trees

\#17 MeSH descriptor: [Carotid Artery Diseases] explode all trees

\#18 MeSH descriptor: [Dementia, Vascular] explode all trees

\#19 MeSH descriptor: [Intracranial Arterial Diseases] explode all trees

\#20 MeSH descriptor: [Intracranial Embolism and Thrombosis] explode all trees

\#21 MeSH descriptor: [Intracranial Hemorrhages] explode all trees

\#22 MeSH descriptor: [Stroke] explode all trees

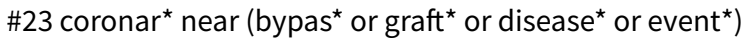

\#24 cerebrovasc* or cardiovasc* or mortal* or angina* or stroke or strokes or tia or ischaem* or ischem*

\#25 myocardi* near (infarct* or revascular* or ischaem* or ischem)

\#26 morbid $^{\star}$ near (heart* or coronar* or ischaem* or ischem or myocard ${ }^{\star}$ )

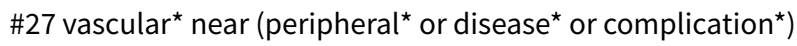

\#28 heart* near (disease* or attack* or bypas*)

$\# 29$ (\#12 or \#13 or \#14 or \#15 or \#16 or \#17 or \#18 or \#19 or \#20 or \#21 or \#22 or \#23 or \#24 or \#25 or \#26 or \#27 or \#28)

\#30 (\#11 and \#29) Date added to CENTRAL trials database: 05/03/2014-15/10/2019

\section{MEDLINE OVID}


1. (lipid\$ adj5 (low\$ or reduc\$ or modifi\$)).mp.

2. (cholesterol\$ adj5 (low\$ or modific\$ or reduc\$)).mp.

\section{1 or 2}

4. exp Nutrition Therapy/

5. (diet\$ or food\$ or nutrition\$).mp.

6.4 or 5

7.3 and 6

8. (fat adj5 (low\$ or reduc\$ or modifi\$ or animal\$ or saturat\$ or unsatur\$)).mp.

9. exp Diet, Atherogenic/

10. exp Diet Therapy/

11.7 or 8 or 9 or 10

12. cardiovascular diseases/ or exp heart diseases/ or exp vascular diseases/

13. cerebrovascular disorders/ or exp brain ischemia/ or exp carotid artery diseases/or exp dementia, vascular/ or exp intracranial arterial diseases/or exp "intracranial embolism and thrombosis"/ or exp intracranial hemorrhages/ or exp stroke/

14. (coronar\$ adj5 (bypas\$ or graft\$ or disease\$ or event\$)).mp

15. (cerebrovasc\$ or cardiovasc\$ or mortal\$ or angina\$ or stroke or strokes).mp.

16. (myocardi\$ adj5 (infarct\$ or revascular\$ or ischaemi\$ or ischemi\$)).mp.

17. (morbid\$ adj5 (heart\$ or coronar\$ or ischaem\$ or ischem\$ or myocard\$)).mp.

18. (vascular\$ adj5 (peripheral\$ or disease\$ or complication\$)).mp.

19. (heart\$ adj5 (disease\$ or attack\$ or bypass\$)).mp.

20.12 or 13 or 14 or 15 or 16 or 17 or 18 or 19

21. 11 and 20

22. randomized controlled trial.pt.

23. controlled clinical trial.pt.

24. randomized.ab.

25. placebo.ab.

26. drug therapy.fs.

27. randomly.ab.

28. trial.ab.

29. groups.ab.

30.22 or 23 or 24 or 25 or 26 or 27 or 28 or 29

31. exp animals/ not humans.sh.

32. 30 not 31

33. 21 and 32

34. limit 33 to ed=20140305-20191015

Reduction in saturated fat intake for cardiovascular disease (Review) 


\section{Embase OVID}

1. cardiovascular diseases/ or exp heart diseases/ or exp vascular diseases/

2. cerebrovascular disorders/ or exp brain ischemia/ or exp carotid artery diseases/ or exp dementia, vascular/ or exp intracranial arterial diseases/ or exp "intracranial embolism and thrombosis"/ or exp intracranial hemorrhages/ or exp stroke/

3. (coronar\$ adj5 (bypas\$ or graft\$ or disease\$ or event\$)).mp.

4. (cerebrovasc $\$$ or cardiovasc $\$$ or mortal\$ or angina\$ or stroke or strokes).mp.

5. (myocardi\$ adj5 (infarct\$ or revascular\$ or ischaemi\$ or ischemi\$)).mp.

6. (morbid\$ adj5 (heart\$ or coronar\$ or ischaem\$ or ischem $\$$ or myocard\$)).mp.

7. (vascular\$ adj5 (peripheral\$ or disease\$ or complication\$)).mp.

8. (heart\$ adj5 (disease\$ or attack\$ or bypass\$)).mp.

9. or/1-8

10. (lipid\$ adj5 (low\$ or reduc\$ or modifi\$)).mp.

11. (cholesterol\$ adj5 (low\$ or modific\$ or reduc\$)).mp.

12. 10 or 11

13. (diet\$ or food\$ or eat\$ or nutrition\$).mp.

14. exp nutrition/

15. 13 or 14

16. 12 and 15

17. (fat adj5 (low\$ or reduc\$ or modifi\$ or animal\$ or saturat\$ or unsatur\$)).mp.

18. exp lipid diet/ or exp fat intake/ or exp low fat diet/

19. 16 or 17 or 18

20.9 and 19

21. random\$.tw.

22. factorial\$.tw.

23. crossover\$.tw.

24. cross over\$.tw.

25. cross-over\$.tw.

26. placebo\$.tw.

27. (doubl\$ adj blind\$).tw.

28. (singl\$ adj blind\$).tw.

29. assign\$.tw.

30. allocat\$.tw.

31. volunteer\$.tw.

32. crossover procedure/

33. double blind procedure/ 
34. randomized controlled trial/

35. single blind procedure/

36.21 or 22 or 23 or 24 or 25 or 26 or 27 or 28 or 29 or 30 or 31 or 32 or 33 or 34 or 35

37. (animal/ or nonhuman/) not human/

38. 36 not 37

39. 20 and 38

40. limit 39 to $d d=20140305-20191015$

\section{Clinicaltrials.gov}

Condition or disease: Cardiovascular Diseases OR CVD OR "heart disease" Intervention/treatment: Dietary Fats OR saturated OR unsaturated OR fat Study type: Interventional Studies (Clinical Trials)

\section{ICTRP}

Condition: Cardiovascular Diseases OR CVD OR heart disease Intervention: Dietary Fats OR saturated OR unsaturated OR fat

WHAT'S NEW

\begin{tabular}{lll}
\hline Date & Event & Description \\
\hline 9 January 2020 & $\begin{array}{l}\text { New citation required but conclusions } \\
\text { have not changed }\end{array}$ & $\begin{array}{l}\text { No new trials included, but four ongoing trials and one study } \\
\text { awaiting assessment, and we found new data for two of the al- } \\
\text { ready included trials (WHI 2006; WINS 2006). We updated assess- } \\
\text { ment of risk of bias, including assessment of summary risk of }\end{array}$ \\
& $\begin{array}{l}\text { bias for each trial, and carrying out sensitivity analyses omitting } \\
\text { trials not at low summary risk of bias. We updated assessment } \\
\text { of small study bias by comparing results of fixed- and random-ef- } \\
\text { fects meta-analyses. Data, results, GRADE assessment and con- } \\
\text { clusions updated. }\end{array}$ \\
\hline
\end{tabular}

\section{H I S T O R Y}

Protocol first published: Issue 2, 1999

Review first published: Issue 6, 2015

\begin{tabular}{lll}
\hline Date & Event & Description \\
\hline 27 March 2015 & $\begin{array}{l}\text { New citation required and conclusions } \\
\text { have changed }\end{array}$ & $\begin{array}{l}\text { We split a previously published review (Reduced or modi- } \\
\text { fied dietary fat for preventing cardiovascular disease, DOI: }\end{array}$ \\
& $\begin{array}{l}10.1002 / 14651858 . C D 002137 . p u b 3) \text { into six smaller review up- } \\
\text { dates. The conclusions are therefore now focused on reduction } \\
\text { in saturated fat intake instead of reducing or modifying fat intake } \\
\end{array}$ & overall on its effect on cardiovascular disease risk.
\end{tabular}




\begin{tabular}{lll}
\hline Date Event Description & Den
\end{tabular}

This split review update includes 15 randomised controlled trials.

\section{CONTRIBUTIONS OF AUTHORS}

All authors were active in the design of the review and in providing critical revisions of the manuscript, all authors took part in assessment of the results of the updated search, and assessment of inclusion of potentially relevant studies. All authors edited, proof-read and agreed the final version of the review.

LH was the principal author of earlier versions (Hooper 2000; Hooper 2001; Hooper 2012; Hooper 2015a), originated and was primarily responsible for planning and carrying out this systematic review, liaising with WHO NUGAG, carrying out the statistical analyses, and writing the first draft of this review.

AA, OFJ, CK, EF and LH were responsible for data extraction and assessment of validity.

\section{DECLARATIONS OF INTEREST}

Lee Hooper: LH is a member of the World Health Organization Nutrition Guidance Expert Advisory Group (NUGAG). WHO paid for her travel, accommodation and expenses to attend NUGAG meetings in Geneva, China and South Korea where the evidence of effects of dietary fats on health was discussed and guidance developed. LH's institution was given grant funding from WHO to carry out the 2019 update of this systematic review, to update a systematic review on the relationship between total fat intake and body weight and a series of systematic reviews on the health effects of polyunsaturated fatty acids.

Nicole Martin: None known

Asmaa Abdelhamid: None known

Oluseyi Florence Jimoh: This review was funded by a grant from the World Health Organization.

Eve Foster: None known

Christian Kirk: None known

\section{SOURCES OF SUPPORT}

\section{Internal sources}

- University of East Anglia, UK

Help with acquiring papers for previous versions of this review, and allowing time for Lee Hooper to work on the review

- University of Manchester, UK

Support with collection of papers for the first version of this review

\section{External sources}

- Studentship, Systematic Reviews Training Unit, Institute of Child Health, University of London, UK Funding to support Lee Hooper to carry out the first version of the systematic review

- World Health Organization, Other

WHO funded the most recent update of this review

\section{DIFFERENCES BETWEEN PROTOCOL AND REVIEW}

This review is the result of updating the searches for Hooper 2015a. The objective and outcomes have been widened since the protocol to address queries by WHO NUGAG and the inclusion criteria have changed to focus on saturated fat and long-term trials ( 24 months instead of six months). 


\section{N D EX TERMS}

\section{Medical Subject Headings (MeSH)}

Cardiovascular Diseases [mortality] [ ${ }^{*}$ prevention \& control]; Cause of Death; Dietary Carbohydrates [administration \& dosage];

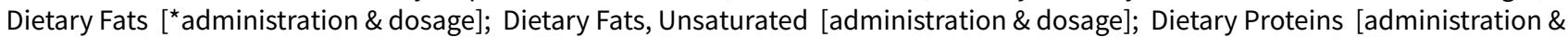
dosage]; Energy Intake; Fatty Acids [ ${ }^{\star}$ administration \& dosage]; Myocardial Infarction [mortality] [prevention \& control]; Randomized Controlled Trials as Topic; Stroke [prevention \& control]

\section{MeSH check words}

Adult; Humans 